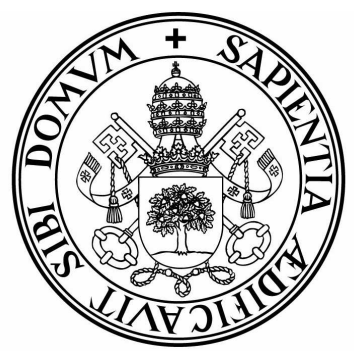

Universidad de Valladolid

Departamento de Física Teórica, Atómica y Óptica

\title{
Climatología de los aerosoles mediante la caracterización de propiedades ópticas y masas de aire en la estación 'El Arenosillo' de la red AERONET.
}

Memoria presentada por Carlos Toledano Olmeda

Para optar al grado de Doctor en Ciencias Valladolid, octubre de 2005 



\section{Agradecimientos}

Quiero dar las gracias en primer lugar a mis padres y mis hermanos, a los que debo casi todo, también mi vocación por la Física. Su apoyo ha sido constante. También quiero dar las gracias a Ana, que me ayudó a reconocer la oportunidad en forma de beca que me ha llevado hasta aquí. Su apoyo (y comprensión) siempre han estado conmigo. Mis amigos Álvaro, Pedro, M. José y por fortuna muchos otros tienen también su parte de culpa en todo esto.

Agradezco de forma muy especial el trabajo de mis directores Victoria Cachorro y Ricardo Vergaz, que ha sido esencial para la realización de esta tesis doctoral, y por supuesto a Ángel de Frutos. A ellos debo mi formación en este camino de cuatro años, con una dedicación ejemplar en lo científico y en lo humano. A ellos debo también que el Grupo de Óptica Atmosférica haya sido mi casa. A mis compañeros Alberto Berjón, Mar Sorribas, Sandra Mogo y Natalia Prats debo agradecerles las horas (incontables) de trabajo compartido y su amistad. Doy las gracias a todos los compañeros y amigos en el departamento de Óptica, en especial a Santiago González y Fernando Muñoz Box.

Quiero agradecer su trabajo a toda la gente de la estación de El Arenosillo, en especial a Benito de la Morena y José Manuel Vilaplana. También agradezco su constante apoyo al personal del Laboratorio de Óptica Atmosférica de la Universidad de Lille, encabezado por Philippe Goloub.

Y a ti lector te agradezco por anticipado tu paciencia... 

Este trabajo ha sido realizado en el

Grupo de Óptica Atmosférica de la Universidad de Valladolid.

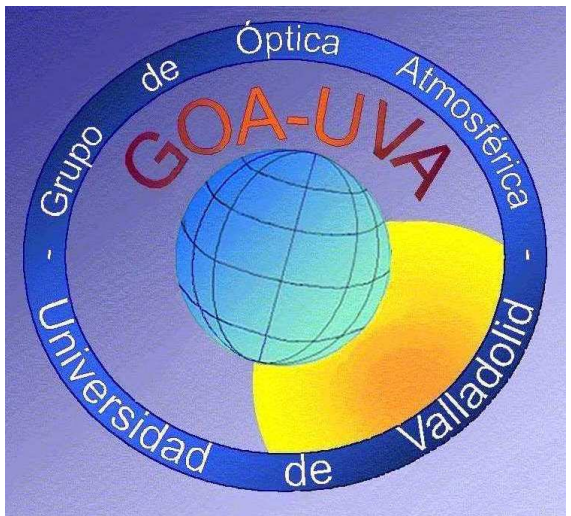





\section{Índice}

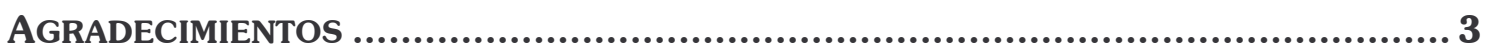

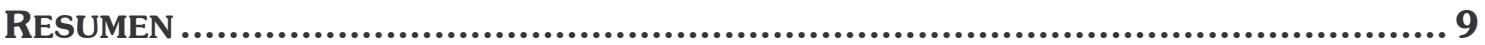

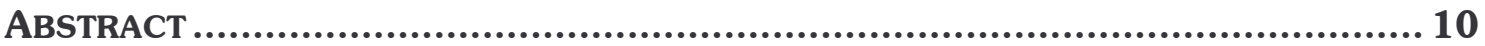

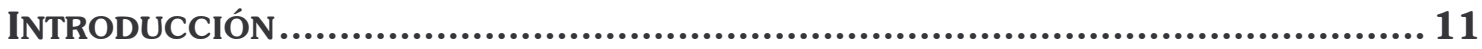

Capítulo 1. Propiedades ópticas de los Aerosoles. IntRoducción............ 13

1.1. AEROSOLES ATMOSFÉRICOS Y ESPECTRORRAdIOMETRÍA SOLAR. ............. 14

1.1.1. Determinación del espesor óptico de aerosoles ........................................... 15

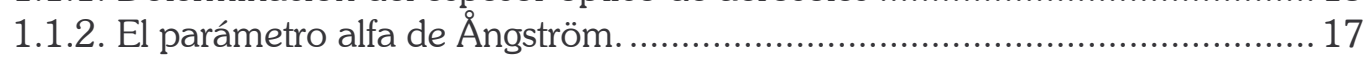

1.2. TIPOS DE AEROSOLES ATMOSFÉRICOS Y PROPIEDADES ÓPTICAS ................ 19

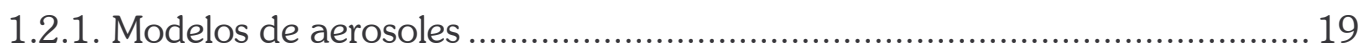

1.2.2. La climatología de aerosoles de AERONET .............................................. 21

1.3. Métodos de Calibración..................................................... 28

1.3.1. Calibraciones de irradiancia en laboratorio ................................................. 28

1.3.2. Calibraciones absolutas por el método de Langley ...................................2.29

1.3.3. Otros métodos basados en el método de Langley ..................................... 33

1.3.4. Intercalibración o calibración por comparación con un instrumento de

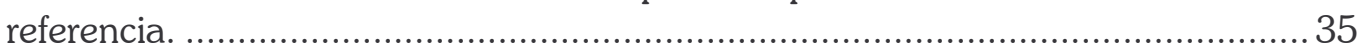

CApítulo 2. InStRUmentación: el fOtómetro Cimel y LA RED AERONET.

LUGAR DE MEDIDA Y SERIE DE DATOS. .................................................... 37

2.1. LA RED AERONET .................................................................. 38

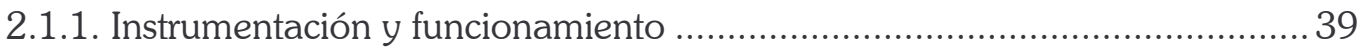

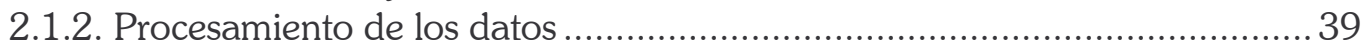

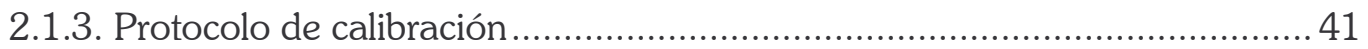

2.2. DESCRIPCIÓN DEL FOTÓMETRO CIMEL 318 .................................. 43

2.2.1. Características del fotómetro Cimel ........................................................... 43

2.2.2. Secuencia automática de medidas............................................................ 45

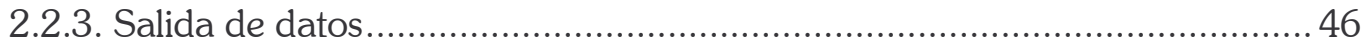

2.3. Estación dE MEdidA: El ARENOSILlo. SERIE de dATOS. ..................... 48

2.3.1. La estación de Sondeos Atmosféricos de El Arenosillo ............................... 48

2.3.2. Calendario de las medidas realizadas ..................................................... 49

2.4. DETECCIÓN DE PROBLEMAS EN LA SERIE DE DATOS: El FALSO CICLO DIURNO DEL AOD. ......................................................................... 52 
Capítulo 3. El método de CAlibración - CORRECción KCIClO. Aplicación y RESULTADOS............................................................................ 5

3.1. FORMULACIÓN ANALÍTICA Y EVALUACIÓN DEL FALSO CICLO DIURNO .........60

3.2. DESCRIPCIÓN DEL MÉTODO PROPUESTO: KCICLO .............................63

3.3. ApliCACión del MÉtodo KCICLO a la SERIE de dATOS de El ARENOSILlo

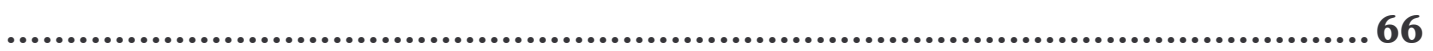

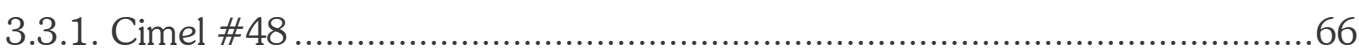

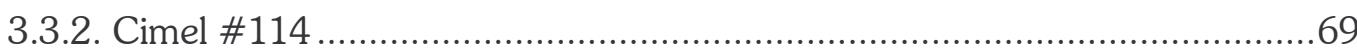

3.3.3. Cimel \#45 ……........................................................................ 71

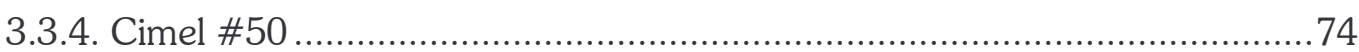

3.4. ANÁLISIS DEL ERROR DEL MÉTODO ................................................. 76

3.5. EQUIVALENCIA CON EL MÉTOdo dE LANGLEY ..................................... 79

3.6. REPROCESADO DE LA SERIE DE DATOS .......................................82

CAPÍtulo 4. ANÁlisis de las MEdidAs de ESPESOR ÓPTICo DE AEROSOlES EN El

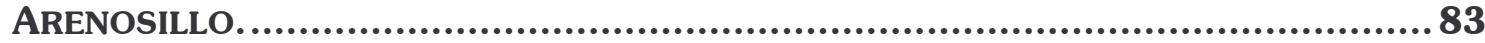

4.1. ANÁLISIS DEL ESPESOR ÓPTICO DE AEROSOLES. ............................84

4.1.1. Análisis de la serie completa de datos 2000-2004 en El Arenosillo .............86

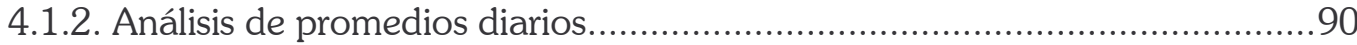

4.1.3. Análisis de promedios mensuales y estacionales ......................................91

4.2. ANÁlISIS DE LA EVOLUCIÓN TEMPORAL DEL PARÁMETRO ALFA................ 95

4.2.1. Análisis de la serie de medidas individuales de alfa(440-870) en el período

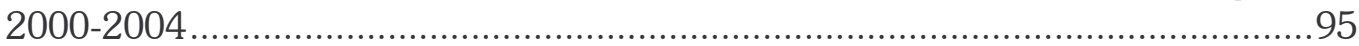

4.2.2. Análisis de promedios diarios, mensuales y estacionales de alfa. ...............98

4.3. CARACTERIZACIÓN DE los AEROSOles MEdiANTE LOS PARÁMETROS AOD Y

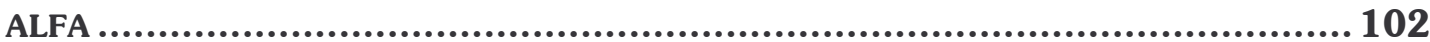

4.4. ANÁLISIS DEL ERROR ASOCIADO AL PARÁMETRO ALFA......................... 105

CAPítulo 5. Comparación de la SERIE de dATOS KCICLO CON LA DE AERONET.

5.1. COMPARACIÓN DE LA SERIE DE MEDIDAS INDIVIDUALES EN LOS PROCESADOS DE AERONET Y KCICLO ............................................................. 112

5.1.1. Diferencias entre los procesados de AERONET y KCICLO en los promedios

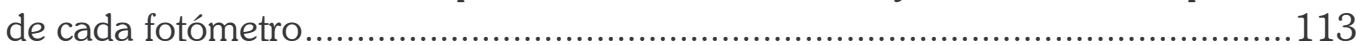

5.1.2. Continuidad de las series temporales de AOD y alfa ...............................119

5.2. COMPARACIÓN DE PROMEDIOS DIARIOS Y MENSUALES EN LOS PROCESADOS DE AERONET Y KCICLO .............................................................. 127

5.2.1. Análisis de las diferencias en los promedios diarios y mensuales para cada

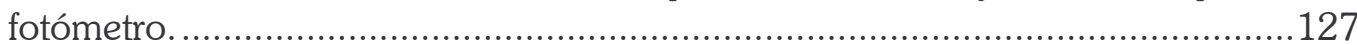

5.2.2. Análisis de diferencias en la climatología derivada de la serie completa ....130 
Capítulo 6. ANÁlisis con masas de AIRE DE LAS PROPIEDAdes DE LOS AEROSOLES.

6.1. DESCRIPCIÓN DE TIPOS DE MASAS de AIRE SOBRE LA PENÍNSUla IbÉRICA 134

6.2. El MODELO HYSPLIT. ELECCIÓN DE LAS RETROTRAYECTORIAS. 137

6.3. Clasificación DE LAS RETROTRAYECTORIAS POR SECTORES DE PROCEDENCIA.................................................................... 141

6.3.1. Definición de los sectores de procedencia. ............................................ 141

6.3.2. Asignación de las trayectorias a un sector de procedencia........................ 143

6.3.3. Análisis de frecuencias de las masas de aire ......................................... 147

6.3.4. Relación entre las medidas espectrales y las trayectorias de masas de aire 149

6.4. Clasificación de las RETROTRAYECTORIAS MEdiante UN ALgoritmo DE CLUSTER NO JERÁRQUICO ......................................................... 160

6.4.1. Descripción del algoritmo de clasificación................................................... 160

6.4.2. Aplicación del algoritmo de cluster no jerárquico a las trayectorias en El

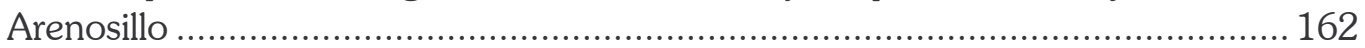

6.4.3. Análisis de frecuencias de las masas de aire .......................................... 167

6.4.4. Relación entre las medidas espectrales y las trayectorias de masas de aire 168

6.5. COMPARACIÓN ENTRE LAS DOS CLASIFICACIONES REALIZADAS. .............. 176

CApítulo 7. CARACTERIzAción Y EVAluación DE EPISOdios DE AEROSOL DESÉRTICO EN EL ARENOSILlO.................................................... 179

7.1. Intrusiones de polvo sahariano en la Península. Mecanismos..... 180

7.2. Metodología PARA El ESTUdio de EVENTOS DE AEROSOl DESÉRTICO ... 184

7.3. Evaluación DE LOS EPISODIOS DE AEROSOLES SAHARIANOS EN EL ARENOSILLO...................................................................... 186

7.3.1. Análisis de la frecuencia y duración de los episodios ............................... 186

7.3.2. Situaciones sinópticas durante los episodios desérticos........................... 188

7.3.3. Características de AOD y alfa durante los episodios desérticos ................. 191

7.3.4. Determinación de un criterio automático para la evaluación de episodios de

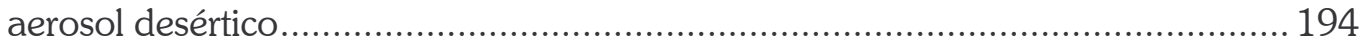

7.3.5. Comparación entre la evaluación de episodios de aerosoles desérticos y el análisis de masas de aire .................................................................................. 198

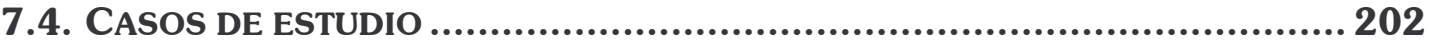

7.4.1. Episodio del 26 de febrero al 15 de marzo de 2000 .............................. 202

7.4.2. Episodio del 30 de julio al 3 de agosto de 2000 ...................................206

7.4.3. Episodio del 29 de julio al 15 de agosto de 2003..................................209

7.4.4. Episodio del 22 al 28 de julio de 2004 ................................................. 213

CONCLUSIONES Y LÍNEAS FUTURAS. ............................................... 217

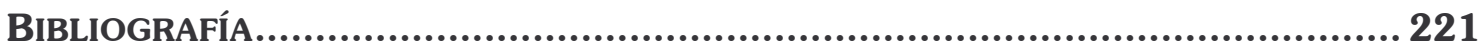

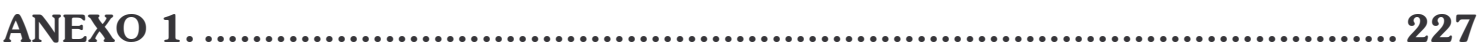

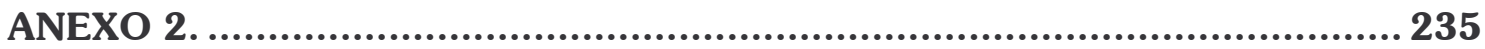




\section{Resumen}

El Grupo de Óptica Atmosférica de la Universidad de Valladolid realiza desde el año 2000 medidas rutinarias de aerosoles en la estación de El Arenosillo, Huelva, propiedad del Instituto Nacional de Técnica Aeroespacial. Las medidas se llevan a cabo con un fotómetro Cimel integrado en la red mundial de seguimiento de aerosoles AERONET, gestionada por NASA y la Universidad de Lille. Esta serie de datos de aerosoles de más de 5 años es la base para la caracterización de las propiedades y clasificación de los aerosoles en la zona de estudio. En este trabajo de tesis doctoral abordamos el análisis del espesor óptico de aerosoles y el parámetro alfa de Ångström, dos parámetros primarios y fundamentales para la caracterización de los aerosoles.

La primera parte de la investigación realizada consiste en el análisis de la calidad de la serie de datos del fotómetro Cimel en El Arenosillo. Se detectaron diversos problemas en los datos de espesor óptico de aerosoles, que se pueden resumir en la aparición de valores negativos y un ciclo diurno. El carácter sistemático del ciclo diurno puso de manifiesto la existencia de errores en la calibración, que han sido identificados y evaluados. El propio efecto del ciclo diurno contiene la información necesaria para poder corregir estos errores por la dependencia con la masa óptica del error del AOD. Esto ha sido aprovechado para desarrollar un método de calibración-corrección para la serie de datos de espesor óptico de aerosoles, el método KCICLO.

En segundo lugar se ha desarrollado un trabajo de análisis exhaustivo de la serie de datos de espesor óptico de aerosoles en la estación de El Arenosillo. Este trabajo de análisis está orientado al establecimiento de una climatología local de los aerosoles. Se ha analizado la procedencia de las masas de aire en la estación, lo que ha permitido establecer las propiedades ópticas de los aerosoles asociados a cada tipo de masa de aire. Para ello se han clasificado mediante dos métodos diferentes las retrotrayectorias de masas de aire, analizando la frecuencia de cada tipo de masa de aire así como las características de AOD y alfa en cada uno de ellos. Por último se ha realizado un estudio pormenorizado de los eventos de aerosol sahariano, con la realización de un inventario completo de los mismos en el período $2000-2004$. 


\section{Abstract}

The Atmospheric Optics Group of Valladolid University performs routine aerosol measurements at El Arenosillo, Huelva (Spain) since February 2000. This station belongs to Instituto Nacional de Técnica Aeroespacial. The measurements are carried out with a Cimel sunphotometer integrated in the global aerosol monitoring network AERONET, managed by NASA and University of Lille. A 5-year aerosol database allows aerosol properties characterization and classification in the site. In this work two basic parameters for aerosol characterization, aerosol optical depth and Angström exponent, are used for aerosol analysis.

As a first part of our study, the quality of the Cimel sunphotometer database has been checked. Several problems were detected in aerosol optical depth data, mainly the occurrence of negative AODs and a diurnal cycle. This systematic diurnal cycle reveals the existence of calibration errors, which were identified and evaluated. The fictitious effect contains the necessary information for an adequate correction, given the airmass dependence of aerosol optical depth errors. Thus the KCICLO method for correction of aerosol optical depth data series has been developed.

On the other hand, a detailed analysis of spectral aerosol optical depth features at El Arenosillo has been performed. The goal is to provide the aerosol local climatology in this region. The study of air mass origin was carried out in order to determine aerosol optical properties for each air mass type. Backtrajectory analysis and classification has been performed with two different methodologies, for origin and frequency of the air masses determination. AOD and alpha features are studied for each air mass type. Last we carry out the evaluation and characterization of Saharan dust outbreaks affecting El Arenosillo, by means of an inventory of dust episodes in the period 2000-2004. 


\section{Introducción}

Los aerosoles atmosféricos juegan un papel fundamental en el clima terrestre, de manera directa por su interacción con la radiación que llega del sol, e indirectamente por los cambios que producen sobre las propiedades microfísicas y ópticas de las nubes. La contribución de los aerosoles constituye una de las principales incertidumbres para los modelos climáticos.

Desde la revolución industrial se produce la emisión continuada y creciente de gases y partículas que alteran la composición de la atmósfera. Estas partículas de origen antropogénico se añaden a los aerosoles de origen natural, y contribuyen al balance radiativo terrestre. Dependiendo de las propiedades de los aerosoles, del albedo de la superficie o de su interacción con las nubes, esta contribución puede dar lugar a calentamiento o enfriamiento de la atmósfera. Las distintas fuentes naturales y antropogénicas, así como los mecanismos atmosféricos de transporte y deposición, hacen que la distribución espacial y temporal de los aerosoles atmosféricos sea enormemente variable.

Para controlar y reducir las incertidumbres sobre los aerosoles, se han venido desarrollando medidas de las propiedades de los aerosoles a nivel global, tanto a partir de medidas de superficie como aerotransportadas y desde satélite. Para ello se han desarrollado redes globales de fotómetros (AERONET, Holben et al., 1998; GAW, Werhli, 2000a; Aerocan, Bokoye et al., 2001; SKYNET, Takamura et al., 2004), y se han llevado a cabo numerosas campañas intensivas para la caracterización de los aerosoles: ACE-ASIA (http://www.ogp.noaa.gov/ace-asia), ACE 2 (Raes et al, 2000), INDOEX (http://wwwindoex.ucsd.edu), SAFARI (http://www.wits.ac.za/fac/engineering/-civil/Safari/), etc. Las medidas de satélite ofrecen una gran cobertura espacial, mientras que las medidas de superficie ofrecen una mayor resolución temporal. Las campañas intensivas presentan la ventaja de poder realizar una evaluación más completa y exhaustiva que en una estación ordinaria de medida, al combinarse las medidas de diversos instrumentos. Podemos afirmar por tanto que las diversas metodologías son complementarias en cuanto a su cobertura espacial, temporal $e$ instrumental.

La red de fotómetros AERONET/PHOTONS nace en 1998 con el objetivo de realizar medidas continuadas a nivel global de las propiedades ópticas de los aerosoles atmosféricos, así como para validar los datos proporcionados sobre los aerosoles por los sensores a bordo de satélites. El Grupo de Óptica Atmosférica de la Universidad de Valladolid (GOA-UVA) participa en la red desde el año 2000 con la instalación de un fotómetro Cimel en la estación de El Arenosillo (Huelva). Esto fue posible gracias a la estrecha colaboración que el GOA-UVA mantiene desde hace años con el INTA (Instituto Nacional de Técnica Aeroespacial), del que depende la estación de El Arenosillo, y el Laboratorio de Óptica Atmosférica de la Universidad de Lille.

Este fotómetro fue el primero de la red AERONET en la Península, y abrió el camino para el seguimiento y la caracterización rutinarios de los aerosoles atmosféricos. Este fotómetro automático continuó la serie de medidas realizadas por el GOA-UVA con un espectrorradiómetro Licor, que se llevaron a cabo en El Arenosillo desde 1996 con una menor resolución temporal. El fotómetro automático Cimel, siguiendo los protocolos de la 
red AERONET, proporciona una base de datos sobre las propiedades ópticas de los aerosoles a partir de medidas de radiación solar y radiancia del cielo.

En este trabajo hemos analizado la serie de más de 5 años de observaciones continuadas del espesor óptico de aerosoles y el parámetro alfa de Ångström en El Arenosillo, como parámetros fundamentales de la base de datos del fotómetro Cimel para la caracterización y clasificación de los aerosoles, y dentro del contexto de la red AERONET. El objetivo último es establecer la climatología de los aerosoles en la zona de estudio.

Este trabajo se ha basado necesariamente en el conocimiento de la red AERONET, sus protocolos y diferentes productos sobre aerosoles, así como su instrumento estándar, el fotómetro Cimel, con el que se han realizado las medidas experimentales que empleamos en este trabajo. Este paso obligado tuvo como resultado la detección de algunos problemas en la serie de datos de AERONET, que fue necesario delimitar e intentar resolver antes de analizar las propiedades de los aerosoles que se derivan de esas medidas. Una primera parte de este trabajo, que se recoge en los capítulos 2, 3 y 5 de la memoria, está por tanto relacionada con la necesidad de conocer y asegurar la calidad de la serie de datos con la que vamos a trabajar.

La segunda parte del trabajo, que se recoge en los capítulos 4, 6 y 7 de la memoria, ha consistido en la evaluación de las propiedades del espesor óptico de aerosoles y parámetro alfa en El Arenosillo, para lo cual se ha desarrollado una metodología de análisis que ya se comenzó a establecer en el GOA-UVA con los trabajos de Durán (1997) y Vergaz (2001). En el capítulo 4 se realiza el análisis de la serie de datos del Cimel, con el objetivo de establecer las características principales de los aerosoles y su evolución diaria, mensual, estacional e interanual. En el capítulo 6 emplearemos el estudio y clasificación de retrotrayectorias para establecer qué relación existe entre el tipo de aerosol y la procedencia de las masas de aire que llegan a la estación. Por último dedicamos el capítulo 7 a evaluar y caracterizar los episodios de aerosol de origen desértico, que son de gran importancia por su frecuencia e intensidad en toda la Península Ibérica y de manera especial en el sur. 


\section{Capítulo 1. Propiedades ópticas de los aerosoles. Introducción}

\section{Introducción}

En trabajos doctorales anteriores dentro del Grupo de Óptica Atmosférica (Durán, 1997; Vergaz, 2001) se puede encontrar una recopilación teórica exhaustiva sobre los aerosoles, así como, por supuesto, en la extensa literatura disponible sobre el tema. En este primer capítulo no queremos detenernos a realizar una introducción teórica de este tipo. Esa parte queda reducida a una definición de los aerosoles y de los parámetros que emplearemos para su descripción a lo largo de esta memoria.

Sin embargo, la interpretación de las medidas experimentales que se analizarán a lo largo de este trabajo necesita una referencia en cuanto a tipos de aerosol. Por ello vamos a incluir un apartado sobre los modelos de aerosoles y la climatología de aerosoles de la red AERONET.

Por último, una parte relevante de este trabajo tiene que ver con la calibración, por lo que haremos un repaso a los métodos de calibración, con énfasis especial en los métodos de campo, sus dificultades y los trabajos de diversos autores sobre la precisión y la mejora de esas calibraciones. 


\subsection{Aerosoles atmosféricos y espectrorradiometría solar}

Los aerosoles se pueden definir como un conjunto de partículas sólidas o líquidas en suspensión en un medio gaseoso, que se mantienen el tiempo suficiente como para permitir su observación y medida (Willeke and Baron, 1993).

El estudio de los aerosoles es muy amplio, y abarca campos muy diversos: procesos de combustión, contaminación atmosférica, los estudios climáticos, los que afectan a la higiene del trabajo y la salud, procesos de fabricación en salas limpias, los aerosoles biogénicos, etc. El estudio de los aerosoles en la atmósfera también presenta varias áreas, desde los procesos básicos de interacción con la radiación, hasta llegar a analizar su impacto climático en lo que se conoce como radiative forcing, pasando por los problemas de la contaminación atmosférica.

Por su origen, los aerosoles atmosféricos se clasifican en naturales, compuestos principalmente por cenizas volcánicas, sal marina, polvo desértico, esporas, polen, etc., y antropogénicos, derivados de la actividad humana, tales como humo de chimeneas, partículas minerales surgidas de procesos industriales... Las partículas producidas fotoquímicamente a partir de contaminantes gaseosos también entran en este apartado.

Existen diversos mecanismos generadores de aerosoles: combustión, reacciones químicas entre sustancias precursoras, movimientos de masas de aire que arrastran consigo partículas marinas o polvo desértico, etc. Las partículas así generadas son eliminadas de la atmósfera mediante acción gravitatoria, coagulación, condensación y su posterior precipitación, etc. Todo ello implica un tiempo de vida de estas partículas en la atmósfera que puede variar de minutos a semanas en la troposfera, y llegar a meses si tales partículas alcanzan la estratosfera (común tras las erupciones volcánicas, por ejemplo), ya que ahí procesos de eliminación tales como condensación o precipitación no se producen.

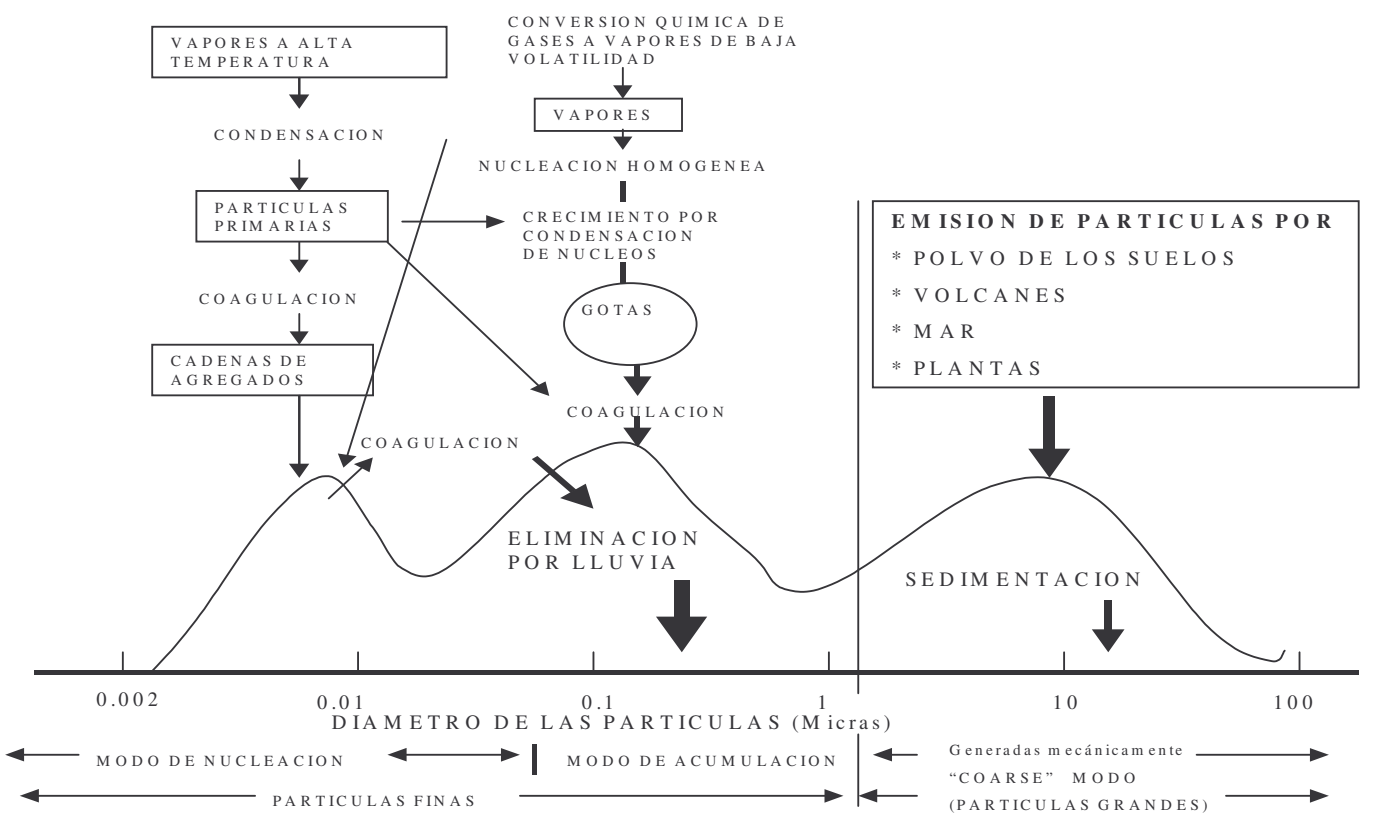

Figura I-1. Forma típica de la función de distribución de tamaños de los aerosoles, con los tres modos y los mecanismos de formación y eliminación en la atmósfera.

Son muchas las características del aerosoles que son objeto de estudio a la hora de determinar sus efectos: forma, tamaño, composición química... La distribución de 
tamaños, que nos da el número de aerosoles que tenemos de cada intervalo de tamaño, es la base para derivar propiedades ópticas de los aerosoles a través de la teoría de scattering. Las partículas en la atmósfera muestran tamaños que podemos delimitar desde unas milésimas de micra hasta $100 \mu \mathrm{m}$. Se suelen distinguir, por su tamaño y densidad numérica, tres modos en la distribución de tamaños de los aerosoles, tal como muestra la Figura I-1, llamados modo de condensación (desde unos nm hasta $0.1 \mu \mathrm{m}$ ), modo de acumulación (entre 0.1 y $1-2 \mu \mathrm{m}$ ) y modo grueso (desde 1 a $100 \mu \mathrm{m}$ ).

Los efectos de los aerosoles sobre la atmósfera y el clima se pueden resumir en los efectos directos de atenuación de la radiación, por scattering y absorción, y los efectos indirectos por su participación en la formación de nubes, y por tanto por su influencia sobre las características radiativas de las mismas, como el albedo.

Los aerosoles atmosféricos pueden ser medidos y estudiados mediante varios métodos. Se puede tomar muestras a lo largo de la columna atmosférica y analizarlas en el laboratorio. Otra alternativa es usar una instrumentación que nos permita analizarlas en el lugar de medida (in situ), tanto a nivel de suelo como embarcada en avión. Por otra parte podemos extraer propiedades de los aerosoles a partir del efecto que la presencia del aerosol está provocando en la radiación solar que atraviesa la atmósfera y llega hasta el suelo. Este tipo de instrumentación es también el que hoy en día se embarca en los satélites artificiales para el estudio de la atmósfera y de la superficie terrestre, con aquellas técnicas que se han empleado en las medidas experimentales de este trabajo: las medidas ópticas.

El efecto de los aerosoles sobre la radiación es diferente para cada región espectral y está íntimamente ligado a las características de los aerosoles. Por tanto la medida y el análisis de la radiación en la atmósfera constituye una de las principales metodologías para el estudio de los aerosoles. En concreto una partícula en la atmósfera introduce una discontinuidad en el índice de refracción con respecto al aire que la rodea, y dispersa luz en todas direcciones aunque no de forma isótropa en general, tal y como explica la teoría de scattering de Mie (1908, 1944). En el caso en que la absorción tenga un peso relativamente grande en los procesos de extinción, este índice de refracción adquiere una forma compleja, siendo la parte imaginaria la que da cuenta de esa contribución. La extinción de la radiación debida a los aerosoles es por tanto la suma del scattering más la absorción.

\subsubsection{Determinación del espesor óptico de aerosoles}

Los aerosoles dejan su "firma" en la radiación que, procedente del sol, llega hasta la superficie de la tierra. El estudio de componentes atmosféricos, no sólo aerosoles sino también el ozono, vapor de agua, oxígeno, etc. mediante medidas radiométricas se basa en la comparación entre el espectro de la radiación solar directa en la superficie de la tierra y el espectro solar extraterrestre. Ambos están representados en la Figura I-2. La diferencia entre ellos se debe a la atmósfera, por atenuación debida a scattering de moléculas (scattering Rayleigh) y aerosoles (scattering Mie), y por absorciones selectivas debidas a diferentes componentes atmosféricos como el ozono, oxígeno, vapor de agua, etc.

Así pues, suponiendo una atmósfera plano-paralela de una sola capa, la atenuación se puede expresar mediante la ley de Beer-Bouguer-Lambert:

$$
I=I_{0} \cdot e^{-\tau m}
$$

I es la irradiancia medida a nivel del suelo, $\mathrm{I}_{0}$ es la irradiancia extraterrestre, $\tau$ es el espesor óptico total de la atmósfera (espesor de toda la columna atmosférica), y m es la masa 
óptica, que se define como la razón entre el espesor óptico en la vertical y el espesor óptico en una dirección determinada.

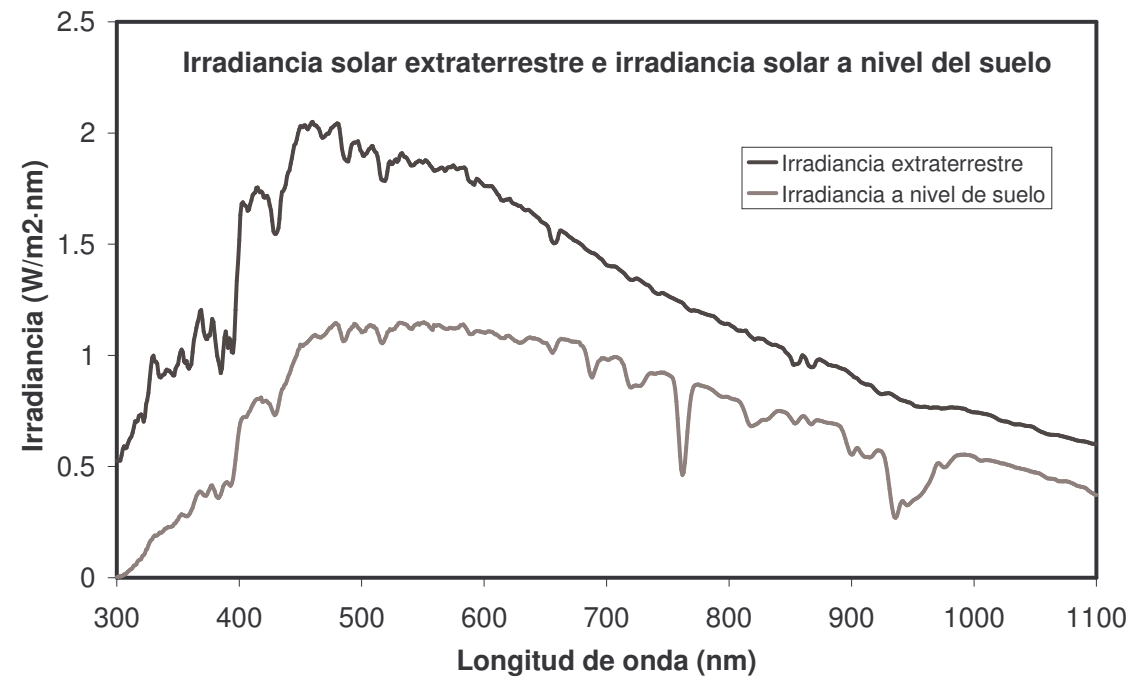

Figura I-2. Irradiancia solar extraterrestre y al nivel del suelo. Espectro a nivel de suelo tomado con una elevación solar de $44^{\circ}$ sobre el horizonte.

Nuestra medida habitual para calcular el espesor óptico de la atmósfera es una medida directa al sol (medida de extinción). En este caso, asumiendo el modelo de atmósfera plano paralela, la masa óptica se puede expresar en función del ángulo cenital solar $(\theta)$ como:

$$
\mathrm{m}=\sec \theta
$$

Esta fórmula para la masa óptica deja de ser una buena aproximación a partir de unos $60^{\circ}$ en el ángulo cenital solar, por diversos motivos: la curvatura de la Tierra, la refracción en el aire y el scattering múltiple. Por eso se emplean otras fórmulas más aproximadas, empíricas, como la de Kasten y Young:

$$
m=1 /\left(\cos \theta+0.50572 \cdot(1.46468-\theta)^{-1.6364}\right)
$$

También aquí $\theta$ es el ángulo cenital solar (en radianes). A partir de la ley de Beer-BouguerLambert, el espesor óptico total de la atmósfera viene dado por:

$$
\tau=-\frac{1}{m} \ln \left(I / I_{0}\right)
$$

Para obtener el espesor óptico de aerosoles $\left(\tau_{\mathrm{a}}\right)$, restamos la contribución al espesor total que se debe al scattering Rayleigh $\left(\tau_{\mathrm{R}}\right)$ y a las absorciones por parte de diferentes gases atmosféricos $\left(\tau_{\mathrm{g}}\right)$ :

$$
\tau_{a}=\tau-\tau_{R}-\tau_{g}
$$

El espesor óptico de aerosoles (AOD, aerosol optical depth) es el parámetro fundamental y primario en el estudio de las propiedades de los aerosoles. Se trata de un indicador del contenido vertical de la cantidad (índice de turbiedad) de aerosoles en la atmósfera, y a partir de sus valores y haciendo uso de la teoría de Mie, se puede utilizar toda una metodología de algoritmos de inversión para obtener la distribución de tamaños de los aerosoles. Podemos encontrar diversos algoritmos de inversión, como por ejemplo el de 
King et al. (1978) que sólo utiliza la información del espesor óptico, o bien aquellos que utilizan conjuntamente medidas de espesor óptico y radiancia del cielo (Nakajima et al, 1983; Dubovik and King, 2000a).

\subsubsection{El parámetro alfa de Ångström}

La dependencia espectral del scattering de Mie está relacionada con el tamaño de las partículas, en concreto por la relación entre el tamaño de la partícula y la longitud de onda de la radiación incidente. Por tanto el análisis de la forma espectral del espesor óptico de aerosoles es fundamental para la caracterización de los aerosoles mediante este tipo de medidas ópticas.

Para describir la forma espectral del espesor óptico de aerosoles, esto es, su dependencia con la longitud de onda, se utiliza otro parámetro muy sencillo, debido a Ångström. Este autor (Ångström, 1929; 1964) propuso la siguiente expresión parametrizada, válida para un cierto rango espectral:

$$
\tau_{a}=\beta \cdot \lambda^{-\alpha}
$$

donde $\lambda$ es la longitud de onda (en $\mu \mathrm{m}), \alpha$ el parámetro o exponente de Ångström (relacionado con el tamaño del aerosol) y $\beta$ es una constante que coincide con el espesor óptico correspondiente a $1 \mu \mathrm{m}$.

Teóricamente el parámetro alfa puede tomar valores entre 0 y 4 . Los valores más bajos están asociados a partículas grandes, en las que la extinción no tiene dependencia espectral. El ejemplo clásico es el color blanco de las nubes, dado por el scattering de luz solar en gotas de nube, que tienen tamaños alrededor de $100 \mu \mathrm{m}$. Frente a esto, el scattering debido a partículas pequeñas tiene una fuerte dependencia espectral, y es mayor para las longitudes de onda cortas. Como ejemplo tenemos el color azul del cielo, dado por el scattering de las moléculas (scattering Rayleigh), que es el límite inferior del tamaño, y cuya extinción depende en una potencia 4 con la longitud de onda. Para los aerosoles lo habitual es que alfa varíe entre 0 y 2.5 .

Para calcular en la práctica el parámetro alfa, se utilizan los datos medidos de AOD en diferentes longitudes de onda. Debido a que esta expresión sólo se cumple de manera aproximada, según el rango en el que calculemos alfa el resultado va a ser distinto, lo cual supone un serio problema a la hora de comparar resultados entre diferentes estudios.

En primer lugar podemos calcular el valor de alfa a partir de AOD en pares de longitudes de onda, que denotaremos como $\alpha\left(\lambda_{1} / \lambda_{2}\right)$. Para ello tomaremos logaritmos en la expresión de Ångström:

$$
\ln \tau_{a}=\ln \beta-\alpha \cdot \ln \lambda
$$

Esta expresión se verificará para cada longitud de onda, por lo que si disponemos del AOD en 2 longitudes de onda sólo tenemos que restar ambas expresiones para encontrar el valor del exponente alfa:

$$
\ln \tau_{a}\left(\lambda_{1}\right)-\ln \tau_{a}\left(\lambda_{2}\right)=-\alpha \cdot\left(\ln \lambda_{1}-\ln \lambda_{2}\right)
$$


Por ejemplo, para el par AOD en 440nm y en 670nm:

$$
\alpha(440 / 670)=-\ln \left(\tau_{a}(440) / \tau_{a}(670)\right) / \ln (0.44 / 0.67)
$$

Para calcular alfa a partir de 3 ó más datos espectrales de AOD, es necesario realizar un ajuste logarítmico a partir de la expresión (1.7). Un conjunto de observaciones espectrales de AOD permite entonces determinar los parámetros $\alpha$ y $\beta$. El resultado es representativo de un rango espectral, y lo denotaremos con las longitudes inicial y final de ese rango. Por ejemplo, si empleamos tres valores de AOD en 440, 670 y $870 \mathrm{~nm}$, el parámetro alfa derivado lo denotaremos como alfa(440-870).

El parámetro alfa y el espesor óptico de aerosoles son las dos magnitudes que vamos a emplear en este trabajo, y nos van a permitir estudiar y clasificar los aerosoles en nuestra zona de estudio. 


\subsection{Tipos de aerosoles atmosféricos y propiedades ópticas}

A la hora de estudiar los aerosoles atmosféricos es necesario realizar una caracterización de sus propiedades ópticas y microfísicas que permitan delimitar el tipo de aerosol en una zona de estudio. Las características microfísicas de los aerosoles están representadas básicamente por la función de distribución de tamaños. Estas propiedades varían además con la humedad relativa del aire. El problema de relacionar las propiedades ópticas y radiativas de los aerosoles con la microfísica y la composición química, es un elemento básico en el estudio de los aerosoles.

\subsubsection{Modelos de aerosoles}

Los modelos de aerosoles se basan en la recopilación de conocimientos sobre los distintos tipos de aerosoles, a partir de las medidas realizadas y mostradas en la extensa literatura sobre la caracterización de los aerosoles atmosféricos. En Vergaz (2001) se dan algunas características para los tipos de aerosol en los modelos de Shettle and Fenn (1979), Standard Radiation Atmosphere del World Climate Programme (WCP-55, 1983; WCP112 , 1986), la climatología global de aerosoles de D'Almeida (1991) y el modelo OPAC (Hess et al., 1998). En general se asigna a cada tipo de aerosol unos valores o rangos esperables para diferentes parámetros microfísicos y radiativos.

La climatología global de los aerosoles de D'Almeida (1991) establece una serie de componentes básicos -polvo, materiales solubles, hollín, sal, minerales, sulfatos, aerosoles volcánicos y biogénicos- que caracterizan mediante la distribución de tamaños. Para ello emplea el radio geométrico y la anchura de una distribución lognormal monomodal. A partir de los tipos básicos, por medio de mezclas adecuadas entre ellos, se establece una distinción de los aerosoles en función de los lugares de origen: aerosol de tipo ártico, antártico, continental, marítimo, desértico, urbano. Estos tipos son caracterizados mediante el rango en determinadas magnitudes ópticas, como el índice de refracción, albedo de scattering simple o parámetro de asimetría.

El modelo OPAC (Optical Properties of Aerosols and Clouds) está en gran medida basado en el trabajo de D'Almeida. El OPAC viene provisto de un software que permite calcular las propiedades ópticas de aerosoles con una composición dada por el usuario, que se establece a partir de mezclas de los componentes básicos. Estos componentes fundamentales y sus características de radio geométrico y anchura de la distribución de tamaños se recogen en la Tabla I-I.

\begin{tabular}{lcc}
\hline \hline Componente & $\mathrm{Rg}$ & $\sigma$ \\
\hline Insolubles & 0.471 & 2.51 \\
Solubles agua & 0.0212 & 2.24 \\
Hollín & 0.0118 & 2.00 \\
Sal (acumul.) & 0.209 & 2.03 \\
Sal (gruesas) & 1.75 & 2.03 \\
Minerales (nucleac.) & 0.07 & 1.95 \\
Minerales (acumul.) & 0.39 & 2.00 \\
Minerales (gruesas) & 1.90 & 2.15 \\
Mineral+transportado & 0.50 & 2.20 \\
Sulfatos & 0.0695 & 2.03 \\
\hline \hline
\end{tabular}

Tabla I-I. Radios geométricos y anchuras de las distribuciones medios (ambas en $\mu \mathrm{m}$ ) para los tipos genéricos de aerosoles considerados en el modelo OPAC. 
En la Tabla I-II mostramos los valores característicos de parámetros radiativos que definen los diferentes tipos de aerosoles según el modelo OPAC. En esta tabla tenemos el espesor óptico de aerosoles a 550nm y el parámetro alfa de Ångström en dos intervalos espectrales.

El análisis del diagrama de dispersión entre AOD y alfa es un procedimiento habitual para identificar tipos de aerosol, según la región de este diagrama en la que se sitúen nuestras medidas. En la Figura I-3 mostramos sobre este diagrama los tipos de aerosol dados por el OPAC. El aerosol continental más o menos polucionado y el aerosol urbano se sitúan en la región de valores de alfa en torno a 1.5, y con más o menos turbiedad en función de la contaminación. La otra situación de turbiedad alta se refiere al aerosol desértico, pero en este caso el alfa asociado es bajo (0.2), puesto que se trata de partículas grandes. Los diferentes tipos de aerosoles marítimos se sitúan en valores bajos de AOD (entre $0.05 \mathrm{y}$ 0.12 en $550 \mathrm{~nm}$ ) y valores de alfa en el rango de 0 a 1.

\begin{tabular}{lccccc}
\hline \hline & $\tau$ & $\omega$ & $\mathrm{g}$ & $\alpha_{350-500}$ & $\alpha_{500-800}$ \\
\hline Continental limpio & 0.064 & 0.972 & 0.709 & 1.10 & 1.42 \\
Continental promedio & 0.151 & 0.925 & 0.703 & 1.11 & 1.42 \\
Continental contaminado & 0.327 & 0.892 & 0.698 & 1.13 & 1.45 \\
Urbano & 0.643 & 0.817 & 0.689 & 1.14 & 1.43 \\
Desértico & 0.286 & 0.888 & 0.729 & 0.20 & 0.17 \\
Marítimo limpio & 0.096 & 0.997 & 0.772 & 0.12 & 0.08 \\
Marítimo contaminado & 0.117 & 0.975 & 0.756 & 0.41 & 0.35 \\
Marítimo tropical & 0.056 & 0.998 & 0.774 & 0.07 & 0.04 \\
Artico & 0.063 & 0.887 & 0.721 & 0.85 & 0.89 \\
Antártico & 0.072 & 1.000 & 0.784 & 0.34 & 0.73 \\
\hline \hline
\end{tabular}

Tabla I-II. Características radiativas medias de los tipos de aerosoles considerados en el modelo OPAC (Hess et al., 1998) dadas en 550nm: $\tau$ es el espesor óptico, $\omega$ el albedo de scattering simple, g el parámetro de asimetría y $\alpha$ el parámetro de Ångström, calculado en los intervalos espectrales $(350-500 \mathrm{~nm})$ y $(500-800 \mathrm{~nm})$.

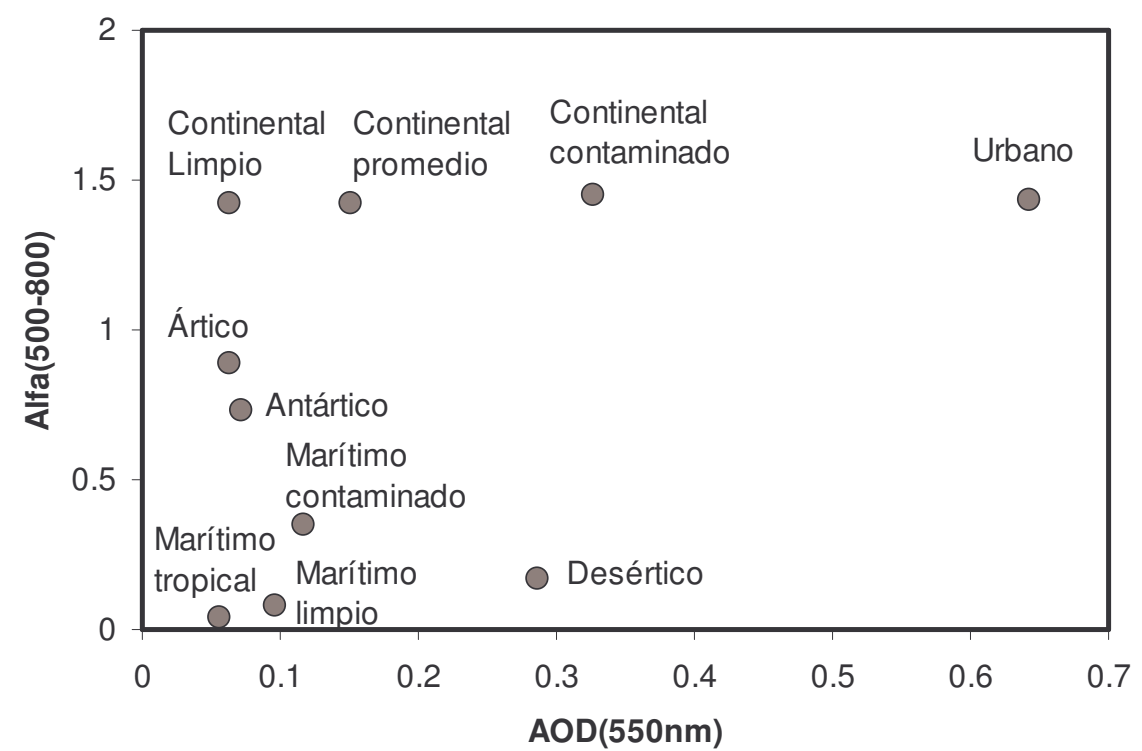

Figura I-3. Diagrama de dispersión $\mathrm{AOD}(550 \mathrm{~nm})$ frente a alfa(500-800) con los tipos de aerosoles dados por el modelo OPAC. 


\subsubsection{La climatología de aerosoles de AERONET}

La red AERONET (Holben et al., 1998), de la que hablaremos en el capítulo siguiente, realiza medidas rutinarias de aerosoles en numerosas estaciones por todo el planeta. Estas series de datos alcanzan ya varios años de medidas en numerosas estaciones, lo que permite establecer climatologías locales de los aerosoles. Las estaciones en las que predomina un tipo determinado de aerosol son idóneas para tratar de establecer las propiedades microfísicas y radiativas fundamentales de ese tipo concreto de aerosol. Varias publicaciones importantes de AERONET en los últimos años se han dirigido en este sentido, analizando en detalle series de datos más o menos largas en estaciones clave para cada tipo de aerosol: marítimo, urbano, desértico, continental, combustión de biomasa, etc. Queremos hacer en este apartado un resumen de la climatología de los aerosoles que se está desarrollando a partir de las medidas de AERONET (Eck et al., 1999; Holben et al., 2001; Dubovik et al., 2002; Smirnov et al., 2002).

Los parámetros básicos empleados para la caracterización de los aerosoles en estos trabajos son fundamentalmente el AOD y el alfa de Ångström, y en segundo lugar, los parámetros obtenidos a partir de los algoritmos de inversión de la red (Dubovik and King, 2000a), esto es, distribución de tamaños, albedo de scattering simple, parámetro de asimetría, índice de refracción. Nosotros nos vamos a centrar en las características de AOD y alfa que se han medido para diferentes tipos de aerosol.

\section{Aerosol marítimo}

El trabajo de Smirnov et al. (2002) muestra las características de AOD y alfa en varias estaciones marítimas remotas, tanto del Atlántico como del Pacífico. Además realiza una extensa recopilación de trabajos sobre medidas de aerosoles marítimos. En general los aerosoles marítimos en estaciones remotas se caracterizan por AOD (500nm) menor de 0.12. A partir de las medidas en 5 estaciones marítimas remotas de la red AERONET se puede concluir que el AOD para las estaciones remotas del Pacífico tiene un promedio de 0.07 , con un parámetro alfa entre 0.3 y 0.7 . En el Atlántico la turbiedad es mayor, también por la mayor presencia de polvo desértico, aerosol antropogénico o de combustión de biomasa. El promedio del AOD en las estaciones atlánticas es de 0.13 en $500 \mathrm{~nm}$, con alfa menor que 1 en general.

La Figura I-4, tomada de Smirnov et al. (2002), es un resumen de las medidas de AOD y alfa en las cinco estaciones mencionadas, tres de ellas situadas en el océano Pacífico y dos en el Atlántico. Podemos observar los valores de fondo con AOD por debajo de 0.1 y alfas por debajo de 1 en general, con una mayor turbiedad en las estaciones atlánticas que en las del Pacífico. Estos diagramas nos servirán de referencia al respecto de las características de AOD y alfa para el aerosol marítimo.

\section{Aerosol continental}

En el trabajo de Holben et al. (2001) se dan las características principales de dos estaciones de carácter continental, en los que se puede encontrar aerosol rural o continental de fondo. En la Figura I-5 encontramos los diagramas AOD-alfa correspondientes a estas dos estaciones. El aerosol rural de fondo se caracteriza por presentar valores de AOD por debajo de 0.1 , con valores altos del parámetro alfa, por encima de 1.3 en promedio. En estas estaciones en concreto se produce una subida de AOD durante el verano debido a la presencia de incendios forestales. También mostramos el diagrama de AOD-alfa para la 
estación de Palencia, gestionada por el GOA-UVA, de marcado carácter continental y situada en las afueras de una ciudad pequeña y con apenas industria. En este caso también hay situaciones que se superponen a la de fondo, como las entradas de aerosol desértico. Podemos observar en general valores de alfa por encima de 1 y AOD variable. No podemos establecer de momento si los valores medios y altos de AOD observados se deben a contaminación local o regional, incendios forestales u otras causas.
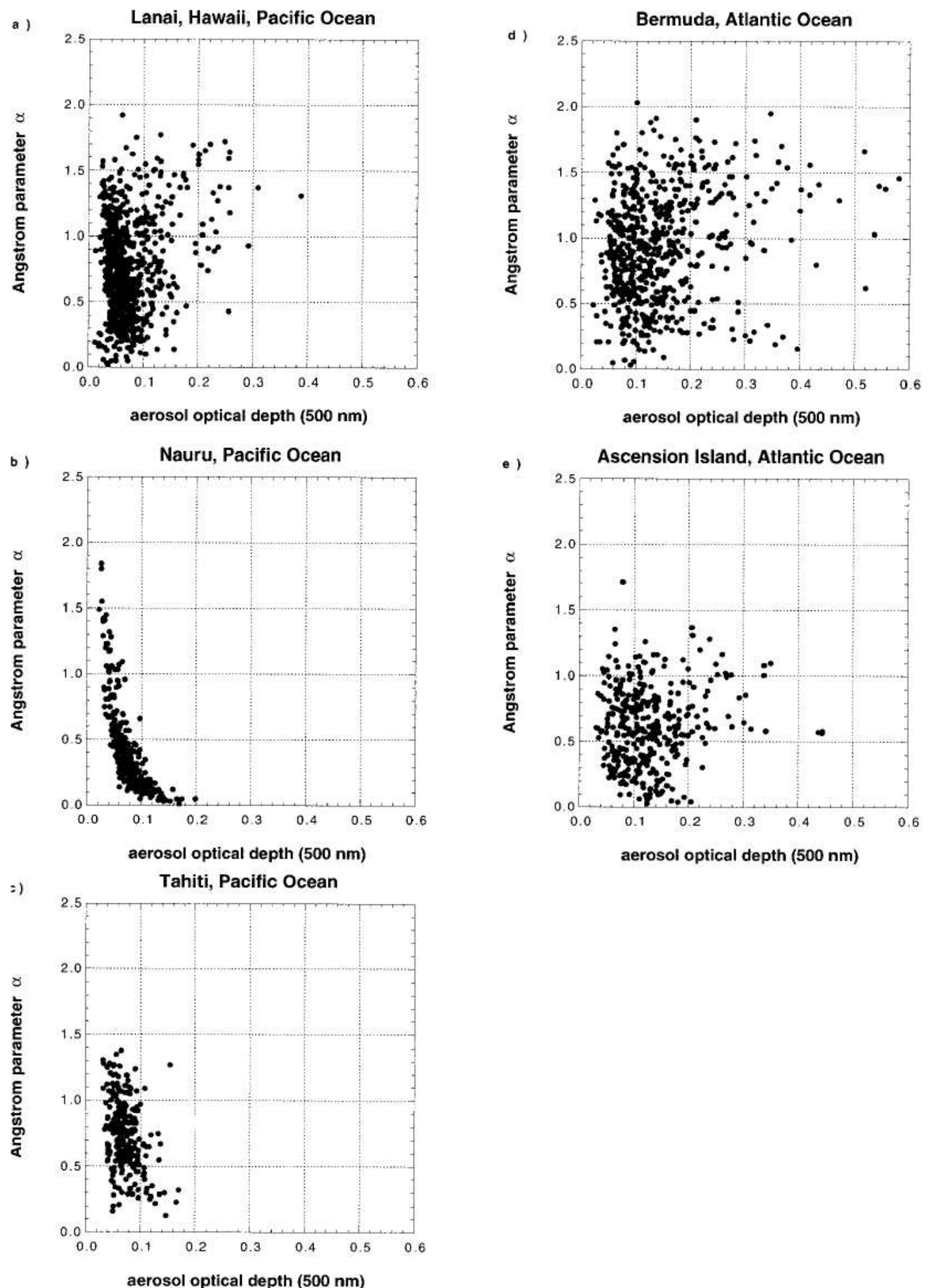

Figura I-4. Diagramas $\mathrm{AOD}(500 \mathrm{~nm})$ frente a alfa para 5 estaciones marítimas remotas de la red AERONET: (a) Lanai; (b) Nauru; (c) Tahiti; (d) Bermuda; (e) Ascension Island (tomada de Smirnov et al., 2002). 

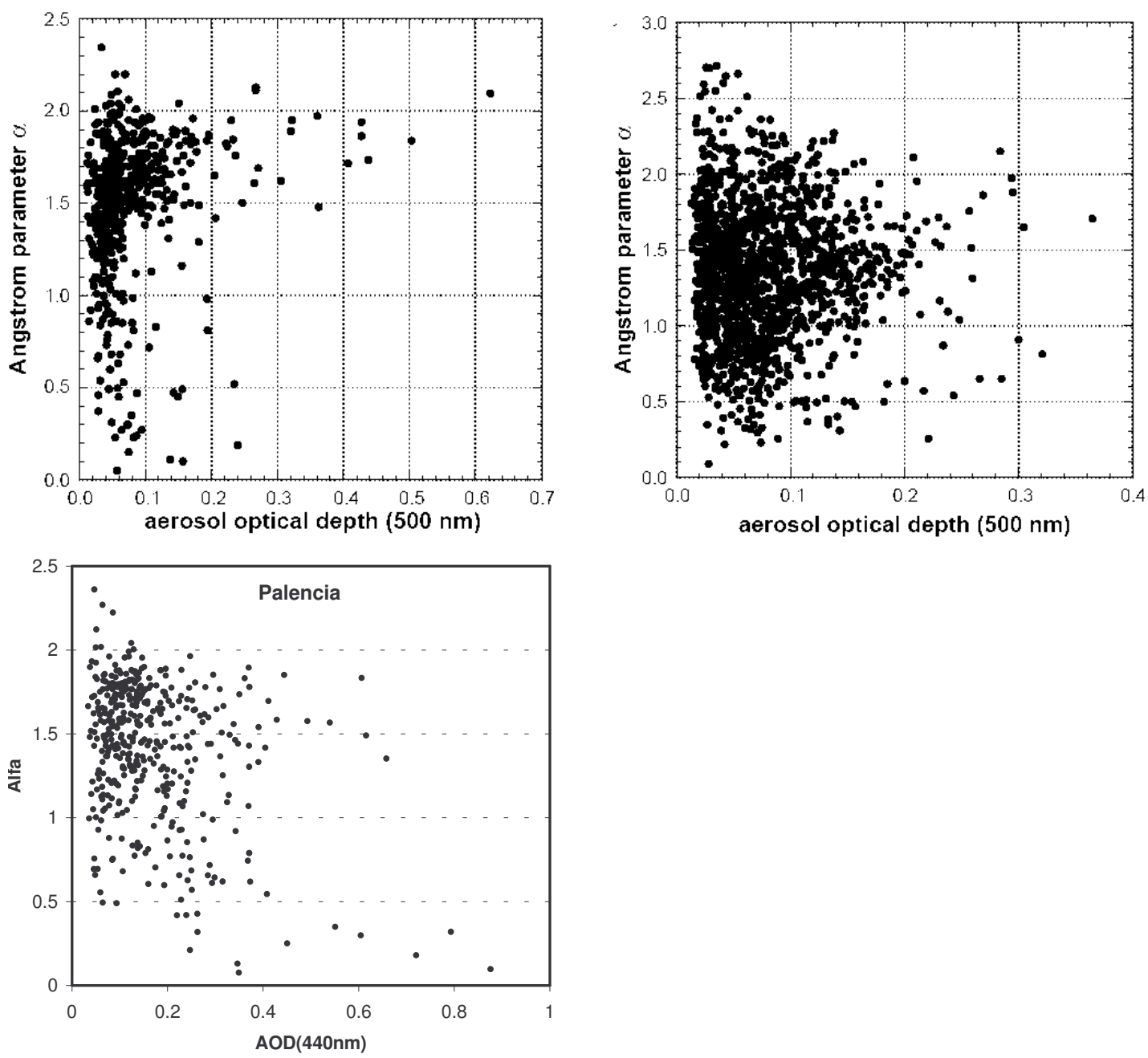

Figura I-5. Diagramas $\mathrm{AOD}(500 \mathrm{~nm})$ frente a alfa para 3 estaciones de carácter continental: HJAndrews, EEUU (arriba izquierda) y Sevilleta, México (arriba derecha), tomadas de Holben et al. (2001); Palencia (abajo), diagrama de AOD(440nm)-alfa, datos de AERONET nivel 1.5.

\section{Aerosol desértico}

El aerosol de origen desértico constituye una de las mayores contribuciones al aerosol atmosférico. Sus fuentes principales son las regiones áridas y semiáridas de África, Asia y Oriente Medio. Las estaciones de este carácter cuyos datos mostramos son Cabo Verde y Agoufou, ambas gestionadas por PHOTONS, y que se ven afectadas directamente por el polvo desértico procedente de los desiertos del Sahara y Sahel. En la Figura I-6 podemos observar las características de AOD y alfa propias de estas estaciones, con una turbiedad alta en general y que alcanza valores de AOD por encima de 1 , junto con un alfa muy bajo, propio de la extinción de partículas gruesas. Se observa además una correlación negativa entre AOD y alfa. Sólo para valores bajos de AOD se han registrado algunas medidas de alfa por encima de 1. La región por tanto que ocupan los aerosoles desérticos en el diagrama AOD-alfa es la parte inferior, con turbiedad alta y alfas bajos.

\section{Aerosol producido por la combustión de biomasa}

Los incendios forestales en vastas zonas de Sudamérica y África aportan enormes cantidades de aerosoles a la atmósfera. Las características de estos aerosoles dependen en principio del tipo de combustible, así como de la edad del aerosol y la humedad. La 
combustión de biomasa inyecta un gran número de partículas finas, alcanzándose espesores ópticos muy altos y variables, en valores similares a los del polvo desértico, pero sin embargo con un parámetro alfa muy alto, puesto que la extinción debida a partículas finas tiene una fuerte dependencia espectral. Por tanto en los diagramas AOD-alfa para dos estaciones con una marcada temporada de incendios (Figura I-7), las medidas de estos aerosoles se encuadran en la zona superior, con AOD y alfas en valores altos.
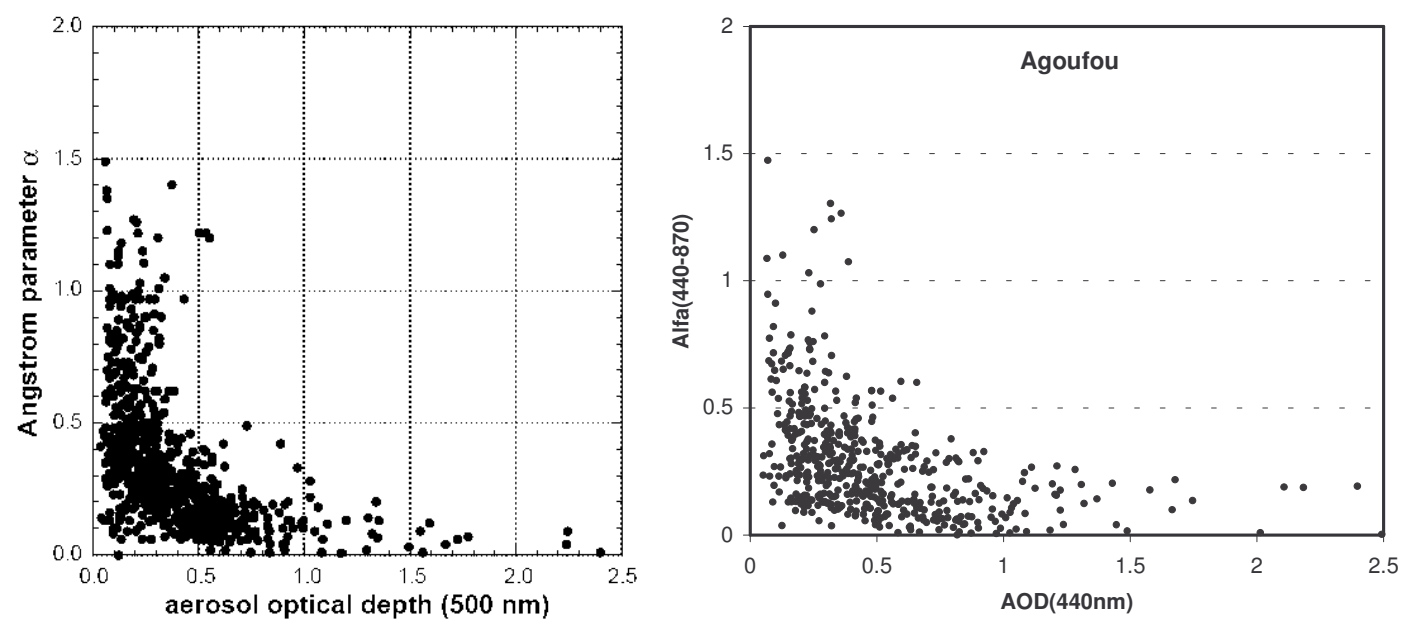

Figura I-6. Diagramas AOD frente a alfa para 2 estaciones en las que predominan los aerosoles desérticos: Cabo Verde (izquierda), tomada de Holben et al. (2001); y Agoufou (derecha), datos tomados de AERONET nivel 2.0 para este trabajo.
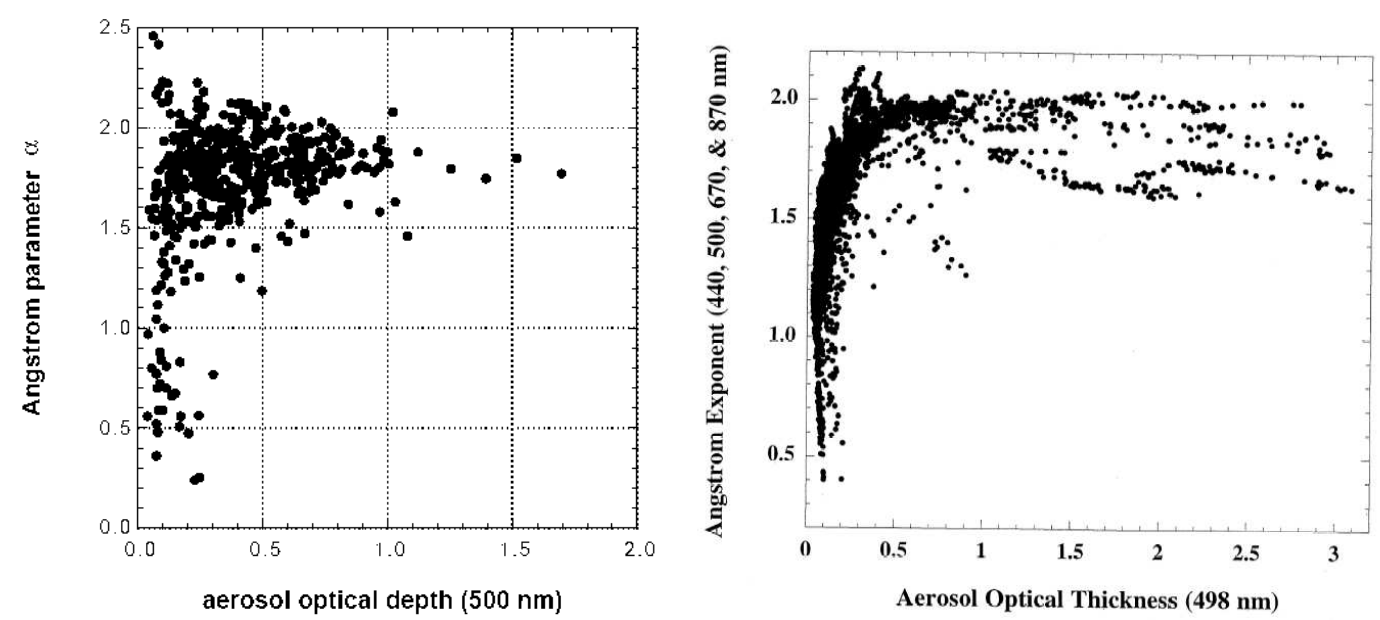

Figura I-7. Diagramas AOD frente a alfa para 2 estaciones en las que predominan los aerosoles generados por combustión de biomasa: Mongu, Zambia (izquierda), tomada de Holben et al. (2001); y Los Fierros, Bolivia (derecha), tomada de Eck et al. (1999).

\section{Aerosol urbano/industrial}

Las industrias y grandes núcleos de población emiten aerosoles que denominamos antropogénicos a la atmósfera. Esto puede tener mayor o menor impacto en función de la meteorología de cada región, puesto que el viento, la humedad, la renovación de las masas de aire o la orografía van a tener gran influencia a la hora de que aumente de forma más o menos severa la concentración de estos aerosoles antropogénicos. Las condiciones sinópticas van a condicionar también el transporte de esos aerosoles a otros lugares situados a gran distancia. 
Como ejemplo de estación con influencia antropogénica mostramos el Goddard Space Flight Center (GSFC), en Washington. Esta estación ubicada en la sede de la red AERONET es obviamente una de las que tiene una base de datos más larga y fiable, que comenzó en 1994. En este caso tenemos un gran número de medidas con valores bajos de AOD y alfas entre 1 y 2 que constituyen el aerosol de fondo en GSFC, típico de los meses de invierno. Junto a esto, durante el verano se producen valores altos de AOD con alfas altos asociados (Figura I-8), debidos a que el cambio en las condiciones meteorológicas favorece la acumulación de aerosoles antropogénicos. También queremos mostrar la estación de México City, una de las ciudades más contaminadas del mundo. En el diagrama correspondiente observamos valores muy altos de AOD y casi mayoritariamente con alfas altos, ocupando una amplia región en la parte superior del diagrama. El rango de valores de AOD que se registran es muy grande, como sucede también en GSFC.
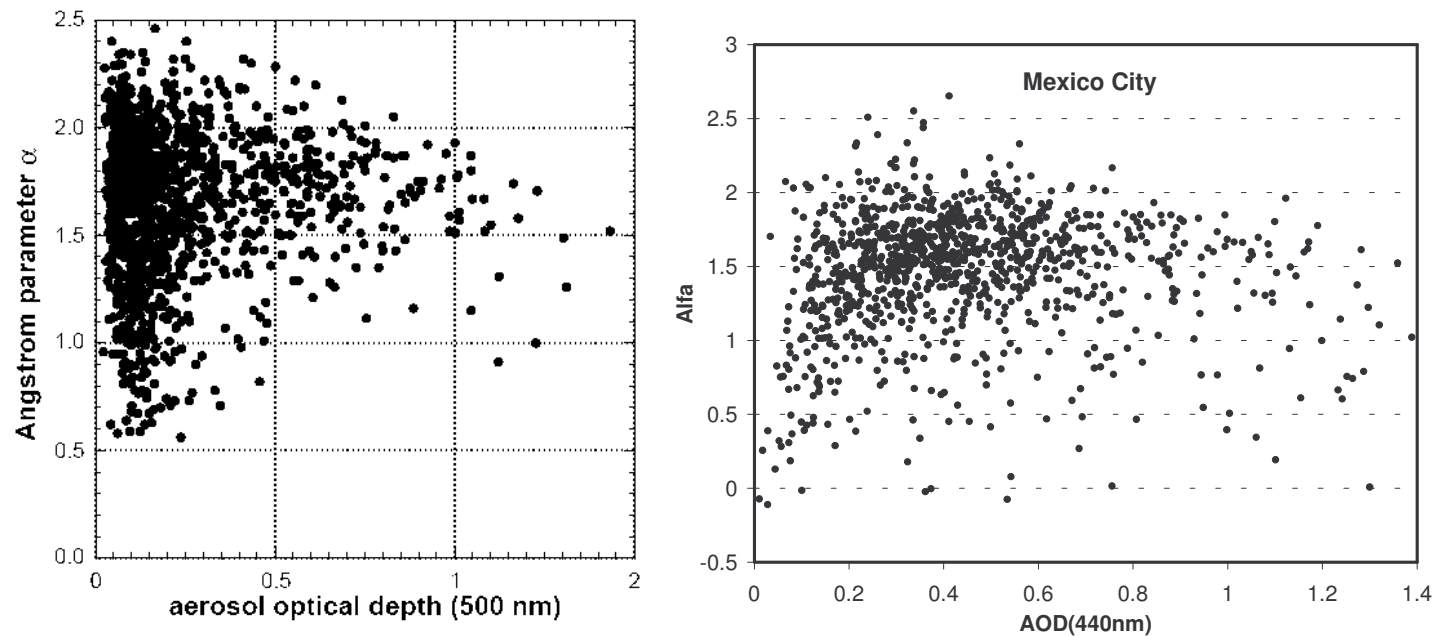

Figura I-8. Diagramas AOD frente a alfa para 2 estaciones en las que predominan los aerosoles antropogénicos: GSFC (izquierda), tomada de Holben et al. (2001); y México City (derecha), datos de AERONET nivel 1.5 .

\section{Aerosol ártico}

Durante los veranos de 2002, 2003 y 2005 el Grupo de Óptica Atmosférica ha realizado campañas para la medida del espesor óptico de aerosoles en ALOMAR (Arctic Lidar Observatory for Middle Atmosphere Research, Andenes, Noruega, $\left.69^{\circ} \mathrm{N} 16^{\circ} \mathrm{E}\right)$. La estación está situada en una isla frente a la costa atlántica noruega, unos $300 \mathrm{Km}$ por encima del círculo polar. Estas medidas permiten una primera aproximación a las propiedades ópticas de los aerosoles en esta región ártica durante el verano. Las medidas de AOD hablan de una atmósfera con una turbiedad muy baja en general, con valores del alfa altos. También se han producido algunos episodios de turbiedad media debida al transporte desde Europa o Rusia, fenómeno más frecuente durante el invierno y primavera, y que se conoce como 'Arctic haze'.

El diagrama AOD-alfa para estas medidas se muestra en la Figura I-9 (izquierda), en la que destacan por una parte la ausencia de valores altos de AOD, pues apenas hay medidas por encima de 0.2 y el promedio es 0.10 , mientras que el parámetro alfa muestra valores altos, en promedio 1.60. Probablemente este es el tipo de aerosol que muestra más discrepancias con lo establecido en el modelo OPAC. En la Figura I-9 (derecha) mostramos las medidas también en verano, en este caso de los veranos de 2003 y 2004 , en otra estación de AERONET situada en el Ártico. Se trata de Longyearbyen $\left(78^{\circ} \mathrm{N}\right.$, $\left.16^{\circ} \mathrm{E}\right)$, en el archipiélago de las Svalbard. Al igual que en Andenes, el período de medida con el fotómetro Cimel está restringido a los meses con luz solar. 

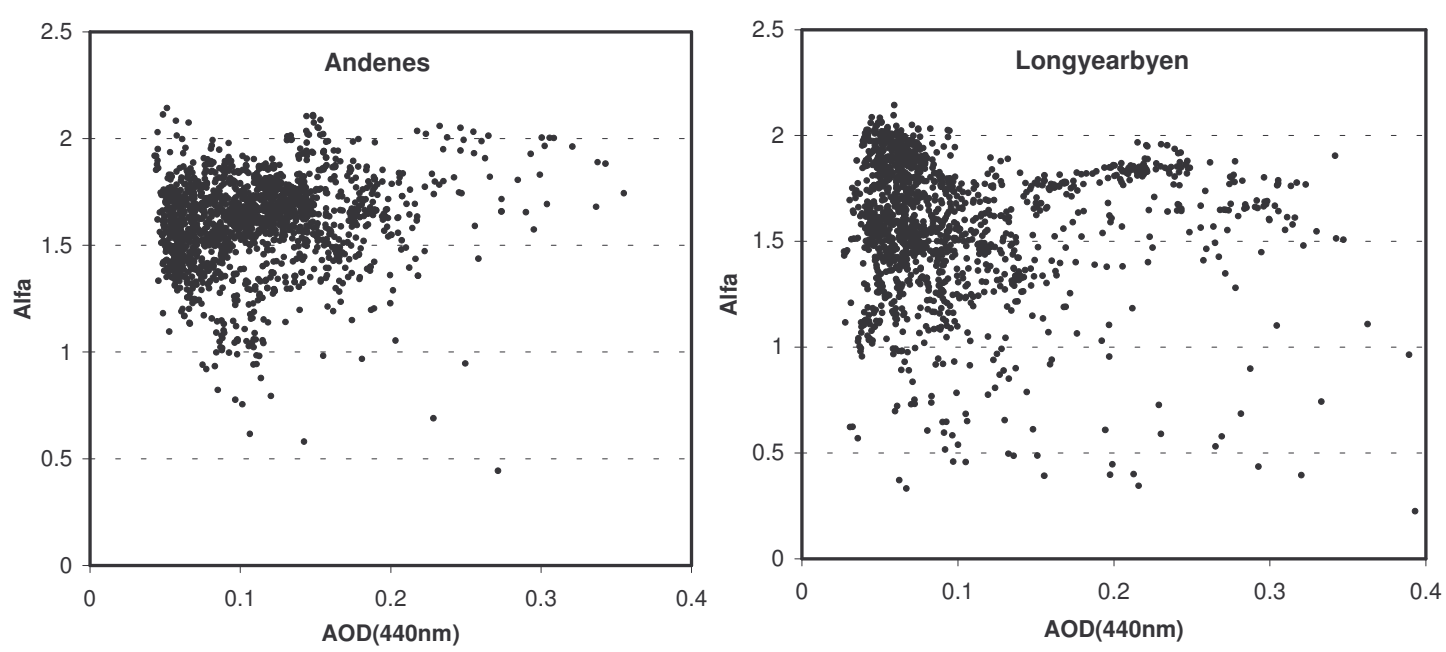

Figura I-9. Diagrama de $\mathrm{AOD}(440 \mathrm{~nm})$ frente a alfa para Andenes, Noruega (izquierda) y Longyearbyen, Noruega (derecha). Datos de AERONET nivel 1.5.

Como vemos se pueden establecer una serie de características generales en cuanto a los rangos de AOD y alfa que se pueden asociar a cada tipo más o menos puro de aerosol. En la Tabla I-III mostramos los valores promedios de estas estaciones tipo que se han ido comentando.

\begin{tabular}{lccccccc}
\hline \hline Estación & Período medida & $\mathrm{N}$ & Tipo aerosol & $\tau \mathrm{a}$ & $\sigma(\tau \mathrm{a})$ & alfa & $\sigma(\mathrm{alfa})$ \\
\hline Andenes & $2002-2003$ & 88 & Ártico (verano) & 0.11 & 0.05 & 1.51 & 0.25 \\
Longyearbyen & $2003-2004$ & 109 & Ártico (verano) & 0.11 & 0.09 & 1.50 & 0.32 \\
Los Fieros & $1998-1999$ & 283 & Combustión biomasa & 0.79 & 0.89 & 1.58 & 0.35 \\
Mongu & $1995-2000$ & 2000 & Combustión biomasa & 0.30 & 0.22 & 1.52 & 0.33 \\
HJ Andrews & $1994-1999$ & 514 & Continental & 0.06 & 0.04 & 1.35 & 0.35 \\
Palencia & $2003-2004$ & 424 & Continental & 0.17 & 0.11 & 1.41 & 0.42 \\
Sevilleta & $1994-1999$ & 1407 & Continental & 0.07 & 0.03 & 1.31 & 0.23 \\
Agoufou & Oct 2002-Abr 2004 & 517 & Desértico & 0.51 & 0.43 & 0.28 & 0.22 \\
Cabo Verde & $1994-1999$ & 726 & Desértico & 0.40 & 0.13 & 0.33 & 0.14 \\
Lanai & Nov 1995-Abr 2000 & 722 & Marítimo & 0.07 & 0.05 & 0.76 & 0.37 \\
Nauru & Jun 1999-Abr 2000 & 276 & Marítimo & 0.08 & 0.03 & 0.43 & 0.35 \\
Tahiti & Jul 1999-May 2000 & 234 & Marítimo & 0.07 & 0.02 & 0.74 & 0.27 \\
Ascension & Nov 1998-Jun 2000 & 338 & Marítimo mezcla & 0.13 & 0.07 & 0.62 & 0.3 \\
Bermuda & Mar 1996-Dic 1999 & 590 & Marítimo mezcla & 0.14 & 0.09 & 0.93 & 0.41 \\
GSFC & $1993-1999$ & 1325 & Urbano/industrial & 0.23 & 0.14 & 1.64 & 0.13 \\
México DF & 1999-2005 & 1228 & Urbano/industrial & 0.47 & 0.34 & 1.45 & 0.44 \\
\hline \hline
\end{tabular}

Tabla I-III. Resumen de las características de AOD y alfa en algunas estaciones clave de la red AERONET. Se indica el período de medida en el que están tomados los promedios, el número de días, el tipo de aerosol que caracteriza la estación, el AOD en 500, su desviación estándar, y el parámetro alfa también con su desviación estándar (promedios y STD calculados a partir de promedios diarios).

Los diagramas AOD-alfa son una referencia para dar una primera caracterización de los aerosoles. Las similitudes son apreciables entre estos datos de estaciones de AERONET y los tipos fundamentales del modelo OPAC, aunque es evidente que los datos experimentales siempre van a mostrar cierta dispersión y tampoco vamos a encontrar una estación con un aerosol estrictamente puro. Los tipos de aerosol marítimo y ártico son los que muestran una mayor discrepancia en los valores estimados por el OPAC y la climatología dada en AERONET. Los tipos de aerosol continental, urbano y desértico coinciden en sus características, aunque el modelo no recoge casos de turbiedad tan alta 
como se puede medir en algunas de las estaciones mencionadas. El aerosol debido a la combustión de biomasa no aparece como tipo fundamental en el modelo OPAC.

Estos resultados de la climatología desarrollada a partir de las medidas de AERONET pretenden ser orientativos y a ellos nos referiremos cuando se analicen las medidas en la estación de El Arenosillo, que constituyen el objeto de este trabajo. 


\subsection{Métodos de calibración}

La instrumentación que se puede emplear para las medidas radiométricas es realmente diversa en cuanto al modo de funcionamiento, rangos de medida, resolución... No vamos a entrar en una descripción. Las características relevantes para describir estos instrumentos son muchas: resolución, paso, rango espectral, anchura de los filtros, campo de iluminación, rango dinámico del detector...

Para las medidas de extinción (medida directa al sol) se limita el campo que ve el detector mediante un tubo limitador de campo o un colimador. El campo de iluminación media (FOV, field of view) es un parámetro importante para la descripción de nuestro sistema de medida.

El procedimiento concreto para calibrar estos instrumentos difiere según los tipos, pero en general hay tres métodos para realizar calibraciones en los instrumentos radiométricos: la calibración en laboratorio, la calibración absoluta por el método de Langley y otros métodos derivados, y la calibración por intercomparación. Dejamos de lado aquellos métodos que impliquen vuelos en globo, avión o satélite (Soufflet et al, 1996; Wehrli, 2000b).

Los parámetros que vamos a analizar en este trabajo (el espesor óptico de aerosoles) se derivan como hemos visto a partir de las medidas de irradiancia directa del sol. Por tanto nos vamos a centrar en los métodos para calibrar en irradiancia instrumentos radiométricos como el fotómetro Cimel. Es necesario tener en cuenta que el cálculo del AOD a partir de la ley de Beer no requiere una calibración absoluta en unidades físicas, sino la determinación de la señal (voltaje, cuentas, etc.) del instrumento fuera de la atmósfera, es decir, lo que denominamos constante extraterrestre del instrumento.

\subsubsection{Calibraciones de irradiancia en laboratorio}

La calibración en el laboratorio se basa en la utilización de lámparas estándar de irradiancia conocida. Se realiza un montaje en el que se coloca la lámpara estándar a una distancia especificada del detector. Se emplean lámparas a las que se ha trasladado la calibración de lámparas referenciadas por el NIST (National Institute of Standards and Technology) u organismos análogos. Realizando una serie de medidas con nuestro instrumento se obtiene un fichero que nos sirve para convertir la medida bruta (en cuentas, voltajes, etc.) del instrumento en irradiancia (en $\mathrm{W} / \mathrm{m}^{2}$ ), es decir, obtenemos una calibración absoluta en irradiancia.

Estas lámparas tienen una vida útil determinada (unas 50h) y la certificación tiene un coste elevado. Requieren una alimentación perfectamente estabilizada y controlada para emitir la potencia deseada, a la vez que unas rampas de encendido y apagado para evitar el deterioro de la lámpara y por tanto de la calibración.

Esta calibración absoluta requiere la caracterización de la función instrumento (por ejemplo la respuesta espectral si se emplean filtros interferenciales), con la que se debe pesar el espectro conocido de la lámpara. El resultado se divide por el voltaje o las cuentas digitales de la medida bruta para obtener el coeficiente de calibración para una determinada longitud de onda o canal. 
En caso de existir elementos intermedios entre la fuente y el detector (fibras ópticas, difusores, lentes, etc.) el montaje para la calibración debe hacerse con todos los elementos del sistema, es decir, el mismo montaje que para realizar medidas, además de evaluar por separado la transmitancia espectral de cada uno de estos elementos, en particular filtros y lentes, para poder disponer de una calibración absoluta en irradiancia. La calibración puede ser sólo relativa, esto es, trasladando la forma del espectro pero no su valor absoluto. Esto puede ser suficiente si se van a emplear posteriormente cocientes entre distintas longitudes de onda. En este caso basta con hacer medidas de la lámpara estándar, sin importar la distancia a la que se coloca la lámpara ni la presencia de lentes, aunque el montaje debe siempre incluir todos los elementos del sistema.

Las fuentes de error son diversas (Slusser et al., 2000):

- La incertidumbre sobre el espectro de la lámpara referenciada, en torno al $1 \%$ para un estándar de $2^{\circ}$ orden.

- Diferencia de magnitud entre la irradiancia de la lámpara y la irradiancia solar, ya que las lámparas pueden emitir una señal mucho menor que la del sol. En este caso estaríamos calibrando el detector bajo unas condiciones diferentes de las de medida, siendo decisiva la linealidad en la respuesta del detector frente al flujo incidente.

- Incertidumbres en el montaje experimental, por ejemplo en la distancia o la alineación del filamento de la lámpara con el instrumento.

- Medida de la respuesta espectral o función instrumento del aparato.

- Errores al interpolar el espectro de la lámpara al paso que precisemos.

- Eliminación del ruido o la corriente oscura.

- Repetitividad en la respuesta del instrumento.

Todas las fuentes de error influyen de forma diferente según la región del espectro en la que estemos trabajando. La evaluación de estos errores debe hacerse en cada caso concreto, aunque en general se suele estimar en torno al 1\%-3\%. Sin embargo se pueden encontrar discrepancias mayores que esa estimación, en torno al $5 \%$, al comparar calibraciones con lámparas frente a calibraciones por el método de Langley, lo que sugiere que estas incertidumbres pueden ser mayores (Schmid and Wehrli, 1995; Wehrli, 2000b; Slusser et al., 2000).

Las calibraciones absolutas en irradiancia realizadas mediante lámparas difícilmente superan la precisión del 3\%, lo cual es insuficiente para evaluar el espesor óptico de aerosoles en condiciones de baja turbiedad, ya que esta precisión en la calibración se traduciría en un posible error del $100 \%$ en el espesor óptico (Forgan, 1994). Por esto el método más empleado a la hora de calibrar instrumentación para la medida del AOD es el método de Langley. Sin embargo, en regiones del espectro en las que haya absorciones debidas a gases, el método de Langley es de difícil aplicación (Reagan et al., 1986), por lo que la calibración en laboratorio sigue jugando un papel importante en los instrumentos espectralmente resueltos.

\subsubsection{Calibraciones absolutas por el método de Langley}

El método de Langley se basa en la ley de Beer-Bouguer-Lambert ya mencionada, en la que indicamos el subíndice $\lambda$ puesto que esta relación se cumple para cada longitud de onda:

$$
I_{\lambda}=I_{0 \lambda} \cdot e^{-\tau_{\lambda} m}
$$


donde I es la irradiancia medida a nivel del suelo, $\mathrm{I}_{0}$ es la irradiancia extraterrestre, corregida de la distancia tierra-sol, $\tau$ es el espesor óptico total de la atmósfera y m es la masa óptica. Tomando logaritmos se obtiene:

$$
\ln I_{\lambda}=\ln I_{0 \lambda}-\tau_{\lambda} \cdot m
$$

Si para una longitud de onda se representa el logaritmo de la irradiancia medida frente a la masa óptica, a lo largo de una serie de medidas sin variación en el espesor óptico total, el resultado es una recta cuya pendiente es el espesor óptico total y la ordenada en el origen corresponde a la irradiancia extraterrestre para esa longitud de onda o canal. Ésta nos dará el coeficiente de calibración para esta longitud de onda o canal. Un ejemplo de esta representación es la Figura I-10.

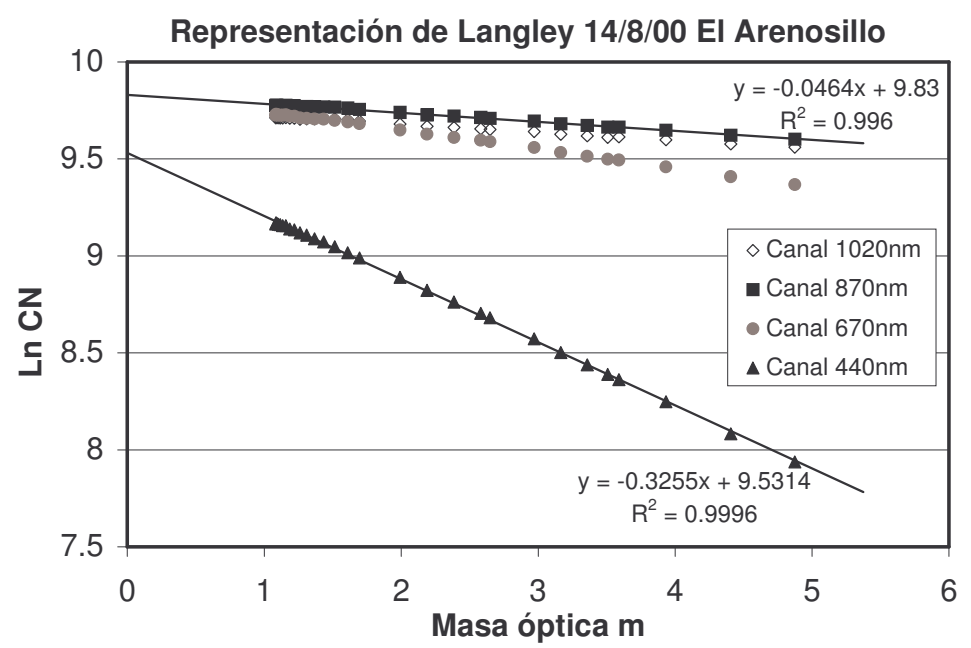

Figura I-10. Ejemplo de representación de Langley con datos del Cimel en El Arenosillo. Mostramos el resultado de las rectas de ajuste para los canales de 440 y $870 \mathrm{~nm}$.

Si comparamos esta señal extraterrestre (en voltaje o cuentas digitales) con el espectro de irradiancia extraterrestre, podemos obtener unos coeficientes de calibración para convertir la señal bruta en unidades físicas $\left(\mathrm{W} / \mathrm{m}^{2}\right)$. En todo caso, para el cálculo de espesores ópticos con la ley de Beer no son necesarios coeficientes de calibración en unidades físicas, tan sólo los voltajes o cuentas digitales extraterrestres que nos da la ordenada en el origen en el ajuste de Langley.

Estos coeficientes, tal y como se obtienen a partir del ajuste de Langley, dependen de la distancia tierra-sol. Por ello se hace una corrección y se deja un coeficiente referido a la distancia media tierra-sol. Cuando se quiera calcular el espesor óptico para un día concreto, se vuelve a aplicar esta corrección, que podemos evaluar, por ejemplo, con la siguiente fórmula (Spencer 1971), que depende simplemente del día del año $\left(\mathrm{N}_{\mathrm{d}}\right)$, donde $\Gamma$ se conoce como el ángulo del día (en radianes), dado por $\Gamma=2 \pi\left(\mathrm{N}_{\mathrm{d}}-1\right) / 365$ :

$d=1.00110+0.034221 \cos \Gamma+0.001280 \operatorname{sen} \Gamma+0.000719 \cos 2 \Gamma+0.000077 \operatorname{sen} 2 \Gamma$

La condición más fuerte que el método requiere es que el espesor óptico total de la atmósfera permanezca constante en la serie de medidas realizadas. Esta es una condición muy restrictiva en la atmósfera.

Las medidas a realizar deben cubrir un rango suficiente de masas ópticas. Generalmente se habla de masas ópticas entre 1 y 7 , aunque las medidas cerca del mediodía no se suelen tener en cuenta por "estropear" el ajuste. Masas superiores a 6 ó 7 tampoco se consideran 
por la dificultad de estimar correctamente la masa óptica cuando el sol está muy bajo. Vamos a discutir todo esto detenidamente, pues la elección del rango de masas ópticas condiciona decisivamente la aplicación del método y el resultado final de la calibración.

El rango dinámico del instrumento nos va a indicar la limitación para las masas ópticas grandes (Slusser et al. 2000). Partimos de la relación (nuevamente la ley de Beer-BouguerLambert):

$$
\ln \left(I / I_{0}\right)=-\tau \cdot m
$$

Si introducimos el valor más pequeño aceptable de I, es decir, el menor con una suficiente relación señal-ruido, la irradiancia extraterrestre del día y el valor de $\tau$, obtenemos el valor de la masa óptica más grande que la sensibilidad del instrumento nos permite alcanzar. Debemos destacar, como ejemplo de esta limitación, que para zonas del espectro como el ultravioleta, en el que la señal es pequeña y el espesor óptico total grande (en comparación con la zona del visible, por ejemplo), esta relación nos puede indicar que no podemos medir más allá de masa óptica 3. Esta limitación es menor en otras regiones del espectro en las que la irradiancia solar es mayor.

Además de esto, hay que tener en cuenta otras limitaciones y fuentes de error cuando se mide con el sol muy bajo: luz que nos llega por scattering múltiple, la influencia de la curvatura de la tierra, la refracción en el aire, la presencia frecuente de brumas o capas con diferente aerosol por el mayor recorrido por la troposfera o el mayor tamaño angular del disco solar. Todo esto hace que aumente la incertidumbre tanto en el cálculo de la masa óptica como en la medida en sí misma, limitando las medidas en masas ópticas muy grandes.

En cuanto a las medidas cerca del mediodía el problema radica en la variabilidad de la atmósfera. En latitudes medias y bajas, las masas ópticas cambian con mucha rapidez al amanecer o al atardecer. En verano en El Arenosillo transcurren 2 horas entre las masas ópticas 2 y 7. Entre la masa óptica 2 y el mediodía solar, con masa cercana a 1, pueden transcurrir 4 horas. La condición más fuerte para la calibración Langley, esto es, que el espesor óptico total permanezca constante, es evidentemente más fácil de asegurar en un intervalo de tiempo menor. Cerca del mediodía podemos tener numerosas medidas cercanas en masa óptica pero que difieren sensiblemente en el espesor óptico, empeorando el ajuste.

Una vez planteado esto, la elección del rango de masas ópticas para el ajuste debe realizarse teniendo en cuenta por un lado la limitación del instrumento y por otro las condiciones de la atmósfera, sin umbrales prefijados a priori. Una vez que hemos realizado la representación de Langley se puede eliminar los puntos que están más alejados que 2 ó 3 veces la desviación estándar, estableciendo un procedimiento iterativo cuya limitación sería, por un lado, un valor de la desviación estándar suficientemente pequeño y por otro un numero mínimo de puntos.

El coeficiente de correlación no es un buen indicativo de la bondad del ajuste, por su fuerte dependencia con la pendiente. Además, como discutiremos más adelante al hablar del método de corrección propuesto en el capítulo 3, puede haber variaciones en el espesor óptico de la atmósfera que no son detectables a la vista del ajuste y que introducen errores difícilmente evaluables. Se advierte claramente (Herman, 1981; Kremser, 1984; Reagan, 1986; Terez and Terez, 2003) que variaciones monótonas en el AOD dan lugar a errores sistemáticos en la calibración por el método de Langley, que además son imposibles de detectar en un solo ajuste. Por ello, para asegurar una precisión del $1 \%$ en la 
calibración (en lugares idóneos, como alta montaña) es necesario realizar un cierto número de ajustes entre los cuales realizar un promedio. Al menos 20 ajustes Langley son necesarios para asegurar una precisión del 1\% (Slusser, 2000) en el voltaje extraterrestre, lo cual es imprescindible para poder determinar el AOD con un error absoluto de 0.010.02. En condiciones de baja turbiedad este error absoluto puede significar un error relativo superior al $10 \%$ en el AOD (Wehrli, 2000b). Si llevamos un poco más lejos este argumento, la bondad de una estación de medida para realizar calibraciones por el método de Langley se puede caracterizar mediante el tiempo que hace falta para llevar a cabo los ajustes necesarios para alcanzar una precisión del 1\% (Wehrli, 2002). Por supuesto la clave para esta condición se encuentra en el número de días estables y soleados de la estación.

Las condiciones ideales para realizar una calibración Langley pasan por la menor variación posible en el espesor óptico total. Esta variación, en las longitudes de onda no afectadas por absorciones, se debe a los aerosoles, por lo que se suelen buscar lugares en alta montaña, en altitudes donde la cantidad de aerosoles es baja y sus variaciones pequeñas en términos absolutos. Las estaciones por encima de la capa límite, como Mauna Loa, son óptimas para este tipo de calibraciones. De hecho numerosas redes de fotómetros realizan la calibración absoluta de sus instrumentos de referencia en Mauna Loa. Por supuesto las zonas urbanas no son adecuadas, y en los lugares dentro de la capa de mezcla continental se requerirá un número grande de ajustes y los coeficientes de calibración estarán afectados por más error. El Observatorio Atmosférico de Izaña, situado en la isla de Tenerife a 2360m sobre el nivel del mar (s.n.m.), es una estación idónea para este tipo de calibraciones, en particular por su accesibilidad e instalaciones, así como el clima y la altitud. La Red Ibérica de Medida de Aerosoles (RIMA), actualmente en desarrollo, realizará la calibración de sus instrumentos de referencia en Izaña, donde ya se calibran de forma rutinaria los instrumentos master de la red PHOTONS (ver sección 2.1.3).

Las fuentes de error para el método de Langley (Reagan et al., 1986; Slusser et al., 2000) se deben a:

- La estabilidad del espesor óptico total durante las medidas, como ya hemos descrito. En el UV las variaciones de ozono durante el día tienen especial importancia. Las variaciones en la cantidad de aerosol afectan a todo el rango espectral.

- El ancho de banda finito del espectro, puesto que la ley de Beer es sólo aplicable monocromáticamente, y su aplicación sobre una banda de cierta anchura espectral (la función instrumento del aparato o la anchura del filtro empleado) conlleva un error. Este error aumenta con la anchura y también depende de la región espectral considerada.

- La inestabilidad del instrumento.

- La linealidad de la respuesta del detector frente al flujo incidente.

- La contribución de una pequeña parte de radiación difusa en la medida directa al sol, en función de lo grande que sea el campo angular que ve el detector.

- Si deseamos convertir la señal a unidades físicas, entonces hay que considerar también el error del espectro de irradiancia extraterrestre que se emplee. Los errores suelen ser menores del $2 \%$ y además son susceptibles de mejora a medida que se desarrollen mejores medidas desde satélites.

- La consideración de una misma masa óptica para moléculas, aerosoles y gases absorbentes (como el ozono). En rigor, al aplicar la ley de Beer-Bouguer-Lambert, cada componente debe tener una masa óptica diferente, puesto que son diferentes los perfiles verticales de concentración de cada uno de ellos. (Thomason et al., 1983; Forgan, 1988). Estas diferencias son mayores para el UV por la fuerte dependencia con el ozono y aumentan con el ángulo cenital solar. 


\subsubsection{Otros métodos basados en el método de Langley}

Numerosos autores se han enfrentado con la dificultad de obtener calibraciones con el método de Langley en estaciones ordinarias, sujetas a la disponibilidad de días estables y despejados, y en todo caso con una variabilidad en el espesor óptico de aerosoles mucho mayor que en estaciones situadas por encima de la capa límite.

Por otra parte el método de Langley implica una serie de suposiciones que se pueden intentar evitar en lo posible, de cara a intentar minimizar la diferencia entre los resultados de calibraciones sucesivas por el método de Langley.

La expresión (1.11) contiene la suposición de que la masas ópticas de las diferentes especies que contribuyen al espesor óptico total son iguales. En realidad es más exacto computar de forma independiente la masa óptica de aerosoles (a), moléculas $(\mathrm{m})$ y gases absorbentes (g):

$$
\ln I_{\lambda}=\ln I_{0 \lambda}-\tau_{a \lambda} \cdot m_{a}-\tau_{m \lambda} \cdot m_{m}-\tau_{g \lambda} \cdot m_{g}
$$

La diferencia entre la masa óptica de aerosoles y las masas ópticas de moléculas y de ozono se ha representado en la Figura I-11. Las diferencias se incrementan con el ángulo cenital solar. Esta expresión (1.14) de Langley 'modificado' (Forgan, 1994) requiere el conocimiento de las diferentes masas ópticas así como el contenido de los gases absorbentes que se desee considerar. Es habitual considerar el ozono por su influencia en las regiones ultravioleta y visible, en las que también tienen mayor influencia los aerosoles. De esta manera se minimizan los errores en la calibración derivados de los cambios en el contenido de ozono, aunque esto obliga a disponer de medidas auxiliares (Reagan et al., 1986).

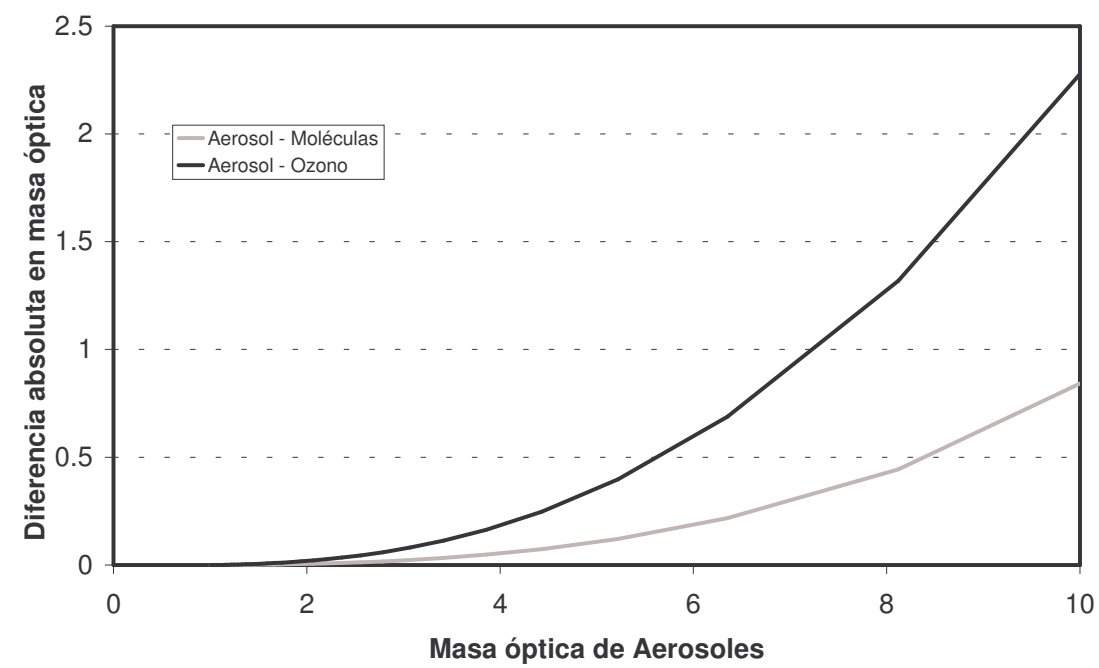

Figura I-11. Diferencia entre la masa óptica de aerosoles y la masa óptica para moléculas y ozono (Robinson, 1966).

Por otra parte la regresión lineal por el método de mínimos cuadrados en el método de Langley da más peso a las medidas en masas ópticas grandes (Herman et al., 1981). Por tanto se puede mejorar el cálculo estadístico mediante una modificación para que todas las observaciones que se consideren en el ajuste de Langley tengan el mismo peso (Langley 'pesado'). En Herman et al. (1981) se propone una metodología de ajuste pesado. También Forgan (2003) propone una pequeña variación sobre el método de Langley para 
evitar este problema estadístico, que consiste en dividir por la masa óptica de aerosoles. Si realizamos esta corrección sobre el Langley modificado, tenemos:

$$
\ln I_{\lambda} / m_{a}=\left(\ln I_{0 \lambda}-\tau_{m \lambda} \cdot m_{m}-\tau_{g \lambda} \cdot m_{g}\right) / m_{a}-\tau_{a \lambda}
$$

Esta modificación intercambia los papeles de pendiente y ordenada respecto del método de Langley clásico. Sin embargo sigue requiriendo una gran estabilidad en el contenido de aerosoles, y en el caso de adoptarse la versión modificada, también requiere el conocimiento del contenido de gases y las mencionadas masas ópticas de aerosoles, moléculas y gases absorbentes por separado.

El trabajo de Korotaev et al. (1993) también propone un método de calibración basado en el método de Langley. En este caso establece que el aerosol de fondo en la estación (background) no debe tener dependencia alguna con la altura solar, lo cual está de hecho implícito en el método de Langley. Partiendo de que un error en la constante de calibración provoca un error en el AOD que depende de la masa óptica, se puede variar el coeficiente de calibración hasta conseguir eliminar la dependencia de AOD con la altura solar, encontrando así el coeficiente de calibración correcto.

Sería deseable la introducción de mejoras que permitan realizar una buena calibración absoluta sin los requerimientos tan fuertes de estabilidad atmosférica. Diversos autores (por ejemplo Tanaka et al., 1986; Nieke et al., 1999) proponen el uso de medidas de radiancia en la aureola solar, bien para el control de la transmitancia atmosférica, bien como medidas complementarias que permitan realizar una calibración cuando no se dan las condiciones para la realización de un Langley. Los mismos autores ponen de relieve la dificultad principal con la que se enfrentan estos métodos, que es la correcta evaluación de la radiancia de la aureola en condiciones de scattering múltiple, lo que requiere complejos modelos de transferencia radiativa y diversas suposiciones. Las medidas de radiancia en la aureola son también muy sensibles a la precisión de posicionamiento del instrumento (Forgan, 1994).

El trabajo de Kremser (1984) propone una metodología basada en permitir que el espesor óptico de aerosoles varíe linealmente con el tiempo durante el ajuste de Langley. Este cambio es sustancial ya que todos los métodos mencionados basan la bondad de los resultados en que las variaciones que se produzcan en el AOD sean pequeñas y además aleatorias. La metodología de Kremser (1984) disminuye notablemente la dispersión en una serie de ajustes de Langley realizados en Tsumeb, Namibia. La variación que introduce sobre el Langley, permitiendo una variación lineal del AOD, consigue reducir el error de los coeficientes de calibración del 3\% al 1\%. De manera similar el trabajo de Terez and Terez (2003) permite una variación de AOD lineal con el tiempo durante el ajuste de Langley, llegando a un conjunto de calibraciones con una dispersión menor que el método de Langley ordinario.

Por otra parte Forgan (1994) propone dos métodos, denominados 'método general' y 'ratio-langley', aunque en este caso se trata de métodos relativos. A partir de un canal del instrumento que esté correctamente calibrado, se puede realizar una calibración de los demás canales aunque las condiciones atmosféricas no sean idóneas para la aplicación del método de Langley. Ambas metodologías están basadas en la fuerte correlación que se observa entre canales de AOD y la variación suave del AOD con la longitud de onda. La razón entre el AOD en dos canales puede permanecer casi inalterada aunque los valores absolutos del AOD cambien. Para ello basta que se mantenga la distribución de tamaños de aerosoles relativa, o lo que es lo mismo, que se mantenga el tipo de aerosol aunque varíe su cantidad. Las condiciones de estos métodos relativos son menos restrictivas por tanto que las calibraciones absolutas por el método de Langley. La expresión matemática 
de estos métodos consiste en la diferencia entre las ecuaciones del método de Langley (9) para dos canales diferentes, el de referencia con calibración conocida, y aquel cuya calibración queremos determinar. El método del 'ratio-langley' (1.16) emplea la diferencia directamente de la expresión del método de Langley (1.11), mientras que el 'método general' (1.17) emplea la ecuación del Langley modificado (1.14), en el que se consideran los diferentes componentes atmosféricos por separado. En este caso, $\Psi_{1 \mathrm{r}}$ es la razón entre el AOD en el canal de referencia ( $\mathrm{r}$ ) y en el canal que queremos calibrar (denotado con subíndice 1). Los subíndices '0' siempre se refieren a constantes de calibración.

$$
\begin{gathered}
\ln \left(I_{1} / I_{r}\right)=\ln \left(I_{01} / I_{0 r}\right)-\left(\tau_{1}-\tau_{r}\right) m \\
\Psi_{1 r} \cdot\left(m_{a} \tau_{r}\right)-\ln I_{01}=-\left(\ln I_{1}+m_{m} \tau_{m}+m_{g} \tau_{g}\right)
\end{gathered}
$$

En el trabajo de Soufflet et al. (1992) se realizaron medidas experimentales en una localidad en la que no se cumplían los requisitos para un Langley clásico. También plantea un procedimiento, en este caso iterativo, para encontrar la calibración de un fotómetro con una variación del método de Langley.

\subsubsection{Intercalibración o calibración por comparación con un instrumento de referencia}

La intercalibración consiste en trasladar a nuestro instrumento la calibración de otro que ya está previamente calibrado, que denominamos instrumento de referencia o master.

Se aplica con mayor éxito y facilidad entre instrumentos idénticos. Dos instrumentos distintos pueden diferir en resolución, tiempo para realizar la medida, campo de iluminación, rango espectral... Esto dificultaría enormemente el traslado de la calibración de uno a otro. Incluso entre instrumentos idénticos hay que extremar, por ejemplo, la exactitud del reloj, pues diferencias de pocos segundos empeoran sensiblemente el resultado, en particular si la transmitancia de la atmósfera no es muy estable.

El método consiste en realizar una serie de medidas simultáneas con los dos instrumentos, asegurando que las condiciones sean idénticas. Después los coeficientes $\mathrm{I}_{0 \lambda}$ se transfieren mediante un ajuste lineal: aplicamos la ley de Beer para el instrumento de referencia (ref) y para el que se quiere calibrar:

$$
\begin{aligned}
& I_{\lambda}(r e f)=I_{0 \lambda}(r e f) \cdot \exp (-\tau m) \\
& I_{\lambda}=I_{0 \lambda} \cdot \exp (-\tau m)
\end{aligned}
$$

Se hace el cociente y se despeja el coeficiente de calibración $\mathrm{I}_{0 \lambda}$ del instrumento que se pretende calibrar, construyendo con las medidas simultáneas realizadas la siguiente recta sin ordenada en el origen:

$$
I_{0 \lambda}=\frac{I}{I(r e f)} \cdot I_{0 \lambda}(r e f)
$$

El conjunto de medidas simultáneas debería abarcar todo el rango posible en la irradiancia solar directa, o lo que es equivalente, de masas ópticas. Sin embargo se tiende a una comparación sólo durante las horas centrales del día, con lo que no se abarca todo el rango dinámico del instrumento. Los errores de este método, en su evaluación analítica a partir de la recta de ajuste, se podrían minimizar teóricamente realizando un número elevado de observaciones simultáneas. Sin embargo traemos como ejemplo la calibración 
de instrumentos de la red AERONET por medio de intercomparación: frente a una precisión del $0,2 \%$ para los instrumentos de referencia (calibrados por el método de Langley) la precisión se evalúa en torno al $2 \%$ para los fotómetros calibrados por comparación con estos instrumentos de referencia, si bien es cierto que se consideran otras fuentes de error adicionales. Es decir, puede haber una incertidumbre mayor de la que teóricamente se deriva de la bondad del ajuste. No es recomendable por tanto trasladar calibraciones utilizando patrones de segundo, tercer, cuarto... orden pues se perdería precisión rápidamente. 


\section{Capítulo 2. Instrumentación: el fotómetro Cimel y la red AERONET. Lugar de medida y serie de datos}

\section{Introducción}

La base de este trabajo de tesis doctoral son las medidas experimentales del fotómetro Cimel en El Arenosillo, dentro de la red mundial de medida de aerosoles AERONET. En este capítulo se recoge la descripción de la red AERONET y su funcionamiento, así como las características de la estación de El Arenosillo y el conjunto de medidas realizadas. Dentro de las medidas de espesor óptico de aerosoles se ha detectado en algunos canales un ciclo diurno sistemático que vamos a analizar en detalle, puesto que su explicación ha resultado fundamental para poder asegurar la calidad de la serie de datos. 


\subsection{La red AERONET}

AERONET (Aerosol Robotic Network) es una federación de redes (AERONET norteamericana, PHOTONS de Francia y Aerocan de Canadá) de fotómetros para la medida de aerosoles con el objeto de estudiar sus propiedades ópticas y validar las medidas de satélite respecto de los aerosoles (Holben et al., 1998). La red está gestionada por el Goddard Space Flight Center (GSFC) de NASA (National Aeronautics and Space Administration) y el Laboratorio de Óptica Atmosférica (LOA) de la Universidad de Lille. Estos objetivos requieren el establecimiento de un estándar para los instrumentos, para la calibración y para el tratamiento de los datos.

La red se creó en 1998, y actualmente cuenta ya con más de 200 estaciones repartidas por todo el planeta, como vemos en la Figura II-1. La base de datos de la red proporciona información sobre el espesor óptico de aerosoles, vapor de agua, y diversos parámetros radiativos y microfísicos obtenidos mediante algoritmos de inversión en estaciones con muy diferentes condiciones y regímenes de aerosoles: marítimo, continental, ártico, de alta montaña, desértico, etc. Los datos recogidos se procesan en el GSFC. Gran parte de las estaciones en Europa y África occidental pertenecen a PHOTONS (PHOtométrie pour le Traitement Opérationnel de Normalisation Satellitaire, http://www-loa.univlille1.fr/photons).

El Grupo de Óptica Atmosférica de la Universidad de Valladolid, en colaboración con el LOA y el INTA instalaron en el año 2000 la primera estación de la red AERONET/PHOTONS de la Península Ibérica, situada en El Arenosillo (Huelva), cuyos datos de AOD son el objeto de este trabajo.

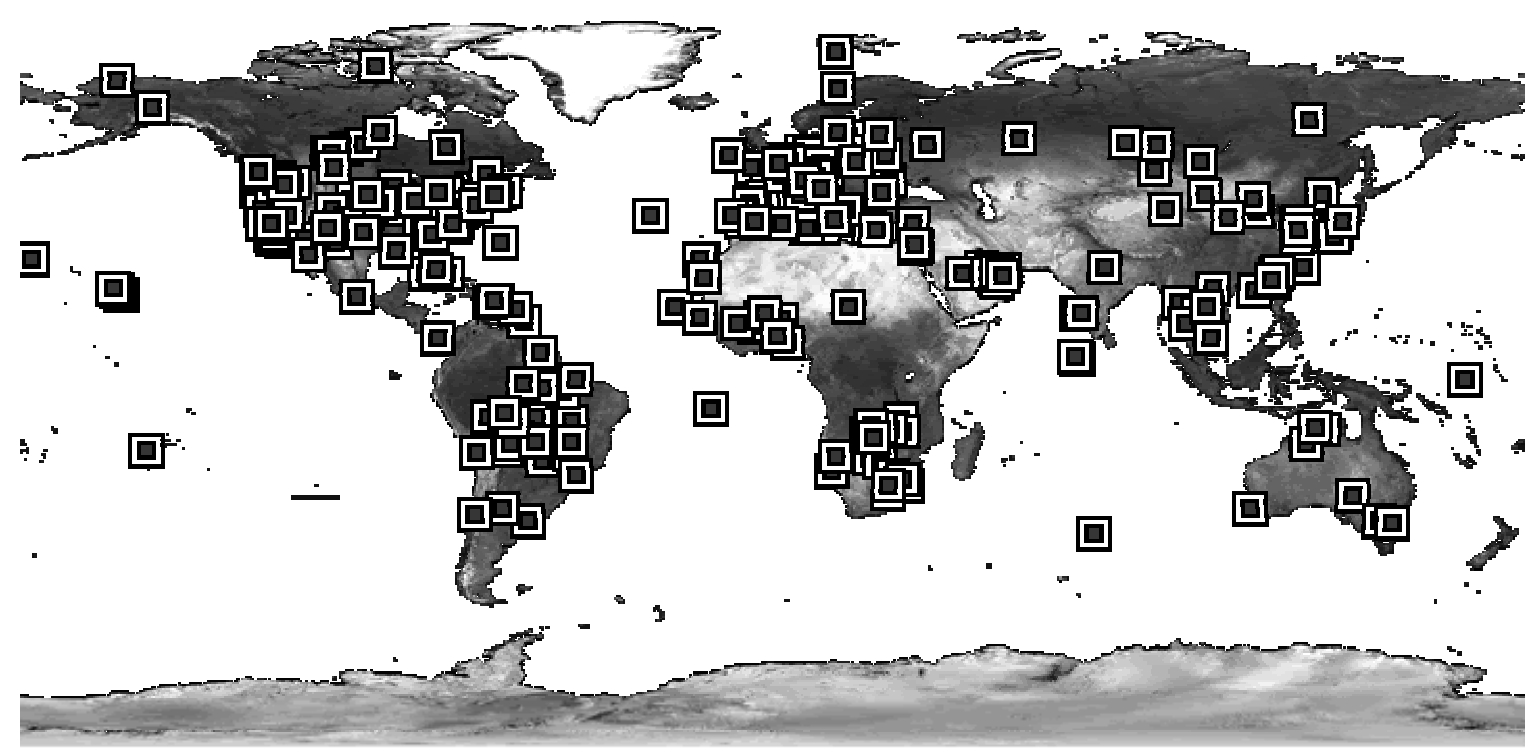

Figura II-1. Situación de los fotómetros CIMEL que pertenecen a la red AERONET (tomado de la web de AERONET).

Los datos de espesor óptico de aerosoles se clasifican en tres niveles de calidad. El nivel 1.0 está constituido por las medidas brutas. El nivel 1.5 es la serie de la que se han eliminado las medidas afectadas por nubosidad, mediante un algoritmo automático (Smirnov et al., 1998) desarrollado por la red -en inglés cloud-screening. El nivel 2.0 son los datos definitivos, una vez que se ha realizado una segunda calibración al instrumento al final del período en que haya estado en estación. Esto se comentará con más detalle al referirnos al protocolo de calibración. 
Los datos en cada uno de los tres niveles son archivados y distribuidos desde el GSFC, y están disponibles a través de la página web http://aeronet.gsfc.nasa.gov, en la que además se puede encontrar información actualizada sobre la red. En este trabajo sólo vamos a estudiar la serie de datos de espesor óptico de aerosoles y el parámetro alfa. El estudio de la serie de datos de radiancia relativa a las medidas de almucántar $^{1}$, plano principal y polarización se está desarrollando en otros trabajos.

\subsubsection{Instrumentación y funcionamiento}

El fotómetro Cimel-318 es el instrumento estándar de la red AERONET. La secuencia de medidas de todas las estaciones es idéntica, y se describirá en detalle posteriormente (Tabla II-III).

Estos datos se transmiten vía satélite hasta el Wallops Receiving Center en el caso del satélite GOES, y a Darmstadt (Alemania) en el caso del MeteoSat, a razón de 2 transmisiones de 1 minuto de duración durante cada hora. Cada estación tiene asignadas estas pequeñas ventanas de transmisión en las redes del GOES o del MeteoSat, lo que permite enviar unos 30kbytes por día. El transmisor empleado es un Vitel VX1004, modificado para ser empleado con el Cimel 318. La antena es cónica y de unos $40 \mathrm{~cm}$ de diámetro y otros $40 \mathrm{~cm}$ de longitud. El transmisor dispone de una batería que se recarga mediante un panel solar de 10W (ver Figura II-2).

Las transmisiones de satélite para cada estación se pueden seguir en tiempo real en la página web de AERONET, así como los avisos sobre posibles problemas, como por ejemplo la exactitud de hora del fotómetro y del transmisor, los voltajes de las baterías, etc.

\subsubsection{Procesamiento de los datos}

Una vez que los datos se encuentran en el GSFC, se les aplican los correspondientes coeficientes de calibración, para convertir los datos brutos (en cuentas digitales) en espesor óptico de aerosoles, vapor de agua para toda la columna y radiancia del cielo en unidades físicas. Estos datos constituyen el nivel 1.0 de AERONET, y pasan inmediatamente a la página web. Menos de dos horas después de ser tomada una medida, ésta puede ser consultada en Internet, por lo que la base de datos en el nivel 1.0 se considera disponible en tiempo real.

El espesor óptico de aerosoles se obtiene para cada longitud de onda a partir de la medida directa al sol, utilizando la Ley de Beer, pero trabajando siempre en cuentas digitales, pues el Cimel nunca llega a medir la irradiancia en unidades absolutas. El espesor óptico debido al scattering Rayleigh y la absorción del ozono y de otros gases atmosféricos se descuenta del espesor óptico total, para así aislar el espesor óptico debido a aerosoles. En el filtro de $1020 \mathrm{~nm}$ se corrige el efecto de la temperatura sobre el detector, a razón de $0,25 \% /{ }^{\circ} \mathrm{C}$ para lo que nos servimos del dato de la temperatura en la cabeza del fotómetro dada por el sensor de temperatura. El filtro de 670nm lleva una corrección por la absorción del ozono, que se calcula a partir de los valores climatológicos mensuales de ozono (1978-2004). E espesor óptico debido a scattering Rayleigh se corrige por la presión según la altura de la estación y mediante datos de análisis meteorológicos (con resolución temporal de 6h). Si

\footnotetext{
${ }^{1}$ En rigor el almucántar solar se denomina en castellano 'almicantarada'.
} 
estos datos no están disponibles se realiza una corrección basada en valores climatológicos mensuales (1993-2001). Durante la primavera de 2005 se ha implantado una nueva versión del algoritmo de AERONET para procesar las medidas de irradiancia directa solar. La comparación entre ambas versiones (nueva y antigua), con las correcciones que se realizan en cada una de ellas para cada canal se recogen en la Tabla II-I. En la actualidad se puede encontrar en la base de datos de AERONET los AOD calculados con ambas versiones (para más detalles se puede consultar Smirnov et al. (2004) y la página web de AERONET).

\begin{tabular}{lll}
\hline \hline Longitud de onda nominal $(\mathrm{nm})$ & Versión 1 & Versión 2 \\
\hline $340(2 \mathrm{~nm})$ & Rayleigh, O3 & Rayleigh, NO2, O3 \\
$380(4 \mathrm{~nm})$ & Rayleigh & Rayleigh, NO2 \\
$440(10 \mathrm{~nm})$ & Rayleigh & Rayleigh, NO2 \\
$500(10 \mathrm{~nm})$ & Rayleigh, O3 & Rayleigh, NO2, O3 \\
$675(10 \mathrm{~nm})$ & Rayleigh, O3 & Rayleigh, O3 \\
$870(10 \mathrm{~nm})$ & Rayleigh & Rayleigh \\
$940(10 \mathrm{~nm})$ & AOD interpolado 870-1020nm & AOD extrapolado 675,870 a 940nm \\
$1020(10 \mathrm{~nm})$ & Rayleigh & Rayleigh, H2O \\
$1640(25 \mathrm{~nm})$ & Rayleigh & Rayleigh, H20, C02, CH4 \\
\hline \hline
\end{tabular}

Tabla II-I. Conjunto de correcciones espectrales para el cálculo del AOD y vapor de agua a partir de las medidas directas al sol del Cimel, para las versiones 1 y 2 del algoritmo de procesado estándar de AERONET. En la primera columna se indican los filtros y entre paréntesis la anchura a altura mitad de los mismos.

Las medidas de radiancia del cielo (almucántar y plano principal) proporcionan información sobre la luz difundida por scattering para diferentes ángulos de dispersión. Éstos se utilizan, en combinación con las medidas de extinción, como datos de entrada en el algoritmo de inversión (Dubovik and King, 2000a; Dubovik et al., 2000b) que se utiliza en la red para obtener la distribución de tamaños de los aerosoles, y otros parámetros microfísicos y radiativos, como el radio efectivo, el radio medio, la función de fase, el índice de refracción, el parámetro de asimetría o el albedo de scattering simple, etc. Actualmente la red proporciona un amplio conjunto de parámetros sobre los aerosoles.

El siguiente paso que se realiza en la red es la eliminación de las medidas afectadas por nubosidad, para alcanzar el nivel 1.5. Se ha desarrollado un algoritmo para seleccionar de manera automática las medidas libres de nubes, como ya se ha mencionado anteriormente. Esto resultaba imprescindible dado el volumen de datos que se maneja diariamente y dado que los Cimel puestos en estación miden, independientemente de que haya nubes o no.

Para ello, en primer lugar, se eliminan las medidas con valores de AOD<-0.01, por considerarse imposible físicamente. Esto se puede deber, según Smirnov et al. (2000) a "problemas de calibración, de corrección de temperatura en el filtro de 1020nm, o por incertidumbre en la medida de la presión, o del ozono". El dato de la presión, necesario para evaluar correctamente el espesor óptico que corresponde al scattering Rayleigh, difícilmente se aleja más de un $3 \%$ de la presión real, por lo que este error sólo sería detectable con valores bajísimos de AOD. También se eliminan las medidas que corresponden a masas ópticas mayores que cinco, que corresponden con las medidas del Langley con el sol más bajo, por la mañana y por la tarde.

En segundo lugar, se sirven de los tripletes de observaciones del Cimel, que toma 3 medidas para cada longitud de onda con un intervalo de 30s entre ellas. La variación temporal de los aerosoles es típicamente mucho menor que la debida a las nubes, por lo que variaciones por encima de un cierto umbral (0.02 en espesor óptico) en un triplete 
indican que está contaminado por nubosidad. En segundo lugar, las medidas están tomadas cada 15 minutos, lo cual permite identificar variaciones de frecuencia más baja. Para evitar que nubes tenues y estables, por ejemplo cirros, escapen a esta selección, el algoritmo utiliza hasta 3 criterios más, que en general parten del principio de que los aerosoles varían con el tiempo de manera más suave que las nubes.

Las medidas libres de nubes constituyen el nivel 1.5 de la base de datos de AERONET. Sólo después de una segunda calibración del fotómetro, una vez que ha sido retirado de la estación y sustituido por otro, se pueden pasar estos datos al nivel 2.0. Éste se consigue reprocesando los datos del nivel 1.5 mediante una interpolación lineal entre los coeficientes de precalibración y poscalibración. Sólo cuando se ha comprobado la calidad de los datos, se elevan al nivel 2.0.

\subsubsection{Protocolo de calibración}

La calibración es otro de los problemas fundamentales que se plantean al gestionar una red mundial con un número grande de estaciones. Todos los instrumentos se calibran bajo el mismo protocolo y, en un principio, en el Goddard Calibration Facility. Desde el año 2003 también está operativo otro centro de calibración dependiente del LOA, y que se encarga de los aparatos integrados en PHOTONS, que es parte de AERONET. Debemos destacar que los procedimientos de calibración relativos al AOD y vapor de agua (medidas de radiación solar directa) son muy diferentes a los relativos a la radiancia del cielo.

Dos Cimel de referencia (master) se calibran por el método de Langley en el Observatorio Atmosférico de Izaña (Tenerife, $2360 \mathrm{~m}$ s.n.m.). Anteriormente estas calibraciones absolutas se llevaban a cabo en Pic du Midi (en el Pirineo francés), donde las condiciones atmosféricas no son tan favorables. La intercalibración entre los master y los instrumentos de campo se realiza en Carpentras, al sur de Francia. La calibración en radiancia y polarización se realizan en el en LOA (Lille, Francia). Realmente es en la red PHOTONS donde está integrado el Cimel de El Arenosillo y también la otra estación Cimel de que disponemos desde 2003 en la E.T.S. de Ingenieros Agrónomos, en Palencia.

Para calibrar el Cimel 318 hay que determinar una serie de coeficientes necesarios para convertir la salida del instrumento (en cuentas digitales, $\mathrm{CN}$ ) en espesor óptico de aerosoles, vapor de agua y radiancia en $\mathrm{W} /\left(\mathrm{m}^{2} \mathrm{sr} \mathrm{nm}\right)$.

Los canales de medida directa al sol se calibra por intercomparación con instrumentos patrón. Éstos a su vez, se calibran cada tres meses por el método de Langley en el observatorio de Mauna Lo ó Izaña. Mediante la representación de Langley se obtiene el voltaje extraterrestre (en cuentas) para cada uno de los filtros. Este voltaje extraterrestre $\left(\mathrm{CN}_{0}\right)$ se determina con un error entre el 0.2 y el $0.5 \%$, dependiendo del canal, para los instrumentos patrón. Esta incertidumbre sobre el $\mathrm{CN}_{0}$ se traduce en un error absoluto de 0.002 en espesor óptico de aerosoles para los instrumentos patrón.

Los fotómetros de las distintas estaciones se calibran por comparación con estos instrumentos patrón, tanto antes como después de su período de operación en una estación determinada. Se considera que el procedimiento de intercalibración se traduce en un error absoluto sobre el espesor óptico de aerosoles de 0.01-0.02, dependiendo de los canales, para los instrumentos ordinarios de las distintas estaciones de la red (Holben et al., 1998). Este error tiene en cuenta, además del procedimiento de intercalibración, los errores derivados de introducir valores climatológicos para el ozono y la presión, en lugar de realizar en el procesado correcciones con valores observados. 
Para asegurar al menos esta precisión los instrumentos se calibran cada 12 meses aproximadamente. Con respecto a la estabilidad de la calibración, los elementos que peor se comportan son los filtros interferenciales. En promedio se degradan entre un $1 \%$ y un $10 \%$ por año. Al aplicar una interpolación entre los calibrados anterior y posterior al período de medidas, se asume un deterioro lineal para estos filtros. Cada tres años se reemplazan por otros nuevos, o también si se han degradado más del $5 \%$ en un año.

Para calibrar los canales que miden la radiancia del cielo se utilizan unos protocolos diferentes. Es necesario utilizar una esfera integradora que proporcione una superficie con radiancia conocida y uniforme. Estas calibraciones se llevan a cabo tanto en GSFC como en LOA. No entramos en detalles sobre este procedimiento ya que no emplearemos las medidas de radiancia en este trabajo. En este caso las medidas in situ no permiten un seguimiento de la calibración, al contrario de lo que ocurre con las mediadas de sol directo. El canal para la medida del vapor de agua, $936 \mathrm{~nm}$, se calibra mediante un procedimiento de Langley modificado, y su error se estima menor del 12\% (Holben et al., 1998).

Como se puede inferir de todo esto, en cada estación opera un instrumento de la red durante un año aproximadamente. Tras este período, desde el GSFC se envía un nuevo fotómetro recién calibrado, y el que estaba instalado se envía de vuelta para una calibración poscampaña. Los instrumentos, todos ellos etiquetados con un número de la red, van pues rotando por diferentes estaciones, y en una misma estación la serie de datos se va conformando con diferentes fotómetros. Esto presenta la ventaja de evitar sesgos por culpa de defectos en un fotómetro concreto, y hace a la vez más exigente la condición de continuidad de la serie a lo largo del tiempo. 


\subsection{Descripción del fotómetro Cimel 318}

El instrumento Cimel-318 (Figura II-2) es un fotómetro automático y portátil, que está diseñado para medir irradiancia solar y radiancia del cielo en ciertas longitudes de onda para obtener distintas propiedades que definen las características microfísicas y radiativas de los aerosoles en la atmósfera, comenzando por el espesor óptico de aerosoles. También se determina el contenido de vapor de agua sobre la vertical atmosférica, aunque nosotros en este trabajo nos centramos en el estudio de los aerosoles.

El fotómetro Cimel Electronique-318 (Cimel, 2004), fabricado en Francia por la firma Cimel, es el instrumento estándar de la red AERONET. Su diseño es adecuado para estar integrado en una red en varios sentidos. En primer lugar, la mayoría de las redes de fotómetros solares han tenido un éxito limitado cuando se requiere que los técnicos realicen observaciones de rutina. Por eso un instrumento automático es un componente fundamental para cualquier red donde se precisen estas observaciones. El fotómetro Cimel es además autónomo, puesto que se alimenta mediante un panel solar y está preparado para soportar la intemperie. En segundo lugar, opera con un protocolo que permite que los datos que no se necesitan puedan ser separados de los que son útiles. La importancia de esto radica en que es un instrumento automático, que va a medir tanto en condiciones de cielo nuboso como despejado. Entre las numerosas medidas se precisa extraer las que no estén afectadas por nubosidad. Por último, el instrumento recoge datos para el seguimiento de su calibrado, cuestión que resulta fundamental para este trabajo, como veremos.

\subsubsection{Características del fotómetro Cimel}

Este instrumento dispone de dos detectores de silicio, uno para la medida directa al sol (irradiancia solar) y otro para la medida de la radiancia del cielo, instalados dentro de la cabeza sensora, que está convenientemente sellada y resecada para prevenir daños en los componentes. Además de los detectores, en el interior de la cabeza hay un sensor de temperatura, que permite medir la temperatura para una posterior corrección en el filtro de $1020 \mathrm{~nm}$, y una rueda con 8 filtros interferenciales, que se mueven con un motor paso a paso. A la cabeza se acoplan dos tubos limitadores de campo de $33 \mathrm{~cm}$. El sistema final tiene un FOV (Field Of View, campo de iluminación media) de $1.2^{\circ}$ para ambos tubos. La cabeza del sensor se monta de tal forma que la óptica se proteja de la lluvia y de la entrada de partículas extrañas en el sistema en la posición no activa (PARK).

El final del tubo limitador de campo para la medida directa al sol está protegido por una ventana de cuarzo, permitiendo la observación con un detector UV con la suficiente relación señal-ruido para observaciones espectrales desde aproximadamente $300 \mathrm{~nm}$ hasta $1020 \mathrm{~nm}$. El colimador para la medida del cielo tiene el mismo campo de iluminación media, pero una apertura aproximadamente 10 veces mayor obtenida con la ayuda de lentes para conseguir un mejor rango dinámico en la medida de la radiancia del cielo (almucántar y plano principal).

El Cimel 318-1, fotómetro estándar (no polarizado), dispone de 8 filtros entre 340 y 1020nm (Tabla II-II). La anchura a altura mitad de estos filtros es de 10nm para los filtros del visible e infrarrojo y menor de $5 \mathrm{~nm}$ para los filtros en el ultravioleta (ver Tabla II-I). La versión 318-2 dispone de 3 filtros de $870 \mathrm{~nm}$ a los que se anteponen polarizadores con $120^{\circ}$ entre sus ángulos de polarización (870 P1, P2 y P3), lo que permite realizar un análisis de polarización de la luz. El filtro de $936 \mathrm{~nm}$ se emplea para evaluar el vapor de 
agua. El resto de filtros están escogidos en zonas del espectro sin apenas absorciones por parte de componentes atmosféricos para facilitar la obtención del espesor óptico de aerosoles.

\begin{tabular}{|l|l|l|l|l|l|l|l|l|}
\hline Cimel 318-1 & 1020 & 870 & 670 & 550 & 440 & 380 & 340 & 936 \\
\hline Cimel 318-2 & 1020 & 870 & 670 & 440 & $\mathbf{8 7 0}$ P1 & $\mathbf{8 7 0}$ P2 & $\mathbf{8 7 0}$ P3 & 936 \\
\hline
\end{tabular}

Tabla II-II. Filtros en las dos versiones del Cimel 318 (en nm)
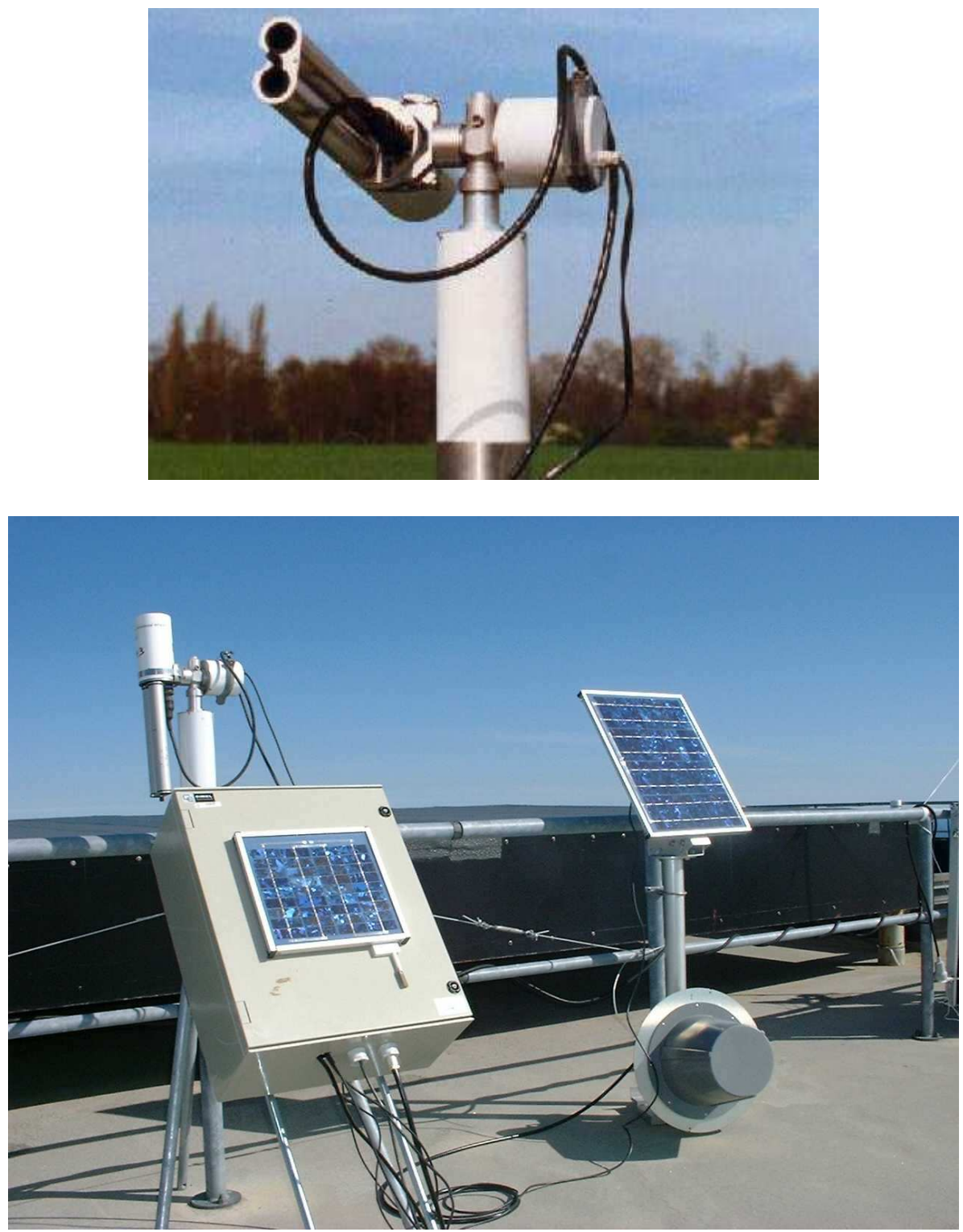

Figura II-2. Fotografías del fotómetro Cimel 318: realizando una medida (imagen superior) y en la posición PARK, junto con el panel solar y la antena para transmisión al satélite (imagen inferior).

Durante 2005 la casa Cimel ha desarrollado una nueva versión de su fotómetro, cuya principal innovación consiste en la incorporación de un nuevo canal en el infrarrojo, en la longitud de onda de $1640 \mathrm{~nm}$. Este canal ha obligado a la incorporación de un detector adecuado para ese rango. Este nuevo modelo introduce también cambios en la electrónica, el FOV de los colimadores y la velocidad para realizar las medidas. De momento este nuevo modelo se está empezando a implantar en la red AERONET. 
El robot seguidor, en el que está montada la cabeza con los tubos limitadores, tiene dos motores de transmisión directa que dirigen la cabeza del sensor en los ángulos de acimut y cenital con una exactitud de $0.05^{\circ}$. Un microprocesador calcula la posición del sol basándose en coordenadas del tiempo y de localización, y dirige la cabeza del sensor hacia el sol con una precisión de $1^{\circ}$, después de lo cual un detector de cuatro cuadrantes apunta al sol con una precisión de $0.1^{\circ}$ precisamente en el instante previo a una medida programada. Después de completar una medida de rutina, el instrumento vuelve a una posición de reposo, esperando la siguiente secuencia de medida. Un sensor de humedad expuesto a la precipitación cancelará cualquier secuencia de medida en caso de activarse, y dejará el instrumento en posición de reposo (PARK), con los tubos mirando hacia abajo para evitar que el agua afecte a las lentes (Figura II-2). El sensor consiste simplemente en un circuito impreso de carácter resistivo, con una serie de pistas que las gotas de agua comunican entre sí, enviando una señal que inhibe la secuencia automática y mantiene el robot con los colimadores apuntando hacia abajo para que la lluvia resbale y no penetre en ellos.

El robot seguidor tiene un tamaño de $40 \mathrm{~cm}$ y un peso de $15 \mathrm{~kg}$. La caja de control, las baterías y el transmisor se instalan en una caja estanca de $30 \times 62 \times 46 \mathrm{~cm}$, lo que supone un peso de otros $22 \mathrm{~kg}$.

\subsubsection{Secuencia automática de medidas}

El fotómetro tiene la suficiente relación señal-ruido para que se puedan hacer observaciones desde 300 hasta 1030nm. La medida básica directa al sol se compone de tres observaciones espectrales (triplete) tomadas con un intervalo de 30 segundos. El motivo para tomar las medidas basándose en tripletes es que la variación temporal de las nubes es típicamente mayor que la de los aerosoles y se manifestaría en una variación observable entre las tres medidas del triplete, lo que sirve para eliminar las medidas afectadas por nubosidad, como ya se ha comentado. La triple observación se hace durante las secuencias de calibrado Langley de la mañana y la tarde, y a intervalos de 15 minutos durante el centro del día. La secuencia Langley consiste en medidas realizadas a masas ópticas fijas, entre $\mathrm{m}=7$ y $\mathrm{m}=2$. Cada noche el fotómetro calcula las horas para las medidas del día siguiente. Las medidas entre la masa óptica 2 de la mañana y la de la tarde se realizan a intervalos regulares de 15 minutos en torno al mediodía solar local, y por tanto sus horas están calculadas a partir de la hora solar verdadera (true solar time, TST). Además del reloj en hora GMT, el fotómetro tiene un reloj en TST con el que gobierna las medidas de la secuencia automática, que se recoge en la Tabla II-III. Esta secuencia con un Langley cada mañana y cada tarde es la que permite el seguimiento de la calibración.

Las secuencias de medidas básicas del cielo son el almucántar y el plano principal. Llamamos almucántar a una serie de medidas tomadas con un ángulo cenital constante, y ángulos de acimut específicos en relación con la posición del sol. Durante la secuencia de medida para uno de los cuatro canales de aerosoles, el instrumento hace una medida del sol, comienza el barrido de la aureola a través del disco solar y continúa $180^{\circ}$ grados de acimut primero hacia la derecha y tras centrarse de nuevo en el sol, repite hacia la izquierda. La secuencia de plano principal opera de la misma forma pero en el plano principal del sol (ángulo acimutal constante), y barriendo distintos ángulos de elevación desde $6^{\circ}$ por debajo del sol hasta $150^{\circ}$. Esto se repite también para cada uno de los cuatro filtros de aerosoles. 


\begin{tabular}{cc}
\hline \hline Denominación & Secuencia de medidas \\
\hline Grupo A & 3 SUN; 3 SKY \\
Grupo B & 3 SUN; 3 SKY; ALMUC; BLACK \\
Grupo C & 3 SUN; 3 SKY; ALMUC; PP; PPP*; BLACK \\
Grupo D & 3 SUN \\
Grupo E & 3 SUN; PP \\
Grupo F & 3 SUN; ALMUC; PP; PPP; BLACK \\
\hline \hline
\end{tabular}

\begin{tabular}{cccc}
\hline \hline Masa óptica & Medida & Hora $($ TST) & Medida \\
\hline $7 ; 6.5 ; 6 ; 5.5 ; 5 ; 4.5$ & Grupo A & $9: 45$ & Grupo D \\
4 & Grupo B & $10: 00$ & Grupo E \\
$3.8 ; 3.6 ; 3.4 ; 3.2$ & Grupo A & $10: 15$ & Grupo D \\
3 & Grupo C & $10: 30$ & Grupo D \\
$2.8 ; 2.6 ; 2.4 ; 2.2$ & Grupo A & $10: 45$ & Grupo D \\
2 & Grupo B & $11: 00$ & Grupo E \\
Hora (TST) & & $11: 15$ & Grupo D \\
$9: 00$ & Grupo E & $11: 30$ & Grupo D \\
$9: 15$ & Grupo D & $11: 45$ & Grupo D \\
$9: 30$ & Grupo D & $12: 00$ & Grupo F \\
\hline \hline
\end{tabular}

Tabla II-III. Secuencia automática de medidas del Cimel 318 hasta el mediodía solar. La secuencia es simétrica por la tarde. SUN: medida directa al sol; SKY: radiancia a $6^{\circ}$ (aureola); ALMUC: almucántar; PP: plano principal; PPP: plano principal polarizado; BLACK: medida de corriente oscura. *PPP sólo para el fotómetro polarizado 318-2.

\subsubsection{Salida de datos}

Los datos se almacenan en primer lugar en la memoria del fotómetro, para pasar luego a un transmisor desde el cual se pueden enviar a uno de los 3 satélites geosíncronos GOES, METEOSAT o GMS, y desde allí a la estación receptora terrestre apropiada. Este transmisor se sirve de una antena como vemos en la Figura II-2, y se alimenta, igual que el fotómetro, mediante un panel solar. Junto con los datos se envía un informe de estado relativo a los voltajes de las baterías, temperatura, sensor de humedad y, si es el caso, de posibles errores en el robot, en la rueda de filtros, etc.

Los datos también se pueden recuperar mediante un PC, utilizando el software ASTP-Win proporcionado por la casa Cimel. Esta descarga de datos se puede hacer manualmente o de manera programada, siempre con una frecuencia diaria, puesto que la memoria del fotómetro almacena aproximadamente un día y medio de medidas. Los datos no se borran al vaciar la memoria, sino que de manera circular se van sobreescribiendo los más antiguos.

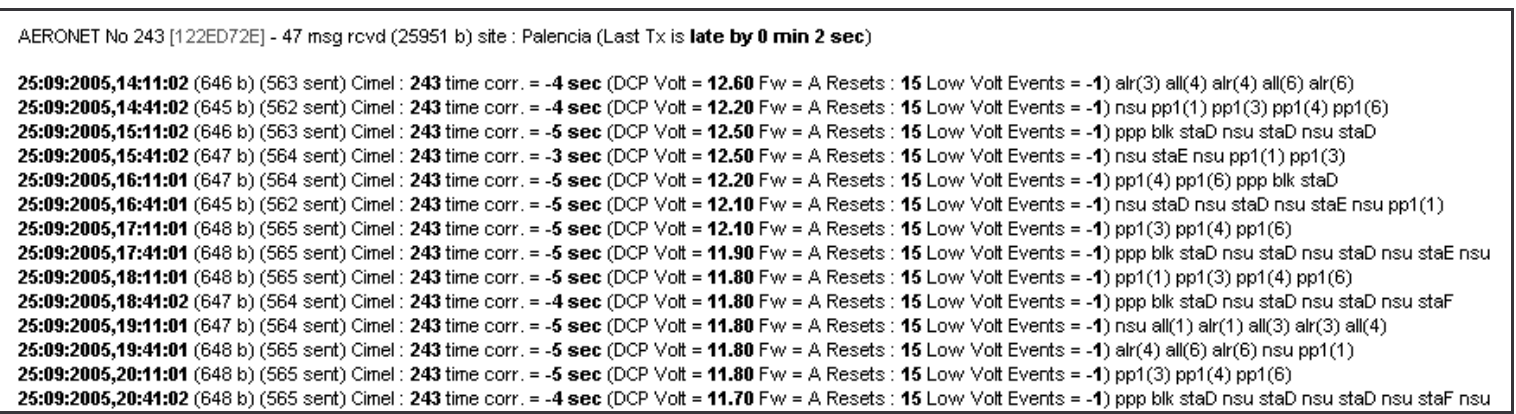

Figura II-3. Ejemplo de informe de transmisiones del satélite en AERONET del Cimel de Palencia.

En la página web de AERONET se pueden consultar los informes de las transmisiones, los cuales se generan cada hora de manera automática. En ellas se puede comprobar el estado de los relojes del fotómetro y del transmisor vía satélite, pues éstos se comparan con la hora del Meteosat. También se puede consultar el número de mensajes enviados en las 
últimas $24 \mathrm{~h}$, los bytes enviados, el voltaje de las baterías del transmisor, y las medidas enviadas en cada transmisión (Figura II-3). Antes de cada medida el Cimel realiza un chequeo ('status') en el que se prepara para hacer la medida. Si es correcto se guarda un pequeño informe, que anuncia el tipo de medida a realizar: staA para una medida del Grupo A, staB para el Grupo B, etc. (ver Tabla II-III). Después del mensaje de 'status' se envían las medidas correspondientes. En caso de detectarse algún error se genera también un mensaje de 'status'. Estos mensajes de error y su significado están en la Tabla II-IV.

\begin{tabular}{ll}
\hline \hline 'Status' aviso & Incidencia \\
\hline stab & Error de Robot \\
star & Error de Reset \\
stah & Sensor de humedad activado* \\
stas & Error de la rueda de filtros \\
\hline \hline
\end{tabular}

Tabla II-IV. Diferentes 'status' de incidencias que pueden aparecer en los informes automáticos de transmisiones de AERONET. *La activación del sensor de humedad no implica la existencia de un problema con el instrumento. Si el sensor está en correcto funcionamiento, indica que la secuencia automática de medidas se ha interrumpido debido a la humedad. Cuando el sensor se seca la secuencia se retoma automáticamente.

Todo este sistema de control de los datos, junto con la visualización y descarga de todas las medidas que realiza el Cimel, es accesible no sólo a través de la web sino a través de una interfaz mucho más potente, llamada 'Demonstrat'. Los servidores del GSFC y LOALille disponen de esta aplicación a través de la cual se puede consultar toda la metabase de datos: magnitudes de control, historial de las calibraciones, tipo de fotómetro, etc. Permite también una visualización cómoda de los datos, incluyendo AOD, parámetro alfa, almucántar y planos principales, productos de las inversiones... También permite realizar ajustes de Langley, comparaciones entre instrumentos que han medido conjuntamente e intercalibraciones. Es una herramienta diseñada para gestionar la red de manera integral. 


\subsection{Estación de medida: El Arenosillo. Serie de datos}

\subsubsection{La estación de Sondeos Atmosféricos de El Arenosillo}

La Estación de Sondeos Atmosféricos (ESAT) de El Arenosillo $\left(37.1^{\circ} \mathrm{N}, 6^{6} .7^{\circ} \mathrm{E}\right)$, que pertenece al INTA (Instituto Nacional de Técnica Aeroespacial), se encuentra unos $30 \mathrm{~km}$ al este de la ciudad de Huelva, en el entorno del Parque Nacional de Doñana, prácticamente al nivel del mar (17m de altitud). Tenemos una vista del edificio en la Figura II-4.

El Arenosillo cuenta con numerosos instrumentos radiométricos instalados permanentemente. El más antiguo es el Dobson \#120, con más de 25 años de observaciones continuadas de ozono. Además opera un Brewer desde 1997, el \#150, para la medida de radiación UV y del ozono; un piranómetro Yankee Environmental System, para la medida de la irradiancia integrada en el UV (eritémica); un radiómetro de la red Eldonet para la medida de la radiación PAR (Photosintetic Active Radiation) y componente A y $\mathrm{B}$ de la radiación ultravioleta; y el fotómetro Cimel 318, objeto de este trabajo.

La instrumentación está instalada en una terraza plana, con excelentes condiciones para las medidas de radiación, en particular por el horizonte sin obstáculos y por el pinar que rodea la estación. Este pinar confiere unas características muy especiales en cuanto al albedo, que se mantiene muy uniforme y en un valor bajo durante todo el año (Figura II$5)$.

Además las condiciones climáticas en El Arenosillo son muy favorables, con un promedio de 156 días despejados por año y un total de 2998 horas de sol al año, lo cual significa el $68 \%$ del número total de horas en las que el sol está por encima del horizonte ${ }^{2}$.

Por otra parte durante los últimos años se ha realizado una fuerte inversión en instrumentación para poner en marcha la medida de aerosoles in situ en la estación. En este momento ya se encuentran operativos dos espectrómetros de la casa TSI para la medida in situ de distribuciones de tamaños de aerosoles, uno en el rango micrométrico partículas mayores de $1 \mu \mathrm{m}$-, llamado APS (Aerodynamic Particle Sizer) y otro en el rango submicrométrico, llamado SMPS (Scanning Mobility Particle Sizer). También operan un impactor en cascada de 4 niveles de la casa Dekati para la medida de niveles másicos (Mogo et al., 2005), y un nefelómetro para la medida del coeficiente de scattering de aerosoles.

Esto aumenta la capacidad de la estación para el estudio integral de los aerosoles atmosféricos en la zona de estudio, a través de la complementariedad de las medidas radiométricas y de las medidas in situ.

En la estación de El Arenosillo se llevan adelante también, en diferentes proyectos, otro tipo de medidas que pueden ser también útiles para nosotros, como la vigilancia continuada del ozono troposférico y otros gases traza, datos meteorológicos, etc.

${ }^{2}$ Fuente: Instituto Nacional de Meteorología, valores climatológicos normales 1971-2000. 


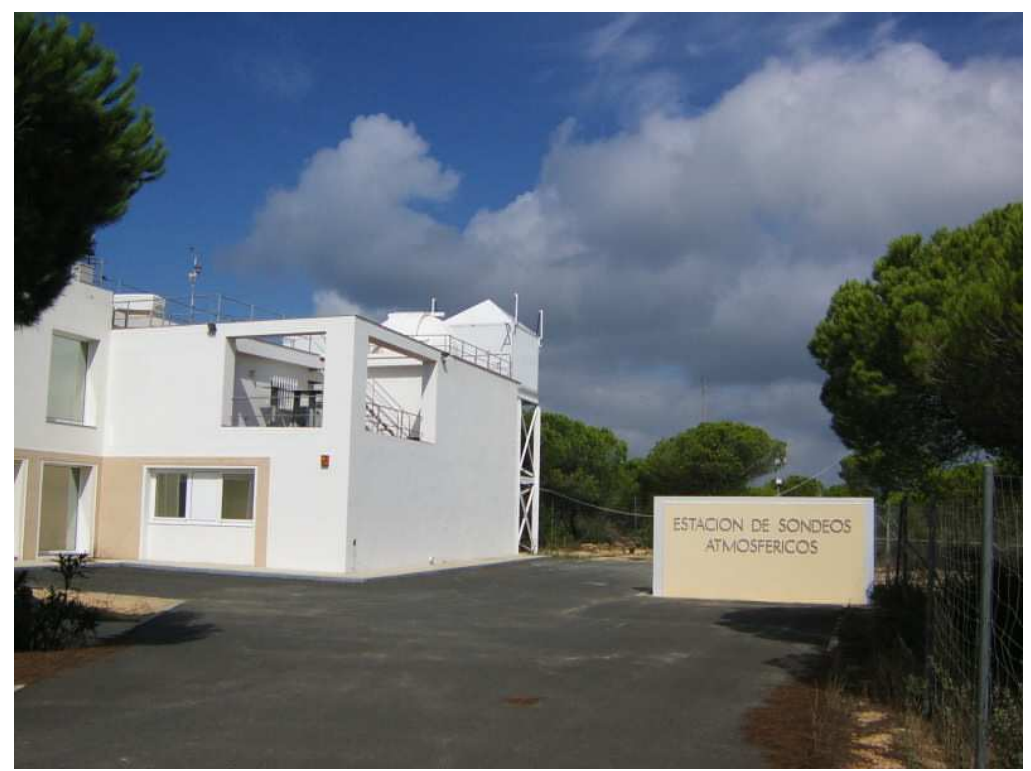

Figura II-4. La estación de Sondeos Atmosféricos de El Arenosillo (Huelva), perteneciente al INTA.

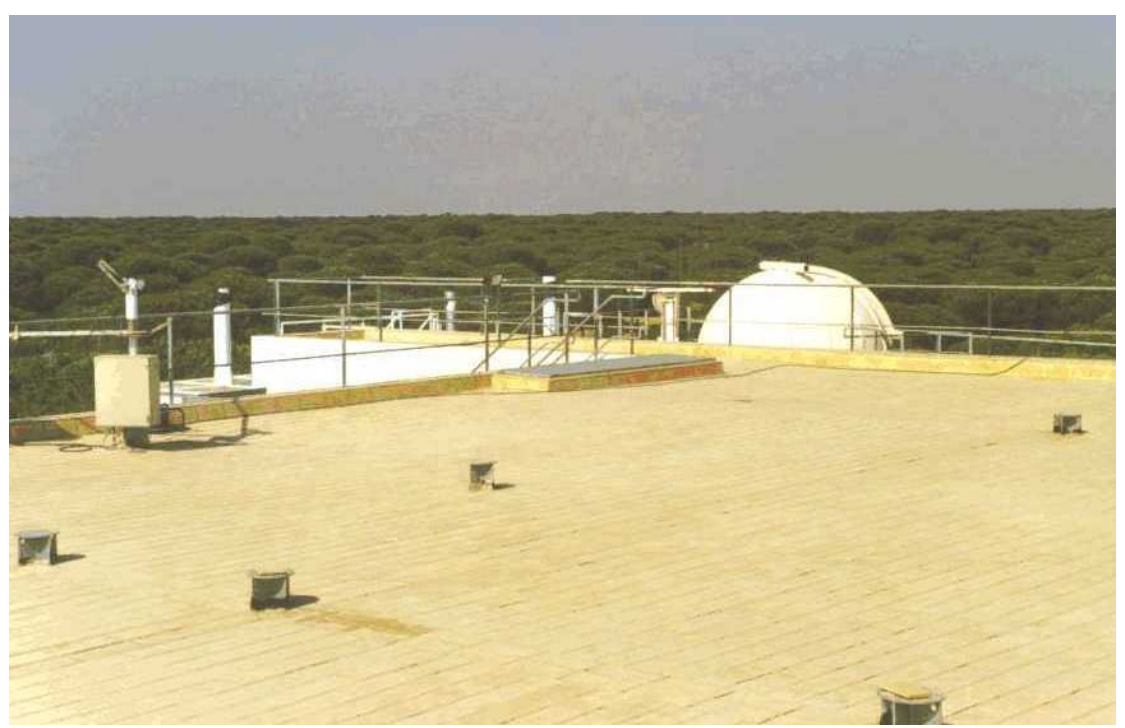

Figura II-5. Vista de la terraza de la estación de El Arenosillo con el fotómetro Cimel.

\subsubsection{Calendario de las medidas realizadas}

El Cimel está operativo desde febrero de 2000. Los distintos aparatos que han estado instalados, con su numeración de AERONET, están en la Tabla II-V.

\begin{tabular}{lccc}
\hline \hline Instrumento & Fecha inicio & Fecha final & Base de datos \\
\hline Cimel \#48 & $16 / 02 / 2000$ & $9 / 07 / 2000$ & Nivel 2.0 \\
Cimel \#114 & $10 / 07 / 2000$ & $21 / 07 / 2001$ & Nivel 1.5 \\
Cimel \#45 & $22 / 07 / 2001$ & $27 / 01 / 2003$ & Nivel 2.0 \\
Cimel \#50 & $27 / 01 / 2003$ & $12 / 05 / 2004$ & Nivel 2.0 \\
Cimel \#45 & $12 / 05 / 2004$ & $\ldots$ & Nivel 1.5 \\
\hline \hline
\end{tabular}

Tabla II-V. Distintos instrumentos Cimel que han operado en la estación de El Arenosillo.

El Cimel \#45 es el fotómetro que está operativo en la actualidad. Es la segunda ocasión que se instala en El Arenosillo, y sus datos están en el nivel 1.5, a la espera de la segunda calibración. Los datos del Cimel \#114 no ha alcanzado la calidad necesaria para el nivel 2.0 incluso después de la poscalibración. Los problemas con este instrumento son uno de 
los puntos clave que vamos a tratar en este trabajo. Los datos tomados con los otros dos instrumentos sí pasaron al nivel 2.0 después de la segunda calibración.

El número de medidas individuales hasta el 31 de diciembre de 2004 supera las 54.000 en el nivel 1.0. Una vez eliminadas las medidas afectadas por nubosidad, quedan en la base de datos del nivel 1.5 (y en su caso del 2.0) unas 39.000 observaciones, lo cual representa el $72 \%$ del total. En septiembre de 2005 ya se han superado las 70.000 observaciones en nivel 1.0 .

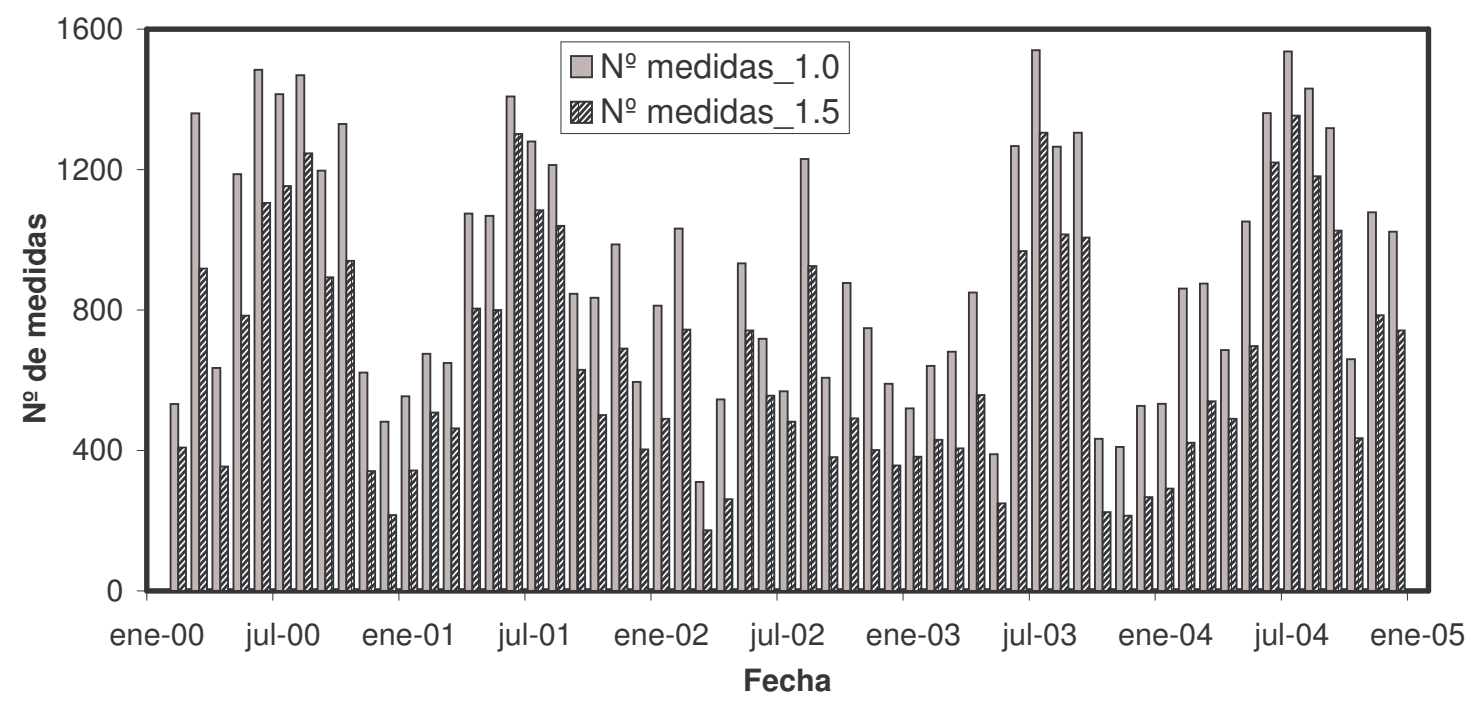

Figura II-6. Número de medidas por mes del Cimel en El Arenosillo hasta el 31 de diciembre de 2004, tanto las medias brutas (nivel 1.0) como las medidas libres de nubosidad (nivel 1.5).

En la Figura II-6 podemos ver el número de medidas del fotómetro Cimel para cada mes desde febrero de 2000 hasta diciembre de 2004. Recordamos que éstas son solamente las medidas directas al sol. Junto a ellas se realizan también las medidas de radiancia de cielo (almucántares, planos principales). Durante los meses de verano, con una mayor disponibilidad de horas, el número de medidas asciende por encima de las 1200 (unas 40 al día en promedio), mientras que en los meses de invierno este número de medidas se reduce a la mitad. El número total de días con medidas brutas en nivel 1.0 es de 1557 respecto al total de 1781 (el 87\%). Una vez eliminadas las nubes tenemos más de 3 medidas libres de nubosidad en 1346 días. Esto quiere decir que en la estación de El Arenosillo existe una medida útil para el AOD en el 75\% de los días.

Como ejemplo mostramos a continuación los datos de un día de medidas, tal y como se puede encontrar directamente en la página web de PHOTONS (Figura II-7). En ella se encuentra una primera gráfica con el parámetro alfa de Ångström, bajo ella la evolución durante el día del AOD para las cuatro longitudes de onda del Cimel, y luego las distribuciones de tamaños obtenidas con la inversión de las medidas de radiancia y el grado de polarización de la luz en función del ángulo de dispersión. 
29/05/2002 El Arenosillo 45

(Lat: $37.105^{\circ}$ Lon: $-6.733^{\circ}$ Elev:0m last sun calibration: 00/00/00)

Angström coefficient
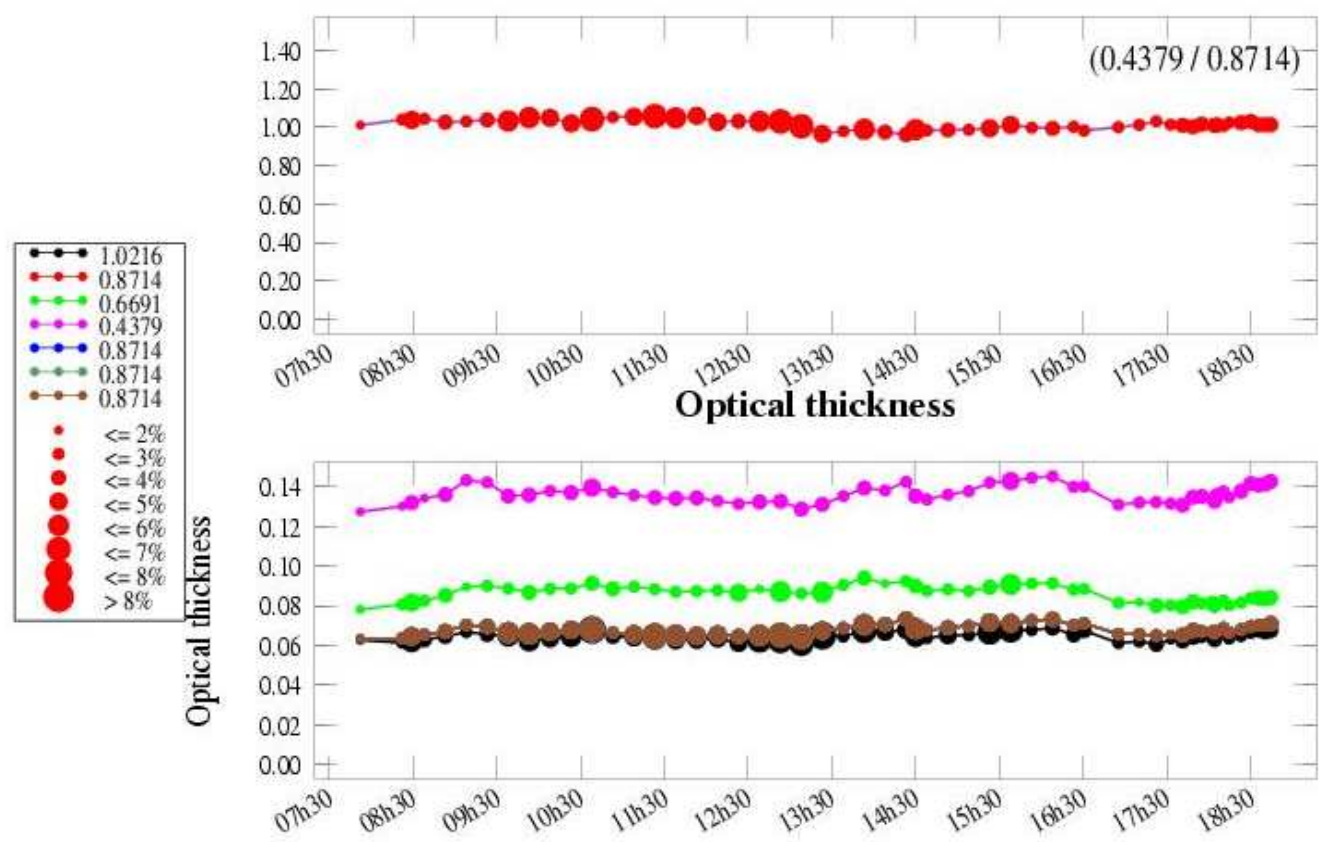

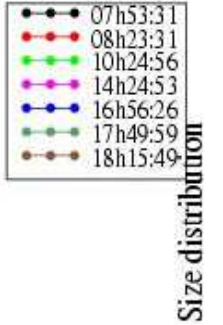

Dubovik size distribution

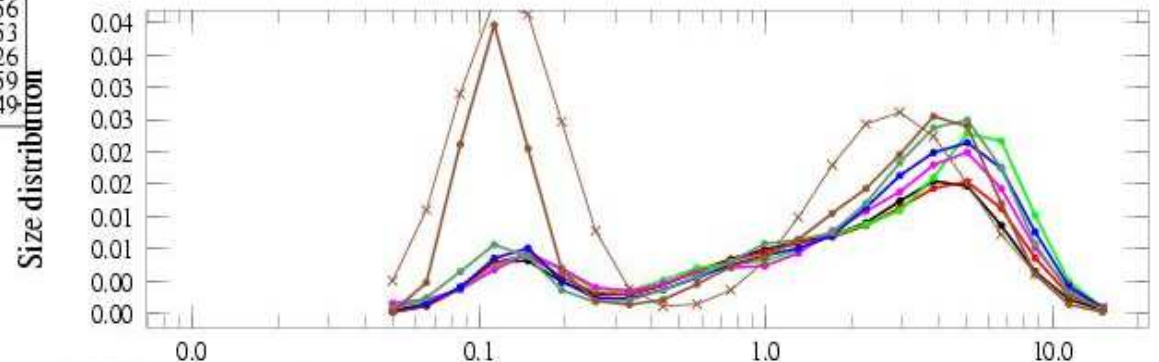

18:15:49:(sza: 75.56) (cor: 0.84) UrbanHighAbsorption Radius

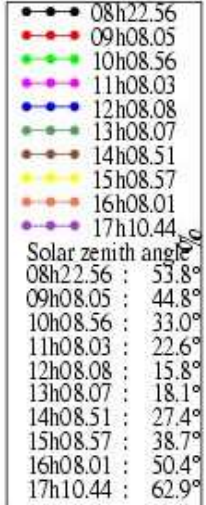

\section{Polarization ratio}

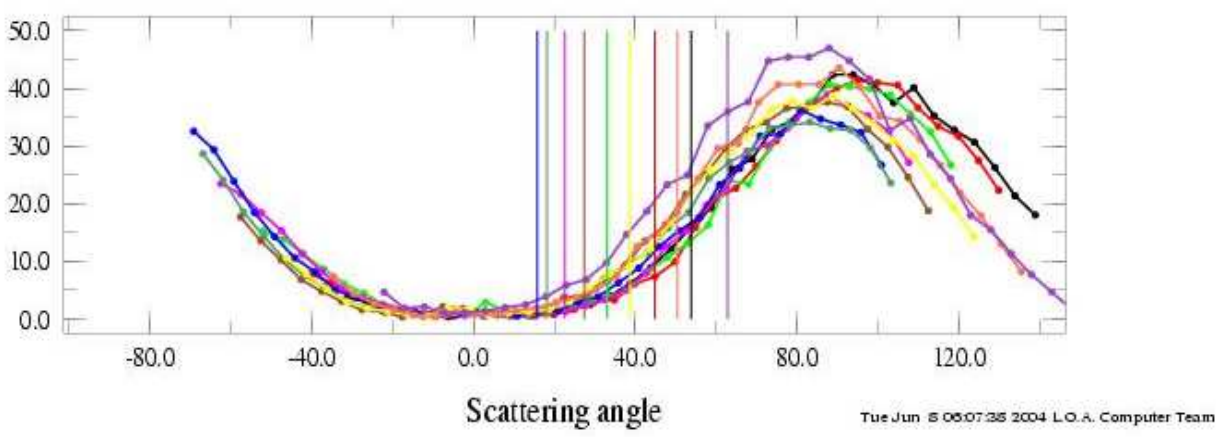

Figura II-7. Medidas del Cimel en El Arenosillo el día 29/5/2002. Tomada de http://www-loa.univlille1.fr/photons/sites.html 


\subsection{Detección de problemas en la serie de datos: el falso ciclo diurno del AOD}

La base de datos de AOD con el fotómetro Cimel en El Arenosillo tiene una gran extensión. Por otra parte la variabilidad del AOD es muy grande como mostraremos posteriormente al analizar los 5 años de medidas en el capítulo 4. Estas dos circunstancias obligan a un análisis detallado si se quiere comprobar la calidad de los datos, la bondad de las calibraciones, etc. La red AERONET gestiona más de 200 instrumentos, por lo que tiene establecidos unos protocolos automáticos para el control de la calidad de los datos. Sin embargo unos protocolos estrictamente automáticos no siempre son capaces de reconocer los numerosos problemas que pueden presentarse, por lo que antes de elevar unos datos al nivel 2.0 de AERONET, los responsables en el GSFC realizan una inspección manual, como queda reflejado en la cabecera de los ficheros que se descargan a través de la web: "Level 2.0. Quality Assured Data. The following data are pre and post field calibrated, automatically cloud cleared and manually inspected" ${ }^{3 "}$.

Una inspección detallada y diaria queda por tanto fuera de las posibilidades de los gestores de la red. En el caso de los fotómetros Cimel del GOA-UVA, nuestro grupo sí puede realizar un seguimiento diario de las medidas de 2 fotómetros, además del mantenimiento de rutina del instrumento. Esto nos ha permitido detectar una serie de problemas en la serie de datos, que pasamos a describir a continuación.

Los problemas principales encontrados al analizar la serie de datos en la estación de El Arenosillo son la aparición de valores negativos de AOD y la aparición de un ciclo diurno de origen incierto en algunos canales de AOD durante ciertos períodos de tiempo y de manera sistemática. Se encontró también que estos períodos de tiempo coincidían en algunos casos con los cambios de instrumento Cimel, por lo que nos encontramos con ciertos saltos entre las series de datos de cada instrumento, tanto para algunos canales de AOD como también de forma acusada al analizar el parámetro alfa de Ångström. Todo ello se plantea desde el conocimiento de las circunstancias de mantenimiento y del seguimiento día a día de cada instrumento.

La existencia de valores negativos en una serie de datos de AOD no es admisible, y por tanto en la mayoría de los casos se eliminan por considerar que se trata de medidas incorrectas, lo que cabe dentro de un posible error aleatorio. Lo mismo podríamos decir de valores aislados demasiado altos. $\mathrm{Si}$ estos son continuados, el problema reside generalmente en un mal funcionamiento del detector (calentamiento, etc.), por lo cual la mayoría de los instrumentos llevan un control de la temperatura. Finalmente ello puede ser debido a una mala calibración, o la degradación de la misma, en particular por una degradación demasiado rápida de los filtros.

Por otra parte hay efectos atmosféricos como las brisas o la contaminación urbana, que pueden dar lugar a un ciclo diurno del AOD. Por tanto el origen o la causa del ciclo diurno en nuestra serie de datos es un problema que debemos resolver.

Las primeras medidas en las que identificamos el ciclo diurno en el AOD fueron las del espesor óptico de aerosoles obtenido con el espectrorradiómetro Brewer. En la tesis

3 "Nivel 2.0. Datos de calidad asegurada. Los siguientes datos han sido calibrados antes y después de su puesta en estación, eliminadas las nubes de manera automática e inspeccionados manualmente." 
doctoral de Vergaz (2001) se advierte de la presencia de un acusado ciclo diurno en las medidas del espesor óptico de aerosoles obtenidas con el Brewer \#150 (que como indicamos anteriormente opera también en El Arenosillo), en longitudes de onda del UV (305 a $320 \mathrm{~nm}$ ). Al estudiar en detalle un evento de aerosol desértico de finales de julio y principio de agosto del 2000, se hacía una comparación de la evolución del AOD obtenido con el Brewer y el Cimel en El Arenosillo (Figura II-8). En esta figura podemos comprobar el fuerte ciclo diurno que experimentan las medidas de AOD del Brewer mientras que este efecto no aparece en los AOD del Cimel.

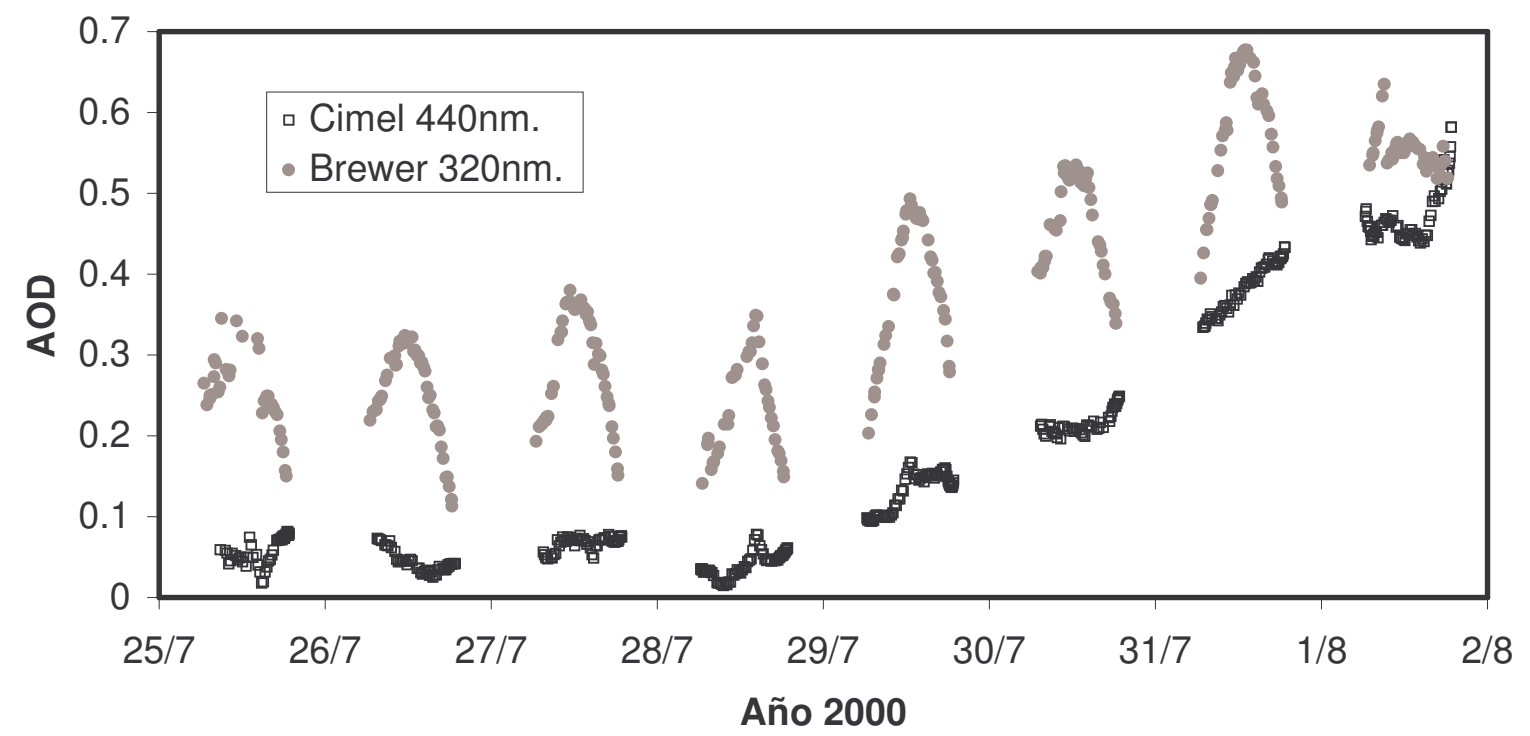

Figura II-8. Evolución del AOD obtenido con Brewer (en 320nm) y Cimel (en 440nm) desde el 25 julio al 1 de Agosto de 2000.

El hecho de que este efecto fuera exactamente simétrico con el mediodía solar local (siguiendo la altura del sol), así como que los datos del Cimel en una longitud de onda no muy lejana no tengan en absoluto una evolución parecida, hacía sospechar que ese ciclo diurno fuera ficticio, debido a algún problema aún por determinar.

Las medidas de AOD con el fotómetro Cimel \#114 en El Arenosillo también resultaron tener un ciclo diurno de similares características, aunque en este caso el comportamiento era contrario, es decir, con un mínimo al mediodía. Este problema se presentó en los canales del infrarrojo, y en días de baja turbiedad daba lugar a espesores ópticos negativos en esos dos canales, como vemos en la Figura II-9. En esta figura hemos recogido los diversos procesados de AERONET para el Cimel \#114 en El Arenosillo: el procesado con los coeficientes de precalibración (Figura II-9a y b), con los coeficientes interpolados entre la pre y la pos calibración (Figura II-9c y d), y el procesado final de AERONET realizado mediante un Langley in situ (Figura II-9f). Para apreciar este ciclo diurno y los valores negativos de AOD ha sido preciso realizar un procesado del AOD a partir de los ficheros brutos del Cimel, los ficheros K7 (acrónimo del francés cassete).

En los datos brutos de AERONET nivel 1.0 de la precalibración (Figura II-9a) había valores eliminados en los canales de 870 y $1020 \mathrm{~nm}$ por ser negativos (en concreto aquellos datos de AOD menores que -0.01). En el nivel 1.5 (Figura II-9b) se habían eliminado esas mismas medidas también en el resto de los canales de AOD, por lo que no es posible reconocer el comportamiento del ciclo diurno a partir de estos datos, y además se eliminan medidas en canales que en principio tenían unos datos correctos.

Cuando el fotómetro se retira de El Arenosillo y se calibra de nuevo, los datos se reprocesan con los coeficientes de calibración interpolados. Sin embargo esto no mejora 
en absoluto la situación (Figura II-9a y b). De nuevo en nivel 1.0 se eliminan medidas en 870 y $1020 \mathrm{~nm}$ por ser negativas, y en nivel 1.5 estos datos se han eliminado en el resto de canales.
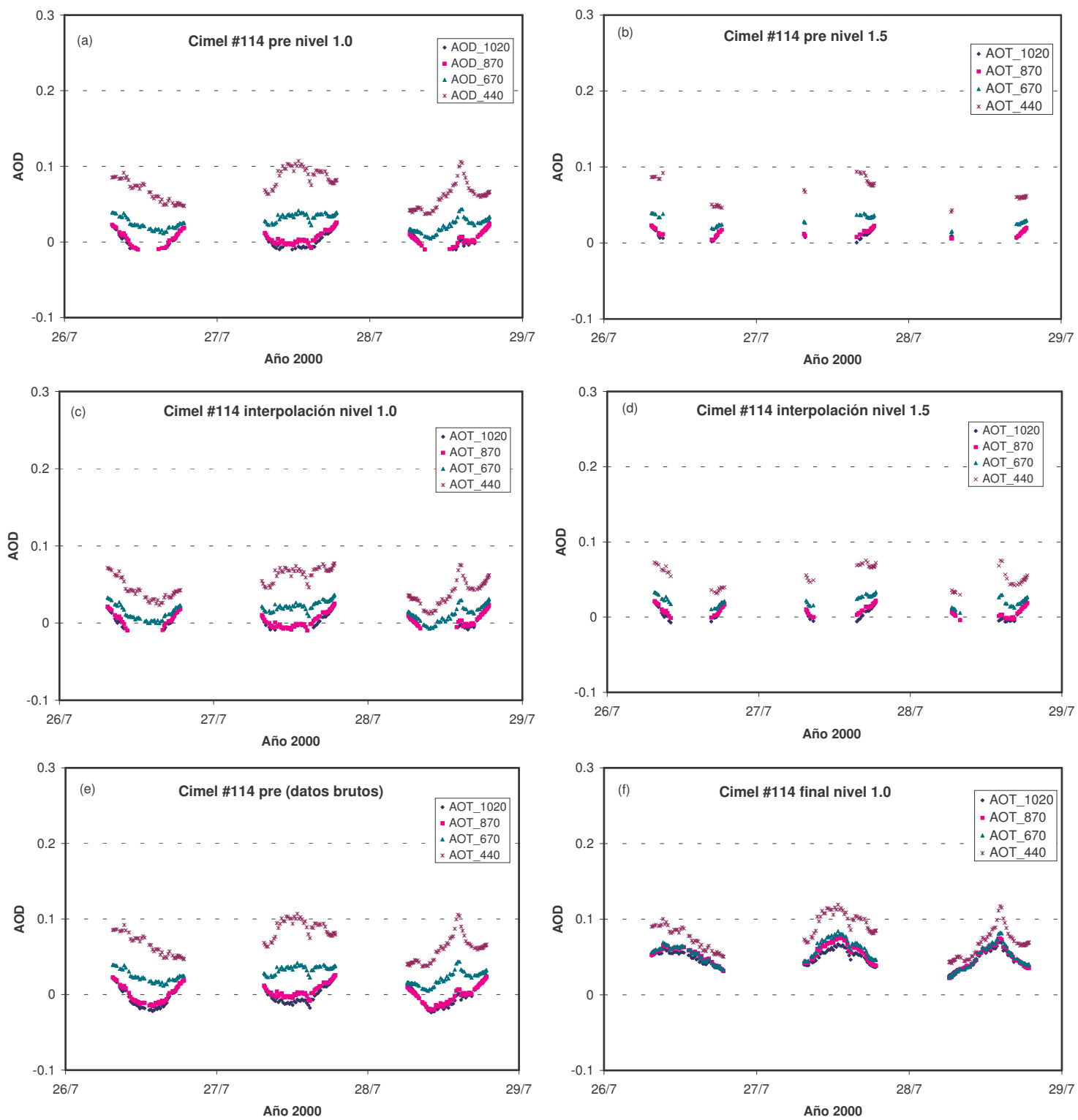

Figura II-9. Diferentes procesados de los datos del Cimel \#114 en El Arenosillo: (a) Precalibración en nivel 1.0; (b) Precalibración en nivel 1.5; (c) interpolación pre-pos en nivel 1.0; (d) interpolación pre-pos en nivel 1.5; (e) Precalibración en nivel 1.0 a partir de los ficheros K7 brutos; (f) Procesado final de AERONET mediante Langley in situ.

El problema que genera esos valores negativos es un ciclo diurno hacia abajo. Para apreciar este ciclo diurno y los datos de AOD que fueron eliminados mostramos el procesado a partir de los datos brutos en la (Figura II-9e). En este caso además no cabe confusión alguna con un efecto real de tipo atmosférico ya que los valores negativos de AOD son imposibles físicamente. Ante estos problemas de valores negativos, AERONET desechó las calibraciones pre y pos, y las sustituyó por una calibración Langley en un día de medida en El Arenosillo (Langley in situ). El resultado de ese procesado, que ha quedado como procesado final de AERONET, muestra un ciclo diurno hacia arriba en todos los canales de AOD (Figura II-9f). De hecho el error en los canales del infrarrojo es similar en valor absoluto aunque de signo contrario al que presentan los datos de la 
precalibración, pero no se producen valores negativos de AOD, por lo que es más difícil de detectar. La evolución diurna del AOD con este procesado final no tiene nada que ver con la que se obtenía con los coeficientes de pre y poscalibración.

Todos estos cambios en la calibración del Cimel \#114 en El Arenosillo dejan claro que los coeficientes de calibración afectan decisivamente al comportamiento diurno del AOD.

Es importante resaltar el hecho de que este ciclo diurno se produce de manera sistemática en los datos, todos los días, aunque es más sencillo identificarlo visualmente en el caso de días estables y despejados, en particular cuando el AOD es bajo. Por otra parte este problema de AOD negativo comienza bruscamente al instalar el Cimel \#114 (en sustitución del \#48), y desaparece cuando se reemplazó el \#114 por el siguiente fotómetro. El hecho de que se trate de un problema sistemático, simétrico respecto del mediodía solar, que no aparece en todos los canales de AOD ni en todos los fotómetros, termina de poner este problema dentro del contexto adecuado. No se trata en absoluto de un efecto real en la atmósfera, sino de un problema de calibración de los fotómetros. Una vez que se sabe qué buscar, y en estaciones con buenas condiciones de cielo despejado, la detección del ciclo diurno es sencilla, pero en caso contrario puede quedar camuflado por la variabilidad natural del AOD.

El trabajo de detección e identificación del ciclo diurno nos ha llevado a consultar en la bibliografía trabajos relativos a este hecho, lo cuales han sido muy útiles para comprender y tratar de resolver el problema.

El trabajo de Killing et al. (1998), relativo a la campaña "Photochemical Activity and Solar Ultraviolet" en dos localidades de Grecia en el verano de 1996, muestra la evolución del AOD obtenidos con un Brewer y un espectrorradiómetro Bentham. En esta evolución del AOD aparece con claridad unos ciclos diurnos que los autores no mencionan. Aunque desconocemos las condiciones atmosféricas en la campaña y el procesado de estos datos, creemos con toda la prudencia que puede ser un problema análogo al de los AOD calculados con el Brewer en El Arenosillo, es decir, algún tipo de problema de calibración.

El trabajo de Kremser et al. (1984) identifica el problema del ciclo diurno del AOD. En él se analiza la influencia que sobre el calibrado por el método de Langley tiene el hecho de que el AOD presente un comportamiento no constante, sino en forma cosenoidal, durante el día, pero además se explica cómo una elección incorrecta del coeficiente de calibración da como resultado un ciclo diurno del AOD perfectamente simétrico respecto del mediodía solar local. Este efecto ficticio puede ser cóncavo o convexo, según el error de calibración sea por defecto o exceso. Esta publicación propone por ello un procedimiento de calibración para reducir los errores del método de Langley, ya que, como otros autores ya citados (ver sección 1.3.3) se centran en el problema de que variaciones monótonas de la transmitancia atmosférica sesgan los resultados del método de Langley e introducen errores sistemáticos en el cálculo del espesor óptico de aerosoles. Una deducción similar se puede encontrar en Korotaev et al. (1993), donde los autores explican cómo un error en la constante de calibración da lugar a un error en el espesor óptico de aerosoles que depende inversamente de la masa óptica.

El trabajo de Romero y Cuevas (2002) presentado en la $3^{\mathrm{a}}$ Asamblea de Geofísica y Geodesia en Valencia, recoge este problema del ciclo diurno del AOD y lo analiza a partir de medidas tomadas en el Observatorio de Izaña con un fotómetro PFR (Precision Filter Radiometer, Wehrli, 2000a). El comportamiento similar del PFR y los datos del Brewer mostrados en Vergaz (2001) están en el origen de su trabajo. El fotómetro PFR, construido por el WRC (World Radiation Center), está dentro del programa GAW (Global 
Atmosphere Wach), con más de 12 fotómetros en todo el mundo. Este programa está financiado por el gobierno suizo y la WMO (Organización Meteorológica Mundial). En este caso las condiciones de medida son totalmente diferentes a las comentadas en El Arenosillo. En el caso del PFR en Izaña se trata de un instrumento muy preciso, calibrado en laboratorio o bien con un master calibrado por la técnica Langley en Jungfraujoch (Suiza) o Mauna Loa (Hawai), y los espesores ópticos con los que se detecta el problema son muy bajos, entre 0.02 y 0.04 , cercanos a la precisión absoluta del aparato (Wehrli, 2000a), debido a la altitud de la estación de Izaña. La Figura II-10 muestra estos datos durante el mes de junio de 2001 en Izaña, donde puede verse el ciclo diurno sistemático. En el citado trabajo se demuestra práctica y teóricamente que ese ciclo diurno desaparece con una calibración correcta realizada en este caso, por las condiciones de la estación, con el método de Langley. Destacamos la importancia de demostrar que ese efecto se debe a un problema de calibración.

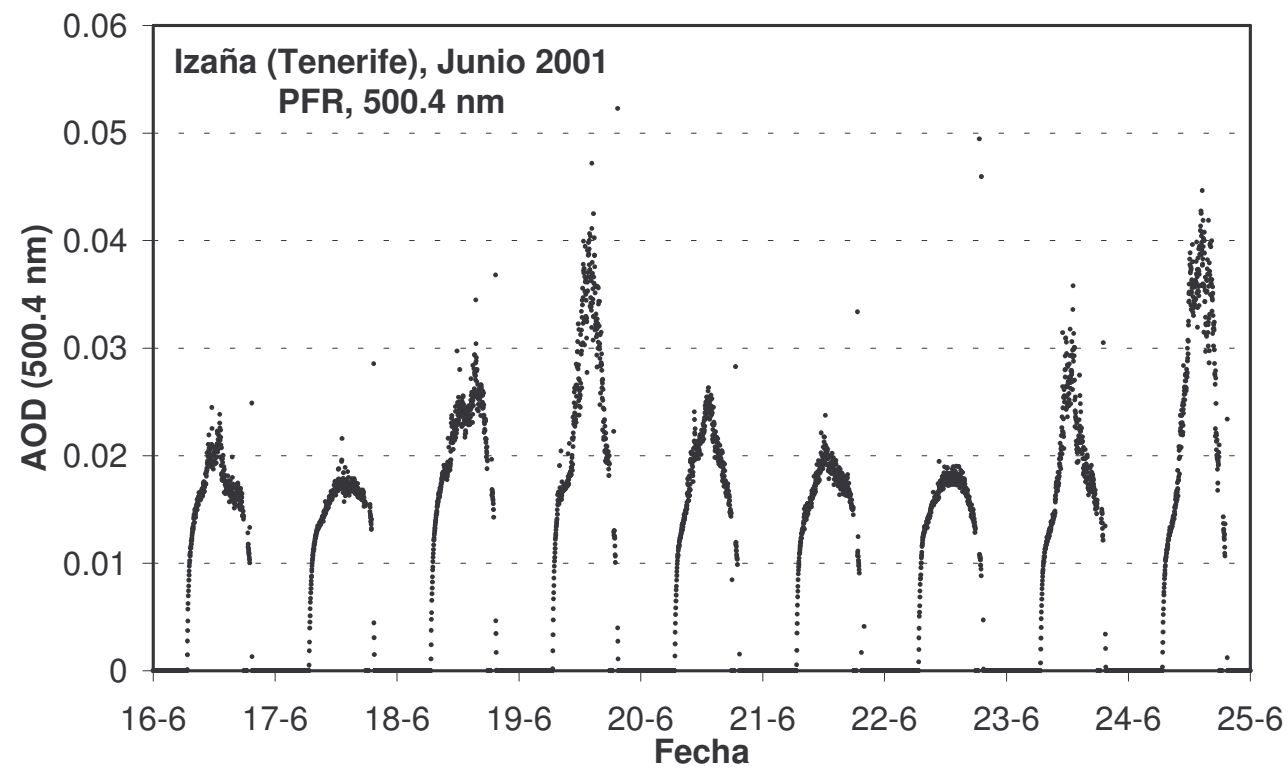

Figura II-10. Ciclo diurno en el AOD medido en Izaña con el PFR en junio de 2001.

El Observatorio de Izaña es idóneo para realizar calibraciones absolutas por el método de Langley. En el citado trabajo se compara la calibración de laboratorio con la obtenida mediante el ajuste de Langley. Las diferencias son del $1.7 \%$ en el canal de $861 \mathrm{~nm}$ y $2.4 \%$ en 500nm. En la Figura II-11 tenemos el espesor óptico con ciclo diurno obtenido con la constante de calibración determinada en laboratorio para el canal de 500nm (500_Lab). Frente a esto, el ciclo diurno no aparece con el AOD calculado con una constante de calibración obtenida con el método de Langley, en este caso el promedio de varios ajustes de Langley (500_Lg_Med). Una vez descartados otros problemas instrumentales como la influencia de la temperatura (el PFR está termostatizado) o un incorrecto alineamiento con el sol por parte del sistema seguidor, el trabajo concluye que el ciclo diurno es ficticio y se debe a un problema de calibración.

A partir de este trabajo desarrollamos conjuntamente con el INM un método de correccióncalibración alternativo al método de Langley, que hemos denominado KCICLO (Cachorro et al., 2004). El método se basa en que el propio efecto del ciclo diurno contiene la información necesaria para corregir la calibración errónea, como veremos en el capítulo siguiente. 


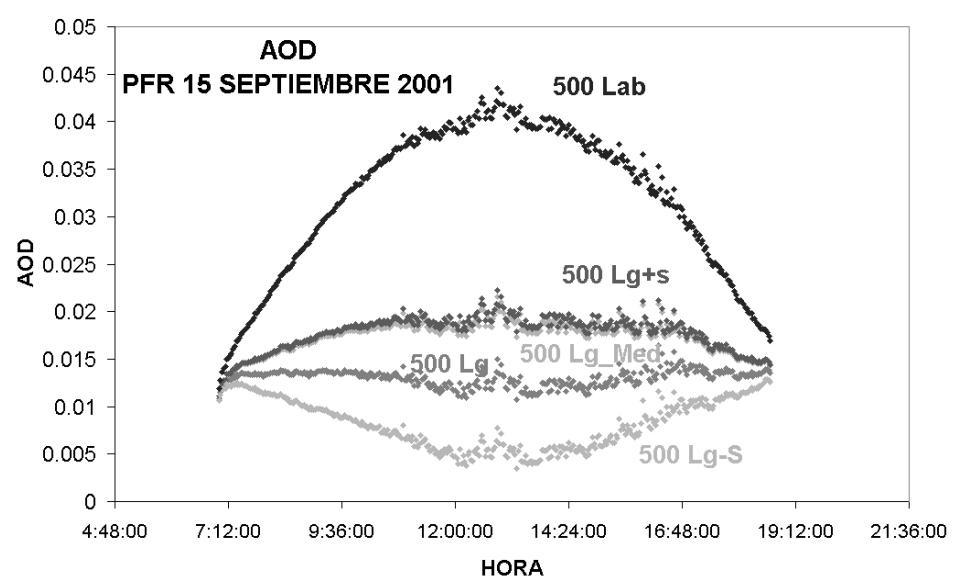

Figura II-11. Evolución diaria del AOD para $500 \mathrm{~nm}$ medido con el PFR el 16 de septiembre y como desaparece el ciclo diurno del AOD con el valor de la constante de calibración obtenido con el método de Langley mediante el promedio de los ajustes de varios días (Lg_Med). Tomada de Romero y Cuevas (2002).

Como conclusión por tanto a este apartado, tenemos que decir que el problema del ciclo diurno del AOD que encontramos en medidas de AOD del Cimel y el Brewer, se debe a una calibración errónea. Este efecto es conocido por las personas dedicadas a la calibración y está planteado en la bibliografía desde hace años. Sin embargo puede ser complicado de detectar debido a la variabilidad del AOD o a las condiciones de nubosidad en una estación ordinaria, por ejemplo de la red AERONET. Queremos además resaltar que sólo desde hace muy pocos años se dispone de series largas de datos de suficiente calidad para poder aislar e interpretar este problema. El error es de tipo sistemático y está presente en todas las medidas, pero su detección es más sencilla si se dispone de varios días consecutivos que hayan sido despejados y estables, como los que empleamos en las gráficas de ejemplo. En todo caso se había tomado este efecto como un problema, mientras que nosotros vamos a tomarlo como una oportunidad para corregir la serie de datos. 


\section{Capítulo 3. El método de calibración - corrección KCICLO. Aplicación y resultados}

\section{Introducción}

En este capítulo vamos a plantear el método KCICLO, que surgió de la necesidad de corregir los problemas en la serie de datos del fotómetro Cimel en El Arenosillo. Este método aprovecha el efecto del falso ciclo diurno descrito en el capítulo anterior, y va a ser aplicado a la serie de AOD en la estación. Cada fotómetro Cimel instalado de manera consecutiva en El Arenosillo debe ser analizado individualmente. Por último se analizará el error del método KCICLO y la precisión que permite alcanzar en los AOD, así como su equivalencia con un método bien establecido para este tipo de medidas como es el método de Langley. 


\subsection{Formulación analítica y evaluación del falso ciclo diurno}

La formulación analítica que desarrollamos en este apartado se puede encontrar en diversos trabajos (Kremser et al., 1984; Korotaev et al., 1993; Romero y Cuevas, 2002), en los que se llega a resultados similares aunque desde enfoques distintos. La base del cálculo es la ley de Beer-Bouguer-Lambert. El espesor óptico total $\tau$ obtenido a partir de esta ley se puede expresar de la siguiente manera:

$$
\tau=\frac{1}{m} \ln \left(I_{0} / I\right)
$$

Si en lugar de la constante $\mathrm{I}_{0}$, que vamos a suponer fuese el valor correcto, introducimos $\mathrm{I}_{0}{ }_{0}=\mathrm{K} \cdot \mathrm{I}_{0}$, donde $\mathrm{K}$ es una constante que cuantifica el error de $\mathrm{I}_{0}$, el valor del espesor total resultante $\tau$ ' estaría relacionado con $\tau$ por la siguiente expresión:

$$
\begin{gathered}
\tau^{\prime}=\frac{1}{m} \ln \left(I_{0}^{\prime} / I\right)=\frac{1}{m} \ln \left(K \cdot I_{0} / I\right)=\frac{1}{m} \ln \left(I_{0} / I\right)+\frac{1}{m} \ln K \\
\tau^{\prime}=\tau+\frac{1}{m} \ln K
\end{gathered}
$$

El error absoluto del espesor óptico queda:

$$
\Delta \tau=\frac{1}{m} \ln K
$$

A partir de estas dos expresiones equivalentes, podemos concluir varias cosas de gran importancia. Antes queremos mencionar que si bien hemos puesto en las ecuaciones anteriores el espesor óptico total de la atmósfera, nos vamos a referir en todo momento al espesor óptico de aerosoles, que como se mostró en el capítulo 1 sólo requiere eliminar el espesor óptico de Rayleigh y el de gases absorbentes del espesor óptico total. Como éstos se restan, las expresiones anteriores se obtienen igualmente:

$$
\tau_{a}{ }^{\prime}=\tau_{a}+\frac{1}{m} \ln K
$$

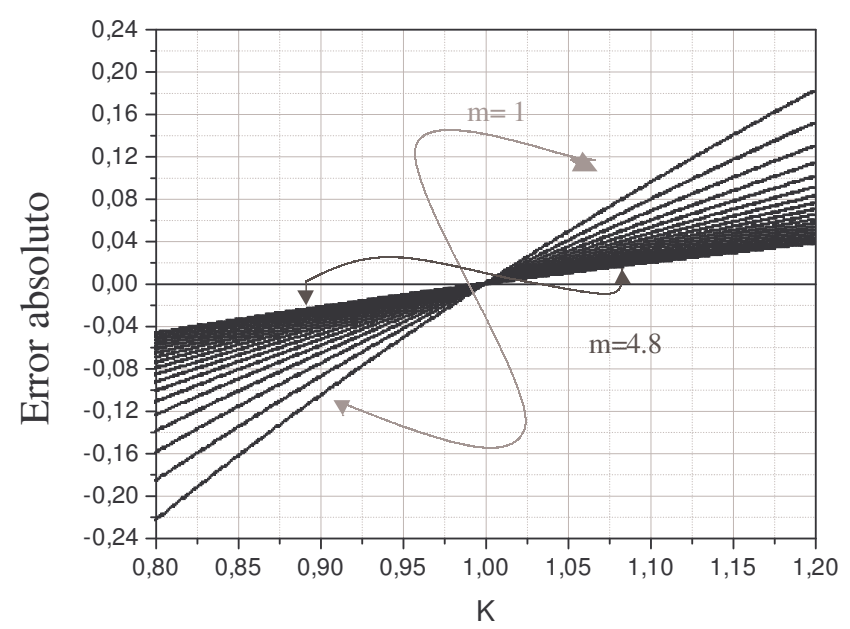

Figura III-1. Evaluación del error absoluto del espesor óptico de aerosoles frente a la constante K para masas ópticas de 1 a 4.8 .

En primer lugar, el error que se traslada al espesor óptico si el coeficiente de calibración dado por $\mathrm{I}_{0}$ tiene un cierto error, no es una cantidad fija sino modulada por el inverso de la masa óptica m, o lo que es igual, directamente proporcional al coseno del ángulo cenital 
solar, lo cual provoca la forma de ciclo diurno. De aquí se deriva inmediatamente que el error es menor para masas ópticas grandes -al principio y al final del día- y máximo en el centro del día, haciendo que $\tau$ ' experimente un ciclo diurno ficticio que no existe en el valor verdadero $\tau$.

La Figura III-1 muestra la evaluación de la expresión (3.4) donde $\Delta \tau$ es evaluado frente a $\mathrm{K}$ para valores de la masa óptica de 1 a 4.8. En la Tabla III-I se dan algunos datos representativos del error del AOD en función del error en la calibración para masa óptica 1. Vemos que un error en la calibración del $1 \%$ supone un error absoluto máximo del espesor de 0.01 , un $5 \%$ nos lleva ya a un error absoluto de 0.05 , etc.

\begin{tabular}{cccccc}
\hline \hline Error en $\mathrm{I}_{0}$ & $\mathrm{~K}$ & $\operatorname{lnK}$ & Error en $\mathrm{I}_{0}$ & $\mathrm{~K}$ & $\operatorname{lnK}$ \\
\hline $1 \%$ & 1.01 & 0.01 & $-1 \%$ & 0.99 & -0.01 \\
$2 \%$ & 1.02 & 0.02 & $-2 \%$ & 0.98 & -0.02 \\
$5 \%$ & 1.05 & 0.05 & $-5 \%$ & 0.95 & -0.05 \\
$10 \%$ & 1.10 & 0.095 & $-10 \%$ & 0.90 & -0.10 \\
\hline \hline
\end{tabular}

Tabla III-I. Error máximo en el AOD $(\mathrm{m}=1)$ en función del error en la determinación de $\mathrm{I}_{0}$

Este ciclo diurno ficticio puede tener forma cóncava o convexa, puesto que el término $(1 / \mathrm{m}) \cdot \operatorname{lnK}$ puede ser positivo o negativo, según el valor de $\mathrm{K}$ : si sobrestimamos el coeficiente de calibración $(\mathrm{K}>1)$, el término contribuye positivamente y el ciclo diurno tiene forma convexa (mayores valores del AOD al mediodía). Si nuestro valor del coeficiente de calibración $\mathrm{I}_{0}$ ' es menor que el verdadero $\mathrm{I}_{0}(\mathrm{~K}<1)$, el término es negativo y el ciclo tiene forma cóncava (menores valores del AOD al mediodía).

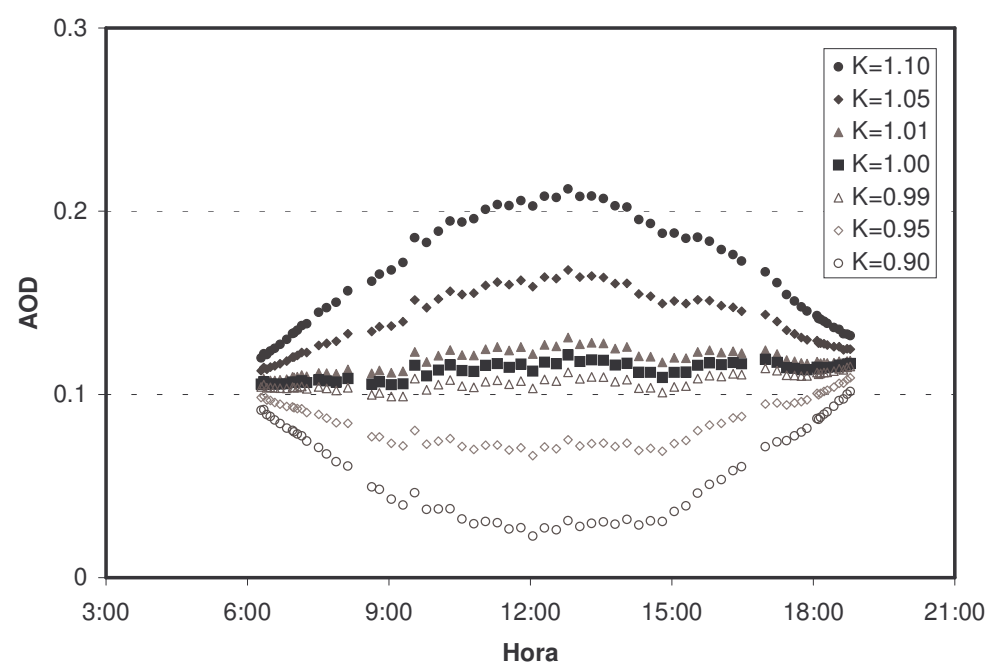

Figura III-2. Falso ciclo diurno en el AOD para distintos valores del error en la constante de calibración, dado por K.

En segundo lugar, este error no depende del valor absoluto del espesor óptico, puesto que sólo depende de $\mathrm{K}$, esto es, de la relación entre la irradiancia extraterrestre verdadera del aparato ( $\mathrm{I}_{0}$ ó $\mathrm{CN}_{0}$ si no está en unidades físicas) y el valor con un cierto error que nosotros utilicemos I' (ó $\mathrm{CN}_{0}{ }_{0}$ ). Esto explica también que el efecto se identifique más fácilmente en días soleados con un espesor óptico muy estable, y también cuando el espesor óptico de aerosoles es muy bajo, puesto que entonces la variación relativa es mucho mayor.

Por tanto, el error máximo se dará a mediodía, y viene dado directamente por $\operatorname{lnK}$, siempre teniendo en cuenta que la masa óptica modula este error. A mediodía en verano, para nuestra latitud, el valor de la masa óptica m es muy cercano a 1. En lugares en los 
que la masa óptica a mediodía sea mayor, el error se ve atenuado y la detección es también más difícil.

Los otros dos problemas planteados en la serie de datos están ciertamente relacionados con el falso ciclo diurno. Cuando el espesor óptico de aerosoles es muy bajo, una subestimación de la constante de calibración $(\mathrm{K}<1)$ puede hacer que el AOD tome valores negativos (forma cóncava con mínimo al mediodía). Este problema difícilmente aparece si el error es por sobrestimación.

Es interesante comparar la expresión (3.4) con el error absoluto del espesor óptico total evaluado a través de la teoría de propagación de errores ( $\varepsilon$ error relativo), que aparece en numerosos trabajos (ver por ejemplo Reagan et al., 1986; Durán, 1997):

$$
\Delta \tau=\frac{1}{m}\left(\tau \Delta m+\varepsilon\left(I_{o}\right)+\varepsilon(I)\right)
$$

Vamos a suponer un sistema fotométrico como el Cimel, donde no se realizan medidas absolutas de la irradiancia $(\Delta \mathrm{I}=0)$, y además supondremos que el error que se introduce por la masa óptica $m$ es despreciable frente a la calibración $(\Delta \mathrm{m}=0)$. Es decir, suponemos que todo el error del espesor óptico va a proceder del error del coeficiente de calibración. En este caso tenemos (donde hemos sustituido $\mathrm{I}_{\mathrm{o}}$ por $\mathrm{CN}_{\mathrm{o}}$, indicando con ello las cuentas digitales del fotómetro Cimel):

$$
\Delta \tau=\frac{1}{m}\left(\frac{\Delta C N_{o}}{C N_{o}}\right)=\frac{1}{m}\left(\frac{C N_{o}-C N_{o}^{\prime}}{C N_{o}}\right)=\frac{1}{m}\left(1-\frac{C N_{o}^{\prime}}{C N_{o}}\right)=\frac{1}{m}(1-K)
$$

Esta última expresión es equivalente en primera aproximación a la expresión (3.4), y nos permite una rápida evaluación mental del error de $\tau$, como puede verse a través de la Tabla III-I

Una vez explicado el ciclo diurno, el problema que se plantea entonces es corregir la calibración de los datos de espesor óptico de aerosoles que presenten este error. Para ello proponemos en el apartado siguiente un método de corrección, que denominamos KCICLO, que aprovecha el efecto del ciclo diurno, esto es, la dependencia con la masa óptica del error en el espesor óptico de aerosoles. 


\subsection{Descripción del método propuesto: KCICLO}

Una vez descritos los problemas de la serie de datos, y en particular el problema del falso ciclo diurno, podemos intentar corregir ese efecto, sacando provecho de él, para lo cual proponemos el método que se expone a continuación. Este método que calificamos de corrección-calibración es por un lado un método de corrección para los datos de espesor óptico de aerosoles, pero también un verdadero método de calibración del instrumento.

Comenzamos en la expresión analítica de ese falso ciclo diurno (3.3), donde el espesor óptico calculado $\left(\tau^{\prime}\right)$ es igual al espesor óptico real $(\tau)$ más el término $\operatorname{lnK}$ multiplicado por el inverso de la masa óptica (o bien aproximadamente el coseno del ángulo cenital solar).

Esta expresión se puede entender como una recta, cuya pendiente es lnK y cuya ordenada en el origen es $\tau$. Es decir: si ajustamos a una recta el espesor óptico que vamos midiendo a lo largo del día, $\tau$ ', frente al inverso de la masa óptica, la pendiente del ajuste nos da el valor de $\operatorname{lnK}$. Vimos que $\mathrm{K}$ es la razón entre la coeficiente de calibración que hemos utilizado $\left(\mathrm{I}_{0}\right)$ y el verdadero $\mathrm{I}_{0}$.

La condición necesaria y suficiente para que este ajuste sea bueno es que el espesor óptico verdadero $\tau$ sea constante durante el día. En realidad basta con que esta condición se cumpla durante medio día. Tampoco es necesario que el AOD tenga un valor bajo, aunque si la turbiedad es alta hay más posibilidades de que varíe el AOD. La pendiente de la recta $(\operatorname{lnK})$ es un valor que teóricamente permanece constante en el ajuste, ya que la variación de la constante $\mathrm{K}$, por una deriva de la calibración, no es relevante a lo largo de un día.
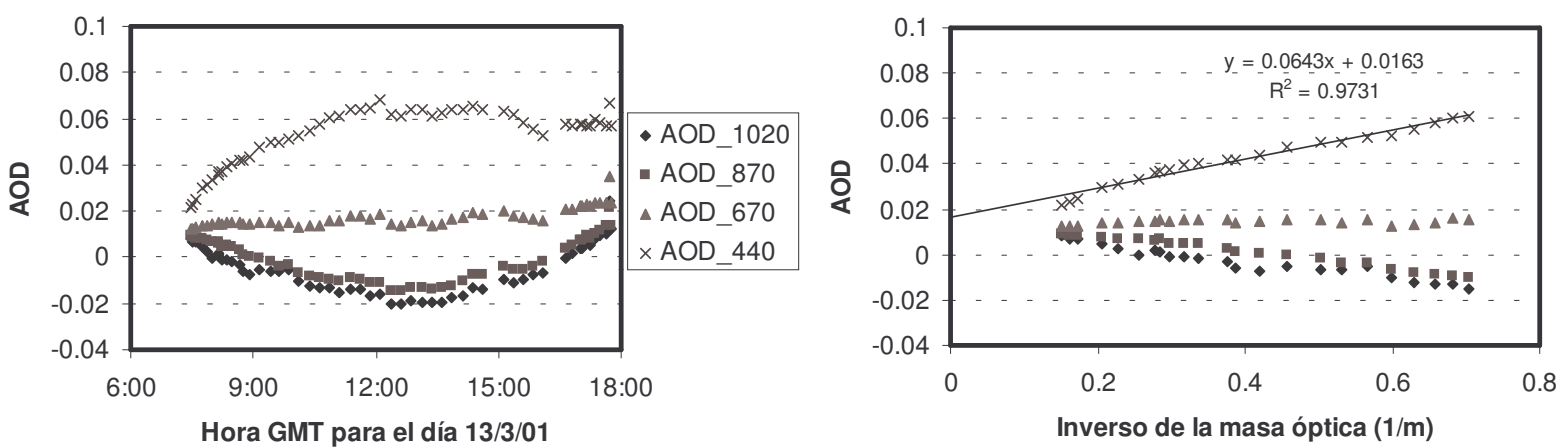

Figura III-3. Espesor óptico de aerosoles el día 13 de marzo de 2001 en El Arenosillo (izquierda) y ajuste del método KCICLO para los datos de la mañana (derecha) en el canal de 440nm. Datos del Cimel \#114 con los coeficientes de precalibración.

En la Figura III-3 tenemos un ejemplo de la aplicación de este tipo de ajuste lineal. Representamos en la gráfica de la derecha el espesor óptico de aerosoles frente al inverso de la masa óptica, lo que da como resultado una recta. Cuando un filtro está correctamente calibrado la pendiente de la recta está muy cerca de cero $(\operatorname{lnK} \approx 0)$ y por tanto $\mathrm{K}=1$. Esto significa que el coeficiente de calibración que estamos utilizando es el correcto: $\mathrm{I}_{0}{ }^{\prime}=\mathrm{I}_{0}$. Cuanto peor calibrado está un filtro, mayor es la pendiente de la recta (en valor absoluto), y por tanto tendremos un valor de $\mathrm{K}$ mayor o menor que 1 , según el error de calibración sea por exceso o por defecto.

En el ejemplo de la Figura III-3 el filtro de 670nm estaría correctamente calibrado, mientras que el filtro de $440 \mathrm{~nm}$ tendría una constante $\mathrm{I}_{0}>\mathrm{I}_{0}$. Los filtros de $870 \mathrm{~nm}$ y $1020 \mathrm{~nm}$ tendrían una constante de calibración menor que la real, $\mathrm{K}<1$. También podemos 
observar el ciclo diurno que presenta el AOD durante el día para los filtros de 440, 870 y $1020 \mathrm{~nm}$ mientras que para el filtro de 670nm el AOD permanece constante durante el día. Por otra parte las condiciones atmosféricas son mejores por la mañana que por la tarde. Estos datos corresponden con la precalibración de AERONET del fotómetro Cimel\#114.

La condición de que el espesor óptico $\tau$ permanezca constante es muy exigente en la atmósfera. La variabilidad del espesor óptico de la atmósfera en las longitudes de onda en las que mide el fotómetro Cimel se deben principalmente a los aerosoles, puesto que las absorciones por parte de otros componentes atmosféricos son mínimas. Pues bien, la variabilidad típica de los aerosoles es muy grande y sin patrones claros, por lo que no es fácil encontrar días tan estables como para garantizar la condición de que $\tau$ sea constante. Tan solo en días excepcionales o en estaciones remotas en altitud, como los observatorios de Izaña o Mauna Loa, se puede cumplir casi plenamente esta condición.

Además es muy difícil asegurar que la condición $\tau=$ cte. se esté cumpliendo incluso dentro de ciertos márgenes. Para eso se necesita un aparato previamente bien calibrado, y eso es lo que precisamente se intenta comprobar. Un segundo instrumento sería de gran ayuda, aunque tampoco esto se debe considerar una garantía definitiva. De hecho, como ya se ha comentado, si el AOD durante el día experimenta una variación con la misma forma que el coseno del ángulo cenital solar, es decir, el inverso de la masa óptica, esta variación no es distinguible en la práctica de un efecto real, ni para este método ni tampoco para el método de Langley.

Pese a estas dificultades, la aplicación del método con éxito es posible buscando un número suficiente de días despejados en los que el espesor óptico sea estable y constante, o bien en los que el AOD presente un ciclo diurno más o menos acentuado y que presente simetría respecto al mediodía solar, o al menos un comportamiento de este tipo durante medio día, la mañana o la tarde. Los resultados de los ajustes para cada día serán previsiblemente distintos, pero estas variaciones se deben al comportamiento de la atmósfera, por lo que podemos asumirlas como un ruido aleatorio en torno al valor verdadero. En la sección 3.4 evaluaremos la precisión o error del método.

En resumen, el método propuesto, que denominaremos 'método KCICLO' consiste en:

1. Seleccionar los días de la serie de datos que cumplan los siguientes criterios:

- Desviación estándar del ajuste $\sigma<0.01$

- Número de puntos $\mathrm{N}>12$

- El rango mínimo de masas ópticas, la diferencia entre la primera y la última, debe ser $m_{\max }-m_{\min }>4$. El rango usual es entre 1.7 y 7 .

Esta selección se realiza de forma automatizada, como explicaremos enseguida.

2. Realizar una segunda selección entre estos días por visualización directa.

Esto es necesario por la dificultad de diseñar un procedimiento automatizado completo: el falso ciclo diurno dificulta la selección de días a partir de un criterio sobre el espesor óptico. No se pueden elegir días estables sólo a partir de la constancia del $\mathrm{AOD}$, puesto que entonces se desecharían precisamente días válidos ya que estos deben presentar ciclo diurno. Tampoco se debe emplear el coeficiente de correlación $\mathrm{R}^{2}$ del ajuste KCICLO, porque en el caso de filtros bien calibrados la pendiente del ajuste estará próxima a cero y $\mathrm{R}^{2}$ también será pequeño, desechando de nuevo días válidos. Se trata de desechar en su caso alguno de los días seleccionados por el procedimiento automático si no es suficientemente estable. 
3. Ajustar para cada mañana o tarde el espesor óptico calculado frente al inverso de la masa óptica. Este ajuste se puede restringir a valores de la masa óptica $\mathrm{m}>1.4$, esto es, prescindiendo del mediodía solar.

Es habitual eliminar estos datos porque en el centro del día la masa óptica varía lentamente, con lo que las variaciones normales del AOD dan lugar a puntos con mucha dispersión, que falsean el resultado del ajuste. Recordemos que la condición primera es que el espesor óptico permanezca constante. Es más fácil asegurarlo en un período corto de tiempo, al amanecer o al atardecer, en el que podemos encontrar un rango suficiente de masas ópticas en un intervalo de tiempo relativamente corto en latitudes medias. Este planteamiento ya se discutió a propósito del rango de masas ópticas en el método de Langley.

4. Hacer el promedio entre los diferentes valores obtenidos para $\mathrm{K}$ con los distintos ajustes. De esta manera se determina una nueva constante de calibración con su error correspondiente. Otra posibilidad es situar las constantes obtenidas frente a la fecha, y hacer un ajuste temporal lineal. Esto tiene sentido si el período es muy largo o para detectar alguna tendencia con el tiempo. Entre los distintos valores de $\mathrm{K}$ obtenidos, se hace una última selección, no teniendo en cuenta aquellos valores que disten del promedio de $\mathrm{K}$ más de dos veces la desviación estándar del conjunto de $\mathrm{K}$ obtenidos:

$$
\left|\bar{K}-K_{i}\right|<2 S T D(K)
$$

5. Utilizar la nueva constante de calibración para reprocesar la serie de datos, obteniendo una nueva serie de datos de espesor óptico.

Los datos de entrada para este procedimiento son las medidas de la serie de AERONET en nivel 1.0 procesados con el coeficiente de precalibración. Esto es necesario por dos motivos: para que los ajustes del método sean comparables necesitamos que todas las constantes ' $\mathrm{K}$ ' estén referidas al mismo coeficiente de calibración $\mathrm{CN}_{0}$, y no a un coeficiente interpolado que varíe cada día. En segundo lugar, el nivel 1.0 mantiene las medidas hasta masa óptica 7 , mientras que en el nivel 1.5 sólo se mantienen las medidas a masas ópticas menores que 5. Por supuesto los datos definitivos de AERONET (nivel 2.0), son procesados con un coeficiente de calibración interpolado entre la precalibración y la poscalibración. Cuando comparemos las series de datos de AERONET y KCICLO (capítulo 5), emplearemos este nivel 2.0 de AERONET. Sin embargo a la hora de aplicar el método KCICLO partimos de los datos de AERONET procesados con la precalibración en nivel 1.0 .

De cara a la selección de días y la realización de ajustes KCICLO de manera automática con nuestra serie de datos, se ha desarrollado un programa informático en lenguaje IDL. Este programa toma los datos de AOD de un fotómetro (en general más de 1 año de datos) y realiza para cada mañana y cada tarde el ajuste del AOD medido frente al inverso de la masa óptica. El programa tiene una serie de filtros y umbrales de calidad de los ajustes que pueden ser modificados para ser más o menos restrictivos, de acuerdo a lo descrito en el método, es decir, sobre la desviación estándar del ajuste, el rango de masas ópticas y el número mínimo de puntos.

Hay además otro filtro adicional que podemos imponer sobre el AOD en 440nm, puesto que los mejores ajustes se dan en condiciones de baja turbiedad. Para cada ajuste realizado se evalúan los criterios mencionados. Si los cumple, el ajuste es aceptado; si no los cumple, se elimina el dato peor y se vuelve a evaluar. Si finalmente el número de puntos o el rango de masas ópticas no cumplen los criterios mínimos, se desecha definitivamente la mañana o tarde. Este programa permite encontrar de manera rápida los días adecuados para la aplicación del método KCICLO en series de datos de varios meses de duración. 


\subsection{Aplicación del método KCICLO a la serie de datos de El Arenosillo}

Vamos a aplicar el método propuesto a la serie de datos del fotómetro Cimel en El Arenosillo. Tenemos varios instrumentos con períodos distintos y cada uno tiene sus propios coeficientes de calibración para cada uno de sus filtros. Esto hace que tengamos que analizar la calibración de cada fotómetro por separado. Para esto es importante la meta base de datos de AERONET, información que está disponible mediante el Demonstrat: historiales de calibración, fechas de instalación de cada fotómetro, etc.

El procedimiento a aplicar, como hemos visto, tiene varios pasos, pero podemos decir que el fundamental es la realización de los ajustes lineales para obtener la constante $\mathrm{K}$ para los diversos días seleccionados y a continuación se obtiene un valor medio de la misma para un determinado período.

\subsubsection{Cimel \#48}

El Cimel \#48 estuvo instalado entre el 16 de febrero de 2000 y el 9 de julio de ese mismo año. En el anexo 1 (Tabla A1-I a Tabla A1-IV) se pueden consultar en detalle los ajustes realizados para cada canal del AOD en los días seleccionados. En ellas se indica la fecha, si el ajuste se realiza con datos de la mañana o la tarde (AM ó PM), el número de puntos, el coeficiente de correlación $\mathrm{R}^{2}$, la desviación estándar (STD), el valor de lnK, con su error, el valor de $\tau$ también con su error, y finalmente $\mathrm{K}$ con su error.

Mostramos a continuación como ejemplo uno de los días seleccionados durante el período de operación del Cimel \#48 (Figura III-4). Se trata del día 29 de mayo de 2000 que presenta espesores ópticos medios, pues los valores de AOD para el filtro de 440nm están entre 0.1 y 0.2 . El ajuste se realiza con los datos de la tarde, que como se ve es muy estable a partir de las 14h. De hecho la masa óptica 1.4 se alcanza a las 15:30h, y el ajuste llega a masa óptica 6.7 alrededor de las $19 \mathrm{~h}$. En la figura se incluyen los resultados del ajuste para los canales de $440 \mathrm{~nm}$ y $670 \mathrm{~nm}$, aunque el resto de canales también se pueden consultar en las tablas anteriores.
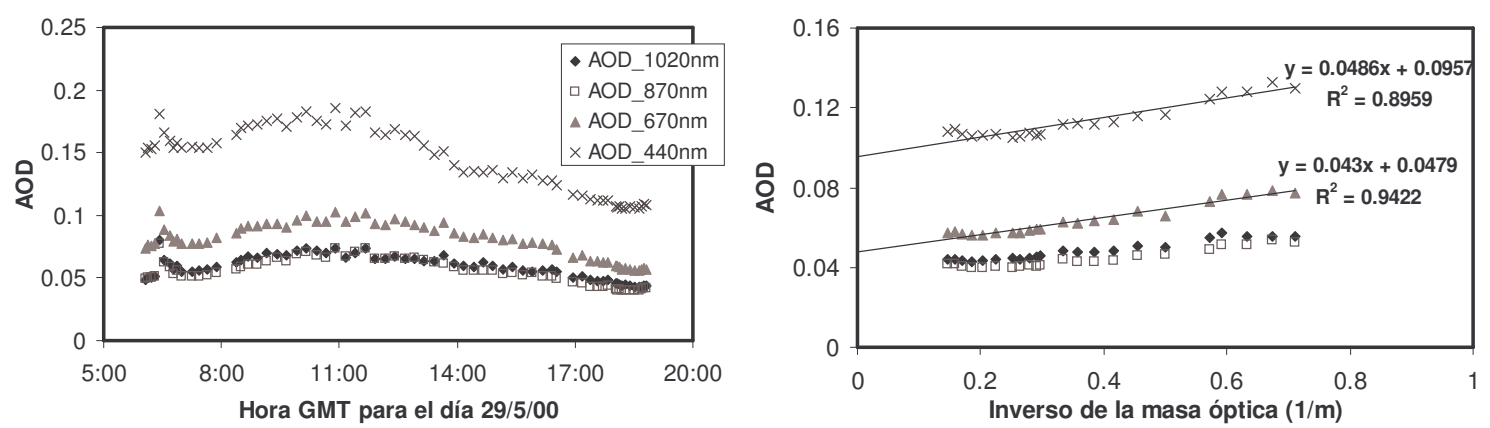

Figura III-4. Espesor óptico de aerosoles (izquierda) y ajustes KCICLO para los datos de la tarde (derecha) del día 29 de mayo de 2000 en El Arenosillo. Medidas del Cimel \#48.

Los coeficientes de precalibración, con los que están calculados estos espesores ópticos, corresponden a la calibración realizada en el GSFC el 1 de mayo de 1999, por intercomparación con el instrumento de referencia (o master) Cimel \#94. La poscalibración se realizó el 12 de octubre de 2000, también en el GSFC, y en este caso por intercomparación con otro master, el instrumento \#101. Esto se recoge en la Tabla III-II. En ella detallamos los coeficientes de calibración, que denominamos $\mathrm{CN}_{0}$ en lugar de $\mathrm{I}_{0}$ 
porque no están en unidades físicas sino en cuentas digitales, tal como se expresan el los ficheros originales de calibración de AERONET. Según la notación que hemos utilizado los coeficientes de precalibración son los designados con la tilde $\left(\mathrm{CN}^{\prime}{ }_{0}\right)$.

\begin{tabular}{lcccc}
\hline \hline Filtro & 1020 & 870 & 670 & 440 \\
\hline CN $_{0}$ PRECALIBRACIÓN & 17648.801 & 19761.714 & 20778.175 & 15802.154 \\
Error relativo \% & 0.292 & 0.204 & 0.242 & 0.243 \\
CN ${ }_{0}$ POSCALIBRACIÓN & 16721.985 & 19165.907 & 19294.611 & 14599.247 \\
Error relativo \% & 0.181 & 0.137 & 0.250 & 0.194 \\
Diferencia absoluta & -926.816 & -595.807 & -1483.564 & -1202.907 \\
Diferencia relativa (\%) & -5.3 & -3.1 & -7.0 & -7.9 \\
\hline \hline
\end{tabular}

Tabla III-II. Coeficientes de pre y poscalibración del Cimel \#48, errores asociados y diferencias entre ambos.

También en esta tabla se adjuntan sus errores, del orden del $0.2 \%$. Estos errores especificados por AERONET para los coeficientes de calibración se obtienen a partir de la recta de ajuste que da la relación entre el instrumento de campo y el master. En realidad están por debajo del error que la propia red AERONET fija en la calibración por intercomparación con los master, que es del orden del 1-2\% (Holben et al., 1998; Eck et al., 1999). Este error da lugar a un error absoluto del AOD de 0.01-0.02, es decir, en torno al $10 \%$ en los valores habituales (0.1-0.2) del AOD en nuestra estación. Las diferencias absolutas y relativas en la Tabla III-II se han evaluado con respecto a la precalibración.

La discusión sobre los errores asociados al método KCICLO es fundamental en este trabajo, ya que nos va a indicar el nivel de precisión con que podemos determinar la constante $\mathrm{K}$ y por tanto los nuevos coeficientes de calibración, y el alcance de la corrección de los espesores ópticos que se propone. Como veremos este método tiene un nivel de precisión que se sitúa alrededor del $0.5 \%$, con capacidad, por tanto, de corregir los errores del orden del $2-8 \%$ que parecen presentar nuestros datos.

Los ajustes $\mathrm{K}$ se han realizado tras calcular los espesores ópticos con los coeficientes de precalibración $\left(\mathrm{CN}_{0}{ }_{0}\right)$. A estos estarán referidos los valores de $\mathrm{K}$ que obtengamos, aunque conviene recordar que los datos del Cimel \#48 están el nivel 2.0 de AERONET, y que por tanto han sido procesados con unos coeficientes de calibración obtenidos por interpolación entre los coeficientes pre y pos.

Los promedios de los ajustes realizados para cada canal se presentan en la Tabla III-III. A partir de los valores de $\mathrm{K}$ de los ajustes individuales se hace un promedio para calcular el valor de $\mathrm{K}$ promedio para cada filtro respecto a un período determinado de tiempo, y su desviación estándar (STD). Los nuevos $\mathrm{CN}_{0}$ se calculan dividiendo por $\mathrm{K}$ los primitivos $\mathrm{CN}_{0}$.

\begin{tabular}{lcccc}
\hline \hline Filtro & $\mathbf{1 0 2 0}$ & $\mathbf{8 7 0}$ & $\mathbf{6 7 0}$ & $\mathbf{4 4 0}$ \\
\hline $\mathrm{CN}_{0}$ (pre) & 17648.801 & 19761.714 & 20778.175 & 15802.154 \\
$\mathbf{K}( \pm \mathbf{S T D})$ & $\mathbf{1 . 0 3 4} \pm \mathbf{0 . 0 0 3}$ & $\mathbf{1 . 0 3 0} \pm \mathbf{0 . 0 0 3}$ & $\mathbf{1 . 0 3 5} \pm \mathbf{0 . 0 0 3}$ & $\mathbf{1 . 0 4 7} \pm \mathbf{0 . 0 0 5}$ \\
Nuevo $_{\mathrm{CN}}$ & 17069 & 19185 & 20075 & 15087 \\
Error $_{0} \mathrm{CN}_{0} \%$ & 0.26 & 0.33 & 0.30 & 0.46 \\
Differencia con Pre $(\%)$ & -3.3 & -2.9 & -3.4 & -4.5 \\
\hline \hline
\end{tabular}

Tabla III-III. Valores de $\mathrm{K}$ con su error junto con los nuevos $\mathrm{CN}_{0}$ para el Cimel \#48.

Los resultados de los distintos ajustes (Figura III-5) o valores de K presentan una dispersión notable. Las fuertes discrepancias entre unos ajustes y otros se deben a la inestabilidad del día, es decir, a que el AOD no fue en realidad constante en los días seleccionados, como podemos esperar en una estación a nivel del mar. Esto, como hemos señalado, puede sesgar el resultado del ajuste KCICLO sin afectar al error del ajuste. Las dificultades para 
obtener una buena calibración mediante un único ajuste de langley in situ son por tanto muy grandes en estaciones no remotas. Las barras de error para los diferentes valores de $\mathrm{K}$, obtenidas a partir de los errores del ajuste, son mucho menores que la variabilidad real de K. Esta variabilidad de la atmósfera afecta a todos los canales, aunque es mayor en términos absolutos para los de 440 y $670 \mathrm{~nm}$. El procedimiento de interpolación de AERONET entre la pre y la poscalibración sugiere que sería esperable una degradación progresiva de los filtros y por tanto en el valor de los $\mathrm{K}$ derivados de los ajustes. Para el caso concreto del Cimel \#48 este efecto se observa sobre todo en el filtro de 670nm.

Para el caso de los filtros de 1020nm y 870nm el comportamiento es muy bueno, y la desviación estándar para el valor promedio de $\mathrm{K}$ se sitúa por debajo del $0.3 \%$. Los ajustes en el canal de 440nm son los que presentan mayor dispersión y por tanto el valor más alto del error de $\mathrm{K}$, con el $0.46 \%$. El filtro de $670 \mathrm{~nm}$ en este instrumento presenta en el valor de $\mathrm{K}$ una tendencia a aumentar, lo cual concuerda con la gran diferencia entre la precalibración y la poscalibración en este filtro.
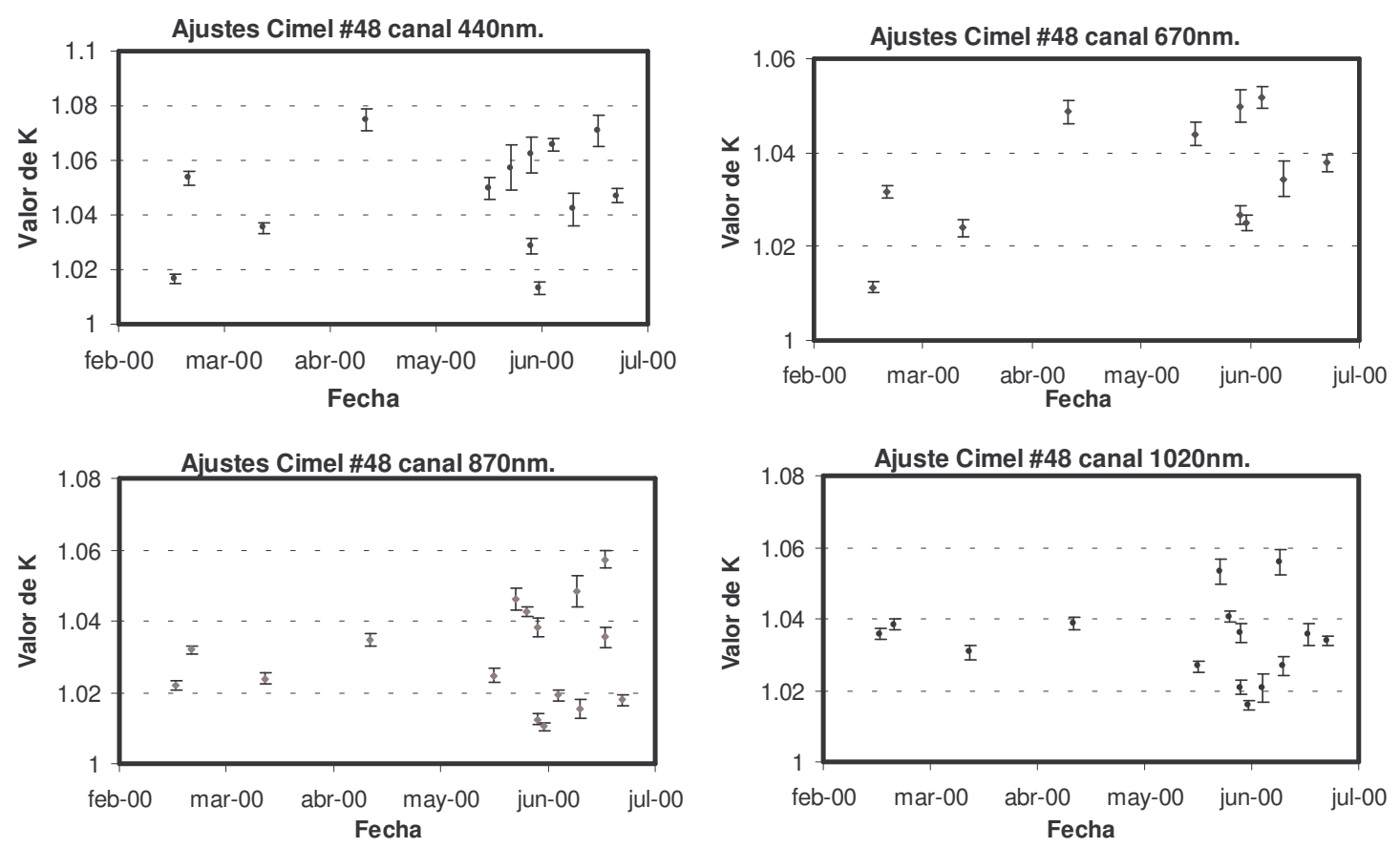

Figura III-5. Ajustes KCICLO para el Cimel \#48 para cada uno de los 4 canales de AOD.

Para finalizar analizamos las diferencias obtenidas al comparar entre sí todos los coeficientes de calibración que tenemos. Lo coeficientes de poscalibración son todos ellos inferiores a los de la precalibración, con diferencias del $5.3 \%$ y 3\% para los filtros $1020 \mathrm{~nm}$ y $870 \mathrm{~nm}$ y $7 \%$ y casi $8 \%$ para $670 \mathrm{~nm}$ y $440 \mathrm{~nm}$ (Tabla III-II).

También hemos representado los coeficientes de calibración en la Figura III-6. El filtro que más se ha degradado es el de 670nm. Los nuevos coeficientes calculados por el método KCICLO tienen en este caso un valor intermedio entre los coeficientes de pre y poscalibración para todos los canales. Nótese el gran número de meses que transcurrieron entre la precalibración y la puesta en estación del instrumento, período en el que teóricamente los filtros no se degradan. Sin embargo la poscalibración se realizó sólo 2 meses después de su retirada de El Arenosillo. Por tanto el coeficiente de calibración final, que es una interpolación temporal entre la pre y la pos, está muy cercano al coeficiente de la poscalibración. Éste último queda muy por debajo del coeficiente que da el método KCICLO para el canal de 670nm. De ahí que los datos en nivel 2.0 de AERONET tengan una fuerte subestimación del AOD en $670 \mathrm{~nm}$, como veremos en el capítulo 5. 


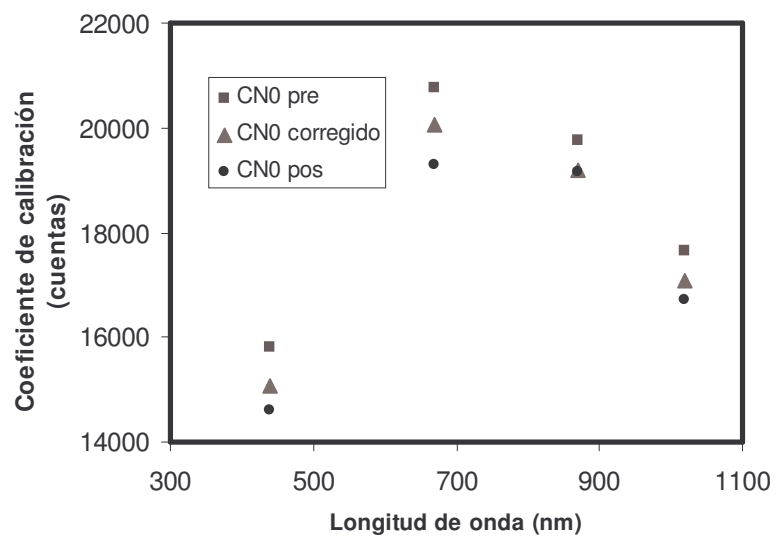

Figura III-6. Diferentes coeficientes de calibración para el Cimel \#48.

Los valores de K que hemos obtenido para cada canal se emplearán para corregir los AOD medidos con este fotómetro, como se explica en la sección 3.5. Vamos a proceder de manera análoga con el resto de los fotómetros que se han ido instalando en El Arenosillo.

\subsubsection{Cimel \#114}

El Cimel \#114 estuvo instalado entre el 10 de julio de 2000 y el 21 de julio de 2001. Este fotómetro ha sido el más problemático de los instalados hasta ahora, lo cual también ha supuesto la oportunidad de desarrollar este trabajo. Las Tabla A1-IV a Tabla A1-VIII del anexo 1 detallan los ajustes realizados para cada filtro en los días seleccionados. En ellas se indica de nuevo los distintos valores de $\mathrm{K}$ con su error.

La Figura III-7 muestra uno de los días seleccionados, en el que podemos observar las deficiencias en la precalibración de este fotómetro, que se traducen en falso ciclo diurno en tres de los canales de AOD. Se trata del día 30 de enero de 2001. El ajuste se realiza con los datos de la mañana, que es muy estable y con valores muy bajos de espesor óptico de aerosoles (entre 0.025 y 0.07 para el filtro de 440nm). Como vemos en la figura de la izquierda, el AOD muestra un ciclo diurno que da lugar a valores de AOD negativos en los canales de 870 y 1020nm, y también un ciclo diurno hacia arriba en el canal de 440nm. En la figura de la derecha se incluyen los resultados del ajuste KCICLO con los parámetros de las rectas de regresión para los filtros de 440nm y 870nm.
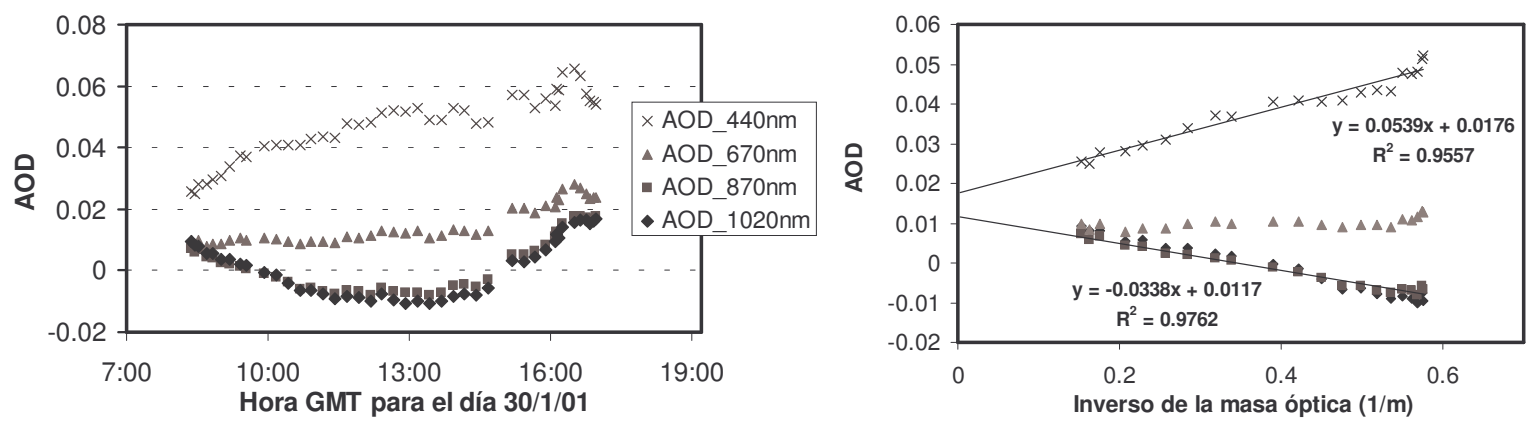

Figura III-7. Espesor óptico de aerosoles (izquierda) y ajustes KCICLO (derecha) para el día 30 de enero de 2001 en El Arenosillo. Datos del Cimel \#114.

Los coeficientes de precalibración, con los que están calculados estos espesores ópticos, corresponden a la calibración realizada en el GSFC el 22 de junio de 1999, por intercomparación con el Cimel \#37. Se realizó por tanto un año antes de la instalación del 
fotómetro. La poscalibración se realizó el 12 de febrero de 2002 en el GSFC, seis meses después de la retirada del fotómetro, y en este caso por comparación con el instrumento \#117. En la Tabla III-IV se recogen todos estos datos, al igual que se hizo con el Cimel \#48. Los filtros $1020 \mathrm{~nm}$ y $870 \mathrm{~nm}$ muestran una deriva alrededor del $2 \%$ con respecto a la precalibración; el filtro de $670 \mathrm{~nm}$ un $4.6 \%$ y el de $440 \mathrm{~nm}$ un $8.5 \%$, siempre con valores menores en la poscalibración.

\begin{tabular}{lcccc}
\hline \hline Filtro & 1020 & 870 & 670 & 440 \\
\hline CN ${ }_{0}$ PRECALIBRACIÓN & 16967.223 & 18390.122 & 19254.920 & 14655.182 \\
Error relativo \% & 0.145 & 0.254 & 0.126 & 0.292 \\
CN ${ }_{0}$ POSCALIBRACIÓN & 16646.405 & 18027.009 & 18294.959 & 13304.502 \\
Error relativo \% & 0.128 & 0.090 & 0.126 & 0.076 \\
Diferencia absoluta & -320.18 & -363.13 & -959.961 & -1350.68 \\
Diferencia relativa (\%) & -1.9 & -1.9 & -4.6 & -8.5 \\
\hline \hline
\end{tabular}

Tabla III-IV. Coeficientes de pre y poscalibración del Cimel \#114, errores asociados y diferencias entre ambos.

Los datos del Cimel \#114 están en el nivel 1.5 de AERONET por los problemas expuestos en la sección 2.4. Ahora veremos que tres de los cuatro filtros tenían una calibración errónea.
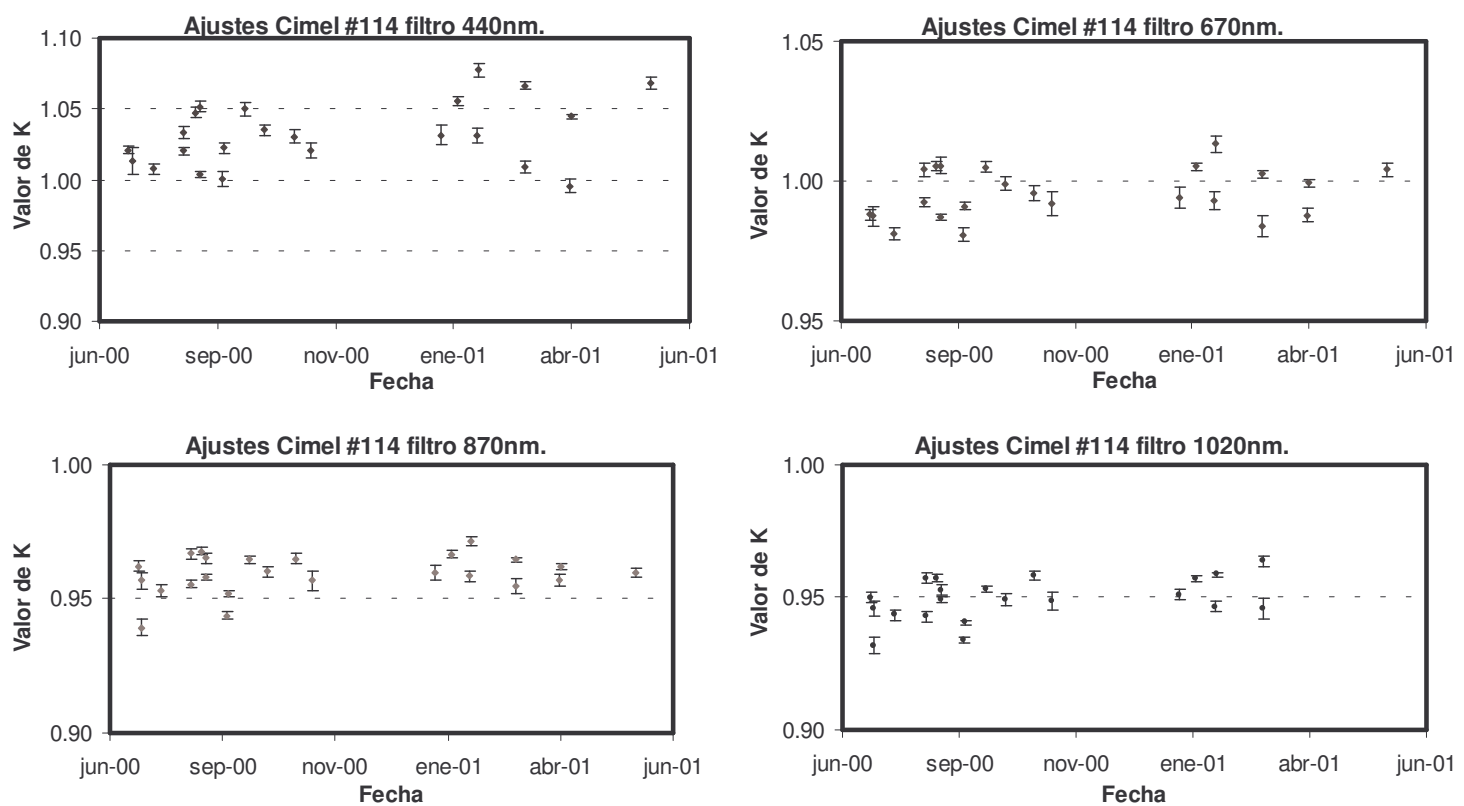

Figura III-8. Ajustes K para el Cimel \#114 para cada uno de los 4 canales de AOD.

Los resultados de los ajustes se presentan en la Tabla III-V. Los valores de $\mathrm{K}$ que obtenemos están referidos a los coeficientes de la precalibración. A partir de ellos, se hace un promedio para calcular el valor de $\mathrm{K}$ para cada filtro. Los nuevos $\mathrm{CN}_{0}$ se calculan dividiendo por $\mathrm{K}$ los primitivos $\mathrm{CN}_{0}$.

\begin{tabular}{lcccc}
\hline \hline Filtro & $\mathbf{1 0 2 0}$ & $\mathbf{8 7 0}$ & $\mathbf{6 7 0}$ & $\mathbf{4 4 0}$ \\
\hline $\mathrm{CN}_{0}$ (pre) & 16967.223 & 18390.122 & 19254.920 & 14655.182 \\
$\mathbf{K}( \pm \mathrm{STD})$ & $\mathbf{0 . 9 4 9 4 \pm 0 . 0 0 1 8}$ & $\mathbf{0 . 9 5 9 3 \pm \mathbf { 0 . 0 0 1 5 }}$ & $\mathbf{0 . 9 9 5 6 \pm 0 . 0 0 1 9}$ & $\mathbf{1 . 0 3 2 \pm \mathbf { 0 . 0 0 5 }}$ \\
Nuevo $\mathrm{CN}_{0}$ & 17872 & 19171 & 19341 & 14200 \\
Error $\mathrm{CN}_{0} \%$ & 0.19 & 0.16 & 0.19 & 0.46 \\
Differencias con Pre(\%) & 4.7 & 4.2 & 0.5 & -3.1 \\
\hline \hline
\end{tabular}

Tabla III-V. Valores de $\mathrm{K}$ con su error junto con los nuevos $\mathrm{CN}_{0}$ para el Cimel \#114

Los resultados de los distintos ajustes se disponen en torno a un cierto valor promedio de forma satisfactoria para los filtros de $670 \mathrm{~nm}$, $870 \mathrm{~nm}$ y $1020 \mathrm{~nm}$, con un error relativo 
asociado al $\mathrm{K}$ y a los nuevos coeficientes de calibración por debajo del $0.2 \%$. Por ejemplo en 1020nm los ajustes presentan una variabilidad claramente menor que la corrección del $5 \%$ que se va a realizar sobre el coeficiente de calibración, ya que los valores de $\mathrm{K}$ son aproximadamente de 0.95 para este canal. El canal de 440nm tiene un comportamiento más irregular, y su error está en el $0.46 \%$.

El caso de este fotómetro nos permite ilustrar todas las posibilidades de ciclo diurno. Tenemos dos filtros (1020 y 870nm) que estaban mal calibrados por defecto, obteniendo un valor de $\mathrm{K}$ menor que 1 . Esto se aprecia muy bien en la gráfica del espesor óptico para el día 30 de enero de 2001 (Figura III-7), en donde se ve que esos dos filtros presentan un ciclo diurno hacia abajo. El filtro de 440nm presenta un ciclo diurno hacia arriba, por sobrestimación del coeficiente de calibración $(\mathrm{K}>1)$. El filtro de $670 \mathrm{~nm}$ se puede decir que está correctamente calibrado en este caso, presentando un valor de $\mathrm{K}$ aproximadamente igual a 1 y un AOD estable durante el día, sin ciclo diurno.

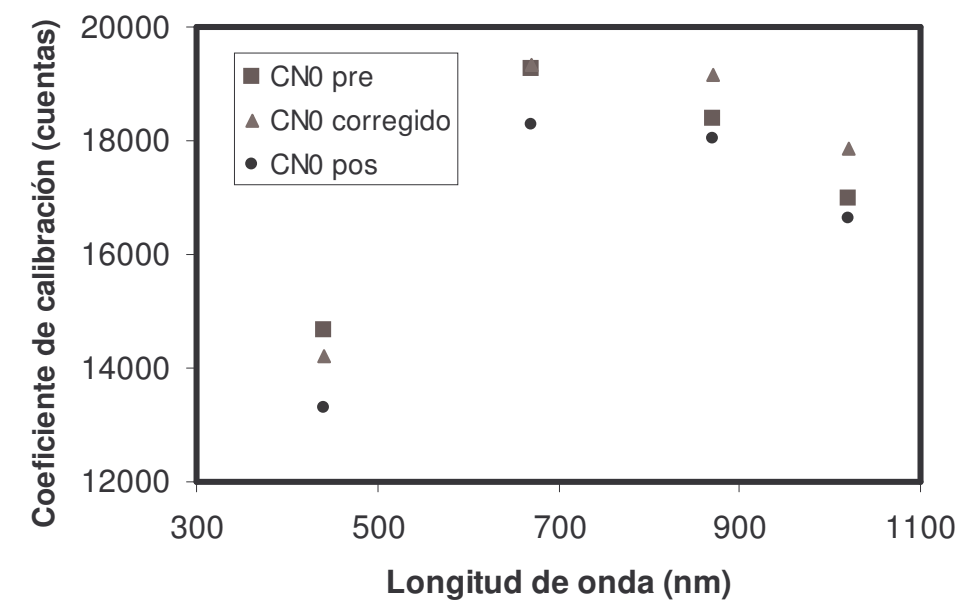

Figura III-9. Diferentes coeficientes de calibración para el Cimel \#114.

Como resumen de los resultados tenemos la comparación de los nuevos coeficientes con los valores de pre-pos calibración, que mostramos en la Figura III-9, la cual pone de manifiesto la defectuosa calibración en los filtros de $870 \mathrm{~nm}$ y $1020 \mathrm{~nm}$. Tanto la pre como la poscalibración están fuertemente subestimadas, más del $4 \%$, lo cual provoca falso ciclo diurno con espesores ópticos negativos durante los días de baja turbiedad. La calibración del canal de 440nm queda razonablemente entre la pre y la poscalibración. El filtro de $670 \mathrm{~nm}$ muestra gran concordancia entre el reprocesado KCICLO y la precalibración, mientras que la poscalibración queda demasiado alejada. Conviene recordar que nuestro coeficiente obtenido por el método KCICLO debe ser comparado con la interpolación entre esas dos calibraciones de AERONET.

\subsubsection{Cimel \#45}

El Cimel \#45 estuvo instalado entre el 22 de julio de 2001 y el 27 de enero de 2003. Las Tabla A1-IX a Tabla A1-XIV del anexo 1 detallan los ajustes realizados para cada canal en los días seleccionados. Es el Cimel que más tiempo ha estado en estación, más del previsto en AERONET, y sin embargo sus datos no presentan muchos problemas y han sido ya elevados al nivel 2.0. 
El día que mostramos como ejemplo en la Figura III-10 es el 6 de diciembre de 2002. Es un día con un espesor óptico de aerosoles muy bajo, en el que se aprecia cómo el filtro de $670 \mathrm{~nm}$ es el que tiene una pendiente (y por tanto un error) más significativo.
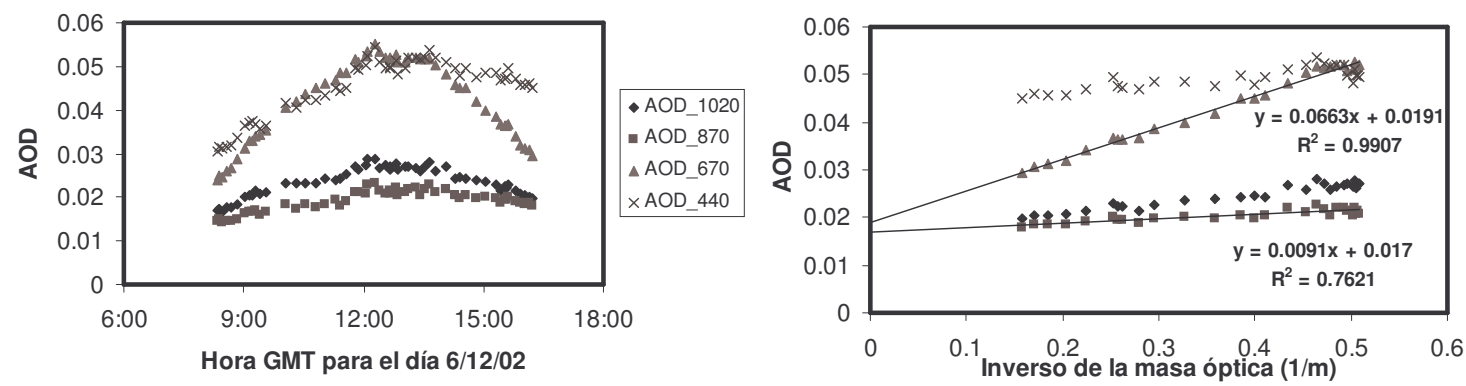

Figura III-10. Espesor óptico de aerosoles el día 6 de diciembre de 2002 en El Arenosillo (izquierda) y ajustes KCICLO para los datos de la tarde (derecha).Medidas del Cimel \#45.

Los coeficientes de precalibración, con los que están calculados estos espesores ópticos, corresponden a la calibración realizada el 23 de mayo de 2001, por intercomparación con el Cimel \#37. La poscalibración se realizó el 18 de marzo de 2003 en LOA-Lille, por intercomparación con el instrumento \#117. El canal de $670 \mathrm{~nm}$ es el único que presenta una deriva importante, superior al $5 \%$ (Tabla III-VI).

\begin{tabular}{lcccc}
\hline \hline Filtro & 1020 & 870 & 670 & 440 \\
\hline CN $_{0}$ PRECALIBRACIÓN & 15630.675 & 19361.243 & 16591.166 & 14085.025 \\
Error relativo \% & 0.097 & 0.095 & 0.113 & 0.115 \\
CN ${ }_{0}$ POSCALIBRACIÓN & 15472.975 & 19289.205 & 15676.887 & 14069.048 \\
Error relativo \% & 0.131 & 0.136 & 0.075 & 0.082 \\
Diferencia absoluta & -157.70 & -72.04 & -914.28 & -15.98 \\
Diferencia relativa (\%) & -1.01 & -0.37 & -5.67 & -0.11 \\
\hline \hline
\end{tabular}

Tabla III-VI. Coeficientes de pre y poscalibración del Cimel \#45, errores asociados y diferencias entre ambos.

Los datos del Cimel \#45 están en el nivel 2.0 de AERONET, esto es, su calidad está certificada. Igual que en el caso del Cimel \#48, también en el nivel 2.0, podemos todavía mejorar la calidad de esta serie por nuestro procedimiento.

Los resultados de los ajustes se presentan en la Tabla III-VII. Los valores de $\mathrm{K}$ que obtenemos están de nuevo referidos a los coeficientes de la precalibración. A partir de ellos, se hace un promedio para calcular el valor de $\mathrm{K}$ para cada canal. Los nuevos $\mathrm{CN}_{0}$ se calculan dividiendo por $\mathrm{K}$ los primitivos $\mathrm{CN}_{0}$.

\begin{tabular}{|c|c|c|c|c|}
\hline Filtro & 1020 & 870 & 670 & 440 \\
\hline CN'0 & 15630.675 & 19361.243 & 16591.166 & 14085.025 \\
\hline K ( \pm STD $)$ & $\begin{array}{c}1.0121 \pm \\
0.0017\end{array}$ & $\begin{array}{c}1.0046 \pm \\
0.0019\end{array}$ & $\begin{array}{c}1.046 \pm 0.004 \\
1.030 \pm 0.003(1 \text { er p. }) \\
1.062 \pm 0.002\left(2^{\circ} \text { p. }\right)\end{array}$ & $\begin{array}{c}1.010 \pm \\
0.004\end{array}$ \\
\hline Nuevo CNO & 15474 & 19329 & $\begin{array}{c}15876 \\
16124 \text { (inicial) } \\
15636 \text { (final) } \\
\end{array}$ & 13828 \\
\hline Error CNO \% & 0.17 & 0.19 & 0.41 & 0.42 \\
\hline Diferencias con pre(\%) & -1.2 & -0.5 & -4.4 & -1.0 \\
\hline
\end{tabular}

Tabla III-VII. Valores de $\mathrm{K}$ con su error junto con los nuevos $\mathrm{CN}_{0}$ para el Cimel \#45 

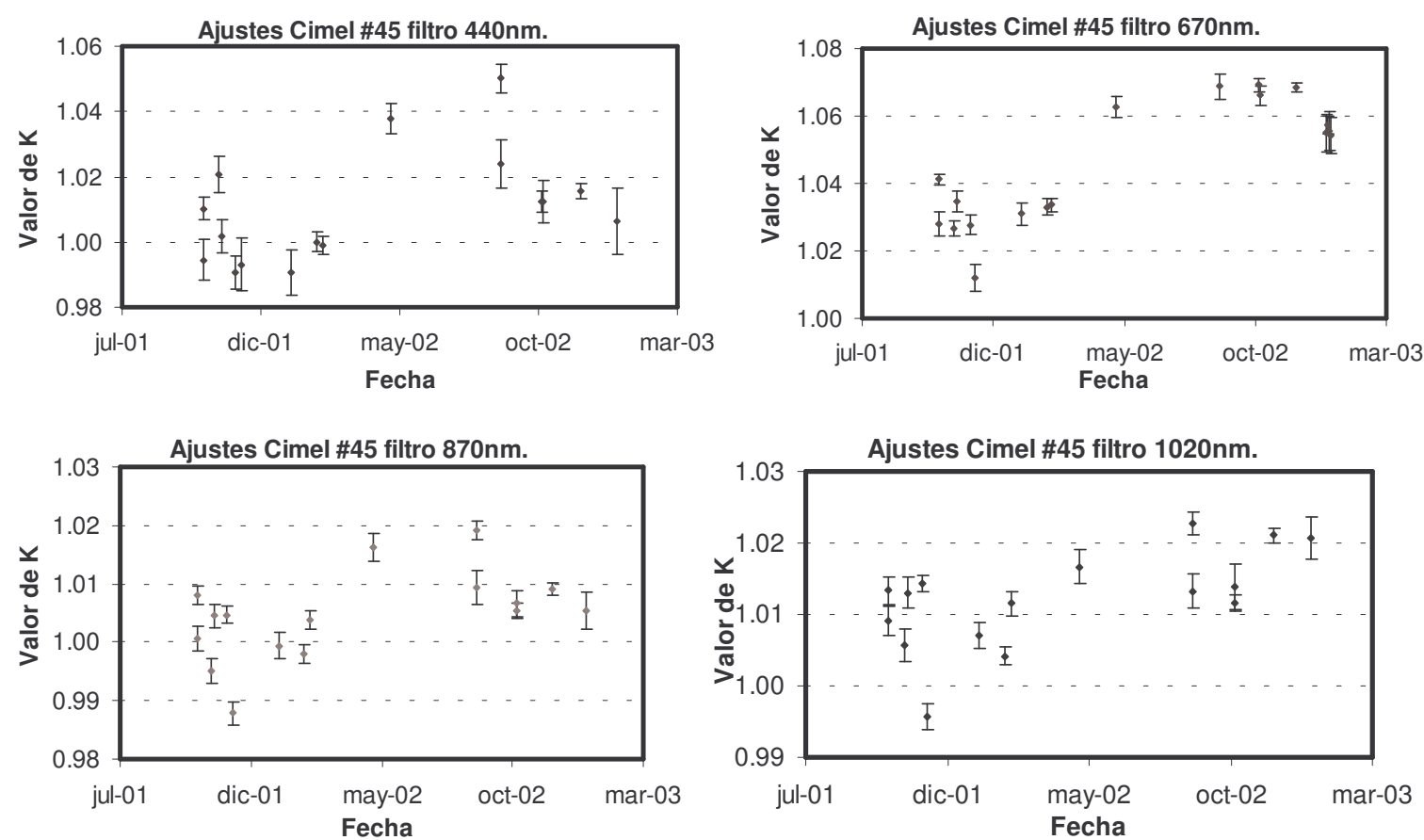

Figura III-11. Ajustes K para el Cimel \#45 en cada uno de los 4 canales de AOD

Los resultados de los ajustes KCICLO tienen menos error en los canales de 1020nm y 870nm. La dispersión en los ajustes en 670nm y 440nm hacen que el error calculado para los nuevos coeficientes de calibración en estos canales esté en torno al $0.4 \%$, el doble que para los canales del infrarrojo. En el Cimel \#45 sólo el filtro de 670nm tiene un error de calibración considerable, en torno al $5 \%$, lo cual le provoca un ciclo diurno muy acusado, con lo que incluso llega a superar en ocasiones el espesor óptico de aerosoles en 440nm en torno al mediodía, algo en principio poco realista en la atmósfera. La deriva tan fuerte del filtro de 670nm nos ha obligado a tomar dos períodos para el coeficiente de calibración, hasta julio de 2002 y desde esa fecha hasta el final, lo cual mejoró notablemente la serie de datos reprocesada.

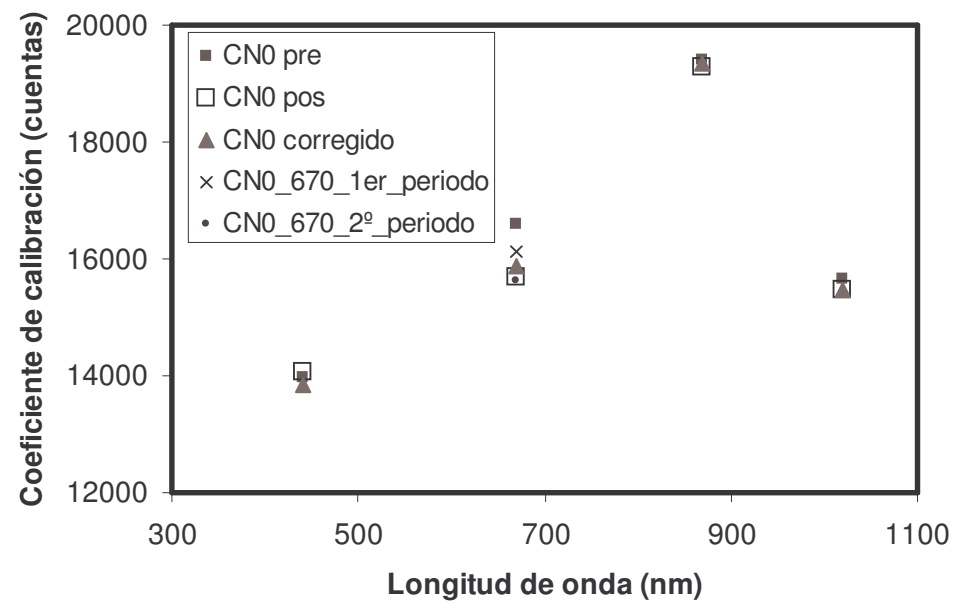

Figura III-12. Diferentes coeficientes de calibración para el Cimel \#45

En la Figura III-12 tenemos los diferentes coeficientes de calibración en cuentas digitales para cada canal. En los canales de 440, 870 y 1020nm prácticamente no hay diferencias entre la pre, la poscalibración y los coeficientes que da el método KCICLO. Nuevamente el filtro de 670nm parece haber experimentado una fuerte degradación (más del 5\% entre pre y pos) y es el único problemático en este fotómetro. Vemos que los coeficientes del 
método KCICLO quedan cerca de la poscalibración, mientras que la precalibración tiene un coeficiente demasiado alto que es el causante del ciclo diurno del AOD en este canal.

\subsubsection{Cimel \#50}

El Cimel \#50 estuvo instalado entre el 27 de enero de 2003 y el 12 de mayo de 2004. En el anexo 1 se detallan de nuevo los ajustes realizados en los días seleccionados para cada canal (Tabla A1-XIII a Tabla A1-XVII). Estos datos han sido ya elevados al nivel 2.0 de AERONET.

En la Figura III-13 mostramos como ejemplo el día 26 de abril de 2004. Durante la mañana el AOD fue muy estable y el ajuste del método KCICLO nos muestra la dependencia con la masa óptica en los canales de 440 y $670 \mathrm{~nm}$, mientras que en 870 y $1020 \mathrm{~nm}$ el AOD permanece prácticamente constante. A partir de las $11 \mathrm{~h}$ el AOD ya no es estable, como se observa en el cambio de la evolución diurna en los cuatro canales.
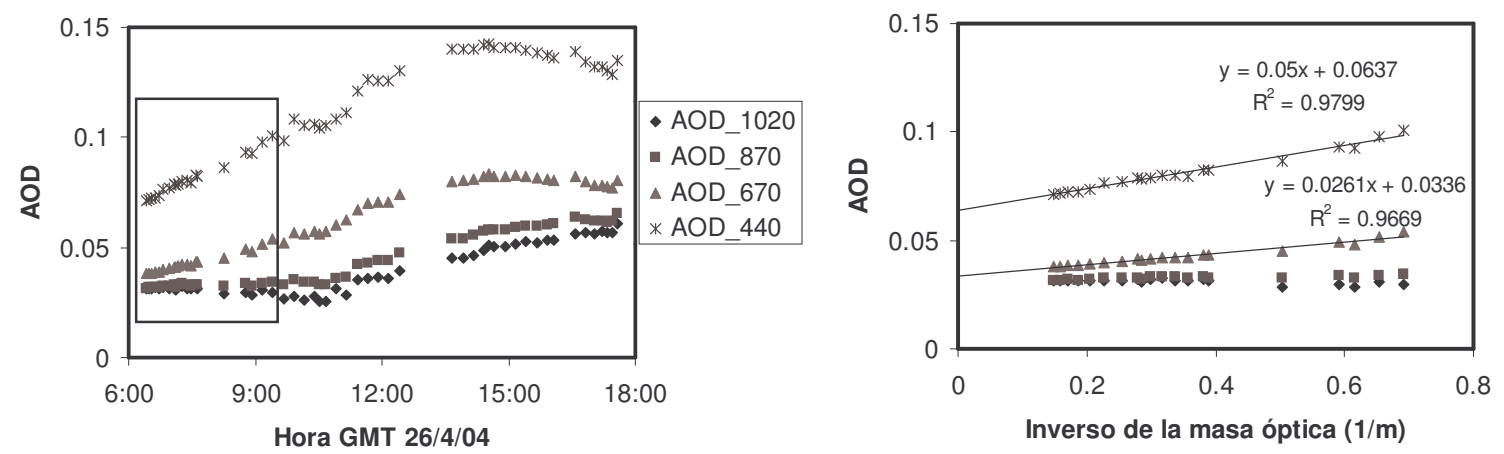

Figura III-13. Espesor óptico de aerosoles y ajustes del método KCICLO para la mañana del día 26 de abril de 2004 en El Arenosillo. Datos del Cimel \#50.

La precalibración fue realizada por el LOA el día 14 de diciembre de 2002 por comparación con el master \#117. La poscalibración se realizó el 15 de julio de 2004 en el LOA, por comparación con el instrumento \#245. Los coeficientes de calibración se encuentran recogidos en la Tabla III-VIII. Queremos resaltar que los canales de 870nm y sobre todo $1020 \mathrm{~nm}$ tienen un coeficiente de calibración más alto en la poscalibración. Lo habitual es que sea menor, es decir, que en su degradación los filtros disminuyan su transmitancia. También es posible que aumenten su transmitancia, aunque esto se ha observado en AERONET sobre todo para los filtros de ultravioleta, que no están instalados en el Cimel de El Arenosillo.

\begin{tabular}{lcccc}
\hline \hline Filtro & 1020 & 870 & 670 & 440 \\
\hline CN $_{0}$ PRECALIBRACIÓN & 15573.233 & 15405.942 & 24179.714 & 14113.657 \\
Error relativo \% & 0.144 & 0.223 & 0.165 & 0.184 \\
CN $_{0}$ POSCALIBRACIÓN & 15789.437 & 15440.084 & 24131.789 & 13989.87 \\
Error relativo \% & 0.558 & 0.314 & 0.257 & 0.615 \\
Diferencia absoluta & 216 & 34 & -48 & -124 \\
Diferencia relativa (\%) & 1.4 & 0.2 & -0.2 & -0.9 \\
\hline \hline
\end{tabular}

Tabla III-VIII. Coeficientes de pre y poscalibración del Cimel \#50, errores asociados y diferencias entre ambos.

El conjunto de ajustes KCICLO realizados durante el período de medidas del Cimel \#50 (Figura III-14) vuelve a mostrar dispersión sobre todo en los canales de 440 y 670nm, con valores de $\mathrm{K}$ entre 1 y 1.08 en 440nm, que en todo caso indican una calibración con error por exceso en este canal. También se da un error de calibración por exceso aunque en menor medida para el canal de 670nm. En 870 y 1020nm los ajustes se agrupan en torno al valor de $\mathrm{K}=1$, es decir, una calibración correcta. 

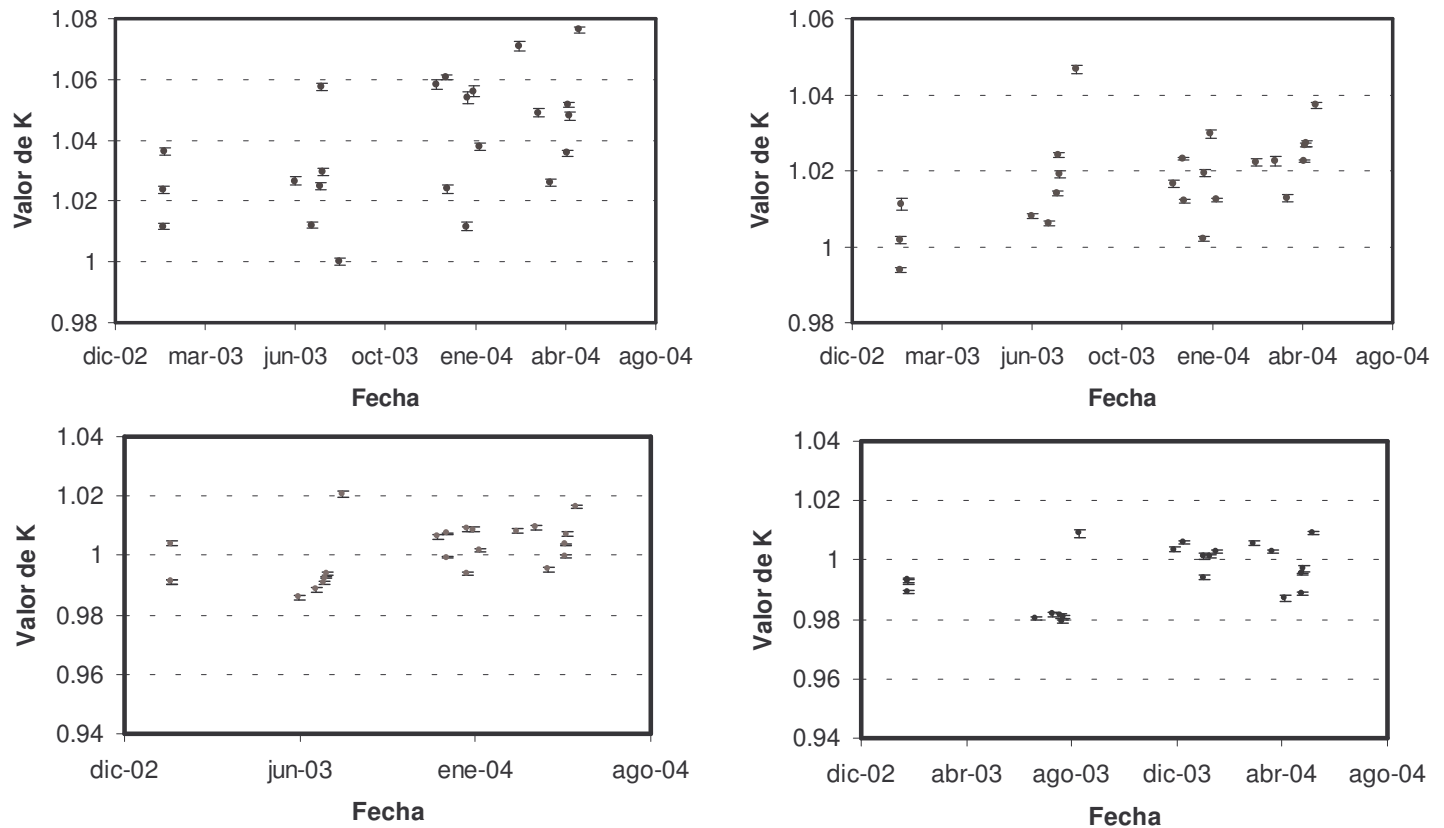

Figura III-14. Ajustes K para el Cimel \#50 en cada uno de los 4 canales para la medida del AOD.

Los resultados estos los ajustes (los coeficientes 'K') se muestran en la Tabla III-IX. Según esto, los canales de $870 \mathrm{~nm}$ y $1020 \mathrm{~nm}$ están correctamente calibrados a lo largo del período de operación de este instrumento, como también podemos ver en la Figura III-15. En $870 \mathrm{~nm}$ y $1020 \mathrm{~nm}$ tanto los coeficientes de la pre, la poscalibración y el que da el método KCICLO presentan pequeñas diferencias. Sin embargo en $440 \mathrm{~nm}$ las calibraciones de AERONET tienen unos valores por encima (más del 3\%) del método KCICLO, lo que implica un error por exceso en el coeficiente de calibración y un ciclo diurno hacia arriba en ese canal. Un resultado análogo aunque con una diferencia por debajo del $2 \%$ se da en el canal de $670 \mathrm{~nm}$. La combinación de ambos errores da como resultado un parámetro alfa con una fuerte sobrestimación (mayor del 25\%), como mostraremos en el capítulo 5.

\begin{tabular}{lcccc}
\hline \hline Filtro & $\mathbf{1 0 2 0}$ & $\mathbf{8 7 0}$ & $\mathbf{6 7 0}$ & $\mathbf{4 4 0}$ \\
\hline CN'0 & 15573.233 & 15405.942 & 24179.714 & 14113.657 \\
K ( \pm STD) & $\mathbf{0 . 9 9 5} \pm \mathbf{0 . 0 0 2}$ & $\mathbf{1 . 0 0 1} \pm \mathbf{0 . 0 0 2}$ & $\mathbf{1 . 0 1 8} \pm \mathbf{0 . 0 0 2}$ & $\mathbf{1 . 0 4 0 \pm 0 . 0 0 4}$ \\
Nuevo CN0 & 15657 & 15389 & 23755 & 13569 \\
Error CNO \% & 0.21 & 0.19 & 0.24 & 0.39 \\
Diferencias con pre(\%) & 0.54 & -0.11 & -1.76 & -3.86 \\
\hline \hline
\end{tabular}

Tabla III-IX. Resultado de los ajustes KCICLO y nuevos $\mathrm{CN}_{0}$ para el Cimel \#50

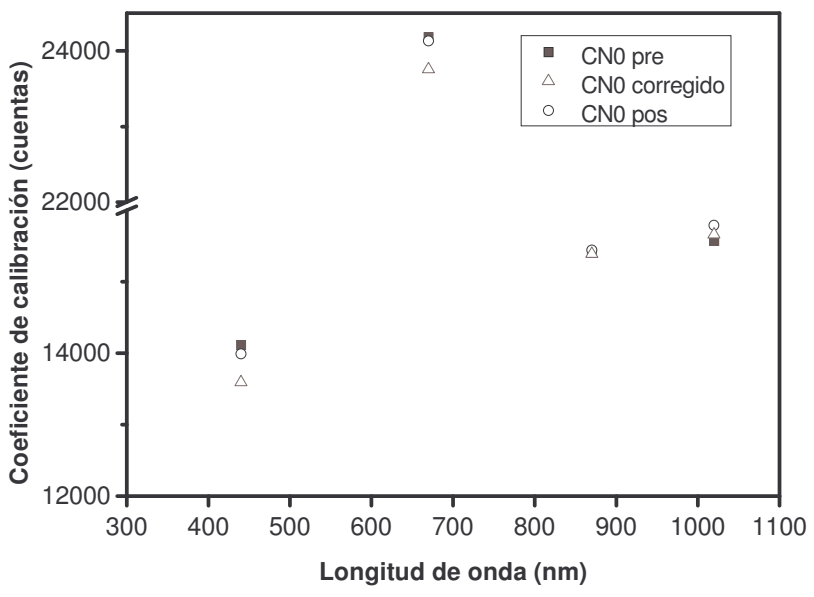

Figura III-15. Diferentes coeficientes de calibración para el Cimel \#50 


\subsection{Análisis del error del método}

Los valores del parámetro $\mathrm{K}$ para cada uno de los ajustes individuales se han presentando con su error en las diversas tablas. También se indica el error para los valores promedio de $\mathrm{K}$ para cada instrumento y para los nuevos coeficientes de calibración. También hemos hecho una comparación entre la precisión de los coeficientes de calibración originales y los que aquí se han calculado según el método propuesto. Vamos a detallar a continuación el análisis de errores realizado para obtener esos resultados.

En cada uno de los ajustes del espesor óptico de aerosoles frente al inverso de la masa óptica, obtenemos $\operatorname{lnK}$ como pendiente del ajuste. El ajuste también nos da el error absoluto de esta pendiente, que en las diversas tablas aparece como 'error $\ln K$ ' $(\Delta \ln K)$. Este error de las $\mathrm{K}$ individuales es del orden de $10^{-3}$. Estos errores se muestran con barras de error en las gráficas de la Figura III-5, Figura III-8, Figura III-11 y Figura III-14. Sin embargo se aprecia a simple vista en estas mismas gráficas que la variabilidad de los $\mathrm{K}$ obtenidos en los distintos días es mucho mayor que el error que se estima para cada una de ellas individualmente. En concreto, la desviación típica de la serie de K obtenidas para cualquiera de los instrumentos es del orden de $10^{-2}$, esto es, un orden de magnitud mayor que el error asociado a las $\mathrm{K}$ individuales.

El motivo de esto ya se ha venido apuntando: las variaciones que se producen en el espesor óptico de aerosoles, propias de la atmósfera, no empeoran necesariamente el coeficiente de correlación o la desviación estándar del ajuste, sino que dan como resultado una variabilidad en los valores de $\mathrm{K}$ manteniendo la bondad del ajuste. Esto supone que los errores no son detectables tan solo a partir de un ajuste. Es necesario repetir el ajuste durante un número suficiente de días, que estimamos en torno al $5 \%$ del número total de días que esté el instrumento en estación. En este caso, como se especificó en los criterios para la selección de días, se trata de días no sólo despejados sino también con un espesor óptico de aerosoles muy estable. Son por tanto días poco frecuentes, y en estaciones con peores condiciones que El Arenosillo, esta escasez de días adecuados podría dificultar la aplicación del método o al menos haría aumentar los errores. En la Tabla III-X vemos el número de días en estación y el número de ajustes que se ha realizado, con un porcentaje de días adecuados entre el $3 \%$ y el $9 \%$.

El valor de $\mathrm{K}$ final para un cierto canal se ha obtenido mediante el promedio de los diferentes ajustes realizados en un período determinado. Asignamos al valor promedio de $\mathrm{K}$ un error $(\Delta \bar{K})$ que es la desviación estándar de la media para el conjunto de valores de $\mathrm{K}$, es decir:

$$
\Delta \bar{K}=S T D(K) / \sqrt{N}
$$

Se asume que por variaciones en la atmósfera estos valores de $\mathrm{K}$ experimentan un ruido aleatorio, y que por tanto se distribuyen en torno al valor verdadero, que hemos estimado mediante el promedio. El error relativo de los coeficientes de calibración que finalmente obtenemos es, de acuerdo con la teoría de errores, el mismo que el error relativo de $\mathrm{K}$. Sólo cuando se observa una tendencia clara a degradarse los filtros con el tiempo es aconsejable dividir el período analizado en diversos tramos.

\begin{tabular}{lccc}
\hline \hline Instrumento & Días en estación & $\mathrm{N}^{\circ}$ ajustes & Porcentaje días \\
\hline$\# 48$ & 144 & 13 & $9 \%$ \\
$\# 114$ & 376 & 23 & $6 \%$ \\
$\# 45$ & 553 & 16 & $3 \%$ \\
$\# 50$ & 472 & 23 & $5 \%$ \\
\hline \hline
\end{tabular}

Tabla III-X. Número de ajustes frente al número de días en estación para cada fotómetro. 
Podemos evaluar en qué se traduce esta precisión en los coeficientes de calibración a la hora de calcular espesores ópticos. La corrección realizada a los primitivos espesores ópticos $\left(\tau^{\prime}\right)$ viene dada por $\ln \mathrm{K}$, modulado por el inverso de la masa óptica. Para obtener el error absoluto sobre el nuevo espesor óptico $(\tau)$ utilizamos la teoría de errores a partir de la expresión (3.5), en la que sólo tenemos en cuenta los errores de $\tau_{\mathrm{a}}$ debidos al error de $\mathrm{K}$, es decir, al error en la calibración:

$$
\Delta \tau_{a}=\frac{1}{m} \cdot \frac{\Delta K}{K}
$$

La Tabla III-XI contiene un resumen de las correcciones efectuadas para cada canal en cada instrumento, así como el error calculado para el AOD corregido. Con esta cota de error nos referimos al error sistemático debido exclusivamente a la calibración. El error sobre los nuevos coeficientes de calibración está en torno a 0.003 (el 0.3\%) para los filtros de $1020 \mathrm{~nm}, 870 \mathrm{~nm}$ y $670 \mathrm{~nm}$ (Tabla III-XI). Para el filtro de 440nm el error es mayor, en torno al $0.5 \%$. Esto se traduce en un error absoluto para el espesor óptico menor que 0.003 para $670 \mathrm{~nm}, 870 \mathrm{~nm}$ y $1020 \mathrm{~nm}$, y menor de 0.005 para el canal de $440 \mathrm{~nm}$. Tanto la evaluación de la corrección efectuada como el error del AOD corregido están dados para masa óptica $\mathrm{m}=1$, es decir, los máximos posibles. La masa óptica modula la corrección, que no es constante durante el día, y que es también distinta a lo largo del año por la distinta declinación solar. También vemos que el error final sobre el AOD, que nos da la precisión del método, está un orden de magnitud por debajo de la corrección que se realiza sobre los espesores ópticos.

\begin{tabular}{lcccc}
\hline \hline Canal $(\mathrm{nm})$ & Corrección $(\operatorname{lnK})$ & Error $\tau_{\mathrm{a}}$ & Corrección $(\operatorname{lnK})$ & Error $\tau_{\mathrm{a}}$ \\
\hline \multicolumn{2}{c}{ Cimel \#48 } & \multicolumn{3}{c}{ Cimel \#114 } \\
\hline 1020 & 0.033 & 0.003 & -0.052 & 0.002 \\
870 & 0.030 & 0.003 & -0.042 & 0.002 \\
670 & 0.034 & 0.003 & -0.004 & 0.002 \\
440 & 0.046 & 0.005 & 0.032 & 0.005 \\
\multicolumn{2}{c}{ Cimel \#45 } \\
Canal $(\mathrm{nm})$ & 0.012 & 0.002 & -0.005 & 0.002 \\
1020 & 0.005 & 0.002 & 0.001 & 0.002 \\
870 & 0.045 & 0.004 & 0.018 & 0.002 \\
670 & 0.010 & 0.004 & 0.039 & 0.004 \\
440 & & & & \\
\end{tabular}

Tabla III-XI. Corrección absoluta y error absoluto para el espesor óptico recalculado en cada instrumento y en cada uno de los filtros.

El error absoluto que estima AERONET para el espesor óptico es 0.01-0.02 para los instrumentos que se calibran por intercomparación. Como se ve, la corrección es en muchos casos mayor (en valor absoluto) que ese valor, lo cual indica que la calibración era peor de lo previsto. Esto coincide con las medidas de AOD que presentan un ciclo diurno claro. En los casos en que la corrección es menor que 0.02 podemos afirmar que la calibración de AERONET es la correcta. En estos casos además no hay un ciclo diurno visible en las medidas procesadas por AERONET.

Los errores que especifica AERONET en los ficheros de calibración están un orden de magnitud por debajo de los errores reales. Mientras que a los coeficientes de calibración se les asocia una precisión del $0.1 \%$, al espesor óptico le asocian un error del $2 \%$ aproximadamente (0.02 en valor absoluto sobre el espesor óptico). Nuestra corrección muestra que en ocasiones superan el 5\% de error en los coeficientes de calibración y 0.05 en error absoluto para el AOD. 
A la vista de estos resultados el método KCICLO es capaz de corregir los espesores ópticos hasta disminuir el error absoluto por debajo de las especificaciones de AERONET, a pesar de la dispersión que hemos podido observar entre los diferentes ajustes KCICLO (ajustes in situ). Hay que aclarar que con esta metodología se reduce el error del AOD debido a la calibración, pero no elimina otras fuentes de error, como pueden ser las correcciones de presión u ozono, problemas de temperatura, o simplemente suciedad del fotómetro. En El Arenosillo se deposita habitualmente sal sobre el fotómetro Cimel, y también arena proveniente de las intrusiones saharianas. El protocolo de limpieza es semanal, y esto puede dar lugar a variaciones difícilmente controlables. Por tanto hay que ser cautos en la evaluación de errores.

Por otra parte hay que destacar que los errores de calibración son relativamente sencillos de detectar y corregir, por tratarse de un error sistemático que provoca un efecto tan visible como un ciclo diurno sistemático en las medidas del AOD. 


\subsection{Equivalencia con el método de Langley}

El método de Langley, que ya hemos descrito, se basa en la ley de Beer-Bouguer-Lambert. Este modelo físico establece que debe haber una relación lineal entre el logaritmo de la irradiancia medida y la masa óptica: cuanto más atmósfera se atraviesa mayor es la atenuación.

El método de corrección KCICLO se basa también en la ley de Beer, y por tanto en su base física coincide exactamente con el método de Langley. Partimos de la expresión (3.3), mediante la cual hemos realizado los ajustes del método KCICLO.

Expresamos el espesor óptico total calculado inicialmente con los coeficientes de calibración $\mathrm{CN}_{0}$ (expresados en cuentas digitales, tal y como trabaja el fotómetro Cimel) según indica la ley de Beer-Bouguer-Lambert. Aunque no incluimos el subíndice $\lambda$, por no sobrecargar la notación, se entiende que esta expresión se verifica para cada longitud de onda:

$$
\tau^{\prime}=-\frac{1}{m} \ln \frac{C N}{C N_{0}^{\prime}}
$$

Sustituyendo en (3.3) tenemos:

$$
-\frac{1}{m} \ln \left(\frac{C N}{C N_{0}^{\prime}}\right)=\tau+\frac{1}{m} \ln K
$$

Los coeficientes de calibración que pretendemos obtener son los $\mathrm{CN}_{0}$, y la razón entre ambos es lo que venimos denominando $\mathrm{K}=\mathrm{CN}_{0} / \mathrm{CN}_{0}$. Multiplicamos todo por la masa óptica y sustituimos $\mathrm{K}$ por su valor:

$$
-\ln C N+\ln C N_{0}^{\prime}=\tau \cdot m+\ln \left(C N^{\prime}{ }_{0} / N_{0}\right)
$$

El $\mathrm{LnCN}_{0}$ aparece en ambos miembros y se puede simplificar. Cambiando todo de signo se llega finalmente a una expresión idéntica al ajuste del método de Langley:

$$
\ln C N=-\tau \cdot m+\ln C N_{0}
$$

La base física es por tanto la misma en ambos casos, así como las condiciones para ser aplicados, puesto que en ambos se exige que el espesor óptico de la atmósfera permanezca constante para que las medidas se ajusten a una recta. Esto hace que la variabilidad de la atmósfera sea la principal fuente de error.

La diferencia se encuentra en las magnitudes de entrada y de salida. Mientras que el método de Langley requiere las medidas brutas del instrumento (en cuentas digitales, voltaje, o irradiancia si se tiene una calibración previa en unidades físicas) para realizar el ajuste, el método que proponemos requiere conocer el espesor óptico de aerosoles. El método de Langley nos da como resultado el coeficiente de calibración (dado por la ordenada en el origen del ajuste). Nuestro método da como resultado la razón entre el coeficiente de calibración utilizado y el valor verdadero (dado por la pendiente del ajuste), que denominamos $\mathrm{K}$. 
Esto tiene ventajas e inconvenientes: el inconveniente es que el espesor óptico de aerosoles es una magnitud derivada de la irradiancia, y que por tanto tiene ya un error asociado. Las ventajas radican en que este método nos permite analizar la calidad de una serie de datos de espesores ópticos, y corregir los errores de calibración que pudiera tener, sin necesidad de conocer la calibración con la que están procesados ni ninguna medida bruta del aparato.

Por otro lado, en el ajuste de Langley obtenemos el coeficiente de calibración en la ordenada en el origen, mientras que en nuestro método sale de la pendiente. Matemáticamente es diferente, como muestra en su trabajo Forgan (1994). La consecuencia principal es que el método de Langley clásico da más peso en el ajuste a las medidas de masas de aire grandes, como ya se ha discutido. El método KCICLO es en su forma muy similar a esta variante del método de Langley.

Una ventaja adicional del método KCICLO consiste en trabajar con espesores ópticos. El ajuste KCICLO nos indica directamente si existe o no un error de calibración, puesto que en un día estable no debería existir dependencia de AOD con la masa óptica. En un ajuste de Langley no es posible conocer directamente la existencia de errores a partir del valor de la ordenada en el origen excepto si el fotómetro dispone ya de una calibración absoluta. En el caso del fotómetro Cimel no se dispone de una calibración absoluta al no ser necesaria para el cálculo de espesores ópticos.

Se han realizado una serie de casos de prueba, con objeto de ver si los resultados sobre los coeficientes de calibración, utilizando el método KCICLO o el método Langley in situ, son equivalentes y las conclusiones son que las variaciones entre ellos son del mismo orden que el error asociado al procedimiento.

Un análisis para verificar la calibración de medidas de datos de varios Cimel de AERONET, pero utilizando el método de Langley in situ, fue realizado por el grupo ULCOLISE (Laboratoire Interdisciplinaire de Sciences de l'Environnement, Université du LittoralCôte d'Opale; Martiny, 2001). La estación de El Arenosillo estaba incluida entre ellas. Los resultados que obtienen para los instrumentos \#48 y \#114 (los datos del Cimel \#45 y el \#50 son posteriores a ese análisis) son muy similares a los que hemos obtenido nosotros, mostrando por un lado la equivalencia en la práctica (además de la teoría) de ambas metodologías, además de confirmar nuestros resultados.

Los coeficientes de calibración se recogen en las Tabla III-XII, en los que se realizan comparaciones con los valores de AERONET y los obtenidos por el método KCICLO en el Cimel \#48. El procesado ULCO-LISE plantea dos períodos, aun cuando el tiempo que el aparato está en estación es bastante reducido (6 meses). Se observa una fuerte deriva en los filtros de 440nm y 670nm a la vista de las diferencias entre la precalibración y la poscalibración de AERONET. En este trabajo también se comenta que los instrumentos más antiguos tienen en los filtros derivas más fuertes que los instrumentos más modernos. La corrección que plantea el citado trabajo sobre el canal de 440nm es excesivamente fuerte en comparación con nuestros resultados, debido a que realizan la selección de días con criterios menos restrictivos que el método KCICLO, y alcanzan en este caso un resultado menos preciso. La mayor variabilidad en $440 \mathrm{~nm}$ que en los demás canales obliga a unos criterios restrictivos para los ajustes (bien sean del método KCICLO o el método de Langley). 


\begin{tabular}{ccccc}
\hline \hline Calibración del Cimel \#48 & 1020 & 870 & 670 & 440 \\
\hline Aeronet precalibración & $\mathbf{1 7 6 4 8 . 8 0}$ & $\mathbf{1 9 7 6 1 . 7 1}$ & $\mathbf{2 0 7 7 8 . 1 8}$ & $\mathbf{1 5 8 0 2 . 1 5}$ \\
Aeronet poscalibración & $\mathbf{1 6 7 2 1 . 9 9}$ & $\mathbf{1 9 1 6 5 . 9 1}$ & $\mathbf{1 9 2 9 4 . 6 1}$ & $\mathbf{1 4 5 9 9 . 2 5}$ \\
Diferencia absoluta (cuentas) & -926.82 & -595.81 & -1483.56 & -1202.91 \\
Diferencia relativa (\%) & -5.4 & -3.1 & -7.4 & -7.9 \\
ULCO-LISE feb-abr & $\mathbf{1 7 2 1 1 . 0 9}$ & $\mathbf{1 9 4 4 0 . 1 9}$ & $\mathbf{2 0 3 7 5 . 9 1}$ & $\mathbf{1 4 7 0 8 . 7 3}$ \\
Diferencia absoluta (cuentas) & -437.71 & -321.52 & -402.26 & -1093.42 \\
Diferencia relativa (\%) & -2.5 & -1.6 & -2.0 & -7.2 \\
ULCO-LISE abr-jul & $\mathbf{1 7 0 3 7 . 3 1}$ & $\mathbf{1 9 1 1 0 . 1 8}$ & $\mathbf{1 9 5 6 9 . 1 9}$ & $\mathbf{1 4 2 6 0 . 5 2}$ \\
Diferencia absoluta (cuentas) & -611.49 & -651.54 & -1208.99 & -1541.63 \\
Diferencia relativa (\%) & -3.5 & -3.4 & -6.0 & -10.3 \\
Método KCICLO & $\mathbf{1 7 0 6 9}$ & $\mathbf{1 9 2 1 8}$ & $\mathbf{1 9 9 5 0}$ & $\mathbf{1 5 0 7 8}$ \\
Diferencia relativa (\%) & -3.3 & -2.8 & -4.0 & -4.6 \\
\hline \hline
\end{tabular}

Tabla III-XII. Comparaciones entre los procesados de AERONET, ULCO-LISE y KCICLO para el Cimel\#48. Todas las diferencias están tomadas respecto del coeficiente de precalibración.

El método KCICLO nos permite comprobar la calibración de los instrumentos, realizando un seguimiento continuo durante el tiempo que un instrumento está en estación. Ya sea como comprobación, como corrección o como calibración, el método se aplica in situ, en el lugar de medida. Esto conlleva inconvenientes relacionados con las condiciones atmosféricas de la estación, ya que la necesidad de un número suficiente de días estables y despejados es la limitación principal de una calibración in situ.

Una ventaja del método KCICLO es que puede permitir la recuperación de series de datos antiguas, en principio poco fiables, siempre que sea posible probar que los errores detectados son debidos a la calibración y no a otro tipo de problemas.

Por último queremos destacar el excelente diseño de la secuencia automática de medidas de AERONET, entre la masa óptica 7 de la mañana y de la tarde, que permite el seguimiento in situ de la calibración y de la estabilidad del instrumento.

Otro punto que aquí no se abordará (y que se deja para la continuación de este trabajo de tesis doctoral) es el de las simulaciones. En particular sería de interés realizar un análisis de la sensibilidad del método KCICLO y del método de Langley frente a variaciones aleatorias y sistemáticas del AOD durante un día, y cómo afecta esto a la pendiente del ajuste. El resultado de este trabajo sería la confección de unos criterios más seguros para la elección de días adecuados para la calibración in situ. También se podría simular el funcionamiento de una variante para nuestro método similar a la variante del método de Langley, el Langley en dos pasos, que aparece en el trabajo de Kremser (1984) y el procedimiento que se plantea en el trabajo de Korotaev et al. (1993). 


\subsection{Reprocesado de la serie de datos}

Hay dos posibilidades para realizar el reprocesado de la serie de datos. La primera de ellas consiste en aplicar los nuevos coeficientes de calibración a los ficheros brutos del Cimel 318, los K7. En este sentido el método KCICLO es un auténtico método de calibración. Con esto se obtiene una serie nueva, pero que incluye todas las medidas afectadas por nubosidad, por lo que necesita algún procedimiento equivalente al algoritmo de AERONET para eliminar esas medidas. La segunda posibilidad consiste en corregir los espesores ópticos de aerosoles de un fichero de AERONET que esté procesado con los coeficientes de precalibración. En este caso sólo hay que restar la cantidad conocida $(1 / \mathrm{m}) \cdot \operatorname{lnK}$ para cada filtro según la ecuación que da el método KCICLO, sin necesidad de información adicional. Ambos procedimientos son equivalentes. Si esta corrección se la aplicamos a un fichero de AERONET que esté en nivel 1.5, es decir, libre de medidas afectadas por nubes, obtenemos directamente una serie reprocesada y libre de nubosidad. Es posible además utilizar un valor no constante sino interpolado para aquellos filtros que muestren una deriva importante durante el período de estación del instrumento.

Sin embargo ha sido necesario partir de los ficheros brutos K7 del Cimel en el caso del Cimel \#114 porque el defecto en la calibración dio lugar a valores negativos del AOD, que fueron eliminados por el algoritmo de eliminación de nubosidad (cloud-screening) de AERONET (ver sección 2.4). Finalmente fue el personal de AERONET quien realizó para nosotros un procesado de este instrumento de acuerdo a nuestros coeficientes de calibración del KCICLO. Se hizo de esta forma para tener unos datos del Cimel\#114 que se pueden comparar directamente con la serie de datos de AERONET, y también por otros problemas menores que aparecieron en el Cimel \#114, como una avería en el sensor de temperatura. Queremos resaltar que nuestro procedimiento ha permitido recuperar muchos datos que se habían perdido por culpa del ciclo diurno hacia abajo, que daba lugar a valores negativos de AOD.

Cada fotómetro es procesado separadamente, de acuerdo con la corrección correspondiente para canal de AOD. También es necesario calcular el parámetro alfa de Ångström, tanto en el rango 440-870 como para los pares de espesores ópticos en los distintos canales.

El método KCICLO permite corregir una serie de espesores ópticos de aerosoles sin necesidad de conocer los coeficientes de calibración empleados para procesarla. Los coeficientes $\mathrm{K}$ obtenidos mediante los ajustes del método son suficientes para corregir la serie de espesor óptico de aerosoles, restando directamente la cantidad $(1 / \mathrm{m}) \cdot \operatorname{lnK}$, como ha quedado descrito. Esto podría permitir la corrección de series de datos con una mínima información sobre el instrumento o su calibración. En este sentido el método se puede considerar como método de corrección. 


\section{Capítulo 4. Análisis de las medidas de espesor óptico de aerosoles en El Arenosillo}

\section{Introducción}

En este capítulo se va a realizar una descripción completa de la serie de datos del fotómetro Cimel en El Arenosillo, en concreto sobre el espesor óptico de aerosoles en los cuatro canales del Cimel (440, 670, 870 y 1020nm) y sobre el parámetro alfa de Ångström que se deriva de ellos. Con todo esto se pretende realizar una climatología local de los aerosoles, apoyada en 5 años de datos del Cimel. Como todo estudio climatológico debe enfocarse a largo plazo, y según vaya aumentando la base de datos la climatología será más consistente. Los datos que se van a presentar corresponden al procesado KCICLO, la serie de datos corregida por nosotros con el método KCICLO descrito en el capítulo anterior.

Incluimos también el análisis de errores a propósito del parámetro alfa, que es de gran importancia dada la sensibilidad de este parámetro a los errores en el espesor óptico de aerosoles, del cual se deriva. Desde los resultados de este estudio se pueden valorar mejor los datos obtenidos para el alfa en ciertas situaciones, particularmente en las de turbiedad baja. 


\subsection{Análisis del espesor óptico de aerosoles}

En este apartado comenzamos el análisis de la serie de datos del Cimel en El Arenosillo, en concreto los datos de espesor óptico de aerosoles. Vamos a realizar la descripción a partir de la serie de datos corregida por el método KCICLO, que hemos desarrollado en el capítulo anterior.

La serie de datos de 5 años de datos del Cimel nos van a permitir una caracterización de los aerosoles en nuestra zona de estudio. Anteriormente nuestro grupo ya había realizado en El Arenosillo otros 4 años de medidas con el espectrorradiómetro Licor (Vergaz, 2001; Vergaz et al., 2005; Cachorro et al., 2005). El objetivo final es la descripción de la climatología local de los aerosoles en esta región. También por este motivo uno de los objetivos fundamentales de este trabajo ha sido asegurar la calidad de la serie de medidas del fotómetro Cimel y su continuidad, ya que esta se ha ido obteniendo mediante varios aparatos idénticos que se van relevando a lo largo de los años.

En todo el análisis se han utilizado los cuatro canales disponibles, aunque a la hora de las figuras emplearemos sobre todo el canal de 440nm.
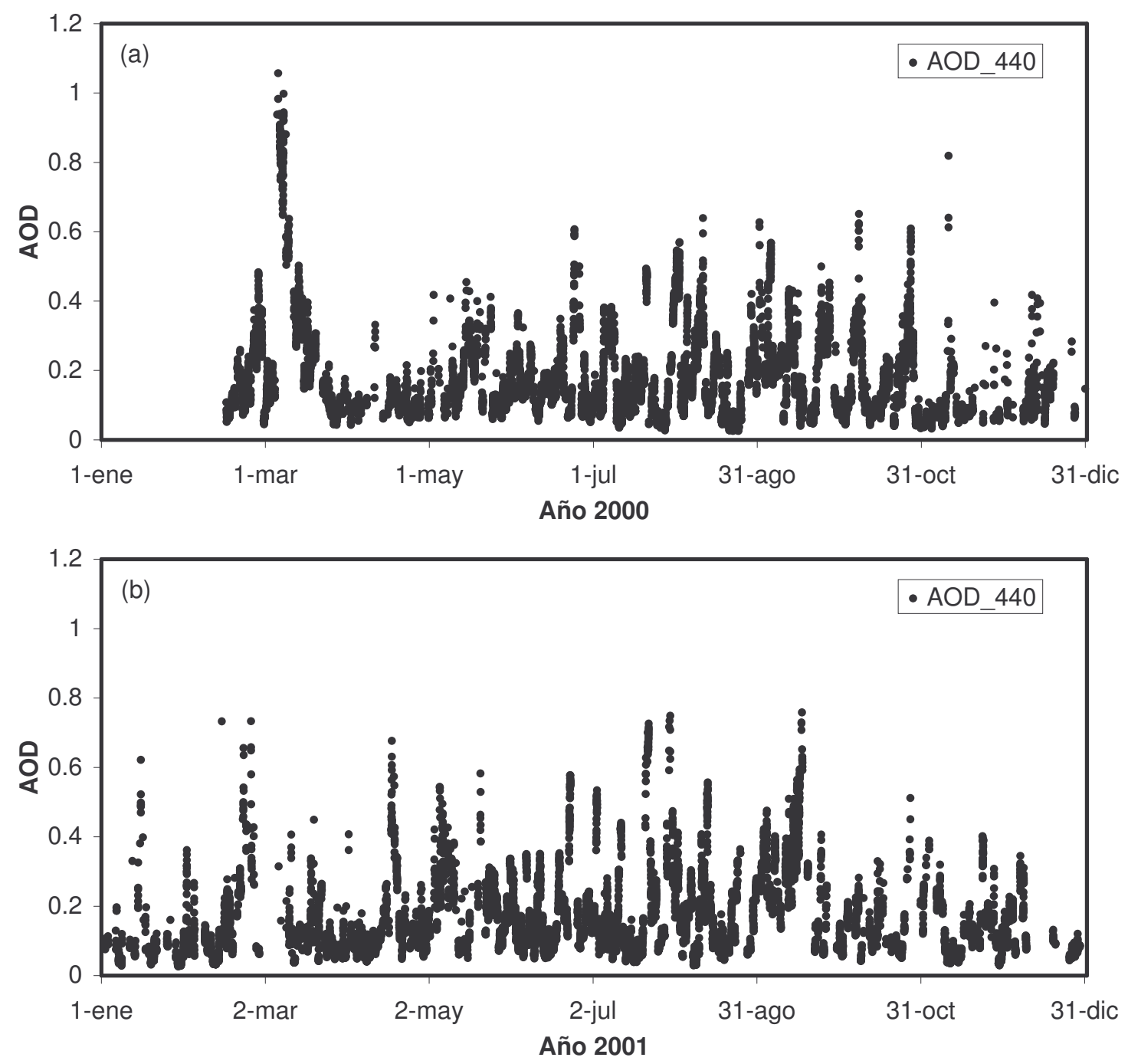

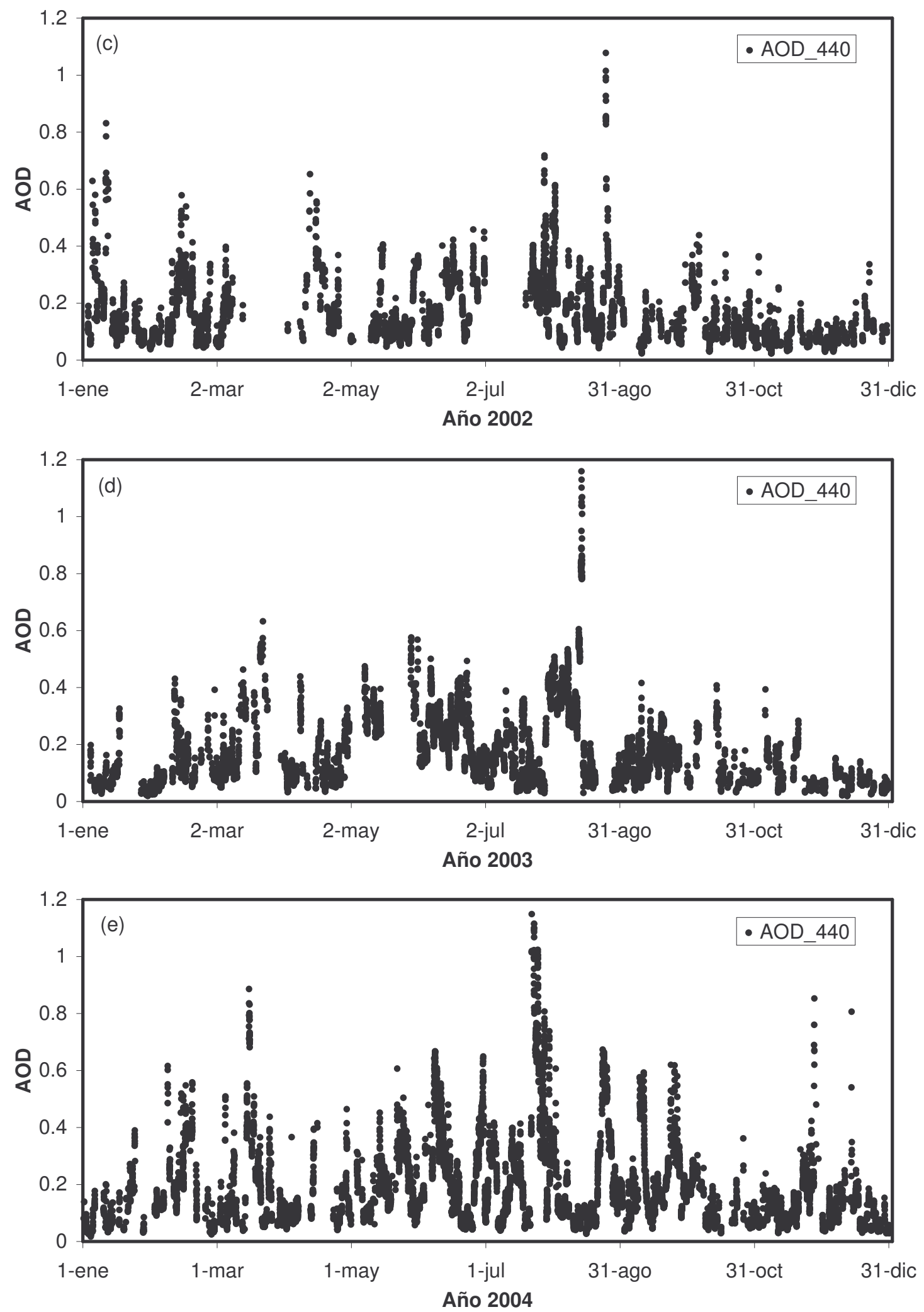

Figura IV-1. Espesor óptico de aerosoles en 440nm en El Arenosillo para el filtro de 440nm: (a) año 2000; (b) año 2001; (c) año 2002; (d) año 2003; (e) año 2004. Datos de la serie reprocesada con el método KCICLO, nivel 1.5.

La serie completa de espesor óptico de aerosoles en El Arenosillo para la longitud de onda de 440nm una vez reprocesada por el método KCICLO se muestra en la Figura IV-1. En cada una de las gráficas $(\mathrm{a}, \mathrm{b}, \mathrm{c}, \mathrm{d}$, y e) se ha representado un año de datos. En primer 
lugar observamos la característica fundamental de las medidas de AOD en la estación de El Arenosillo: la variabilidad. Excepto en 2001, durante todos los años se alcanzan durante algún episodio valores superiores a 1 en $\mathrm{AOD}(440 \mathrm{~nm})$, y en contraste, valores tan bajos como 0.03. El máximo absoluto en la estación se registró durante un episodio desértico extraordinario a finales de julio de 2004, con 2.69 en 440nm. Este máximo no se muestra en la Figura IV-1e porque todas las gráficas tienen la misma escala vertical para poder comparar mejor entre sí los diferentes años. Este máximo en julio de 2004 se muestra en detalle en la sección 7.4. La variabilidad en 440nm es mayor que en los canales en longitudes de onda más largas, alcanzándose valores más altos y con mayor frecuencia. La frecuencia de episodios de alta turbiedad es mayor durante los meses de verano, en los que además el número de medidas disponibles es también mayor.

Lo segundo que se puede observar al respecto de la serie es la existencia de dos situaciones fundamentales: una con valores bajos, por debajo de 0.1 en $440 \mathrm{~nm}$, que corresponde con el aerosol de fondo en la estación, que podemos etiquetar como aerosol marítimo y que se ve afectado por la influencia de aerosoles de origen continental y también antropogénico. A su vez aparecen numerosos episodios de alta turbiedad, con picos muy acusados en episodios de varios días, que en general están relacionados con la entrada de aerosoles desérticos procedentes del norte de África. El año 2004 es un buen ejemplo de esta presencia de aerosol desértico, con agudos picos y continuas subidas y bajadas entre mayo y octubre (Figura IV-1e).

La presencia de todos estos tipos de aerosol hacen de la estación de El Arenosillo un lugar privilegiado para el estudio de este componente atmosférico, y su situación costera en el golfo de Cádiz es idónea para la caracterización del aerosol en el sur de la península, en particular de cara al seguimiento permanente y la caracterización de las intrusiones saharianas.

A la vista de este análisis de las medidas de aerosoles en El Arenosillo, parece obligado abordar también el estudio de las trayectorias de masas de aire que llegan a la estación, por la relación que -a priori- se intuye entre la masa de aire y el tipo de aerosol presente en la estación. Este análisis se realizará en el capítulo 6.

\subsubsection{Análisis de la serie completa de datos 2000-2004 en El Arenosillo}

Nuestro análisis comienza con la serie de datos de todas las observaciones individuales del Cimel, es decir, sin realizar ningún tipo de promedios. Esto se traduce en observaciones cada 15 minutos aproximadamente, con un tiempo de observación entre 7 horas al día en invierno y hasta 13 horas al día en verano, en el que se alcanza una elevación solar máxima de $76^{\circ}$ que equivale a una masa óptica de 1.03 .

El valor promedio del espesor óptico de aerosoles en El Arenosillo, que recogemos en la Tabla IV-I, presenta unos valores acordes con la condición marítima y costera de la estación (Smirnov, 2002). El valor promedio del AOD en $440 \mathrm{~nm}$ es 0.17 , con una desviación estándar de 0.13 que representa un $75 \%$ de variación típica. Para las demás longitudes de onda su desviación típica supone un $100 \%$ o más de variación sobre los valores promedio. El AOD promedio disminuye con la longitud de onda hasta 0.08 en $1020 \mathrm{~nm}$. En esta tabla podemos encontrar también el máximo y mínimo absolutos de cada canal, así como los percentiles 10, 25, 50 (mediana), 75 y 90 para toda la serie de datos. El máximo absoluto es similar en los cuatro canales y se alcanzó el 22 de julio de 2004. 


\begin{tabular}{lcccc}
\hline \hline & AOD_1020 & AOD_870 & AOD_670 & AOD_440 \\
\hline Promedio & 0.089 & 0.096 & 0.113 & 0.177 \\
Desviac. est. & 0.102 & 0.105 & 0.112 & 0.135 \\
P25 & 0.036 & 0.039 & 0.046 & 0.084 \\
P75 & 0.104 & 0.114 & 0.140 & 0.230 \\
P10 & 0.025 & 0.027 & 0.030 & 0.059 \\
P90 & 0.189 & 0.201 & 0.236 & 0.344 \\
Mínimo & 0.009 & 0.008 & 0.003 & 0.017 \\
Máximo & 2.541 & 2.623 & 2.691 & 2.697 \\
Mediana & 0.058 & 0.063 & 0.077 & 0.137 \\
\hline \hline
\end{tabular}

Tabla IV-I. Estadística de la serie completa de AOD en El Arenosillo para los 4 filtros del Cimel. Datos para la serie 2000-2004 reprocesada con el método KCICLO.

La situación más frecuente en la estación, que relacionamos con el aerosol marítimo, está caracterizada por un AOD inferior al promedio que se muestra en la Tabla IV-I. A su vez, las entradas de aerosoles desérticos presentan un AOD claramente superior a este promedio. Por tanto los valores promedio de la estación son el resultado de las dos situaciones principales, una de baja y otra de alta turbiedad, pero no corresponden exactamente con ninguna de ellas. Vemos que la mediana para el AOD en 440nm es 0.137. La mediana nos da el valor que deja el $50 \%$ de las observaciones por debajo, y es claramente inferior al promedio correspondiente, puesto que la situación predominante en El Arenosillo es de baja turbiedad. Dado que las intrusiones desérticas son frecuentes y de variable intensidad, parece lógico intentar caracterizar y evaluar dichas situaciones de manera separada. Este análisis se realizará en el capítulo 7.

En la Tabla IV-II mostramos una estadística análoga a la tabla anterior pero dividida por años, en este caso sólo para el canal de $440 \mathrm{~nm}$. El promedio para cada año oscila entre 0.158 para 2002 y 0.196 en 2004. Este año 2004, como veremos en el capítulo 7, fue excepcional en cuanto al número de entradas de aerosoles desérticos. Durante 2004 también destacan la desviación estándar (0.173) y sobre todo el máximo de AOD con 2.697, más del doble que los otros años. El año 2001 tiene el máximo más bajo, con 0.758 en $\operatorname{AOD}(440 \mathrm{~nm})$.

\begin{tabular}{lccccc}
\hline \hline & $\begin{array}{c}\text { AOD(440nm) } \\
\text { Año 2000 }\end{array}$ & $\begin{array}{c}\text { AOD(440nm) } \\
\text { Año 2001 }\end{array}$ & $\begin{array}{c}\text { AOD(440nm) } \\
\text { Año 2002 }\end{array}$ & $\begin{array}{c}\text { AOD(440nm) } \\
\text { Año 2003 }\end{array}$ & $\begin{array}{c}\text { AOD(440nm) } \\
\text { Año 2004 }\end{array}$ \\
\hline N $^{\circ}$ días de medida & $279^{*}$ & 300 & $237^{* * *}$ & 255 & 311 \\
N $^{\circ}$ observaciones & 8358 & 8567 & 6003 & 7024 & 9186 \\
Promedio & 0.181 & 0.164 & 0.158 & 0.181 & 0.196 \\
Desviac. est. & 0.129 & 0.114 & 0.109 & 0.136 & 0.173 \\
P25 & 0.091 & 0.081 & 0.080 & 0.081 & 0.086 \\
P75 & 0.230 & 0.215 & 0.215 & 0.258 & 0.252 \\
P10 & 0.065 & 0.058 & 0.061 & 0.054 & 0.056 \\
P90 & 0.344 & 0.320 & 0.304 & 0.370 & 0.394 \\
Min & 0.025 & 0.026 & 0.023 & 0.019 & 0.017 \\
Max & 1.057 & 0.758 & 1.078 & 1.159 & 2.697 \\
Mediana & 0.144 & 0.126 & 0.123 & 0.138 & 0.149 \\
\hline \hline
\end{tabular}

Tabla IV-II. Estadística de la serie de AOD(440nm) por años entre el 2000 y el 2004.

* Año 2000: los datos comienzan el 16 de febrero

** Año 2002: hubo 6 semanas sin datos por problemas de instrumentación 

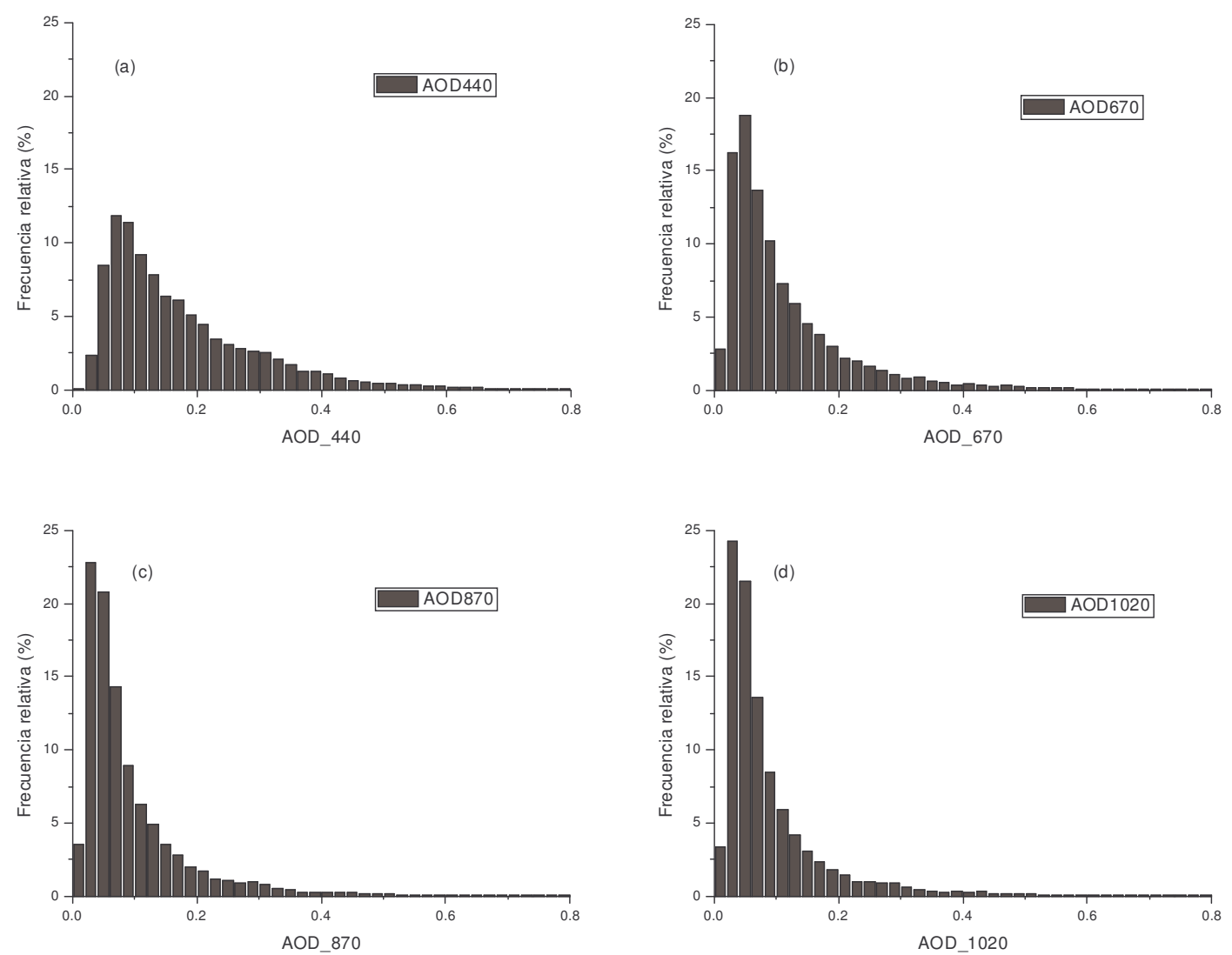

Figura IV-2. Histograma de frecuencias relativas del AOD para la serie completa 2000-2004 en El Arenosillo: (a) en 440nm; (b) en 670nm; (c) en 870nm; (d) en 1020nm.

En 440nm, y sobre una serie de 5 años de datos y más de 39000 medidas individuales, el AOD en $440 \mathrm{~nm}$ presenta un $55 \%$ de valores por debajo de 0.15 , esto es, más de la mitad de las observaciones corresponden con los valores que podemos asignar al aerosol marítimo. En la Figura IV-2 podemos observar la distribución de frecuencias para todas las medidas individuales de El Arenosillo. La frecuencia de valores de AOD en 440nm por encima de 0.3 en $440 \mathrm{~nm}$ es del $14 \%$. Este porcentaje es ya una primera aproximación sobre la presencia de aerosol sahariano en El Arenosillo.

En 670nm el histograma aparece más desplazado hacia la izquierda, es decir, hacia valores más bajos de AOD. Por debajo de 0.15 está el $77 \%$ de los datos, un porcentaje sensiblemente mayor que en 440nm. Si comparamos los valores más bajos, en $670 \mathrm{~nm}$ tenemos el $62 \%$ de datos por debajo de 0.1 mientras que en $440 \mathrm{~nm}$ sólo está el $35 \%$. Por el contrario sólo el $5 \%$ de los datos queda por encima de 0.3 para el AOD en $670 \mathrm{~nm}$. En $870 \mathrm{~nm}$ el porcentaje de valores por debajo de 0.15 es del $84 \%$ y sólo un $3 \%$ está por encima de 0.3 . En $1020 \mathrm{~nm}$ el $71 \%$ de los datos queda por debajo de 0.1 , y sólo el $3 \%$ está por encima de 0.3. Vemos que conforme aumentamos la longitud de onda los histogramas son más estrechos y están más desplazados hacia los valores bajos del AOD. En 1020nm el $43 \%$ de los datos de AOD están entre 0.02 y 0.06.

El análisis de la correlación entre canales nos da una idea de cuánta información podemos obtener de un canal a partir de otro. El resumen de las diferentes correlaciones está en la Tabla IV-III. La mayor correlación se da entre canales contiguos, en particular 670 y $870 \mathrm{~nm}$, con $\mathrm{R}^{2}=0.975$. Cuanto más alejados están los canales en longitud de onda menor es la correlación, descendiendo hasta una correlación de 0.718 para los canales de 440 y 
1020nm. Las pendientes nos dan una idea, en promedio, de la relación entre los valores en uno y otro canal.

\begin{tabular}{lccc}
\hline \hline Canales & Pendiente & Ordenada & Coef. Correlación $\mathrm{R}^{2}$ \\
\hline $440-670$ & 0.755 & -0.022 & 0.904 \\
$440-870$ & 0.650 & -0.021 & 0.798 \\
$440-1020$ & 0.611 & -0.022 & 0.718 \\
$670-870$ & 0.905 & -0.007 & 0.975 \\
$670-1020$ & 0.869 & -0.011 & 0.917 \\
$870-1020$ & 0.971 & -0.005 & 0.961 \\
\hline \hline
\end{tabular}

Tabla IV-III. Correlaciones entre los 4 canales de AOD del Cimel

Sin embargo lo más importante de estas correlaciones se ve en la representación gráfica (Figura IV-3). En ellas se puede apreciar que el diagrama presenta dos ramas para los valores altos de AOD. Estas dos ramas delimitan una región en la que todos los datos están confinados, que -como veremos más adelante- corresponde con los extremos del parámetro alfa. La rama superior, cercana a la diagonal, nos indica un AOD similar en los dos canales representados, es decir, una dependencia pequeña del AOD con la longitud de onda o un espectro muy plano de AOD. Para AOD altos este espectro de extinción tan plano es característico de la extinción debida a los aerosoles desérticos.

La rama inferior nos indica una diferencia muy grande entre el AOD de los dos canales representados, es decir, una fuerte pendiente del espectro de AOD, propio del predominio de partículas finas. En situaciones de alta turbiedad esta rama corresponde con los episodios de contaminación urbana o industrial, o humo de incendios, pero no al aerosol desértico.
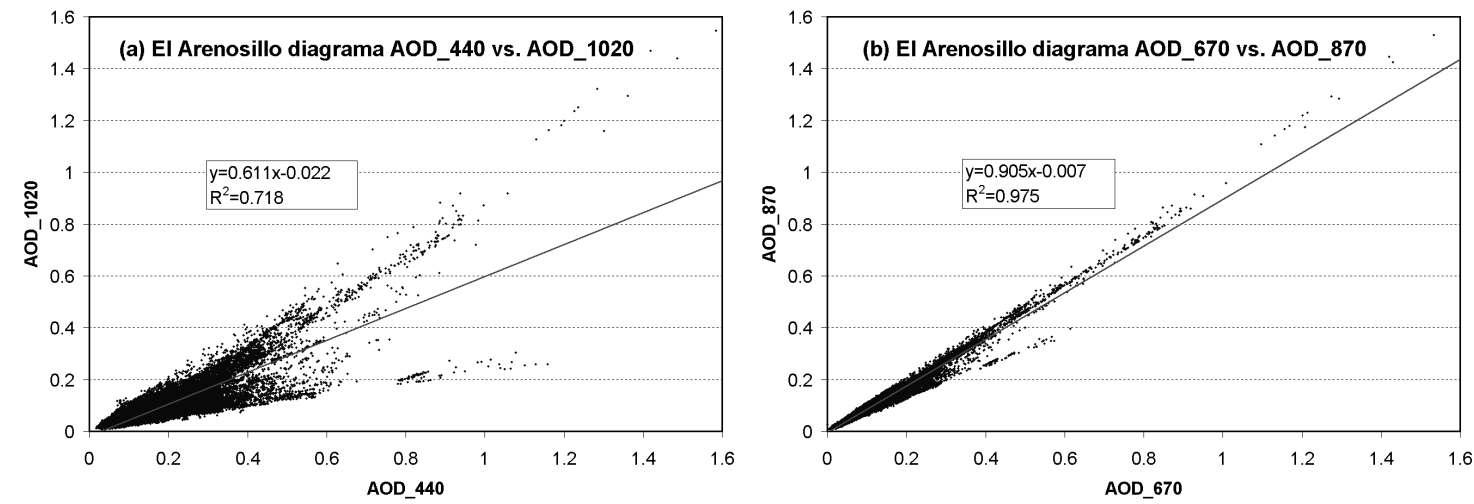

Figura IV-3. Correlaciones entre los canales de (a) AOD en 440 y 1020nm, y (b) AOD en 670 y $870 \mathrm{~nm}$

En la gráfica Figura IV-3 mostramos los dos casos con mayor y menor correlación entre canales. En el caso de los canales de 440 y 1020nm (Figura IV-3a) las dos ramas están muy separadas por la gran distancia espectral entre los dos canales. Esto explica la baja correlación entre ellos. Vemos en la rama inferior que valores de 0.8 en $440 \mathrm{~nm}$ se corresponden con valores de 0.2 en $1020 \mathrm{~nm}$, mientras que en la rama superior los mismos valores de 0.8 en $440 \mathrm{~nm}$ se corresponden con valores de 0.7 en $1020 \mathrm{~nm}$. Para la representación de los canales de 670 y 870nm (Figura IV-3b) la correlación es muy superior al ser canales contiguos. Por la misma razón las dos ramas están mucho más cerca aunque siguen siendo distinguibles. En conclusión, aunque la correlación entre los diferentes canales sea alta en general, uno solo de los canales no sirve para describir totalmente el $\mathrm{AOD}$ en los demás canales, pues la relación entre ellos (la pendiente del espectro) depende del tipo de aerosol. 


\subsubsection{Análisis de promedios diarios}

El análisis de los promedios diarios tiene el inconveniente de enmascarar la evolución durante el día del espesor óptico de aerosoles, ofreciendo un valor que puede ser más o menos significativo en función de la variabilidad del día. Sin embargo presenta la ventaja de destacar las situaciones de mayor persistencia, frente a observaciones individuales que pueden ser menos significativas.

La evolución de los promedios diarios en El Arenosillo se muestra en la Figura IV-4 para el filtro de 440nm. Se puede apreciar la variabilidad de la carga de aerosol, con valores entre 0.02 y 1.17 en $440 \mathrm{~nm}$. Hasta un 55\% de los días quedan con AOD por debajo de 0.15 en $440 \mathrm{~nm}$, que son los que corresponden con la situación ordinaria de la estación, esto es, aerosoles principalmente marítimos. El 15\% de los días presentan un promedio por encima de 0.3 .

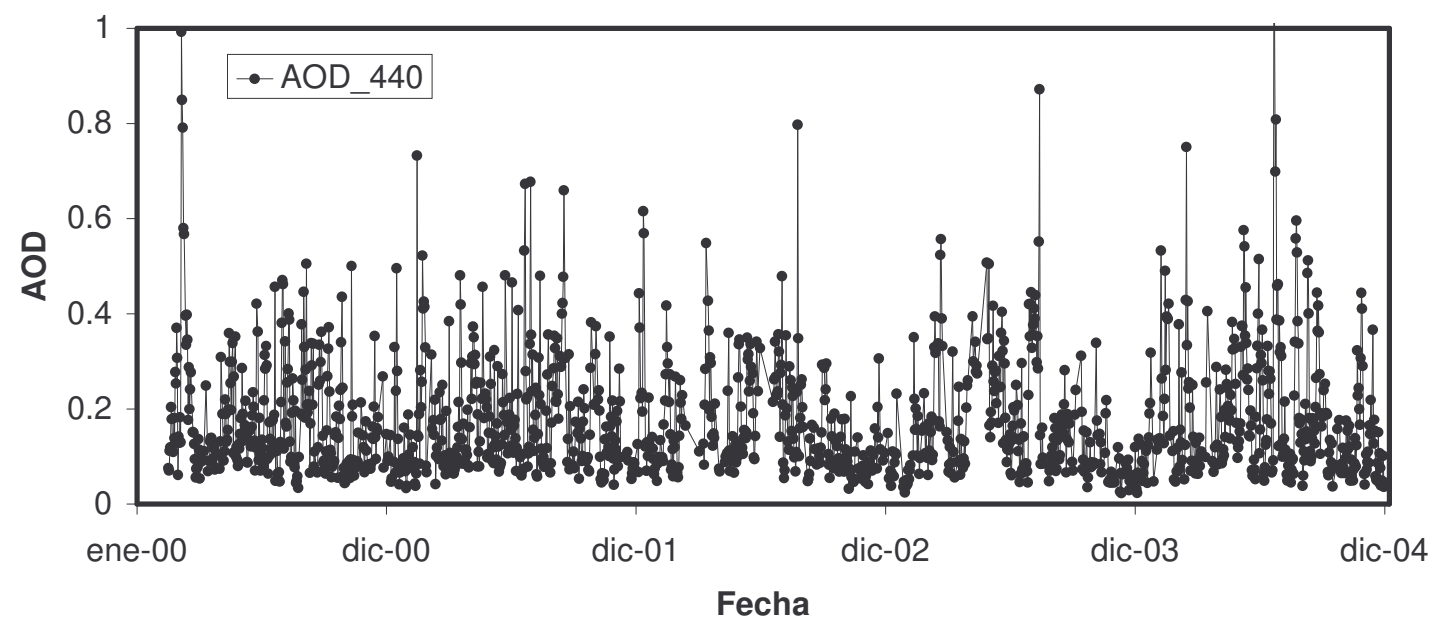

Figura IV-4. Evolución del promedio diario del AOD en 670nm en El Arenosillo

En esta gráfica de evolución de los promedios diarios para los 5 años (Figura IV-4) podemos observar también una menor frecuencia de días con valores altos durante los inviernos, en especial los inviernos de 2002-2003 y 2003-2004. Durante los veranos se pueden observar tanto los agudos picos por los numerosos días con valores altos como un aumento en general del valor de fondo (background) respecto del invierno.

La distribución de frecuencias de los promedios diarios en 440nm y 870nm se recoge en el histograma de la Figura IV-5. Como se ve, la distribución es muy similar a la obtenida para todas las observaciones individuales. La frecuencia de valores bajos aumenta con la longitud de onda. En 870nm un 83\% de los días están por debajo de 0.15, frente al 55\% en $440 \mathrm{~nm}$. La escala horizontal se ha dejado en 0.6 aunque haya valores hasta 1.2 porque así podemos apreciar mejor un segundo pico de frecuencia, aunque pequeño, en 0.29 para los valores de $\mathrm{AOD}(440 \mathrm{~nm})$. El pico principal de frecuencia en $440 \mathrm{~nm}$ está en 0.08 mientras que en $870 \mathrm{~nm}$ el valor del pico está en 0.05 .

La variabilidad durante el día del AOD se puede analizar mediante la desviación estándar (STD) de las medidas durante el día, que mostramos en la Figura IV-6, que completan la información sobre los promedios diarios. La desviación estándar diaria tiene un valor en promedio de 0.03 , y toma valores en general por debajo de 0.05 , hasta el $85 \%$ de los casos. Por encima de 0.1 sólo queda el $2 \%$ de los días. Los días mas estables, con una desviación estándar por debajo de 0.025 constituyen el 56\% de los días. La variabilidad que se registra en El Arenosillo está por encima de la registrada en estaciones marítimas remotas, que se estima en torno a 0.01-0.02 para la desviación estándar diaria (Smirnov et 
al., 2002), lo que nos recuerda de nuevo la influencia del carácter costero de la estación, que no afecta sólo a la carga de aerosol sino también a su variabilidad.

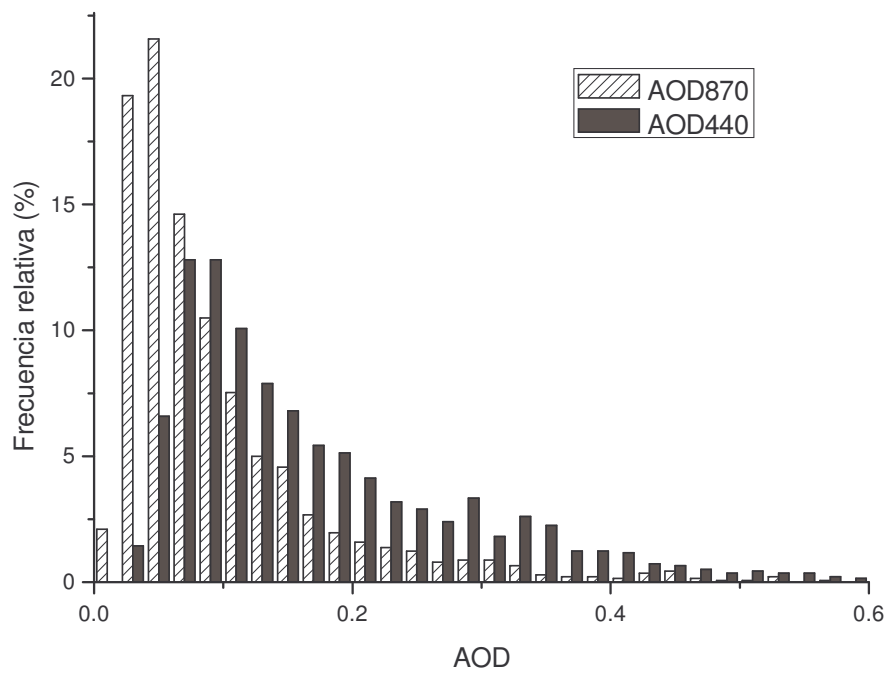

Figura IV-5. Frecuencia relativa del AOD en 440 y 870nm en El Arenosillo (procesado KCICLO).

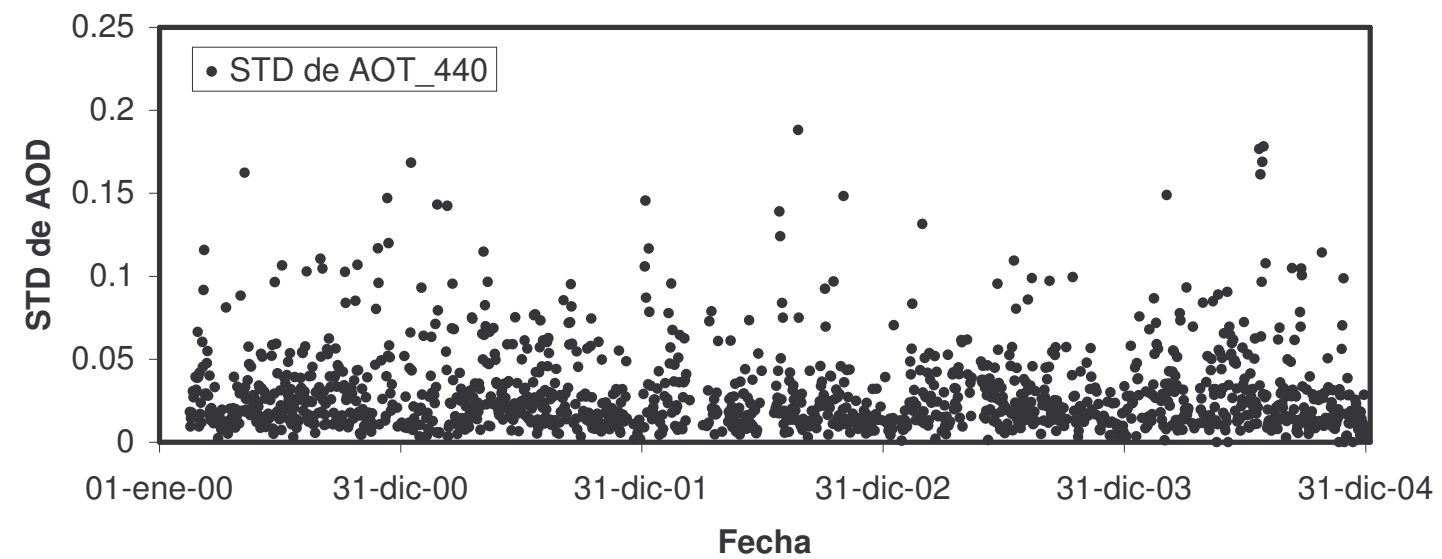

Figura IV-6. Desviación estándar de las medidas diarias en 440nm para la serie de datos de El Arenosillo

El análisis a partir de los promedios diarios resulta ser muy similar al realizado anteriormente a partir de todas las medidas individuales, tanto cualitativa como cuantitativamente. Los porcentajes de días correspondientes a las situaciones fundamentales (marítima y desértica, alta y baja turbiedad) son muy similares a los realizados a partir de todas las medidas. Además queda recogida la influencia continental y antropogénica en los valores de AOD y en su variabilidad (promedio y desviación estándar de las medidas diarias), ambos más elevados que los correspondientes al aerosol marítimo más puro, propio de estaciones remotas.

\subsubsection{Análisis de promedios mensuales y estacionales}

La estadística de promedios a más largo plazo nos acerca a la climatología de los aerosoles en la zona de estudio. En primer lugar mostramos la evolución durante los cuatro años del promedio mensual para el filtro de 440nm del Cimel (Figura IV-7). Estos promedios mensuales han sido evaluados a partir de los promedios diarios. Podemos observar cómo en los meses de invierno, el AOD alcanza los valores más bajos, cercanos o incluso por 
debajo de 0.1. Estos niveles tan bajos de carga de aerosol se deben a la ausencia de eventos saharianos y a la mayor frecuencia de lluvias, que limpian la atmósfera.

Los meses de verano presentan valores promedio más altos, en muchos casos entre 0.2 y 0.3 , lo cual nos indica la mayor turbiedad de la atmósfera por ser la estación más seca, y también la entrada frecuente de aerosoles desérticos. Todos los veranos se han registrado episodios de mayor o menor intensidad. Además de los episodios durante el verano, hay que destacar la ocurrencia de episodios severos de aerosol desértico durante los meses de febrero y marzo, al final del invierno (marzo de 2000, marzo 2003 ó febrero 2004 por citar los más significativos). Esto afecta a los promedios en esos meses y nos da como resultado dos máximos a lo largo del año: al final del invierno ó inicio de la primavera por una parte, y después en verano.

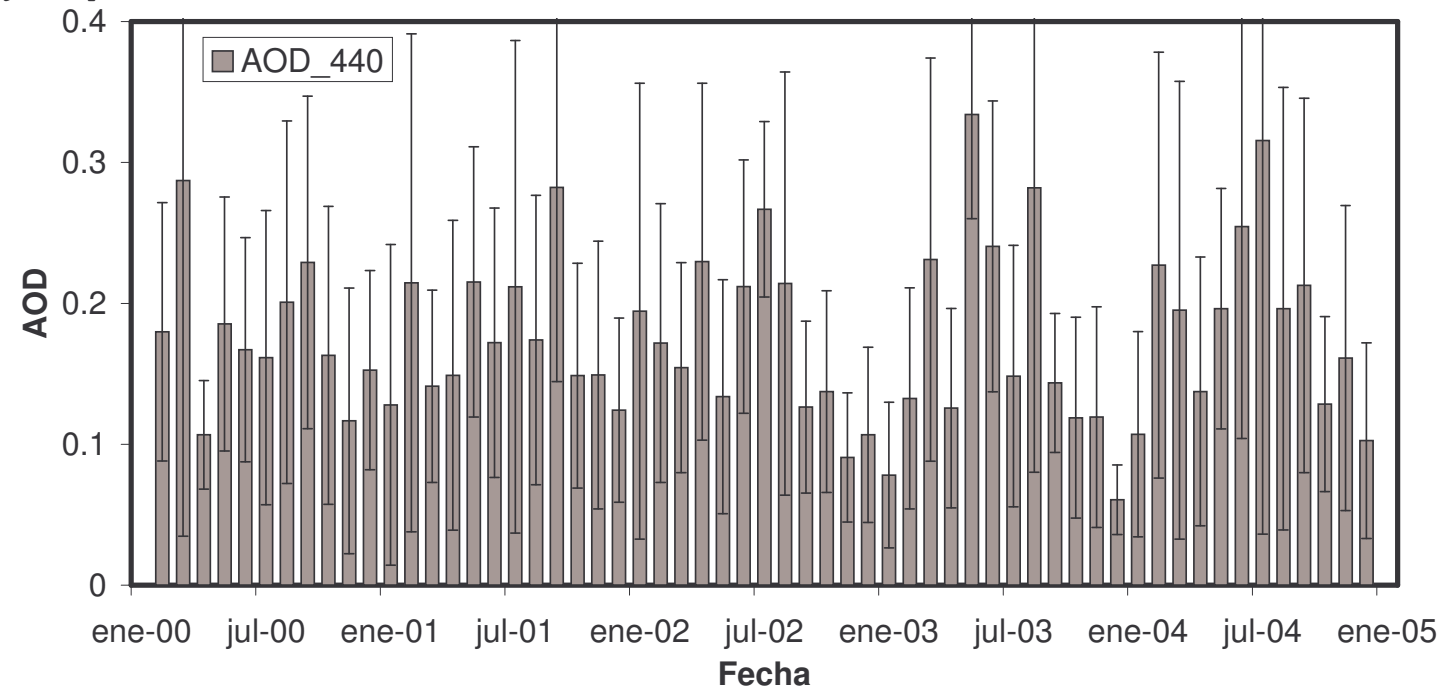

Figura IV-7. Evolución temporal del promedio mensual del AOD en 440nm en El Arenosillo, período 2000-2004.

Al analizar la desviación estándar asociada a los promedios mensuales, se comprueba que existe una cierta correlación $\left(\mathrm{R}^{2}=0.44\right)$ entre el promedio mensual y la desviación estándar mensual de AOD en 440nm, es decir, entre turbiedad y variabilidad, para los 59 meses analizados. Aproximadamente la desviación estándar es 0.5 veces el valor del promedio. Por tanto se verifica el hecho de que los valores altos están asociados a mayor variabilidad, pues se deben a la ocurrencia de eventos concretos y no a situaciones persistentes a escala mensual.

\begin{tabular}{lcccccc}
\hline \hline & AOT_1020 & AOT_870 & AOT_670 & AOT_440 & N $^{\circ}$ días & Meses \\
\hline enero & $0.08( \pm 0.05)$ & $0.08( \pm 0.05)$ & $0.09( \pm 0.05)$ & $0.13( \pm 0.05)$ & 80 & 4 \\
febrero & $0.09( \pm 0.02)$ & $0.10( \pm 0.02)$ & $0.11( \pm 0.03)$ & $0.19( \pm 0.04)$ & 101 & 5 \\
marzo & $0.12( \pm 0.04)$ & $0.13( \pm 0.05)$ & $0.14( \pm 0.05)$ & $0.20( \pm 0.06)$ & 108 & 5 \\
abril & $0.07( \pm 0.01)$ & $0.07( \pm 0.01)$ & $0.09( \pm 0.02)$ & $0.15( \pm 0.05)$ & 106 & 5 \\
mayo & $0.10( \pm 0.03)$ & $0.11( \pm 0.03)$ & $0.13( \pm 0.05)$ & $0.21( \pm 0.07)$ & 116 & 5 \\
junio & $0.10( \pm 0.04)$ & $0.12( \pm 0.03)$ & $0.14( \pm 0.03)$ & $0.21( \pm 0.04)$ & 140 & 5 \\
julio & $0.11( \pm 0.05)$ & $0.13( \pm 0.05)$ & $0.15( \pm 0.05)$ & $0.22( \pm 0.07)$ & 134 & 5 \\
agosto & $0.11( \pm 0.03)$ & $0.12( \pm 0.03)$ & $0.14( \pm 0.03)$ & $0.21( \pm 0.04)$ & 140 & 5 \\
septiembre & $0.09( \pm 0.03)$ & $0.10( \pm 0.03)$ & $0.12( \pm 0.04)$ & $0.20( \pm 0.06)$ & 123 & 5 \\
octubre & $0.08( \pm 0.01)$ & $0.08( \pm 0.01)$ & $0.09( \pm 0.01)$ & $0.14( \pm 0.02)$ & 116 & 5 \\
noviembre & $0.08( \pm 0.01)$ & $0.08( \pm 0.01)$ & $0.09( \pm 0.01)$ & $0.13( \pm 0.03)$ & 116 & 5 \\
diciembre & $0.07( \pm 0.03)$ & $0.07( \pm 0.03)$ & $0.07( \pm 0.03)$ & $0.11( \pm 0.03)$ & 102 & 5 \\
\hline \hline
\end{tabular}

Tabla IV-IV. Promedios mensuales de AOD( \pm STD) para el período 2000-2004 en El Arenosillo 
El promedio mensual interanual para cada mes en 440nm está recogido en la Figura IV-8, en la que se ha considerado el período 2000-2004. Estos promedios mensuales interanuales y sus desviaciones estándar se han evaluado a partir de los promedios mensuales. En ella podemos observar más claramente valores altos de AOD en febrero y marzo, similares a los del máximo del verano. También se aprecia la baja turbiedad durante los meses de noviembre a enero, que es consistente a lo largo de los cuatro años. Todos estos datos promedio y los del resto de filtros, junto con las correspondientes desviaciones estándar, están recogidos en la Tabla IV-IV. Después del máximo estival (con 0.22 en el mes de julio), el AOD decrece paulatinamente durante el otoño hasta alcanzar el mínimo durante el invierno ( 0.11 en $440 \mathrm{~nm}$ para el mes de diciembre). Al final del invierno estos valores vuelven a aumentar, aunque en abril el valor es bastante menor ( 0.15 en $440 \mathrm{~nm})$ que los meses anteriores y siguientes, que se mueven en torno al 0.20 en $440 \mathrm{~nm}$ del régimen de verano. En los cuatro canales el comportamiento es análogo.

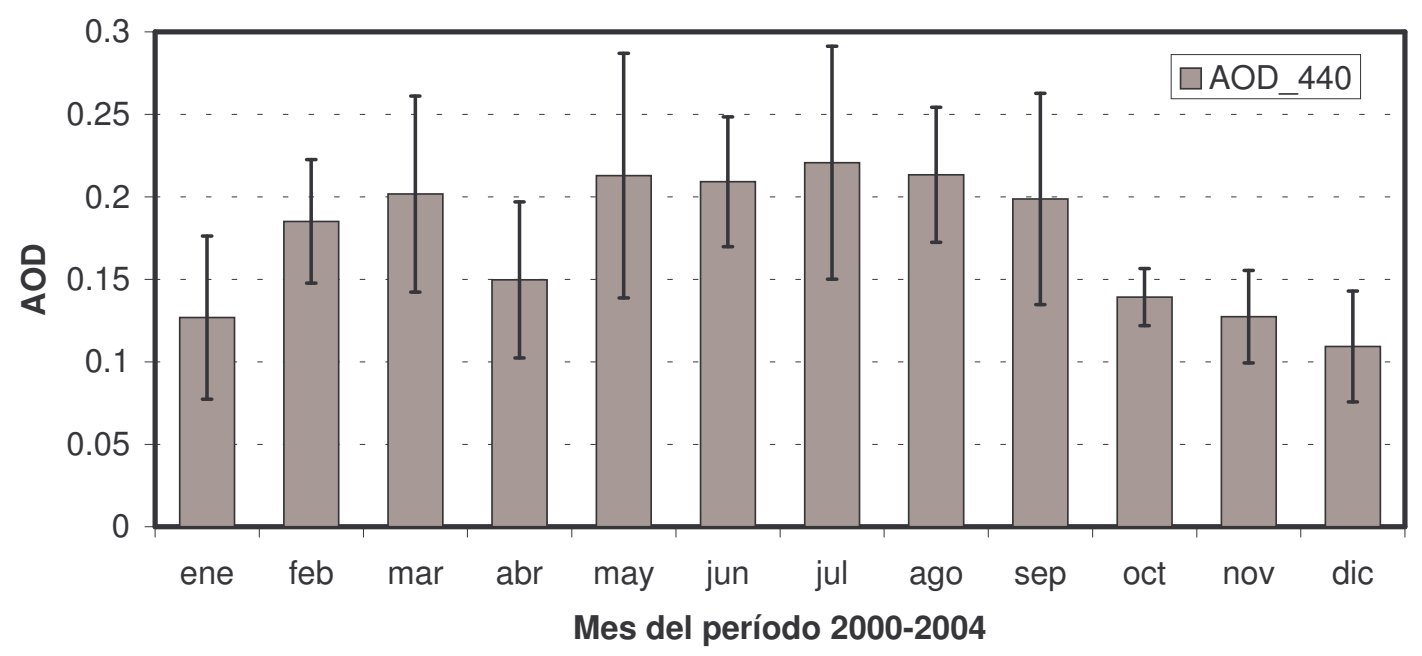

Figura IV-8. Promedio mensual interanual del AOD en 440nm para el período 2000-2004 en El Arenosillo. Las barras indican la desviación estándar $( \pm 1 S T D)$.

El promedio estacional del AOD (Figura IV-9) para los cuatro filtros del Cimel resulta quizá un poco engañoso en cuanto al mínimo invernal. Al considerarse los meses de diciembre, enero y febrero el promedio de invierno queda muy elevado (por encima de 0.1 ) cuando ya hemos visto que entre los meses de noviembre y enero los valores son más bajos. El mes de febrero, por causa de las entradas de aerosol desértico, es el responsable de este valor para el invierno. En todo caso se sigue apreciando un mínimo invernal (0.13 en $440 \mathrm{~nm})$ y un máximo estival $(0.20$ en $440 \mathrm{~nm})$. Los promedios estacionales con las correspondientes desviaciones estándar se muestran en la Tabla IV-V.

\begin{tabular}{lccccc}
\hline \hline & Meses & AOT_1020 & AOT_870 & AOT_670 & AOT_440 \\
\hline Invierno & 14 & $0.08( \pm 0.03)$ & $0.08( \pm 0.03)$ & $0.09( \pm 0.04)$ & $0.14( \pm 0.05)$ \\
Primavera & 15 & $0.10( \pm 0.04)$ & $0.10( \pm 0.04)$ & $0.12( \pm 0.05)$ & $0.19( \pm 0.06)$ \\
Verano & 15 & $0.11( \pm 0.04)$ & $0.12( \pm 0.04)$ & $0.14( \pm 0.04)$ & $0.21( \pm 0.05)$ \\
Otoño & 15 & $0.08( \pm 0.02)$ & $0.09( \pm 0.02)$ & $0.10( \pm 0.03)$ & $0.16( \pm 0.05)$ \\
\hline \hline
\end{tabular}

Tabla IV-V. Promedios estacionales de AOD( \pm STD) para el período 2000-2004 en El Arenosillo.

El resultado más significativo en el análisis mensual y estacional lo constituye el hecho de haber encontrado una serie de pautas y un comportamiento que se repite dentro de los 5 años de datos, cuando hemos podido comprobar la enorme variabilidad de la carga de aerosol en la estación. Este comportamiento estacional está asociado en primer lugar a la mayor presencia de lluvias en otoño e invierno, y en segundo lugar por la mayor frecuencia de intrusiones saharianas en primavera y verano. 


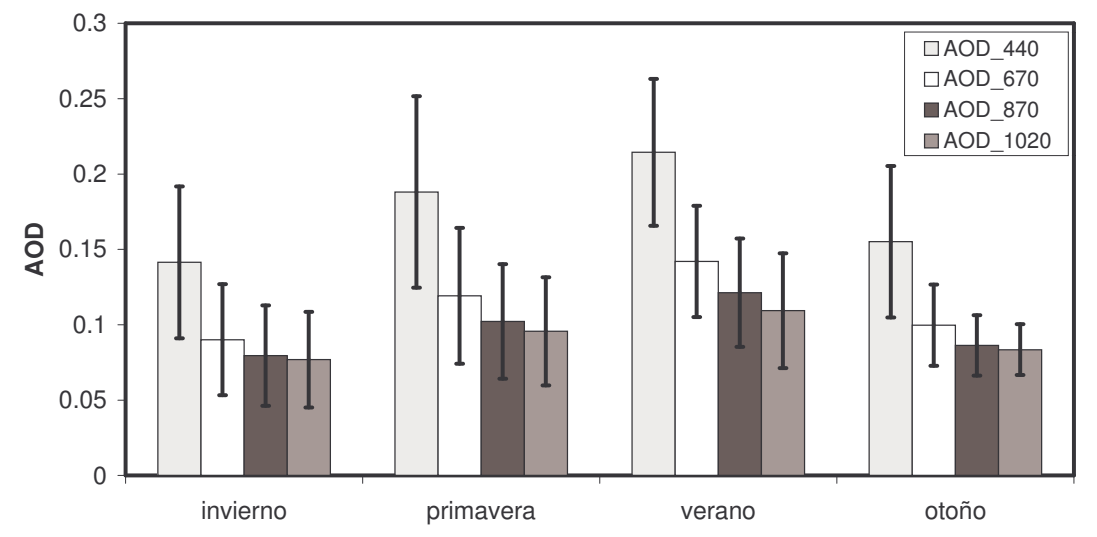

Figura IV-9. Promedio estacional de AOD en El Arenosillo para el período 2000-2004 en los cuatro canales del Cimel. Las barras de error indican la desviación estándar ( $\pm 1 \mathrm{STD})$. 


\subsection{Análisis de la evolución temporal del parámetro alfa}

Para el análisis del parámetro alfa de Ångström (ver sección 1.1.2 para aclarar notación) vamos a seguir un esquema análogo al utilizado para el espesor óptico de aerosoles: en primer lugar se analiza la serie de medidas individuales, para pasar luego al análisis de promedios y la búsqueda de comportamientos estacionales o interanuales.

El parámetro alfa que vamos a utilizar para la descripción general del aerosol en la estación es el derivado a partir del AOD en 3 longitudes de onda: 440, 670 y 870nm, y que se calcula mediante un ajuste logarítmico según la expresión de Ångström.

\subsubsection{Análisis de la serie de medidas individuales de alfa(440-870) en el período $2000-2004$}

La estadística básica de los valores de alfa en El Arenosillo se recoge en la primera columna de la Tabla IV-VI, en la que se incluyen además otros tres parámetros alfa calculados con pares de longitudes de onda para poder compararlos con el parámetro alfa que hemos elegido.

La primera y la cuarta columna ofrecen valores muy similares. Sin embargo el alfa(440/670) presenta un valor superior al de alfa(440-870) por la mayor pendiente del espectro de AOD en ese primer tramo. De forma análoga, el alfa(670/870) presenta un valor inferior al alfa(440-870). Estos valores para los cuatro parámetros alfa son consistentes a lo largo de la serie de 5 años y de los diferentes instrumentos Cimel para el procesado KCICLO. No es así para los datos de AERONET, que presentan numerosos problemas como abordaremos en el capítulo siguiente.

\begin{tabular}{lcccc}
\hline \hline & alfa(440-870) & alfa(440/670) & alfa(670/870) & alfa(440/870)par \\
\hline Promedio & 1.04 & 1.28 & 0.67 & 1.05 \\
Desviac. est. & 0.43 & 0.50 & 0.54 & 0.43 \\
P25 & 0.71 & 0.96 & 0.29 & 0.72 \\
P75 & 1.37 & 1.63 & 1.07 & 1.39 \\
P90 & 0.39 & 0.52 & 0.03 & 0.40 \\
P10 & 1.56 & 1.87 & 1.36 & 1.57 \\
Min & -0.27 & -0.59 & -2.72 & -0.24 \\
Max & 2.34 & 3.47 & 3.36 & 2.31 \\
Mediana & 1.11 & 1.36 & 0.66 & 1.12 \\
\hline \hline
\end{tabular}

Tabla IV-VI. Estadística de los distintos parámetros alfa en El Arenosillo con el procesado KCICLO

El valor promedio de 1.04 se puede considerar alto para el aerosol marítimo, que en las estaciones marítimas remotas presenta valores de 0.5-0.6 en promedio. Esto concuerda muy bien con la situación costera de la estación de El Arenosillo y la influencia de aerosoles de origen urbano o industrial y de origen continental, como ya se ha venido comentando. La mediana está en 1.11 lo que indica que la situación habitual es de alfa por encima del promedio. El 14\% de las observaciones está por encima de 1.5, mientras que el $19 \%$ está por debajo de 0.6. La distribución completa de frecuencias relativas de alfa(440-870) la encontramos en la Figura IV-10. En esta distribución podemos observar dos modos de frecuencia. El modo principal tiene el pico en 1.2, mientras que aparece un modo secundario cuyo pico de frecuencia corresponde con un valor de alfa de 0.50. Este modo secundario se corresponde con los valores medidos durante las entradas de 
aerosoles desérticos, como iremos mostrando en los capítulos 6 y 7 , mientras que el modo principal se corresponde con la situación ordinaria en la estación. La frontera entre ambas situaciones se puede situar en torno a un valor de alfa de 0.8-0.9.

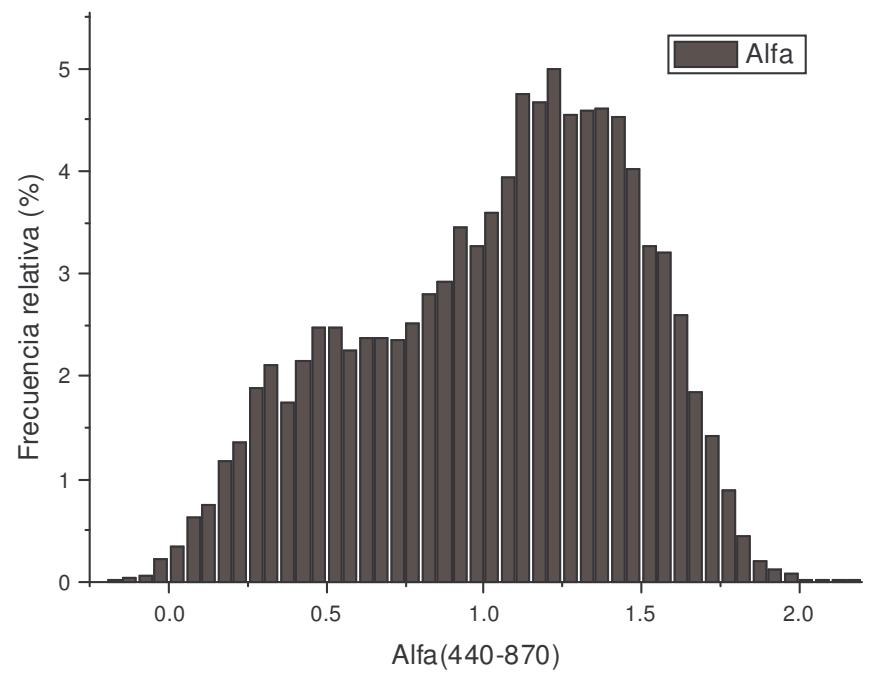

Figura IV-10. Histograma de frecuencias relativas de alfa(440-870) para la serie 2000-2004 en El Arenosillo con el procesado KCICLO

La evolución temporal en El Arenosillo del parámetro alfa(440-870) se muestra en la Figura IV-11. Este parámetro muestra una gran variabilidad, ocupando prácticamente todo el rango entre 0 y 2 . Se puede apreciar que en invierno hay un número mucho menor de medidas que en los veranos, por la mayor nubosidad y por el menor número de horas de sol al día. En estos meses más nubosos y lluviosos, con menos medidas y, como hemos visto, un AOD bajo en general, encontramos algunos valores muy bajos de alfa, incluso valores negativos, aunque esto se debe a que están sujetos a errores grandes. En concreto los datos negativos en el alfa(440-870) se concentran en una serie pequeña de días muy dudosos, en los que el algoritmo de eliminación de nubosidad puede haber dejado pasar medidas malas, por ejemplo con bruma ligera o cirros finos, etc. Los valores negativos de alfa se concentran en unos pocos días, suponen sólo el $0.4 \%$ de las observaciones, todos ellos son días de otoño o invierno en los que hay pocas medidas en general, lo cual indica la presencia de nubosidad.

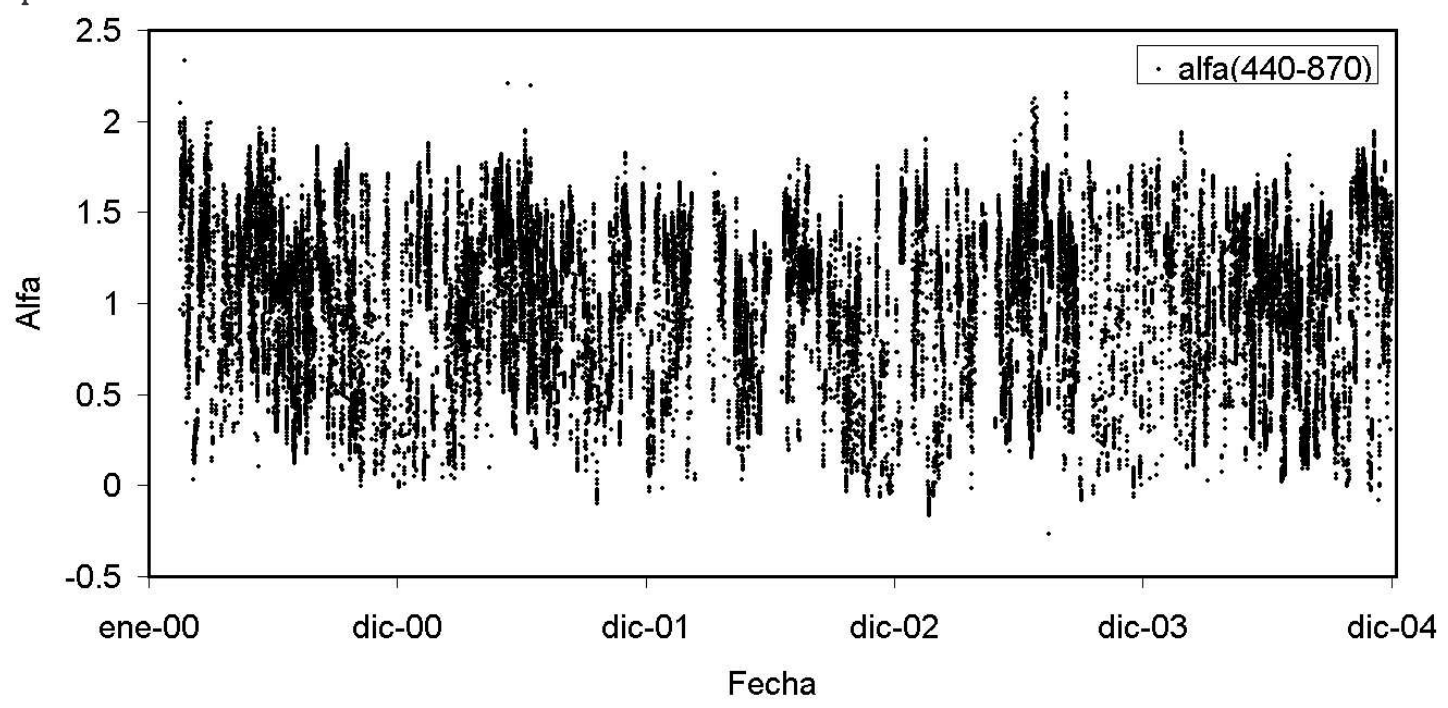

Figura IV-11. Evolución del parámetro alfa(440-870) en El Arenosillo con el procesado KCICLO

La situación habitual, con alfa entre 1 y 1.5, nos indica la presencia de un aerosol mezclado, con presencia de aerosoles de fuentes y tamaños típicos distintos, como son el 
aerosol marítimo y el antropogénico. Los valores por debajo de 0.5-0.6 corresponden con las entradas de aerosol desértico cuando están asociados a AOD alto. Los valores bajos de alfa en situaciones de baja turbiedad podrían corresponder con aerosol marítimo poco contaminado, aunque en este caso es muy difícil asegurar nada debido a los errores tan altos asociados a alfa. A este respecto nos remitimos a la sección 4.4 en la que se tratan los errores asociados al parámetro alfa.

Los alfas medidos durante las intrusiones desérticas son bajos, entre 0 y 0.6. Cuando entra el aerosol desértico el valor de alfa desciende desde los valores promedio (1-1.2) hasta valores en torno a 0.2-0.4 a la vez que el AOD va ascendiendo. Al final del episodio se recuperan valores cercanos a los iniciales. Sin embargo en los episodios de alta turbiedad debidos a contaminación, incendios, etc. este descenso del alfa no se produce, sino que incluso aumenta por encima del valor promedio hasta un valor en torno a 1.5.

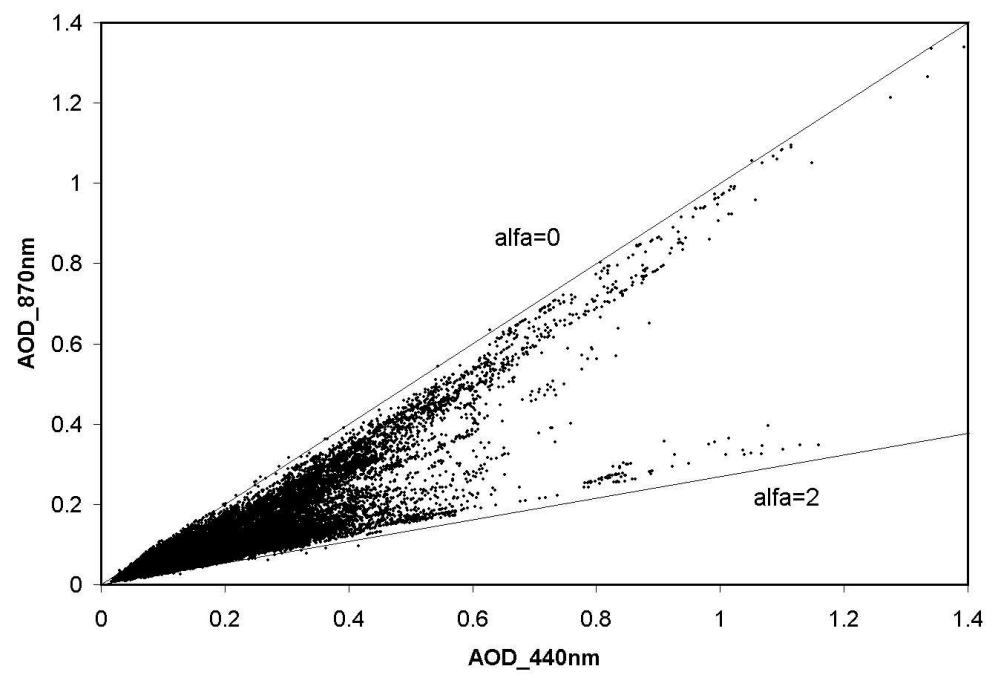

Figura IV-12. Diagrama de dispersión entre AOD en 440nm y en 870nm en El Arenosillo.

\begin{tabular}{lccccc}
\hline \hline alfa(440-870) & Año 2000 & Año 2001 & Año 2002 & Año 2003 & Año 2004 \\
\hline Promedio & 1.09 & 1.06 & 0.96 & 1.06 & 1.00 \\
Desviac. est & 0.44 & 0.40 & 0.40 & 0.45 & 0.45 \\
P25 & 0.75 & 0.75 & 0.66 & 0.70 & 0.67 \\
P75 & 1.43 & 1.37 & 1.27 & 1.41 & 1.36 \\
P10 & 0.42 & 0.48 & 0.35 & 0.41 & 0.31 \\
P90 & 1.64 & 1.55 & 1.45 & 1.59 & 1.55 \\
Min & -0.01 & -0.10 & -0.07 & -0.27 & -0.08 \\
Max & 2.34 & 2.21 & 1.79 & 2.16 & 1.95 \\
Mediana & 1.14 & 1.11 & 1.05 & 1.16 & 1.08 \\
\hline \hline
\end{tabular}

Tabla IV-VII. Estadística por años de alfa(440-870) en El Arenosillo para la serie 2000-2004.

Estas dos situaciones de alta turbiedad se pueden identificar muy bien gracias al parámetro alfa, lo cual consiste simplemente en tener en cuenta el comportamiento espectral del AOD. En la Figura IV-12 podemos observar el diagrama de dispersión de AOD en 870nm frente al AOD en 440nm. Las dos ramas en las que se separa el diagrama a medida que aumenta la turbiedad se corresponden con estas dos situaciones tipo: AOD y alfa altos (alta turbiedad debida a partículas finas) o bien AOD altos pero alfa bajo (las partículas gruesas del aerosol desértico). Los valores quedan confinados como ya se ha comentado entre esos dos límites: alfa $=0$ para las partículas gruesas; y alfa $=2$ para las partículas finas. Este análisis es más ilustrativo a partir del diagrama AOD-alfa, como mostraremos posteriormente (Figura IV-19). 
Se ha observado una cierta variabilidad en el parámetro alfa de unos años a otros. En la Tabla IV-VII tenemos la estadística por años de este parámetro. El promedio anual varía entre 0.96 en 2002 y 1.09 en el año 2000, es decir, alrededor del 13\%. Las desviaciones estándar se mantienen altas y suponen en general un 40-45\% de variación típica.

\subsubsection{Análisis de promedios diarios, mensuales y estacionales de alfa}

A la hora de describir el comportamiento del parámetro alfa mediante promedios, la información más útil viene proporcionada por los promedios diarios, ya que los días pueden ser más significativos que una observación en concreto. El 25\% de los días tienen un promedio de alfa inferior a 0.6 , mientras que el $10 \%$ tienen un promedio superior a 1.5. Seguimos teniendo algunos valores negativos ( $1 \%$ de los días) que son el promedio de las pocas medidas en días muy localizados que ya se ha comentado.

La distribución de frecuencias de los promedios diarios (Figura IV-13) es muy similar a la de las observaciones individuales, aunque en este caso el promedio es 0.94 (con una desviación estándar de 0.45), es decir, ligeramente inferior por el distinto peso que se da a las observaciones. En todo caso alfa presenta un modo principal con el pico de frecuencia en 1.14, mientras que el modo secundario se da en torno a 0.7 , es decir valores algo más altos que cuando analizamos las medidas individuales. Esto se puede deber a que el promedio diario camufla la variabilidad del día.

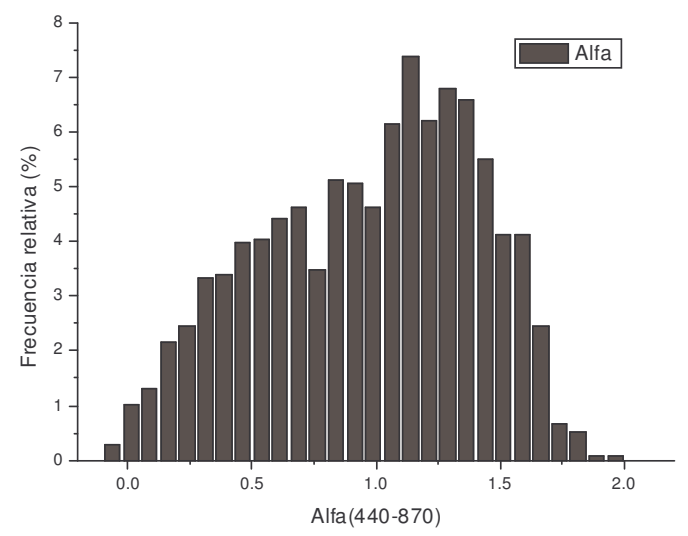

Figura IV-13. Distribución de frecuencias relativas del promedio diario de alfa(440-870) en El Arenosillo

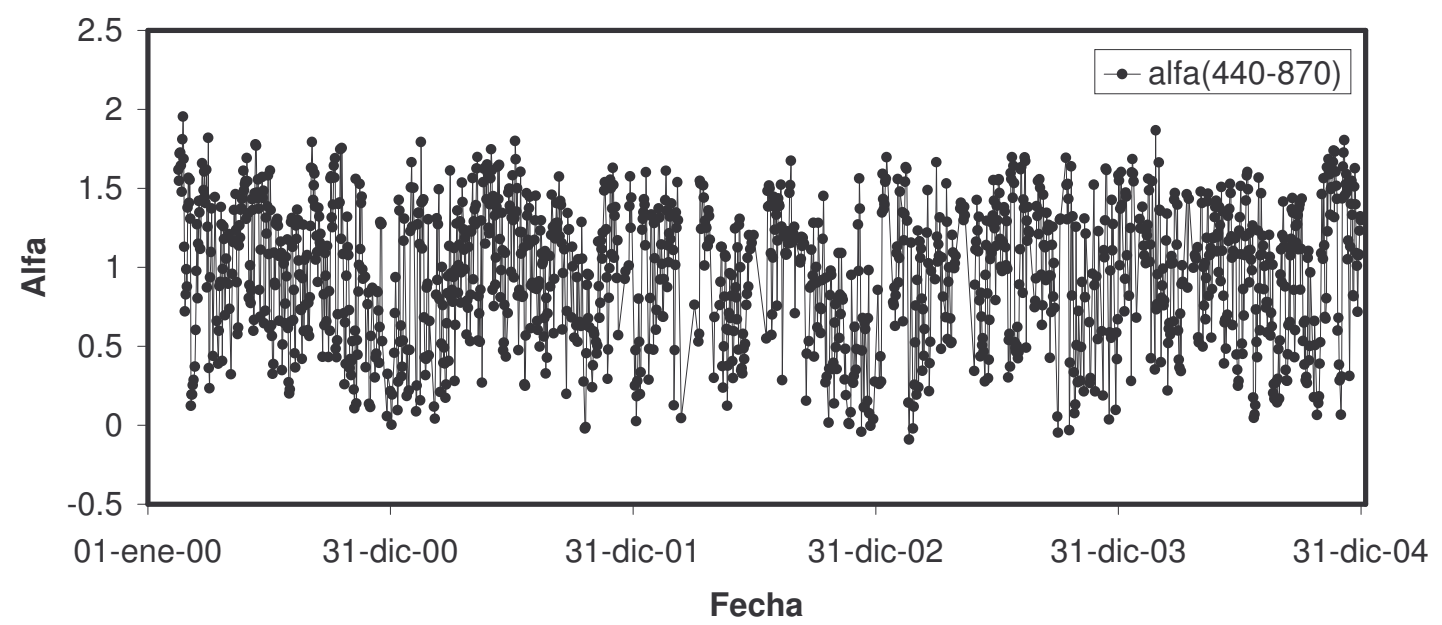

Figura IV-14. Evolución temporal del promedio diario de alfa(440-870) en El Arenosillo 
La Figura IV-14 presenta la evolución temporal del promedio diario de alfa(440-870). Al igual que en las observaciones individuales, el promedio diario ocupa todo el rango de variación. La realización de promedios hace desaparecer los pocos valores mayores que 2 . Algunos de los valores negativos se mantienen aunque suponen un porcentaje despreciable y con causas conocidas. Este tipo de gráficas generales dan a entender la variabilidad de este parámetro y el rango de valores que toma, así como la consistencia de la serie de datos reprocesada por el método KCICLO.

Se puede apreciar una cierta estacionalidad en los valores bajos de alfa, que presentan mínimos en invierno que no se alcanzan en verano. Frente a este resultado hay dos posibles explicaciones. La primera es que el aerosol se acerque más al aerosol marítimo más puro en invierno. Ya vimos que el $\mathrm{AOD}$ es menor en otoño e invierno, y si está asociado a un alfa menor de 0.5 lo podríamos calificar de aerosol marítimo casi sin mezcla. La segunda posibilidad es que el alfa medido durante el invierno, con un AOD bajo, lleve asociados errores grandes, con lo cual alcanza valores muy bajos pero que no son tan fiables. Por el contrario en verano los alfas serían más fiables por estar asociados a AOD más altos en general, y aunque se alcanzan valores bajos, éstos no llegan a igualar a los del invierno.

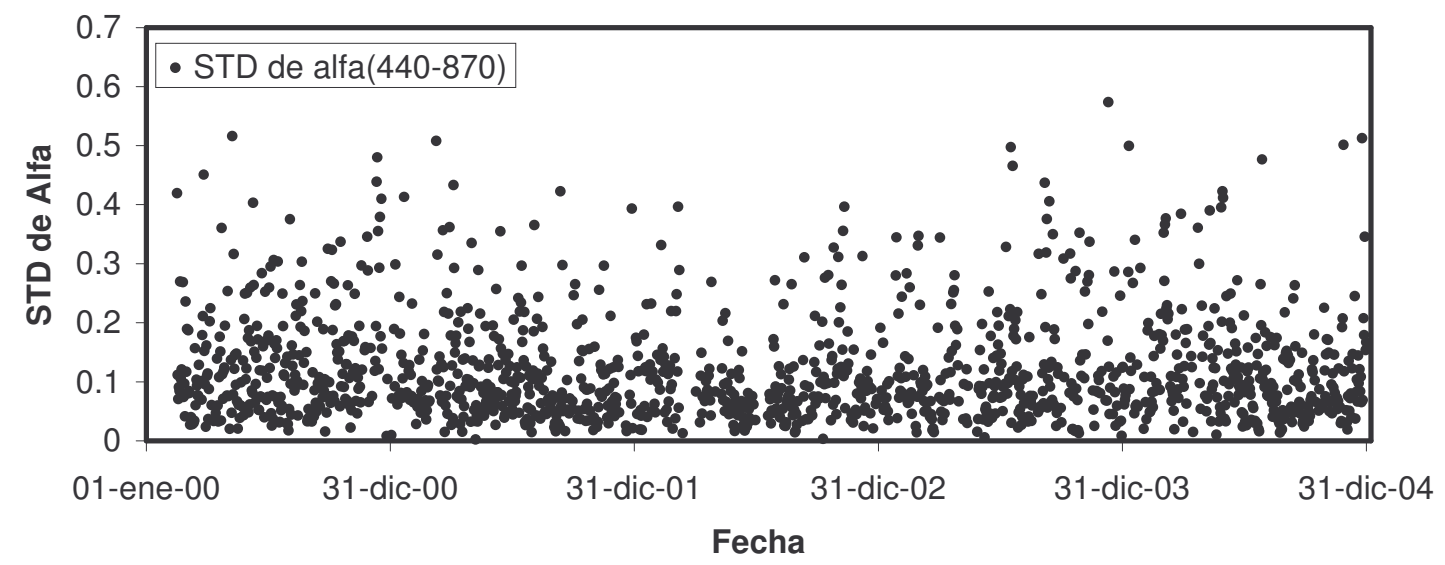

Figura IV-15. Desviación estándar diaria de alfa(440-870) en El Arenosillo

La desviación estándar diaria de alfa (Figura IV-15) nos indica que este parámetro va a presentar variaciones del orden del 0.1 (aproximadamente del 10\%) durante un día, aunque en ocasiones estas desviaciones típicas pueden ser incluso superiores a 0.5 en valor absoluto, lo cual estaría relacionado bien con un cambio muy fuerte durante el día, por ejemplo por un cambio del tipo de aerosol o gran variabilidad durante esa jornada, o bien, como veremos en la sección de errores, con errores grandes en alfa.

El promedio mensual del parámetro alfa (Figura IV-16) se mueve en un rango mucho menor que los promedios diarios, típicamente entre 0.8 y 1.2 con un promedio de 1.02 , sin alcanzar en ningún mes valores por encima de 1.5 o por debajo de 0.5 . En este caso el promedio de las situaciones tan diversas que pueden ocurrir durante todo un mes no aporta excesiva información. Nos describe, lógicamente, cómo es la situación típica, en promedio, pero no hay matices ni tampoco correlación significativa con la evolución del AOD. La gran variabilidad de unos días a otros que acabamos de mostrar se ve suavizada por los promedios mensuales, lo cual nos permite detectar una cierta estacionalidad. En concreto se observa un descenso del alfa en los meses de otoño que se repite con mayor o menor claridad durante los cinco años. Como ya se ha comentado, esto puede deberse a que el aerosol sea más cercano al marítimo limpio, sin tanta presencia de mezclas o influencias locales. 


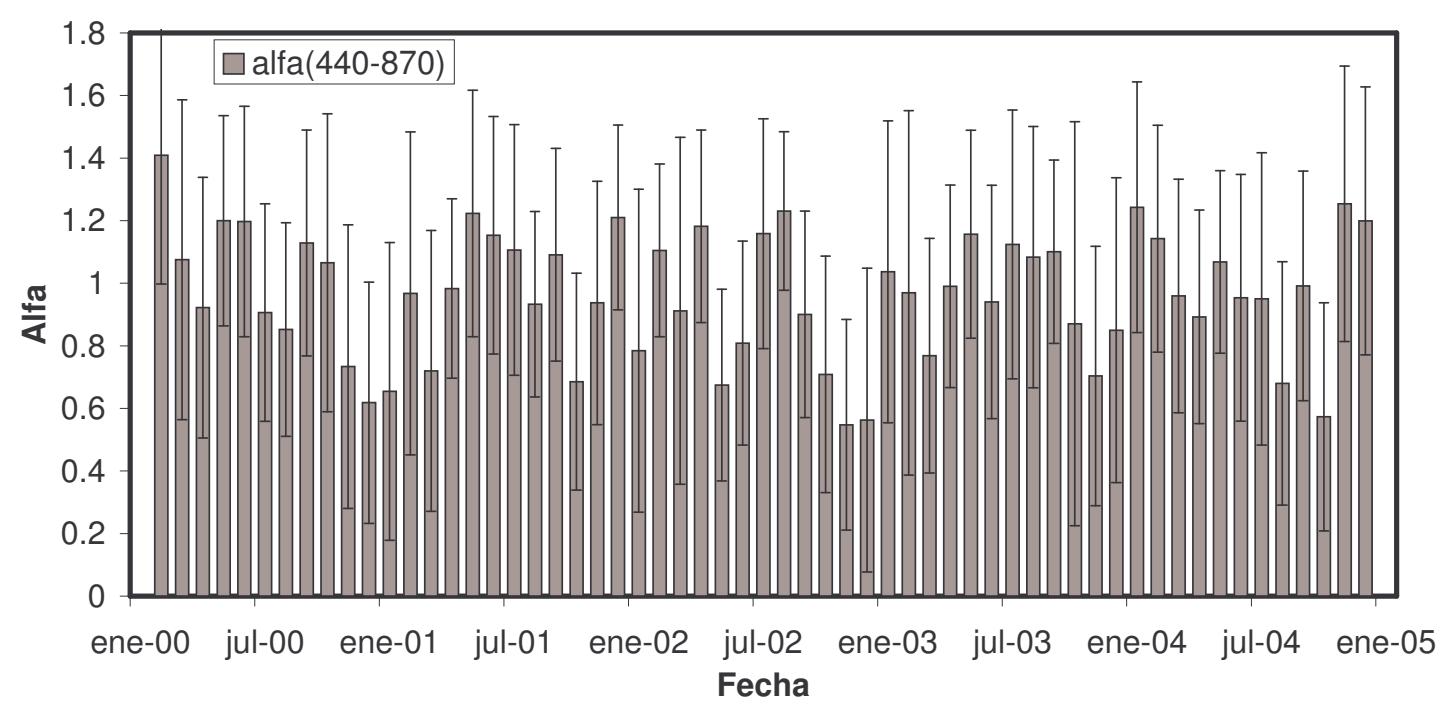

Figura IV-16. Evolución temporal del promedio mensual de alfa(440-870) en El Arenosillo

El promedio para cada mes a lo largo del período de medidas (Figura IV-17) y el promedio estacional del parámetro alfa en El Arenosillo (Figura IV-18) tienen el mismo problema de falta de matices o correlación con el AOD. Sólo nos indican cómo es la situación ordinaria, con un valor en torno a 1 , y que en los meses de otoño el parámetro alfa toma un valor algo inferior. Este descenso creemos que está relacionado por una parte con situaciones de baja turbiedad y por otra con malas condiciones de medida, es decir, mayor presencia de nubes o lluvia. El análisis de otro tipo de datos, como las trayectorias de masas de aire o las medidas de aerosoles in situ, ayudará a aclarar este punto.

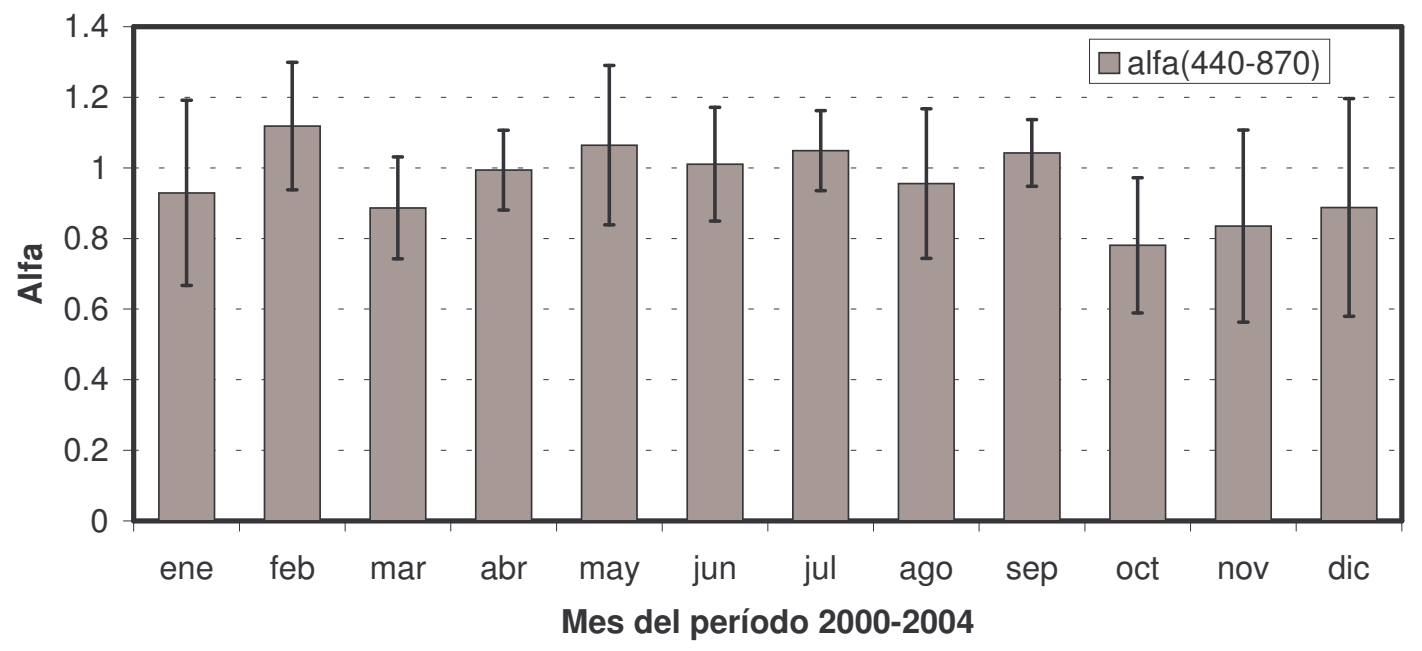

Figura IV-17. Valores mensuales de alfa(440-870) en El Arenosillo durante el período 2000-2004

La desviación estándar de estos promedios mensuales interanuales se refiere a los 4 ó 5 meses que se promedian para dar un valor interanual. Esta desviación es pequeña en verano y más grande en invierno, lo que nos indica que los meses de verano han sido más uniformes a lo largo de los cuatro años. Los meses de enero, mayo y diciembre son los que han presentado mayores variaciones de unos años a otros.

En la Tabla IV-VIII se recogen todos los datos interanuales de alfa, con su desviación estándar. Además podemos comprobar el número de días de medida, que en los meses de verano es superior al invierno. El promedio de alfa oscila entre el 0.78 en octubre y 1.12 en febrero. Es estable durante toda la primavera y el verano en torno a 1, para luego descender durante el otoño. 


\begin{tabular}{lcccc}
\hline \hline & alfa(440-870) & Desv. Est. & $\mathrm{N}^{0}$ días & Meses \\
\hline enero & 0.93 & 0.26 & 80 & 4 \\
febrero & 1.12 & 0.18 & 101 & 5 \\
marzo & 0.89 & 0.14 & 108 & 5 \\
abril & 0.99 & 0.11 & 106 & 5 \\
mayo & 1.06 & 0.23 & 116 & 5 \\
junio & 1.01 & 0.16 & 140 & 5 \\
julio & 1.05 & 0.11 & 134 & 5 \\
agosto & 0.96 & 0.21 & 140 & 5 \\
septiembre & 1.04 & 0.09 & 123 & 5 \\
octubre & 0.78 & 0.19 & 116 & 5 \\
noviembre & 0.84 & 0.27 & 116 & 5 \\
diciembre & 0.89 & 0.31 & 102 & 5 \\
\hline \hline
\end{tabular}

Tabla IV-VIII. Promedios mensuales para alfa(440-870) para el período 2000-2004 en El Arenosillo.

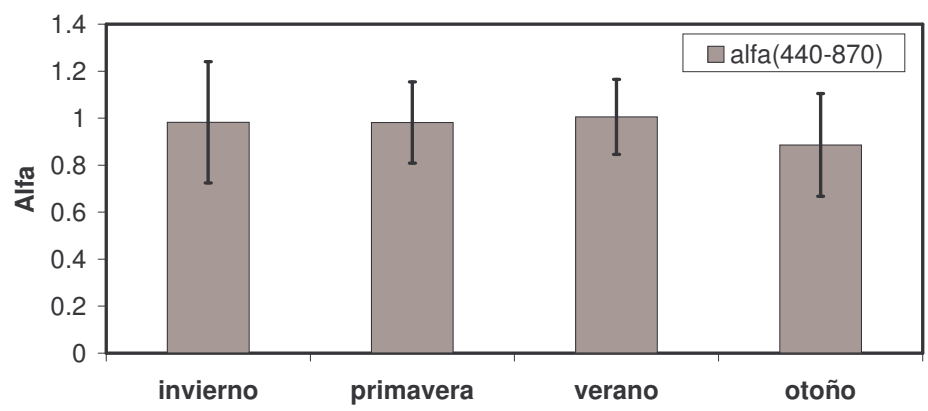

Figura IV-18. Valores estacionales de alfa(440-870) en El Arenosillo

El análisis estacional, como resumen final, nos indica de nuevo el mínimo otoñal de alfa, así como la mayor variabilidad de los meses de invierno. Como vemos en la Tabla IV-IX, los meses de verano son los más uniformes, pues tienen una desviación estándar menor. En la misma tabla podemos ver el número de meses que se ha utilizado para calcular cada promedio estacional.

\begin{tabular}{cccc}
\hline \hline & $\mathrm{N}^{\circ}$ meses & alfa(440-870) & Desviación est. \\
\hline invierno & 14 & 0.98 & 0.26 \\
primavera & 15 & 0.98 & 0.17 \\
verano & 15 & 1.01 & 0.16 \\
otoño & 15 & 0.89 & 0.22 \\
\hline \hline
\end{tabular}

Tabla IV-IX. Promedios y desviaciones estándar estacionales para alfa(440-870) en el Arenosillo durante el período 2000-2004.

En resumen, el parámetro alfa presenta una gran variabilidad, al igual que lo hace el AOD, y toma valores en un rango amplio. Los promedios mensuales tienen un rango de variación mucho menor, entre 0.6 y 1.4. Por último los promedios estacionales son bastante uniformes, y se mueven en un rango bastante estrecho, entre 0.9 y 1. 


\subsection{Caracterización de los aerosoles mediante los parámetros AOD y alfa}

Los diagramas de dispersión entre AOD y alfa son de gran ayuda a la hora de distinguir tipos de aerosol, y por tanto son útiles para caracterizar los aerosoles en El Arenosillo. Aunque el espesor óptico de aerosoles proporciona información sobre la cantidad de aerosol, y el parámetro alfa nos da información sobre el tipo, por ser un indicativo de la predominancia de partículas gruesas o finas, es la conjunción de ambos lo que posibilita una mejor interpretación de las observaciones.

En la Figura IV-19 se muestra el diagrama de dispersión para AOD en 440nm frente a alfa (calculado en el rango 440-870). Cuando el AOD es bajo tenemos todo un abanico de valores de alfa que ocupa el rango entre 0 y 2 , mientras que para valores altos de AOD la situación está más definida: o bien valores por encima de 1.5 o bien por debajo de 0.5. Por tanto hay dos situaciones diferenciadas para alta turbiedad. Sin embargo por debajo de 0.2 en $\mathrm{AOD}(440 \mathrm{~nm})$, cuando tenemos la situación ordinaria en El Arenosillo, podemos encontrar cualquier valor de alfa. El valor más bajo encontrado en la estación -aerosol de fondo o background- se sitúa aproximadamente en 0.03 a $440 \mathrm{~nm}$ y con unos valores de alfa en torno a 1-1.5.

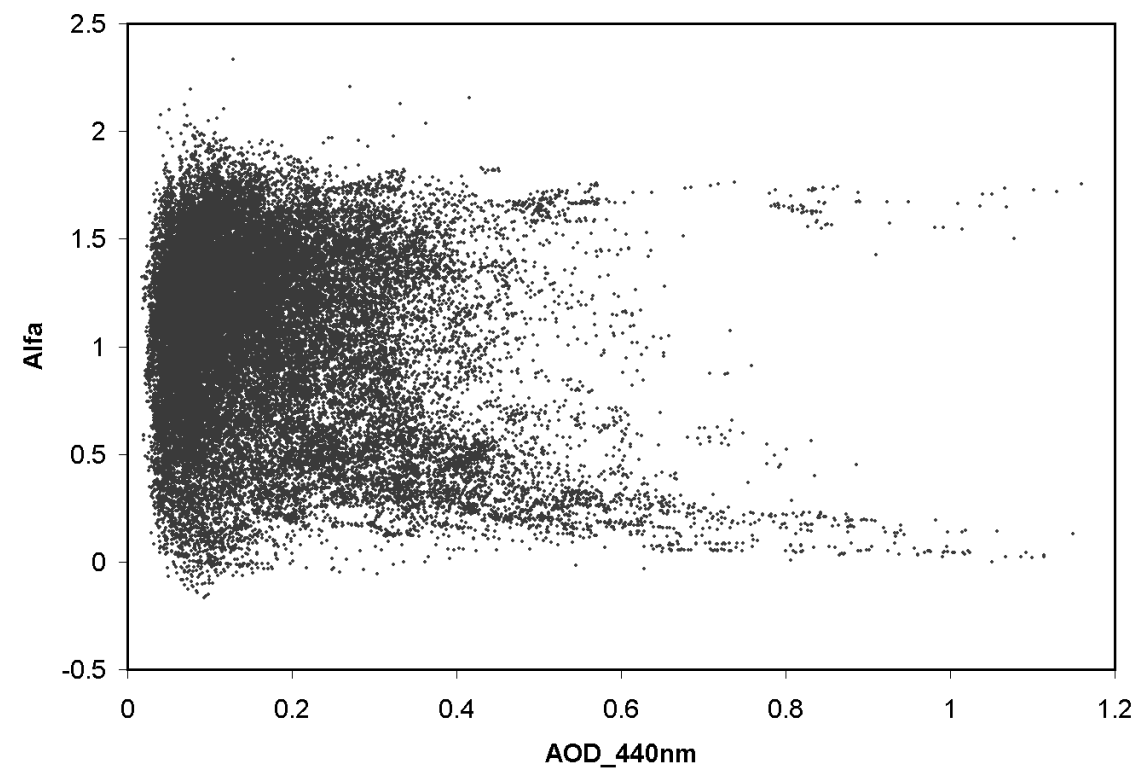

Figura IV-19. Diagrama de dispersión AOD 440 frente a alfa(440-870) en El Arenosillo

La rama inferior del diagrama, con AOD creciente y alfas bajos, es la región correspondiente al aerosol desértico: aproximadamente AOD por encima de 0.2-0.3 y alfa por debajo de 1 . Se trata de medidas que indican una fuerte extinción por aerosoles que es muy plana en longitud de onda, es decir, propia de partículas gruesas, de acuerdo con la literatura sobre aerosoles desérticos. La otra situación de alta turbiedad (región superior del diagrama), con valores altos de AOD y también valores altos de alfa, se corresponde con los valores propios del aerosol antropogénico o bien con incendios cercanos u otras situaciones de alta turbiedad debida a partículas finas. Las trayectorias de masas de aire, mapas de satélite u otro tipo de datos además de los que proporciona el fotómetro Cimel pueden ayudar a establecer el origen de estos episodios. Como ejemplo podemos citar el episodio de alta turbiedad de agosto de 2003, con valores de AOD superiores a 1 y por encima de alfa de 1.7, que coincidió con los numerosos incendios en Portugal y España 
durante el verano extraordinariamente caluroso de 2003. Este episodio se estudia en detalle en el capítulo 7.

Sin embargo, y una vez comentada la excepción, la situación más característica de alta turbiedad en El Arenosillo se debe al aerosol desértico, presente un 15-20\% de los días en la estación. En el capítulo 7 se realiza una evaluación de estos episodios desérticos a la vez que se muestran varios ejemplos de intrusiones en la estación. Durante estos episodios el AOD aumenta de forma similar en todos los canales, y el parámetro alfa desciende en consecuencia. La entrada y la salida de estos episodios presenta por lógica unos valores intermedios, de transición, que hacen difícil delimitar un umbral estricto en AOD o alfa para denominar al aerosol desértico. Además se pueden presentar eventos de muy diferente intensidad, con un rango entre 0.3 y 2 en el AOD y los valores más bajos (y también más fiables) de alfa.

Cuando la turbiedad es baja, la interpretación de las medidas a partir del diagrama AODalfa es más complicada. Para analizar mejor este diagrama se ha realizado en la Figura IV20 una representación alternativa, que consiste en evaluar la densidad de puntos que hay en el diagrama AOD-alfa. La representación se ha realizado a partir del número de medidas contadas en celdas de 0.01 de ancho en AOD y en alfa. La mayor densidad de puntos se concentra en una región muy concreta, con alfa entre 0.5 y 1.7 y AOD $(440 \mathrm{~nm})$ menor que 0.2. Esta región con más de 10 medidas para cada celda (en color azul más claro hasta el naranja) agrupa aproximadamente el $47 \%$ de las medidas en la estación, y corresponde por tanto con la situación ordinaria. Dentro de esta región con más densidad de datos, cuando el AOD es menor de 0.1 (las masas de aire más limpias), encontramos valores de alfa entre 0.55 y 1.65 . Sin embargo para AOD entre 0.1 y 0.2 los valores de alfa ocupan un rango menor, entre 1 y 1.5. Por tanto estas deben de ser las características de los aerosoles marítimos mezclados con las diferentes influencias, como continentales, antropogénicas y aquéllas que podemos catalogar como locales, sobre todo por las industrias cercanas y la resuspensión de partículas del suelo.

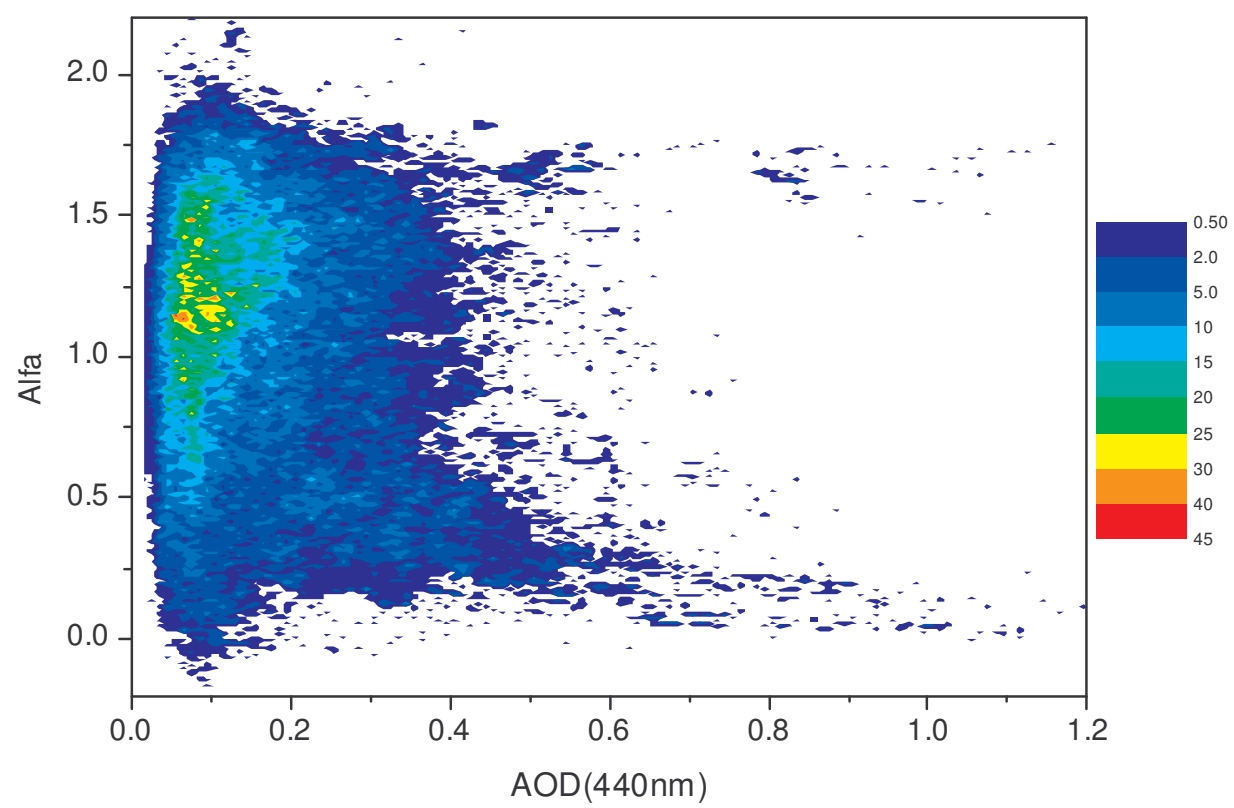

Figura IV-20. Diagrama AOD de $\mathrm{AOD}(440 \mathrm{~nm})$ frente al parámetro alfa, en el que se muestra la densidad de observaciones a través del número de datos registrados en celdas de $0.01 \times 0.01$ en AOD y alfa.

Esta influencia de aerosoles de origen continental o industrial tiene su reflejo, como ya se ha comentado, en el valor promedio de alfa 1.04, claramente más alto que el 0.5-0.6 
característico del aerosol marítimo puro, propio de estaciones oceánicas remotas. Los aerosoles continentales se situarían dentro del diagrama de la Figura IV-19 en unos valores de AOD entre 0.15 y 0.30 , es decir, valores por encima de los propios del aerosol marítimo, y valores de alfa por encima de 1 (Vergaz, 2001).

El análisis del diagrama de dispersión entre AOD en 440nm y alfa (440-870) para los promedios diarios, que se muestra en la Figura IV-21, nos muestra una gran densidad de puntos en la región correspondiente a la situación ordinaria en la estación, esto es, aerosoles marítimos mezclados. Esta región se puede acotar con AOD menor de 0.2 y alfa entre 0.5 y 1.6 aproximadamente, y en ella se concentra el $50 \%$ de los días. También se puede apreciar la región correspondiente a los aerosoles desérticos, con valores crecientes de AOD a partir de 0.2 aproximadamente y alfas bajos, por debajo de 1 .

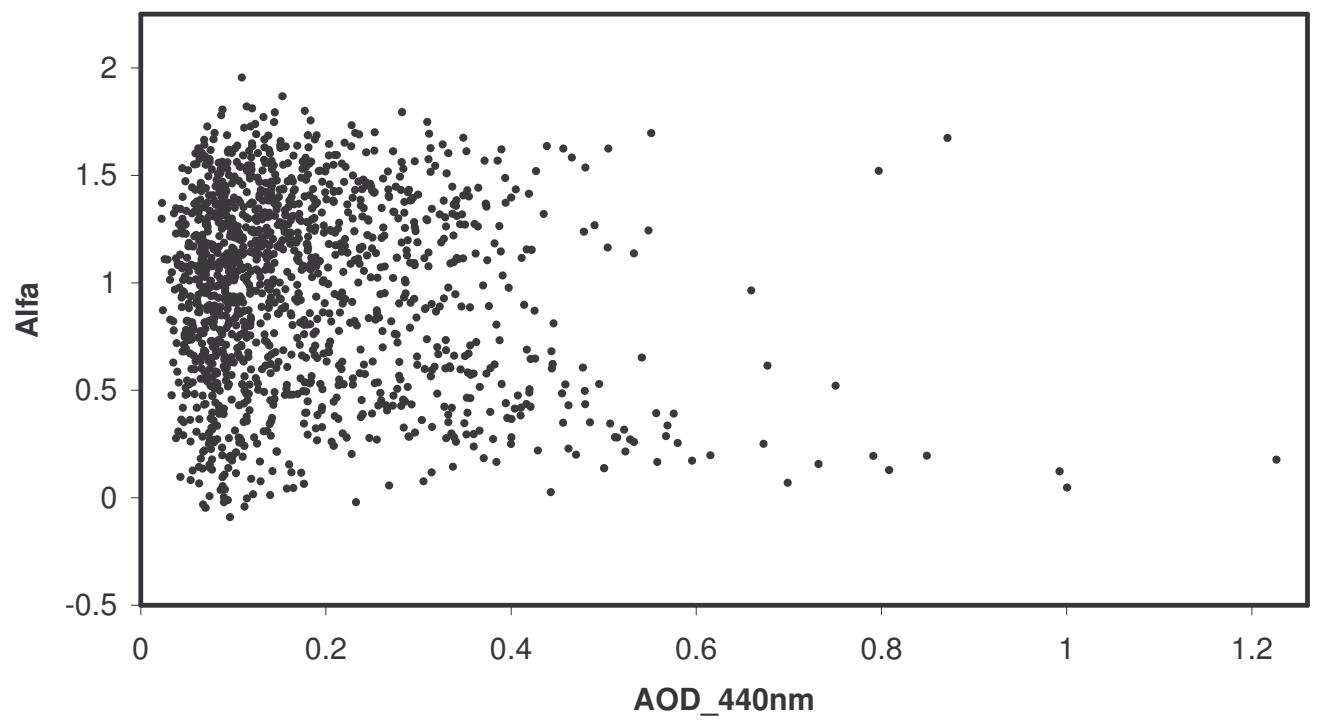

Figura IV-21. Diagrama de dispersión de AOD en 440nm frente a alfa para los promedios diarios de la serie 2000-2004 en El Arenosillo.

Este diagrama de promedios diarios también nos permite identificar los días con AOD alto con valores altos de alfa, es decir, los eventos de alta turbiedad debidos a contaminación, incendios, etc. En general el análisis que se puede hacer mediante los promedios diarios es análogo al que hemos realizado mediante las medidas individuales. 


\subsection{Análisis del error asociado al parámetro alfa}

Durante el análisis de los datos en El Arenosillo del parámetro alfa de Ångström, nos hemos encontrado con una serie de valores que podríamos calificar como defectuosos, en particular debido a la aparición de algunos valores negativos y de un ciclo diurno que habitualmente es mucho más acusado que para el AOD. Esto nos obliga a realizar un análisis de errores para alfa, con el objetivo de determinar si los errores asociados dan cuenta o son capaces de explicar estas observaciones defectuosas. Éstas observaciones negativas están asociadas en general a situaciones de AOD bajos o muy bajos, del mismo orden de la exactitud absoluta para el AOD calculado con el Cimel (0.02). En ellas el valor de alfa es extraordinariamente sensible a los errores en el AOD, como vamos a analizar.

Además los diagramas de AOD frente a alfa nos muestran cómo, para valores bajos de $\mathrm{AOD}$, podemos encontrar todo tipo de valores de alfa, y por tanto el diagrama pierde utilidad a la hora de establecer unos criterios o valores tipo para las masas de aire más limpias, que habitualmente son de origen marítimo.

El valor de alfa(440-870) se ha calculado mediante un ajuste lineal de $\ln \tau_{\mathrm{a}}$ frente a $\ln \lambda$, tomando logaritmos en la fórmula de Ångström (ec. 1.7). La estadística del ajuste nos da un error para la pendiente. Sin embargo este error es muy pequeño si no consideramos que los puntos con los que se realiza el ajuste tienen un error asociado. En efecto los AOD en 440, 670 y 870nm, a partir de los cuales se calcula el alfa, llevan un error asociado de 0.01-0.02. Este error puede significar un $100 \%$ de error relativo en las longitudes de onda más largas en condiciones de baja turbiedad. Además el dar un valor absoluto constante para el error resulta engañoso puesto que el error del espesor óptico de aerosoles depende de la masa óptica, como hemos visto al analizar el falso ciclo diurno del AOD en el capítulo 3 (Toledano et al., 2003; Cachorro et al., 2004). Esto se puede traducir en un error muy grande al calcular el alfa (superior al $100 \%$ ) y que no es constante sino modulado por la masa óptica, dando lugar a un ciclo diurno en el alfa.

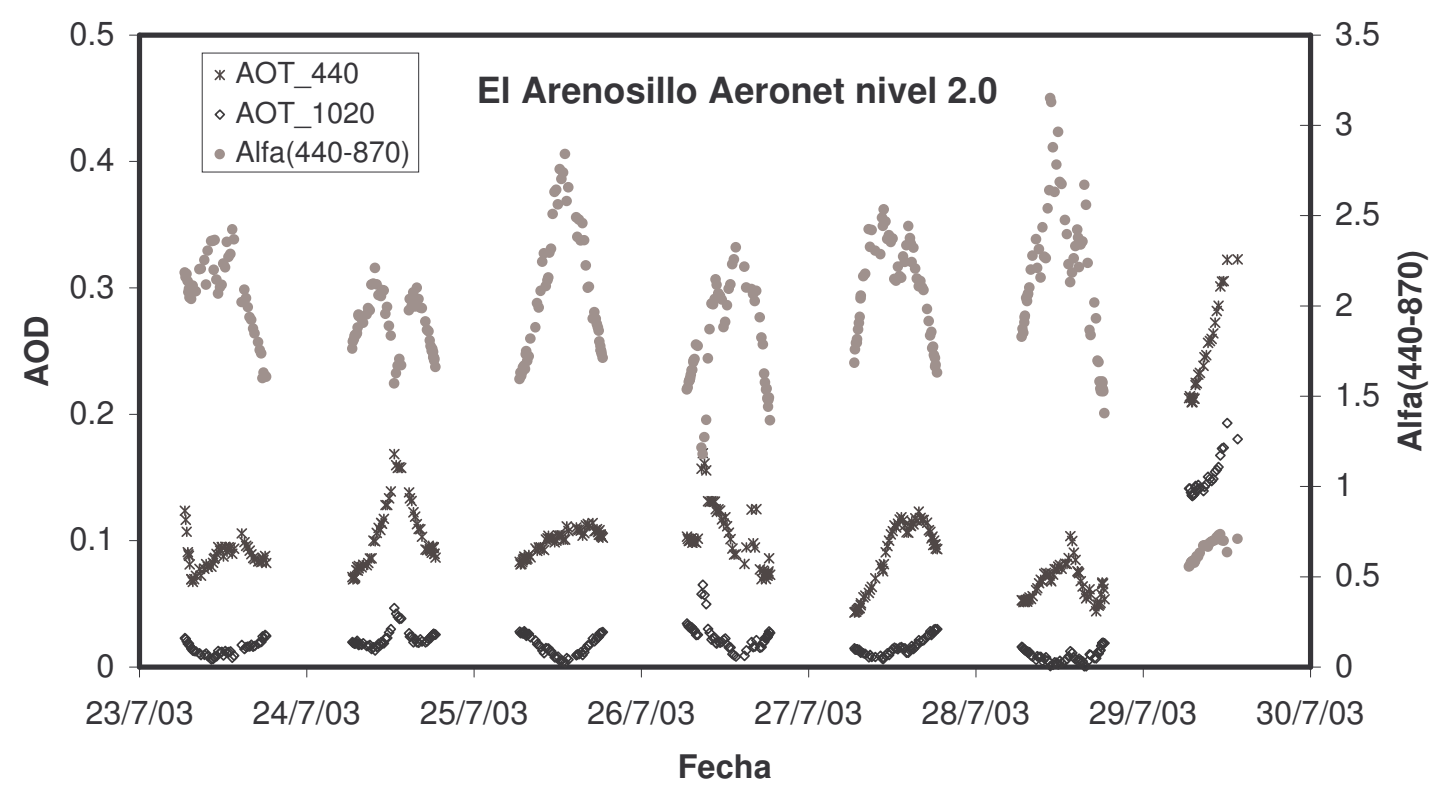

Figura IV-22. Ejemplo de alfa con errores grandes y falso ciclo diurno en la base de datos nivel 2.0 de AERONET. 
Parece lógico que si el AOD tiene un problema de calibración y presenta ciclo diurno, también lo presente el alfa derivado del AOD. Como ejemplo, mostramos en la Figura IV22 datos en El Arenosillo de AERONET en nivel 2.0. Estas medidas fueron tomadas por el Cimel\#50 durante julio de 2003. El AOD en 440nm presenta un ligero ciclo diurno por una sobrestimación de la constante de calibración, el cual se traduce en un error enorme en el parámetro alfa en situaciones de baja turbiedad. Vemos que el alfa presenta un acusado ciclo diurno y además alcanza valores muy por encima de 2 , con un rango de variación superior a 1 durante el día. Este tipo de resultados en el alfa nos hablan de problemas con la calibración en el AOD.

Es necesario dar cuenta de estos errores tan abultados en el parámetro alfa, pues de lo contrario dejará de ser un parámetro fiable. Por tanto hay que delimitar qué influye en el parámetro alfa y la sensibilidad de éste dentro de la precisión que estamos manejando. En primer lugar debemos estimar el error del parámetro alfa teniendo en cuenta que éste se calcula mediante un ajuste a partir de tres puntos (AOD en 440, 670 y 870nm) que llevan un error asociado. Para estimar el error de alfa $(\Delta \alpha)$ hemos derivado de acuerdo a la teoría de errores la expresión que nos da la pendiente en un ajuste lineal. Los $\mathrm{x}_{\mathrm{i}}$ son $\ln \lambda_{\mathrm{i}}$ mientras que $y_{\mathrm{i}}$ son $\operatorname{los} \ln \tau_{\mathrm{i}}$. La expresión a la que se llega es algo larga aunque sencilla de interpretar:

$$
\Delta \alpha=\frac{\left[\left|2 \ln \lambda_{1}-\ln \lambda_{2}-\ln \lambda_{3}\right| \cdot \frac{\Delta \tau_{1}}{\tau_{1}}+\left|2 \ln \lambda_{2}-\ln \lambda_{1}-\ln \lambda_{3}\right| \cdot \frac{\Delta \tau_{2}}{\tau_{2}}+\left|2 \ln \lambda_{3}-\ln \lambda_{1}-\ln \lambda_{2}\right| \cdot \frac{\Delta \tau_{3}}{\tau_{3}}\right]}{3 \sum \ln \lambda_{i}^{2}-\left(\sum \ln \lambda_{i}\right)^{2}}
$$

En esta expresión $\Delta \alpha$ es el error de alfa, $\lambda_{\mathrm{i}}$ son las longitudes de onda de los canales del Cimel y $\tau_{\mathrm{i}}$ son los espesores ópticos de aerosoles medidos en cada canal 'i'. Sólo hemos tenido en cuenta el error asociado a alfa por causa del error de los AOD. De momento no vamos a considerar la contribución al error de alfa por el error en longitud de onda. Esta expresión indica que el error de alfa va a depender de dos parámetros: el error asociado a los AOD y el propio valor del AOD. El error de alfa será mayor cuanto mayor sea la incertidumbre en el AOD, y también será mayor cuanto menor sea la turbiedad.

La evaluación de este error se ha realizado sobre las propias medidas experimentales en lugar de realizar una curva teórica mediante simulaciones porque nos ha parecido más ilustrativo. En la Figura IV-23 (a y b) se muestran los cálculos del error de alfa realizados para las medidas en El Arenosillo, tanto en escala lineal como logarítmica. Esta segunda gráfica nos ayuda a apreciar la fuerte dependencia del error de alfa con el AOD. Ambas gráficas muestran el error de alfa(440-870) en función del valor del AOD en 440, para dos casos distintos, según el error que asociemos al AOD en cada canal:

a) Caso 1 (en negro), $\Delta \tau_{440}=0.02 \quad \Delta \tau_{670}=0.015 \quad \Delta \tau_{870}=0.01$ (errores absolutos especificados en AERONET).

b) Caso 2 (en gris), $\Delta \tau_{440}=0.005 \Delta \tau_{670}=0.005 \Delta \tau_{870}=0.003$ (errores absolutos para la serie de datos reprocesada con el método KCICLO).

El caso 1 (puntos negros en la Figura IV-23) corresponde a considerar unos errores en AOD del orden de los que considera AERONET: 0.01-0.02. El cálculo indica que en este caso, el error absoluto de alfa se dispara cuando el AOD es bajo. Por ejemplo este error vale 0.7 cuando el AOD baja hasta 0.1 en $440 \mathrm{~nm}$, lo cual concuerda con los alfas mostrados en la Figura IV-22, es decir, con los datos reales. En el caso 2 (puntos grises en la Figura IV-23a y b) hemos tomado los errores para los AOD que se derivan del método KCICLO (0.003-0.005). Para estos errores del AOD el alfa tiene un error absoluto menor, aunque también llega a valores altos en los casos de más baja turbiedad (por ejemplo el error de alfa vale 0.5 cuando el AOD es de 0.04). Como se puede observar el error 
depende críticamente del AOD, y disminuye enormemente en términos absolutos cuando el AOD es alto. Sin embargo el parámetro clave para controlar el error en el alfa es el error en los AOD.
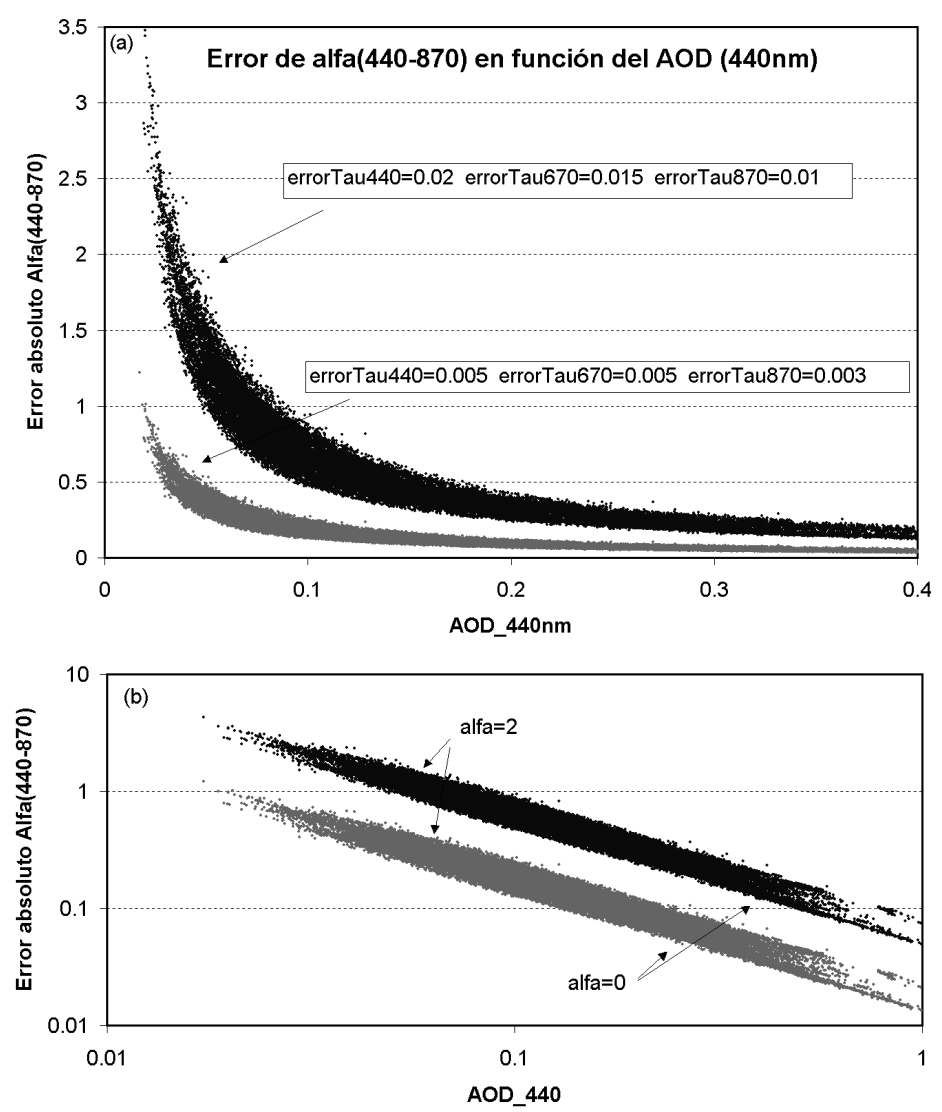

Figura IV-23. Error del parámetro alfa en función del AOD en dos casos distintos, según los errores de los AOD especificados en AERONET (en negro) y los estimados para la serie KCICLO (en gris): (a) en escala lineal; (b) en escala logarítmica. Los bordes superior e inferior de las nubes de puntos corresponden con alfa $=0$ y alfa $=2$, como se indica sobre las figuras.

La anchura que encontramos en estas franjas, que nos dan el error de alfa en función del $\mathrm{AOD}$, corresponde con las diferentes relaciones de valores que puede existir entre el AOD en los tres canales considerados, es decir, depende del valor de alfa. La parte superior de cada franja se corresponde con alfa $=2$ mientras que la parte inferior se corresponde con valores de alfa $=0$.

Este comportamiento del error de alfa se puede observar en las medidas del fotómetro Cimel. En la Figura IV-24 mostramos dos episodios de alta turbiedad en El Arenosillo, uno de ellos debido a combustión de biomasa, y por tanto con alfas altos, y el otro debido a una entrada de aerosol desértico, y por tanto con una bajada en el parámetro alfa. En ambos casos al aumentar la turbiedad se puede observar una disminución en el error de absoluto de alfa, que indicamos mediante las barras de error. En el ejemplo de la Figura IV-24a tenemos unos primeros días con AOD alto y alfa también muy alto (obsérvese la gran diferencia entre el AOD en $440 \mathrm{~nm}$ y en $1020 \mathrm{~nm}$ ). El error asociado a alfa durante estos días es pequeño, tanto en términos absolutos como relativos (menor de 0.05 ó 3\%). Además se puede observar que alfa es muy estable los días 13 y 14 de agosto. Cuando la turbiedad desciende a valores de fondo (días 16 y 17 de agosto) el error de alfa aumenta, y podemos observar que alfa es menos estable, con variaciones en forma de diente de sierra que no se dan cuando la turbiedad es alta. El valor de alfa que se aleja mucho del resto durante el día 15 coincide con un mínimo acusado en el $\mathrm{AOD}$, que da lugar a un alfa 
con mucho error (0.3 ó 20\%). En la Figura IV-24b podemos observar un valor de alfa estable en torno a 1.2 durante el día 29/7, con AOD en 440nm en torno a 0.15. El error absoluto de alfa está en 0.15. Cuando el AOD sube y alfa desciende, también desciende el error de alfa. El día 1 de agosto, con alfa en torno a 0.2 y AOD en 440 en 0.5 , el error de alfa ha bajado un orden de magnitud y se sitúa en 0.03. Aunque aquí el error relativo sea similar en ambos casos (10-15\%) la información que obtenemos de alfa es mucho más fiable en el caso de alta turbiedad.
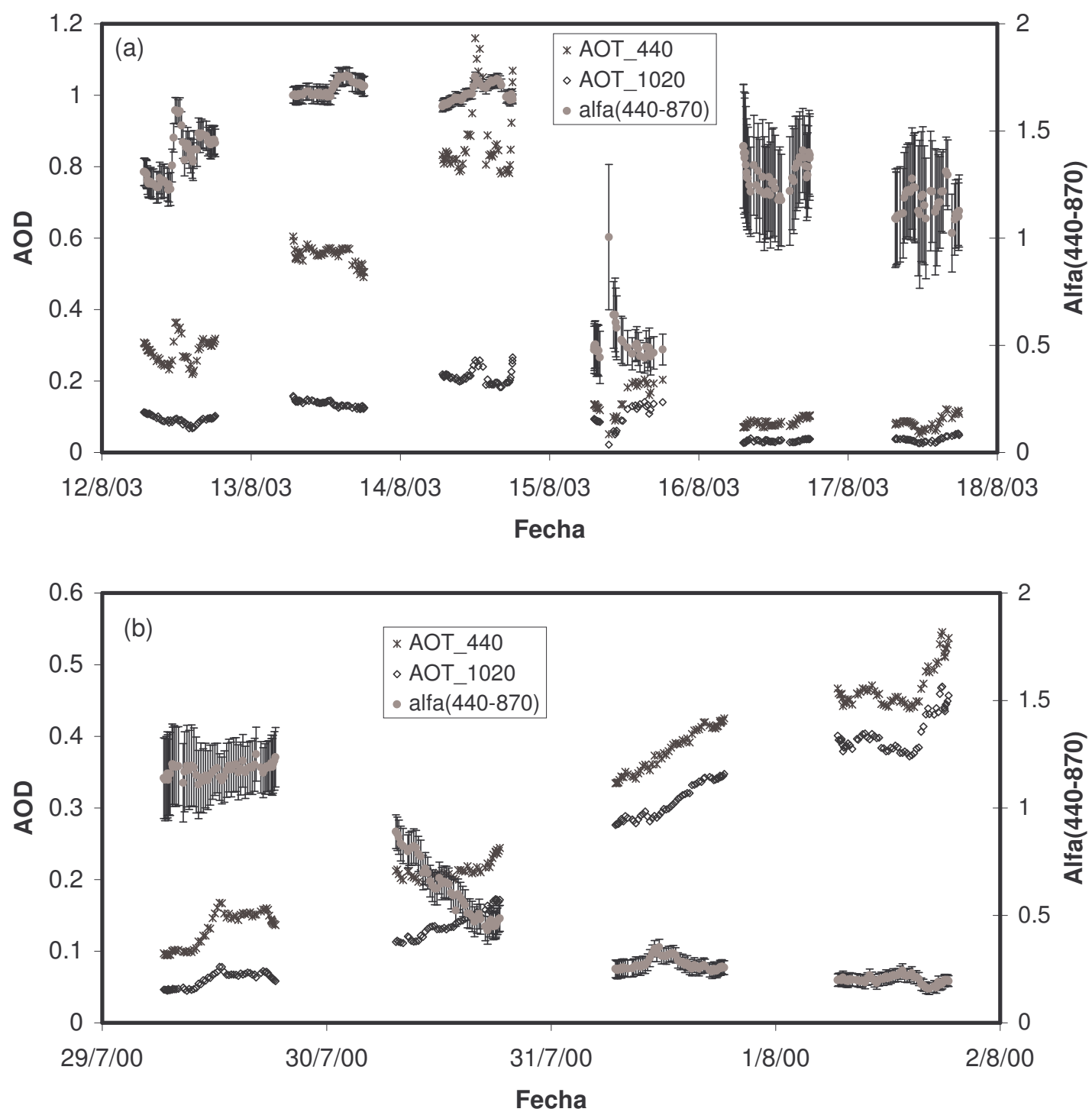

Figura IV-24. Parámetro alfa con su error junto con el AOD en 440nm y 1020nm en El Arenosillo con el procesado KCICLO: (a) episodio de combustión de biomasa en agosto de 2003; (b) episodio de aerosoles desérticos en agosto de 2000.

En definitiva los alfas se van a evaluar con más error cuanto mayor sea el error de los AOD, cualquiera que sea el valor de alfa, y en particular en el caso de AOD bajos. La dependencia del error de alfa con el error del AOD es tal que, en caso de haber errores grandes en el AOD, estos se manifiestan en el alfa produciendo un enorme ciclo diurno durante los días de baja turbiedad. Es decir, aumentan los errores y además tienen fuerte dependencia con la altura solar. Esto supone una ventaja: la ausencia de ese ciclo diurno en el valor de alfa es un buen indicativo de la calidad de los datos, aunque no sea condición suficiente. 
La estimación del error de alfa tal y como se ha calculado, da perfecta cuenta de esos pocos valores negativos o por encima de 2 que aparecen en la serie de datos de El Arenosillo con el procesado KCICLO. También explica los errores en los datos de AERONET si se tiene en cuenta el mayor error asociado al AOD.

El error de alfa mantiene una buena correlación $\left(R^{2}=0.63\right)$ con la desviación estándar del ajuste que realizamos para calcular alfa(440-870). Si la desviación estándar es pequeña, también lo es el error de alfa, y viceversa. Sin embargo esto no ocurre con el coeficiente de correlación del ajuste: para valores cercanos a 1 en el coeficiente de correlación encontramos errores de alfa tanto grandes como pequeños. Esto se debe a que el coeficiente de correlación tiende a cero si la pendiente tiende a cero. Esto no ocurre con la desviación estándar. Resulta pues más fiable la desviación estándar del ajuste a la hora de evaluar el error del parámetro alfa resultante (Vergaz, 2001, p.VI-11).

Por otra parte, el error de los alfas calculados a partir del AOD en pares de longitudes de onda es mayor que el error del parámetro alfa(440-870), y se dispara para valores bajos del AOD. Esto pone de manifiesto la extrema sensibilidad de los alfas calculados a partir de pares de AOD, lo cual será muy útil para detectar errores de calibración en el AOD. Para los alfas de los pares el error se calcula mediante la expresión siguiente:

$$
\Delta \alpha\left(\lambda_{1} / \lambda_{2}\right)=\left|\frac{1}{\ln \left(\lambda_{2} / \lambda_{1}\right)}\right| \cdot\left(\frac{\Delta \tau_{1}}{\tau_{1}}+\frac{\Delta \tau_{2}}{\tau_{2}}\right)+\left|\frac{\ln \left(\tau_{2} / \tau_{1}\right)}{\left(\ln \left(\lambda_{2} / \lambda_{1}\right)\right)^{2}}\right| \cdot\left(\frac{\Delta \lambda_{1}}{\lambda_{1}}+\frac{\Delta \lambda_{2}}{\lambda_{2}}\right)
$$

En esta fórmula se tiene en cuenta tanto el error introducido por el error en los AOD (primer sumando) como el error que se debe a la incertidumbre sobre la longitud de onda (segundo sumando). Es fácil comprobar que el error de alfa derivado de la incertidumbre en la longitud de onda depende del error en la longitud de onda del canal y también es directamente proporcional a alfa. En todo caso su valor no excede de 0.1 como error absoluto si estimamos en $10 \mathrm{~nm}$ el posible error en longitud de onda. La contribución por tanto de error a alfa por una mala evaluación de la longitud de onda central de los filtros del Cimel es pequeña, sobre todo en comparación con el error de alfa que se produce por errores en el AOD cuando la turbiedad es baja.

En resumen los errores asociados al parámetro alfa pueden ser tan grandes como el $100 \%$, en particular en situaciones de baja turbiedad, lo cual puede disminuir mucho la fiabilidad de este parámetro. Hay que ser por tanto cautos a la hora de manejar datos de alfa, pues en ocasiones no se podrá pasar de una interpretación cualitativa debido al error asociado. Dejamos aquí una puerta abierta para un estudio en profundidad sobre la interpretación cuantitativa y cualitativa de alfa, en particular en relación con la microfísica de las partículas. Se trataría de evaluar cuánta información y con qué margen de fiabilidad se puede obtener a partir de alfa al respecto, por ejemplo, del radio geométrico o el radio efectivo de la distribución de tamaños de los aerosoles mediante técnicas de inversión (Vergaz, 2001, p. VI-10, figura VI-5). 


\section{Capítulo 5. Comparación de la serie de datos KCICLO con la de AERONET}

\section{Introducción}

En este capítulo vamos a analizar en detalle las diferencias entre el procesado estándar de AERONET y la serie de datos reprocesada con el método KCICLO. El objetivo de este capítulo es evaluar y comparar la calidad de estos dos procesados diferentes. Para poder apreciar mejor las diferencias entre dos procesados distintos conviene analizar en detalle episodios o días concretos respecto a los datos de espesor óptico de aerosoles por una parte, y por otra parte analizar las estadísticas del AOD y del parámetro alfa de Ångström. 


\subsection{Comparación de la serie de medidas individuales en los procesados de AERONET y KCICLO}

La aplicación de diferentes coeficientes de calibración a unos mismos datos brutos da lugar a resultados sustancialmente diferentes, tanto en lo que se refiere a los propios valores del espesor óptico de aerosoles, como en lo que se refiere al comportamiento durante el día, puesto que el error depende de la masa óptica. En la Figura V-1 podemos observar este hecho para las medidas del día 9 de agosto de 2000. Las mismas medidas brutas del Cimel, en cuentas digitales, han sido procesadas con diferentes coeficientes de calibración: los coeficientes de la precalibración y la poscalibración de AERONET, el coeficiente interpolado para ese día (entre el de precalibración y el de poscalibración de AERONET), el coeficiente de calibración calculado por el grupo ULCO-LISE en el trabajo de Martiny (2001), el del método KCICLO y el correspondiente al procesado final de AERONET para el fotómetro Cimel \#114, realizado a partir de un Langley in situ.

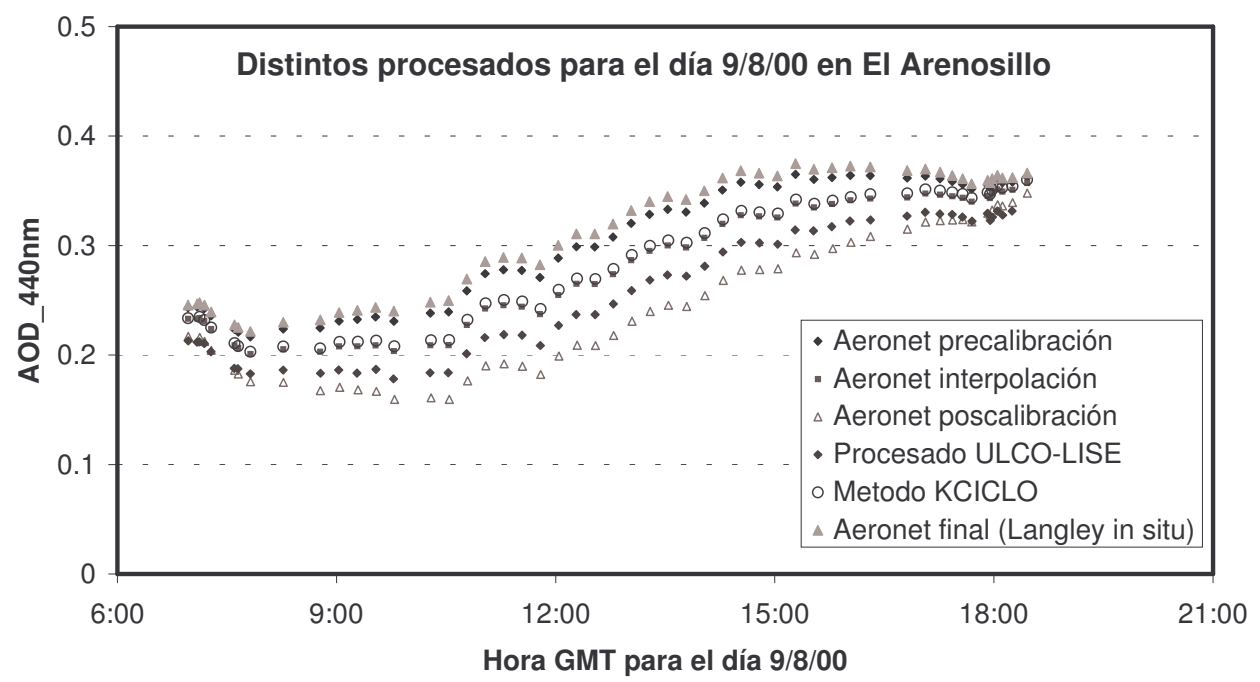

Figura V-1. Datos AOD 440nm para el 9 de agosto de 2000 procesados con diferentes coeficientes de calibración: procesados de AERONET, el grupo ULCO-LISE y el procesado KCICLO.

A la vista de los resultados gráficos se aprecia lo decisivo de la calibración tanto para los valores absolutos o las estadísticas que se puedan realizar en el sitio de medida, como para el análisis detallado de la evolución diurna del espesor óptico de aerosoles. Obsérvese cómo las diferencias son pequeñas en los extremos del día y se amplifican en el centro del día, exactamente moduladas por el inverso de la masa óptica. El promedio diario para el día mostrado, 9 de agosto de 2000, con cada uno de los procesados se recoge en la Tabla V-I. En ella podemos ver que el efecto que los distintos procesados trasladan al promedio diario, y en particular las diferencias entre los diferentes procesados respecto del KCICLO. Al igual que se ve en la gráfica, el filtro de 440nm tenía unas calibraciones pre y pos correctas, por lo que al realizar el interpolado apenas hay diferencia con el KCICLO. Sin embargo en el procesado final del Langley in situ el AOD es mucho más alto y la diferencia en el promedio diario supera el 0.02 . Al mediodía la diferencia absoluta con el procesado KCICLO llega a 0.04 .

Estas diferencias tan notables a simple vista tienen también un reflejo claro en las estadísticas realizadas sobre la serie completa de datos. Pero estas diferencias se aprecian mucho más claramente si la estadística, además de realizarse en general para toda la serie de 5 años, se detalla por instrumentos. Por esto vamos a analizar y comparar los 
procesados de AERONET y del método KCICLO para cada instrumento y en diferentes niveles: con todas las medidas individuales, promedios diarios y promedios mensuales. De esta forma podremos comparar la evolución de días concretos en detalle, de episodios, de cada instrumento por separado y de la serie completa. El procesado de AERONET al que nos referimos es siempre el nivel definitivo que se puede encontrar en la web de AERONET, es decir, nivel 2.0 excepto para el Cimel \#114 que está en nivel 1.5.

\begin{tabular}{lcccccc}
\hline \hline \multicolumn{1}{c}{ Día 9/ago/00 } & $\begin{array}{c}\text { AERONET } \\
\text { pre }\end{array}$ & $\begin{array}{c}\text { AERONET } \\
\text { interpolado }\end{array}$ & $\begin{array}{c}\text { AERONET } \\
\text { pos }\end{array}$ & $\begin{array}{c}\text { AERONET final } \\
\text { (Langley) }\end{array}$ & $\begin{array}{c}\text { ULCO- } \\
\text { LISE }\end{array}$ & KCICLO \\
\hline AOD(440nm) & 0.304 & 0.282 & 0.247 & 0.312 & 0.258 & 0.287 \\
Dif. abs. con KCICLO & 0.018 & -0.004 & -0.040 & 0.025 & -0.028 & - \\
Dif. relat. con KCICLO & 5.9 & -1.4 & -16.2 & 8.0 & -10.9 & - \\
\hline \hline
\end{tabular}

Tabla V-I. Promedios diarios del AOD en 440 para el día 9 de agosto de 2000 con diferentes procesados, y diferencias absolutas y relativas entre estos promedios y el que se obtiene con el procesado KCICLO.

\subsubsection{Diferencias entre los procesados de AERONET y KCICLO en los promedios de cada fotómetro}

A partir de las más de 39000 medidas individuales del fotómetro Cimel-318 libres de nubosidad, vamos a realizar una comparación entre los procesados de AERONET y del método KCICLO. En primer lugar mostramos en las Tabla V-II a Tabla V-IX los valores promedio y las desviaciones típicas para los AOD y alfa de Ångström, por separado para cada uno de los instrumentos que ha operado en El Arenosillo, tanto para el procesado de AERONET como para el KCICLO, así como las diferencias entre ellos.

\section{Cimel \#48}

Este fue el primer instrumento que estuvo operativo en El Arenosillo. Presenta unas diferencias entre los dos procesados que son notables para los canales de 440 y $670 \mathrm{~nm}$. En efecto los promedios de AOD difieren en 0.014 y 0.015 respectivamente, al límite de lo especificado por la red, en particular para el filtro de 670nm (Tabla V-II). Este filtro tiene un error de calibración por defecto y en promedio tiene valores casi iguales a los del filtro de 870nm.

\begin{tabular}{lcccc}
\hline \hline \multicolumn{1}{c}{$\# 48$} & AOD_1020 & AOD_870 & AOD_670 & AOD_440 \\
\hline KCICLO & $0.097 \pm 0.114$ & $0.100 \pm 0.120$ & $0.120 \pm 0.127$ & $0.186 \pm 0.137$ \\
AERONET & $0.094 \pm 0.115$ & $0.102 \pm 0.120$ & $0.106 \pm 0.128$ & $0.171 \pm 0.138$ \\
Diferencia absoluta & -0.004 & 0.003 & -0.014 & -0.015 \\
Diferencia relativa(\%) & 4.1 & 3.0 & -11.6 & 8.1 \\
\hline \hline
\end{tabular}

Tabla V-II. Estadística del AOD del Cimel \#48 con el procesado KCICLO

Este problema de calibración de AERONET en el filtro de $670 \mathrm{~nm}$ se traduce en notables discrepancias en las alfas. Como se recoge en la Tabla V-III, el alfa(440/670) de AERONET tiene un promedio de 1.6 frente al 1.3 del KCICLO, y frente al 1.2 de la serie completa de AERONET. Todavía más espectacular es la diferencia en el promedio de alfa(670/870), con un valor de -0.157 para AERONET frente al 0.9 del KCICLO y el 0.8 de la serie completa de AERONET. Además la desviación estándar es más del doble (1.000 frente a 0.406). 


\begin{tabular}{lccc}
\hline \hline \multicolumn{1}{c}{$\# 48$} & alfa(440-870) & alfa(440/670) & alfa(670/870) \\
\hline KCICLO & $1.188 \pm 0.428$ & $1.338 \pm 0.483$ & $0.908 \pm 0.406$ \\
AERONET & $1.003 \pm 0.398$ & $1.611 \pm 0.767$ & $-0.157 \pm 1.000$ \\
Diferencia absoluta & -0.185 & 0.274 & -1.065 \\
Diferencia relativa(\%) & -15.6 & 20.5 & 117.3 \\
\hline \hline
\end{tabular}

Tabla V-III. Estadística de alfa del Cimel \#48 con el procesado KCICLO

Esto nos obliga a remitirnos al apartado de análisis de errores de alfa, en el que se explicó por qué al tener un cierto error en el AOD, esto se traducía en errores muy grandes para los alfas de los pares, así como en un ciclo diurno de esos alfas que aumenta la desviación estándar de la serie. También podemos comprobar que el alfa(440-870) del ajuste con 3 filtros es el que menos se ve afectado por las deficiencias de calibración.

Podemos observar en detalle el problema del procesado de AERONET del Cimel \#48, y cómo afecta a los valores de AOD y de alfa (Figura V-2). En cuanto al AOD se aprecia cómo el AOD en 670nm se cruza con el de 870nm debido a la deficiente calibración, alcanzando en el centro del día valores de AOD más bajos en $670 \mathrm{~nm}$ que en $870 \mathrm{~nm}$. Esto tiene como consecuencia el abultado ciclo diurno de los alfas obtenidos a partir de los pares (440/670) y (670/870). Teniendo en cuenta la escala de la derecha, veremos que estos parámetros sobrepasan los valores realistas, en particular por los valores fuertemente negativos. Sin embargo hay que destacar que el alfa calculado en el rango (440-870) no se ve afectado significativamente y sigue siendo un indicador válido aunque su error asociado pueda ser grande. En este sentido podemos concluir que para detectar problemas de calibración el alfa calculado a partir de pares de longitudes de onda es una herramienta muy útil. Por otra parte, para caracterizar o clasificar el aerosol, el parámetro más fiable parece ser el alfa calculado con las tres longitudes de onda.

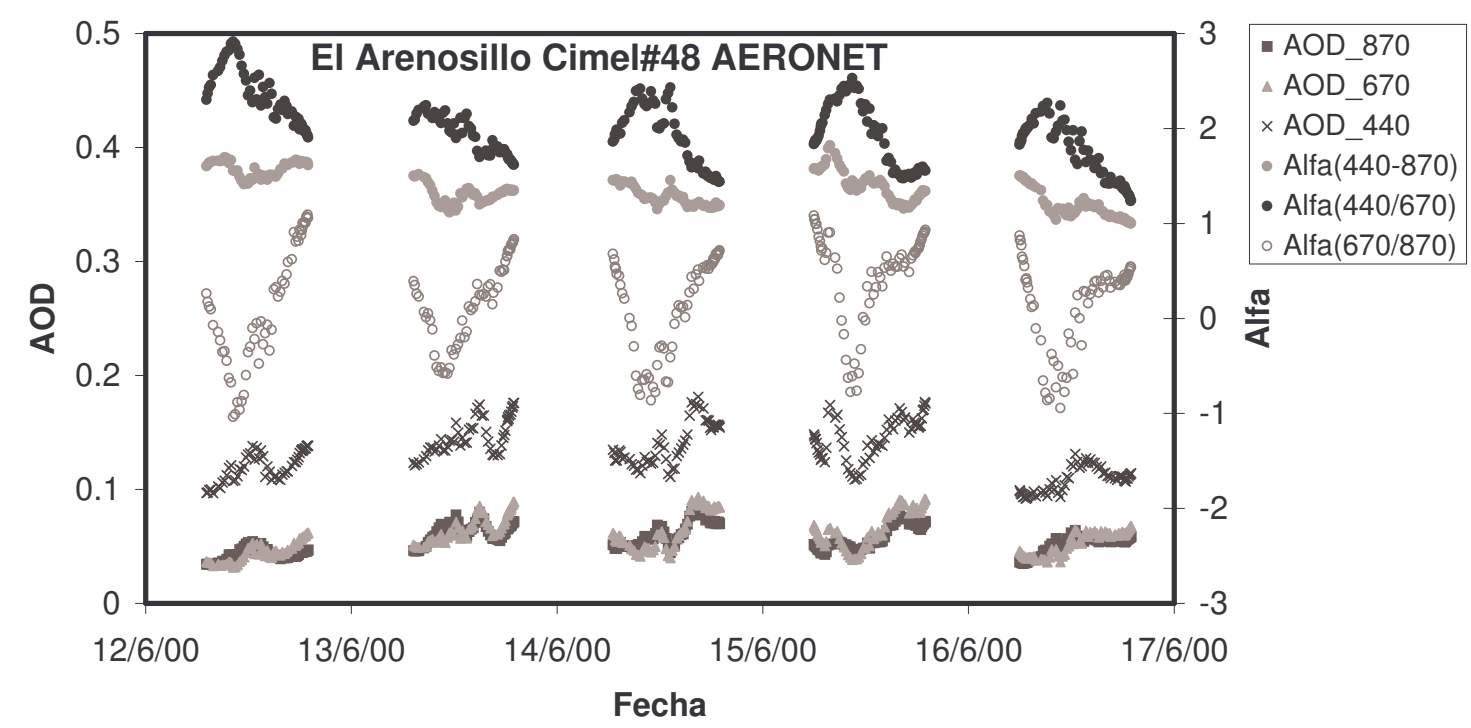

Figura V-2. Espesor óptico y alfa de Ångström en El Arenosillo con el Cimel \#48 procesado por AERONET.

En la Figura V-3 encontramos los mismos días de ejemplo del Cimel \#48 pero con el procesado KCICLO. Al conservar la misma escala en ambas gráficas se puede apreciar la sustancial mejora que introduce el KCICLO, puesto que el AOD en 670nm pasa a tener un comportamiento mucho más realista $y$, sobre todo, porque desaparecen los abultados ciclos diurnos en los alfas de los pares. Con todo, esta corrección del \#48 no es completamente satisfactoria debido a la escasez de días estables y despejados en los meses 
que estuvo en operación, imprescindibles para una óptima aplicación del método $\mathrm{KCICLO}$, a lo que se une la fuerte deriva de algunos de los filtros hasta del $7 \%$ en 6 meses según la calibración de AERONET, aunque ya hemos visto que no era del todo fiable en este caso.

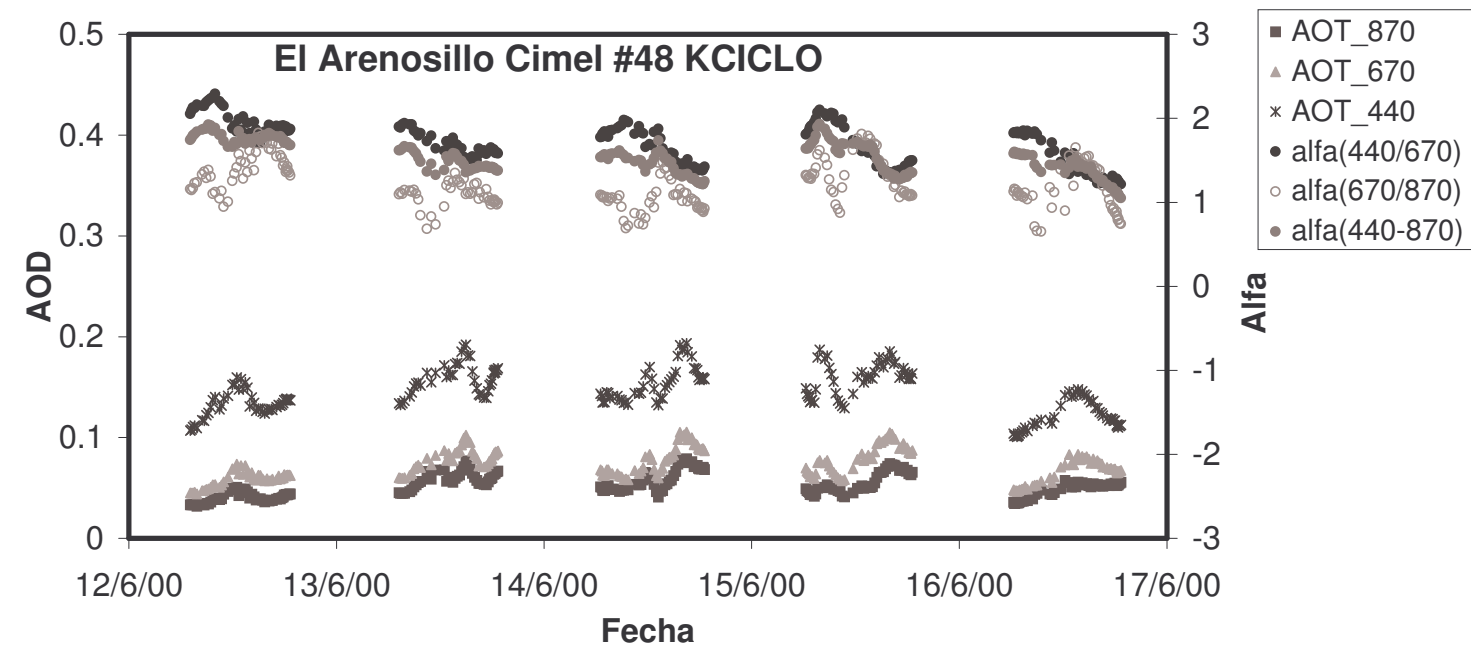

Figura V-3. Espesor óptico y alfa de Ångström en El Arenosillo con el Cimel \#48 procesado $\mathrm{KCICLO}$

Queremos aclarar que el comportamiento de AOD o alfa en estos días de ejemplo se da de manera sistemática en toda la serie de datos de este fotómetro, no sólo en ciertos días. Obviamente elegimos días despejados y estables para poder apreciar más claramente los ciclos diurnos. Esta misma observación es aplicable a todas las gráficas de ejemplo que iremos mostrando a continuación.

\section{Cimel \#114}

Este es sin duda el instrumento que más problemas ha presentado (Tabla V-IV). Todos los filtros presentan, en el procesado de AERONET, unas diferencias con los promedios del KCICLO que son mayores que el error que especifica AERONET (desde 0.01 para las longitudes de onda largas hasta 0.02 en el ultravioleta). Este instrumento no alcanzó el nivel 2.0 de AERONET por los problemas de ciclo diurno y valores negativos, y la red acabó por adoptar un procesado con un Langley in situ en un día que no era estable, dando el resultado que detallamos a continuación.

\begin{tabular}{lcccc}
\hline \hline \multicolumn{1}{c}{$\# 114$} & AOD_1020 & AOD_870 & AOD_670 & AOD_440 \\
\hline KCICLO & $0.084 \pm 0.081$ & $0.087 \pm 0.081$ & $0.103 \pm 0.089$ & $0.164 \pm 0.117$ \\
AERONET & $0.096 \pm 0.082$ & $0.105 \pm 0.082$ & $0.123 \pm 0.091$ & $0.189 \pm 0.119$ \\
Diferencia absoluta & 0.011 & 0.018 & 0.020 & 0.024 \\
Diferencia relativa(\%) & 13.9 & 20.7 & 19.4 & 14.6 \\
\hline \hline
\end{tabular}

Tabla V-IV. Estadística del AOD del Cimel \#114 con el procesado KCICLO

Sin embargo, con unos AOD sobrestimados por AERONET y que presentan además un claro ciclo diurno, los valores de alfa de este aparato (Tabla V-V) no muestran grandes diferencias en los promedios. En cualquier caso todos los alfas tienen en el procesado de AERONET un valor más bajo que para el KCICLO porque presentan un ciclo con mínimo al mediodía cuando el AOD es bajo (ver Figura V-4). 


\begin{tabular}{lccc}
\hline \hline \multicolumn{1}{c}{$\# 114$} & alfa(440-870) & alfa(440/670) & alfa(670/870) \\
\hline KCICLO & $1.056 \pm 0.430$ & $1.274 \pm 0.474$ & $0.623 \pm 0.541$ \\
AERONET & $0.928 \pm 0.367$ & $1.107 \pm 0.389$ & $0.580 \pm 0.395$ \\
Diferencia absoluta & -0.128 & -0.168 & -0.043 \\
Diferencia relativa(\%) & 12.1 & 13.2 & 6.9 \\
\hline \hline
\end{tabular}

Tabla V-V. Estadística de alfa del Cimel \#114 con el procesado KCICLO

El hecho de que los alfas tengan un ciclo diurno hacia abajo tiene también el efecto de reducir su desviación estándar, que es claramente inferior en el procesado de AERONET respecto al KCICLO para este Cimel \#114 y respecto a la serie general de AERONET.

Para mostrar estos problemas gráficamente, elegimos una situación de baja turbiedad en El Arenosillo, con valores bajos de AOD en todos los filtros y valores de alfa medios-altos (Figura V-4). El día 13/3/01 es excelente para verificar cómo el procesado de AERONET tiene un ciclo diurno con máximo al mediodía en los filtros de 440 y 870nm, lo cual produce también un ciclo diurno en el parámetro alfa. El tercero de los días mostrados $(15 / 3 / 01)$ es mucho más inestable y el incremento del AOD da lugar a que el alfa calculado tanto a partir de los AOD de AERNOET como del KCICLO sean parejos.

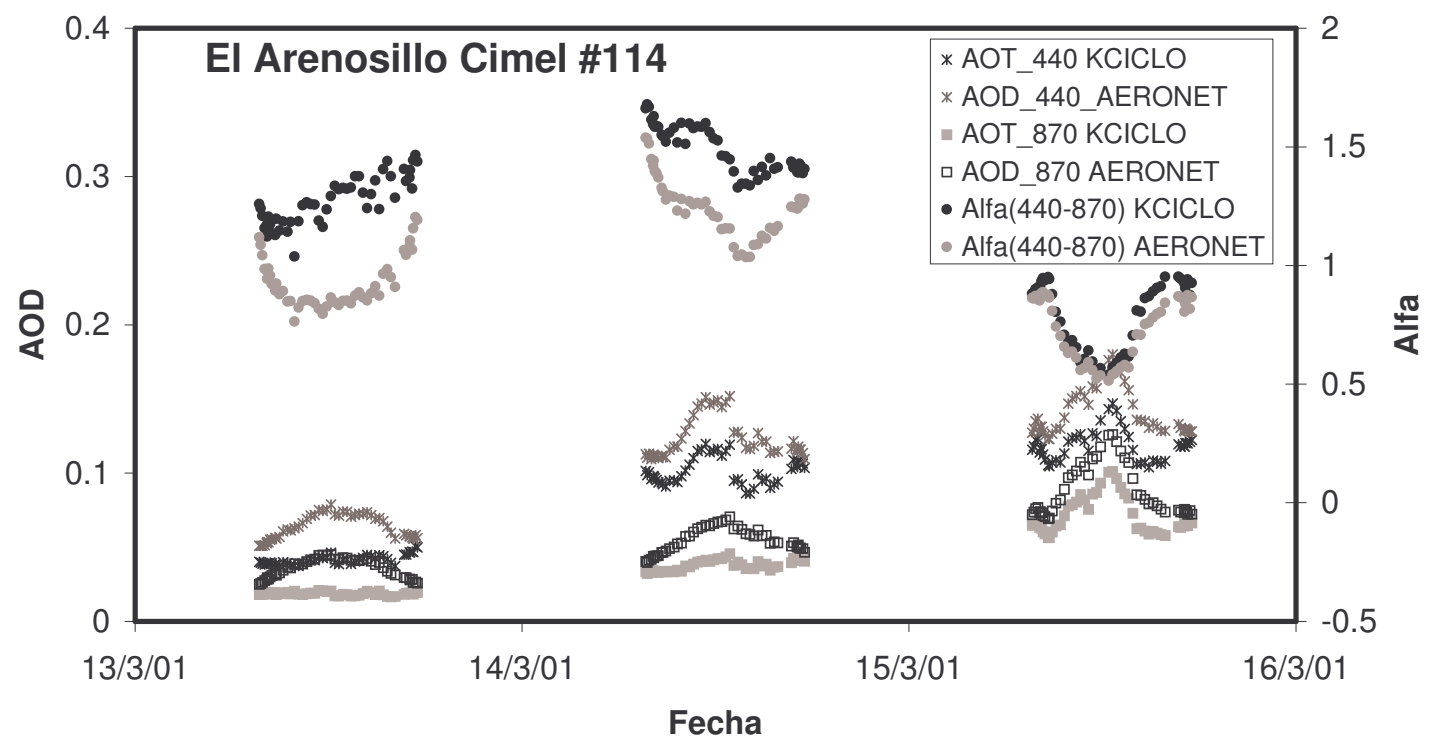

Figura V-4. Comparación de los procesados de AERONET y del KCICLO en El Arenosillo para el Cimel \#114 en los filtros de 440nm y 870nm y del alfa. Ejemplo de días con baja turbiedad.

\section{Cimel \#45}

El Cimel \#45 es el aparato mejor calibrado hasta el momento de los que han estado en el Arenosillo. Las diferencias en los promedios de AOD (Tabla V-VI) son pequeñas excepto en el filtro de 670nm, que es el más problemático, como estamos viendo. Este filtro tiene una discrepancia entre los promedios de 0.009, que es apreciable para los valores de alfa.

\begin{tabular}{lcccc}
\hline \hline \multicolumn{1}{c}{$\# 45$} & AOD_1020 & AOD_870 & AOD_670 & AOD_440 \\
\hline KCICLO & $0.079 \pm 0.069$ & $0.084 \pm 0.073$ & $0.100 \pm 0.083$ & $0.165 \pm 0.113$ \\
AERONET & $0.081 \pm 0.065$ & $0.084 \pm 0.069$ & $0.108 \pm 0.080$ & $0.165 \pm 0.109$ \\
Diferencia absoluta & 0.002 & 0.000 & 0.009 & 0.000 \\
Diferencia relativa(\%) & 2.5 & 0.0 & 9.0 & 0.0 \\
\hline \hline
\end{tabular}

Tabla V-VI. Estadística del AOD del Cimel \#45 con el procesado KCICLO. 
En efecto, en la Tabla V-VII vemos que el alfa(440/670) tiene un valor para AERONET de 1.075 frente al 1.309 del KCICLO y el 1.21 de la serie completa de AERONET. A su vez el alfa(670/870) tiene para AERONET un valor de 1.060 que está muy por encima del KCICLO (0.716) y del promedio de la serie completa de AERONET (0.81). Nuevamente el alfa(440-870) es el que menos se ve afectado, y es muy similar entre los dos procesados y con el promedio general de la serie.

\begin{tabular}{lccc}
\hline \hline \multicolumn{1}{c}{$\# 45$} & alfa(440-870) & alfa(440/670) & alfa(670/870) \\
\hline KCICLO & $0.970 \pm 0.406$ & $1.309 \pm 0.488$ & $0.716 \pm 0.489$ \\
AERONET & $1.060 \pm 0.404$ & $1.075 \pm 0.425$ & $1.060 \pm 0.478$ \\
Diferencia absoluta & 0.090 & -0.234 & 0.344 \\
Diferencia relativa(\%) & 9.3 & 17.9 & 48.0 \\
\hline \hline
\end{tabular}

Tabla V-VII. Estadística de alfa del Cimel \#45 con el procesado KCICLO.

También podemos confirmar gráficamente estos resultados de los promedios de AERONET y del procesado KCICLO. En la Figura V-5 se han representado cinco días medidos con el Cimel \#45 poco después de ser instalado en El Arenosillo. El AOD en $440 \mathrm{~nm}$ es prácticamente idéntico en ambos procesados, mientras que el AOD en 670nm muestra diferencias del orden de 0.02 al mediodía, con un cierto ciclo diurno del $\mathrm{AOD}(670 \mathrm{~nm})$ de AERONET. Este ciclo diurno se aprecia mejor durante el día 6 de agosto, que es bastante estable, y durante el día 9 de agosto, en el que la turbiedad es baja y el $\mathrm{AOD}(670 \mathrm{~nm})$ de AERONET casi se iguala con el $\mathrm{AOD}(440 \mathrm{~nm})$ por culpa del falso ciclo diurno. Éste queda también reflejado en el Alfa(440/670) de AERONET, que presenta un claro ciclo diurno hacia abajo, el cual no aparece para este mismo parámetro con el procesado KCICLO. Este ciclo diurno del alfa de AERONET es especialmente fuerte para los días 8 y 9 de agosto, cuando el AOD es muy bajo.

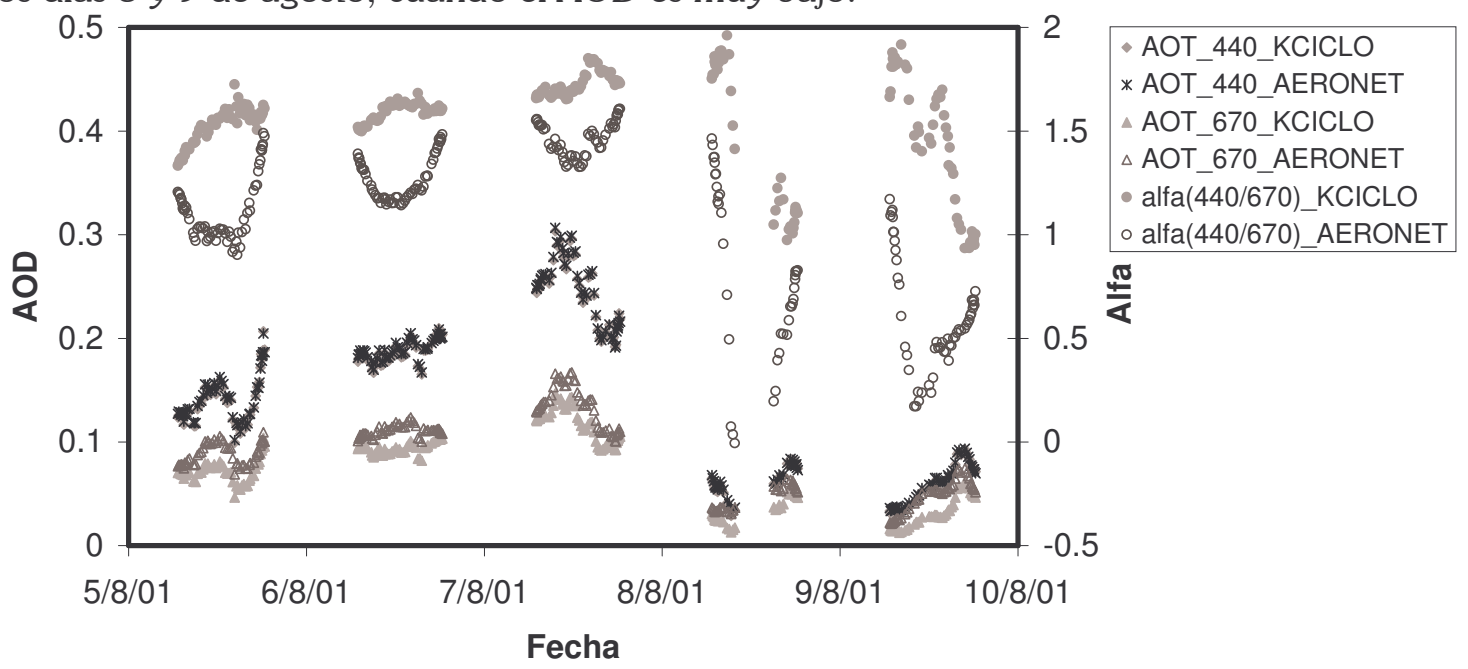

Figura V-5. Comparación de los procesados de AERONET y del KCICLO en El Arenosillo para el Cimel \#45 en los canales de 440nm y 670nm y del alfa(440/670).

\section{Cimel \#50}

El Cimel \#50 presenta de nuevo discrepancias en los promedios de AOD que rozan las especificaciones de AERONET (Tabla V-VIII ). En filtro de 440nm los promedios de AOD tienen una diferencia absoluta de 0.02 y en $670 \mathrm{~nm}$ de 0.008 . De nuevo estas diferencias se trasladan al parámetro alfa, que tiene valores demasiado altos en el procesado de AERONET para todos los alfas que hemos definido. 


\begin{tabular}{lcccc}
\hline \hline \multicolumn{1}{c}{$\#$ 50 } & AOD_1020 & AOD_870 & AOD_670 & AOD_440 \\
\hline KCICLO & $0.089 \pm 0.081$ & $0.096 \pm 0.086$ & $0.116 \pm 0.098$ & $0.179 \pm 0.136$ \\
AERONET & $0.085 \pm 0.080$ & $0.095 \pm 0.085$ & $0.124 \pm 0.098$ & $0.199 \pm 0.137$ \\
Diferencia absoluta & -0.005 & -0.001 & 0.008 & 0.020 \\
Diferencia relativa(\%) & -5.6 & -1.0 & 6.9 & 11.2 \\
\hline \hline
\end{tabular}

Tabla V-VIII. Estadística del AOD del Cimel \#50 con el procesado KCICLO.

En la Tabla V-IX recogemos estos promedios de alfa. El alfa(440/670) tiene unos valores similares en ambos procesados, y similares también al promedio general de la serie. No ocurre lo mismo para el alfa(670/870), que en el procesado de AERONET tiene un valor demasiado alto (1.261), por encima del KCICLO (0.754) y del promedio general de la serie, por el defecto en $670 \mathrm{~nm}$. Esto hace que el alfa(670/870) del procesado de AERONET tenga un fuerte ciclo diurno. Sin embargo lo más notable en este instrumento es el elevado valor del promedio de alfa(440-870) de AERONET (1.281). Este promedio es muy superior al de la serie general y también al nuestro del KCICLO para este instrumento (1.048). Este alfa de 3 canales, que normalmente no se ve muy afectado, sí se ve alterado en este caso al combinarse los errores por exceso en 440 y $670 \mathrm{~nm}$.

\begin{tabular}{lccc}
\hline \hline \multicolumn{1}{c}{$\# 50$} & alfa(440-870) & alfa(440/670) & alfa(670/870) \\
\hline KCICLO & $1.048 \pm 0.432$ & $1.204 \pm 0.480$ & $0.754 \pm 0.575$ \\
AERONET & $1.281 \pm 0.481$ & $1.309 \pm 0.456$ & $1.261 \pm 0.690$ \\
Diferencia absoluta & 0.233 & 0.105 & 0.507 \\
Diferencia relativa(\%) & 22.2 & 8.7 & 67.2 \\
\hline \hline
\end{tabular}

Tabla V-IX. Estadística de alfa del Cimel \#50 con el procesado KCICLO.

Tenemos en la Figura V-6 unos días de ejemplo de este fotómetro Cimel \#50, en abril de 2004, poco antes de ser reemplazado. Estos datos están en el nivel 2.0 de AERONET. El AOD en 440nm tiene un ciclo diurno hacia arriba, y en menor medida también el AOD en el canal de 670nm. Como resultado el alfa(440-870) tiene un ciclo diurno claro, lo que explica ese promedio de 1.28 muy por encima del promedio para este mismo parámetro en el procesado KCICLO, con 1.05. Este último valor es mucho más acorde con el promedio general de la serie.

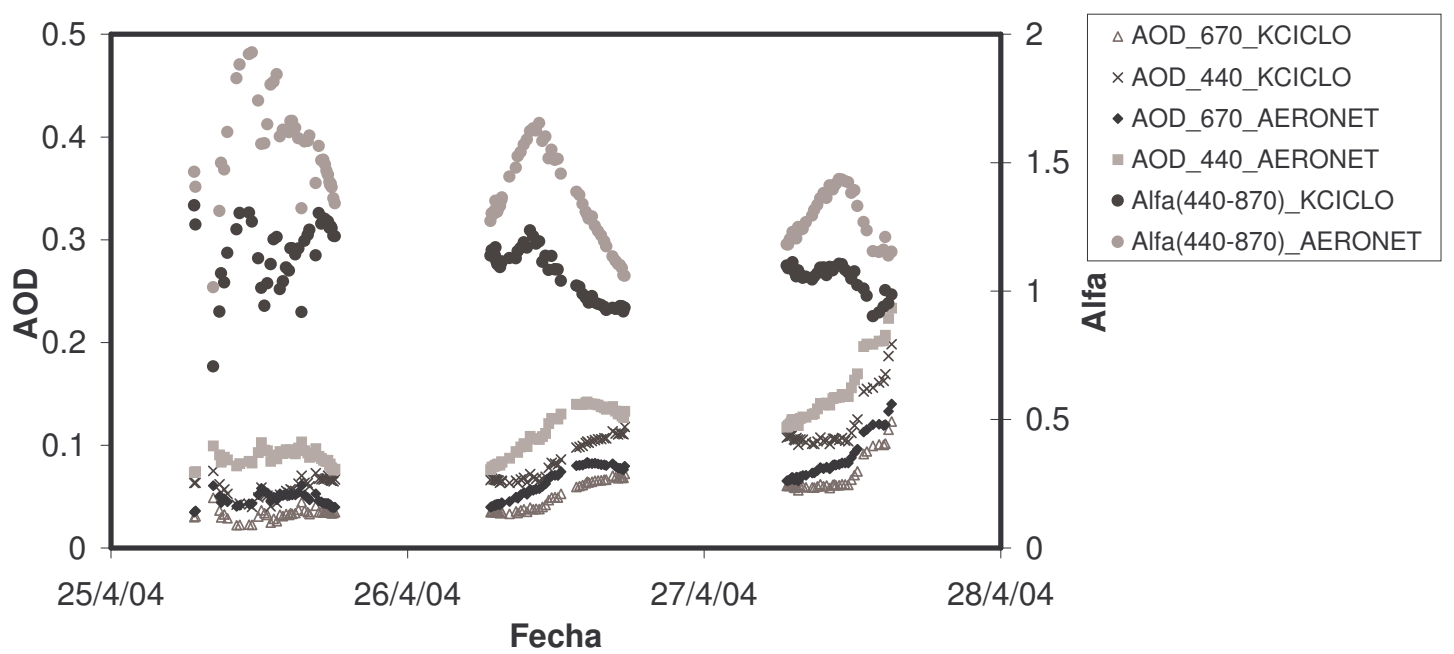

Figura V-6. Comparación de los procesados de AERONET y del KCICLO en El Arenosillo para el Cimel \#50 del AOD en los canales de 440nm y 870nm y del alfa(440-870). 


\section{Cimel \#45 (año 2004)}

En el momento de redactar esta memoria el Cimel \#45 está todavía operativo en El Arenosillo, por lo que no está disponible el procesado definitivo de AERONET. Sin embargo queremos señalar que este fotómetro tiene de nuevo un problema severo de calibración, con algunas medidas de AOD negativos en 1020nm y un falso ciclo diurno muy marcado tanto en algunos canales de AOD como en el parámetro alfa. Por este motivo se ha aplicado el método KCICLO y se ha realizado una primera corrección de las medidas de este fotómetro hasta el 31 de diciembre de 2004. Una vez que el instrumento sea retirado para realizar la poscalibración se podrá realizar una evaluación definitiva de esos datos. También por este motivo la comparación entre la serie de datos KCICLO y la de AERONET se realiza sólo hasta el 12 de mayo de 2004, fecha en la que se retiró el fotómetro Cimel \#50 y se instaló el \#45.

Los problemas del Cimel \#45 en esta segunda ocasión en la que ha sido instalado en El Arenosillo se pueden observar en la Figura V-7. El AOD en los canales de 870nm y $1020 \mathrm{~nm}$ tienen un ciclo diurno hacia abajo, por lo que durante los días con turbiedad muy baja (7, 8 y 9 de julio) se alcanzan AOD negativos en estos canales. El parámetro alfa(440$870)$ presenta también un claro ciclo diurno, en este caso hacia arriba.

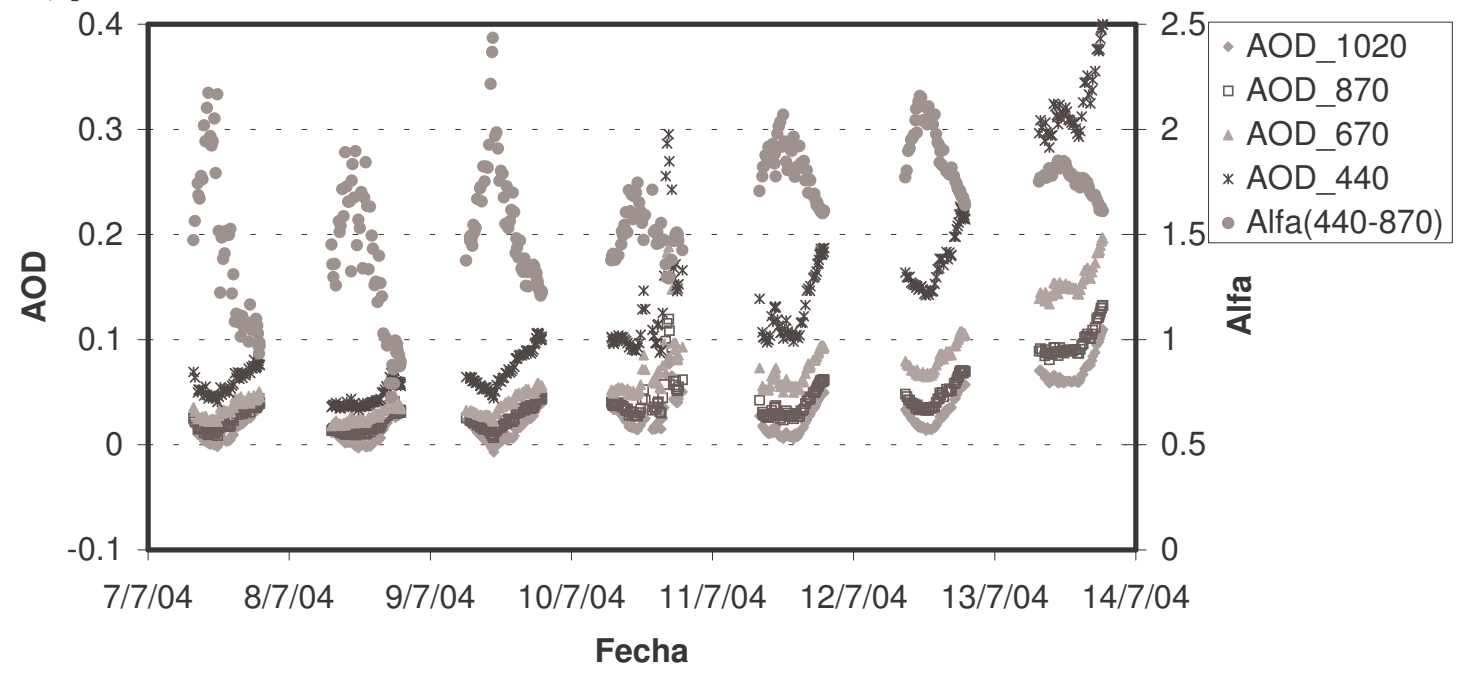

Figura V-7. Medidas del Cimel \#45 en julio de 2004 para los cuatro canales en el procesado de AERONET nivel 1.0.

En resumen, hay varias discrepancias notables entre la estadística correspondiente al procesado KCICLO y la estadística sobre el procesado de AERONET. En primer lugar el método KCICLO nos ha permitido corregir los graves problemas de calibración de canales concretos de instrumentos concretos: todos los filtros del Cimel \#114; los filtros de 670nm del Cimel \#48, del \#45 y el \#50; y el filtro de 440nm del Cimel \#50. El Cimel \#45, actualmente operativo, también necesitará una corrección severa en algunos de sus canales.

\subsubsection{Continuidad de las series temporales de AOD y alfa}

En primer lugar vamos a analizar en su conjunto los datos de AOD para los procesados de AERONET y KCICLO. A la hora de analizar la continuidad de la serie de datos hay que discriminar las diferencias reales en los aerosoles de las diferencias debidas a problemas de calibración. El criterio fundamental en nuestro análisis será lo analizado en la sección 
anterior, es decir, los errores de calibración que se traducen en falsos ciclos diurnos y que alteran significativamente los promedios de cada fotómetro.

$\mathrm{Al}$ analizar las estadísticas de la serie completa de datos de AOD (Tabla V-X) observamos que las diferencias absolutas entre las dos series de datos son pequeñas $y$, en todo caso, quedan dentro del error absoluto que AERONET especifica para el AOD de los instrumentos de campo (0.01-0.02). Vemos que el error disminuye para los filtros de longitud de onda más larga. Esto nos podría hacer pensar que no merece la pena realizar una corrección como la del KCICLO. Sin embargo hay dos argumentos decisivos a favor de la necesidad de verificar la calidad de la serie: en primer lugar el análisis por aparatos revela diferencias mucho mayores en ciertos canales para el AOD y el alfa, por encima de las especificaciones de AERONET; y en segundo lugar porque la serie de AERONET presenta saltos no justificables entre unos aparatos y otros.

\begin{tabular}{lcccc}
\hline \hline & AOD_1020 & AOD_870 & AOD_670 & AOD_440 \\
\hline KCICLO & $0.086 \pm 0.082$ & $0.090 \pm 0.085$ & $0.107 \pm 0.095$ & $0.171 \pm 0.124$ \\
AERONET & $0.088 \pm 0.082$ & $0.096 \pm 0.086$ & $0.117 \pm 0.096$ & $0.183 \pm 0.125$ \\
Diferencia absoluta & 0.002 & 0.007 & 0.010 & 0.012 \\
Diferencia relativa(\%) & 2.3 & 7.3 & 8.5 & 6.5 \\
\hline \hline
\end{tabular}

Tabla V-X. Promedios generales ( \pm STD) de la serie completa de AOD en El Arenosillo y diferencia entre los procesados de AERONET y KCICLO.

En las diferencias sobre los promedios generales de AOD los datos de AERONET quedan siempre por encima. Sin embargo al comparar los promedios de los diferentes parámetros alfa, Tabla V-XI, en dos de ellos quedan por encima los alfas de AERONET -alfa(440-870) y alfa(670/870) - mientras que en los otros dos queda por debajo. La discrepancia más notable entre los dos procesados se encuentra precisamente en los promedios de alfa(670/870), que difieren en 0.094 (el 13\% aproximadamente), y en las desviaciones estándar, que son mucho mayores para los datos de AERONET (0.766 frente a 0.527, más del $30 \%$ de diferencia). Un buen indicio sobre los problemas que hay detrás de estas diferencias nos lo da el hecho de que casi en la misma medida que este alfa(670/870) es superior en el procesado de AERONET, el promedio de alfa(440/670) queda por debajo. Como hemos visto, estos alfas de los pares muestran anticorrelación si hay un problema de calibración en el filtro que tienen en común, es decir, el de 670nm.

\begin{tabular}{lcccc}
\hline \hline & alfa(440-870) & alfa(440/670) & alfa(670/870) & alfa(440/870) \\
\hline KCICLO & $1.047 \pm 0.426$ & $1.277 \pm 0.481$ & $0.724 \pm 0.527$ & $1.065 \pm 0.428$ \\
AERONET & $1.072 \pm 0.440$ & $1.214 \pm 0.516$ & $0.817 \pm 0.766$ & $1.062 \pm 0.448$ \\
Diferencia absoluta & 0.025 & -0.063 & 0.094 & -0.003 \\
Diferencia relativa(\%) & 2.4 & -4.9 & 13.0 & -0.3 \\
\hline \hline
\end{tabular}

Tabla V-XI. Estadística de la serie completa de alfa en El Arenosillo y diferencia entre procesados

En la Figura V-8a y b mostramos los valores promedio de AOD para cada aparato en función de la longitud de onda, para dar una idea de la forma del espectro que obtenemos como resultado del procesado KCICLO (a) o de AERONET (b). En la Figura V-8b se aprecia el motivo de la anticorrelación entre el alfa(440/670) y alfa(670/870) para el Cimel \#48 en el procesado de AERONET: se combina un error por exceso en el AOD a 870nm con un error por defecto del AOD en $670 \mathrm{~nm}$. El resultado es un espectro muy poco realista. También se aprecia el valor excesivamente alto del Cimel \#114 en todos los filtros, con una forma del espectro poco realista por la excesiva pendiente en el infrarrojo. Por el contrario, en el procesado KCICLO (Figura V-8a) el Cimel \#114 presenta unos valores cercanos al promedio de toda la serie. El valor tan alto del Cimel \#48 para el KCICLO se justifica por el corto período de tiempo de operación y la presencia de la fuerte 
intrusión sahariana de marzo de 2000. En el Cimel \#50 los valores en 440 y 670nm del AOD quedan por encima del promedio, mientras que en 870 y $1020 \mathrm{~nm}$ quedan por debajo del promedio, dando lugar a un espectro con excesiva pendiente (ya hemos visto los valores de alfa anormalmente altos).
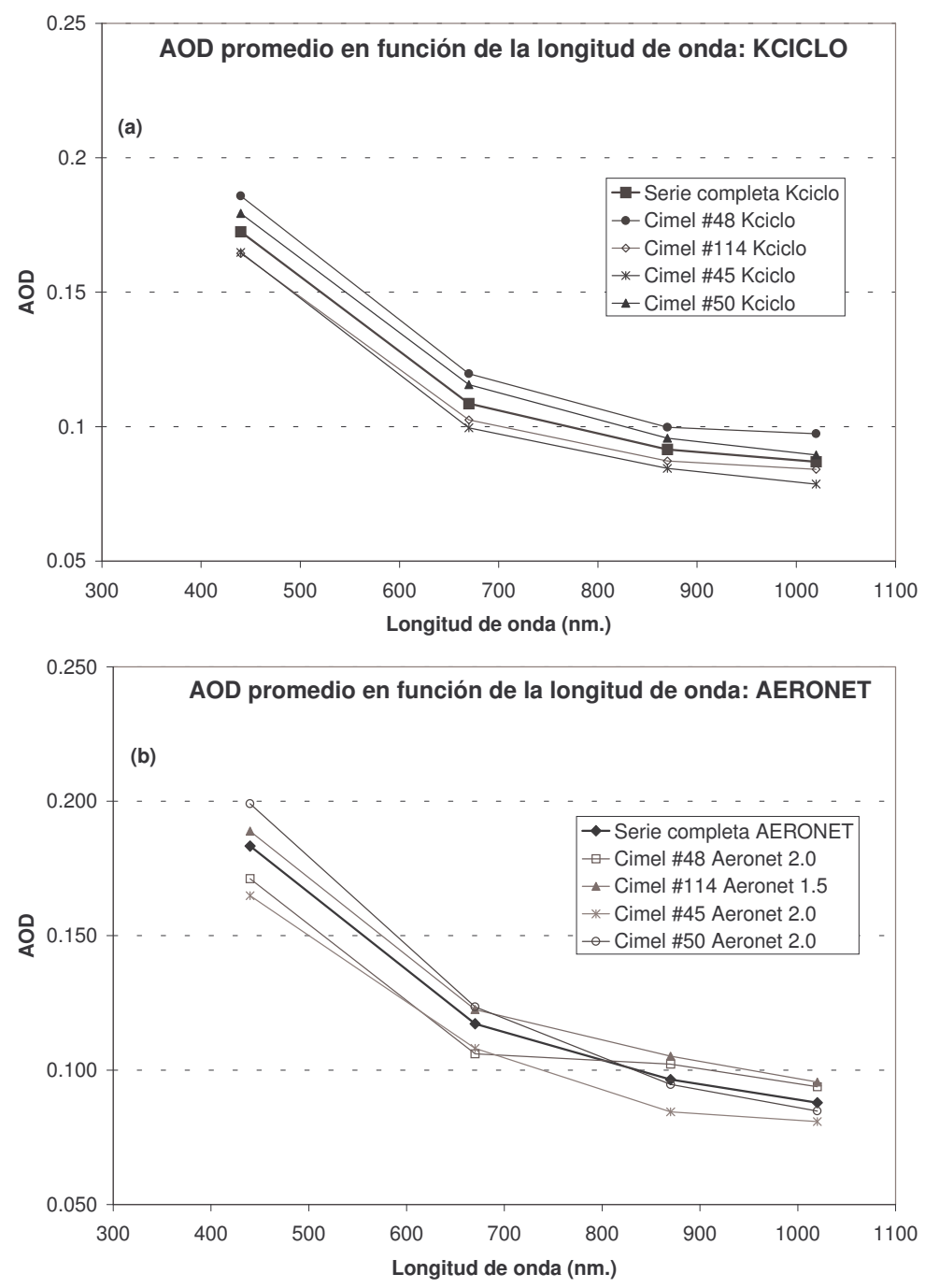

Figura V-8. Gráficas del espesor óptico de aerosoles promedio en función de la longitud de onda para (a) el procesado KCICLO y (b) procesado de AERONET.

Como resumen de las tablas de AOD para cada instrumento, mostramos en la Figura V-9 cada uno de los cuatro canales de AOD para todos los instrumentos, en concreto, la razón del promedio de cada filtro respecto del promedio general de la serie, siempre para el procesado AERONET y KCICLO por separado. Esta gráfica nos sirve para mostrar que en los datos de AERONET de los Cimel \#48 y \#50 unos canales de AOD quedan por encima del promedio general de la serie y otros por debajo, lo cual habla de lo inconsistente y erróneo de esos datos: la atmósfera no se va a comportar de manera opuesta para distintas longitudes de onda, o dicho de otra manera, si había más o menos turbiedad durante un cierto período respecto a la serie completa, será así en todos los canales y no sólo en algunos. Para el procesado KCICLO se observa que cada instrumento corresponde a un período con valores por encima o por debajo del promedio general, pero siempre igual para todos los filtros. 

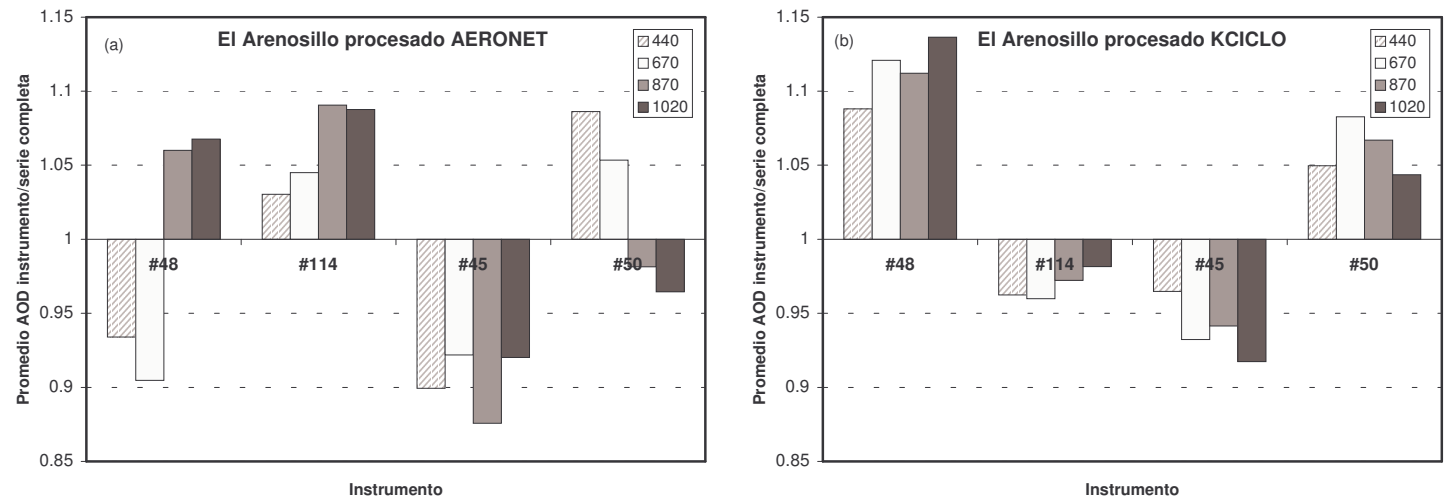

Figura V-9. Razón entre el promedio de cada filtro de cada fotómetro y el de la serie completa para el procesado de AERONET (a) y del KCICLO (b).

El parámetro alfa, derivado del espesor óptico de aerosoles, es muy sensible a variaciones de éste, y sirve como un excelente indicador de la calidad del procesado. Diferencias entre los valores medios del AOD entre uno y otro procesado, que en principio parecen pequeñas, resultan luego ser fundamentales para la consistencia de la serie de datos, puesto que la continuidad en el parámetro alfa de Ångström es necesaria para poder realizar una interpretación correcta de las medidas.

Las discrepancias más fuertes entre los dos procesados se encuentran en los valores del parámetro alfa de Ångström, tanto para el par (440/670nm) como para el par (670/870nm). En nuestra serie reprocesada los valores de alfa son similares entre los tres instrumentos, tanto en el valor promedio como en su desviación estándar, para ambos pares de longitudes de onda. Sin embargo para el procesado de AERONET los valores de alfa cambian radicalmente con el cambio de instrumento, tanto en los promedios como en las desviaciones estándar. Esto se puede comprobar de manera global en la Tabla V-XII. El parámetro alfa(440-870) es el que menos discrepancias muestra a lo largo de la serie dentro de cada procesado. En el procesado de AERONET el Cimel\#50 tiene un alfa(440870 ) de 1.28 que se aleja más del $20 \%$ del promedio de la serie, pero el resto de aparatos sí parecen mantener la continuidad. Esto también se verifica en el procesado KCICLO, aunque el promedio del Cimel \#48 es algo elevado.

\begin{tabular}{l|cc|cc|cc}
\hline \hline & \multicolumn{2}{|c|}{ alfa(440-870) } & \multicolumn{2}{c|}{ alfa(440/670) } & \multicolumn{2}{c}{ alfa(670/870) } \\
\hline \multirow{3}{*}{ Serie completa } & AERONET & KCICLO & AERONET & KCICLO & AERONET & KCICLO \\
$\# 48$ & $1.07 \pm 0.44$ & $1.05 \pm 0.43$ & $1.21 \pm 0.52$ & $1.28 \pm 0.48$ & $0.82 \pm 0.77$ & $0.72 \pm 0.53$ \\
$\# 114$ & $1.00 \pm 0.40$ & $1.19 \pm 0.43$ & $1.61 \pm 0.77$ & $1.34 \pm 0.48$ & $-0.16 \pm 1.00$ & $0.91 \pm 0.41$ \\
$\# 45$ & $0.93 \pm 0.37$ & $1.06 \pm 0.43$ & $1.11 \pm 0.39$ & $1.27 \pm 0.47$ & $0.58 \pm 0.40$ & $0.62 \pm 0.54$ \\
$\# 50$ & $1.06 \pm 0.40$ & $0.97 \pm 0.41$ & $1.08 \pm 0.43$ & $1.31 \pm 0.49$ & $1.06 \pm 0.48$ & $0.72 \pm 0.49$ \\
\hline \hline
\end{tabular}

Tabla V-XII. Comparación entre AERONET y KCICLO de los promedios y STD de los diferentes parámetros alfa.

El parámetro alfa(440/670) tiene un promedio de AERONET de 1.21, pero ha oscilado entre 1.08 y 1.61 entre los diferentes fotómetros. Frente a esto, el procesado KCICLO tiene un promedio de 1.28, y oscila entre 1.20 y 1.34 entre los diferentes instrumentos. Además la STD es muy similar en todos los fotómetros para el procesado KCICLO, en torno a 0.48 , mientras que en el procesado de AERONET la STD para la serie completa es 0.52 pero oscila entre 0.39 en el Cimel \#114 y 0.77 en el Cimel \#48. Los problemas de continuidad de la serie de datos de AERONET son todavía más claros en alfa(670/870). El promedio de este parámetro para toda la serie de AERONET es 0.82 , pero no ha habido dos instrumentos con promedios similares, oscilando entre -0.16 en el Cimel \#48 hasta 1.26 
en el Cimel\#50. Esto también tiene su reflejo en la alta STD de la serie completa, con 0.77. En la serie KCICLO este parámetro presenta también ciertas variaciones, entre 0.62 y 0.91, pero éstas no son tan agudas como en el procesado de AERONET.

La corrección del método KCICLO consigue mejorar sustancialmente los problemas debidos a la calibración, pero no llega obviamente a la perfección. No se producen saltos bruscos con el cambio de aparato, aunque se mantienen algunos problemas, como valores negativos de alfa en casos de baja turbiedad. Creemos que en este contexto es importante tener acotado el error y ser prudentes con las interpretaciones derivadas del parámetro alfa, en particular de los alfas calculados a partir de pares de AOD.

En la Figura V-10, Figura V-11 y Figura V-12 encontramos la serie completa de los tres parámetros alfa que estamos manejando, tanto para el procesado de AERONET como para el KCICLO. Los problemas más graves de continuidad en la serie se detectan a simple vista en el alfa obtenido a partir de los pares de longitudes de onda. En la Figura V-11b y Figura V-12b vemos la anticorrelación y los valores afectados de un gran error en el Cimel \#48, el primero instalado, y también cómo esto se interrumpe al cambiar al instrumento \#114. Este salto no aparece en la serie de datos KCICLO (Figura V-11a y Figura V-12a), en las que por otra parte tenemos un cierto número de medidas, correspondientes con los valores de AOD más bajos, para los cuales los alfas presentan valores negativos.

Por otra parte el parámetro alfa de Ångström calculado con las tres longitudes de onda (440, 670 y 870), esto es, en el rango (440-870), da como resultado unas diferencias mucho menores entre ambos procesados y la desaparición de los saltos entre instrumentos del procesado de AERONET (Figura V-10). Este parece ser el parámetro alfa más fiable aunque de nuevo urge la realización de un análisis de errores, incluyendo la correlación del ajuste realizado, para evaluar la bondad de este parámetro. Estas gráficas generales de varios años de datos no nos permiten apreciar los problemas de ciclo diurno en el alfa, por lo que nos remitimos a las gráficas de ejemplo que hemos ido mostrando de cada fotómetro (Figura V-2 a Figura V-7) y las tablas de valores promedio y desviaciones estándar para justificar que la serie de datos KCICLO tiene una mejor calidad y continuidad entre los datos tomados con los diferentes instrumentos Cimel. 

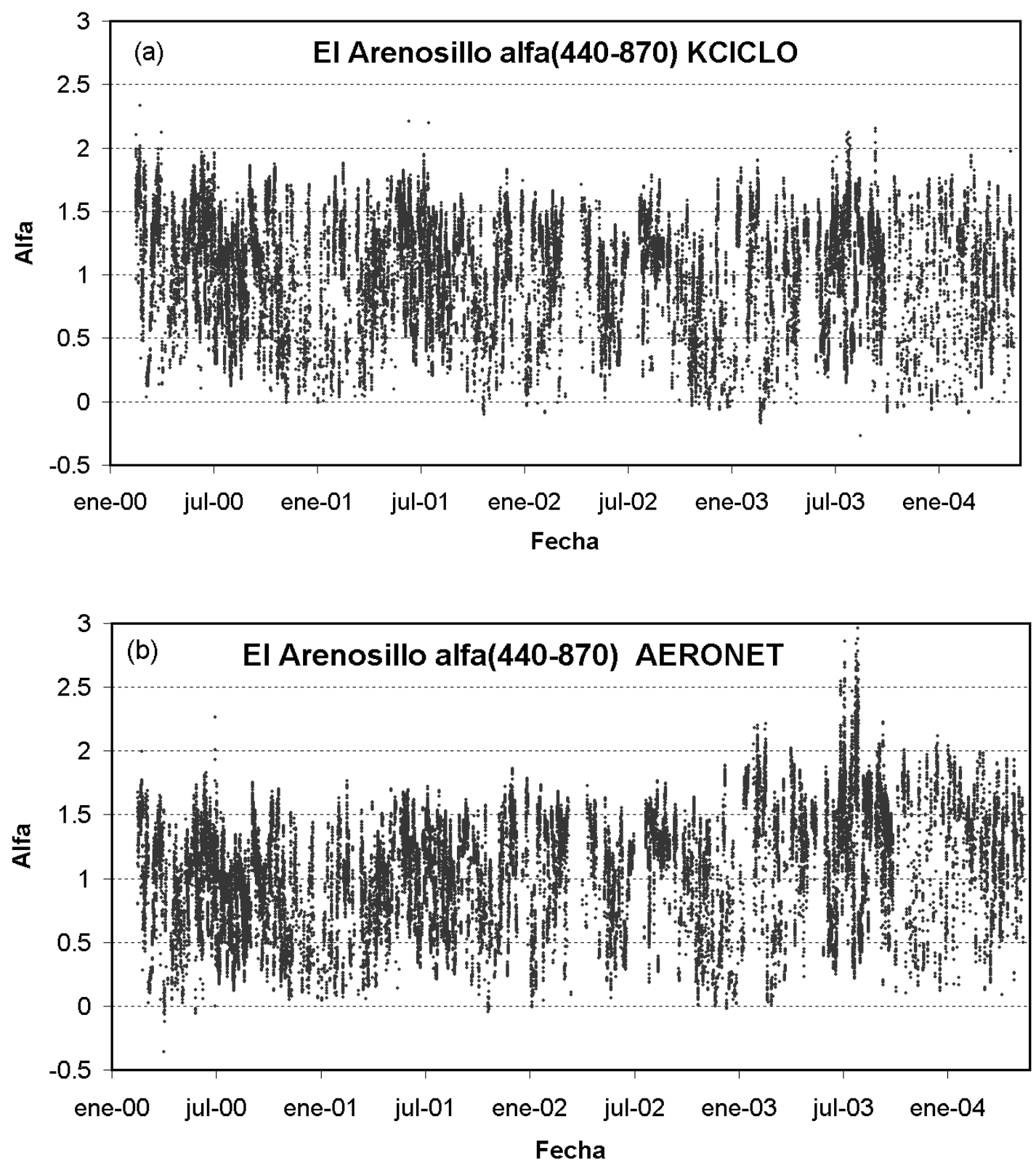

Figura V-10. Serie completa de alfa(440-870). en El Arenosillo para (a) el procesado KCICLO y (b) de AERONET. 

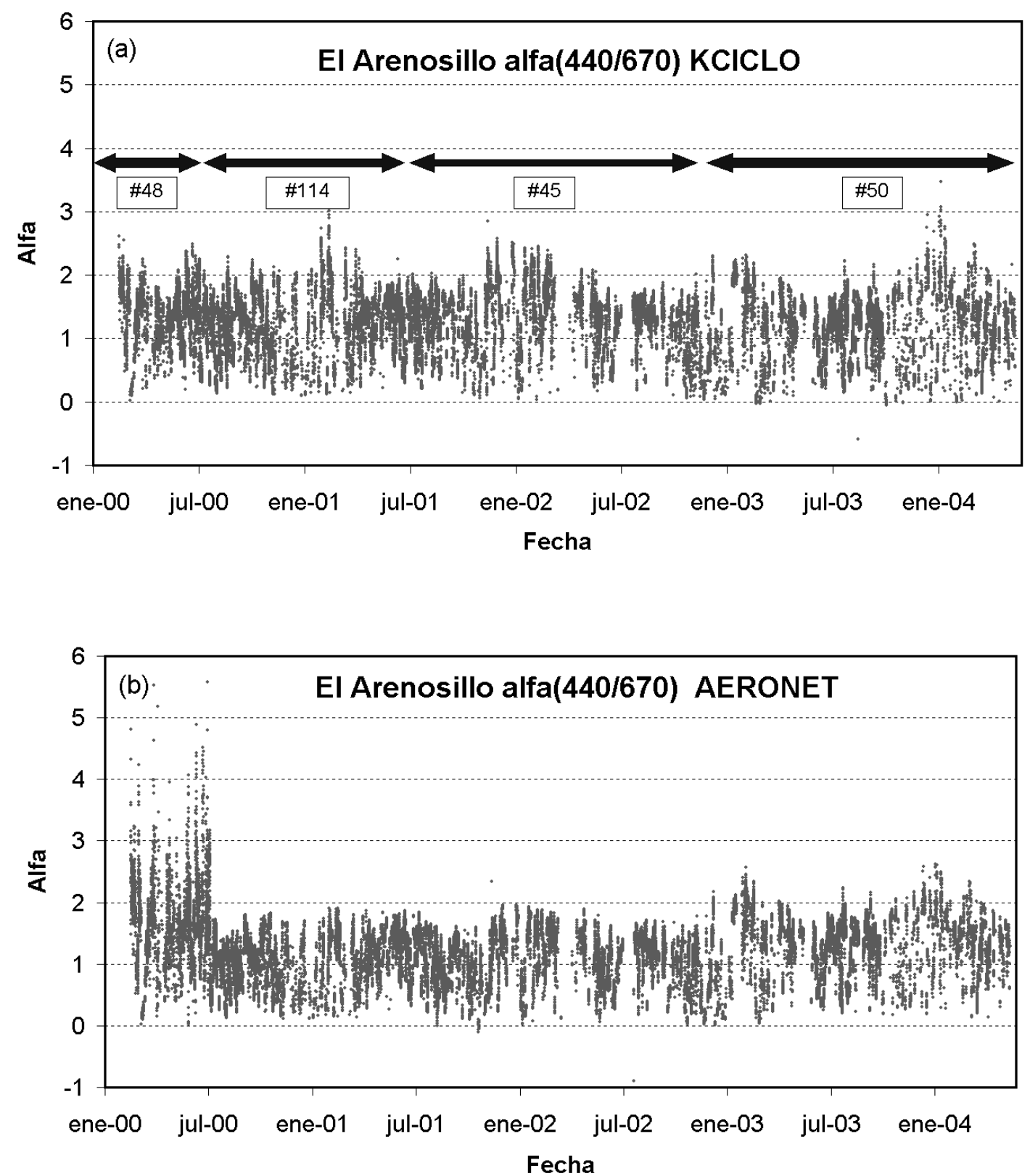

Figura V-11. Serie completa de alfa(440/670). en El Arenosillo para (a) el procesado KCICLO y (b) de AERONET. 

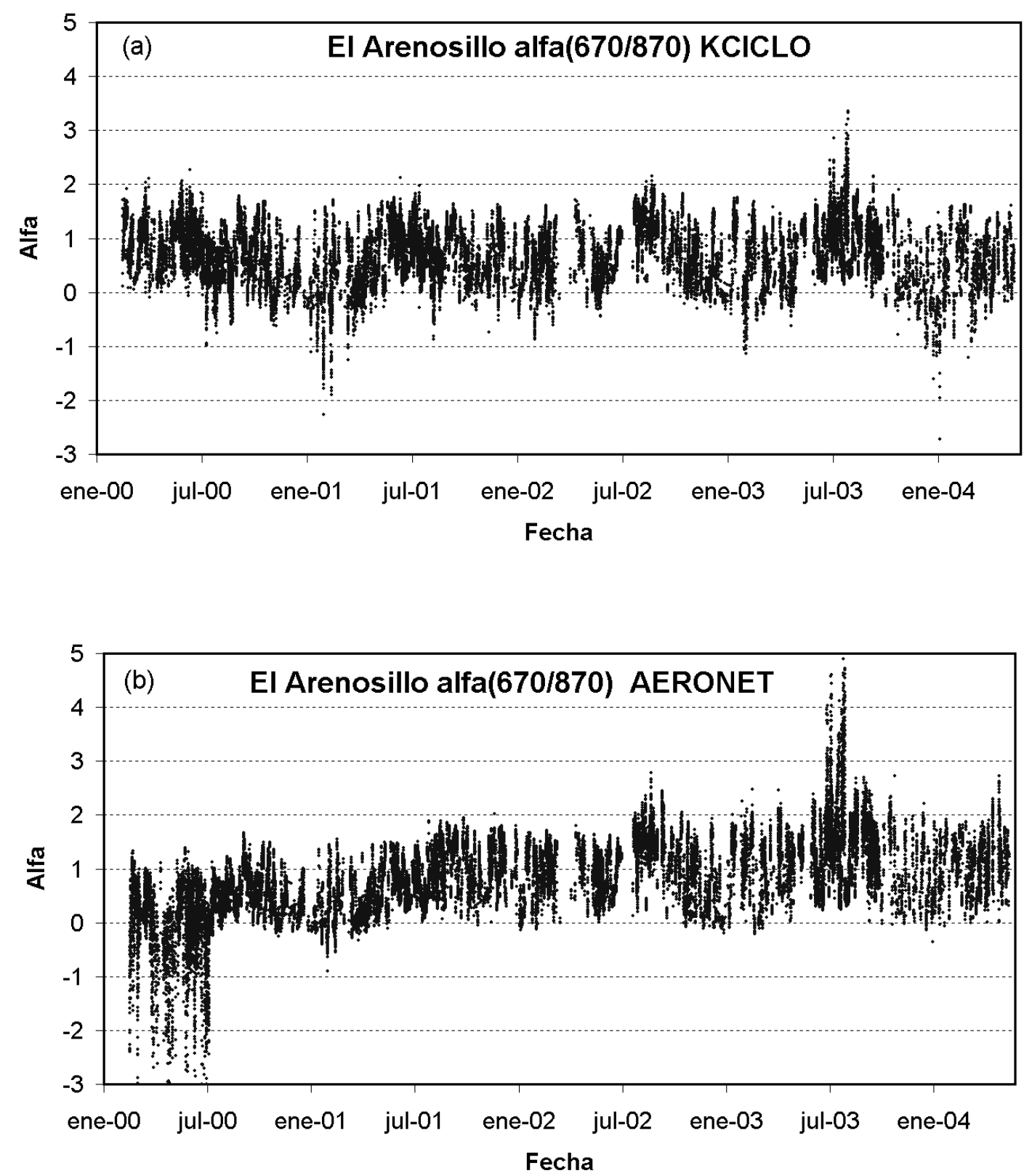

Figura V-12. Serie completa de alfa(670/870). en El Arenosillo para (a) el procesado KCICLO y (b) de AERONET. 


\subsection{Comparación de promedios diarios y mensuales en los procesados de AERONET y KCICLO}

\subsubsection{Análisis de las diferencias en los promedios diarios y mensuales para cada fotómetro}

A la hora de abordar la caracterización del aerosol en una zona concreta, en este caso en la zona del golfo de Cádiz, recurrimos a los promedios diarios, mensuales, estacionales, etc. Los problemas de calibración afectan muy claramente a las medidas individuales y a la evolución durante el día, como ya se ha mostrado. La aparición de un ciclo diurno más o menos acusado falsea el comportamiento de la atmósfera. Sin embargo, al abordar un estudio mediante promedios las diferencias pueden ser más o menos significativas, $e$ incluso estar dentro de la tolerancia requerida por la red, y sin embargo están poniendo de manifiesto una posible falta de calidad en los datos.

Los errores debidos a problemas con la calibración tienen la dependencia mostrada con la masa óptica, siendo máximos al mediodía. Cuando realizamos los promedios diarios el error es también un promedio del error variable durante el día. Por esto las diferencias entre los promedios diarios de AERONET y KCICLO no son tan grandes como la diferencia al mediodía, ni tan pequeñas como al principio o al final del día. Lo más importante a tener en cuenta en este caso es que el error debido a la calibración es un error de tipo sistemático. Está en todas las medidas, y se traslada luego a los promedios diarios, mensuales, etc. siempre en la misma proporción. Como vamos a ver a continuación, la diferencia absoluta entre los procesados de AERONET y KCICLO es la misma tanto para los promedios diarios como los mensuales, y sólo se ve modulada a lo largo del año por el rango de la masa óptica, que es distinto en verano que en invierno, y por la posible degradación de la calibración. En este segundo caso observamos una deriva de tipo lineal a lo largo del tiempo. La cantidad ' $(1 / \mathrm{m}) \cdot \operatorname{lnK}$ ' nos da el error de una observación individual, del promedio diario y finalmente del promedio mensual. No es un error de tipo aleatorio que se pueda solventar por la realización de un alto número de medidas, ni que se atenúe por la realización de suavizados o promedios. Se trata de un error sistemático, intrínsecamente unido al resultado de la medida, y que es necesario eliminar para conseguir una serie de datos consistente. Es fundamental evaluar cada instrumento por separado, pues la corrección que da el método KCICLO es diferente para cada canal de cada fotómetro que ha operado en El Arenosillo.

Las diferencias absolutas del promedio diario de AOD en el filtro de 440nm entre el procesado KCICLO y el de AERONET se muestra en la 0. En ella podemos distinguir fácilmente los cuatro aparatos a partir de las diferencias absolutas entre el procesado KCICLO y el de AERONET. La mayor discrepancia se da con los datos del \#114 (en torno al $15 \%$ y superando el umbral de 0.02 ), aunque también el \#48 presenta una discrepancia al final en torno al 0.02 pero de signo contrario (por defecto), lo cual acentúa más el salto entre aparatos. El Cimel\#45 es el que muestra mayor acuerdo entre los dos procesados (por debajo de 0.005 casi todo el tiempo). Las diferencias con el Cimel \#50 son de nuevo grandes y superiores a la estimación del error de AERONET. Se puede observar que las diferencias absolutas son mayores en verano que en invierno, por la modulación que introduce la masa óptica. En el Cimel \#48 y en el \#50 el efecto de la degradación del filtro (procesado con interpolación) hace que las diferencias vayan cambiando de forma lineal con el tiempo. 
Al respecto del parámetro alfa, las diferencias entre los dos procesados son grandes, en torno a -0.2 para el \#48, en torno a -0.1 para el \#114, menor de 0.1 en el \#45 y superior a 0.4 en el \#50 (Figura V-14b). Con un valor promedio muy cercano a 1 en toda la serie, estas diferencias (o errores) oscilan entre $-20 \%$ y $40 \%$. También destacamos en este caso el hecho de que el Cimel \#45, con un muy buen acuerdo entre los dos procesados para el AOD, mantenga sin embargo en el alfa discrepancias superiores en muchos días al $10 \%$, aunque los días más discrepantes se dan a partir de septiembre de 2002. Anteriormente el comportamiento de ambos procesados es más cercano y las diferencias menores.

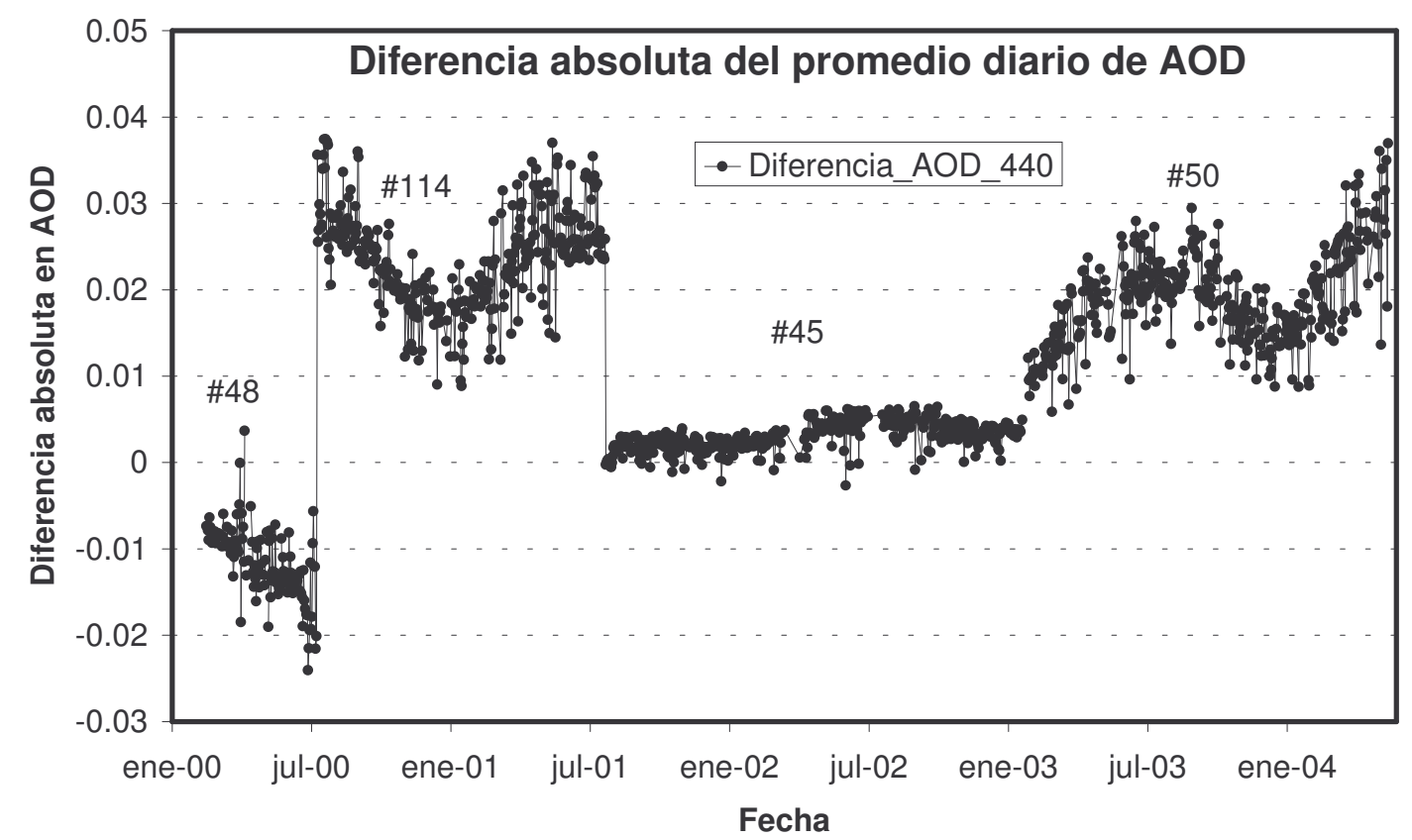

Figura V-13. Diferencia absoluta del promedio diario de AOD en 440nm entre el KCICLO y AERONET, calculado como el promedio diario de AERONET menos el promedio diario de la serie $\mathrm{KCICLO}$.

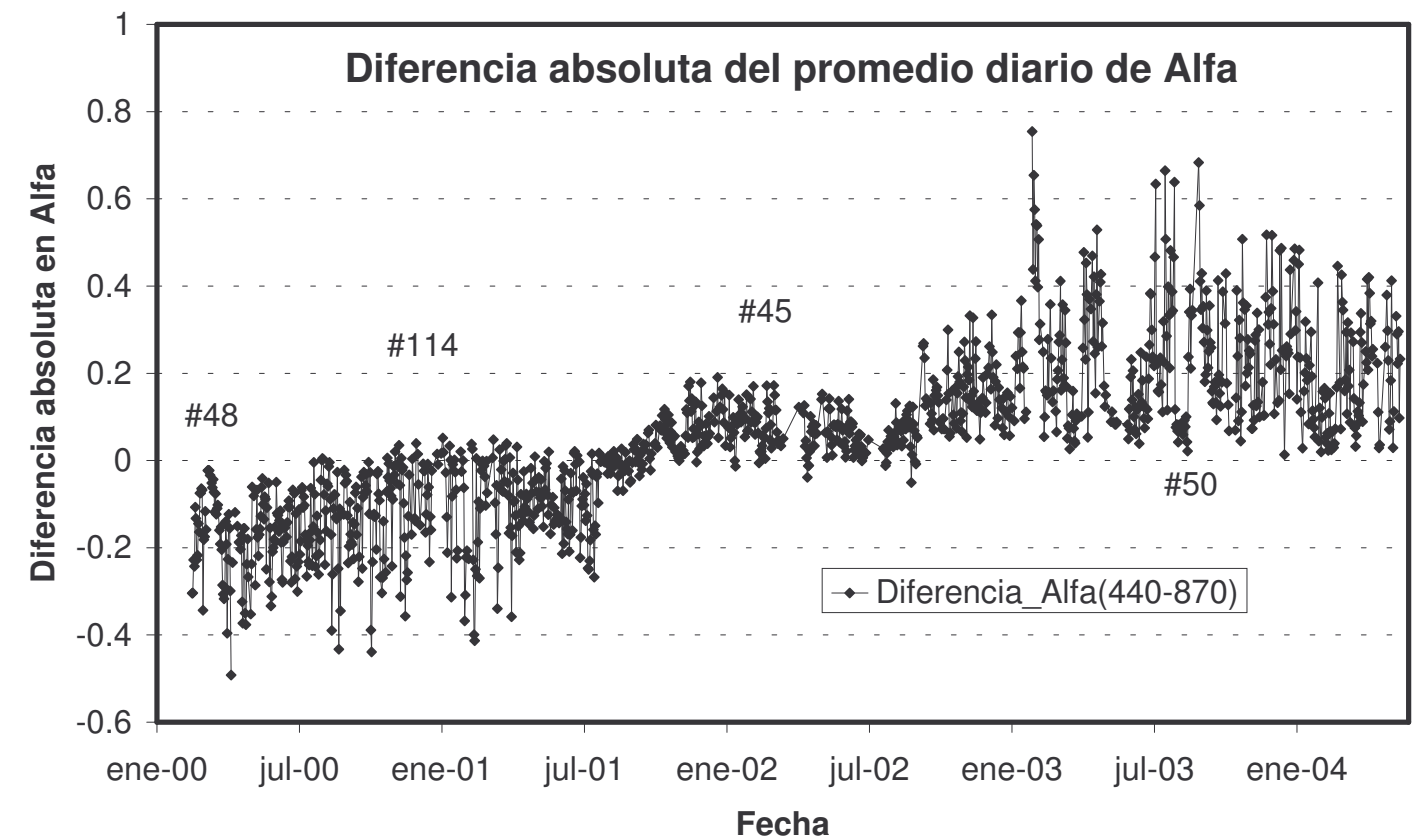

Figura V-14. Diferencia absoluta del promedio de alfa(440-870) entre los procesados KCICLO y AERONET, calculado como el promedio diario de AERONET menos el promedio diario de la serie KCICLO. 

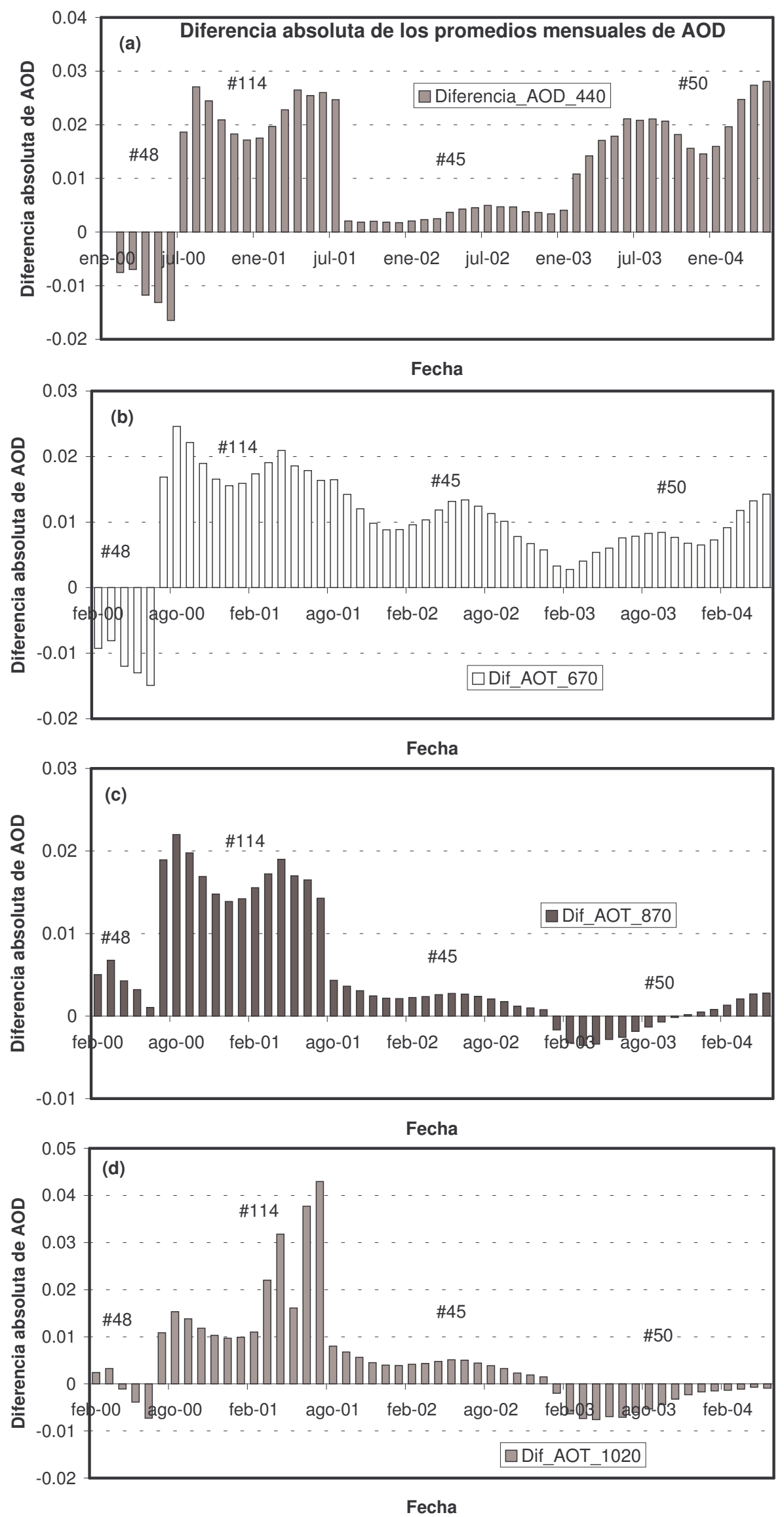

Figura V-15. Diferencia absoluta en los cuatro filtros del Cimel entre el promedio mensual de AERONET y del KCICLO, calculado como el promedio mensual de AERONET menos el promedio mensual de la serie KCICLO. 
Las diferencias absolutas en los promedios mensuales del AOD para cada uno de los filtros se presentan en la Figura V-15. Lo más importante a destacar es que los errores son los mismos que en los promedios diarios, puesto que el error es de naturaleza sistemática. El error absoluto tolerado para los instrumentos de campo $(0.01$ en 670,870 y $1020 \mathrm{~nm}$ y 0.02 en $440 \mathrm{~nm}$ ) se ve superado debido a los problemas de calibración del \#48, del \#114 y del \#50. Las diferencias entre marzo y julio de 2000 en el filtro de $1020 \mathrm{~nm}$ se deben a una avería del sensor de temperatura en el Cimel\#114, y se trata por tanto de una cuestión puntual no relacionada con la calibración.

Por último mostramos en la Figura V-16 la diferencia relativa entre los promedios mensuales del parámetro alfa (440-870) para ambos procesados. Las diferencias relativas son negativas en el cimel\#48 y \#114, al igual que se vio para los promedios diarios. Para el cimel\#45 las diferencias son positivas, acentuándose en su tramo final, y son todavía mayores en el caso del Cimel\#50, de forma paralela al resultado de los promedios diarios.

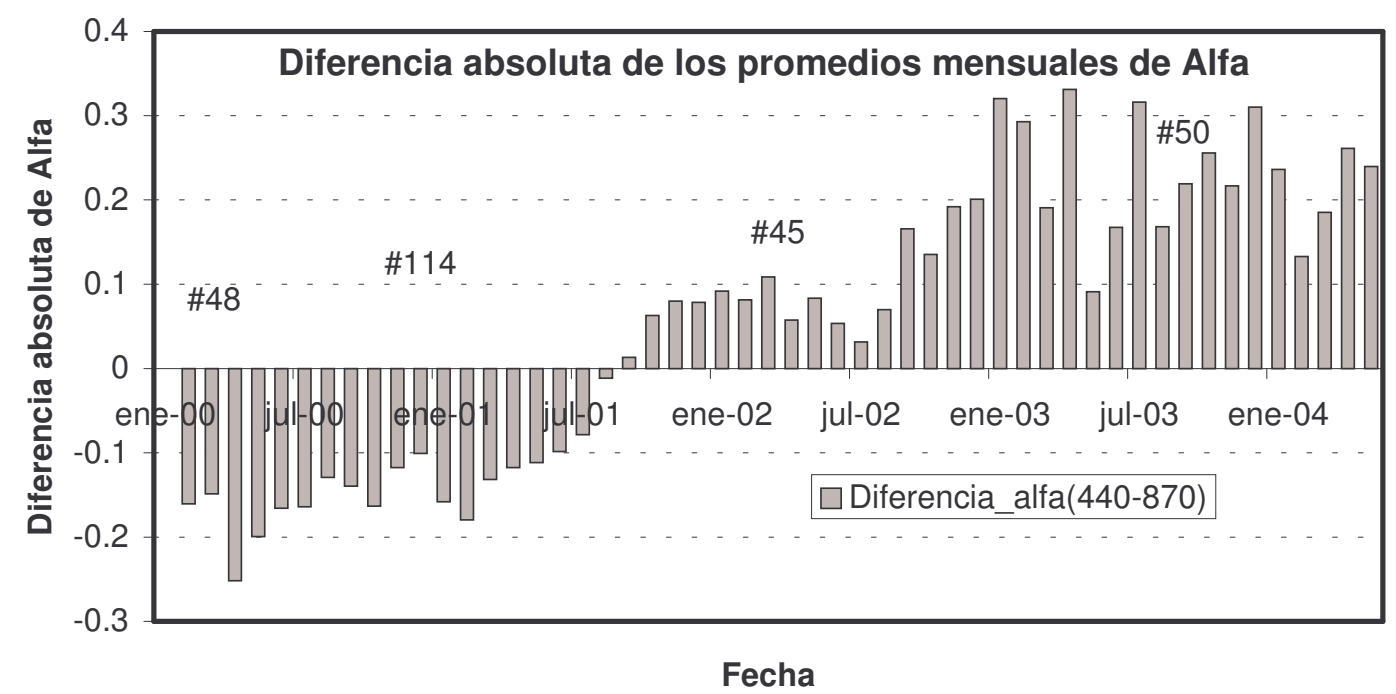

Figura V-16. Diferencia absoluta del promedio mensual de alfa(440-870) entre los procesados de AERONET y KCICLO, calculado como el promedio mensual de AERONET menos el promedio mensual de la serie KCICLO.

\subsubsection{Análisis de diferencias en la climatología derivada de la serie completa}

$\mathrm{Al}$ analizar los promedios mensuales interanuales para todo el período de medidas se puede ver cómo estos errores repercuten en la climatología de la estación. En realidad este promedio interanual está basado en los promedios mensuales (ver Figura V-15 y Figura V16), con 4 ó 5 datos por cada mes a lo largo de la serie, que al estar tomados con instrumentos diferentes, dan como resultado unas diferencias absolutas que no se corresponden con las de ningún momento concreto. Se mezcla un período en el que hubo un error por exceso con otro en que hubo un error por defecto o con otro en el que no hubo apenas error. El resultado (Figura V-17, Figura V-18) y Tabla V-XIII) es un compromiso entre los errores que han ido presentando los instrumentos sucesivos.

Como vemos en la Figura V-17, las diferencias entre AERONET y KCICLO son mayores durante el verano, cuando la masa óptica alcanza valores menores a mediodía. Las compensaciones entre unos aparatos y otros hacen que en ningún mes la diferencia supere el 0.02 en valor absoluto de AOD, aunque se acerca a este valor en verano en los filtros de 
440 y $670 \mathrm{~nm}$. Durante algunos meses las diferencias en $670 \mathrm{~nm}$ son incluso mayores que en $440 \mathrm{~nm}$. Las diferencias relativas oscilan entre el $2 \%$ en lo mejores casos y el $12 \%$ en los peores, en concreto en el canal de $670 \mathrm{~nm}$, que parece ser el más problemático (Tabla VXIII).

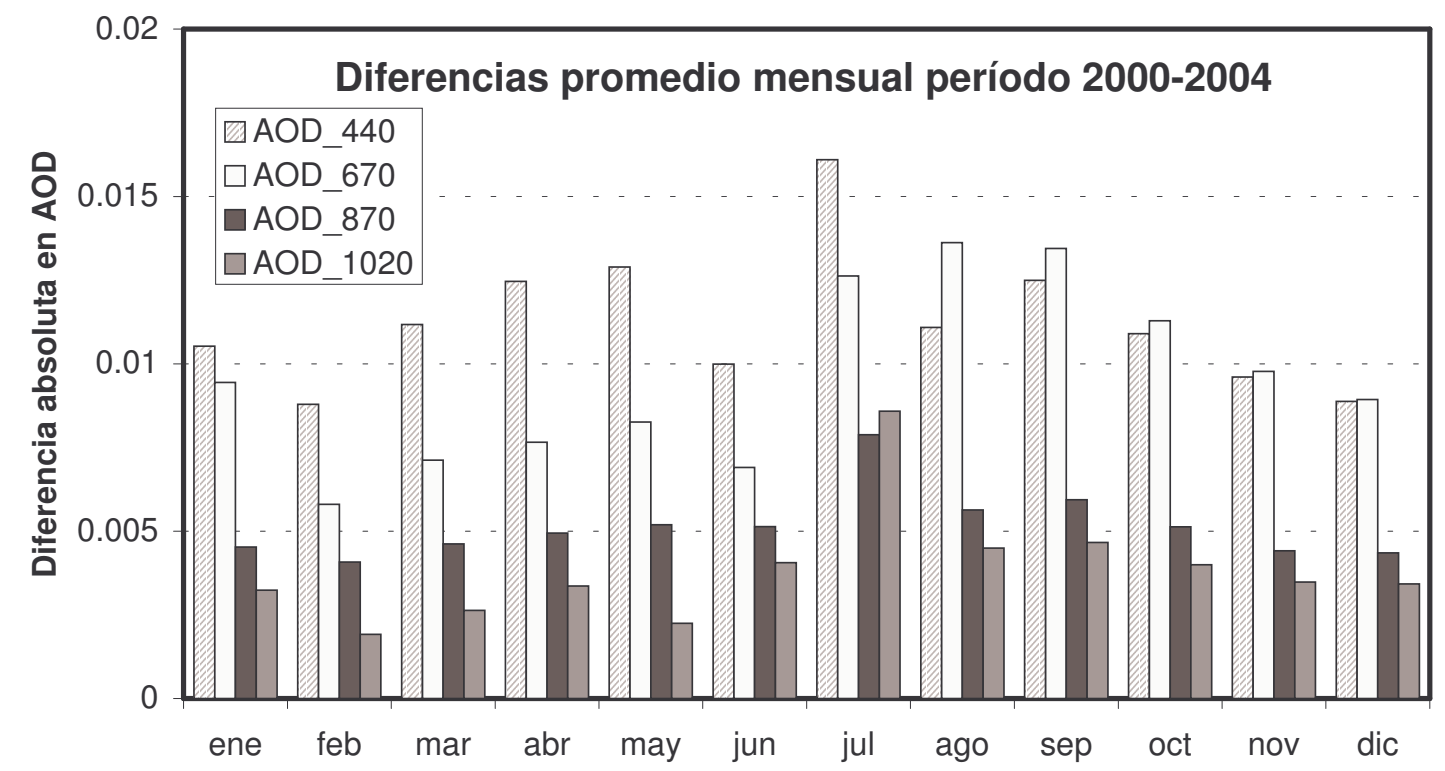

Figura V-17. Diferencias absolutas (AERONET-KCICLO) en los AOD para los promedios mensuales interanuales.

\begin{tabular}{cccccc}
\hline \hline & AOT_1020 & AOT_870 & AOT_670 & AOT_440 & alfa(440-870) \\
\hline ene & $0.003(4.3 \%)$ & $0.005(5.9 \%)$ & $0.009(11.3 \%)$ & $0.011(8.3 \%)$ & $0.135(14.6 \%)$ \\
feb & $0.002(2.2 \%)$ & $0.004(4.3 \%)$ & $0.006(5.1 \%)$ & $0.009(4.7 \%)$ & $0.033(3.0 \%)$ \\
mar & $0.003(2.2 \%)$ & $0.005(3.6 \%)$ & $0.007(5.0 \%)$ & $0.011(5.5 \%)$ & $0.045(5.1 \%)$ \\
abr & $0.003(5.0 \%)$ & $0.005(6.7 \%)$ & $0.008(8.7 \%)$ & $0.012(8.3 \%)$ & $0.055(5.6 \%)$ \\
may & $0.002(2.3 \%)$ & $0.005(5.1 \%)$ & $0.008(6.7 \%)$ & $0.013(6.4 \%)$ & $0.019(1.8 \%)$ \\
jun & $0.004(4.3 \%)$ & $0.005(4.7 \%)$ & $0.007(5.3 \%)$ & $0.010(5.0 \%)$ & $-0.013(-1.2 \%)$ \\
jul & $0.009(9.3 \%)$ & $0.008(7.4 \%)$ & $0.013(9.9 \%)$ & $0.016(8.2 \%)$ & $0.020(1.8 \%)$ \\
ago & $0.004(4.5 \%)$ & $0.006(5.1 \%)$ & $0.014(10.0 \%)$ & $0.011(5.1 \%)$ & $0.033(3.2 \%)$ \\
sep & $0.005(5.3 \%)$ & $0.006(6.3 \%)$ & $0.013(11.5 \%)$ & $0.012(6.4 \%)$ & $0.066(6.3 \%)$ \\
oct & $0.004(5.3 \%)$ & $0.005(6.6 \%)$ & $0.011(12.5 \%)$ & $0.011(7.7 \%)$ & $0.076(9.1 \%)$ \\
nov & $0.003(4.4 \%)$ & $0.004(5.6 \%)$ & $0.010(11.7 \%)$ & $0.010(8.1 \%)$ & $0.098(13.4 \%)$ \\
dic & $0.003(4.9 \%)$ & $0.004(6.3 \%)$ & $0.009(12.0 \%)$ & $0.009(8.0 \%)$ & $0.121(14.9 \%)$ \\
\hline \hline
\end{tabular}

Tabla V-XIII. Diferencias absolutas (AERONET-KCICLO) en los AOD y alfa para los promedios interanuales

Al respecto de las diferencias en el parámetro alfa(440-870) que se recogen en la tabla anterior y en la Figura V-18, destaca el hecho de que las diferencias son mucho menores en verano, es decir, en los meses que hemos visto que presentan valores más altos del $\mathrm{AOD}$, tanto para las diferencias absolutas como relativas, que llegan a alcanzar el $15 \%$ en los meses de invierno. Esto es consistente con el análisis de errores realizado para el parámetro alfa, que nos dice que alfa es mucho menos sensible a los errores cuando el $\mathrm{AOD}$ es alto. De esta forma, durante el verano los alfas derivados de los AOD de AERONET o del KCICLO tienen un valor similar. En todo caso, el alfa es un parámetro derivado del AOD y que se puede ver alterado de distinta manera en función de los posibles errores en el $\mathrm{AOD}$, por lo que conviene sacar conclusiones fundamentalmente sobre las diferencias en los espesores ópticos. Como ilustración sirva de ejemplo el caso del Cimel \#114, en el que las diferencias en los AOD son superiores a 0.03 en 440nm entre AERONET y KCICLO, pero sin embargo los alfas no muestran grandes desacuerdos en 
promedio. Este buen acuerdo en los alfas, sin embargo, carece de sentido al ser tan grandes las diferencias entre los AOD de los que se derivan.

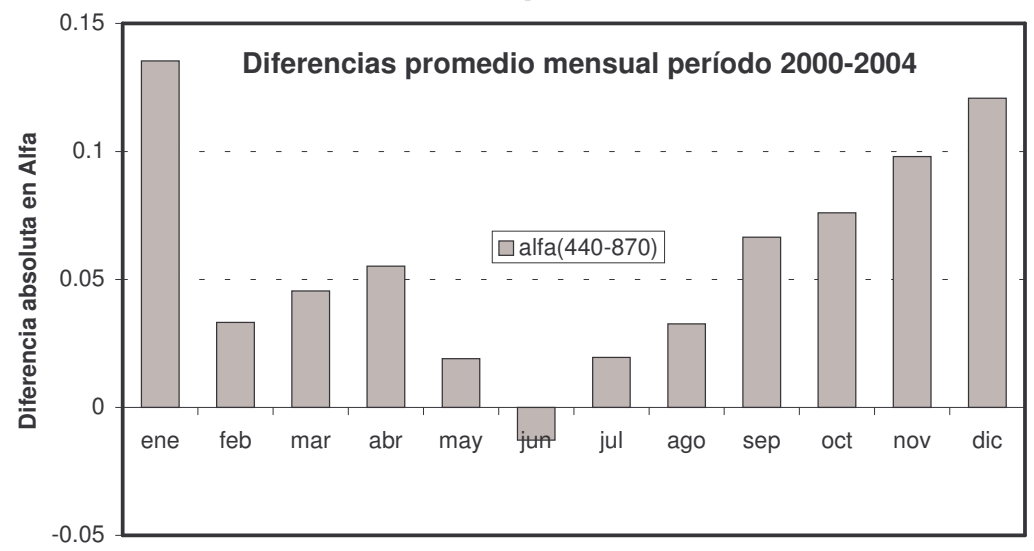

Figura V-18. Diferencias absolutas interanuales AERONET-KCICLO del parámetro alfa

En resumen, se ha delimitado el alcance y la importancia de la corrección llevada a cabo sobre la serie de datos de AERONET, tanto en el detalle del comportamiento diurno como de los valores promedio y su distribución estadística. Las conclusiones fundamentales son que el error de calibración detectado es de tipo sistemático y por tanto su eliminación es imprescindible; y en segundo lugar que la serie corregida es más consistente en cuanto a la continuidad entre los diferentes aparatos, cuestión que resulta problemática con el procesado de AERONET.

También se pone de manifiesto la dificultad de mantener una serie larga de datos de AOD que sea consistente y con errores bajos, por lo que métodos como el KCICLO, que permiten un seguimiento in situ de la calibración, pueden tener gran utilidad. 


\section{Capítulo 6. Análisis con masas de aire de las propiedades de los aerosoles}

\section{Introducción}

En este capítulo se va a realizar una clasificación y análisis de las masas de aire que llegan a la estación de El Arenosillo. El objetivo es estudiar el aerosol asociado a cada tipo de masa de aire, a través de las medidas del fotómetro Cimel. Para ello hemos realizado un estudio de clasificación de retrotrayectorias mediante dos metodologías diferentes, que nos van a aportar informaciones complementarias. Posteriormente se analizan estas clasificaciones en relación con las medidas espectrales del Cimel. Para todo ello utilizaremos como base la serie de datos de 5 años de espesor óptico medido con el Cimel en El Arenosillo, y las retrotrayectorias de masas de aire en ese mismo período calculadas mediante el modelo HYSPLIT. Se trata de realizar una aproximación global para la serie de 5 años, por lo que no nos hemos detenido a explicar excepciones o episodios en detalle, sino que tratamos de encontrar unos patrones generales de los aerosoles en relación con las masas de aire. 


\subsection{Descripción de tipos de masas de aire sobre la Península Ibérica}

El estudio de retrotrayectorias de masas de aire es un recurso muy utilizado en los últimos años y relativamente nuevo en la investigación de los aerosoles atmosféricos. La mejora en los modelos ha estado ligada a la mayor capacidad de cálculo, y en la actualidad están incluso disponibles a través de Internet. Se han publicado por ejemplo trabajos en los que se relaciona el espesor óptico de aerosoles con masas de aire (Vergaz, 2001; Vergaz et al., 2002; Vergaz et al., 2005). También se ha tratado de relacionar la concentración de aerosoles a nivel de suelo con masas de aire (Sánchez et al., 1990; Rodríguez et al., 2001), o la historia de la masa de aire con la concentración de elementos traza (Bahrmann et al., 1998; Cape et al., 2000; Methven et al., 2001). En general numerosos trabajos se apoyan en retrotrayectorias de masas de aire para el análisis de aerosoles u otros componentes atmosféricos (Smirnov et al., 1995; Bonasoni et al., 2001; Sancho and Romero, 2002; Heintzenberg, et al. 2003).

El axioma fundamental que se asume al analizar conjuntamente retrotrayectorias y aerosoles es que existe una relación entre el tipo de masa de aire y el aerosol asociado, como se comprueba por ejemplo con las entradas de aerosol desértico procedente del Sahara. Sin embargo no es sencillo clasificar las retrotrayectorias de forma objetiva ni tampoco establecer una relación cuantitativa entre una determinada trayectoria de la masa de aire y el contenido de aerosol. Existen varios modelos que ofrecen este tipo de productos. Estos modelos son muy complejos, pues han de tener en cuenta una serie de lugares y mecanismos fuente para cada tipo de aerosol, el tipo de suelo, la humedad del mismo, los vientos en superficie, la temperatura y la estabilidad atmosférica, y finalmente las condiciones sinópticas para el transporte. Estos modelos se utilizan con éxito para predecir la llegada de aerosol sahariano a la Europa Mediterránea, pues este es uno de los fenómenos relacionados con los aerosoles más relevantes en cuanto a los efectos climáticos, meteorológicos o sobre la salud. El modelo DREAM (Dust Regional Atmospheric Model, Nickovic et al., 2001) proporciona concentración a nivel de suelo, espesores ópticos y carga de aerosoles en la vertical. El modelo NAAPS (Navy Aerosol Analysis and Prediction System, http://www.nrlmry.navy.mil/aerosol) ofrece concentración a nivel de suelo y espesor óptico debido a polvo, sulfatos y humo.

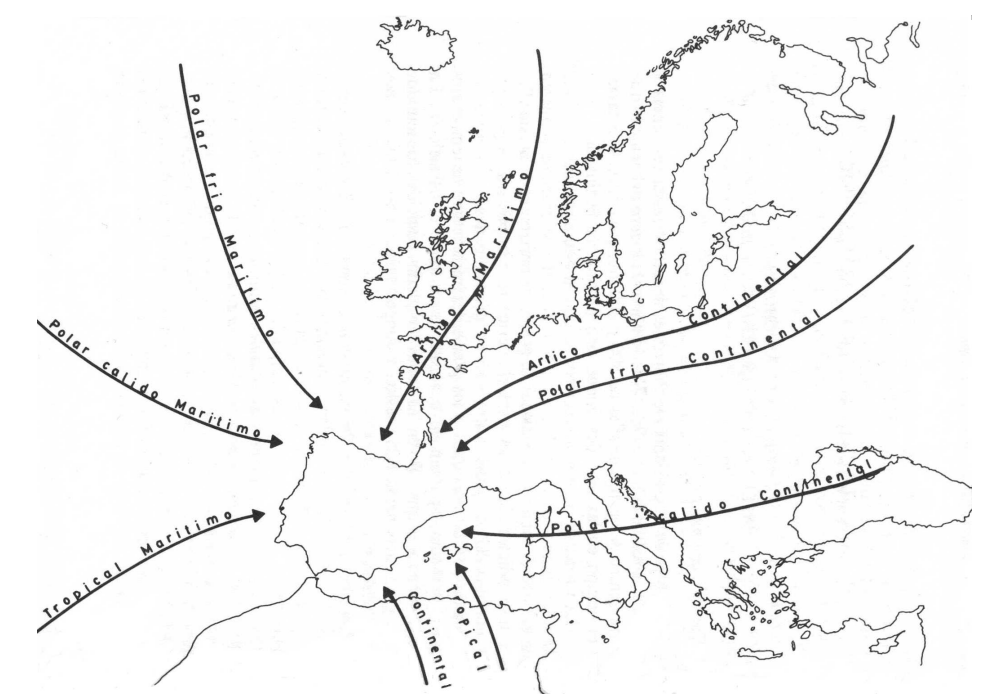

Figura VI-1. Tipos de masas de aire en la Península (tomada de Capel, 1981) 
En trabajos anteriores (Vergaz, 2001; Vergaz, 2005) se realizó un primer estudio para analizar la relación entre retrotrayectorias de masas de aire y medidas radiométricas de aerosoles. La clasificación de las masas de aire se realizó visualmente de acuerdo con los tipos de masas de aire de la Península Ibérica en Capel (1981) que mostramos en la Figura VI-1, y se estableció una primera relación entre el tipo de masa de aire y los parámetros espectrales que proporciona el Cimel. Ya en este trabajo se apuntaba la necesidad de un procedimiento objetivo de clasificación y de realizar el estudio con una serie de datos larga como la que nos proporciona el fotómetro Cimel.

El objetivo del estudio de retrotrayectorias es determinar si existe una relación significativa entre el tipo de masa de aire y las medidas radiométricas de aerosoles a partir de la serie de datos de 5 años con el fotómetro Cimel en El Arenosillo. Es decir, vamos a tratar de relacionar la procedencia de la masa de aire con las medidas espectrales de AOD del Cimel en el período 2000-2004, mediante una trayectoria por día y el promedio diario del AOD y el parámetro alfa. En caso de que exista, se tratará de determinar esa relación tanto para el tipo de aerosol como para la cantidad, mediante unos intervalos de los valores espectrales del AOD y de alfa que se puedan asociar a cada tipo de masa de aire. Para ello el sistema de análisis de retrotrayectorias deberá hacerse por un procedimiento lo más objetivo y automático posible.

La clasificación de masas de aire en la Península, y por tanto, en nuestra zona de estudio, ha sido muy estudiada y su complejidad es bien conocida (Jorba, 2005). De manera muy simplificada podemos establecer varios tipos fundamentales de acuerdo a la procedencia, basadas principalmente en las características de temperatura y humedad, y que se corresponden con situaciones sinópticas típicas (Sánchez, 1993; Capel, 2000; Font, 2000). Estos sectores de procedencia se muestran de manera esquemática en la Figura VI-2.

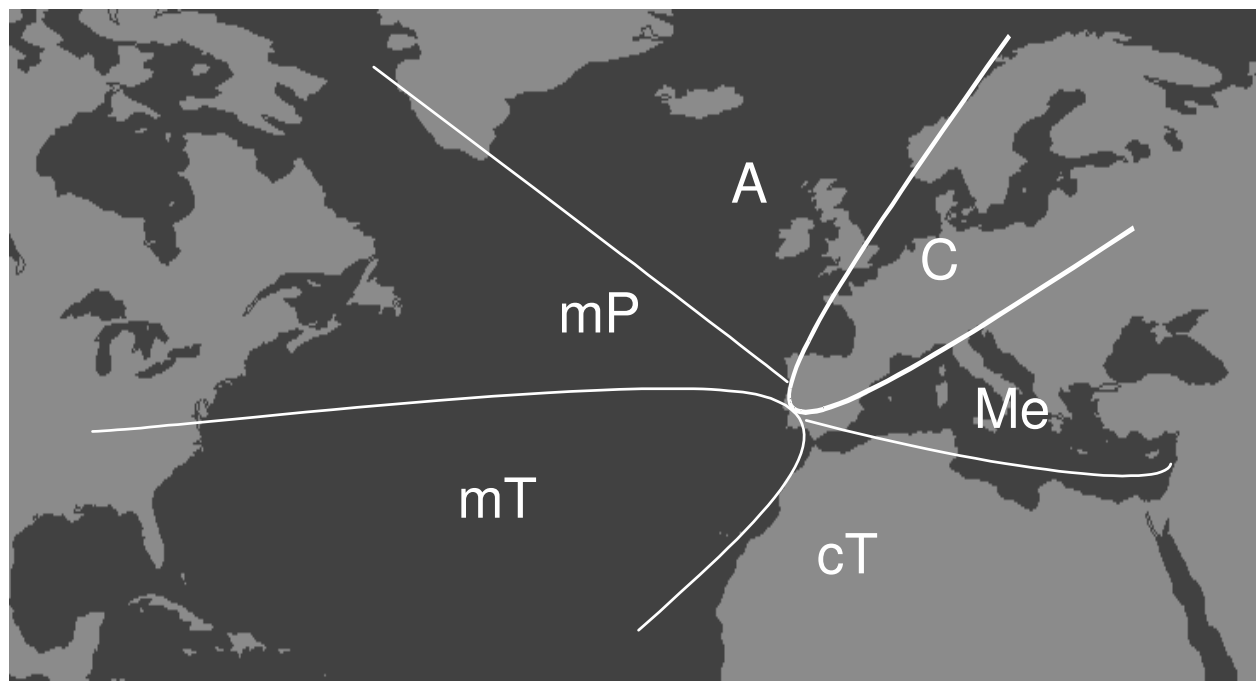

Figura VI-2. Principales sectores de procedencia de masas de aire en la Península Ibérica.

Las masas de aire marítimo tropical son muy frecuentes y tienen su origen en el anticiclón de las Azores. Su carácter es cálido y húmedo. Están presentes durante todo el año.

Las masas de aire marítimo polar proceden del Atlántico norte, y tienen su origen en los flujos del W ó NW debidos a situaciones ciclónicas. Su carácter es húmedo y más o menos frío dependiendo de la trayectoria. Están presentes durante todo el año. 
Las masas de aire ártico son frías y secas. Proceden de regiones árticas o ó subárticas y están asociadas a bajas presiones que se sitúan al NE ó al E de la península. Se dan principalmente en invierno.

También son frías y muy secas las masas de aire polar continental, que llegan a la Península procedentes de Rusia y Siberia, con vientos del NE. Son poco frecuentes y se dan sobre todo en invierno.

Las masas de aire mediterráneas no son muy frecuentes y afectan sobre todo a la vertiente mediterránea peninsular. Conviene distinguirlas del marítimo tropical del Atlántico porque corresponden con situaciones sinópticas diferentes.

Las masas de aire continental tropical tienen su origen en el norte de África, y tienen particular importancia para nosotros por estar asociadas a las entradas de aerosol desértico procedente del Sahara. Dependiendo de la época del año, hay varias situaciones sinópticas típicas que favorecen la llegada de estas masas de aire (Escudero et al., 2005; Rodríguez et al., 2001; Querol et al., 2002). Esto se comentará más en detalle en el capítulo siguiente. 


\subsection{El modelo HYSPLIT. Elección de las retrotrayectorias}

El modelo elegido para el cálculo de las retrotrayectorias es el modelo HYSPLIT (HYbrid Single-Particle Lagrangian Integrated Trajectory; Draxler and Rolph, 2003; Rolph, 2003), desarrollado por el NOAA (National Oceanic and Atmospheric Administration). En estos trabajos se describe en detalle el funcionamiento del modelo, cuestión en la que no vamos a detenernos en este trabajo. Se trata de un modelo de tipo lagrangiano, cuyos datos de entrada son mapas de análisis meteorológico, y que describen el movimiento de una parcela de aire dentro de la atmósfera. La base de datos meteorológicos utilizada es la que se denomina FNL (acrónimo de 'Final') del NOAA. La salida del modelo es un conjunto de puntos (1 por hora) que permiten reconstruir la posición de la partícula en latitud, longitud y altura.

El modelo HYSPLIT es, como hemos dicho, un modelo de tipo lagrangiano, lo que supone una representación más realista del transporte en la capa límite planetaria, donde existe turbulencia. Además no requiere grandes recursos de computación. Puede ser utilizado para el cálculo de trayectorias (tanto hacia delante como hacia atrás) y para modelizar dispersión de contaminantes en la atmósfera. El origen de este modelo fue la necesidad de predecir la dispersión de contaminantes, en particular para casos de emergencia, como el accidente nuclear de Chernobil, o las plumas de erupciones volcánicas, incendios, etc.

\begin{tabular}{|c|c|c|c|}
\hline (a) Campo & Unidades & (b) Nivel & Altura \\
\hline Presión en superficie & $\mathrm{hPa}$ & 13 & $20 \mathrm{hPa}$ \\
\hline Presión reducida al nivel medio del mar & $\mathrm{hPa}$ & 12 & $50 \mathrm{hPa}$ \\
\hline Temperatura en superficie & $\mathrm{K}$ & 11 & $100 \mathrm{hPa}$ \\
\hline Precipitación total acumulada en $6 \mathrm{~h}$ & $\mathrm{~m}$ & 10 & $150 \mathrm{hPa}$ \\
\hline Componente u (zonal) del momento & $\mathrm{N} / \mathrm{m} 2$ & 9 & $200 \mathrm{hPa}$ \\
\hline Componente v (meridional) del momento & $\mathrm{N} / \mathrm{m} 2$ & 8 & $250 \mathrm{hPa}$ \\
\hline Flujo de calor sensible neto en superficie & $\mathrm{W} / \mathrm{m} 2$ & 7 & $300 \mathrm{hPa}$ \\
\hline Flujo de calor latente en superficie & $\mathrm{W} / \mathrm{m} 2$ & 6 & $400 \mathrm{hPa}$ \\
\hline Flujo descendente de radiación de onda corta & $\mathrm{W} / \mathrm{m} 2$ & 5 & $500 \mathrm{hPa}$ \\
\hline Temperatura a 2 metros del nivel de suelo & $\mathrm{K}$ & 4 & $700 \mathrm{hPa}$ \\
\hline Humedad relativa a $2 \mathrm{~m}$ del suelo & $\%$ & 3 & $850 \mathrm{hPa}$ \\
\hline Componente u del viento a $10 \mathrm{~m}$ del suelo & $\mathrm{m} / \mathrm{s}$ & 2 & $925 \mathrm{hPa}$ \\
\hline Componente $\mathrm{v}$ del viento a $10 \mathrm{~m}$ del suelo & $\mathrm{m} / \mathrm{s}$ & 1 & $1000 \mathrm{hPa}$ \\
\hline $\begin{array}{l}\text { Contenido volumétrico de humedad en la } \\
\text { capa } 0-10 \mathrm{~cm} \text { del suelo }\end{array}$ & Fracción & 0 & superficie \\
\hline Cubierta total de nubes en la atmósfera & $\%$ & & \\
\hline Componente u del viento respecto a la rejilla & $\mathrm{m} / \mathrm{s}$ & & \\
\hline Componente $\mathrm{v}$ del viento respecto a la rejilla & $\mathrm{m} / \mathrm{s}$ & & \\
\hline Altura geopotencial & gpm* & & \\
\hline Temperatura & $\mathrm{K}$ & & \\
\hline Velocidad vertical de presión ** & $\mathrm{hPa} / \mathrm{s}$ & & \\
\hline Humedad relativa $* * *$ & $\%$ & & \\
\hline $\begin{array}{l}\text { * metros geopotenciales } \\
* * \text { Velocidad vertical disponible sólo en } 100 \\
* * * \text { Humedad relativa disponible sólo en } 30\end{array}$ & & & \\
\hline
\end{tabular}

Tabla VI-I. Descripción de los datos meteorológicos disponibles con la base de datos FNL: (a) variables meteorológicas; (b) descripción de niveles verticales

Este modelo está disponible a través de Internet tanto para ejecutarlo de forma remota a través de la web (http://www.arl.noaa.gov/ready.html), como para instalarlo y ejecutarlo 
localmente en un PC. Esta segunda opción nos ha permitido automatizar el proceso de cálculo, lo que resultaba imprescindible para realizar un estudio de varios años, que implica el cálculo de muchos cientos de retrotrayectorias.

El archivo de datos meteorológicos que utilizamos como entrada para el modelo FNL empezó a archivarse en 1997. Esta base de datos está disponible por ftp (ftp://www.arl.noaa.gov/pub/archives/fnl/ ) en ficheros quincenales para cada hemisferio, y los datos forman una rejilla de $1^{\circ}$ de latitud por $1^{\circ}$ de longitud cada 6 horas, con 21 variables meteorológicas y 13 niveles de presión desde la superficie hasta $20 \mathrm{hPa}$ (ver Tabla VI-I ), como se describe en Stunder (1997).

La elección fundamental a realizar cuando se quiere calcular retrotrayectorias con el modelo HYSPLIT es el tipo de movimiento vertical. El viento se mide rutinariamente en dirección e intensidad, pero siempre en sus componentes horizontales. Este viento horizontal es del orden de metros por segundo. El viento vertical no se mide de forma rutinaria y por tanto debe ser modelizado, teniendo en cuenta además que es del orden de $\mathrm{cm} / \mathrm{s}$. El movimiento vertical se calcula, por tanto, a través de ciertas hipótesis, a saber: que la masa de aire tenga un movimiento a presión constante (trayectorias isobáricas) o que tenga un movimiento adiabático (isentrópicas). Por último en las trayectorias tridimensionales el propio modelo suministra las velocidades verticales, bien a partir de la divergencia horizontal del campo de viento en cada nivel, aunque esta posibilidad es poco precisa, o bien a través de un modelo dinámico consistente, siendo esta última posibilidad la que ofrece HYSPLIT.

HYSPLIT ofrece estas tres posibilidades: isobáricas, isentrópicas o velocidad vertical del modelo (denominadas tridimensionales), por lo que hay que decidirse por una de ellas. El trabajo de Stohl (1998) realiza una revisión acerca del cálculo y uso de trayectorias. Uno de los puntos de discusión es precisamente el movimiento vertical. Según este autor, las trayectorias isobáricas son las menos realistas. La duda está por tanto entre emplear trayectorias isentrópicas o tridimensionales, que en principio tienen una precisión parecida. Martin et al. (1990), Draxler (1996) y también Stohl (1998) afirman que las trayectorias tridimensionales son las más precisas, siempre que se disponga de un campo preciso de velocidad vertical como el que proporciona la base de datos FNL y el modelo HYSPLIT. Cuevas (1995), Sancho y Romero (2002) o Querol et al., (2004) se inclinan por las isentrópicas, como una mejor opción que las isobáricas, aunque tampoco aportan una justificación frente a las tridimensionales o de velocidad vertical del modelo.

Finalmente nos hemos inclinado por comparar los resultados de nuestra metodología utilizando retrotrayectorias isentrópicas y tridimensionales. De esta comparación concluimos en primer lugar que no hay diferencias sustanciales en cuanto a los resultados de los métodos de clasificación que se describirán en las secciones 5.3 y 5.4. En segundo lugar, ciertas trayectorias isentrópicas (2-3\% del total) presentan situaciones no realistas en el movimiento vertical de la masa de aire, en particular ascensos de la misma por encima del tope del modelo (10000m de altura). Estos hechos, junto con las observaciones de Stohl (1998), nos han hecho inclinarnos definitivamente por las trayectorias tridimensionales, es decir, las que utilizan la velocidad vertical dada por el modelo.

También es necesario acomodar la resolución temporal de las medidas de aerosoles del Cimel con las retrotrayectorias de masas de aire, para poder realizar nuestro estudio. El modelo HYSPLIT permite una resolución temporal de 1 hora, si bien los archivos meteorológicos tienen datos cada 6 horas, mientras que el Cimel realiza medidas rutinarias durante las horas de sol cada 15 minutos aproximadamente. Las situaciones sinópticas tienen una persistencia del orden de horas a varios días. De hecho al analizar 
retrotrayectorias calculadas de hora en hora, se observa muy poco cambio entre ellas excepto -lógicamente- si se da un cambio en la masa de aire, por ejemplo por el paso de un frente. Si tomamos una trayectoria por día a las 12GMT, las medidas del Cimel durante ese día no distarán más de 6 horas de la trayectoria correspondiente. Por esto nos hemos inclinado a tomar una trayectoria por día, y la compararemos con el promedio diario de las medidas espectrales de AOD del Cimel.

El tiempo de vuelo elegido para las retrotrayectorias es de 5 días hacia atrás (120 horas). El error típico de las trayectorias es del orden de cientos de Km por día, o en términos relativos, de un $20 \%$ al día (error en distancia en relación con la distancia recorrida) aunque depende mucho de las situaciones sinópticas. Los errores de las trayectorias en situaciones de altas presiones son mayores que en situaciones de vientos fuertes y estables en régimen de bajas presiones, bien delante o detrás de un frente frío (Stohl, 1998). En diversos estudios que buscan relacionar situaciones sinópticas con ciertos componentes atmosféricos, las trayectorias han sido calculadas con 5 días hacia atrás (Cape et al., 2000; Methven et al., 2001; Sancho y Romero, 2002; Querol et al., 2004), cuatro días (Dorling et al., 1992; Jorba et al, 2004), dos días (Bahrmann and Saxena, 1998) y hasta 10 días (Rodríguez et al., 2001; Heintzenberg et al., 2003). Las retrotrayectorias de más de 5 días parecen estar indicadas en caso de analizar transporte a escala sinóptica, pero el error asociado será muy grande. La elección de cinco días responde por tanto al compromiso entre un tiempo suficientemente largo para determinar la procedencia de la masa de aire, y el error de la trayectoria.

Finalmente el modelo permite calcular las retrotrayectorias a distintos niveles. En nuestro estudio hemos calculado las trayectorias que llegan a la zona de estudio a 500, 1500 y $3000 \mathrm{~m}$ sobre el nivel del mar, que corresponde con 950, 850 y $700 \mathrm{hPa}$. aproximadamente. Aunque los aerosoles se hallen mayoritariamente por debajo de los $1000 \mathrm{~m}$ de altitud, los aerosoles desérticos son transportados a mayores alturas (hasta $5000 \mathrm{~m}$, Escudero et al., 2005) por lo que el análisis de trayectorias a 1500 y 3000m está orientado a una mejor detección de las intrusiones saharianas.

El resumen de todos los parámetros empleados para el cálculo de trayectorias con el modelo HYSPLIT está recogido en la Tabla VI-II.

\begin{tabular}{ll}
\hline \hline Versión HYSPLIT & 4.7 \\
\hline Frecuencia & 1 por día a las 12 GMT \\
Recorrido & Hacia atrás (backward) \\
Tiempo de vuelo & 120 horas \\
Tipo de movimiento vertical & Omega (velocidad vertical del modelo ó 3D) \\
Alturas de llegada & 500,1500 y 3000m s.n.m. \\
Base de datos meteorológica & FNL \\
Límite superior del modelo & $10000 \mathrm{~m}$ \\
Otras variables meteorológicas & Presión, temperatura potencial, humedad relativa, \\
& lluvia y profundidad de la capa de mezcla. \\
\hline \hline
\end{tabular}

Tabla VI-II. Parámetros de las trayectorias calculadas con el modelo HYSPLIT

El modelo nos proporciona finalmente un fichero con los datos de la trayectoria, esto es, el dato de posición de la parcela de aire en longitud, latitud, presión, temperatura potencial y altura de hora en hora para cada uno de los tres niveles y las 120 horas de vuelo, además de un dibujo con las tres trayectorias (ver Figura VI-3) en el que se incluye el movimiento vertical a lo largo de la trayectoria. 


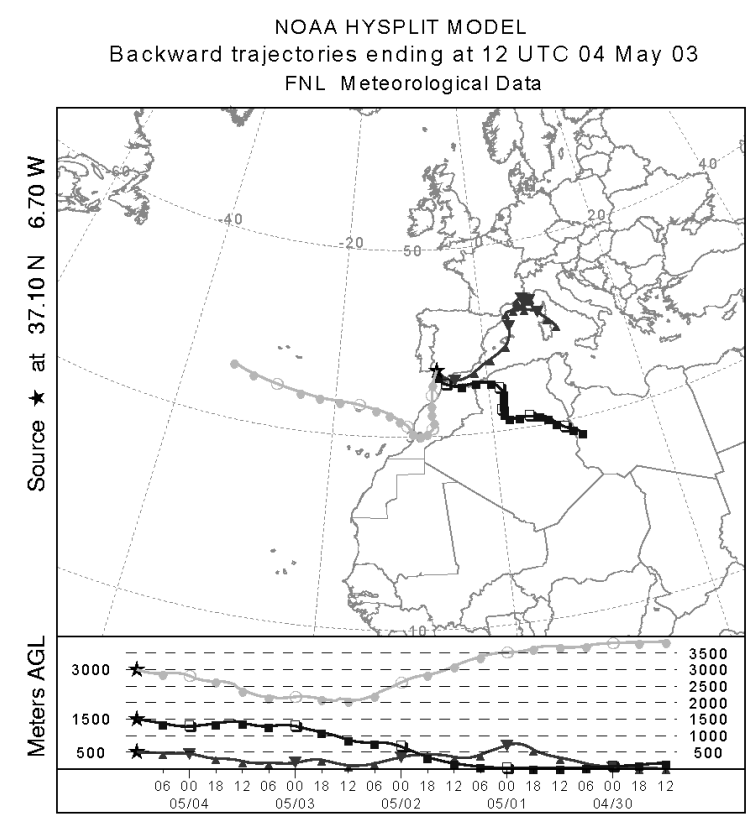

Figura VI-3. Ejemplo de la retrotrayectoria calculada para las 12GMT del día 4/3/05 con el modelo HYSPLIT

Además el modelo puede proporcionar otra serie de variables meteorológicas a lo largo de la trayectoria, como son la humedad relativa (en \%), lluvia (en $\mathrm{mm} / \mathrm{h}$ ) y profundidad de la capa de mezcla (en $\mathrm{m}$ ). Estas variables se han calculado también, aunque de momento no se han incluido en el estudio. La idea es que se puedan emplear en estudios o mejoras posteriores.

Con estos parámetros se han calculado las trayectorias hasta un total de 1781, desde el 16 de febrero de 2000 hasta el 31 de diciembre de 2004. De ellas, un total de 1337 días (el $74 \%)$ cuentan con medidas del Cimel. En realidad hay 1346 días de medida del fotómetro Cimel, pero en la base de datos FNL faltan algunos días por lo que las trayectorias no se pueden calcular. Esto hace que finalmente tengamos tanto retrotrayectorias como medidas del Cimel en 1337 días. Con estos datos se afrontará la clasificación en la sección siguiente. 


\subsection{Clasificación de las retrotrayectorias por sectores de procedencia}

El modelo HYSPLIT nos ha permitido calcular las retrotrayectorias de masas de aire que llegan a la estación de El Arenosillo durante los cinco años en los que se dispone de datos del Cimel. El siguiente paso es la clasificación por un método objetivo y automático de todas esas trayectorias. Existen diversas posibilidades, desde clasificarlas en función del origen de las mismas (Cuevas, 1995), del tiempo de residencia en determinados sectores (Sancho y Romero, 2002, Bahrmann and Saxena, 1998), climatologías de flujo (Miller, 1981), hasta procedimientos más o menos sofisticados de campos de probabilidad o análisis de clusters (Jorba et al., 2004).

Hemos realizado dos tipos de clasificación de nuestra serie de datos de 5 años de trayectorias. Por una parte hemos diseñado un método para clasificar por sectores de procedencia, de acuerdo con los tipos de masas de aire de la Figura VI-2. Esta clasificación se ha comparado con las medidas espectrales de AOD del fotómetro Cimel, y está recogida en esta sección 5.3. En segundo lugar hemos realizado una clasificación de las trayectorias mediante un algoritmo de cluster basado en la distancia geométrica entre trayectorias. Esta segunda clasificación ha sido también relacionada con las medidas del Cimel y se recoge en la sección 5.4 .

\subsubsection{Definición de los sectores de procedencia}

La clasificación de las trayectorias por sectores de procedencia se basa en el tiempo de residencia de la masa de aire sobre distintas zonas predefinidas, de acuerdo con la Figura VI-2. Para ello se ha definido una rejilla de $1^{\circ} \times 1^{\circ}$ en latitud y longitud, cuyas celdas tienen asignado uno de los siguientes caracteres:

1. Marítimo polar o frío (MP): océanos por encima de $40^{\circ} \mathrm{N}$

2. Marítimo tropical (MT): océano Atlántico por debajo de $40^{\circ} \mathrm{N}$

3. Ártico (A): océanos por encima de $60^{\circ} \mathrm{N}$ y continentes por encima de $67^{\circ} \mathrm{N}$ (círculo polar).

4. Continental $(\mathrm{C})$ : todos los territorios continentales por encima de la latitud de $\mathrm{El}$ Arenosillo

5. Continental Tropical (CT): territorios continentales en África sahariana

6. Local (L): las celdas adyacentes a la estación de El Arenosillo hasta $2^{\circ}$ de longitud o latitud

7. Mediterráneo(Me): zona marítima tropical del Mar Mediterráneo

El rango de esta tabla de clasificación es $15^{\circ}$ a $90^{\circ} \mathrm{N}$ de latitud y de $70^{\circ} \mathrm{W}$ a $35^{\circ} \mathrm{E}$ de longitud, lo cual comprende la casi totalidad de puntos de las trayectorias calculadas. Los puntos fuera de la rejilla no son considerados. En la Figura VI-4 mostramos la parte de la rejilla más cercana a la estación, que consiste simplemente en una matriz de números en los que cada celda tiene el número que corresponde los sectores definidos (del 1 al 7), y cuya posición en la matriz se relaciona directamente con una posición en la cuadrícula latitud-longitud. 


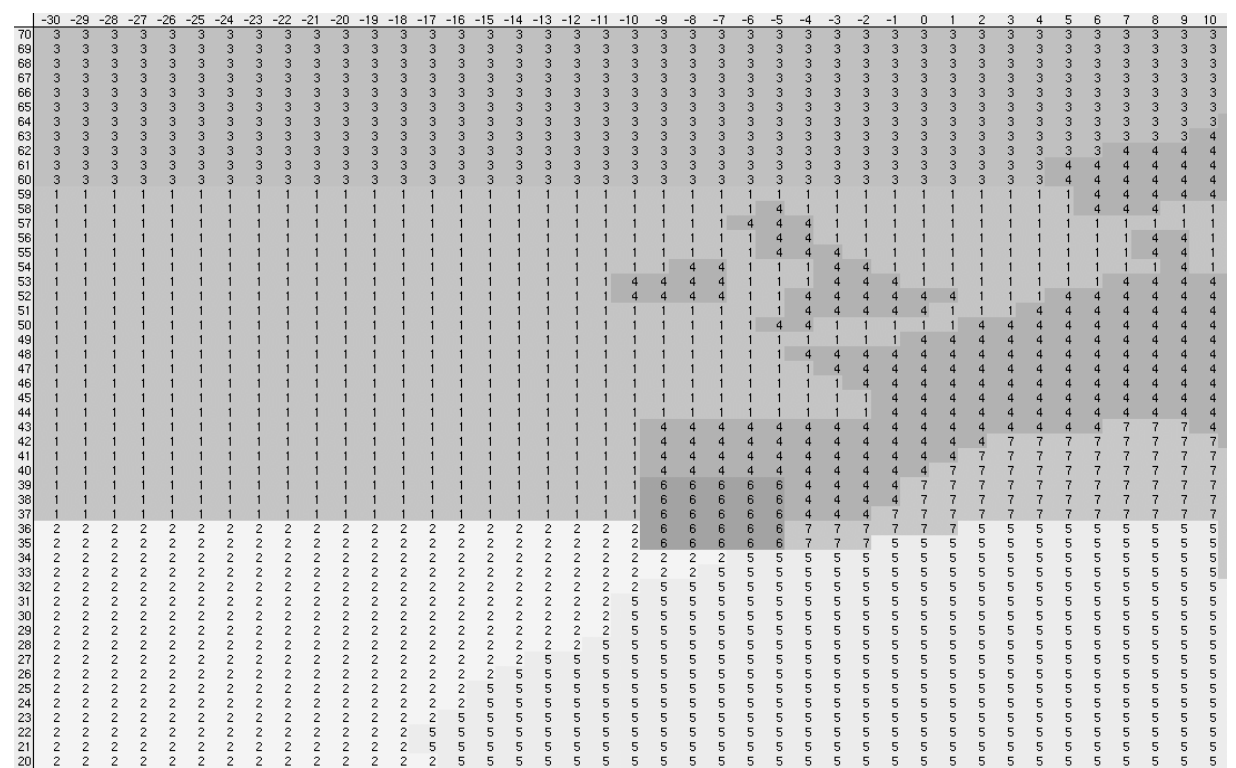

Figura VI-4. Detalle de la rejilla de clasificación para la definición de los sectores de procedencia. El eje vertical es la latitud entre $20^{\circ} \mathrm{N}$ y $70^{\circ} \mathrm{N}$, y en horizontal tenemos la longitud entre $30^{\circ} \mathrm{W}$ y $10^{\circ} \mathrm{E}$. Cada número se refiere a un sector: marítimo polar (1), marítimo tropical (2), ártico (3), continental (4), continental tropical (5), local (6) y mediterráneo (7).

Se ha realizado un programa de clasificación que asigna un vector de 7 componentes a cada trayectoria. Cada una de las 7 componentes del vector se refiere a los 7 sectores detallados, y nos indica el número de puntos, de los 121 en total, que han sobrevolado cada uno de los sectores. Para ello el programa lee del fichero de salida del modelo HYSPLIT la posición de la masa de aire, en latitud y longitud, y consulta sobre la rejilla el sector al que pertenece, sumando un punto a la componente del sector que corresponda. La rejilla de clasificación está organizada como una Look-Up-Table (LUT) para mayor rapidez de cálculo. Como ejemplo mostramos en la Tabla VI-III el vector asociado a la trayectoria de ejemplo de la Figura VI-3.

\begin{tabular}{llllllllc}
\hline \hline Nivel & MP & MT & A & C & CT & L & Me & N $^{\circ}$ puntos \\
\hline 500 & 0 & 0 & 0 & 23 & 0 & 13 & 85 & 121 \\
1500 & 0 & 0 & 0 & 0 & 95 & 17 & 9 & 121 \\
3000 & 0 & 78 & 0 & 0 & 34 & 9 & 0 & 121 \\
\hline \hline
\end{tabular}

Tabla VI-III. Ejemplo de clasificación por sectores en los tres niveles para la trayectoria del día 4 de marzo de 2005 en El Arenosillo (ver Figura VI-3).

Una octava componente nos recoge el número de puntos clasificados, que será 121 excepto en trayectorias muy rápidas y lejanas del oeste, que en ocasiones dejan algunos puntos fuera de nuestra rejilla o LUT de clasificación. Esta clasificación se aplica simultáneamente para los tres niveles, que en general pueden llevar trayectorias más o menos divergentes entre sí. El resultado final es un total de 24 variables que nos permiten calcular el porcentaje o contribución de cada uno de los sectores en los 3 niveles. Cada día tiene entonces asociado su vector que caracteriza la masa de aire en la estación. Todos estos vectores conforman lo que denominamos 'subespacio de trayectorias'.

Con esta metodología de clasificación se asume que las masas de aire toman propiedades del sector que han atravesado hasta llegar a El Arenosillo. Podemos considerar que el tiempo de residencia así calculado da cuenta de la historia de la masa de aire mejor que el punto final de procedencia o una asignación directa (visual) a uno de los sectores. Esto nos permite separar las trayectorias más puras de cada tipo para poder cruzar esta información con las medidas espectrales AOD del Cimel. Se trata de comprobar si las trayectorias que 
se agrupan en la clasificación de masas de aire (subespacio trayectorias) presentan también agrupaciones en cuanto a las medidas del fotómetro Cimel que podemos denominar 'subespacio espectral').

\subsubsection{Asignación de las trayectorias a un sector de procedencia}

Una vez que cada trayectoria tiene asociado un vector que da cuenta del tiempo de residencia sobre cada sector, y de cara a relacionar esta información con las medidas espectrales, hemos asignado las trayectorias a un cierto número de grupos (clusters). Es decir, finalmente se etiqueta cada trayectoria al incluirla dentro de un cluster. En principio los vectores asociados a cada trayectoria son puntos dentro del espacio con 7 componentes (que corresponden a los 7 caracteres definidos). Se puede aplicar por tanto un algoritmo de cluster para agruparlos. La metodología para esta agrupación obliga a tomar determinadas decisiones: número de grupos o clusters, realizar una clasificación supervisada o no supervisada, definir una distancia, etc. Este método, por tanto, es automático pero no un método objetivo, como se señala en Stohl (1998). Los centroides de esos clusters nos van a dar una idea de cómo es el recorrido de una trayectoria representativa de cada grupo. Por ejemplo, una trayectoria de tipo Ártico tendrá una fuerte componente ártica, pero además tendrá marítimo polar y continental, puesto que ha de viajar hasta El Arenosillo. En el otro extremo, la trayectoria de una masa de aire norteafricana llevará asociado un vector con una componente continental tropical más o menos grande y además contribución de marítimo tropical o mediterráneo, según el camino que haya llevado hasta la estación. Todas las trayectorias tendrán una componente local al atravesar las celdas contiguas a la estación. Sin embargo, las trayectorias que podamos etiquetar como locales tendrán una componente local muy grande, ya que la masa de aire se habrá mantenido en ese área durante bastante tiempo.

Nuestra opción para asignar cada trayectoria a un determinado sector va a consistir en realizar una clasificación supervisada, para lo cual necesitamos encontrar el vector representativo de cada uno de los 7 sectores definidos, que utilizaremos de centroide. Para tener una idea sobre cómo se distribuyen los vectores que representan las trayectorias en este subespacio, hemos realizado una serie de análisis de clusters no supervisados. Para ello se ha empleado el conocido algoritmo de $\mathrm{k}$-medias ${ }^{1}$, disminuyendo el número de clusters desde 15 hasta 1 . Se han analizado los centroides que se van encontrando. Éstos son vectores con una o dos componentes muy grandes y el resto casi testimoniales, lo que permite asignar el cluster a un cierto tipo o, a lo sumo, a una mezcla de dos tipos. También se observa que los centroides que aluden a un solo tipo determinado son casi idénticos aunque se varíe el número de clusters. A medida que vamos disminuyendo el número de clusters, siempre encontramos un centroide MP, MT, etc. muy parecidos y en ocasiones idénticos. Es decir, podemos establecer cómo es el vector o centroide característico de cada uno de los caracteres que hemos considerado, que son los que presentamos en la Tabla VI-IV. Éstos se han obtenido promediando los centroides que tienen contribución de un solo tipo entre los que obtenemos en las clasificaciones no supervisadas entre 12 y 6 clusters.

\footnotetext{
${ }^{1}$ El algoritmo de k-medias es un procedimiento de cluster no supervisado para clasificar un conjunto de datos. El número de clusters $(\mathrm{k})$ debe ser introducido a priori. Se parte de una asignación inicial aleatoria de $\mathrm{k}$ centroides, uno para cada cluster. Asociamos cada punto al centroide más cercano, con lo que tenemos una primera asignación. Recalculamos los k nuevos centroides como los baricentros de los clusters que hemos obtenido. Se vuelve a asignar cada punto a estos nuevos centroides, con lo que podemos iniciar un bucle en el que los k centroides se van desplazando paso a paso. Se repite el procedimiento hasta que los centroides ya no cambien.
} 
$\mathrm{Si}$ atendemos por ejemplo a los centroides en 1500m (Tabla VI-IVb) el centroide del cluster número 1 (que podemos etiquetar como Marítimo Polar) tiene una componente alta de MP y una pequeña contribución de Continental. El centroide número 2, etiquetado como Marítimo Tropical, tiene la contribución clara de MT, con el resto casi testimonial. Quizá parezca menos claro el cluster 3 (Ártico), pero debemos tener en cuenta que es el único que tiene una contribución del sector Ártico, y que lógicamente debe tener una contribución de MP y $\mathrm{C}$ en el trayecto de la masa de aire desde las latitudes que hemos designado como Árticas (mayor de $60^{\circ} \mathrm{N}$ ) hasta la estación $\left(37^{\circ} \mathrm{N}\right.$ ) por la lejanía de la zona Ártica. El cluster 4 (Continental) tiene una contribución principal de Continental, pero también MP, Me y sobre todo Local, casi el doble que los clusters anteriores. El cluster 5 agrupa las masas que podríamos llamar norteafricanas, con alta componente de tipo Continental Tropical, y además Local y MT. Naturalmente todas los centroides tienen una contribución Local, pero ésta es claramente mayor en los clusters 4, 5, 6 y 7, es decir, los que están asociados en principio a situaciones anticiclónicas. En el cluster 6 la contribución más importante es Local, mientras que en el cluster 7 predomina el carácter Mediterráneo.

Con estos centroides realizamos una clasificación supervisada, por el criterio de mínima distancia, asignando cada trayectoria a uno de los clusters, y por tanto a uno de los sectores de masas de aire que llegan a la estación. El resultado se puede ver gráficamente en la Figura VI-5, en la que hemos dibujado las trayectorias agrupadas en cada uno de los sectores para el nivel de $1500 \mathrm{~m}$. Los centroides que hemos empleado, y que son diferentes para cada nivel, se recogen en la Tabla VI-IV. En ella se han resaltado las componentes importantes de cada tipo.

\begin{tabular}{lllllllcc}
\hline \hline MP & MT & A & C & D & L & Me & Frecuencia(\%) & Cluster \\
\hline 96 & 1 & 1 & 8 & 0 & 14 & 0 & 41.4 & $1(\mathrm{MP})$ \\
16 & 88 & 0 & 0 & 1 & 14 & 1 & 12.4 & $2(\mathrm{MT})$ \\
49 & 1 & 42 & 13 & 0 & 10 & 0 & 10.1 & $3(\mathrm{~A})$ \\
17 & 1 & 5 & 67 & 0 & 22 & 7 & 14.5 & $4(\mathrm{C})$ \\
0 & 2 & 0 & 2 & 74 & 23 & 21 & 5.2 & $5(\mathrm{D})$ \\
8 & 11 & 0 & 6 & 6 & 79 & 11 & 4.6 & $6(\mathrm{~L})$ \\
1 & 0 & 0 & 8 & 11 & 19 & 81 & 11.8 & $7(\mathrm{Me})$ \\
\hline \hline
\end{tabular}

(a) Centroides en $500 \mathrm{~m}$ para los 7 tipos de masas de aire.

\begin{tabular}{lllllllcc}
\hline \hline MP & MT & A & C & D & L & Me & Frecuencia(\%) & Cluster \\
\hline 93 & 2 & 4 & 8 & 0 & 11 & 0 & 41.6 & $1(\mathrm{MP})$ \\
9 & 95 & 0 & 0 & 5 & 13 & 0 & 19.5 & $2(\mathrm{MT})$ \\
43 & 4 & 40 & 14 & 0 & 8 & 0 & 9.3 & $3(\mathrm{~A})$ \\
18 & 0 & 5 & 66 & 2 & 24 & 5 & 6.6 & $4(\mathrm{C})$ \\
0 & 4 & 0 & 2 & 88 & 22 & 5 & 12.7 & $5(\mathrm{D})$ \\
15 & 3 & 0 & 7 & 8 & 80 & 8 & 5.5 & $6(\mathrm{~L})$ \\
7 & 0 & 2 & 36 & 2 & 17 & 56 & 4.8 & $7(\mathrm{Me})$ \\
\hline \hline
\end{tabular}

(b) Centroides en $1500 \mathrm{~m}$ para los 7 tipos de masas de aire.

\begin{tabular}{lllllllcc}
\hline \hline MP & MT & A & C & D & L & Me & Frecuencia(\%) & Cluster \\
\hline 95 & 1 & 3 & 6 & 0 & 12 & 0 & 38.4 & $1(\mathrm{MP})$ \\
3 & 108 & 0 & 0 & 3 & 7 & 0 & 30.8 & $2(\mathrm{MT})$ \\
42 & 5 & 41 & 11 & 0 & 7 & 0 & 11.9 & $3(\mathrm{~A})$ \\
11 & 0 & 3 & 63 & 3 & 18 & 13 & 3.5 & $4(\mathrm{C})$ \\
1 & 12 & 0 & 4 & 77 & 19 & 8 & 10.4 & $5(\mathrm{D})$ \\
30 & 2 & 0 & 12 & 3 & 74 & 3 & 3.9 & $6(\mathrm{~L})$ \\
1 & 3 & 0 & 24 & 10 & 17 & 65 & 1.1 & $7(\mathrm{Me})$ \\
\hline \hline
\end{tabular}

(c) Centroides en $3000 \mathrm{~m}$ para los 7 tipos de masas de aire.

Tabla VI-IV. Centroides para la clasificación supervisada de las masas de aire mediante sectores (a) para las trayectorias en $500 \mathrm{~m}$; (b) para las trayectorias en $1500 \mathrm{~m}$; (c) para las trayectorias en $3000 \mathrm{~m}$. 
Los centroides que corresponden a un tipo determinado son muy similares en los tres niveles, aunque con ciertos matices. Cabe destacar que la componente Mediterránea es mayor en las trayectorias de $500 \mathrm{~m}$, y que el centroide del sector Continental Tropical en $500 \mathrm{~m}$ tiene además una contribución importante de Mediterráneo. En 3000m el carácter Continental Tropical tiene una cierta relación con el Marítimo Tropical, mientras que en $1500 \mathrm{~m}$ estas influencias casi desaparecen. Los caracteres Mediterráneo, Continental y Local aparecen muy mezclados entre sí, y con presencia mayor o menor de las tres componentes en los clusters que etiquetamos como C, L y Me. Esto se puede deber, como hemos apuntado ya, a la permanencia de una misma masa de aire durante cierto tiempo sobre la Península (recirculaciones), asociada a situaciones sinópticas muy estables. La componente Local es mucho mayor para este tipo de trayectorias más lentas $(\mathrm{C}, \mathrm{D}, \mathrm{L}, \mathrm{Me})$ que para las trayectorias más rápidas (MP, MT, A).

En los mapas de las trayectorias de cada sector (Figura VI-5) podemos ver el resultado de nuestro método de clasificación. Las trayectorias tienen la mayor parte de sus puntos sobre el sector al que han sido asignadas, sobre todo en la parte más cercana a la estación. Es decir, estamos agrupando las trayectorias según el sector sobre el que hayan pasado más tiempo, independientemente del camino seguido o la velocidad (longitud) de la trayectoria. El sector Marítimo Polar agrupa una gran cantidad de trayectorias, pues a este sector pertenecen la mayoría de trayectorias atlánticas. En otros sectores como el Continental, Local o Mediterráneo, la zona que ocupan mayoritariamente las trayectorias reproduce el dibujo de la rejilla de clasificación. El sector Continental Tropical tiene un número considerable de casos, agrupando las masas de aire procedentes del norte de África en este nivel de $1500 \mathrm{~m}$. El sector Ártico agrupa trayectorias rápidas que nos llegan desde regiones septentrionales por diferentes caminos, aunque predomina el noroeste. Finalmente el sector Marítimo Tropical tiene tanto trayectorias cortas del oeste y sudoeste como una segunda rama de trayectorias algo más largas, también de componente oeste o sudoeste.

Una vez que las trayectorias han sido asignadas a un determinado sector, podremos en primer lugar describir la frecuencia de masas de aire de cada sector y su comportamiento estacional, y en segundo lugar comparar la clasificación que hemos realizado con las medidas espectrales del Cimel. 

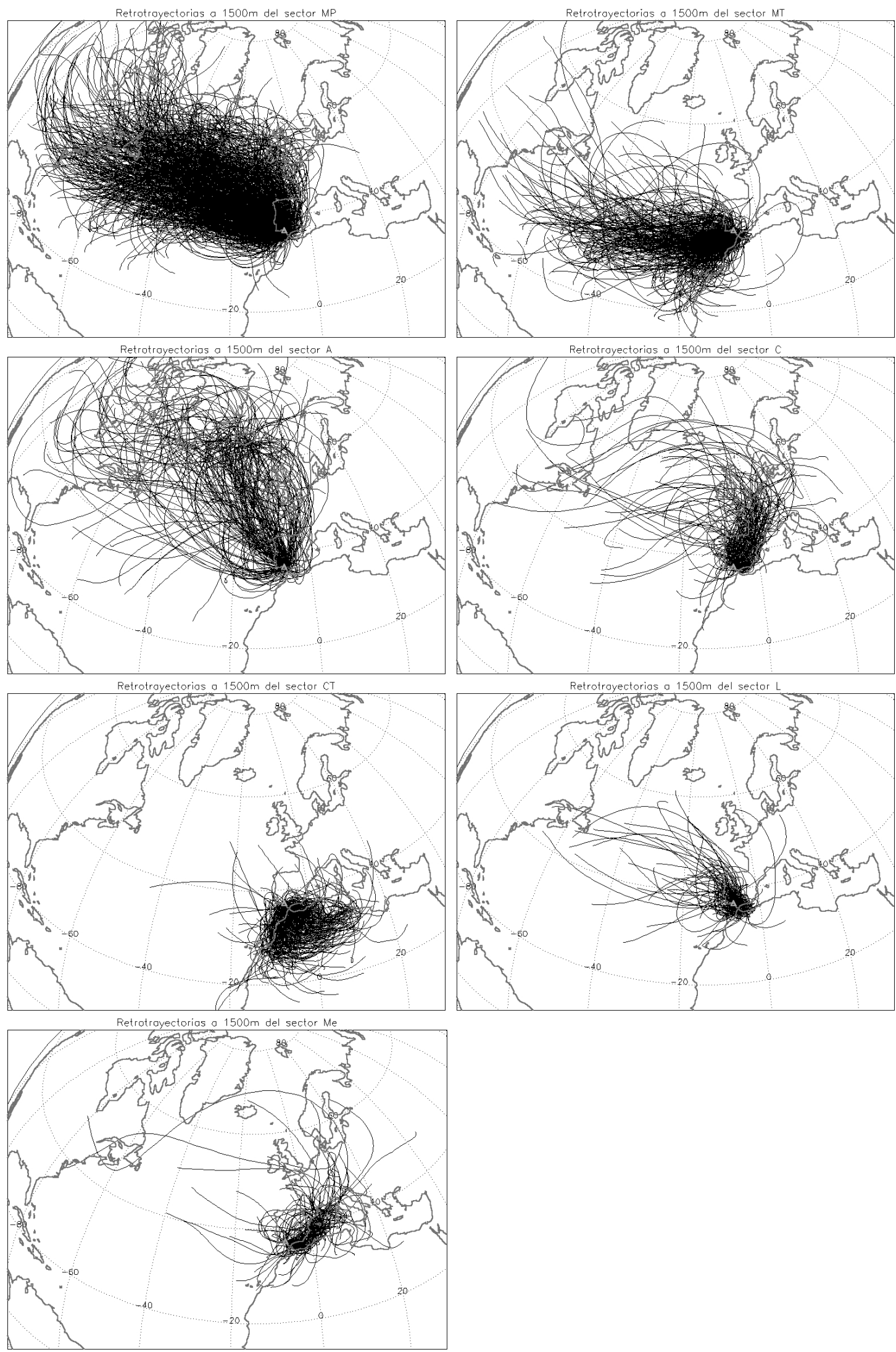

Figura VI-5. Mapa con las trayectorias de cada uno de los 7 clusters según la clasificación por sectores. 


\subsubsection{Análisis de frecuencias de las masas de aire}

Una vez asignadas las trayectorias a un sector determinado, podemos analizar la frecuencia de las masas de aire en cada uno de esos sectores. En la Tabla VI-V tenemos las frecuencias de las masas de aire, anuales y por estaciones, en cada uno de los tres niveles. Los diagramas de las Figura VI-6 y Figura VI-7 muestran esos mismos valores para las frecuencias anuales y estacionales respectivamente.

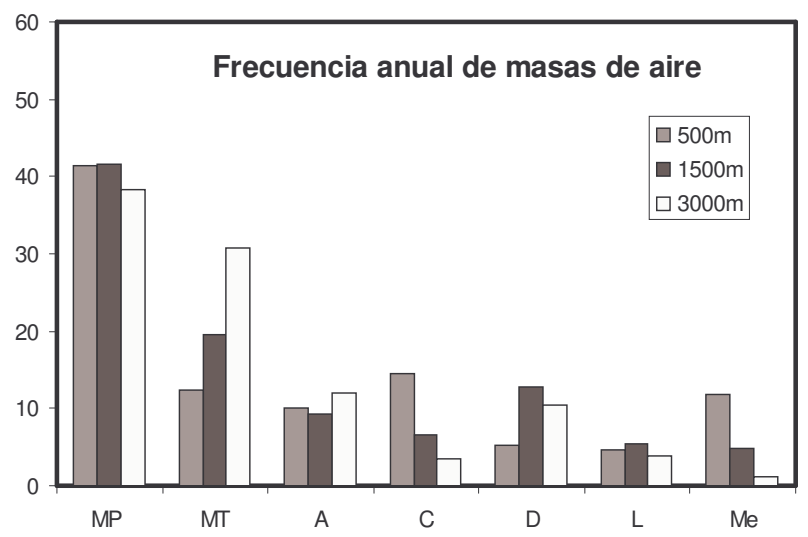

Figura VI-6. Frecuencias anuales de masas de aire en El Arenosillo, clasificadas por sectores

En primer lugar podemos observar que el carácter marítimo polar (MP) es el predominante, con una frecuencia del $40 \%$ similar en los tres niveles. Le sigue en importancia el marítimo tropical (MT) con una frecuencia del $20 \%$ en $1500 \mathrm{~m}$ y $30 \%$ en $3000 \mathrm{~m}$. Sin embargo en el nivel de $500 \mathrm{~m}$ el continental (C), mediterráneo (Me) y MT tienen una importancia similar (12-14\%). El carácter Ártico (A) mantiene una frecuencia del $10 \%$ aproximadamente en los tres niveles. Tanto el carácter Continental como el Mediterráneo disminuyen su frecuencia con la altura de la trayectoria, y son por tanto mucho más frecuentes en $500 \mathrm{~m}$ ( $14.5 \%$ para C y $12 \%$ para Me) que en $3000 \mathrm{~m}$ ( $3.5 \%$ de $\mathrm{C}$ y $1 \%$ de $\mathrm{Me}$ ). Por el contrario el carácter Continental Tropical (CT) es más frecuente en $1500 \mathrm{~m}$ y $3000 \mathrm{~m}$ con un $13 \%$ y $10 \%$ que en $500 \mathrm{~m}$, donde es del $5 \%$. Por último el carácter Local (L) agrupa un 4-5\% de las trayectorias, y es similar en los tres niveles.

Si observamos las frecuencias estacionales (Tabla VI-V y Figura VI-7) de las masas de aire, encontramos también variaciones que conviene destacar. El carácter MP mantiene una frecuencia alta durante todo el año, que es mayor en verano y especialmente en el nivel de $500 \mathrm{~m}$. El MT tampoco presenta grandes diferencias estacionales excepto la disminución en verano (hasta el $5.1 \%$ ) en $500 \mathrm{~m}$, justo cuando aumenta la frecuencia de masas mediterráneas a ese nivel. Estas masas MT siguen siendo más frecuentes para las trayectorias de los niveles más altos.

Las masas Árticas tienen la mitad de frecuencia en verano que durante el resto del año. En invierno llegan a ser un $15 \%$ en $3000 \mathrm{~m}$ y en verano son tan solo del $3 \%$ en $1500 \mathrm{~m}$. El carácter continental es mayor en primavera, mientras que las masas de tipo Continental Tropical son más frecuentes en verano. Cabe destacar que durante el invierno las masas saharianas tienen una frecuencia similar en los tres niveles (10-12\%), mientras que el resto del año son más frecuentes en $1500 \mathrm{~m}$ y $3000 \mathrm{~m}$, llegando hasta el $17 \%$ en verano a $1500 \mathrm{~m}$. Esto puede estar relacionado con los distintos mecanismos para la llegada de masas de aire norteafricanas que se comentarán en el capítulo siguiente. 


\begin{tabular}{lcccccc}
\hline \hline (a) 500m & ANUAL & Primavera & Verano & Otoño & Invierno & N $^{\circ}$ casos \\
\hline MP & 41.4 & 36.7 & 53.2 & 36.9 & 38.7 & 738 \\
MT & 12.4 & 14.9 & 5.1 & 13.8 & 16.2 & 221 \\
A & 10.1 & 10.5 & 6.2 & 12.2 & 11.4 & 179 \\
C & 14.5 & 21.1 & 9.5 & 13.8 & 13.8 & 259 \\
CT & 5.2 & 2.4 & 2.4 & 6.2 & 10.0 & 92 \\
L & 4.6 & 4.6 & 5.7 & 4.7 & 3.3 & 82 \\
Me & 11.8 & 9.7 & 18.0 & 12.4 & 6.7 & 210 \\
\hline \hline
\end{tabular}

\begin{tabular}{lcccccc}
\hline \hline (b) 1500m & ANUAL & Primavera & Verano & Otoño & Invierno & N $^{\circ}$ casos \\
\hline MP & 41.6 & 40.7 & 47.5 & 37.6 & 40.6 & 741 \\
MT & 19.5 & 18.5 & 15.8 & 21.8 & 22.3 & 348 \\
A & 9.3 & 11.0 & 3.1 & 10.4 & 13.1 & 166 \\
C & 6.6 & 10.5 & 4.2 & 7.1 & 4.3 & 117 \\
CT & 12.7 & 8.1 & 16.7 & 14.0 & 11.9 & 226 \\
L & 5.5 & 5.9 & 7.0 & 5.1 & 3.8 & 98 \\
Me & 4.8 & 5.3 & 5.7 & 4.0 & 4.0 & 85 \\
\hline \hline & \multicolumn{7}{c}{} & & & & \\
\hline \hline (c) 3000m & ANUAL & Primavera & Verano & Otoño & Invierno & N $^{\circ}$ casos \\
\hline MP & 38.4 & 42.9 & 38.2 & 36.0 & 36.3 & 684 \\
MT & 30.8 & 25.5 & 35.2 & 33.3 & 29.0 & 548 \\
A & 11.9 & 12.3 & 6.4 & 13.8 & 15.4 & 212 \\
C & 3.5 & 4.2 & 2.6 & 3.1 & 4.3 & 63 \\
CT & 10.4 & 8.6 & 14.1 & 9.3 & 9.7 & 186 \\
L & 3.9 & 4.8 & 2.9 & 4.0 & 3.8 & 69 \\
Me & 1.1 & 1.8 & 0.7 & 0.4 & 1.4 & 19 \\
\hline \hline
\end{tabular}

Tabla VI-V. Frecuencias relativas anuales y estacionales y número de casos en cada sector para las masas de aire en El Arenosillo en los tres niveles: (a) 500m; (b) 1500m; (c) 3000m.
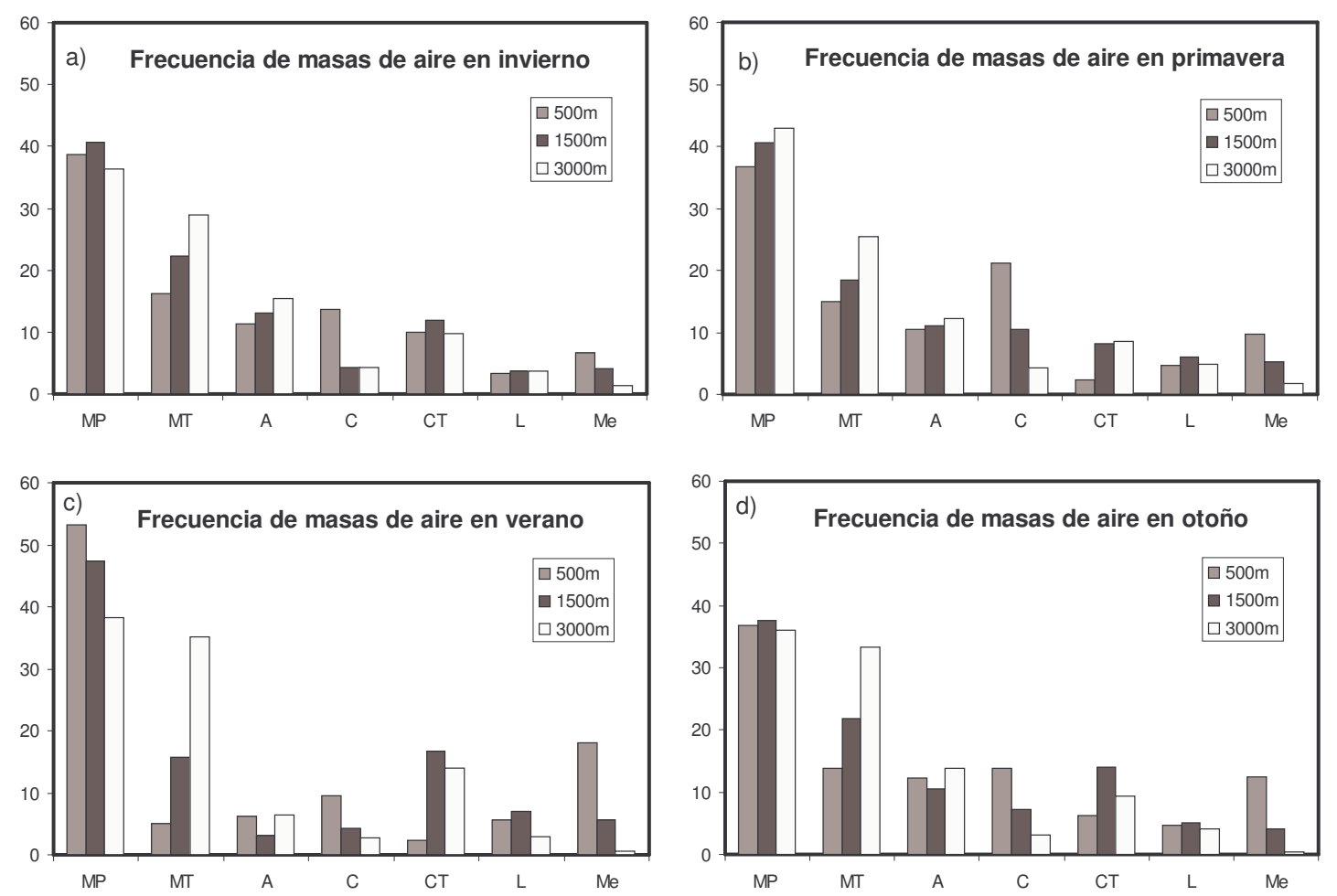

Figura VI-7. Frecuencias relativas de las masas de aire en El Arenosillo, clasificadas por sectores, para cada uno de los tres niveles de trayectorias. Análisis estacional: (a) primavera; (b) verano; (c) otoño; y (d) invierno. 
Las masas de aire que denominamos Locales presentan una frecuencia similar a lo largo del año, algo menor en invierno y mayor en verano para los niveles de 500 y $1500 \mathrm{~m}$. Las masas de aire de origen Mediterráneo son muy frecuentes en verano y a $500 \mathrm{~m}$ de altura $(18 \%)$ frente al $0.4-0.7 \%$ de frecuencia en verano y otoño a $3000 \mathrm{~m}$, una frecuencia prácticamente despreciable.

\subsubsection{Relación entre las medidas espectrales y las trayectorias de masas de aire}

Una vez clasificadas las trayectorias en clusters por este método de los sectores, vamos a analizar las medidas espectrales del espesor óptico de aerosoles y del parámetro alfa medidos con el Cimel para cada uno de los clusters de trayectorias que hemos obtenido. Este apartado tiene como objetivo discriminar qué posible huella espectral puede tener una cierta trayectoria, o también, dada una medida espectral, sugerir el tipo de masa de aire que puede causarla. Para ello vamos a utilizar en primer lugar el promedio diario de AOD (440nm) y alfa de Ångström para realizar un diagrama AOD-alfa con los datos de cada cluster. Este diagrama es muy útil para la discriminación del tipo de aerosol y nos permite ver la huella espectral de los aerosoles asociados a cada tipo de masa de aire. En total disponemos de 1337 días con medidas del Cimel para este análisis.

El diagrama de dispersión AOD-alfa para cada uno de los 7 clusters en los que hemos separado las trayectorias se presenta en la Figura VI-8, para cada uno de los 3 niveles. Son por tanto 21 diagramas que tenemos que comentar detalladamente. En todos ellos se ha representado el AOD en 440nm frente al parámetro alfa para los 1337 días de la serie de datos, y son el resultado a nivel de medidas del fotómetro Cimel de la clasificación por sectores cuando ésta se realiza a partir de las trayectorias en $500 \mathrm{~m}, 1500 \mathrm{~m}$ y 3000m. Como las trayectorias son diferentes en cada nivel, las clasificaciones que se obtienen son también diferentes. Más adelante discutiremos cuál de los niveles resulta más útil o para qué nos puede servir esta diferenciación.

En primer lugar hay que tener muy presente que el fotómetro Cimel nos da una medida integrada a toda la columna atmosférica. Esto implica que en el caso de que las trayectorias tengan un origen diferente en cada nivel, nos podemos encontrar con aparentes discrepancias. Por ejemplo, la llegada de sahariano en $1500 \mathrm{~m}$ puede ser detectada por el Cimel mientras que la trayectoria en $500 \mathrm{~m}$ puede ser Mediterránea ó MT y no tener nada que ver. Como las trayectorias pueden ser muy diferentes en los tres niveles (ver ejemplo de la Figura VI-3), un mismo día puede estar catalogado dentro de un cierto tipo si atendemos a la trayectoria de $500 \mathrm{~m}$ y a otro cierto tipo si atendemos al nivel de $1500 \mathrm{~m}$ ó de $3000 \mathrm{~m}$.

En segundo lugar estos diagramas indican qué medidas espectrales tenemos para las trayectorias que hemos agrupado en un sector, por ejemplo el continental. Como en el nivel de $500 \mathrm{~m}$ hay más trayectorias continentales, tenemos más puntos en ese diagrama que en los de $1500 \mathrm{~m}$ y $3000 \mathrm{~m}$. Análogamente en el cluster Mediterráneo hay muchas trayectorias (y por tanto muchos puntos en el diagrama) para el nivel de $500 \mathrm{~m}$, mientras que en $3000 \mathrm{~m}$ casi no hay puntos. Lo que pretendemos es extraer la máxima información posible, por lo que disponer de tres niveles de trayectorias resulta muy útil. 

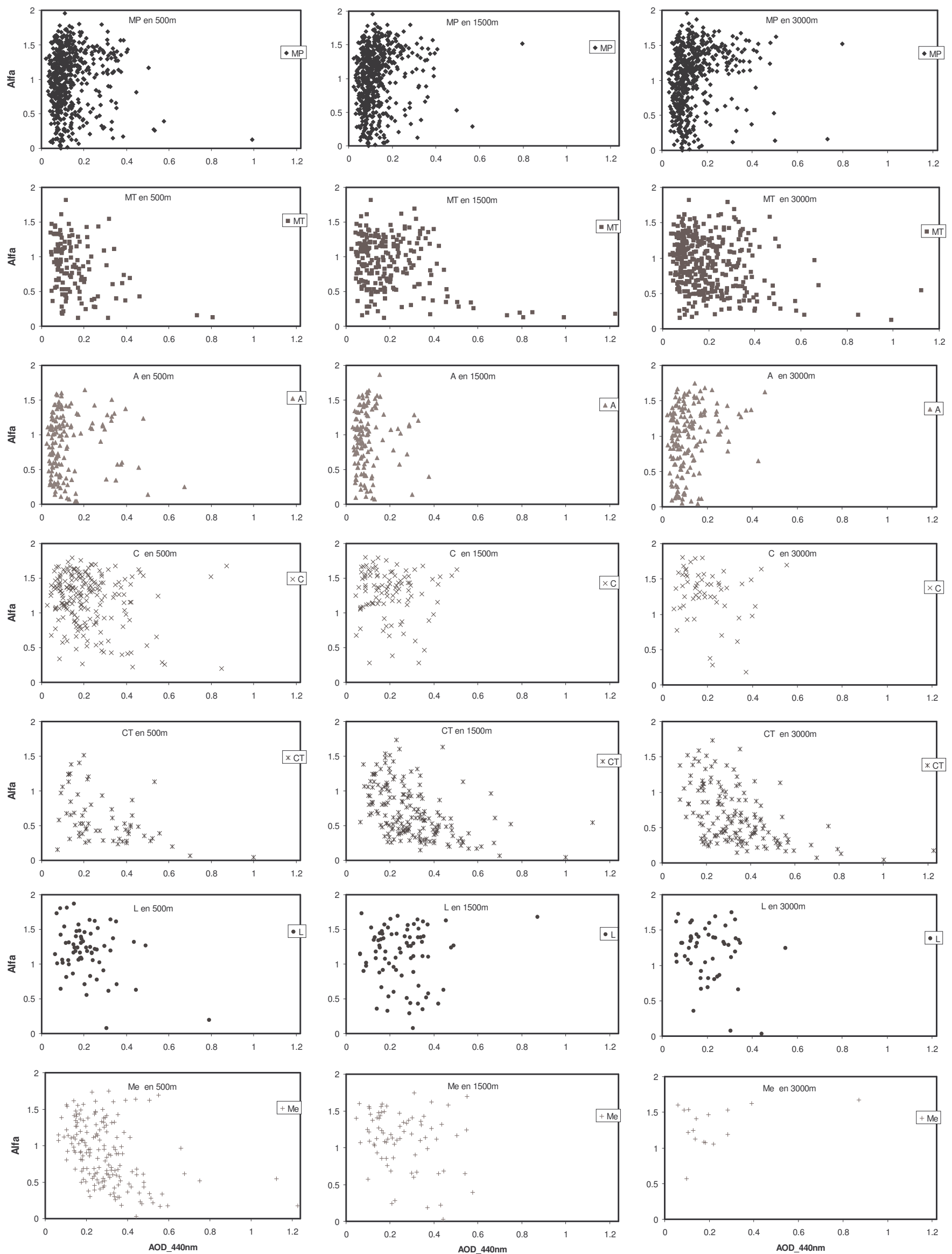

Figura VI-8. Diagramas AOD(440nm)-alfa para cada cluster de masas de aire y para los tres niveles de trayectorias. 
En tercer lugar queremos dejar clara la dificultad de este tipo de análisis, en particular por las posibles mezclas o diferencias entre los niveles, y por el error que llevan asociadas las trayectorias. Como ejemplo de esta dificultad mostramos en la Figura VI-9 las retrotrayectorias calculadas para los días 9 y 10 de noviembre. Ambas quedan clasificadas como Árticas en los 3 niveles excepto el día 10 en 3000m que se clasifica como MP. El AOD promedio el día 9 es 0.09 en 440nm con un alfa de 1.56, es decir, unos valores típicos de aerosol ártico o al menos una situación de muy baja turbiedad. Sin embargo el día 10 el promedio de AOD es 0.30 en 440nm y con un alfa de 0.16 , es decir, valores típicos de aerosol sahariano. Podemos observar cómo la trayectoria de $1500 \mathrm{~m}$ durante el día 10 nos muestra una recirculación por el norte de Marruecos antes de llegar a la estación, que en este caso trae aerosol sahariano como se ve en la medida del Cimel. En resumen dos caracteres, Ártico y Sahariano, con masas de aire tan diferentes y que provienen de sectores opuestos, pueden mezclarse y confundirse. Al día siguiente, día 11 (no mostrado), las trayectorias ya muestran que la situación ha cambiado a masa de aire norteafricana, y el día 10 se trataba por tanto de una transición.
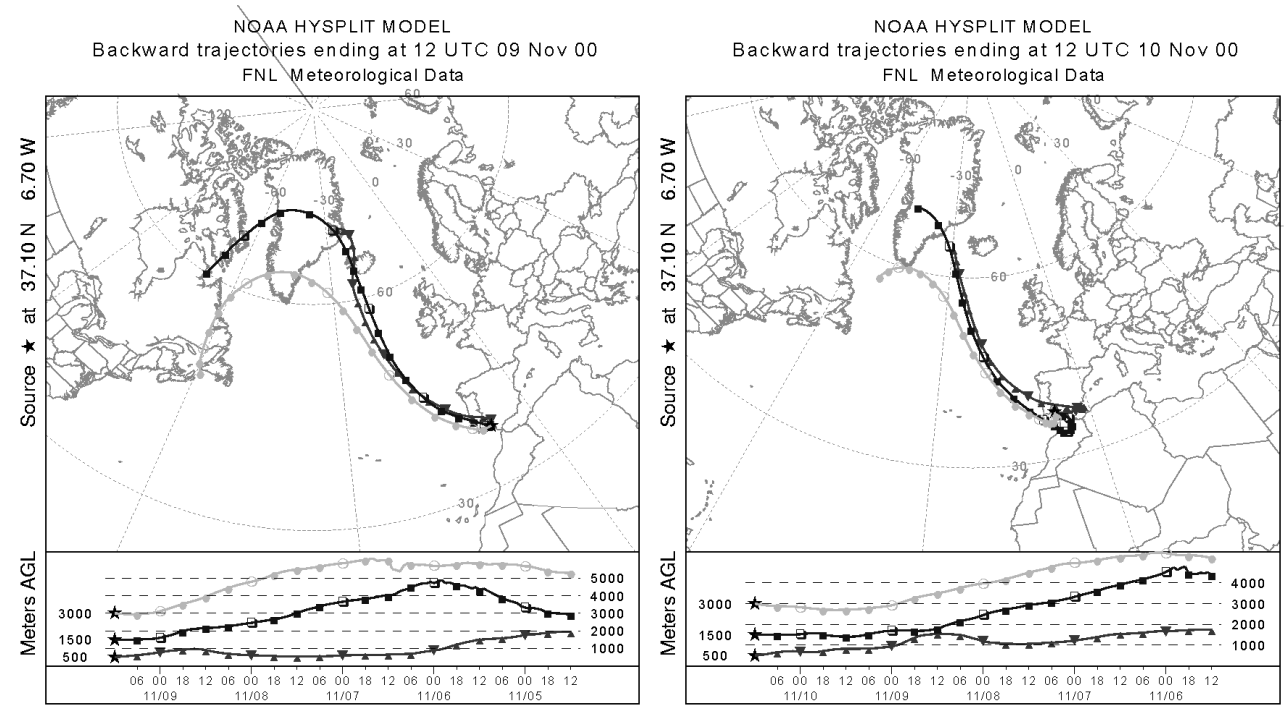

Figura VI-9. Ejemplo de trayectorias de masas de aire durante los días (a) 9, (b) 10 y (c) 11 de noviembre de 2000

De nuevo sobre la Figura VI-8 observamos que las medidas espectrales muestran esencialmente el mismo aspecto, con diagramas AOD-alfa muy parecidos, ya sea la clasificación realizada con las trayectorias a 500, 1500 ó 3000m de altura. Esto es debido a que hay un gran número de trayectorias que son muy similares en los tres niveles, y que por tanto quedan clasificadas en el mismo sector. Las trayectorias de un grupo también se agrupan en una cierta región espectral, más o menos amplia, que se puede definir en términos de AOD y alfa, si bien es cierto que algunos de estos grupos se solapan entre sí. Esto nos permite establecer una huella espectral bastante determinada para cada uno de los sectores de masas de aire. La estadística para los AOD y el parámetro alfa en cada uno de los clusters según la clasificación dada por las trayectorias en $1500 \mathrm{~m}$ está en la Tabla VI-VI a Tabla VI-XII. En estas tablas se recogen el promedio y la desviación estándar, junto con el máximo y el mínimo, más los percentiles 25, 50 (mediana), 75 y 90 de la distribución.

En este análisis y en adelante hemos elegido sólo el nivel de $1500 \mathrm{~m}$ para las tablas estadísticas y para los histogramas de frecuencias que iremos mostrando. Esto se ha hecho por varios motivos. En primer lugar la clasificación de las medidas del fotómetro Cimel utilizando las trayectorias de $1500 \mathrm{~m}$ ha resultado ser más clara y representativa en general. El nivel de $500 \mathrm{~m}$, en principio dentro de la capa límite y con mayor carga de aerosoles, no 
es tan representativo de las situaciones sinópticas principales, ya que presenta numerosas recirculaciones y trayectorias locales por ser menor la velocidad del viento en este nivel. Esto hace además que las trayectorias en $500 \mathrm{~m}$ estén afectadas por errores mayores, relacionados también con la turbulencia en la capa de mezcla, topografía, brisas, etc. El nivel de $3000 \mathrm{~m}$ es demasiado alto para los aerosoles excepto en el caso del aerosol desértico. Queremos relacionar masas de aire y medidas de aerosoles a escala sinóptica, por lo que el nivel de 1500m es adecuado. Con esto también buscamos en lo posible una mayor sencillez en la exposición.

El cluster 1 (sector Marítimo Polar, Tabla VI-VI) presenta en general valores bajos de espesor óptico de aerosoles, prácticamente siempre por debajo de 0.2 , mientras que alfa recorre todo el rango de 0 a 2 . De hecho para alfas altos este cluster y el continental se solapan, dejando clara la influencia costera de la estación, que también se traduce en algunas medidas (menos del 10\%) con AOD medio (entre 0.2 y 0.4 ). El promedio de $\mathrm{AOD}(440 \mathrm{~nm})$ es 0.127 , lo cual significa una turbiedad baja que es característica de este cluster, así como un alfa promedio de 1.00 , pero muy variable (STD 0.44). Aunque este cluster agrupa claramente masas de aire Atlánticas, y por tanto de carácter marítimo, estos promedios de AOD y alfa no parecen coincidir con los típicos de aerosol de tipo marítimo. Según lo expuesto en la sección 1.2, la dependencia espectral del AOD debido a aerosol marítimo es pequeña, puesto que se supone formado por partículas gruesas. En nuestro caso tenemos valores de $\mathrm{AOD}(440 \mathrm{~nm})$ muy bajos y similares a los planteados por estos dos trabajos, pero sin embargo el valor de alfa tiene en nuestro caso unos valores mucho mayores. Probablemente la influencia continental y de fuentes locales de polución sea responsable de esta pendiente del espectro de AOD mayor que la esperada.

\begin{tabular}{lccccc}
\hline \hline Cluster 1 (MP) & AOD_1020 & AOD_870 & AOD_670 & AOD_440 & alfa(440-870) \\
\hline Media & 0.061 & 0.065 & 0.076 & 0.127 & 1.00 \\
Desv. Estándar & 0.045 & 0.045 & 0.053 & 0.079 & 0.44 \\
P25 & 0.036 & 0.038 & 0.045 & 0.077 & 0.68 \\
P75 & 0.073 & 0.077 & 0.088 & 0.147 & 1.34 \\
P90 & 0.109 & 0.113 & 0.129 & 0.220 & 1.53 \\
Mín. & 0.009 & 0.010 & 0.008 & 0.023 & -0.09 \\
Máx. & 0.444 & 0.467 & 0.506 & 0.797 & 1.95 \\
Mediana & 0.052 & 0.053 & 0.062 & 0.106 & 1.08 \\
\hline \hline
\end{tabular}

Tabla VI-VI. Estadística espectral del cluster 1 con los promedios diarios según la clasificación de trayectorias a $1500 \mathrm{~m}$ (sector Marítimo Polar)

El cluster 2 (sector Marítimo Tropical, Tabla VI-VII) tiene una frecuencia como vimos menor en $500 \mathrm{~m}$ (a favor del Mediterráneo) y mayor en el nivel de $3000 \mathrm{~m}$, ya que en este nivel el flujo del sudoeste es muy intenso (Jorba et al., 2004). Los valores de AOD van desde turbiedad baja, semejante al cluster 1 (MP) hasta los valores más altos $(\operatorname{AOD}(440)=1.23)$. Estos días con turbiedad alta corresponden a intrusiones saharianas que vienen desde el Atlántico. Como ejemplo mostramos en la Figura VI-10 la intrusión sahariana de febrero-marzo de 2000 con el modelo NAAPS y la imagen SeaWIFS (Seaviewing Wide Field-of-view Sensor). Este mecanismo parece ser más frecuente en el nivel de $1500 \mathrm{~m}$. Trataremos en detalle el tema de las intrusiones saharianas en el capítulo 6. Frente a estos casos, el $65 \%$ de los días el AOD en $440 \mathrm{~nm}$ está por debajo de 0.2, representando una situación similar a la Marítima Polar aunque con mayor turbiedad. Los valores de alfa son más frecuentes entre 0.5 y 1.5 , es decir, valores intermedios, excepto en los casos de sahariano, en los que alfa presenta valores bajos. 


\begin{tabular}{lccccc}
\hline \hline Cluster 2 (MT) & AOD_1020 & AOD_870 & AOD_670 & AOD_440 & alfa(440-870) \\
\hline Media & 0.114 & 0.122 & 0.140 & 0.202 & 0.91 \\
Desv. Estándar & 0.139 & 0.144 & 0.151 & 0.164 & 0.38 \\
P25 & 0.046 & 0.047 & 0.054 & 0.095 & 0.63 \\
P75 & 0.123 & 0.134 & 0.154 & 0.253 & 1.20 \\
P90 & 0.213 & 0.223 & 0.255 & 0.382 & 1.34 \\
Mín. & 0.009 & 0.013 & 0.011 & 0.026 & 0.00 \\
Máx. & 1.114 & 1.152 & 1.187 & 1.227 & 1.82 \\
Mediana & 0.082 & 0.086 & 0.106 & 0.165 & 0.95 \\
\hline \hline
\end{tabular}

Tabla VI-VII. Estadística espectral del cluster 2 con los promedios diarios según la clasificación de trayectorias a 1500m (sector Marítimo Tropical)

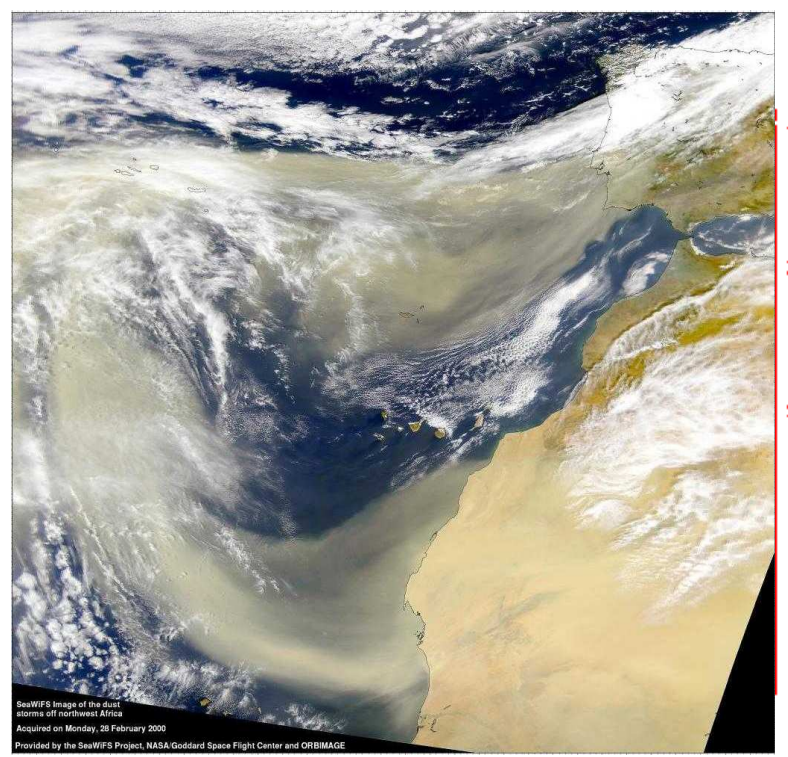

Nh.APS Surface Concentration (ug-m**a) for 12:00Z 26 Feb 2000 Dust

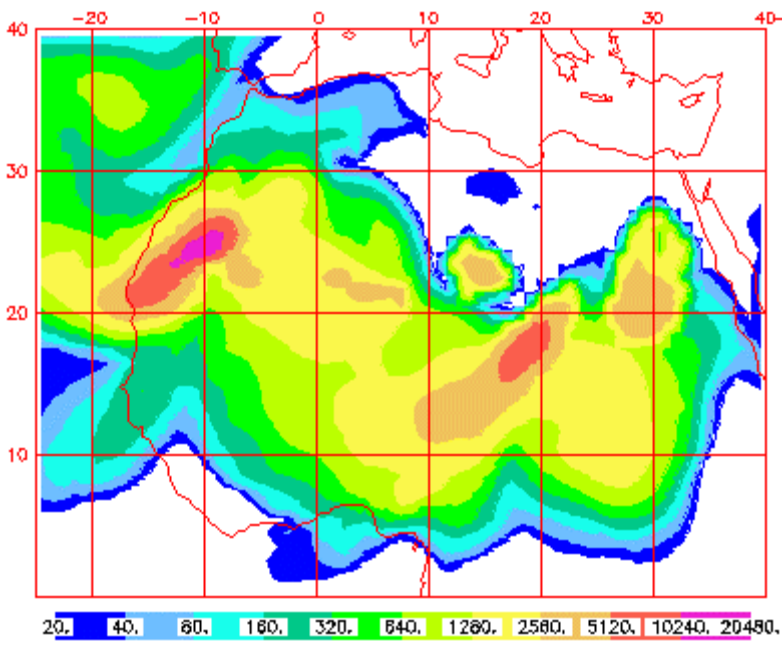

Figura VI-10. Ejemplo de llegada de aerosol sahariano desde el sector Marítimo Tropical. Evento de febrero de 2000: (a) Imagen SeaWIFS del día 28 de febrero de 2000; (b) Análisis de concentración de aerosol mineral dado por el modelo NAAPS para el mismo día 28 de febrero de 2000 a las $12 \mathrm{~h}$. Las medidas del fotómetro Cimel alcanzaron un AOD(440nm) de 0.48.

El cluster 3 (sector Ártico, Tabla VI-VIII) queda muy bien definido por las trayectorias de $1500 \mathrm{~m}$. Se trata de masas de aire muy limpias, con el promedio de AOD más bajo con 0.10 en $440 \mathrm{~nm}$. Alfa toma valores en casi todo el rango, desde casi 0 e incluso algunos negativos (cuestión que ya se ha comentado al respecto de los errores de este parámetro) hasta 1.6. El promedio es de 0.91, algo inferior al del cluster 1 (MP). Destacamos aquí que en el citado modelo OPAC, el valor de alfa para el aerosol de tipo Ártico es 0.89, con un AOD de 0.063 en 550nm (0.08 en 440nm aproximadamente), los cuales son valores bastante similares a los que hemos medido en estas masas de aire rápidas del norte o noroeste.

\begin{tabular}{lccccc}
\hline \hline Cluster 3 (A) & AOD_1020 & AOD_870 & AOD_670 & AOD_440 & alfa(440-870) \\
\hline Media & 0.056 & 0.059 & 0.064 & 0.101 & 0.91 \\
Desv. Estándar & 0.058 & 0.055 & 0.058 & 0.067 & 0.44 \\
P25 & 0.031 & 0.032 & 0.033 & 0.059 & 0.57 \\
P75 & 0.069 & 0.069 & 0.070 & 0.113 & 1.29 \\
P90 & 0.108 & 0.110 & 0.122 & 0.172 & 1.46 \\
Mín. & 0.012 & 0.015 & 0.011 & 0.029 & -0.05 \\
Máx. & 0.472 & 0.464 & 0.460 & 0.378 & 1.87 \\
Mediana & 0.041 & 0.042 & 0.049 & 0.083 & 0.96 \\
\hline \hline
\end{tabular}

Tabla VI-VIII. Estadística espectral del cluster 3 con los promedios diarios según la clasificación de trayectorias a 1500m (sector Ártico). 
El cluster 4 (sector Continental, Tabla VI-IX) recoge los casos más claros de trayectorias continentales aunque este carácter afecta a todos los clusters. Espectralmente se caracterizan por alfas altos y AOD medio, y tiene mayor frecuencia en los niveles bajos que en los niveles altos. El promedio de AOD es 0.196 con una desviación de 0.10, es decir, una turbiedad media en general. El promedio de alfa es 1.29 , el más alto de todos los clusters, rasgo que define este tipo de aerosol. Espectralmente es muy similar al cluster 6 (Local), lo cual es lógico pues las masas de aire que recirculen por encima de la Península tendrán un carácter continental más o menos marcado.

\begin{tabular}{lccccc}
\hline \hline Cluster 4 (C) & AOD_1020 & AOD_870 & AOD_670 & AOD_440 & alfa(440-870) \\
\hline Media & 0.074 & 0.085 & 0.107 & 0.196 & 1.29 \\
Desv. Estándar & 0.051 & 0.053 & 0.066 & 0.104 & 0.34 \\
P25 & 0.036 & 0.044 & 0.055 & 0.111 & 1.13 \\
P75 & 0.097 & 0.108 & 0.145 & 0.255 & 1.53 \\
P90 & 0.139 & 0.160 & 0.214 & 0.345 & 1.65 \\
Mín. & 0.016 & 0.018 & 0.022 & 0.044 & 0.28 \\
Máx. & 0.266 & 0.274 & 0.290 & 0.505 & 1.80 \\
Mediana & 0.066 & 0.075 & 0.099 & 0.184 & 1.37 \\
\hline \hline
\end{tabular}

Tabla VI-IX. Estadística espectral del cluster 4 con los promedios diarios según la clasificación de trayectorias a $1500 \mathrm{~m}$ (sector Continental)

El cluster 5 (sector Continental Tropical, Tabla VI-X) presenta como hemos dicho una frecuencia mayor y similar entre sí para los dos niveles altos, lo cual concuerda con el transporte de aerosol sahariano entre $1-4 \mathrm{~km}$ de altura. Los valores de AOD oscilan entre medios y altos, con un promedio de 0.30 que es el más alto entre todos los sectores. Los valores de alfa son bajos, con un promedio de 0.69 que es también el más bajo. Además el alfa disminuye al aumentar el AOD, lo cual es un rasgo típico del aerosol desértico. En el diagrama de dispersión se sitúan en la zona que corresponde a turbiedad moderada-alta debida a partículas gruesas.

\begin{tabular}{lccccc}
\hline \hline Cluster 5 (CT) & AOD_1020 & AOD_870 & AOD_670 & AOD_440 & alfa(440-870) \\
\hline Media & 0.198 & 0.208 & 0.229 & 0.302 & 0.69 \\
Desv. Estándar & 0.149 & 0.152 & 0.154 & 0.159 & 0.37 \\
P25 & 0.095 & 0.100 & 0.121 & 0.191 & 0.42 \\
P75 & 0.264 & 0.274 & 0.298 & 0.360 & 0.95 \\
P90 & 0.371 & 0.385 & 0.415 & 0.485 & 1.24 \\
Mín. & 0.026 & 0.027 & 0.034 & 0.065 & 0.05 \\
Máx. & 1.136 & 1.145 & 1.118 & 1.123 & 1.73 \\
Mediana & 0.166 & 0.175 & 0.198 & 0.273 & 0.61 \\
\hline \hline
\end{tabular}

Tabla VI-X. Estadística espectral del cluster 5 con los promedios diarios según la clasificación de trayectorias a $1500 \mathrm{~m}$ (sector Continental Tropical)

El cluster 6 (trayectorias Locales, Tabla VI-XI) agrupa a masas de aire que han recirculado cerca de nuestra zona de estudio, y tienen una turbiedad media con un promedio de AOD de 0.25 y más del $30 \%$ de medidas por encima de 0.3 en $440 \mathrm{~nm}$. El promedio de alfa es 1.12 lo que indica una cierta influencia continental. La huella espectral es muy similar para la clasificación en los tres niveles de trayectorias. Este efecto local con presencia de aerosol de tipo continental o de origen urbano parece influir bastante en los episodios de alta turbiedad debidos a aerosol desértico, puesto que es frecuente que esas masas tengan un tiempo de residencia grande en la zona de estudio, con recirculaciones en las que adquieren un carácter más continental. Esta influencia se traduciría en un aumento del parámetro alfa por encima de los valores típicos de aerosol desértico, tal y como se ha observado con el fotómetro Cimel durante episodios desérticos confirmados. 


\begin{tabular}{lccccc}
\hline \hline Cluster 6 (L) & AOD_1020 & AOD_870 & AOD_670 & AOD_440 & alfa(440-870) \\
\hline Media & 0.113 & 0.123 & 0.152 & 0.255 & 1.12 \\
Desv. Estándar & 0.065 & 0.069 & 0.080 & 0.124 & 0.40 \\
P25 & 0.063 & 0.067 & 0.088 & 0.165 & 0.89 \\
P75 & 0.135 & 0.146 & 0.189 & 0.327 & 1.43 \\
P90 & 0.229 & 0.245 & 0.276 & 0.374 & 1.57 \\
Mín. & 0.023 & 0.022 & 0.031 & 0.064 & 0.08 \\
Máx. & 0.302 & 0.316 & 0.439 & 0.871 & 1.73 \\
Mediana & 0.101 & 0.111 & 0.144 & 0.248 & 1.23 \\
\hline \hline
\end{tabular}

Tabla VI-XI. Estadística espectral del cluster 6 con los promedios diarios según la clasificación de trayectorias a $1500 \mathrm{~m}$ (sector Local)

El cluster 7 (sector Mediterráneo, Tabla VI-XII) es el que presenta una mayor disparidad entre el análisis realizado a cada una de las 3 alturas, no sólo por la frecuencia sino también espectralmente. Si atendemos a las trayectorias en $500 \mathrm{~m}$ diríamos que este cluster agrupa masas de aire con turbiedad media e incluso casos de AOD alto. Sin embargo este análisis no se ve corroborado por los niveles altos. La respuesta parece estar en que la llegada de masas de aire saharianas en los niveles altos, que traen como consecuencia valores altos de AOD y alfas bajos, vienen a menudo acompañadas por trayectorias mediterráneas en el nivel de 500m, como en los ejemplos de las Figura VI-3 y Figura VI-9c. Si atendemos al nivel de $1500 \mathrm{~m}$ para este cluster Mediterráneo, el promedio de $\mathrm{AOD}(440 \mathrm{~nm})$ es 0.25 , un valor intermedio y similar al de los cluster Continental y Local. Los valores de alfa, con un promedio de 1.12 son también similares a los C y L. En $3000 \mathrm{~m}$ hay muy pocos casos, por ser el flujo más zonal en este nivel. Esos pocos casos mantienen sobre todo la característica de un alfa alto (entre 1 y 1.5) y turbiedad mediabaja.

\begin{tabular}{lccccc}
\hline \hline Cluster 7 (Me) & AOD_1020 & AOD_870 & AOD_670 & AOD_440 & alfa(440-870) \\
\hline Media & 0.119 & 0.130 & 0.157 & 0.253 & 1.12 \\
Desv. Estándar & 0.092 & 0.093 & 0.100 & 0.131 & 0.39 \\
P25 & 0.057 & 0.063 & 0.080 & 0.150 & 0.90 \\
P75 & 0.147 & 0.168 & 0.205 & 0.341 & 1.40 \\
P90 & 0.211 & 0.231 & 0.314 & 0.443 & 1.56 \\
Mín. & 0.020 & 0.018 & 0.020 & 0.046 & 0.03 \\
Máx. & 0.437 & 0.447 & 0.470 & 0.576 & 1.75 \\
Mediana & 0.093 & 0.106 & 0.132 & 0.217 & 1.18 \\
\hline \hline
\end{tabular}

Tabla VI-XII. Estadística espectral del cluster 7 con los promedios diarios según la clasificación de trayectorias a $1500 \mathrm{~m}$ (sector Mediterráneo)

Queremos añadir un comentario al respecto de los puntos sueltos que podemos observar en los diagramas AOD-alfa de la Figura VI-8. Este es un tipo de análisis global de la relación entre masas de aire y medidas espectrales. Por supuesto en ciertos casos quedan puntos totalmente fuera de las regiones espectrales que hemos definido dentro de unos percentiles del conjunto de puntos. Frente a este análisis global, y para abordar esos casos será necesario realizar un análisis detallado en concreto y que se apoye en otras fuentes de información como mapas de satélite, análisis de modelos o instrumentos de medida in situ como PM10.

Para completar este análisis sobre los valores espectrales dados por el Cimel en cada uno de los clusters, tenemos desde la Figura VI-11 hasta la Figura VI-17 los histogramas de frecuencias de los valores de AOD en 440nm y alfa para cada uno de los 7 clusters, también para la clasificación por sectores con el nivel de $1500 \mathrm{~m}$. Los histogramas han sido realizados a partir de todas las medidas ( $\mathrm{y}$ no a partir de los promedios diarios) porque 
frente a 1337 días tenemos más de 40.000 observaciones individuales, que nos permiten construir unos histogramas más significativos.

Los histogramas de alfa nos pueden ayudar a discriminar en cierto modo el tipo de aerosol, ya que el parámetro alfa nos da información sobre la predominancia de partículas finas o gruesas. El sector Continental (Figura VI-14b) tiene un histograma que claramente muestra la predominancia de valores altos de alfa, con pico de frecuencia en 1.50, habituales para el aerosol de tipo continental. Menos clara pero igualmente presente es la mayor frecuencia de valores altos de alfa en los sectores Local y Mediterráneo (Figura VI$16 \mathrm{~b}$ y Figura VI-17b). En el sector local hay además un segundo pico de frecuencias en 0.50 que relacionamos con el aerosol desértico. Sólo en el caso del sector Continental Tropical (Figura VI-15b) los valores bajos de alfa predominan en la distribución de frecuencias, con un pico de frecuencia en 0.48 , lo cual define muy bien el aerosol desértico que llega de este sector. Esta misma figura tiene un segundo pico de frecuencia de alfa en 0.93, que puede estar relacionado con la influencia local o continental y las recirculaciones de las masas de aire de este grupo. Frente a esto, los histogramas de frecuencia del parámetro alfa de los sectores MP (Figura VI-11b), MT (Figura VI-12b) y A (Figura VI-13b) tienen los picos de frecuencia en valores altos (en torno a 1-1.3) pero toman valores en todo el rango. Esto no parece indicar un tipo de aerosol marítimo claro, aunque probablemente se deba a la situación costera de la estación, con fuerte influencia continental $e$ industrial. Sin embargo hay ciertas diferencias entre ellos. El histograma de alfa para el sector MP presenta muchos más casos por encima de 1.5 que el MT, en el que prácticamente no hay valores altos. A su vez el histograma del sector MT tiene un pequeño modo secundario en el valor de 0.30 , quizá relacionado con los casos de aerosol desértico en este sector. Todavía es más clara la presencia de un segundo modo en el sector Ártico, pero en este caso es de difícil interpretación pues se trata de medidas con AOD muy bajo, esto es, alfas sujetos a errores grandes, lo cual se ve confirmado también por los casos de alfas negativos en este sector.

Los histogramas de AOD en 440nm definen muy bien los clusters Marítimo Polar (Figura VI-11a) y Ártico (Figura VI-13a), que muestran la gran mayoría de datos en valores muy bajos de AOD y distribuciones muy estrechas, particularmente en el cluster Ártico, donde el pico de frecuencia está en un valor tan bajo como 0.06 para el AOD en $440 \mathrm{~nm}$. El pico de frecuencia en el cluster MP esté en 0.08. La distribución de AOD(440nm) en el cluster MT (Figura VI-12a) muestra las dos situaciones que concurren en este sector, con un grupo de valores con baja turbiedad (con pico de frecuencia en 0.1 ) y un segundo pico más bajo de frecuencia para valores medios en torno a 0.3 y con una pequeña fracción de casos que alcanzan valores altos en torno a 1 de espesor óptico de aerosoles en este canal de 440nm, que están relacionadas, como hemos comentado, con las entradas de aerosol sahariano por este sector Marítimo Tropical. La distribución es también más ancha, como se refleja en la desviación estándar del AOD de 0.16, el doble que para el cluster MP (con STD de 0.08) y más del doble que en el cluster Ártico (STD de 0.067 para AOD en 440nm).

$\mathrm{El} \mathrm{AOD(440nm)} \mathrm{en} \mathrm{el} \mathrm{cluster} \mathrm{Continental} \mathrm{(Figura} \mathrm{VI-14a)} \mathrm{tiene} \mathrm{una} \mathrm{frecuencia} \mathrm{que} \mathrm{se}$ mantiene estable entre 0.1 y 0.25 para luego decaer hasta 0.6. Es una distribución de una anchura intermedia, con STD de 0.10 para AOD en 440. Como hemos destacado ya, el rasgo más característico de este cluster son los altos valores de alfa que van asociados a estos valores intermedios de AOD.

El sector Continental Tropical (Figura VI-15a) presenta los valores de AOD más altos, con la distribución de frecuencias más ancha junto con la MT (STD de 0.16) y más desplazada hacia valores altos de AOD. El pico de frecuencia está en 0.2 pero ésta se mantiene alta hasta 0.35 y luego decae lentamente hasta valores de 0.75 . Igual que en sector MT, en el 
sector CT sigue habiendo un cierto número de casos hasta valores de $\mathrm{AOD}(440 \mathrm{~nm})$ superiores a 1.1, que se corresponden con las entradas más fuertes de polvo sahariano. El sector Local (Figura VI-16a) tiene dos picos de frecuencia en 0.15 y 0.30 respectivamente, aunque también se aprecien ciertos episodios con AOD alto. En realidad las observaciones en torno a 0.8 de AOD corresponden a un solo día, pero al tener este cluster un número pequeño de casos, las medidas de un día tienen una presencia significativa. Por último, la distribución de $\mathrm{AOD}(440 \mathrm{~nm})$ en el sector Mediterráneo (Figura VI-17a) tiene el pico de frecuencia en 0.16 , aunque al igual que en el sector Local la distribución tiene un segundo pico de frecuencia, más bajo, en 0.3. Encontramos frecuencias significativas hasta 0.7 de espesor óptico de aerosoles en este canal de $440 \mathrm{~nm}$, lo cual sugiere que las masas de aire mediterráneas pueden llegar muy sucias a la estación.
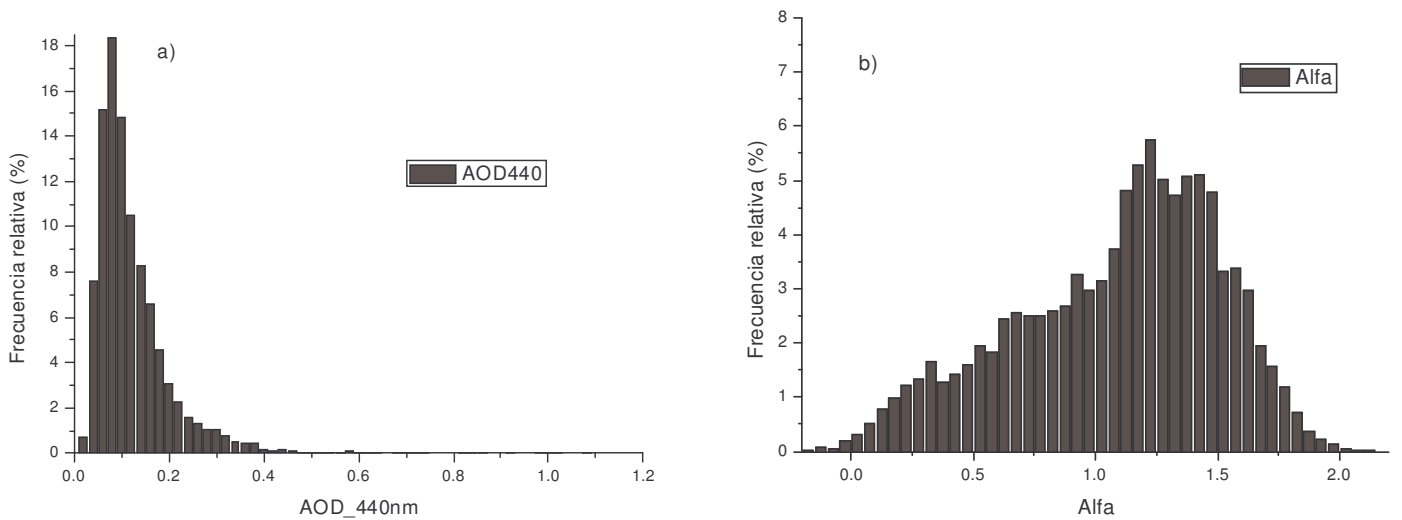

Figura VI-11. Histogramas de frecuencias relativas (\%) de (a) $\mathrm{AOD}(440)$ y (b) alfa para el sector 1 (Marítimo Polar) según la clasificación con las trayectorias en $1500 \mathrm{~m}$.
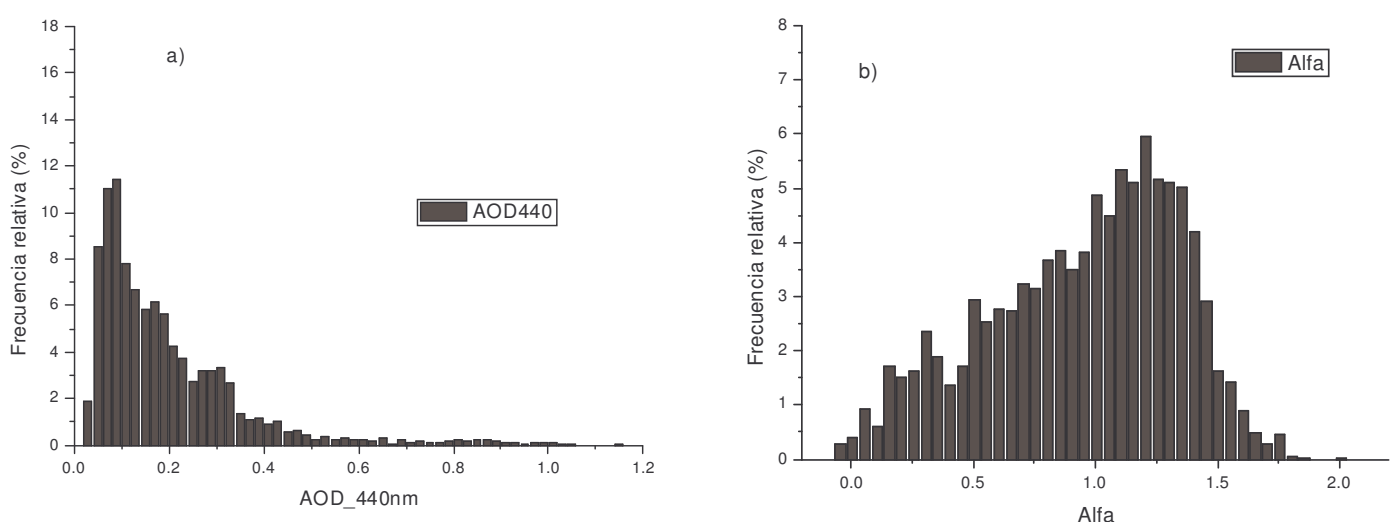

Figura VI-12. Histogramas de frecuencias relativas (\%)de (a) $\mathrm{AOD}(440)$ y (b) alfa para el sector 2 (Marítimo Tropical) según la clasificación con las trayectorias en 1500m.
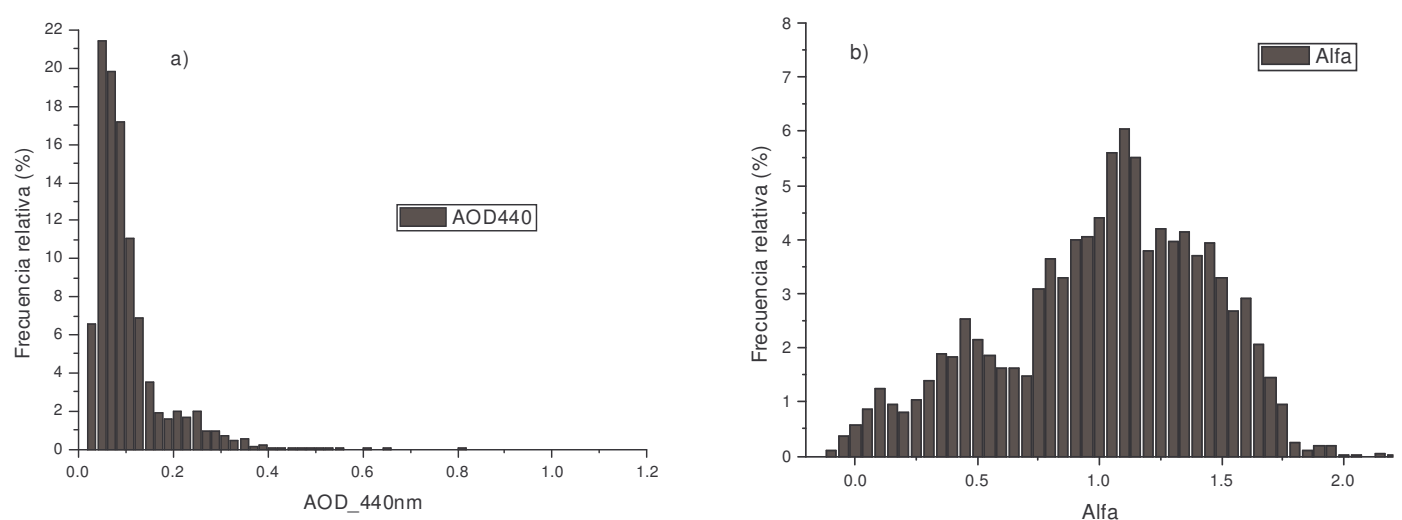

Figura VI-13. Histogramas de frecuencias relativas (\%)de (a) $\mathrm{AOD}(440)$ y (b) alfa para el sector 3 (Ártico) según la clasificación con las trayectorias en 1500m. 

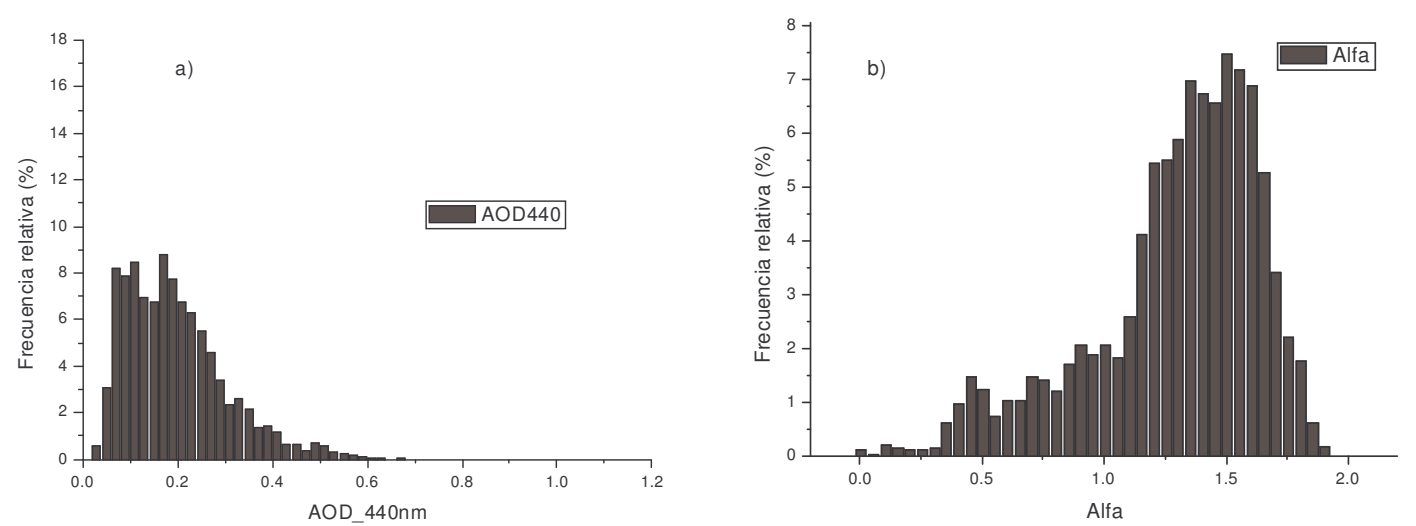

Figura VI-14. Histogramas de frecuencias relativas (\%) de (a) AOD(440) y (b) alfa para el sector 4 (Continental) según la clasificación con las trayectorias en $1500 \mathrm{~m}$.
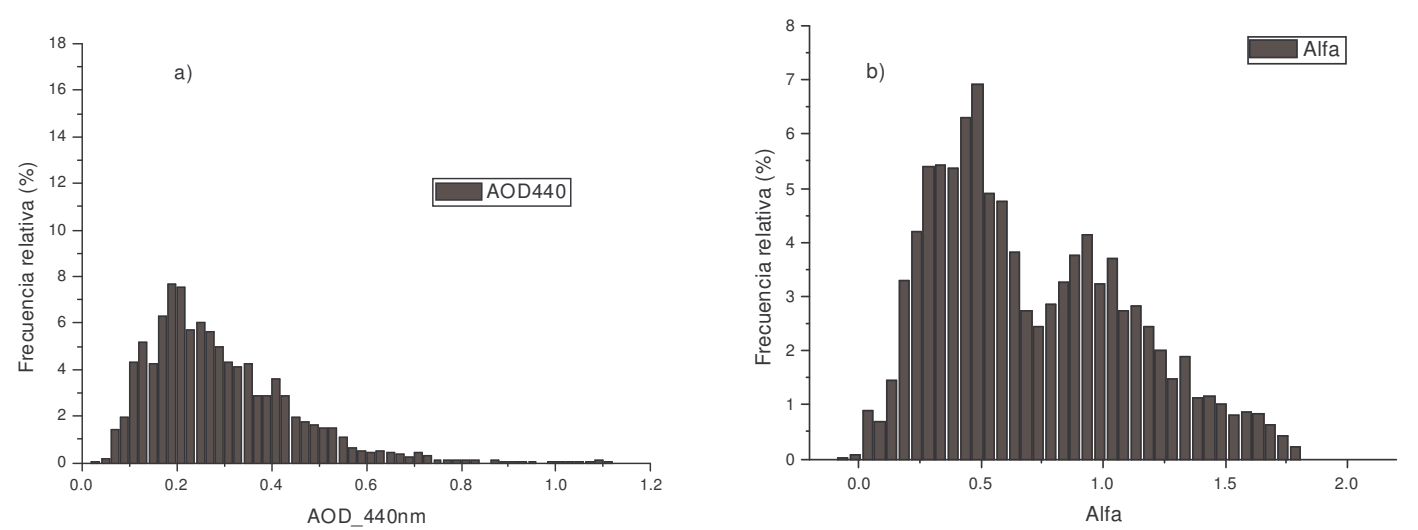

Figura VI-15. Histogramas de frecuencias relativas (\%) de (a) $\mathrm{AOD}(440)$ y (b) alfa para el sector 5 (Continental Tropical) según la clasificación con las trayectorias en $1500 \mathrm{~m}$.
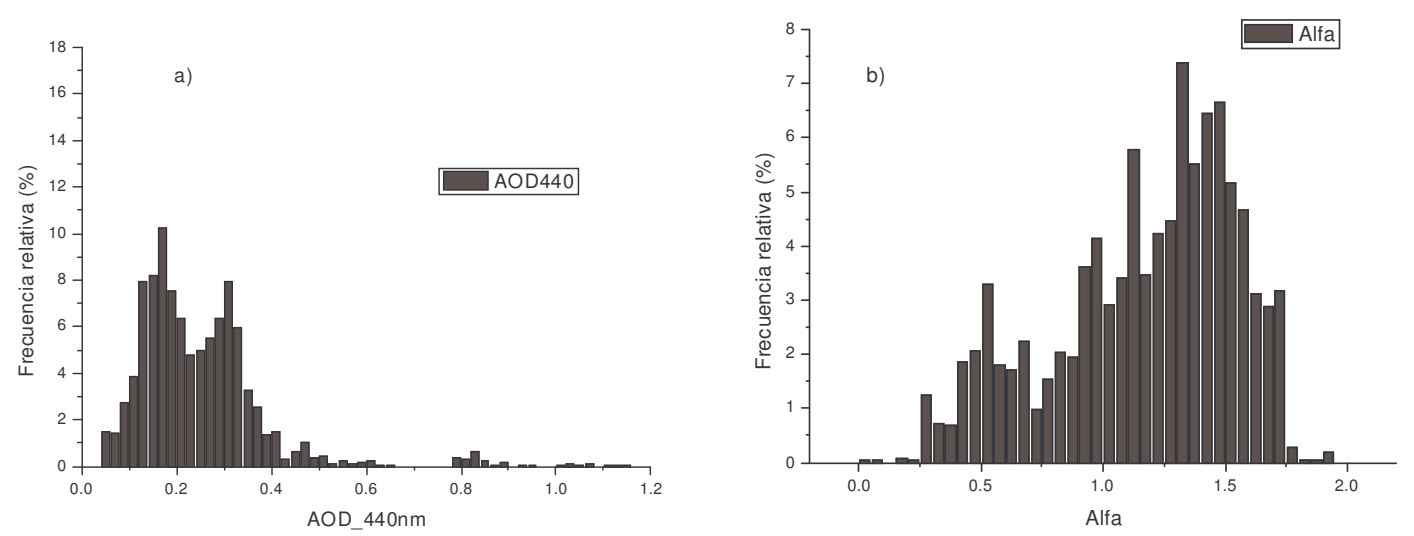

Figura VI-16. Histogramas de frecuencias relativas (\%)de (a) AOD(440) y (b) alfa para el sector 6 (Local) según la clasificación con las trayectorias en 1500m. 

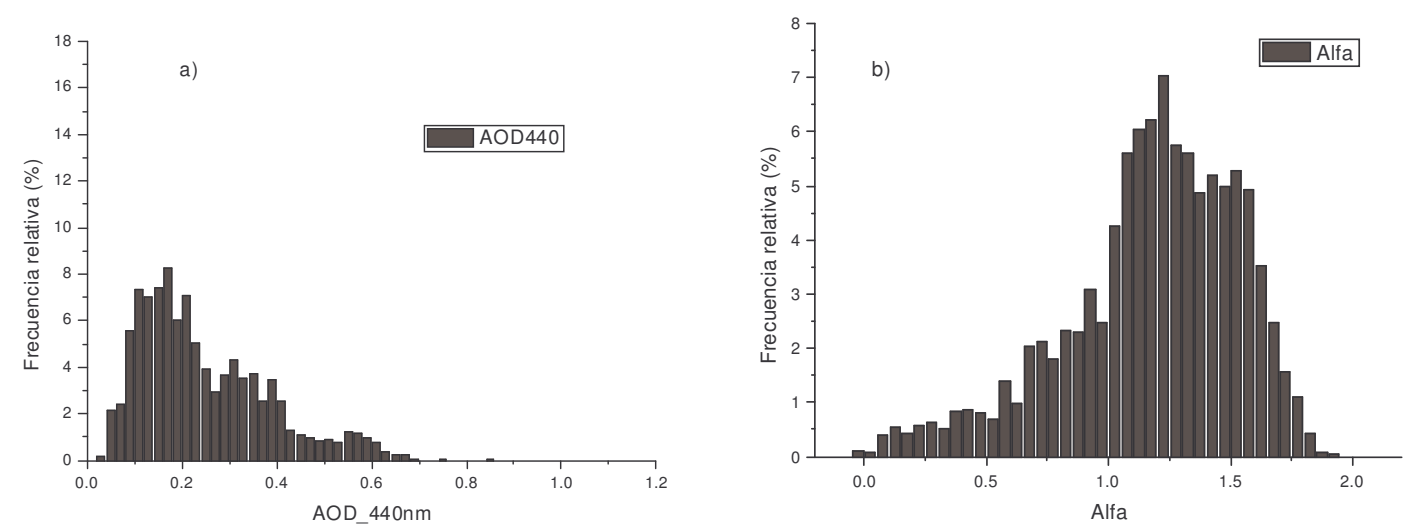

Figura VI-17. Histogramas de frecuencias relativas (\%)de (a) AOD(440) y (b) alfa para el sector 7 (Mediterráneo) según la clasificación con las trayectorias en $1500 \mathrm{~m}$. 


\subsection{Clasificación de las retrotrayectorias mediante un algoritmo de cluster no jerárquico}

En esta sección abordamos una clasificación de las masas de aire a partir de un algoritmo de cluster que vamos a aplicar a las trayectorias calculadas con el modelo HYSPLIT. Esto nos va a permitir agrupar trayectorias que son similares entre sí, es decir, que corresponden con situaciones sinópticas análogas.

Esta metodología de clasificación es completamente diferente a la anterior, que estaba basada en sectores de masas de aire que definimos a priori mediante una rejilla de clasificación. Esta clasificación mediante el algoritmo no jerárquico de cluster está basada en la distancia geométrica entre las trayectorias, en dos dimensiones -latitud y longitud. De esta manera se tiene en cuenta tanto la velocidad como la dirección, obteniéndose clusters de longitud y curvatura similares (Stohl, 1998).

\subsubsection{Descripción del algoritmo de clasificación}

El procedimiento de análisis de clusters se puede considerar más objetivo que el método expuesto en la sección anterior, pues no parte de ninguna asignación subjetiva de sectores. Sin embargo el procedimiento de clasificación mediante un algoritmo de cluster requiere varias elecciones por parte del usuario: el tipo de algoritmo a utilizar, la definición de la distancia, las trayectorias semilla y el número de clusters.

Al respecto de la elección del algoritmo, consideramos adecuado un algoritmo de tipo no jerárquico. Esto permite que los distintos elementos se agrupen en el cluster más apropiado para cada paso del proceso, hasta que finalmente todas las trayectorias quedan en un único cluster. Los trabajos de Dorling et al., (1992) y Jorba et al. (2004) plantean una clasificación de masas de aire mediante un algoritmo de este tipo. El procedimiento se inicia mediante una serie de trayectorias semilla, con las cuales se realiza una asignación inicial de cada trayectoria a un cluster. Sin embargo los autores no inciden en el problema de que el resultado final pueda depender de esta asignación inicial, como advierte Peña (2002). Esta cuestión la abordaremos enseguida. Por otra parte este algoritmo no tiene grandes requerimientos de computación y es por tanto adecuado para tratar una serie de datos de 5 años como la nuestra.

La distancia en la que se basa el algoritmo ha sido definida a partir de la distancia euclídea entre puntos en un plano, ya que sólo hemos considerado las variables latitud y longitud. Cada trayectoria consta de 121 puntos sobre ese plano, y la distancia con los centroides se realiza punto a punto desde la hora de llegada $(\mathrm{t}=0)$ hasta el punto de origen cinco días antes, es decir, $\mathrm{t}=120$ horas:

$$
d(1,2)^{2}=\sum_{i=0}^{120}\left[\left(\lambda_{1 i}-\lambda_{2 i}\right)^{2}+\left(\varphi_{1 i}-\varphi_{2 i}\right)^{2}\right]
$$

En esta expresión ' $d$ ' es la distancia que hemos definido entre trayectorias, $\lambda_{1 \mathrm{i}}$ y $\lambda_{2 \mathrm{i}}$ son las latitudes de las curvas ' 1 ' y ' 2 ' para la hora 'i' de vuelo mientras que $\varphi_{1 \mathrm{i}}$ y $\varphi_{2 \mathrm{i}}$ son las correspondientes coordenadas de longitud. Aquí es fácil intuir la posibilidad de realizar variaciones sobre la definición de la distancia, por ejemplo dando mayor peso a los puntos del principio o del final de la retrotrayectoria, o considerando además de éstas otras de las variables proporcionadas por el modelo HYSPLIT, como la altura, la presión, etc. 
Las trayectorias semilla que hemos utilizado para arrancar el proceso se han generado de forma sintética, y son 32 radiales desde el punto de llegada, es decir, una trayectoria recta cada $11,25^{\circ}$ y de radio $50^{\circ}$. También se han utilizado otras semillas iniciales, rotando $5^{\circ}$ esta configuración, otra aumentando el radio y finalmente otra con una variación lineal de longitud y latitud. Se trata de evaluar la sensibilidad del método a las trayectorias semilla que se utilicen. El resultado que se obtiene es idéntico en todos los casos a partir de 4 clusters. Entre 5 y 9 clusters el resultado es muy similar, con clusters análogos y más del $90 \%$ de trayectorias en los mismos clusters. Las pequeñas diferencias se deben tan solo a que algunas trayectorias quedan asignadas a algún cluster contiguo, pero esencialmente el resultado no cambia.

El funcionamiento general del algoritmo es el siguiente: generamos un número grande de trayectorias semilla, 32 en nuestro caso, que se distribuyen uniformemente por toda la superficie que ocupan nuestras trayectorias. Asignamos cada trayectoria a la trayectoria semilla más cercana según la distancia que hemos definido previamente (6.1), con lo que formamos unos clusters iniciales. A partir de las trayectorias (reales) de cada grupo calculamos los centroides como la trayectoria promedio del cluster. Estos primeros centroides son distintos de las semillas, por lo que habrá trayectorias que no estén asignadas al cluster más cercano. Volvemos por tanto a asignar cada trayectoria al centroide más cercano y volvemos a calcular los nuevos centroides de cada grupo. Este proceso se repite hasta que todas las trayectorias estén correctamente asignadas. Ésta es la asignación definitiva para 32 clusters.

Una vez hecho esto, vamos reduciendo el número de clusters. Para ello se unen los dos clusters cuyos centroides están más cercanos entre sí y se calcula el nuevo centroide. Comprobamos que todas las trayectorias estén correctamente asignadas mediante el proceso ya indicado de asignación, cálculo de los nuevos centroides y volver a asignar, etc. Cuando la asignación ya no cambia, se vuelve a unir los dos clusters más cercanos. De esta manera se va reduciendo el número de clusters hasta 1.

Para la elección del número de clusters hemos empleado el procedimiento descrito en Dorling et al. (1992). Para ello nos valemos de la raíz de la desviación cuadrática media (rmsd del inglés Root Mean Square Deviation) de cada trayectoria, que no es más que la distancia entre una trayectoria y el centroide del cluster al que pertenece. La suma de estas distancias para todas las trayectorias nos da una desviación cuadrática media total. Ésta se evalúa para cada paso, y se enfrenta en un diagrama al número de clusters. Según el algoritmo va agregando trayectorias, y el número de clusters se va reduciendo, esta desviación cuadrática total crece pero no de manera constante. Cuando dos clusters muy distintos entre sí son agregados, el cambio en la rmsd total es mayor en términos relativos. Cuando en un cierto paso del algoritmo ese cambio es mayor de un cierto umbral (un $5 \%$ en Jorba et al., 2004) nos quedamos con ese número de clusters. Nuestro criterio va a ser, sin embargo, el $4 \%$ de cambio en la desviación cuadrática media total. Para ello nos hemos basado también en los resultados que se obtienen posteriormente al comparar con las medidas espectrales del Cimel, y en el hecho de que nos hemos encontrado varios pasos seguidos con un cambio en rmsd entre el $4 \%$ y el $5 \%$, por lo que el número de clusters se reducía demasiado hasta que en un cierto paso se superaba el $5 \%$ de cambio.

Para aplicar este algoritmo de cluster a la serie de datos de 5 años de retrotrayectorias, se ha realizado un programa en lenguaje IDL. Las entradas del programa son: los 1781 ficheros de texto de trayectorias del modelo HYSPLIT tal y como salen del modelo; y un fichero de texto con las 32 trayectorias semilla. A partir de ahí un Pentium III emplea unas 2 horas para realizar el proceso completo. Las salidas son un fichero que contiene la rmsd para cada paso, y luego pares de ficheros para cada número de clusters del proceso, uno 
con la asignación de cada trayectoria (con la trayectoria identificada por la fecha y cluster al que pertenece) y otro con los centroides (dados por puntos hora a hora de latitud y longitud).

\subsubsection{Aplicación del algoritmo de cluster no jerárquico a las trayectorias en El Arenosillo}

Utilizamos este algoritmo para clasificar la serie de trayectorias de 5 años en El Arenosillo para los niveles de 500, 1500 y 3000m. En primer lugar analizamos la desviación cuadrática media total (que hemos llamado rmsd) para cada paso del algoritmo, de cara a elegir el número de clusters apropiados. Esto ha de hacerse independientemente para cada nivel.

El criterio que hemos elegido para seleccionar un número de clusters es que el cambio relativo en $r$ msd sea superior al 4\%. Este umbral se supera al pasar de 7 a 6 clusters en todos los niveles (500m, 1500m y 3000m). Esta gráfica para el nivel de $1500 \mathrm{~m}$ se muestra en la Figura VI-18, en la que vemos cómo el cambio relativo de rmsd no es constante al reducir el número de clusters, y que supera nuestro umbral del $4 \%$ por primera vez al reducir de 7 a 6 clusters.

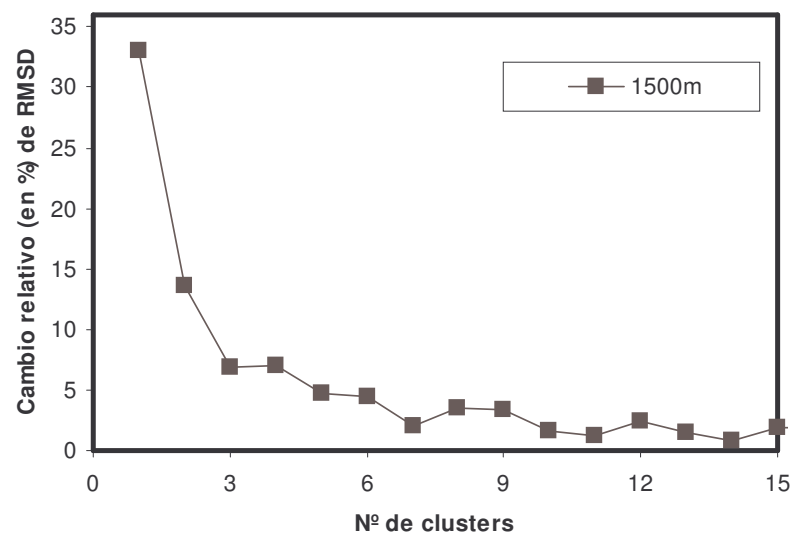

Figura VI-18. Diagrama del cambio relativo de RMSD en función del número de clusters para el nivel de $1500 \mathrm{~m}$.

Con esto ya podemos mostrar el resultado de la clasificación. En primer lugar tenemos en la Figura VI-19 los centroides, calculados como el promedio de cada cluster, para los tres niveles de trayectorias. En los niveles bajos, en $500 \mathrm{~m}$, tenemos un mayor número de trayectorias procedentes del norte o del este, lo que se traduce en unos centroides promedio de masas de aire del norte (cluster 3 y 7 en $500 \mathrm{~m}$ ) y otro centroide de masas de aire mediterráneas (cluster 4 en $500 \mathrm{~m}$ ), que llegan entre el nordeste y el sudeste. Por el contrario, el flujo zonal Atlántico es más lento que en los niveles superiores (cluster $2,5 \mathrm{y}$ 6). Por último, el cluster 1 agrupa trayectorias cortas (y por tanto lentas) de masas de aire y recirculaciones que llegan entre el oeste y el sur.

En 1500m el número de trayectorias mediterráneas disminuye mucho, por lo que ya no tenemos un centroide que provenga claramente del este. El cluster 3 en 1500m agrupa trayectorias entre el este y el sur, es decir, las mediterráneas de este nivel y las norteafricanas. El flujo del norte es más zonal a esta altura, y pasa a ser noroeste (cluster 5). Sin embargo se mantiene un centroide que llega desde el norte-nordeste, desde el continente europeo (cluster 4). El flujo zonal Atlántico es más rápido que en $500 \mathrm{~m}$ (cluster 1,2 y 6), y las trayectorias lejanas del noroeste llegan desde los $100^{\circ} \mathrm{W}$ de longitud (cluster 
1 en $1500 \mathrm{~m}$ ). Las trayectorias Atlánticas del cluster 6 llegan curvadas, con clara influencia de un anticiclón en estas masas de aire. Finalmente tenemos un centroide (cluster 7) de trayectorias cortas desde el oeste y sudoeste.
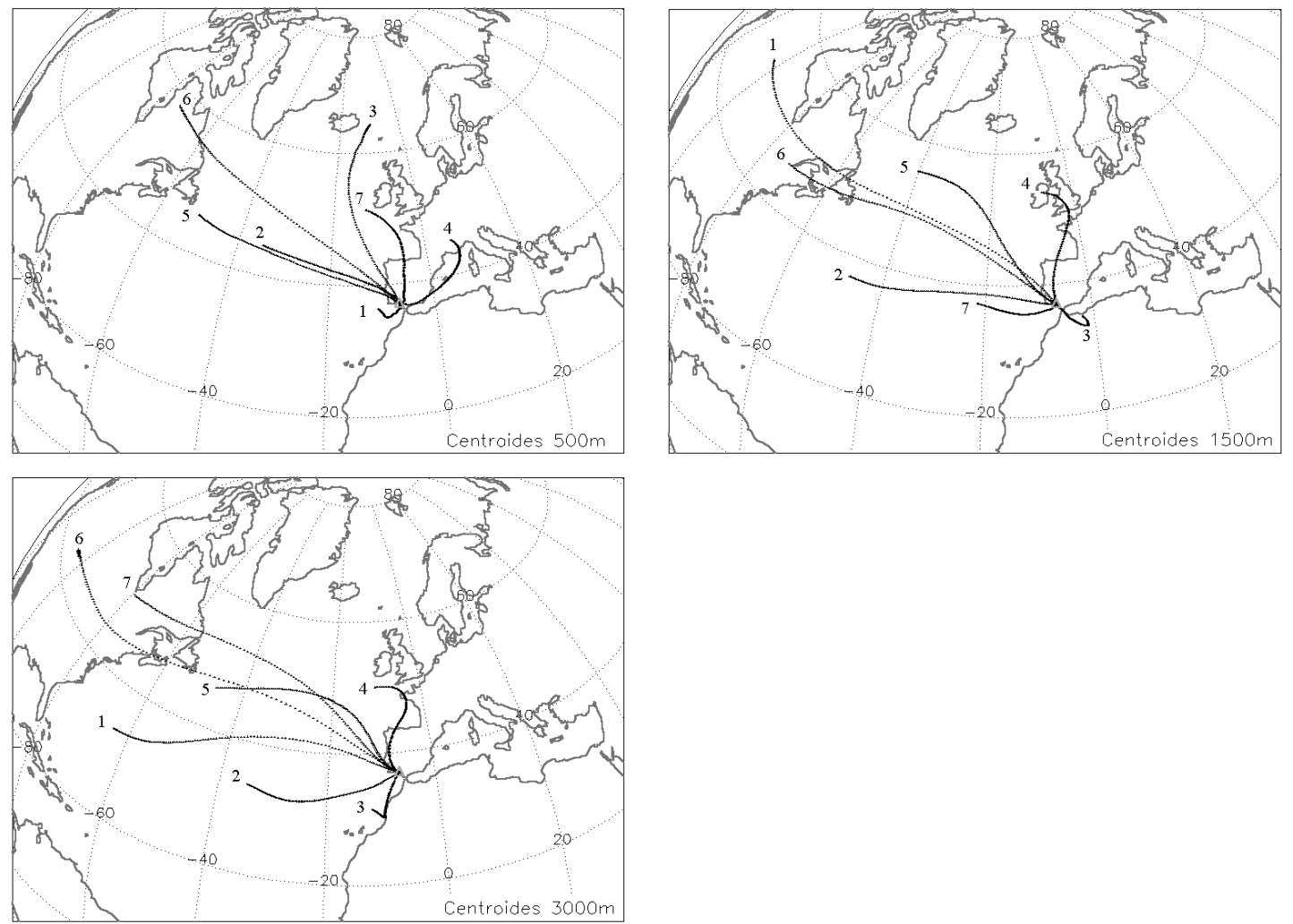

Figura VI-19. Centroides de la clasificación mediante el algoritmo de cluster no jerárquico: (a) en 500m; (b) en 1500m; (c) en 3000m.

En $3000 \mathrm{~m}$ el flujo zonal domina en casi todos los clusters, y es más rápido que en los niveles bajos. Tiene además una acusada curvatura (cluster 1, 5, 6 y 7). Las trayectorias atlánticas del sudoeste son también más rápidas en $3000 \mathrm{~m}$, y por tanto su centroide es más largo (cluster 2). Se mantiene el cluster del norte (cluster 4) que hemos visto en $500 \mathrm{~m}$ $y$ en $1500 \mathrm{~m}$. Por último el cluster 3 recoge las trayectorias que llegan del sur, norteafricanas, mientras que las trayectorias mediterráneas han desaparecido casi totalmente en este nivel.

En segundo lugar mostramos en la Figura VI-20 todas las trayectorias pertenecientes a cada cluster para el nivel de $1500 \mathrm{~m}$, que de nuevo es la altura que hemos elegido para mostrar los resultados de la clasificación. Los diferentes grupos se pueden etiquetar como sigue:

1. Noroeste lejanas

2. Atlánticas Oeste

3. Norteafricanas

4. N-NE cercanas

5. Ártico

6. Atlánticas curvadas

7. Sudoeste cercanas

En estos mapas de la Figura VI-20 se puede observar que los clusters agrupan trayectorias de similar longitud y curvatura, cuestión que no sucedía en la clasificación por sectores (ver la Figura VI-5). Se trata por tanto de una clasificación mucho más relacionada con situaciones sinópticas comunes y que reproduce 7 situaciones meteorológicas típicas en El 
Arenosillo. De hecho, salvo matices que tienen que ver con las masas de origen Mediterráneo, la clasificación es muy similar a la que muestra Jorba et al. (2004) para la zona de Barcelona.

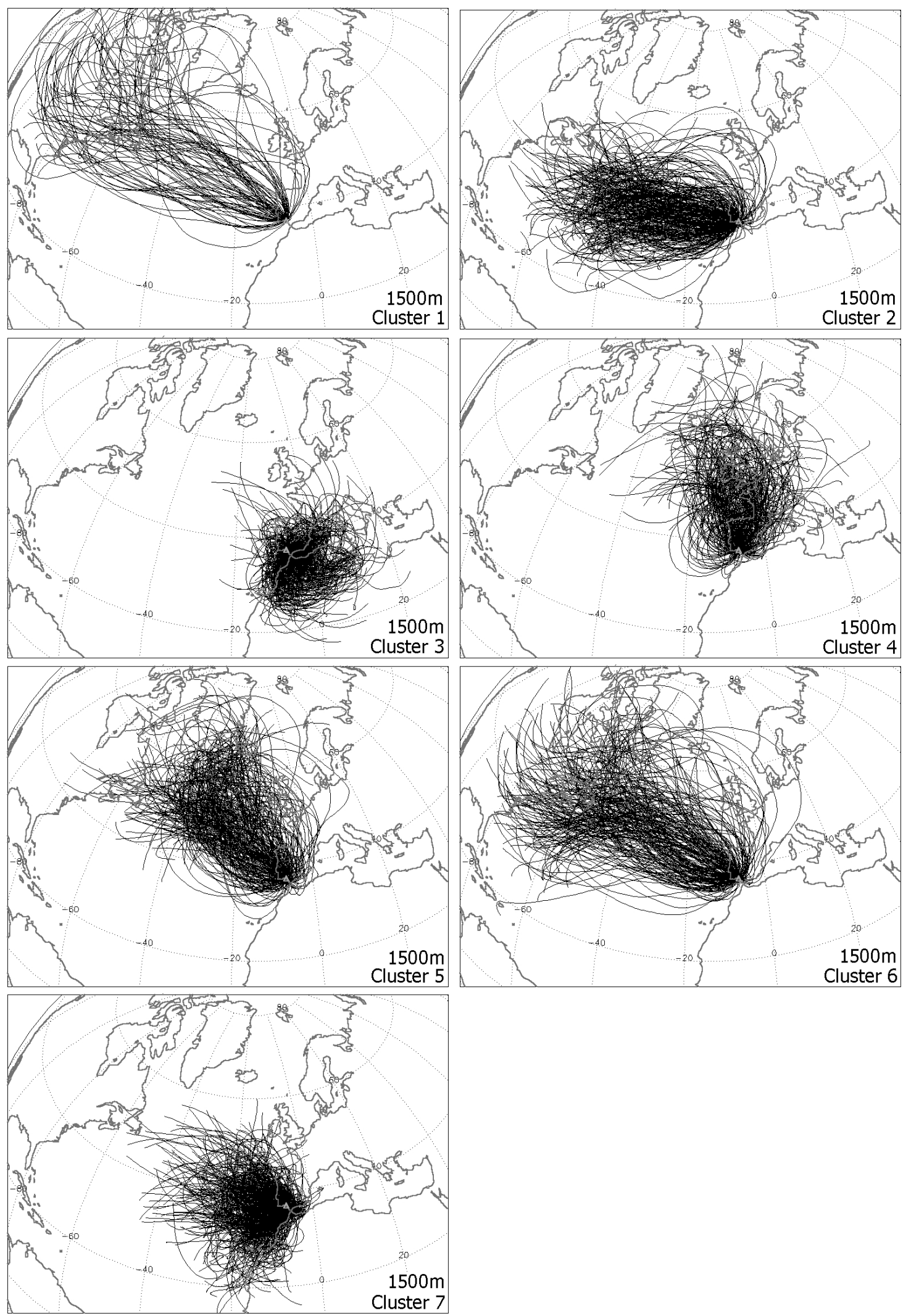

Figura VI-20. Trayectorias de cada cluster para la clasificación en $1500 \mathrm{~m}$ mediante el algoritmo de cluster no jerárquico. 
Con esto podemos ver el grupo de trayectorias que da lugar a cada centroide mostrado en la Figura VI-19. Las trayectorias cortas, relacionadas con situaciones estables y recirculaciones de masas de aire, son las más complicadas de clasificar. En este caso se encuentran repartidas por los clusters 3, 4 y 7 . En el cluster 3 tenemos las trayectorias entre el este y el sur, desde el norte de África, y a priori este grupo estará asociado a las entradas de aerosol desértico. Del mismo modo el cluster 7 agrupa trayectorias atlánticas del SW muy cortas, lo cual se relaciona también con cierto tipo de intrusiones de polvo sahariano (ver Figura VI-10). El cluster 4 agrupa las trayectorias cortas que llegan desde el norte, desde el continente europeo y a través de la Península, lo que a priori podría estar relacionado con aerosol de tipo continental. Las trayectorias más largas del oeste y noroeste están relacionadas con situaciones de vientos más fuertes y flujos más definidos, ya sean ciclónicos o anticiclónicos. Este flujo del Atlántico puede ser más o menos rápido, zonal y curvado, lo que da lugar a cuatro clusters diferentes.

Podemos mostrar como ejemplo algunas situaciones sinópticas mediante el análisis de presión en superficie. Estos mapas corresponden con días que han sido agrupados dentro de cada cluster. No pretendemos introducir aquí una descripción exhaustiva de la climatología peninsular, lo que por otra parte presenta numerosas complicaciones como se describe en la recopilación de este tipo de estudios realizada por Jorba (2005). Simplemente queremos acompañar los mapas de las trayectorias de cada cluster con una situación sinóptica típica como mostramos en Figura VI-21. Esto tampoco significa que todas las trayectorias del cluster respondan a situaciones análogas a las de los ejemplos mostrados, ya que en general hay varias situaciones posibles dentro de cada cluster y nuestro objetivo no es describirlas todas.

(a)

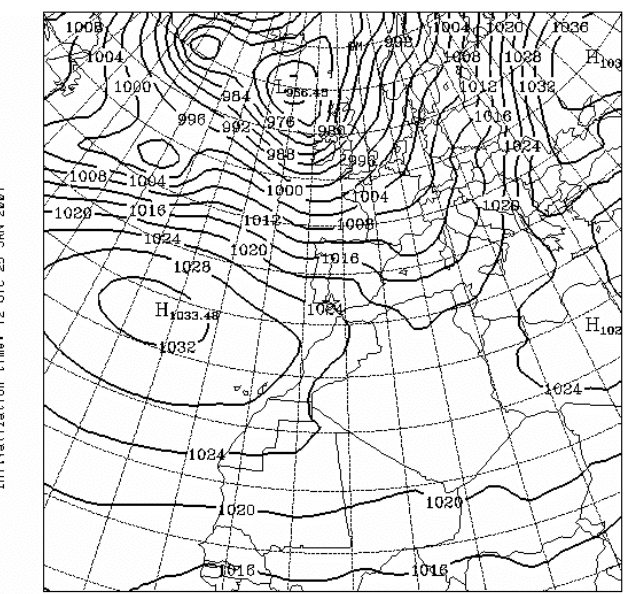

(c)

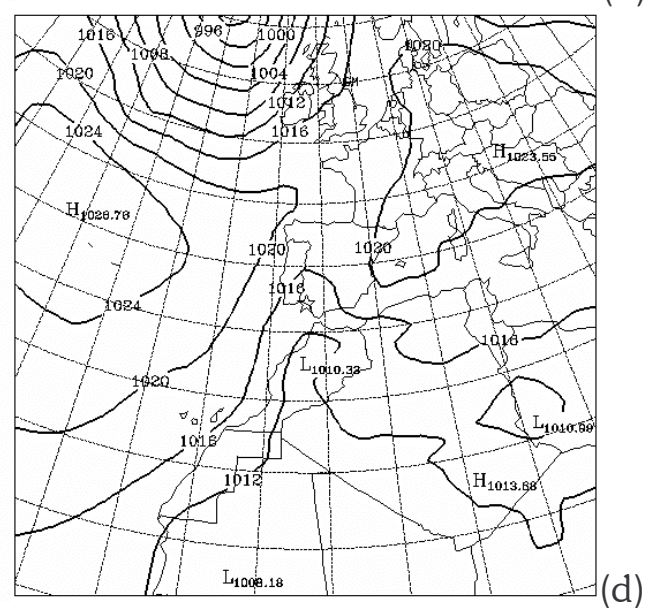

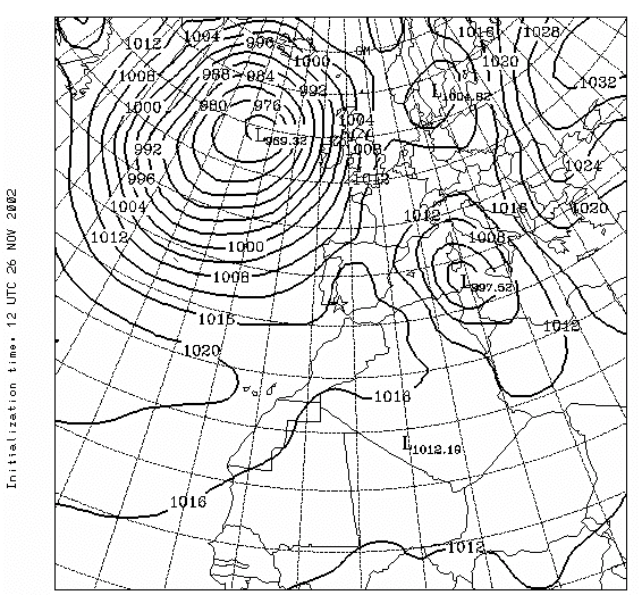

(b)

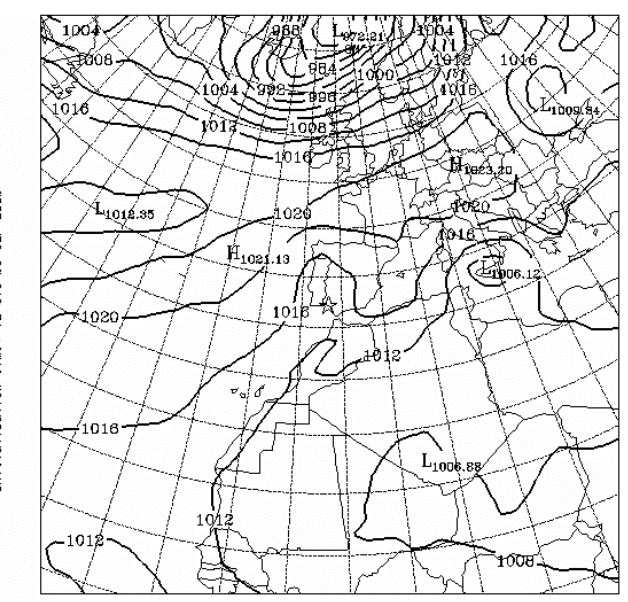



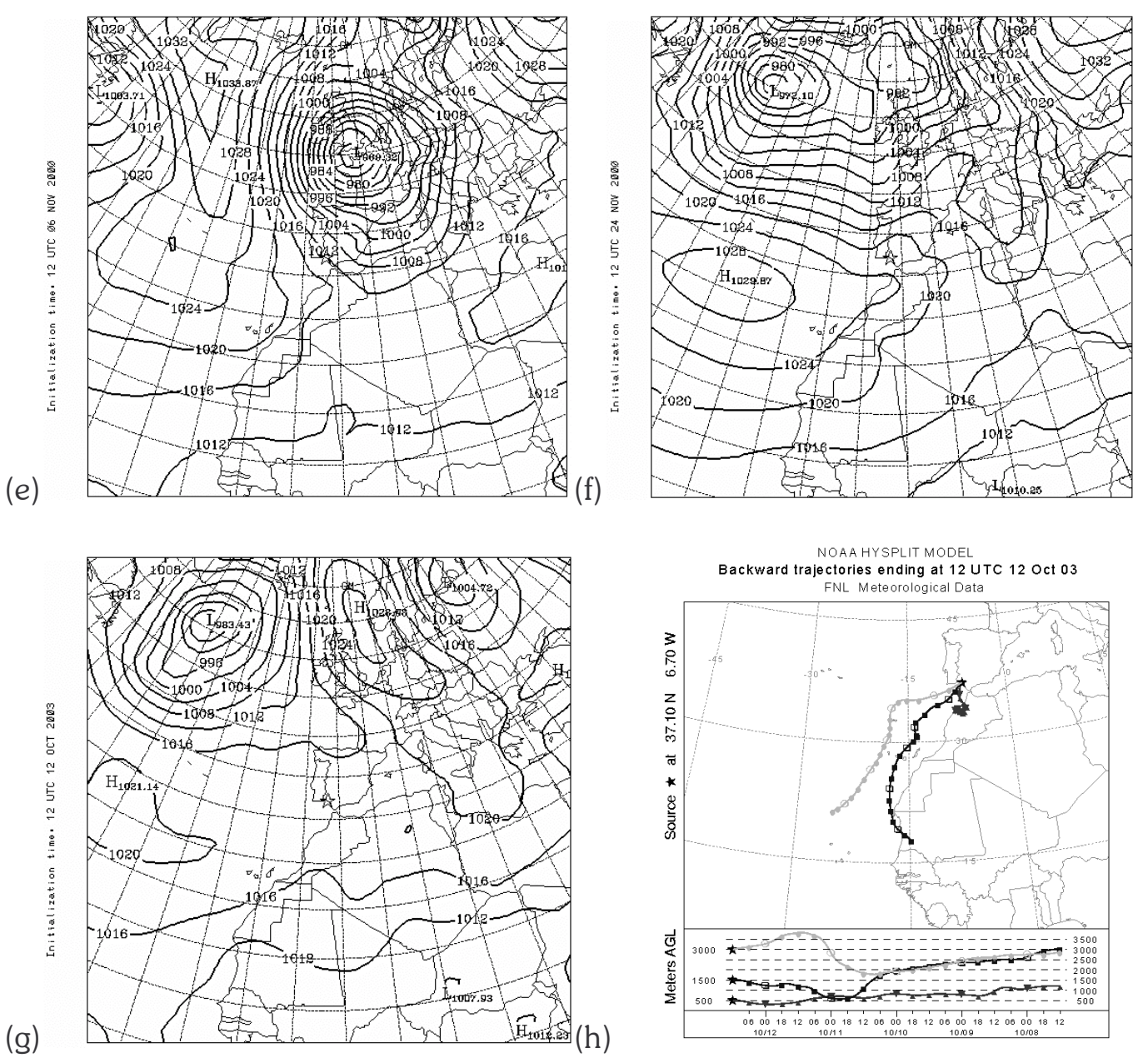

Figura VI-21. Situaciones sinópticas de ejemplo para cada cluster de trayectorias, mediante el mapa de presión en superficie: (a) día 25 de enero de 2001; (b) día 26 de noviembre de 2002; (c) día 15 de mayo de 2000; (d) día 8 de septiembre de 2000; (e) día 6 de noviembre de 2000; (f) día 24 de noviembre de 2000; (g) día 12 de octubre de 2003; (h) retrotrayectorias correspondientes al 12 de octubre de 2003.

La Figura VI-21a corresponde al día 25 de enero de 2001, ejemplo del cluster 1 (noroeste lejanas). La presencia del anticiclón Atlántico y una baja profunda al noroeste de las islas Británicas provoca un intenso flujo zonal, por lo que las trayectorias son muy rápidas y nos llegan desde zonas muy alejadas al oeste y noroeste. La Figura VI-21b, análisis del día 26 de noviembre de 2002, es un ejemplo de situación sinóptica del cluster 2 (atlánticas del oeste). La posición de un borrasca sobre el Atlántico nos hace llegar masas de aire templado desde el oeste o sudoeste. También nos llegan masas atlánticas en el cluster 5, en este caso desde el noroeste. Como vemos en el ejemplo de la Figura VI-21e (mapa del día 6 de noviembre de 2000) estas masas de aire pueden llegar detrás del frente frío, una vez que las bajas presiones se van internando en el continente europeo. El flujo zonal Atlántico del cluster 6 es similar al del cluster 1, aunque menos intenso. La situación que mostramos, del día 24 de noviembre de 2000 (Figura VI-21f) es también similar a la del cluster 1. Estas masas de aire muestran una cierta curvatura al cruzar el Atlántico que podemos relacionar con el anticiclón de las Azores.

Las situaciones sinópticas en los clusters 3, 4, y 7 son quizá menos claras que las mencionadas en el párrafo anterior. Se trata de trayectorias cortas, por lo que encontramos muchas situaciones con escasa variación de las presiones, y también bastantes escenarios diferentes para cada cluster. El ejemplo de situación sinóptica del cluster 3 (día 15 de mayo de 2000, Figura VI-21c) nos muestra la baja térmica norteafricana. En este caso la masa de aire en $1500 \mathrm{~m}$ nos llega desde el sur, impulsada por altas presiones en altura. 
Estos y otros mecanismos para la entrada de masas norteafricanas se detallan en el capítulo 7. En la Figura VI-21d tenemos un ejemplo de situación sinóptica para las masas de aire del cluster 4, correspondiente al día 8 de septiembre de 2000. En él tenemos altas presiones sobre centroeuropa, que nos hace llegar lentamente una masa de aire continental. Por último en el cluster 7 tenemos masas de aire que llegan lentamente desde el oeste o sudoeste. Como ejemplo mostramos la situación del día 12 de octubre de 2003 acompañada de sus retrotrayectorias (Figura VI-21g y h). Este día la situación era de gran uniformidad barométrica en superficie. En altura la masa de aire proviene del sudoeste, mientras que en los niveles bajos prácticamente no se ha movido en 5 días.

\subsubsection{Análisis de frecuencias de las masas de aire}

Las distintas trayectorias atlánticas largas suman una frecuencia del $46 \%$, desde el noroeste hasta el sudoeste, y constituyen por tanto la gran mayoría de las masas de aire en la estación. Frente a ellas, tenemos un $22 \%$ de trayectorias atlánticas cortas, sobre todo del sudoeste, un $12 \%$ de trayectorias del norte o nordeste desde el continente europeo, y un $20 \%$ de trayectorias norteafricanas, locales y mediterráneas. Todas estas frecuencias anuales y también estacionales para el nivel de $1500 \mathrm{~m}$ se encuentran recogidas en la Tabla VI-XIII y en la Figura VI-22.

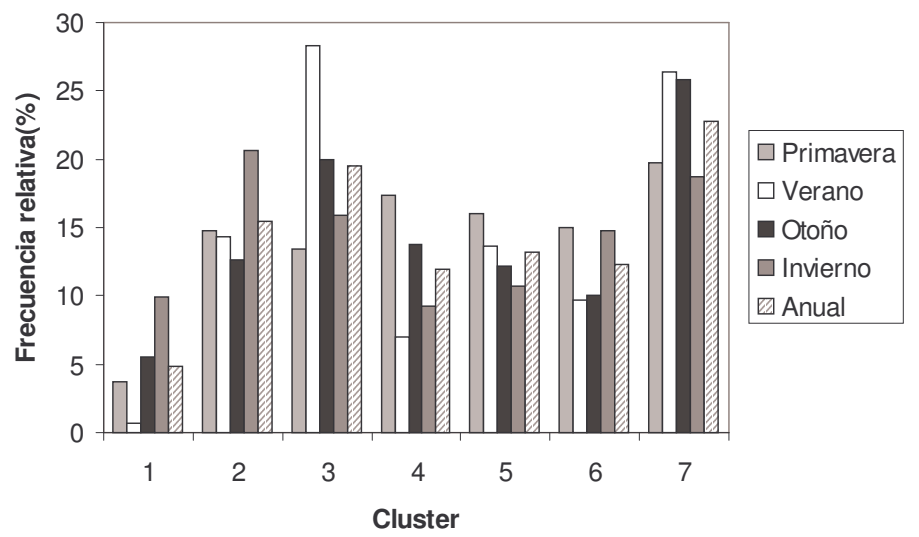

Figura VI-22. Frecuencia relativa (en \%) de ocurrencia para cada cluster, tanto anual como estacional, para el nivel de $1500 \mathrm{~m}$.

Entre las masas de aire Atlánticas, las menos frecuentes son las del cluster 1 (noroeste lejanas), con un $4.9 \%$ de casos. Estas trayectorias se dan principalmente en otoño y sobre todo en invierno, donde alcanzan un $10 \%$ de frecuencia. Sin embargo en verano no llegan al $1 \%$. Las trayectorias del cluster 5 , que hemos denominado Ártico, tienen una frecuencia del $13 \%$ y están presentes durante todo el año. Las trayectorias del oeste se han dividido en dos clusters ( 2 y 6) con un $15.5 \%$ y un $12.3 \%$ de frecuencia respectivamente. Ambas están presentes durante todo el año, y son más frecuentes en primavera y sobre todo en invierno. Las trayectorias del sudoeste (cluster 7) son las que presentan una mayor frecuencia, con un $22 \%$, que es mayor en verano y otoño, donde se alcanza el $26 \%$ de los casos.

Las trayectorias continentales del norte o nordeste tienen una frecuencia promedio anual del $12 \%$, y se dan sobre todo en primavera con un $17 \%$, frente al $7 \%$ tan solo de los casos en verano. En general podemos decir que en verano aumenta mucho la frecuencia de trayectorias cortas del sudoeste y del sur, que suman un 55\% de los casos, mientras que en invierno el flujo Atlántico rápido es más intenso, con un $56 \%$, y si consideramos también las Atlánticas más cortas, del cluster 7, el flujo Atlántico en invierno llega al $75 \%$ de frecuencia. 


\begin{tabular}{llcccccc}
\hline \hline Cluster & & Anual & Primavera & Verano & Otoño & Invierno & N $^{\circ}$ casos \\
\hline 1 & Noroeste lejanas & 4.9 & 3.7 & 0.7 & 5.6 & 10.0 & 87 \\
2 & Atlánticas Oeste & 15.5 & 14.7 & 14.3 & 12.7 & 20.7 & 276 \\
3 & Norteafricanas & 19.5 & 13.4 & 28.4 & 20.0 & 15.9 & 347 \\
4 & N-NE cercanas & 11.9 & 17.4 & 7.0 & 13.8 & 9.3 & 212 \\
5 & Ártico & 13.2 & 16.0 & 13.6 & 12.2 & 10.7 & 235 \\
6 & Atlánticas curvadas & 12.3 & 14.9 & 9.7 & 10.0 & 14.7 & 219 \\
7 & Sudoeste cercanas & 22.7 & 19.8 & 26.4 & 25.8 & 18.8 & 405 \\
\hline \hline
\end{tabular}

Tabla VI-XIII. Frecuencia relativa (en \%) de ocurrencia para cada cluster, tanto anual como estacional, para el nivel de $1500 \mathrm{~m}$ y número total de días ( $\mathrm{N}^{\circ}$ casos) clasificados en cada cluster.

\subsubsection{Relación entre las medidas espectrales y las trayectorias de masas de aire}

$\mathrm{Al}$ igual que hicimos con la clasificación por sectores, el objetivo de esta clasificación de las trayectorias es la comparación con las medidas de aerosoles dadas por el fotómetro Cimel. Se trata de buscar relaciones entre cada uno de los cluster de trayectorias y la huella espectral dada por el promedio diario de AOD en los cuatro canales del Cimel y el parámetro alfa de Ångström.

Para ello nos vamos a servir en primer lugar de los diagramas de dispersión AOD(440nm)alfa realizados con los promedios diarios para aquellos días que pertenecen a cada cluster de trayectorias, que tenemos en la Figura VI-23. Lo primero que llama la atención es la similitud espectral de los clusters 1, 2, 5 y 6, es decir, todos los que agrupan trayectorias atlánticas excepto las más cercanas. Estos cuatro clusters Atlánticos tienen en común una turbiedad muy baja, con un promedio en torno a 0.1 para el AOD en 440nm para todos ellos, y la práctica totalidad de los días por debajo de 0.2. Aunque como veremos hay un cierto predominio de los valores altos de alfa, en general este parámetro puede tomar cualquier valor entre 0 y 2 para estos clusters Atlánticos.

El cluster 3 (norteafricanas) presenta muy alta turbiedad, con numerosos casos de AOD alto asociado a alfas bajos, que reconocemos como aerosol desértico. También encontramos numerosos casos con turbiedad media y alfas altos, que pueden estar asociados tanto a recirculaciones locales, pues se trata de masas de aire muy lentas, como a masas norteafricanas de carácter continental, probablemente en relación con los casos en que no traen polvo desértico. El cluster 4 de trayectorias que llegan desde el continente europeo o la Península, tienen una frecuencia muy alta de los valores más altos de alfa, por encima de 1.2 en general, y con turbiedad media, que podemos etiquetar como aerosol de tipo continental.

Finalmente, el diagrama AOD-alfa del cluster 7 es una mezcla entre la huella espectral del marítimo, del continental o local, y del continental tropical. Aunque una mayoría de valores de $\mathrm{AOD}(440 \mathrm{~nm})$ quedan por debajo de 0.2 , tenemos también numerosos casos de turbiedad muy alta asociada a los episodios de polvo sahariano que entran por este sector, siempre con alfas bajos. Además podemos reconocer también la huella espectral del aerosol de tipo continental (como en el cluster 4), probablemente debido a recirculaciones y masas de aire locales o muy lentas, que adquieren carácter continental sobre la Península.

En general podemos encontrar una separación clara entre los casos de baja turbiedad, que se concentran en los clusters Atlánticos más rápidos (clusters 1, 2, 5 y 6); los eventos de 
polvo desértico, presentes en su mayoría en los clusters 3 y 7 ; y por último el aerosol de tipo continental y las recirculaciones locales, con presencia mayoritaria en el cluster 4 , y también en los clusters 3 y 7.
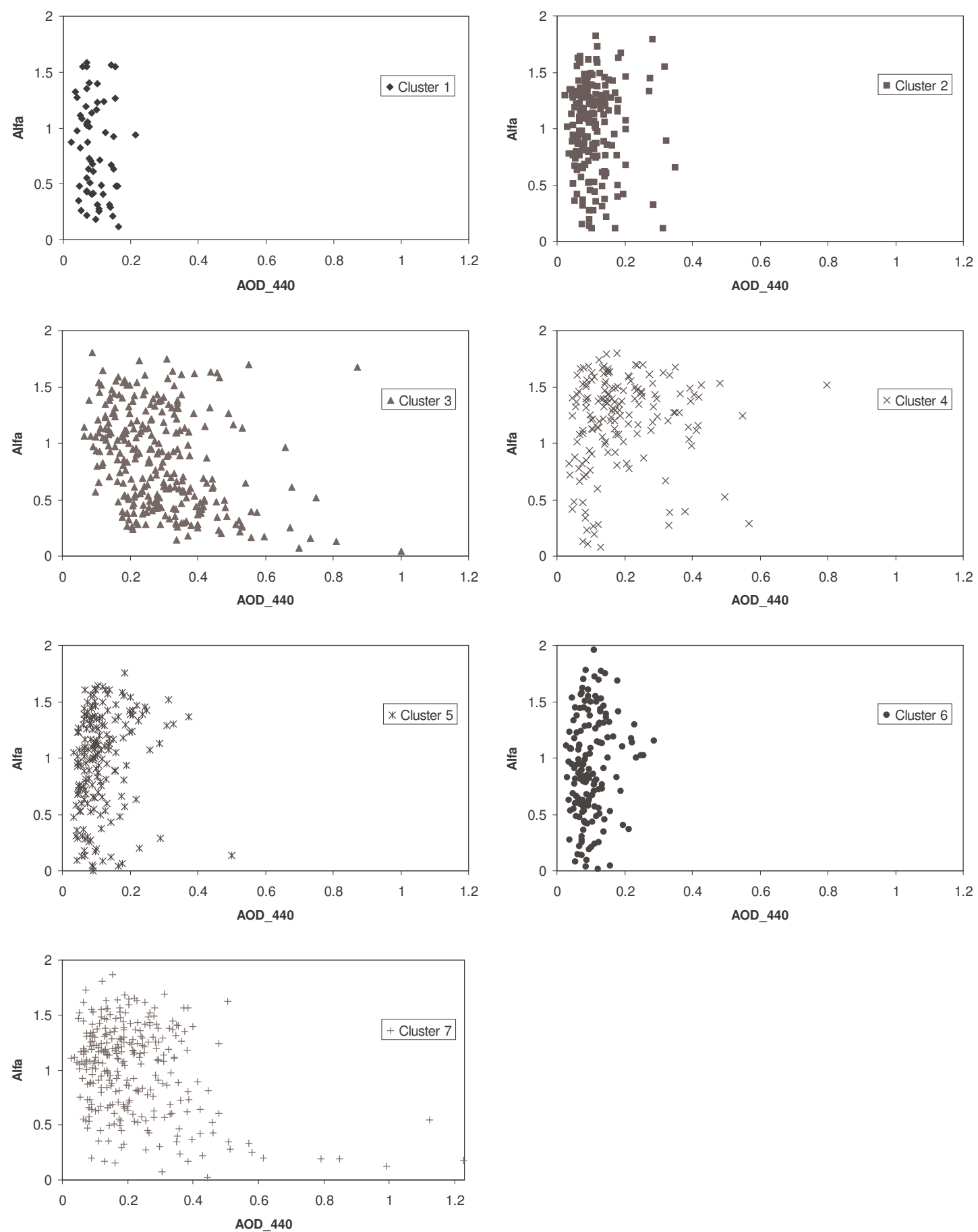

Figura VI-23. Diagramas AOD(440nm)-alfa de los promedios diarios del Cimel para los 7 clusters de la clasificación mediante el algoritmo de cluster no jerárquico con las trayectorias de $1500 \mathrm{~m}$.

Para cuantificar esta relación entre los clusters de trayectorias, realizados mediante el algoritmo no jerárquico de clasificación, y las medidas de AOD espectral del fotómetro Cimel, vamos a emplear la descripción estadística que mostramos en las Tabla VI-XIV a Tabla VI-XX, realizada a partir de los promedios diarios, y los histogramas de frecuencias mostrados desde la Figura VI-24 hasta la Figura VI-30, para cada uno de los siete clusters de trayectorias en el nivel de $1500 \mathrm{~m}$. Estos histogramas se han elaborado a partir de todas 
las medidas, y no a partir de los promedios diarios, como ya se hizo en la clasificación por sectores de la sección anterior.

El cluster 1 (Noroeste lejanas, Tabla VI-XIV) tiene el promedio más bajo en AOD(440nm) con 0.094, también con una desviación estándar muy pequeña (0.04), lo cual da lugar a una distribución de frecuencias de AOD muy estrecha (Figura VI-24), con pico en 0.07. El parámetro alfa promedio es 0.81 , aunque las frecuencias se distribuyen por todo el rango entre 0.2 y 1.8 , con pico de frecuencia en torno a un alfa de 1.1. Se trata de masas de aire de carácter marítimo y muy limpias.

\begin{tabular}{lccccc}
\hline \hline Cluster 1 & AOD_1020 & AOD_870 & AOD_670 & AOD_440 & alfa(440-870) \\
\hline Media & 0.057 & 0.057 & 0.061 & 0.094 & 0.81 \\
Desv. Estándar & 0.032 & 0.032 & 0.033 & 0.040 & 0.43 \\
P25 & 0.032 & 0.034 & 0.037 & 0.069 & 0.44 \\
P75 & 0.080 & 0.080 & 0.084 & 0.121 & 1.16 \\
P90 & 0.106 & 0.106 & 0.113 & 0.154 & 1.41 \\
Mín. & 0.014 & 0.014 & 0.014 & 0.024 & 0.12 \\
Máx. & 0.153 & 0.152 & 0.149 & 0.215 & 1.59 \\
Mediana & 0.050 & 0.050 & 0.054 & 0.083 & 0.73 \\
\hline \hline
\end{tabular}

Tabla VI-XIV. Estadística espectral del cluster 1 (NW lejanas) con los promedios diarios según la clasificación de trayectorias a 1500m mediante el algoritmo no jerárquico de cluster

El cluster 2 (Atlánticas Oeste, Tabla VI-XV) agrupa también masas de aire marítimas limpias, con un AOD promedio en 440nm de 0.109, aunque con una desviación de 0.056 que es algo mayor que en el cluster anterior, por culpa de algunos días con AOD medio. Esto hace que el histograma de frecuencias de AOD $(440 \mathrm{~nm})$, mostrado en la Figura VI25a, no sea tan estrecho, y algunas observaciones llegan casi a 0.4, aunque de nuevo el pico de frecuencia se da para un valor tan bajo como 0.07. El valor promedio de alfa es también mayor que en el cluster 1, llegando a 1.01. Además predominan los valores altos de alfa, con pico de frecuencia en 1.2, como se ve en la correspondiente distribución de frecuencias (Figura VI-25b).

\begin{tabular}{lccccc}
\hline \hline Cluster 2 & AOD_1020 & AOD_870 & AOD_670 & AOD_440 & alfa(440-870) \\
\hline Media & 0.054 & 0.058 & 0.066 & 0.109 & 1.01 \\
Desv. Estándar & 0.042 & 0.038 & 0.042 & 0.056 & 0.39 \\
P25 & 0.034 & 0.035 & 0.039 & 0.071 & 0.76 \\
P75 & 0.068 & 0.071 & 0.082 & 0.133 & 1.30 \\
P90 & 0.097 & 0.102 & 0.123 & 0.179 & 1.45 \\
Mín. & -0.072 & 0.010 & 0.008 & 0.023 & 0.00 \\
Máx. & 0.292 & 0.291 & 0.293 & 0.350 & 1.82 \\
Mediana & 0.046 & 0.046 & 0.054 & 0.095 & 1.08 \\
\hline \hline
\end{tabular}

Tabla VI-XV. Estadística espectral del cluster 2 (Atlánticas W) con los promedios diarios según la clasificación de trayectorias a $1500 \mathrm{~m}$ mediante el algoritmo no jerárquico de cluster

El cluster 3 (Norteafricanas, Tabla VI-XVI) posee, como podríamos esperar, el promedio de $\mathrm{AOD}(440 \mathrm{~nm})$ más alto, con 0.29 , con una desviación estándar también grande de 0.14. La fuerte presencia de aerosol desértico en este cluster se deja notar sobre todo en la alta turbiedad, con un histograma de AOD que es el más ancho y desplazado a la derecha (ver Figura VI-26), con muchas observaciones hasta valores de 0.8 y el pico de frecuencia en 0.22 , que es el más alto de todos los clusters. El promedio de alfa es de 0.86 , aunque en este caso el promedio esconde las dos situaciones presentes en este cluster, es decir, aerosol de tipo desértico con alfas bajos, y aerosol de tipo continental con alfas más altos. Esto se puede observar muy bien en la distribución de frecuencias de alfa (ver Figura VI26), en el que se aprecia un primer pico de frecuencia en 0.45 y un segundo pico en 0.90 . 
El límite entre ambas está bien definido por una caída de la frecuencia de casos en torno a alfa $=0.7$, valor que nos puede servir para discriminar ambas situaciones.

\begin{tabular}{lccccc}
\hline \hline Cluster 3 & AOD_1020 & AOD_870 & AOD_670 & AOD_440 & alfa(440-870) \\
\hline Media & 0.167 & 0.178 & 0.204 & 0.290 & 0.86 \\
Desv. Estándar & 0.124 & 0.127 & 0.131 & 0.143 & 0.43 \\
P25 & 0.085 & 0.093 & 0.118 & 0.189 & 0.49 \\
P75 & 0.210 & 0.222 & 0.251 & 0.354 & 1.20 \\
P90 & 0.320 & 0.330 & 0.350 & 0.462 & 1.44 \\
Mín. & -0.030 & 0.026 & 0.034 & 0.064 & 0.05 \\
Máx. & 0.941 & 0.968 & 0.990 & 1.001 & 1.80 \\
Mediana & 0.137 & 0.148 & 0.176 & 0.272 & 0.86 \\
\hline \hline
\end{tabular}

Tabla VI-XVI. Estadística espectral del cluster 3 (Norteafricanas) con los promedios diarios según la clasificación de trayectorias a $1500 \mathrm{~m}$ mediante el algoritmo no jerárquico de cluster

El cluster 4 (N-NE cercanas, Tabla VI-XVII), presenta una turbiedad media en general, con un promedio de $\mathrm{AOD}(440 \mathrm{~nm})$ de 0.19 , con una desviación de 0.12 . La distribución de frecuencia de AOD de la Figura VI-27 nos muestra que hay un número relevante de observaciones hasta valores por encima de 0.4 , con un pico de frecuencia en 0.17 , que es el segundo más alto, y un histograma bastante más ancho que en el caso de los cluster Atlánticos. Sin embargo lo más significativo en este grupo es el elevado promedio de alfa, 1.20 con una mediana de 1.29. El histograma de frecuencias de alfa (la Figura VI-27) es el más inclinado hacia los valores altos, con un pico de frecuencia en alfa $=1.5$. La gran mayoría de casos están entre 1 y 1.7, es decir, valores muy altos que asociamos a aerosol con predominancia de partículas finas (de tipo continental o polucionado).

\begin{tabular}{lccccc}
\hline \hline Cluster 4 & AOD_1020 & AOD_870 & AOD_670 & AOD_440 & alfa(440-870) \\
\hline Media & 0.078 & 0.086 & 0.107 & 0.189 & 1.20 \\
Desv. Estándar & 0.060 & 0.066 & 0.080 & 0.122 & 0.40 \\
P25 & 0.041 & 0.048 & 0.059 & 0.099 & 1.02 \\
P75 & 0.091 & 0.103 & 0.129 & 0.241 & 1.49 \\
P90 & 0.135 & 0.156 & 0.214 & 0.365 & 1.63 \\
Mín. & 0.007 & 0.018 & 0.020 & 0.035 & 0.08 \\
Máx. & 0.444 & 0.467 & 0.506 & 0.797 & 1.80 \\
Mediana & 0.063 & 0.068 & 0.084 & 0.151 & 1.29 \\
\hline \hline
\end{tabular}

Tabla VI-XVII. Estadística espectral del cluster 4 (N-NE cercanas) con los promedios diarios según la clasificación de trayectorias a 1500m mediante el algoritmo no jerárquico de cluster

\begin{tabular}{lccccc}
\hline \hline Cluster 5 & AOD_1020 & AOD_870 & AOD_670 & AOD_440 & alfa(440-870) \\
\hline Media & 0.059 & 0.062 & 0.070 & 0.116 & 0.95 \\
Desv. Estándar & 0.047 & 0.045 & 0.049 & 0.068 & 0.46 \\
P25 & 0.035 & 0.035 & 0.040 & 0.069 & 0.65 \\
P75 & 0.072 & 0.074 & 0.085 & 0.138 & 1.33 \\
P90 & 0.106 & 0.105 & 0.121 & 0.201 & 1.47 \\
Mín. & -0.079 & 0.017 & 0.017 & 0.034 & -0.05 \\
Máx. & 0.472 & 0.464 & 0.460 & 0.501 & 1.75 \\
Mediana & 0.050 & 0.050 & 0.058 & 0.097 & 1.05 \\
\hline
\end{tabular}

Tabla VI-XVIII. Estadística espectral del cluster 5 (Ártico) con los promedios diarios según la clasificación de trayectorias a $1500 \mathrm{~m}$ mediante el algoritmo no jerárquico de cluster

El cluster 5 (Ártico, Tabla VI-XVIII), nos devuelve a masas de aire limpias, provenientes del Atlántico Norte, que tienen un AOD bajo, en promedio 0.12 en 440nm. Tanto este AOD promedio como su desviación son algo mayores que en los clusters 1 y 2 , y el histograma 
de AOD(440nm) de la Figura VI-28 tiene observaciones hasta 0.3, es decir, es algo más ancho, aunque el pico de frecuencia está en 0.08 que es un valor muy bajo. El promedio de alfa sí es sensiblemente mayor que en los otros cluster mencionados, pues alcanza un valor de 0.95 . Esta relativa mayor turbiedad y promedio de alfa más alto pueden indicar la contaminación de estas masas de aire de componente norte al tener que atravesar toda la Península Ibérica antes de llegar a la estación de El Arenosillo. Las frecuencias de alfa en la Figura VI-28 se distribuyen principalmente entre 1 y 1.5, lo cual puede indicar esta influencia de tipo continental.

El cluster 6 (Atlánticas curvadas, Tabla VI-XIX), contiene también masas de aire limpias de procedencia Atlántica, con un promedio de AOD en 440nm muy bajo, de 0.10. Además la práctica totalidad de observaciones están por debajo de 0.2 , y el histograma de frecuencias de la Figura VI-29 es muy estrecho y desplazado a valores bajos, con el pico de frecuencia en 0.07. El promedio de alfa es de 0.91, pero como vemos en el histograma correspondiente de la Figura VI-29, el parámetro alfa toma valores en todo el rango, con la máxima frecuencia en 1.15 que disminuye paulatinamente hacia los extremos del rango. Tanto este histograma de alfa como el del cluster 1 (noroeste lejanas) tienen en común que no muestran una acumulación de datos entre 1 y 1.5 tal y como muestran las frecuencias de los demás histogramas, lo cual puede estar relacionado con que se trate de masas de aire con menor influencia continental. Conviene tener también en mente que el error asociado a alfa aumenta para los valores bajos de AOD, hasta incertidumbres por encima del $50 \%$ cuando se trata de AOD muy bajos.

\begin{tabular}{lccccc}
\hline \hline Cluster 6 & AOD_1020 & AOD_870 & AOD_670 & AOD_440 & alfa(440-870) \\
\hline Media & 0.054 & 0.056 & 0.062 & 0.102 & 0.91 \\
Desv. Estándar & 0.033 & 0.031 & 0.033 & 0.048 & 0.45 \\
P25 & 0.034 & 0.035 & 0.037 & 0.070 & 0.58 \\
P75 & 0.070 & 0.068 & 0.078 & 0.125 & 1.28 \\
P90 & 0.097 & 0.100 & 0.114 & 0.158 & 1.53 \\
Mín. & -0.030 & 0.015 & 0.011 & 0.029 & -0.09 \\
Máx. & 0.166 & 0.171 & 0.180 & 0.287 & 1.95 \\
Mediana & 0.048 & 0.048 & 0.055 & 0.092 & 0.90 \\
\hline \hline
\end{tabular}

Tabla VI-XIX. Estadística espectral del cluster 6 (Atlánticas curvadas) con los promedios diarios según la clasificación de trayectorias a 1500m mediante el algoritmo no jerárquico de cluster

\begin{tabular}{lccccc}
\hline \hline Cluster 7 & AOD_1020 & AOD_870 & AOD_670 & AOD_440 & alfa(440-870) \\
\hline Media & 0.114 & 0.123 & 0.144 & 0.216 & 1.01 \\
Desv. Estándar & 0.135 & 0.138 & 0.142 & 0.153 & 0.40 \\
P25 & 0.050 & 0.053 & 0.064 & 0.118 & 0.69 \\
P75 & 0.124 & 0.139 & 0.170 & 0.279 & 1.30 \\
P90 & 0.217 & 0.223 & 0.263 & 0.374 & 1.48 \\
Mín. & -0.033 & 0.013 & 0.011 & 0.026 & 0.03 \\
Máx. & 1.136 & 1.152 & 1.187 & 1.227 & 1.87 \\
Mediana & 0.080 & 0.089 & 0.108 & 0.180 & 1.09 \\
\hline \hline
\end{tabular}

Tabla VI-XX. Estadística espectral del cluster 7 (SW cercanas) con los promedios diarios según la clasificación de trayectorias a $1500 \mathrm{~m}$ mediante el algoritmo no jerárquico de cluster

El cluster 7 (Sudoeste cercanas, Tabla VI-XX) tiene un promedio de AOD(440nm) igual a 0.216 , un valor muy elevado para tratarse de trayectorias marítimas si las comparamos con el resto de cluster Atlánticos. Sin embargo ya se ha subrayado el hecho de que numerosas entradas de polvo desértico están asociadas a estas masas de aire del sudoeste. De hecho los casos de AOD más alto, con promedios diarios superiores a 1 se han dado con masas de este tipo, en el día central de los episodios de marzo de 2000 y julio de 2004. El 
histograma de AOD en 440nm (Figura VI-30) es muy ancho, como refleja el hecho de que la desviación estándar de AOD sea la más alta de todos los cluster, con 0.15 , y presenta numerosas observaciones hasta 0.6 . El promedio de alfa es de 1.01, es decir, un valor alto, puesto que hay un gran número de casos con alfas entre 1 y 1.5 , con pico de frecuencia en 1.2. Sin embargo hay también un número relevante de alfas bajos, que están asociados a los AOD más altos (y que por tanto tienen poco error) como vimos en el diagrama AODalfa de este cluster.
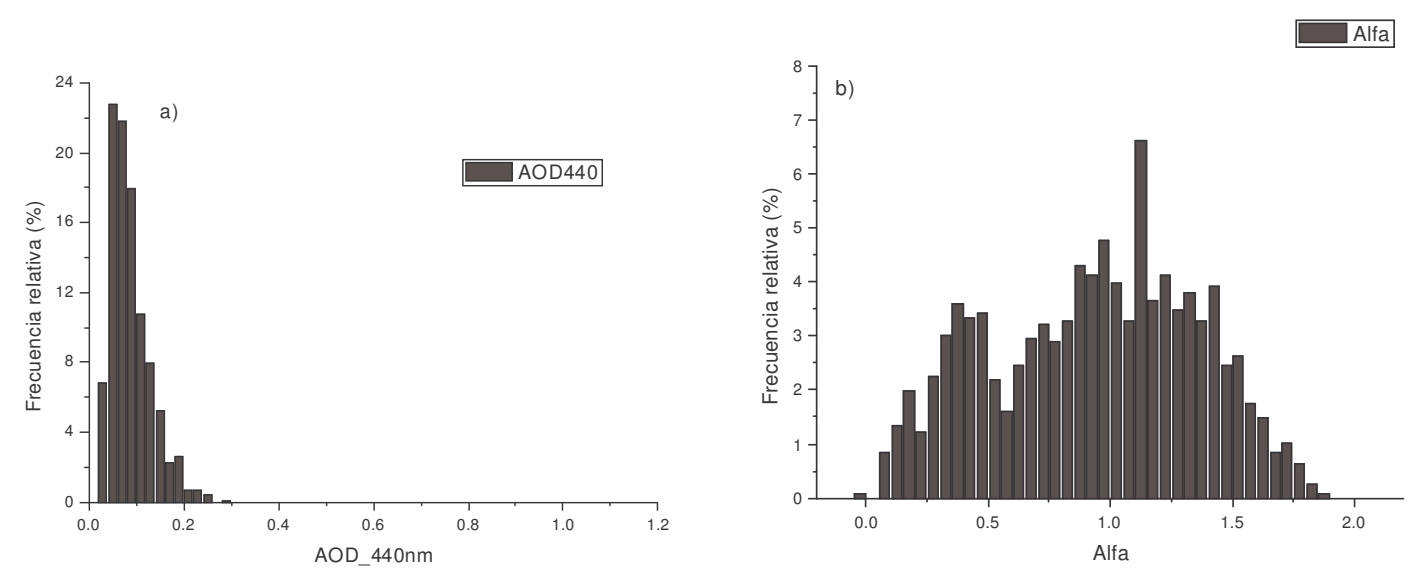

Figura VI-24. Histogramas de frecuencias relativas(\%) de (a) AOD en 440nm y (b) alfa para el cluster 1 (Noroeste lejanas) según la clasificación del algoritmo no jerárquico de cluster con las trayectorias en $1500 \mathrm{~m}$.
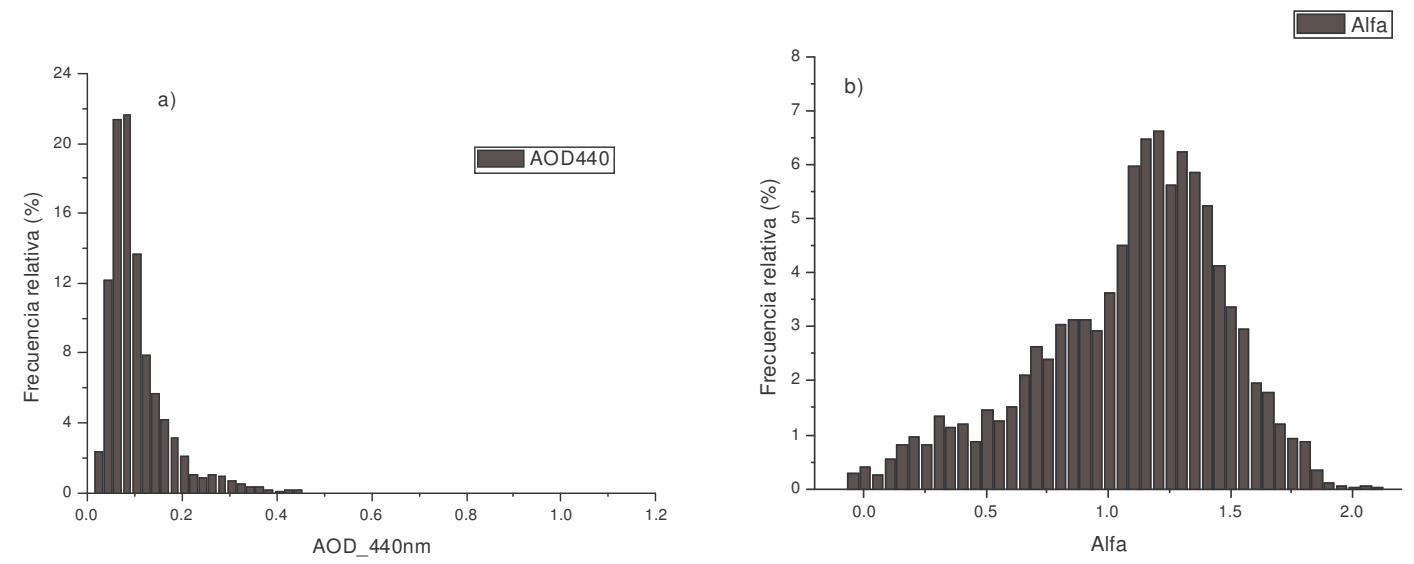

Figura VI-25. Histogramas de frecuencias relativas(\%) de (a) AOD en 440nm y (b) alfa para el cluster 2 (Atlánticas Oeste) según la clasificación del algoritmo no jerárquico de cluster con las trayectorias en $1500 \mathrm{~m}$.
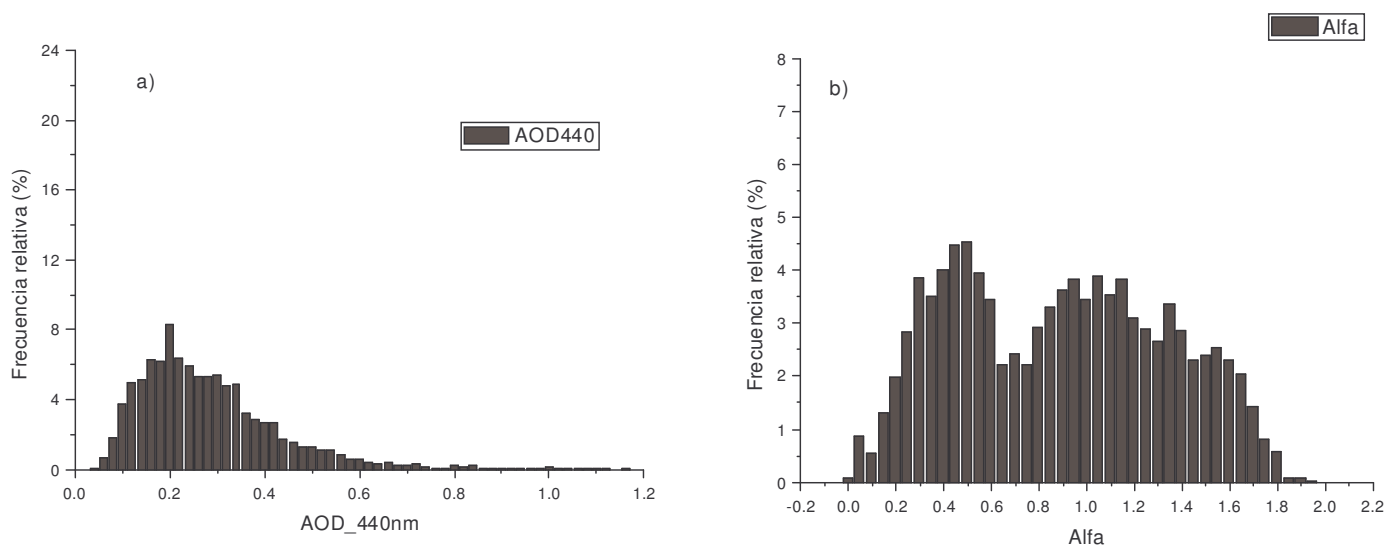

Figura VI-26. Histogramas de frecuencias relativas(\%) de (a) AOD en 440nm y (b) alfa para el cluster 3 (Norteafricanas) según la clasificación del algoritmo no jerárquico de cluster con las trayectorias en $1500 \mathrm{~m}$. 

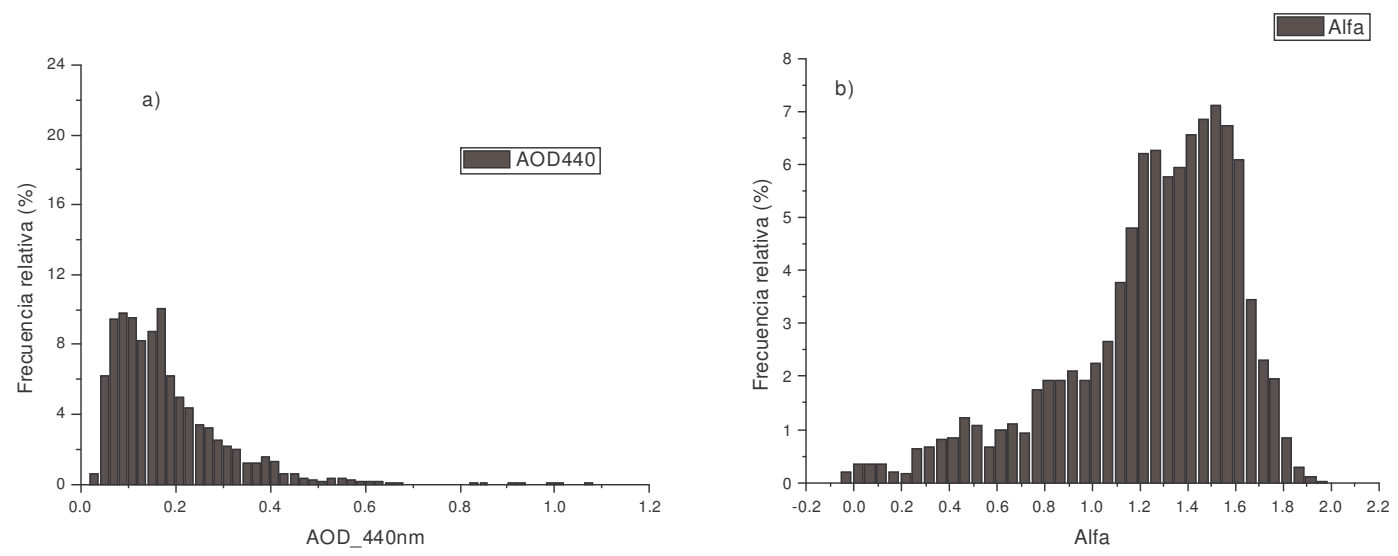

Figura VI-27. Histogramas de frecuencias relativas(\%) de (a) AOD en 440nm y (b) alfa para el cluster 4 (Norte-Nordeste cercanas) según la clasificación del algoritmo no jerárquico de cluster con las trayectorias en $1500 \mathrm{~m}$.
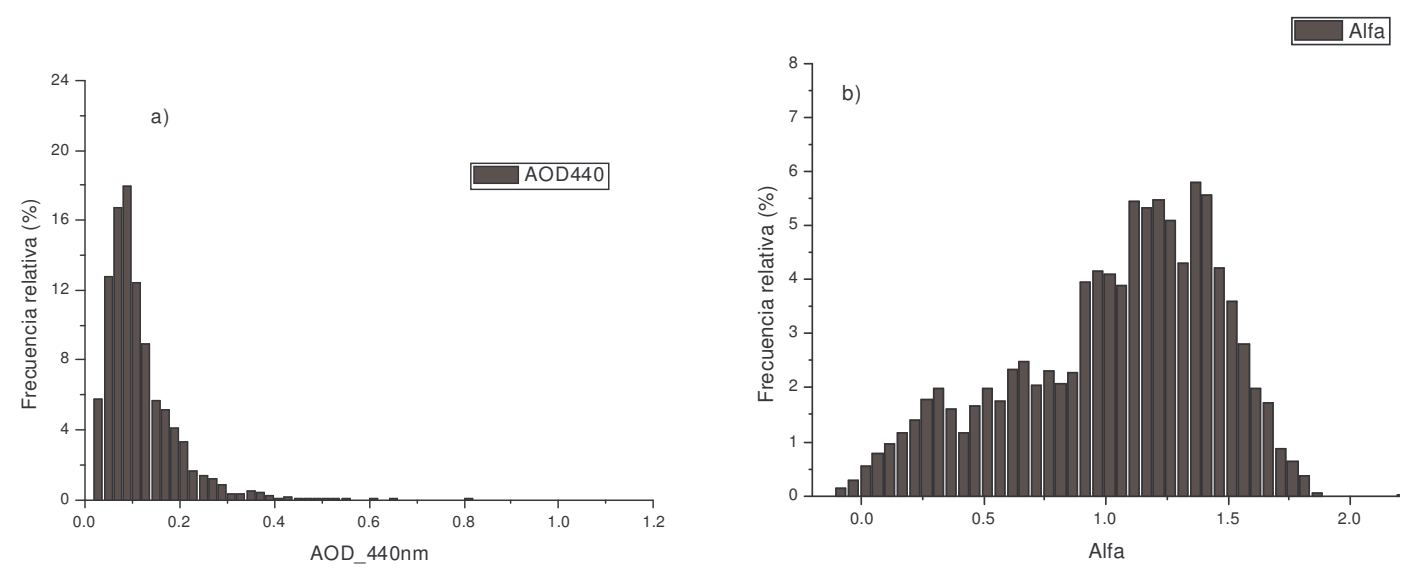

Figura VI-28. Histogramas de frecuencias relativas(\%) de (a) AOD en 440nm y (b) alfa para el cluster 5 (Ártico) según la clasificación del algoritmo no jerárquico de cluster con las trayectorias en $1500 \mathrm{~m}$.
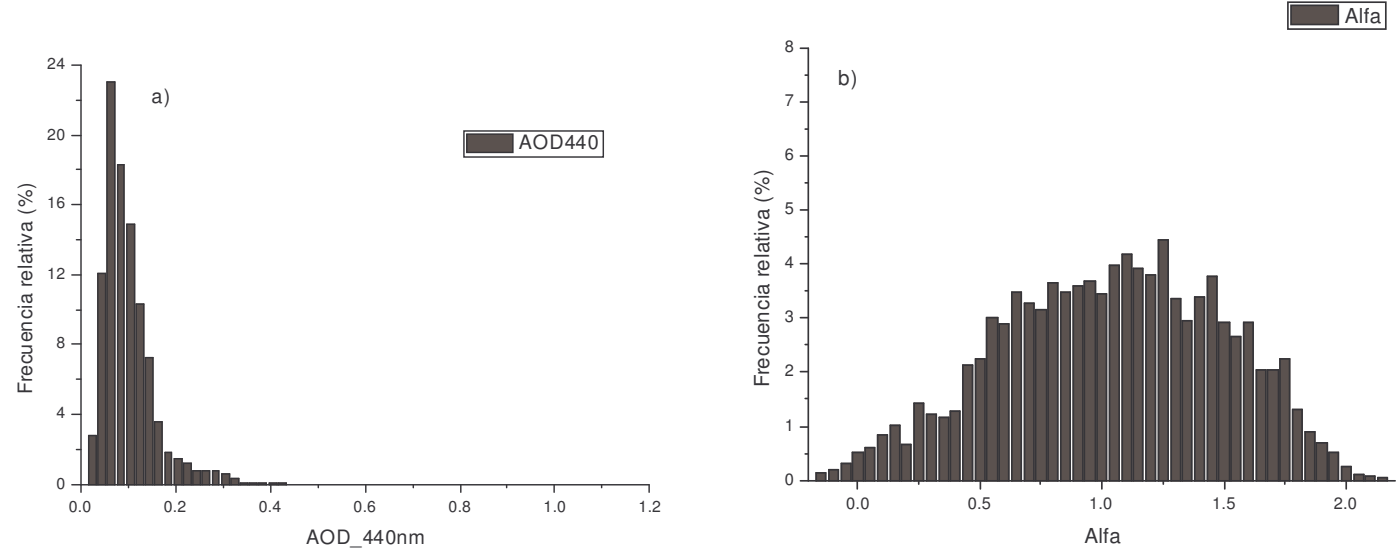

Figura VI-29. Histogramas de frecuencias relativas(\%) de (a) AOD en 440nm y (b) alfa para el cluster 6 (Atlánticas curvadas) según la clasificación del algoritmo no jerárquico de cluster con las trayectorias en $1500 \mathrm{~m}$. 

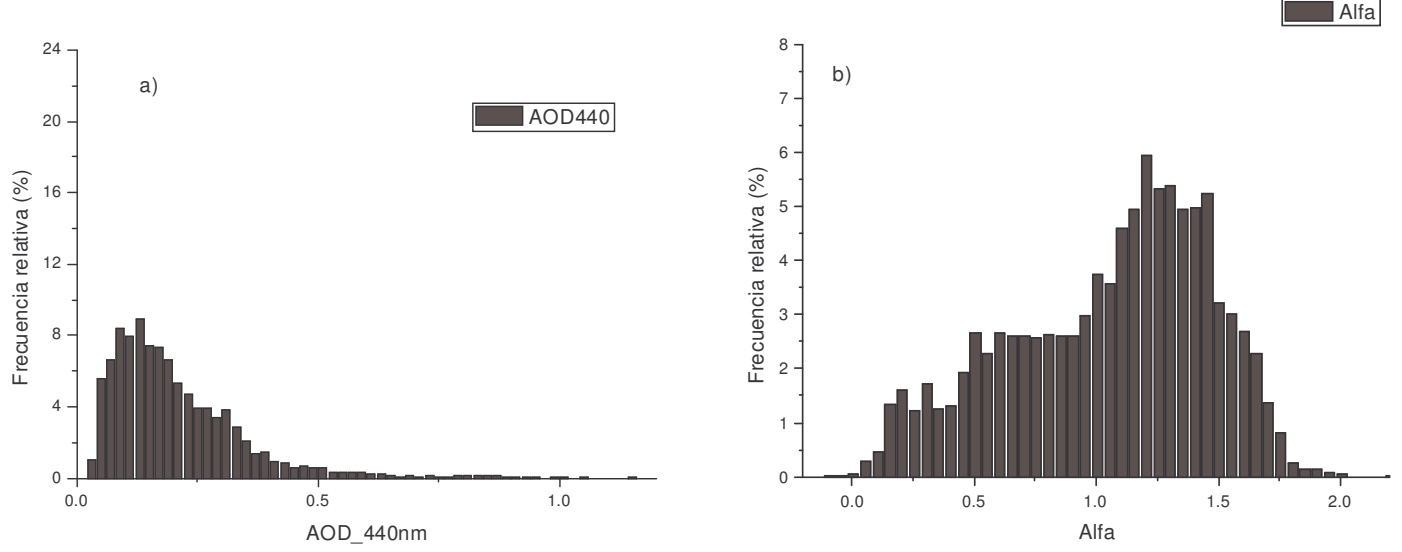

Figura VI-30. Histogramas de frecuencias relativas(\%) de (a) AOD en 440nm y (b) alfa para el cluster 7 (Sudoeste cercanas) según la clasificación del algoritmo no jerárquico de cluster con las trayectorias en $1500 \mathrm{~m}$. 


\subsection{Comparación entre las dos clasificaciones realizadas}

Hemos planteado en los apartados anteriores dos clasificaciones de las masas de aire en nuestra zona de estudio por metodologías diferentes. Por una parte se ha planteado una clasificación por sectores de procedencia y por otra parte un algoritmo de cluster no jerárquico basado en la distancia geométrica entre trayectorias. En ambos casos se ha realizado en primer lugar una clasificación de las masas de aire, analizando el tipo de trayectorias que se agrupaban en cada cluster y sus frecuencias anuales y por estaciones en tres niveles $(500 \mathrm{~m}, 1500 \mathrm{~m}$ y $3000 \mathrm{~m})$. En segundo lugar se ha relacionado esa clasificación con las medidas del fotómetro Cimel, buscando las características de AOD espectral en cada cluster. Parece lógico entonces realizar una comparación entre ambas clasificaciones, que las relacione y ponga de manifiesto los puntos fuertes y débiles de cada una.

Para comparar los dos métodos, en primer lugar hemos construido la matriz de confusión entre ambas clasificaciones, que mostramos en la Tabla VI-XXI para el nivel de $1500 \mathrm{~m}$. En esta tabla se muestran por columnas la clasificación por sectores y por filas la del algoritmo de cluster no jerárquico. De esta manera podemos evaluar cómo se han clasificado por un método las que se han considerado un grupo por el otro método. En esta tabla hemos resaltado en gris las celdas con menos de 3 casos, para ayudar a visualizar los grupos que son disjuntos.

\begin{tabular}{rccccccc}
\hline \hline & MP & MT & A & C & CT & L & Me \\
\hline Noroeste lejanas (1) & 52 & 2 & 31 & 2 & 0 & 0 & 0 \\
Atlánticas Oeste (2) & 149 & 119 & 7 & 0 & 0 & 1 & 0 \\
Norteafricanas (3) & 1 & 19 & 1 & 13 & 204 & 48 & 61 \\
Norte cercanas (4) & 69 & 0 & 39 & 80 & 0 & 5 & 19 \\
NW-Ártico (5) & 171 & 1 & 51 & 8 & 0 & 4 & 0 \\
Atlánticas curvadas (6) & 172 & 12 & 30 & 3 & 0 & 0 & 2 \\
Oeste-SW cercanas (7) & 127 & 195 & 7 & 11 & 22 & 40 & 3 \\
\hline \hline
\end{tabular}

Tabla VI-XXI. Matriz de confusión entre la clasificación de trayectorias por sectores (en columnas) y por el algoritmo no jerárquico de cluster (en filas). Nivel 1500m.

En primer lugar vamos a mirar desde la clasificación dada por el algoritmo de cluster, que aparece por filas. Si atendemos a las trayectorias atlánticas del noroeste, en los cluster 1, 5 y 6, vemos que todas pertenecen a los sectores MP y A, como era de esperar. A su vez las atlánticas del oeste, en los cluster 2 y 7, se dividen entre los sectores MP y MT. El cluster 7 también contiene algunas trayectorias de los sectores $\mathrm{CT}$ y sobre todo $\mathrm{L}$, ya que este cluster incluye trayectorias cercanas por recirculaciones en la zona del golfo de Cádiz. El cluster 3, que hemos denominado masas norteafricanas, agrupa sobre todo masas del sector Continental Tropical, aunque vemos que también contiene trayectorias de los sectores Local y Mediterráneo, incluso unas pocas de los sectores Marítimo Tropical y Continental. Por último, el cluster 4, con trayectorias del norte cercanas, queda bastante mezclado entre los sectores MP, A y C, y también aunque en menor medida en el Me. Los 69 casos en común con el sector MP nos revelan una diferencia fundamental entre las clasificaciones: para la clasificación por sectores no influye la longitud de las trayectorias. El sector MP contiene 741 trayectorias, y entre ellas hay tanto trayectorias largas, como hemos visto en los cluster 1, 2, 5 y 6, y también trayectorias cortas, en los cluster 4 y 7. La longitud de las trayectorias habla mucho de la situación sinóptica, pues significa mayor velocidad del viento y menor tiempo de residencia en el entorno de la estación. En este sentido la clasificación por el algoritmo de cluster proporciona mayor información. 
Si analizamos desde la clasificación por sectores, mostrada por columnas, llama la atención el sector MP, que está muy repartido entre todos los cluster como acabamos de comentar. Excepto en el cluster 3 (norteafricanas) tiene casos en común con todos los cluster, que por otra parte no hacen sino distinguir los distintos tipos de masas atlánticas. El sector MT incluye trayectorias más o menos largas del oeste o sudoeste, y están muy repartidas entre los cluster 2 y 7 . Esto nos indica que en este sector hay dos tipos de trayectorias: unas del oeste bastante rápidas, y otras del oeste o sudoeste que son más cortas, entre las que se encuentran las que traen aerosol desértico. Las trayectorias del sector Ártico no son numerosas y están muy repartidas entre varios cluster, sobre todo de trayectorias del noroeste y norte, mezclándose trayectorias rápidas zonales (cluster 1, 5, y 6) con trayectorias del norte cercanas (cluster 4).

Los sectores restantes son sin embargo mucho más claros, pues sus trayectorias se corresponden claramente con uno o dos grupos de la clasificación del algoritmo de cluster. El sector Continental tiene casi todas las trayectorias en común con el cluster 4 (norte cercanas), como era lógico. Más contundente es la coincidencia del sector CT con el cluster 3 (norteafricanas), ya que todas las demás celdas son cero excepto el cluster 7, con el que comparte algunas trayectorias del sudoeste. Las trayectorias del sector Local se distribuyen entre los 2 cluster de trayectorias más cortas (3 y 7). El sector Mediterráneo no tiene un cluster propio en $1500 \mathrm{~m}$ según la clasificación del algoritmo. Estas trayectorias se han distribuido entre el cluster 3 (norteafricanas) y 4 (norte cercanas). En el nivel de 500m sí hay un cluster Mediterráneo (ver Figura VI-19a) en la clasificación del algoritmo, el cual tiene en común más del $80 \%$ de trayectorias con el sector Mediterráneo, y el resto con el sector Continental, que son trayectorias del NE que llegan desde Europa y pasan sobre el Mediterráneo para llegar a la Península.

Ya se ha comentado que si atendemos a los mapas con las trayectorias que tenemos en la Figura VI-5 y la Figura VI-20, podemos apreciar la manera diferente de clasificar, pues el criterio de distancia geométrica entre trayectorias nos agrupa las de similar longitud y curvatura, y no sólo tiene en cuenta la dirección como en el caso de los sectores. Por eso en los mapas de la Figura VI-20 tenemos trayectorias similares entre sí y se han separado las largas y cortas, que a su vez se corresponden con escenarios diferentes. Por su parte la clasificación por sectores mezcla las trayectorias lentas y rápidas de cada dirección. A la luz de esto dejamos abierta la posibilidad de definir una zona Local más extensa en la rejilla de clasificación, lo cual podría ayudar a separar las trayectorias más cortas.

En general podemos concluir que las dos clasificaciones intercambian trayectorias entre grupos contiguos, por la diferencia de metodología, pero tan solo en un número anecdótico de casos se clasifica de manera discordante una cierta trayectoria, como sería por ejemplo mezclar el sector CT con el cluster 1 (noroeste lejanas). Si tan sólo atendiéramos a las trayectorias y la manera de agruparlas se podría decir que la clasificación mediante el algoritmo de cluster es más objetiva, pues no depende de información a priori, y que además da más información y más precisa sobre la meteorología en la estación, esto es, reconoce las situaciones sinópticas principales. Pero a nosotros nos interesa una segunda parte, la cual consiste en ver si las clasificaciones son capaces de distinguir tipos de aerosol según las medidas de AOD espectral dadas por el fotómetro Cimel.

$\mathrm{Si}$ analizamos las características del AOD para los grupos en ambas clasificaciones, encontramos conclusiones comunes. En primer lugar las masas de aire atlánticas más o menos rápidas traen asociada baja turbiedad. En cuanto a los sectores se trata del Marítimo Polar y del Ártico, en los que el promedio de AOD es 0.13(STD 0.08) y 0.10(STD 0.07) respectivamente, con desviaciones estándar pequeñas porque las 
distribuciones de frecuencias son estrechas y con el pico de frecuencia por debajo de 0.1 en 440nm. Esto mismo ocurre con los cluster 1, 2, 5, y 6 en la otra clasificación, que comprenden diferentes trayectorias atlánticas pero todas ellas rápidas. Los promedios son similares en todos estos cluster: $0.09,0.11,0.12$ y 0.10 en $440 \mathrm{~nm}$ respectivamente, y las distribuciones de frecuencias son siempre estrechas y con un pico de frecuencia en valores bajos, por debajo de 0.1. También encontramos como característica común que el alfa para estas masas atlánticas recorre todo el rango de valores.

Los episodios de alta turbiedad se concentran en las trayectorias cortas, tanto norteafricanas como atlánticas del sudoeste, europeas o mediterráneas. El parámetro alfa ayuda a discriminar el tipo de aerosol, encontrando que en las masas de aire con fuerte carácter continental el parámetro alfa toma valores altos, por encima de 1 en general (promedio 1.2-1.3). Por el contrario, las masas de aire norteafricanas, con aerosol desértico, muestran valores de alfa más bajos, por debajo de 1 en general y con promedio de 0.7-0.8. En el caso de la clasificación por sectores esta discriminación de las medidas espectrales es muy clara, como vimos en la Figura VI-8: el sector continental contiene la mayoría de los casos de alta turbiedad con alfas altos, mientras que el sector Continental Tropical contiene la mayoría de casos con alfas bajos, compartido con el marítimo tropical. Las trayectorias más cortas y locales (sector Local y Mediterráneo) también tienen alta turbiedad de ambos tipos, aunque sobre todo de alfas altos. Es decir, en las recirculaciones locales las masas de aire adquieren un cierto carácter continental y aumentan su turbiedad.

Frente a esto, la discriminación espectral que da la clasificación del algoritmo de cluster es algo más confusa. En particular el cluster 3 (norteafricanas) hemos visto que incluye también trayectorias de los sectores Local y Mediterráneo, todas ellas cortas y asociadas a recirculaciones. Esto hace que tengamos mezclados (ver Figura VI-23) los casos de alfas altos (asociados a masas Locales) y bajos (de las masas Saharianas) en este cluster 3. De esta forma el histograma de frecuencias en este cluster muestra dos modos como en el caso del sector $\mathrm{CT}$, pero el modo en torno a valores bajos de alfa no predomina sobre el de valores altos, sino que tienen una frecuencia similar (ver Figura VI-26). También podemos observar en esta clasificación del algoritmo que el cluster 4 (norte cercanas) reúne días con alfas altos, los que no son debidos a masas de aire locales que como hemos dicho están en el cluster 3 junto con las norteafricanas. Sin embargo también tiene casos que pertenecen al sector Ártico en la otra clasificación, y que se observan muy bien en el diagrama AOD-alfa de la Figura VI-23, puesto que son días con AOD muy bajo y alfas en todo el rango. Por último encontramos en la clasificación del algoritmo hasta cuatro clusters de trayectorias atlánticas, que son diferentes en cuanto a meteorología, pero que en cuanto a las medidas de los aerosoles son muy similares. La clasificación por sectores tiene casi todas estas trayectorias en sólo dos grupos, MP y A, lo cual es más sencillo a la hora de analizar las medidas del Cimel pero se tiene menos información meteorológica.

En general creemos que la clasificación por sectores es más clara en cuanto a las medidas espectrales porque esta metodología de clasificación tiene en cuenta el tiempo de residencia en un cierto sector, lo cual supone en principio que los aerosoles sean de un cierto tipo de manera más marcada. Por su parte la clasificación del algoritmo es más objetiva y no requiere información a priori, y nos da una idea sobre las situaciones sinópticas principales y los aerosoles asociados a cada uno de esos escenarios. Por último creemos que la información que proporcionan las dos clasificaciones es complementaria para describir la climatología de los aerosoles en el Arenosillo, y por eso hemos descrito ambas y no hemos tomado ninguna de ellas como 'mejor' que la otra. 


\section{Capítulo 7. Caracterización y evaluación de episodios de aerosol desértico en El Arenosillo}

\section{Introducción}

En este capítulo vamos a realizar una evaluación de los episodios de aerosol desértico que han tenido lugar en El Arenosillo. El objetivo será caracterizar este aerosol desértico a través de las intrusiones registradas en el período 2000-2004 con el fotómetro Cimel. Para ello emplearemos como base las medidas de AOD espectral del Cimel, y nos ayudaremos de otras fuentes para el análisis. Tendremos en cuenta el análisis de retrotrayectorias del capítulo anterior, las observaciones mediante imágenes de satélite, y mapas sinópticos. También consideramos interesante incluir los mapas de predicción que realizan diversos modelos de transporte de polvo desértico, tomando esta información a nivel ilustrativo.

Las intrusiones de aerosol desértico constituyen los episodios más severos a nivel de carga de aerosol, y por su frecuencia constituyen el escenario de mayor interés en la zona de estudio. 


\subsection{Intrusiones de polvo sahariano en la Península. Mecanismos}

Los episodios más severos en cuanto al aerosol atmosférico que hemos registrado en estos 5 años de datos en El Arenosillo son de dos tipos fundamentales: entradas de aerosol desértico procedente del norte de África y episodios de combustión de biomasa en grandes incendios forestales. Las entradas de aerosol desértico son mucho mas frecuentes y en general pueden afectar a muy extensas zonas de Europa desde el Atlántico hasta el Mediterráneo oriental.

La estación de El Arenosillo tiene una ubicación idónea para la detección y seguimiento de las entradas de aerosol desértico en la Península. Las medidas remotas del Cimel se han mostrado muy útiles para esta función, ya que al ser medidas columnares no se ven perjudicadas por la altura de transporte del aerosol. En ocasiones sucede que a nivel de suelo no hay un gran aumento de concentración de partículas por ser el transporte de polvo muy por encima de la capa límite. Frente a esta ventaja del Cimel, está el inconveniente de no poder medir durante los episodios asociados con nubosidad o lluvia. Estos episodios con deposición húmeda son también de gran interés científico, aunque en ellos el efecto radiativo de los aerosoles juega un papel menor frente a las nubes.

La literatura al respecto de evaluación de fuentes, caracterización y transporte de aerosol sahariano es muy abundante en los últimos años, por lo que renunciamos aquí a presentar una extensa bibliografía, que podríamos empezar a considerar desde principios de los años 70 (Carlson and Prospero, 1972). Sin embargo nos encontramos con que no existe ninguna evaluación de episodios desérticos sobre la Península Ibérica basada en medidas de detección remota, en particular medidas de fotómetros. En todo caso queremos citar los inventarios recientes que aparecen en el trabajo de Sánchez de la Campa (2004) y Escudero (2005), basados en el estudio de retrotrayectorias y concentraciones de masa de partículas, y que tomaremos como elementos de referencia en este trabajo.

Nos interesa describir los mecanismos que a escala sinóptica provocan la llegada de estas masas de aire africanas a la Península Ibérica. Éstos dependen de varios factores y poseen un marcado carácter estacional. Por una parte son necesarias unas zonas fuente de polvo desértico, en nuestro caso el desierto del Sahara y la zona del Sahel (al sur del Sahara). La escasa precipitación, humedad relativa muy baja y viento en superficie en estas zonas fuente, favorecen el aporte de polvo a la atmósfera. Los movimientos convectivos debidos a la insolación, acompañados de fuerte inversión térmica durante las horas nocturnas favorecen la acumulación de material particulado en la atmósfera. Determinadas condiciones sinópticas son luego responsables del transporte de estas partículas a zonas remotas, hasta miles de kilómetros de distancia. Estas masas de aire cargadas de polvo desértico llegan tanto a la zona del Caribe, desde Canarias y atravesando el océano Atlántico (Prospero, 1999), como al norte de Europa o al Mediterráneo oriental.

Podemos distinguir cuatro escenarios típicos que favorecen la llegada de polvo desértico a la Península Ibérica (Rodríguez et al., 2001; Querol et al., 2002; Escudero et al., 2005; Pérez et al., 2005), y que denominamos siguiendo la nomenclatura dada en Escudero et al. (2005):

\section{- Escenario 1: anticiclón sobre el norte de África (NAH-S)}

Este primer escenario se produce por la presencia de un anticiclón en el norte de África o sur de la Península Ibérica (Figura VII-1a), tanto en superficie como en niveles altos. 
Las masas de aire cargadas de polvo, procedentes de la zona Occidental del Sahara y del Sahel son desplazadas hacia Canarias y luego hacia el norte por el Atlántico, siguiendo el movimiento anticiclónico. Esto da lugar a la formación de un arco atlántico, es decir, una pluma de polvo estrecha y curva, que puede llegar hasta el norte de Europa. Este tipo de eventos afecta a la Península llegando desde el oeste o noroeste, y se da principalmente a finales de invierno o principios de primavera. El material particulado es transportado en los niveles bajos de la atmósfera, y en pocos casos vienen acompañados de lluvia (deposición húmeda). La detección de estos episodios mediante retrotrayectorias es difícil porque hay que retroceder hasta 10 días, con el consiguiente error.

\section{- Escenario 2: depresión atlántica frente a Portugal (AD)}

Este segundo escenario tiene lugar por la presencia de una depresión atlántica más o menos profunda frente a las costas de Portugal o el Golfo de Cádiz. En estas condiciones se produce una advección de aire en dirección norte por delante de la borrasca desde las zonas occidentales del Sahara o Sahel, que puede verse reforzada por la presencia de altas presiones en el Mediterráneo oriental (Figura VII-1 b). Estas intrusiones afectan sobre todo al este de la Península, y en un alto porcentaje ( $41 \%$ en Escudero et al., 2005) vienen acompañadas por deposición húmeda, también conocidas como lluvias de barro o lluvias rojas (Avila et al., 1997, 1998). Esto hace que tanto la llegada de las plumas de polvo, empujadas por masas atlánticas, como la salida sea brusca en general, con una duración de los eventos de 2-3 días. El aerosol es transportado tanto en niveles bajos como altos, y la mayor parte de estos eventos se da entre enero y junio.

\section{- Escenario 3: depresión norteafricana (NAD)}

Este tercer escenario tiene lugar por la presencia de bajas presiones en superficie sobre el norte de África o el Mediterráneo occidental, con altas presiones sobre la Península. Esta depresión viene acompañada por un pequeña vaguada en los niveles altos (Figura VII-1 c). Con esta situación llegan a través del Mediterráneo hasta el este peninsular masas de aire cargadas de polvo. En estos casos las regiones fuente de aerosol desértico están en el Sahara más oriental, y las partículas son transportadas sobre todo en los niveles bajos de la atmósfera. Muchos de estos eventos vienen acompañados por deposición húmeda, ya que esta situación de vientos húmedos y cálidos del Mediterráneo suele dar lugar a precipitaciones en la costa este peninsular.

- Escenario 4: altas presiones en los niveles altos sobre el sudeste de la Península (NAH-A)

Este cuarto escenario es responsable de la mayoría de intrusiones y se da sobre todo en verano. El intenso calentamiento de la superficie da lugar a la formación de una baja térmica en superficie sobre el norte de África, con altas presiones en los niveles altos. Cuando estas altas presiones en altura se desplazan hacia el este, se produce en altura la advección de masas de aire hacia la Península (Figura VII-1 d). El aerosol se transporta a gran altura, en ocasiones sin ser detectado a nivel de suelo, y las masas de aire tienen en general una gran carga de aerosol. La zona fuente es todo el desierto del Sahara, en el que los fuertes movimientos convectivos en verano inyectan grandes cantidades de polvo en la troposfera libre, hasta $5000 \mathrm{~m}$ de altura. Las intrusiones ocupan extensas zonas y se desplazan de sur a norte cubriendo toda la Península, y pudiendo afectar a gran parte de Europa. Además el polvo en suspensión suele tener un tiempo de residencia bastante largo, debido a la gran extensión de la masa de aire, la lenta renovación, los movimientos convectivos que mantienen el polvo en suspensión y la escasez de lluvias. 
(a)

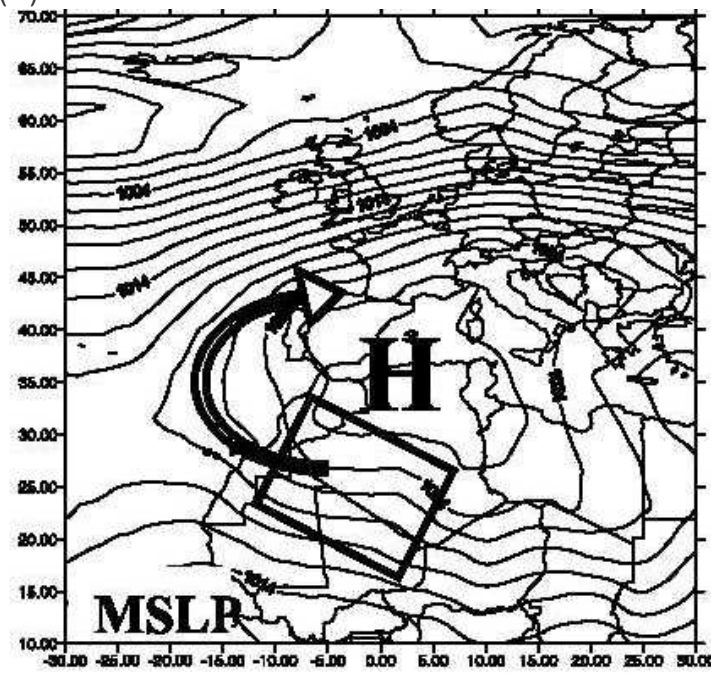

(b)

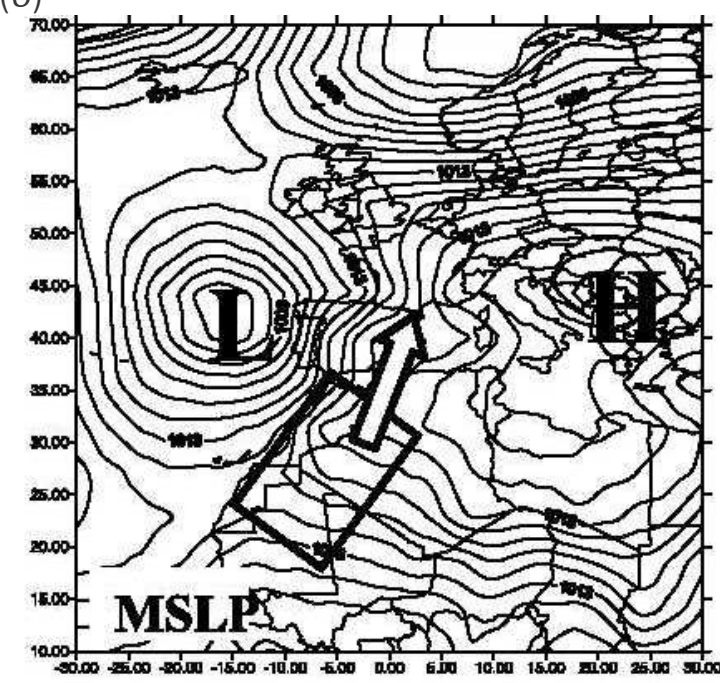

(c)

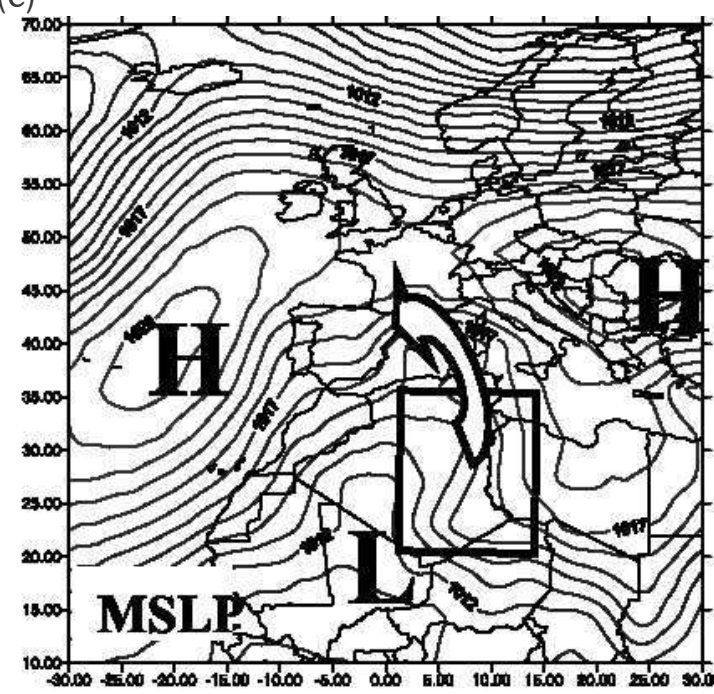

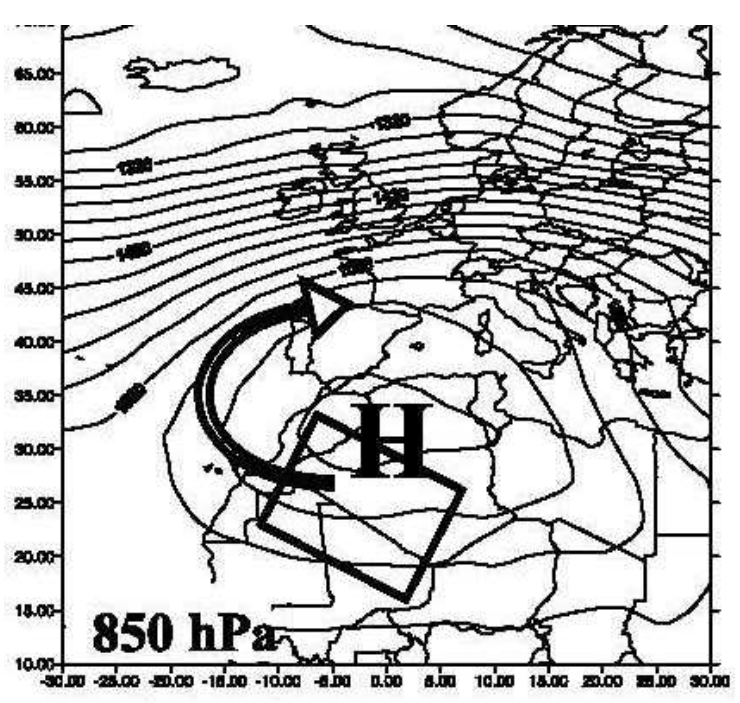
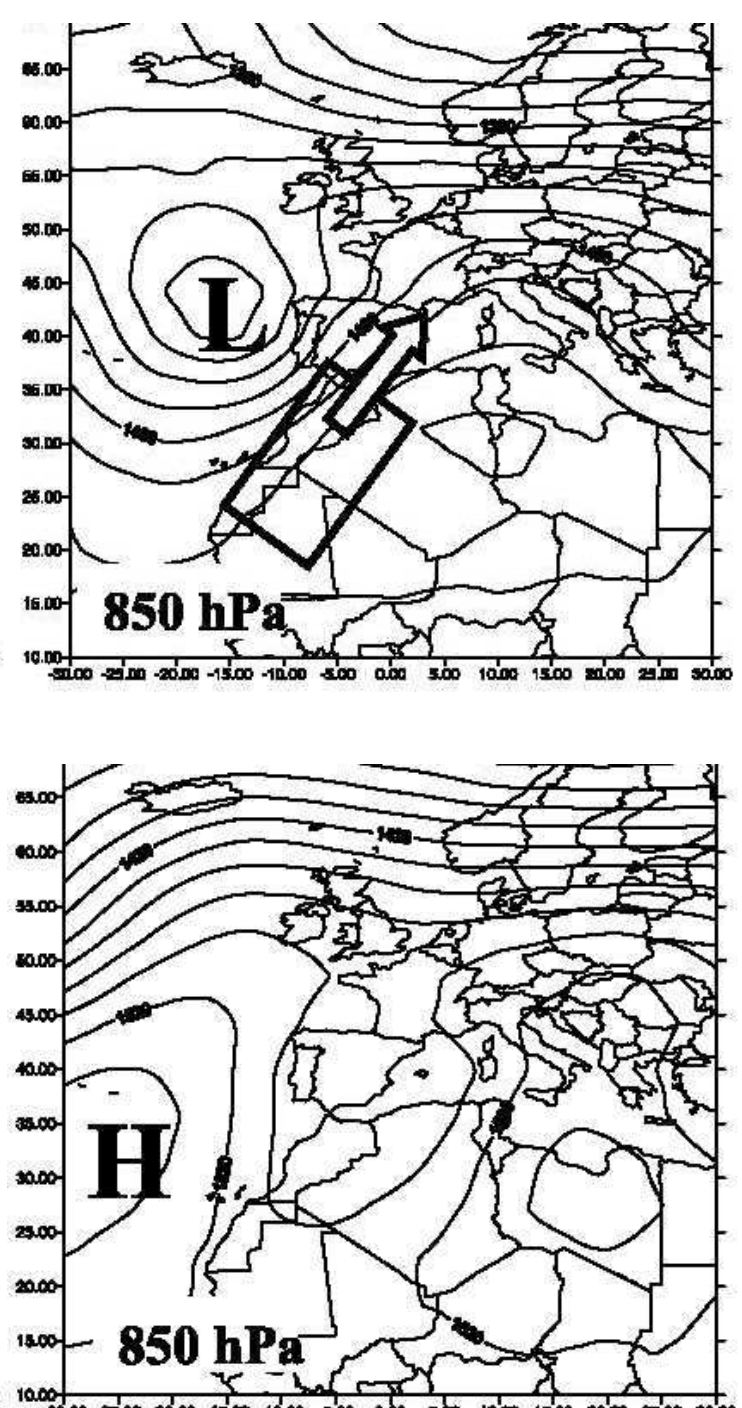
(d)
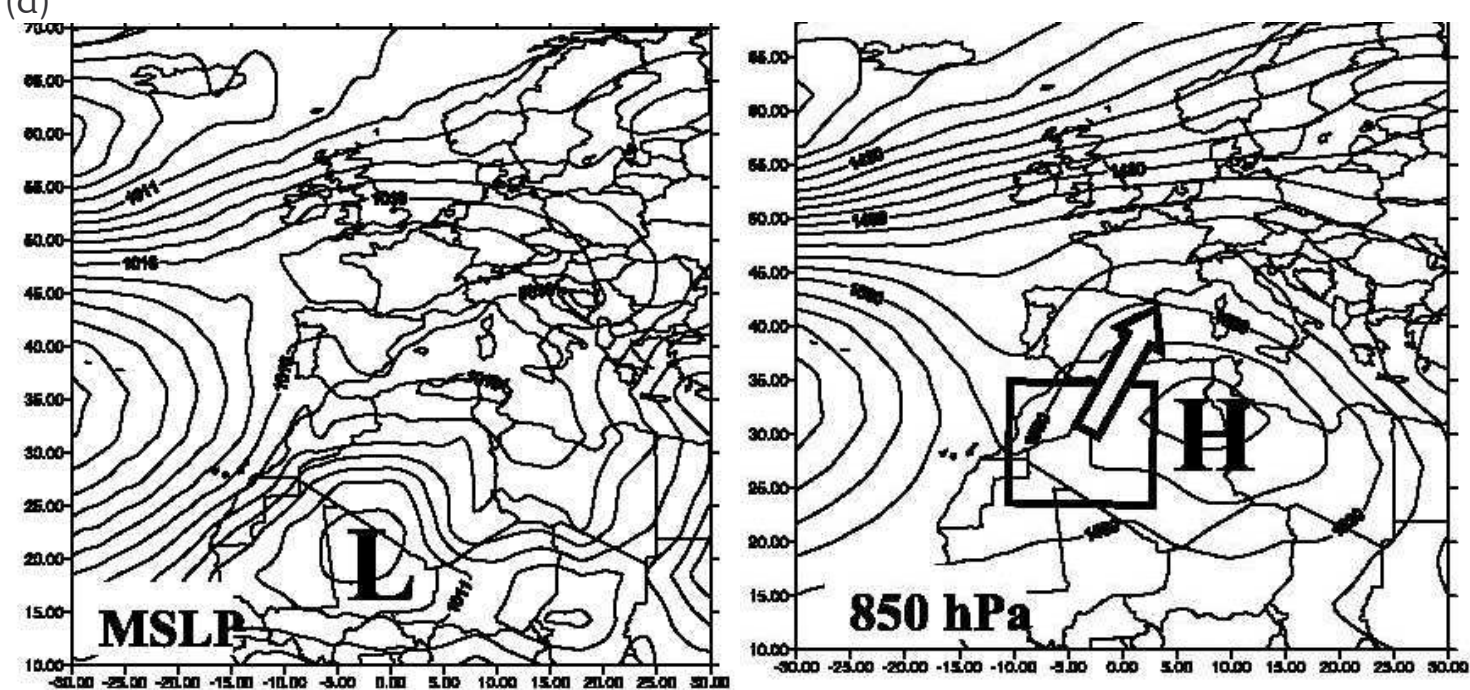

Figura VII-1. Situaciones sinópticas correspondientes a los cuatro escenarios para la llegada de aerosol desértico, con la presión a nivel de superficie (izquierda) y altura geopotencial en 850hPa (derecha): (a) Escenario 1, con anticiclón sobre el norte de África; (b) Escenario 2, depresión atlántica; (c) Escenario 3, depresión norteafricana a nivel de superficie y de 850hPa;

(d) Escenario 4, baja norteafricana con anticiclón en los niveles altos (tomadas de Escudero et al., 2005). 


\subsection{Metodología para el estudio de los eventos de aerosol desértico}

La herramienta básica para el estudio de los eventos de aerosol desértico en El Arenosillo ha sido el fotómetro Cimel, en concreto a través de las medidas espectrales del espesor óptico de aerosoles, y el parámetro derivado alfa. Además los episodios han sido confirmados por otras fuentes, como son el análisis de retrotrayectorias y el análisis de imágenes de satélite.

Como ya se desarrolló en el capítulo 5, se han calculado para cada día a las $12 \mathrm{~h}$ GMT, mediante el modelo HYSPLIT, las retrotrayectorias a 500, 1500 y 3000m s.n.m. de cinco días hacia atrás, lo que permite identificar la procedencia de la masa de aire y también la altura a la que ha viajado hasta la estación. Estas retrotrayectorias se utilizarán para fijar el origen de las supuestas masas de aire desérticas, y confirmar el comienzo y el final de los episodios.

El trabajo de Sánchez de la Campa (2004) realiza un inventario de episodios de aerosol desértico basado en el análisis del origen de las masas de aire, que determinan de manera visual, y que posteriormente relacionan con medidas de concentración a nivel de suelo (PM10 -partículas con tamaño aerodinámico menor de 10 micras-y TSP -total suspended particles). Este inventario entre los años 1996 y 2000 únicamente solapa con nuestra serie de datos durante unos meses. En el trabajo reciente de Escudero et al. (2005) se extiende dicho inventario hasta 2002, pero relativo a la zona del levante español. Volvemos a encontrarnos como en el trabajo anterior con que no se establece un criterio sobre los niveles de PM10 que delimite el episodio desértico. Estos inventarios se siguen realizando en el marco de varios proyectos coordinados de ámbito nacional (PANDORA, TROMPETA) realizados por los grupos de investigación del Instituto de Ciencias de la Tierra Jaume Almera (CSIC), Centro de Investigación Ecológica y Aplicación Forestal de la Universidad Autónoma de Barcelona, Observatorio Atmosférico de Izaña (INM) y el departamento de Geología de la Universidad de Huelva. Disponemos de datos no publicados proporcionados por dichos grupos.

Para identificar las situaciones sinópticas asociadas a las masas de aire con aerosol desértico, se han utilizado los mapas sinópticos de presión en superficie (MSLP, mean sea level pressure) y altura geopotencial en $700 \mathrm{hPa}$. Estos mapas se han obtenido de la base de datos FNL del NOAA, a través de la web: http://www.arl.noaa.gov/ready/amet.html.

Por último, los sensores a bordo de satélites pueden facilitar la detección de las masas cargadas de aerosol. El índice de aerosoles dado por el TOMS (Total Ozone Mapping Spectrometer) y las imágenes SeaWIFS y MODIS (Moderate Resolution Imaging Spectroradiometer) han sido utilizadas también en este trabajo. Algunas de las imágenes son muy espectaculares, como por ejemplo las del arco atlántico de polvo que acompaña a numerosos eventos. También hay que señalar, como advierten varios autores (Herman et al., 1997; Torres et al., 2002), que el sensor TOMS no detecta los aerosoles contenidos en las capas más bajas de la atmósfera, por debajo de los $1000 \mathrm{~m}$. Por ello el índice de aerosoles (AI) podemos tomarlo sólo como un indicador cualitativo en la zona de El Arenosillo. En los trabajos (Vergaz, 2001; Vergaz et al., 2003; Cachorro et al., 2005) se ha hecho uso de este índice, analizando su correlación con el espesor óptico de aerosoles, observándose una muy mala correlación en general. 
La metodología empleada para la identificación de episodios desérticos ha sido la siguiente:

1.- Para la detección de episodios desérticos se ha tomado el siguiente criterio sobre las medidas espectrales: $\operatorname{AOD}(440 \mathrm{~nm})>0.25$ y alfa $<0.8$. La elección de este criterio de detección (que no evaluación) está basado en nuestra experiencia de trabajos anteriores (Vergaz, 2001; Vergaz et al., 2003; Vergaz et al., 2005; Cachorro et al., 2005) y en la evaluación de los promedios de la serie completa de AOD en El Arenosillo realizada en el Capítulo 4. Teniendo en cuenta los resultados del promedio del AOD en 440nm, de 0.17, con su $\mathrm{STD}=0.13$, podríamos tomar un primer valor de corte en 0.3 para el $\mathrm{AOD}$ (promedio+1STD). De manera análoga, el promedio de alfa resultó ser 1.04 (STD 0.43), por lo que el límite para el alfa sería 0.60 (promedio-1STD). Aunque sería igualmente válido para la detección, consideramos que este criterio resulta demasiado restrictivo, y por ello hemos tomado el criterio dicho anteriormente.

También hemos tenido en cuenta las características conocidas en la literatura para los aerosoles desérticos (Hess et al., 1998; Holben et al., 2001; Dubovik et al., 2002). La mayor discrepancia con los valores usuales para estaciones 'desérticas' está en el parámetro alfa, dada la influencia continental o industrial en nuestra estación. Con este criterio se consigue identificar la mayoría de episodios, pero en general encontramos así los días centrales de los mismos, pero no las entradas (y las salidas), que corresponden a las subidas (bajadas) del AOD y al contrario con el parámetro alfa.

2.- Una vez detectado el evento, mediante identificación visual se encuentra el día de comienzo y el de final de los episodios, como complemento al paso $1^{\circ}$. Cuando entra la masa de aire desértica se detecta con relativa facilidad una subida del AOD en los cuatro canales, con la correspondiente bajada en el parámetro alfa. Cuando el episodio termina se recuperan los valores de fondo, con un descenso en el AOD y subida del alfa. Con esto disponemos de un primer inventario de episodios según los datos del Cimel, y que tenemos que confirmar por otras fuentes.

3.- Confirmación de los episodios por retrotrayectorias. Se analizan las retrotrayectorias para los días seleccionados, idénticas a las utilizadas en el capítulo anterior, es decir, en los tres niveles de 500, 1500 y 3000m, y con una duración de 5 días hacia atrás.

4.- Visualización de las imágenes de los satélites SeaWIFS, MODIS y TOMS, sobre todo en los episodios más fuertes, puesto que en episodios débiles la visualización de la imagen no es de gran ayuda. Por otra parte podríamos tomar los datos de AOD dados por estos sensores, pero en esta caso nuestros propios datos serían más fiables. En estas imágenes se observan fácilmente las manchas de polvo en tonos amarillos, frente a las nubes de color blanco, lo que además nos permite detectar si ha habido nubosidad durante el episodio. En caso de dudas sobre la presencia de nubosidad también se ha consultado las imágenes del Meteosat, más frecuentes que las de los sensores mencionados.

5. Consulta de los mapas del modelo NAAPS. El modelo NAAPS ha mostrado una gran fiabilidad al comparar nuestro inventario (realizado con los pasos 1-4) con las predicciones dadas por el mismo. Estos mapas son importantes por cubrir una extensa área alrededor de nuestra zona de estudio, y pueden ser útiles a la hora de interpretar causas de episodios de alta turbiedad (por ejemplo humos de incendios forestales).

6.- Finalmente se realiza el análisis de los mapas sinópticos en $700 \mathrm{hPa}$ y nivel de superficie para catalogar el evento dentro de los cuatro tipos especificados en el punto 6.1, como complemento a los puntos anteriores. 


\subsection{Evaluación de los episodios de aerosoles saharianos en El Arenosillo}

El análisis de la serie de datos 2000-2004 del Cimel en El Arenosillo con la metodología descrita ha dado como resultado un inventario de episodios que se ha incluido íntegro en el anexo 2. En él podemos encontrar un total de 75 episodios confirmados y 319 días con aerosol desértico medido por el Cimel, unos 64 días al año aproximadamente, aunque muy variable de unos años a otros como vamos a ir detallando. El inventario consta de los días de inicio y fin del episodio, la duración, el AOD máximo alcanzado, el alfa mínimo y el tipo de escenario sinóptico durante el episodio entre los cuatro especificados anteriormente. También se incluye un apartado para comentarios.

\subsubsection{Análisis de la frecuencia y duración de los episodios}

En primer lugar resumimos en la Tabla VII-I todos los episodios que hemos medido con el Cimel y comprobado mediante las otras fuentes mencionadas. En esta tabla hemos detallado la información por años y luego para la serie completa. Se incluye el número de episodios y el número de días, con el promedio de duración de los episodios y los promedios de los valores extremos de AOD y alfa. En realidad es más fiable el número de días con desértico que el número de eventos, pues en ocasiones es muy complicado separar el final de un episodio y el comienzo del siguiente, o definir cuándo se trata del mismo o se trata de dos diferentes. Esto es particularmente complicado en verano, cuando el tiempo de residencia del polvo en suspensión puede ser muy largo o se producen recirculaciones de las masas de aire.

Por una parte, tanto la duración como la intensidad de los episodios (dados por el AOD máximo en 440nm que se ha alcanzado y el alfa mínimo) son muy similares entre unos años y otros, con un AOD máximo en torno a 0.5 y alfa mínimo en torno a 0.3 , ambos claramente propios del aerosol desértico, y una duración en torno a 4 días por episodio. Sólo destaca el 0.70 de promedio de AOD máximo en 2004, provocado por el fuerte episodio en el mes de julio, en el que se alcanzó 2.7 de AOD en 440nm. Frente a estas similitudes, el número de episodios y por tanto el número de días por año con aerosol desértico varía de unos años a otros. Mientras que en 2002 se registraron 10 episodios y 51 días, en 2004 se llegó a 19 episodios, casi el doble, y 77 días con aerosol desértico, y números parecidos tenemos para el año 2000. Esto supone que la presencia de aerosol desértico ha variado entre el $14 \%$ y el $21 \%$ de los días según el año. En promedio para toda la serie de 5 años encontramos 15 episodios al año y 64 días al año con desértico (el $17.5 \%$ de los días).

Al respecto del año 2002 queremos señalar que el número tan bajo de días registrados puede ser debido también a la falta de algunos datos, ya que por problemas de instrumentación no se pudieron realizar medidas durante 3 semanas en marzo y otras 3 en julio, dos meses que registran numerosos episodios. De cara a la estadística, en el inventario se ha intentado compensar este problema incluyendo 6 días durante el mes de marzo y 10 días de aerosoles desérticos durante el mes de Julio cuando el aparato no estuvo en funcionamiento. Estos días han sido confirmados por las demás fuentes disponibles, aunque no existe dato del fotómetro Cimel.

También hemos destacado del inventario los episodios de más de 5 días de duración, es decir episodios grandes y que afectan a la zona de estudio de manera continuada durante 
6 ó más días. Se han registrado un total de 17 en los 5 años, entre 2 y 5 por año. El episodio más largo duró 18 días y tuvo lugar entre julio y agosto de 2003. En la Figura VII2a podemos encontrar el número de episodios según la duración, con 16 episodios de 1 solo día hasta el episodio ya mencionado que duró 18 días. El promedio de duración es de unos 4 días por episodio, aunque también se ha contabilizado un número alto de episodios de 1 día de duración. Después los más frecuentes son los de 4 días, y esta frecuencia disminuye para episodios más largos, hasta los 8 días. A partir de ahí destacan los 4 episodios de 10 días y luego tenemos 3 episodios aislados con una duración de 12, 15 y 18 días.

\begin{tabular}{lccccccc}
\hline \hline & 2000 & 2001 & 2002 & 2003 & 2004 & TOTAL & Por año \\
\hline $\mathrm{N}^{\circ}$ episodios & 18 & 16 & 10 & 12 & 19 & 75 & 15 \\
$\mathrm{~N}^{\circ}$ episodios >5 días & 4 & 3 & 1 & 2 & 5 & 15 & 3 \\
$\mathrm{~N}^{\circ}$ días & 71 & 64 & 51 & 56 & 77 & 319 & 63.8 \\
Porcentaje días (\%) & 19.5 & 17.5 & 14.0 & 15.3 & 21.1 & 17.5 & 17.5 \\
Días por episodio & 3.9 & 4.0 & 3.5 & 4.7 & 4.1 & 4.0 & - \\
Promedio AOD_440máx & 0.47 & 0.53 & 0.49 & 0.46 & 0.70 & 0.54 & - \\
Promedio Alfa mín & 0.36 & 0.33 & 0.24 & 0.25 & 0.30 & 0.31 & - \\
\hline \hline
\end{tabular}

Tabla VII-I. Resumen de episodios desérticos del inventario del anexo 2, tanto por años como para el total de los 5 años. La última columna da el promedio anual de número de episodios, número de episodios grandes (de más de 5 días) y el número de días con aerosol desértico.

El análisis del número de episodios para cada mes del año (Figura VII-2b) deja claro el máximo del verano, con 15 episodios durante el mes de julio, y que disminuye en número de casos tanto hacia el otoño, de manera suave, como hacia la primavera, más bruscamente. El mes con menos episodios registrados es abril, con tan solo 2. Frente a este mínimo primaveral, destaca el segundo pico de frecuencia que tiene lugar en febrero y marzo, con 6 y 7 episodios respectivamente. Como veremos en el apartado siguiente, los episodios de febrero y marzo son además diferentes en cuanto a situaciones sinópticas que los de verano. En cuanto a los episodios largos, con una duración superior a 5 días, se concentran sobre todo en los meses de verano, aunque hay que destacar los 2 episodios largos del mes de marzo y del mes de octubre. El mes con más episodios largos ha resultado ser junio, con un total de 4 , lo que supone casi uno por año.
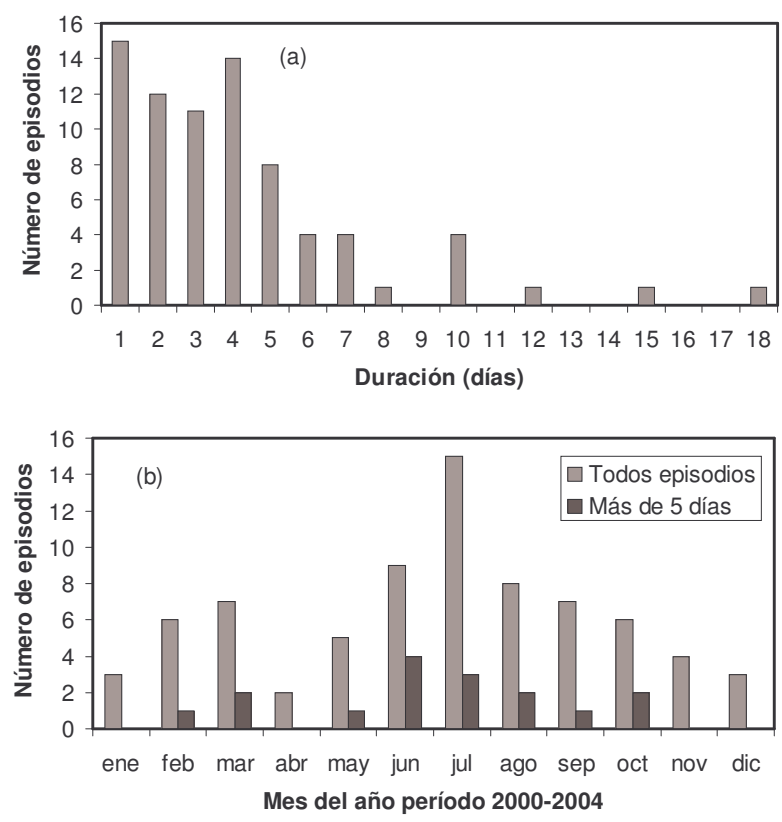

Figura VII-2. (a) Frecuencias absolutas ( $\mathrm{n}^{\circ}$ de episodios desérticos) según la duración en días de los eventos; (b) Distribución mensual del número de episodios para el período 2000-2004. 
Durante estos 5 años el Cimel ha medido un total de 1346 días sobre los 1781 posibles. Esto significa que durante los días con lluvia o muchas nubes no se ha podido analizar la presencia de aerosol desértico. Por tanto el número de episodios y de días al año con aerosol desértico en la estación debe ser mayor que las cifras que estamos dando, en particular durante los meses a priori más lluviosos, entre octubre y abril, en los que se dan episodios con deposición húmeda como los que aparecen en el inventario de Sánchez de la Campa (2004). Sí hemos encontrado en nuestra serie de datos varios episodios en los que las medidas del Cimel se interrumpen bruscamente por la llegada de nubes o lluvia, los cuales quedan contabilizados. Sin embargo aparecen en el citado inventario algunos episodios en días sin medida alguna del Cimel con la que poder comparar. Con respecto al trabajo de Escudero et al. (2005) la diferencia fundamental en cuanto al inventario mensual de episodios está en el mes de mayo, al que asignan el mayor número de episodios, por encima de los meses de verano. La mayoría de esos episodios están relacionados con situaciones de lluvia (tipos $\mathrm{AD}$ y $\mathrm{NAD}$ ), y el análisis está realizado sobre la costa Mediterránea, lo cual explicaría esta diferencia. El resto del estudio muestra acuerdo con nuestros resultados en cuanto a los dos modos con máximos en marzo y julio, al que en ese trabajo se añade un tercero en mayo. Abril es también un mes con muy pocos episodios, así como noviembre y diciembre.

Los episodios desérticos modulan el comportamiento anual del espesor óptico de aerosoles. Como podemos observar en la Figura IV-8 el promedio mensual sigue la misma tendencia que la frecuencia de los episodios (Figura VII-2b), con un máximo principal en verano y otro máximo secundario en febrero-marzo. En el resto de la primavera y el otoño-invierno, cuando menos episodios desérticos se producen, los valores de AOD son también menores.

\subsubsection{Situaciones sinópticas durante los episodios desérticos.}

El análisis de las situaciones sinópticas se ha realizado mediante los citados mapas de análisis dados por la base de datos FNL. Analizando visualmente estos mapas para los días de comienzo de los episodios, se han clasificado dentro de los cuatro tipos descritos anteriormente. Aunque en ocasiones resulta complicado, en general hemos podido asignar una de las situaciones tipo a cada episodio. El número de episodios de cada tipo se resume en la Tabla VII-II.

\begin{tabular}{lcccc}
\hline \hline & $\begin{array}{c}\text { NAH-S } \\
\text { (Tipo 1) }\end{array}$ & $\begin{array}{c}\text { AD } \\
\text { (Tipo 2) }\end{array}$ & $\begin{array}{c}\text { NAD } \\
\text { (Tipo 3) }\end{array}$ & $\begin{array}{c}\text { NAH-A } \\
\text { (Tipo 4) }\end{array}$ \\
\hline Número episodios & 10 & 10 & 11 & 44 \\
Episodios/año & 2 & 2 & 2 & 9 \\
Duración promedio (días) & 3.4 & 3.3 & 3.8 & 4.4 \\
Episodios más de 5 días & 1 & 1 & 4 & 9 \\
\hline \hline
\end{tabular}

Tabla VII-II. Resumen de episodios desérticos clasificados por tipos sinópticos.

En la Figura VII-3 hemos separado estos episodios por meses, para poder analizar también la estacionalidad de los mecanismos que dan lugar a los episodios desérticos, como vamos a detallar a continuación.

La situación sinóptica más frecuente que favorece la llegada de aerosol desértico es la que hemos denominado tipo 4 (NAH-A), que consiste en la baja presión norteafricana en superficie con altas presiones en los niveles altos al sudeste de la Península, sobre el noreste africano. Esta situación da lugar a 44 episodios, esto es, casi 9 episodios al año en promedio, pero con gran variabilidad: 6 episodios de este tipo hubo en 2002 y hasta 13 en 2000. La época del año en la que se da este tipo de eventos es el verano, siendo el único 
mecanismo sinóptico encontrado para el alto número de intrusiones en los meses de junio, julio y agosto y casi el único en septiembre (ver Figura VII-3). Un resultado similar se encuentra en el trabajo de Escudero et al. (2005), en el que prácticamente todos los episodios entre junio y septiembre (50 de 52 en el período 1996-2002) son del tipo NAHA. Entre los meses de noviembre y abril no se ha registrado ningún evento de este tipo. Esto es lógico dado que la formación de la baja térmica norteafricana está relacionada con el intenso calentamiento del suelo en los meses de verano. Como ejemplo mostramos en la Figura VII-4d el mapa sinóptico para el día 28 de agosto de 2000, en el que podemos apreciar las altas presiones en altura sobre Argelia y Túnez, mientras que en superficie hay bajas presiones. Los episodios de este tipo tienen una duración promedio de 4.4 días, es decir, ligeramente por encima del promedio, y conforman 9 de los 15 episodios largos, la mayor parte. Esto es lógico por el mayor tiempo de residencia del polvo en suspensión durante el verano, debido a los movimientos convectivos, recirculaciones y escasez de lluvias como ya se ha comentado. Tres episodios de este tipo se analizarán con detalle en la sección 7.4 .

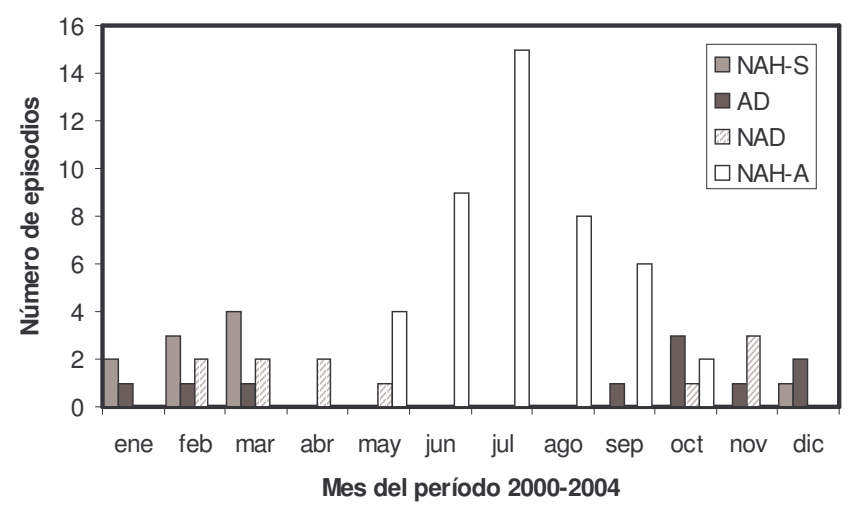

Figura VII-3. Distribución mensual de los episodios de aerosol desértico según el tipo de situación sinóptica.

En segundo lugar, los episodios del tipo 3 (NAD) suman un total de 11, aproximadamente 2 al año. En el ejemplo de episodio de este tipo, correspondiente al 22 de octubre de 2000 (Figura VII-4c) observamos bajas presiones en superficie y también una vaguada en altura sobre el norte de África, que empuja la masa de aire a través del Mediterráneo hasta la Península. La duración promedio de estos episodios de tipo NAD es de 3.8 días, y este grupo contiene 4 episodios largos. Se han registrado en primavera y en otoño, desde febrero hasta abril y en octubre y noviembre. Es posible que el número de episodios de este tipo esté subestimado por estar asociados generalmente con lluvia y deposición húmeda, y que además llega desde el Mediterráneo. Por ejemplo al comparar con el inventario de Escudero et al. (2005), éste registra unos 4 episodios de este tipo al año, principalmente en el mes de mayo, lo cual no ha podido ser medido con el fotómetro Cimel, y se indica también que el $87 \%$ de ellos vinieron acompañados por deposición húmeda.

Los episodios de tipo $2(\mathrm{AD})$, con advección del sur provocada por una depresión atlántica frente a Portugal, suma un total de 10 casos, esto es, un promedio de 2 episodios por año. Nuevamente el número de casos de este tipo puede estar subestimado por la posibilidad de episodios asociados a lluvia. Sólo se han registrado intrusiones de este tipo entre septiembre y marzo, cuando también son más frecuentes las borrascas atlánticas. La duración promedio de estos episodios es de 3.3 días, y dentro de este grupo sólo se ha registrado 1 episodio largo. En el ejemplo de la Figura VII-4b, del día 27 de noviembre de 2004, tenemos una borrasca frente a las costas de Portugal, acompañada también por una vaguada en altura, con viento del sur por delante de la depresión, que se ve reforzado por la presencia de altas presiones sobre el Mediterráneo. 
(a)

NoRA Air Resources Laboratory

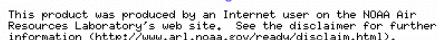

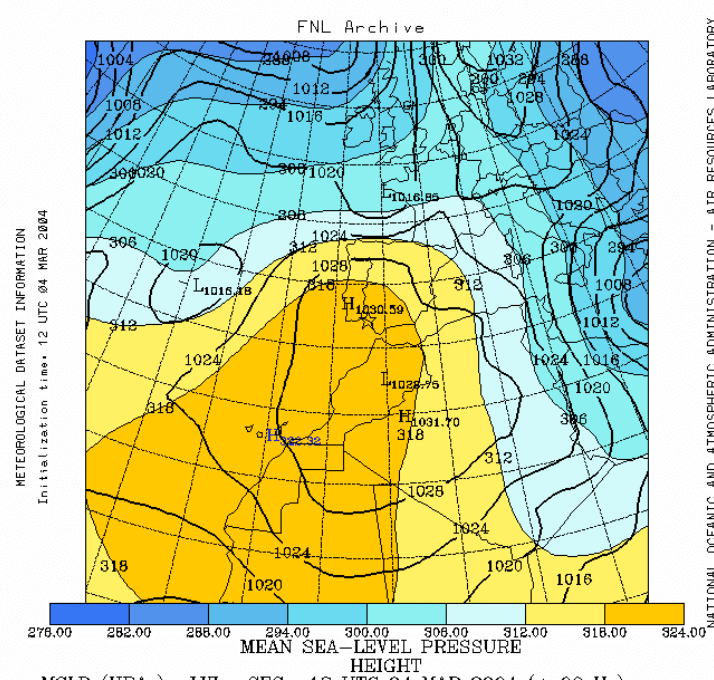

(c)

MSLP (HPA ): LVL $=$ SFC, 12 UTC 04 MAR $2004(+00 \mathrm{H})$

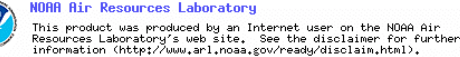

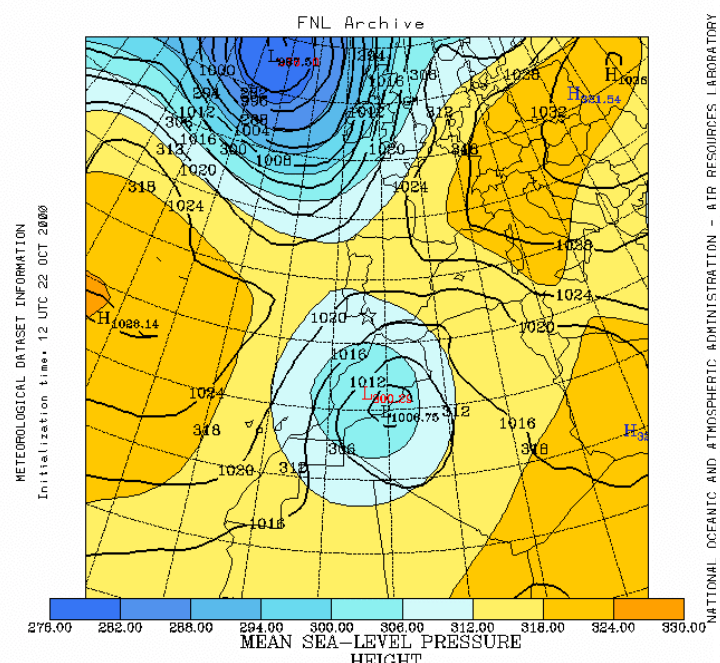

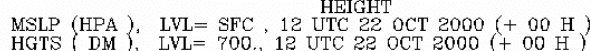

(b)

NOAR Air Resources Laboratory

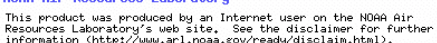

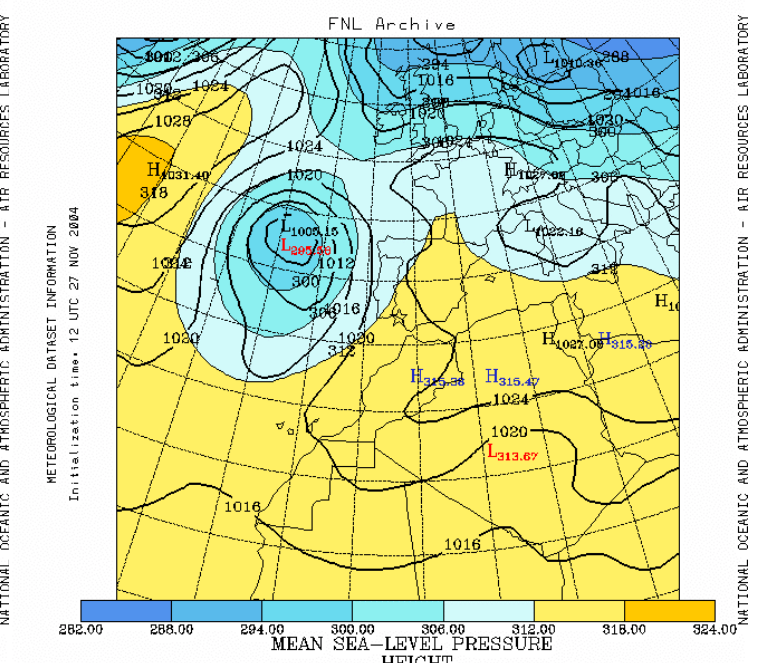

(d)

MSLP (HPA ). LVL $=$ SFC, 12 UTC 27 NOV $2004(+00 \mathrm{H})$

NOAR Air Resources Laboratory

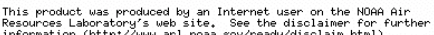

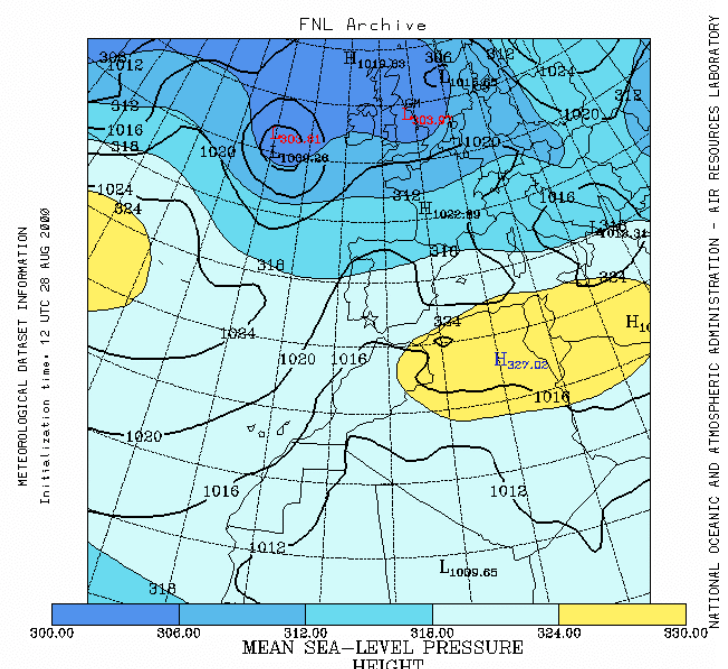

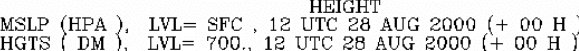

Figura VII-4. Situaciones sinópticas que han dado lugar a episodios de aerosol desértico. Mapas de presión en superficie y altura geopotencial a $700 \mathrm{hPa}$, todos a las 12 GMT: (a) Día 4 de marzo de 2004, ejemplo de tipo 1 (NAH-S); (b) Día 27 de noviembre de 2004, ejemplo de tipo 2 (AD); (c) Día 22 de octubre de 2000, ejemplo de tipo 3 (NAD); (d) Día 28 de agosto de 2000, ejemplo de tipo 4 (NAH-A).

Por último, los episodios del tipo 1 (NAH-S), es decir, altas presiones sobre el norte de África, suman un total de 10, unos 2 episodios al año. La duración media es de 3.4 días y sólo se ha registrado un episodio largo de este tipo, el de marzo de 2000. Este tipo de episodios se da entre diciembre y marzo, lo que provoca ese pico secundario de frecuencia de episodios a finales de invierno y principios de primavera. El ejemplo de la Figura VII$4 \mathrm{a}$, perteneciente al 4 de marzo de 2004, muestra las altas presiones tanto a nivel de superficie como en altura, que empujan las masas de polvo con movimiento anticiclónico que suele dar lugar al arco atlántico. En el apartado 6.4 se analizará en detalle uno de estos episodios de tipo NAH-S. 


\subsubsection{Características de AOD y alfa durante los episodios desérticos}

Una vez seleccionados los días en los que efectivamente hemos tenido aerosoles desérticos en El Arenosillo, podemos analizar las medidas de AOD y alfa dadas por el fotómetro Cimel, que nos van a dar las características de este tipo de aerosol.

Una estadística resumen de las medidas del Cimel durante estos episodios está en la Tabla VII-III. Lo primero que tenemos que destacar en estos datos es el promedio del AOD en los 4 canales, por encima (más de una STD) del promedio general de AOD en 440nm para toda la serie, que es 0.17(STD 0.13), y muy por encima de los promedios alrededor de 0.1 en 440nm y de 0.06 en los otros tres canales, para los días en los que con el análisis con retrotrayectorias identificamos la llegada de masas de aire Atlánticas (ver por ejemplo Tabla VI-XIV). Es decir, la primera y lógica característica de los episodios desérticos es la elevada turbiedad. También la variabilidad del AOD, que hemos caracterizado con la desviación estándar, es entre 4 y 5 veces superior a la que presentan las masas atlánticas limpias. El promedio de AOD para estos días con aerosoles desérticos es de 0.32 en $440 \mathrm{~nm}$, mientras que en los otros tres canales son muy similares entre sí, $0.24,0.21$ y 0.20 según vamos de 670 a 1020nm.

\begin{tabular}{lccccc}
\hline \hline & AOD_1020 & AOD_870 & AOD_670 & AOD_440 & Alfa(440-870) \\
\hline Promedio & 0.201 & 0.212 & 0.238 & 0.318 & 0.69 \\
Desv. Estándar & 0.147 & 0.151 & 0.155 & 0.165 & 0.38 \\
P25 & 0.114 & 0.124 & 0.147 & 0.212 & 0.39 \\
P75 & 0.247 & 0.260 & 0.287 & 0.385 & 0.97 \\
P90 & 0.363 & 0.376 & 0.410 & 0.511 & 1.24 \\
P10 & 0.078 & 0.087 & 0.102 & 0.157 & 0.25 \\
Mín. & 0.017 & 0.016 & 0.019 & 0.030 & -0.03 \\
Máx. & 2.541 & 2.623 & 2.691 & 2.697 & 1.76 \\
Mediana & 0.161 & 0.172 & 0.200 & 0.292 & 0.611 \\
\hline \hline
\end{tabular}

Tabla VII-III. Estadística de AOD y alfa para los días confirmados con aerosoles desérticos a partir de todas las observaciones: promedio, desviación estándar, percentiles 25, 75, 10 y 90 de la distribución, mínimo, máximo y mediana ó percentil 50, para el AOD en los 4 canales del Cimel y el alfa de Ångström.

El AOD alcanza durante determinados episodios desérticos los valores más altos registrados en la estación, alcanzándose 2.7 en 440nm (y 2.5 en 1020nm) durante el episodio de julio de 2004 que comentaremos en la sección siguiente. Estos son los valores extremos, aunque tan sólo se ha alcanzado espesores superiores a 1 durante los días centrales de 3 episodios, y sólo el 10\% de los datos está por encima de 0.5 entre estos días de episodios seleccionados. La mayoría de las observaciones se concentra entre 0.20 y 0.40 para $440 \mathrm{~nm}$, mientras que el AOD máximo registrado en cada episodio está en el $66 \%$ de los casos por encima de 0.4 , siendo 0.54 el promedio de este máximo en $440 \mathrm{~nm}$ (ver Tabla VII-I).

Como ya se ha podido ver en algunos ejemplos a lo largo de esta memoria, cuando se produce la entrada de una masa de aire con aerosol desértico se produce una subida del AOD similar en todas las longitudes de onda. La subida del AOD en las longitudes de onda más largas es reveladora de la entrada de aerosol desértico, puesto que para ellas el AOD suele ser bajo con excepción de estos episodios. Esta subida del AOD viene acompañada por un descenso del parámetro alfa, desde los valores promedio en torno a 1 hasta valores por debajo de 0.5 una vez que el episodio ha entrado completamente. En estos días centrales el espectro de AOD es muy plano, con valores muy similares en las 4 longitudes de onda. Al retirarse la masa de aire los valores de AOD y alfa vuelven los niveles de fondo, aunque no siempre de la misma manera. 
En efecto, en unos episodios desérticos el AOD sube y baja de forma brusca, a veces interrumpido por lluvia; en otros sube bruscamente y luego permanece alto muchos días hasta que se recuperan poco a poco los valores de fondo. Por último hay entradas en las que el AOD sube y baja lentamente. Es decir, no existe un patrón determinado como hemos podido comprobar analizando el promedio para los primeros días de los episodios, segundos días, terceros, etc. La alta variabilidad es por tanto una característica muy acusada en este tipo de eventos.

Este análisis sobre lo brusca o suave que sea la entrada o salida del aerosol desértico podemos hacerla también por tipos sinópticos. Lamentablemente no tenemos un número suficiente de casos de cada tipo excepto para el tipo NAH-A, el propio de verano. En los demás tipos no es posible este análisis. Para las intrusiones de tipo NAH-A se aprecia que el $\mathrm{AOD}$ es en promedio algo más bajo el primer día $(0.27$ en $440 \mathrm{~nm})$ que los días siguientes $\left(0.35\right.$ entre los días $2^{\circ}$ y $\left.5^{\circ}\right)$ y luego disminuye paulatinamente a partir del día $6^{\circ}$. A su vez el alfa vale en promedio 0.75 el primer día de los episodios de este tipo, para bajar a 0.57 el $2^{\circ}$ y $3^{\circ}$. Luego sube lentamente hasta 0.73 entre los días $4^{\circ}$ a $6^{\circ}$.

El parámetro alfa de Ångström tiene un promedio de 0.69 en los episodios desérticos. Este es un valor alto en comparación con la literatura sobre aerosoles desérticos. Entendemos que esto se debe a dos factores: en primer lugar el inventario incluye los días de entrada y salida de los episodios, en los que encontramos valores de fondo en la estación con AOD bajo y alfas en torno a 1 en promedio. Luego, durante los días centrales de los episodios, el valor de alfa suele descender hasta valores entre 0.2 y 0.4 , con un promedio de 0.31 y con un $72 \%$ de episodios con alfa mínimo por debajo de 0.4. Estos valores sí son los habituales para aerosol desértico, aunque como decimos se dan sobre todo los días centrales de cada episodio. En segundo lugar, creemos que la mezcla con aerosoles de origen local, continental o industrial, puede hacer que el parámetro alfa resultante sea mayor. Hemos podido observar esta situación durante episodios largos en los que la masa de aire cargada de polvo permanece muchos días sobre la zona de estudio. También en casos excepcionales, como el episodio de julio de 2003 que se analiza en la sección siguiente, la mezcla con aerosol debido a la combustión de biomasa da como resultado un parámetro alfa cercano a 1.8 y que nada tiene que ver con el propio del aerosol desértico.

En los diagramas de dispersión AOD-alfa de la Figura VII-5 hemos representado tanto el AOD $(440 \mathrm{~nm})$ como el AOD(870nm) frente a alfa. En la Figura VII-5a podemos apreciar el grueso de aerosol desértico con AOD alto, normalmente por encima de 0.2 , y una franja con alfas bajos que se extiende por la parte inferior del diagrama. Sin embargo tenemos un número elevado de casos con AOD medio y alfas por encima de 1 que no son típicos del desértico. Estas son las observaciones que pueden corresponder con las entradas y salidas de los episodios o en las que hay mezcla con otro tipo de aerosoles. También se puede apreciar una franja en la parte superior, con alfa en torno a 1.7 AOD alto, que corresponde con el episodio de julio de 2003 en el que se mezcló gran cantidad de aerosol de combustión de biomasa con el desértico.

El diagrama de AOD(870nm) frente a alfa de la Figura VII-5b es más claro a nuestro entender para describir el aerosol de tipo desértico. Es una figura menos ruidosa, en la que se aprecia la rama inferior que corresponde al aerosol desértico. A lo largo de ella, de izquierda a derecha, el AOD sube y desciende el alfa, mostrando una clara anticorrelación. Esta rama inferior es muy similar a la del diagrama de AOD $(440 \mathrm{~nm})$ frente a alfa (no así la mitad superior), puesto que para los aerosoles desérticos el espectro de AOD es muy plano por tratarse de partículas gruesas. Esta rama muestra una gran similitud con la mostrada para una estación tipo de aerosol desértico de la climatología de aerosoles dada por Holben et al. (2001). 

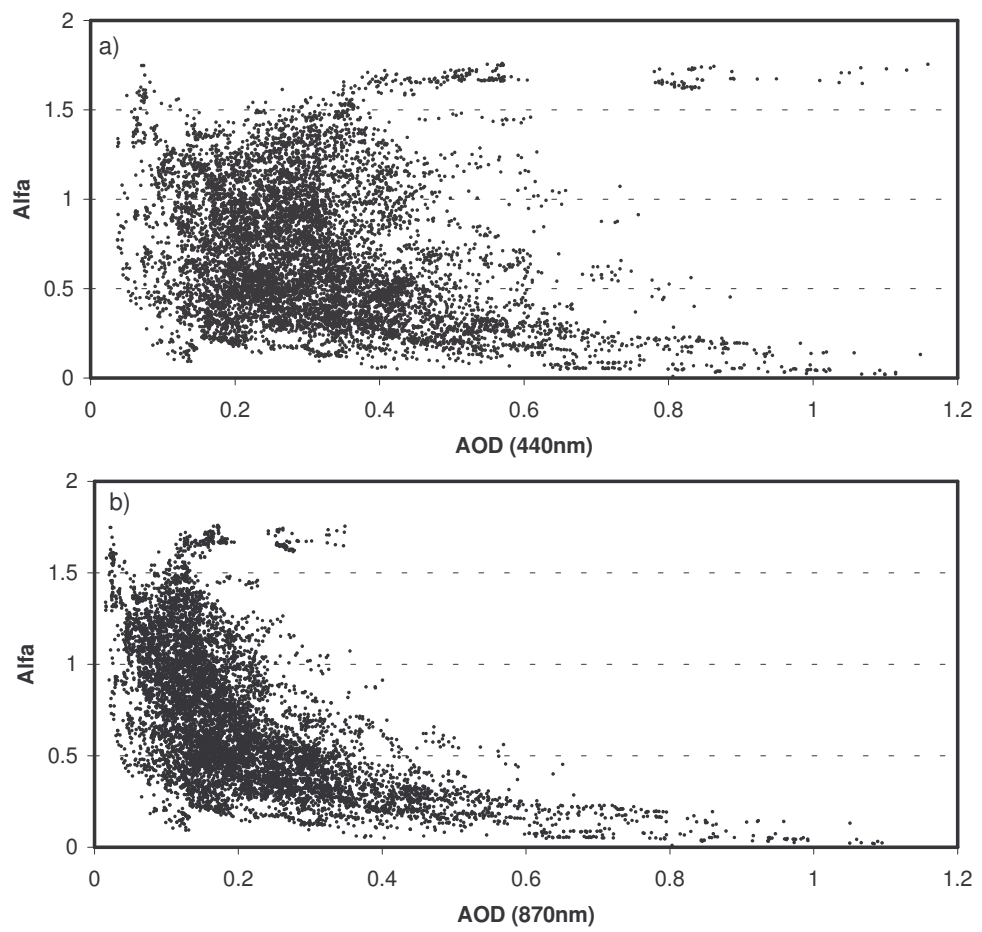

Figura VII-5. Diagramas de dispersión AOD-alfa para los episodios de aerosol desértico en El Arenosillo: (a) AOD(440nm) frente a alfa; (b) AOD (870nm) frente a alfa.

El análisis de las distribuciones de frecuencias de AOD y alfa se muestra en la Figura VII-6. De nuevo vamos a comparar los datos de AOD en 440nm y 870nm. La distribución de frecuencias de $\operatorname{AOD}(870 \mathrm{~nm})$, Figura VII-6a, es más estrecha que en 440nm, y tiene el pico de frecuencia en 0.14. En 440nm este pico de frecuencia está en 0.30 (Figura VII-6b), que es más del doble. Ésta última aparece por tanto más desplazada hacia los valores altos de AOD aunque la desviación estándar de ambas distribuciones es similar. El motivo de esta diferencia no está en las medidas de la rama inferior (en la Figura VII-5), esto es, los desérticos más puros, sino en la parte superior del diagrama, con las medidas realizadas con aerosoles más mezclados. Vemos que el grueso de observaciones en $870 \mathrm{~nm}$ está entre 0.10 y 0.30 , mientras que en $440 \mathrm{~nm}$ está entre 0.20 y 0.40 . El 'escalón' que se aprecia en los diagramas en 0.20 para $870 \mathrm{~nm}$ y 0.35 para $440 \mathrm{~nm}$ coincide con la práctica desaparición de aerosoles con alfas altos (por encima de 1 aproximadamente), con la excepción de algunas medidas en episodios de alta turbiedad que se han mezclado con los aerosoles desérticos. Por encima de este umbral la mayoría de medidas se concentran en la mencionada rama inferior (alta turbiedad con alfas bajos).

La distribución de frecuencias del parámetro alfa (Figura VII-6c) muestra claramente 2 modos. El primero de ellos corresponde a la acumulación de medidas con alfas bajos, lo que hemos denominado la rama inferior del diagrama AOD-alfa, y tiene el pico de frecuencia en 0.46. Hacia valores bajos la frecuencia decrece progresivamente, dándose incluso algunos valores de alfa ligeramente negativos. Hacia los valores altos el descenso de frecuencia se ve interrumpido por el otro modo, que tiene un pico de frecuencia en 0.90, valor cercano al promedio de la estación. Hacia valores más altos se llega hasta 1.5, y el último pico (en alfa=1.67) corresponde al episodio de combustión de biomasa. Las frecuencias de alfa permiten distinguir bien los valores propios del aerosol desértico que llega a El Arenosillo, con valores que oscilan entre 0 y 0.8 , mientras que por encima de este valor ya podemos afirmar que se trata de aerosol más mezclado o simplemente de las entradas y salidas de los episodios. 

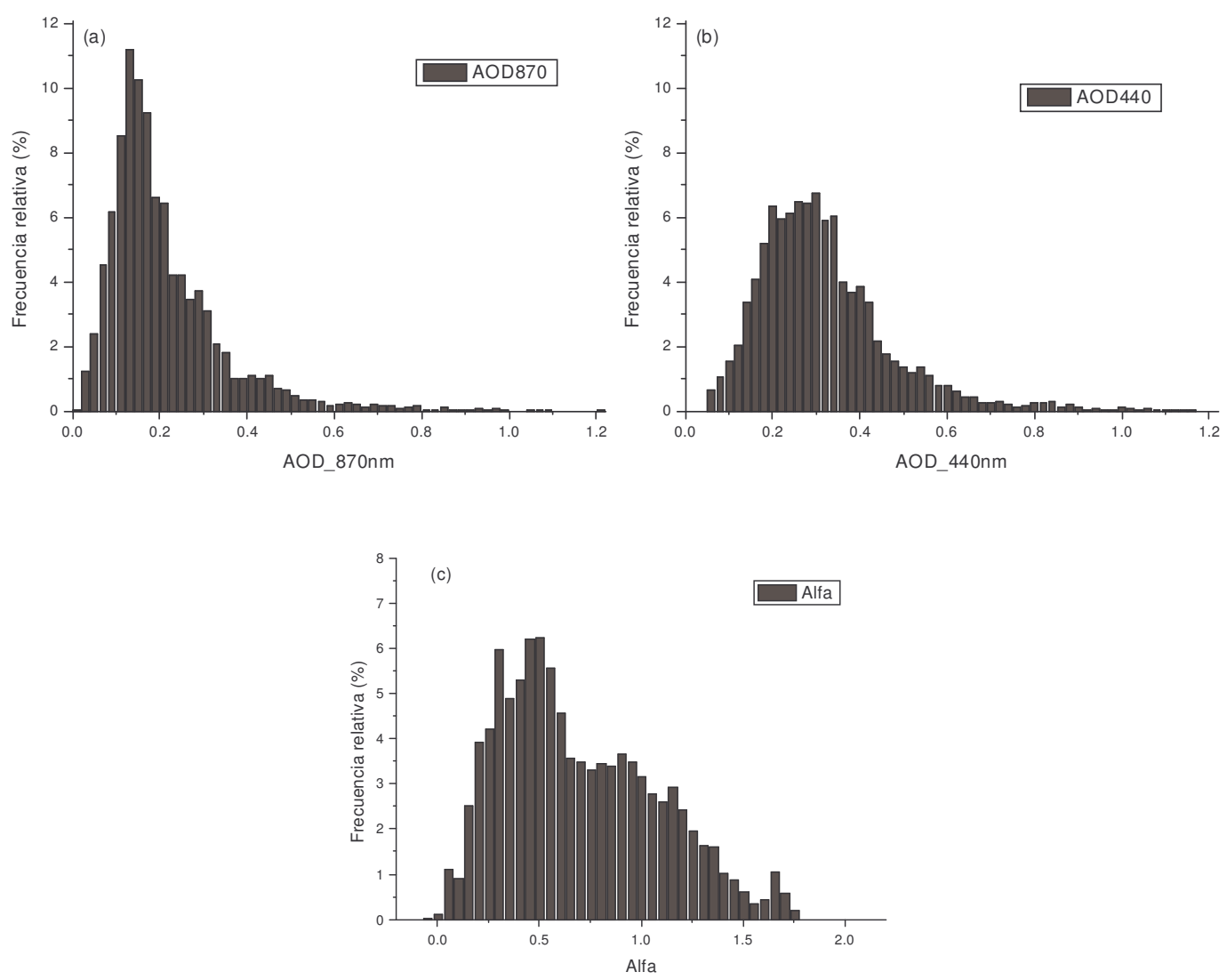

Figura VII-6. Histogramas de distribución de frecuencias relativas para las medidas del Cimel durante los episodios desérticos: (a) AOD en 870nm; (b) AOD en 440nm; (c) Alfa para el rango (440-870nm).

\subsubsection{Determinación de un criterio automático para la evaluación de episodios de aerosol desértico}

Una vez analizadas las medidas de AOD durante los episodios desérticos, podemos intentar dar un criterio que nos permita evaluar de manera automática estos eventos. Esto tiene gran importancia a la hora de analizar series largas de datos. El criterio que se va a plantear es local, válido para el aerosol desértico que llega a El Arenosillo, aunque puede ser de gran utilidad para reconocer el aerosol desértico en otras estaciones en la Península.

El trabajo de Escudero et al. (2005) proporciona un criterio para reconocer el aerosol desértico en los casos de deposición húmeda o lluvias rojas, en concreto un $\mathrm{pH}>6$ y $\left[\mathrm{Ca}^{+2}\right]>80 \mu$ eq/l. Sin embargo no se ofrece un criterio análogo para el caso de episodios sin lluvia. Aunque la diferencia de PM10 con las masas atlánticas limpias es grande, la concentración de PM10 ó TSP es similar en el caso de episodios desérticos y recirculaciones locales $\left(21-22 \mu \mathrm{g} / \mathrm{m}^{3}\right)$, es decir, sólo el dato de PM10 no es capaz de discriminar el aerosol desértico aunque se trate de estaciones de fondo, por lo que los autores se refieren al análisis de retrotrayectorias para identificar episodios, en particular aquellos eventos con PM10 por encima del umbral diario dado por la directiva comunitaria $\left(50 \mu \mathrm{g} / \mathrm{m}^{3}\right)$.

Las medidas de AOD espectral del Cimel nos van a permitir establecer un criterio para distinguir el aerosol desértico que llega a la estación. Para ello vamos en primer a lugar a observar sobre el diagrama AOD-alfa la región que ocupan los aerosoles desérticos de 
nuestro inventario, frente al resto de medidas en la serie de 5 años en El Arenosillo (Figura VII-7). Las medidas de los días con desértico se muestran en negro mientras que el resto se muestran en gris. Se puede apreciar cómo los aerosoles desérticos se agrupan en una determinada región del diagrama, la que venimos denominando 'rama inferior'. También encontramos algunas medidas que están fuera de esta región, que entendemos se deben a las entradas y salidas de los episodios y al evento de julio de 2003 que se comentará en detalle en la sección 7.4.

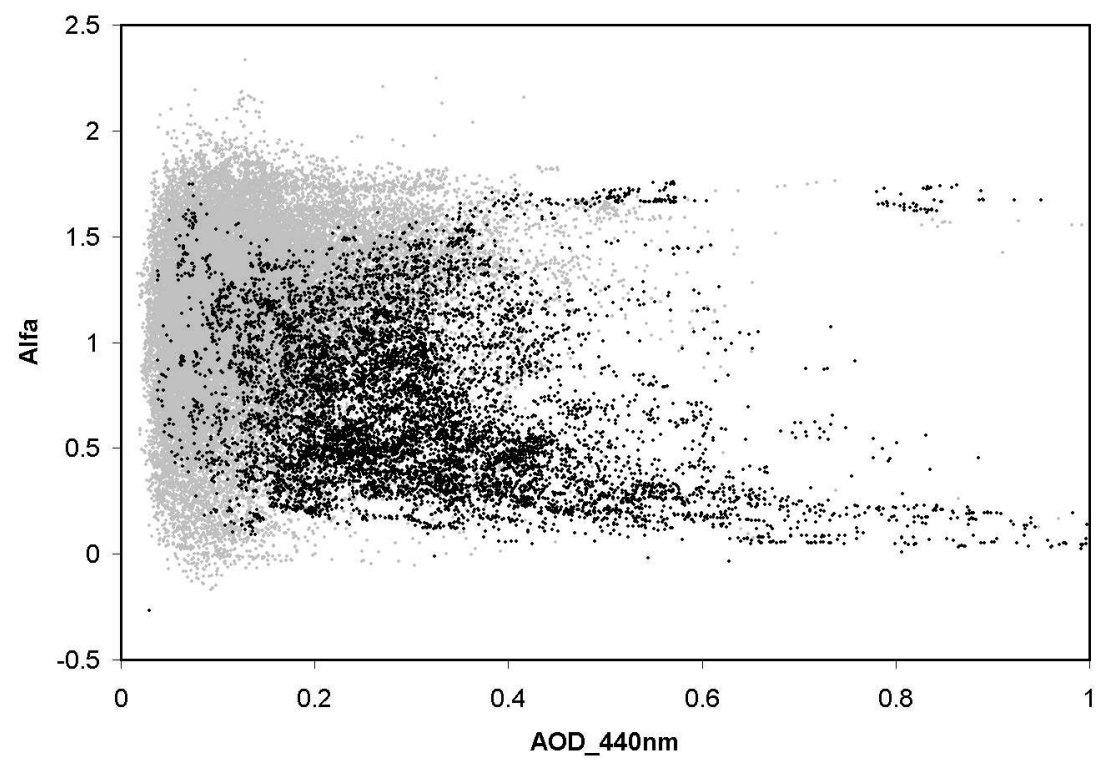

Figura VII-7. Diagrama de $\mathrm{AOD}(440 \mathrm{~nm})$ frente a alfa para las medidas de la serie de datos 2000-2004 en El Arenosillo, en el que están marcadas en negro las medidas correspondientes al inventario de desérticos y en gris el resto.

Para definir el criterio nos apoyaremos en varias de las conclusiones que hemos ido extrayendo del análisis de los episodios de aerosol desértico. En primer lugar, el aumento del AOD en las longitudes de onda largas es una característica muy marcada en estos eventos. En segundo lugar, podemos definir la región que corresponde a los aerosoles desérticos en términos de AOD y alfa. Por último, la presencia de puntos dudosos nos obliga a mantener un cierto margen a partir de los datos del inventario.

El criterio elegido es el siguiente: entre los datos del inventario de desérticos tomamos como límites el percentil 20 (P20) del AOD en 870nm y el percentil 80 (P80) de alfa para medidas individuales:

- $\operatorname{AOD}(870 \mathrm{~nm})>0.11$

- Alfa $<1.05$

Con esto dejamos un margen del $20 \%$ en el límite de AOD y alfa, aunque este límite se deja por debajo en el caso del AOD (P20), mientras que el margen se debe dejar por encima (P80) en el parámetro alfa. Esto define una región dentro del diagrama AOD-alfa en la que se incluye el $69 \%$ de las medidas de nuestro inventario de aerosoles desérticos. Esta región está delimitada por la línea gruesa sobre el diagrama de la Figura VII-8, tanto para el diagrama AOD(870nm)-alfa (Figura VII-8a) como para el diagrama AOD(440nm)alfa (Figura VII-8b). En estos diagramas la región dada por el criterio está en naranja, mientras que el resto de medidas durante los días del inventario desértico se muestran en negro y todas las demás medidas en gris. 

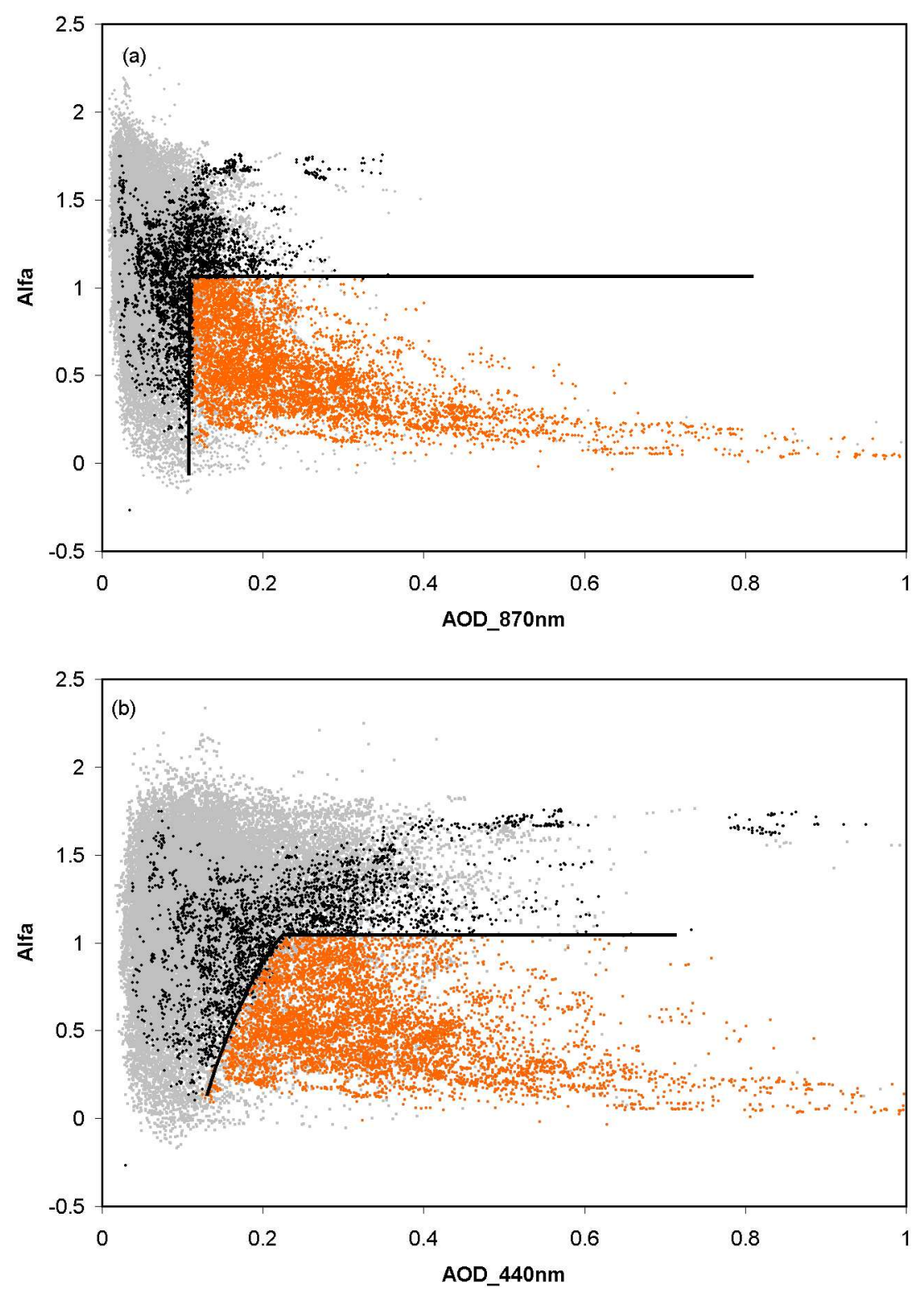

Figura VII-8. Diagrama AOD $(440 \mathrm{~nm})$-alfa en la que se detalla en naranja la región delimitada según el criterio automático para la detección de aerosoles desérticos: (a) diagrama AOD(870nm)-alfa; (b) diagrama AOD(440nm)-alfa

El criterio elegido da como resultado un acierto en cuanto a días identificados del $89 \%$ entre los días del inventario, y si no consideramos los días del episodio de julio de 2003, que coincidió con incendios, el acierto es del 92\%. Es decir, este criterio automático, aplicado sobre las medidas individuales, es capaz de reconocer el $92 \%$ de los días con aerosoles desérticos. La variabilidad durante estos episodios, las mezclas o las transiciones hacen que a nivel de medidas individuales el acierto sea menor, pero nos interesa detectar días y episodios en los que haya medidas dentro del criterio. Los días que tienen medidas dentro del criterio son siempre desérticos, con la excepción de unos pocos días contaminados por nubes. En principio el algoritmo automático de cloud-screening de AERONET elimina las medidas afectadas por nubosidad, pero este filtro no es perfecto. Cuando algunas medidas se han realizado con presencia de nubes tenues y estables el algoritmo no las detecta, y en estos casos la medida espectral es similar a la del aerosol desértico (alto AOD y alfa bajo). En todo caso se trata de excepciones. El bajo número de 
medidas en esos días con nubes y sobre todo la imagen del satélite nos sirven para detectar rápidamente esos posibles errores.

El resultado de la aplicación de este criterio se muestra gráficamente en la Figura VII-9, en la que se muestran en gris todas las medidas del año 2004, en negro las medidas del inventario desértico, y sobre éstas en naranja las que identifica el criterio automático. En la Figura VII-9a tenemos todas las medidas individuales, y en ella comprobamos que la mayoría de medidas son identificadas por el criterio, salvo dos episodios en mayo y en septiembre de 2004 en los que los valores de alfa eran muy altos, y también por algunas medias al comienzo o final de los episodios. En la Figura VII-9b se muestran los mismos resultados para los promedios diarios. Los días han sido identificados por tener medidas que cumplen el criterio mencionado, con un total de 71 de 77 días desérticos identificados por el criterio en 2004. Conviene recordar que el AOD no es el único parámetro que hemos utilizado para identificar los aerosoles desérticos, sino también el alfa, aunque no esté representado en estas figuras (para mayor claridad). Por tanto no debe extrañarnos que existan subidas de AOD que no correspondan con aerosoles desérticos.
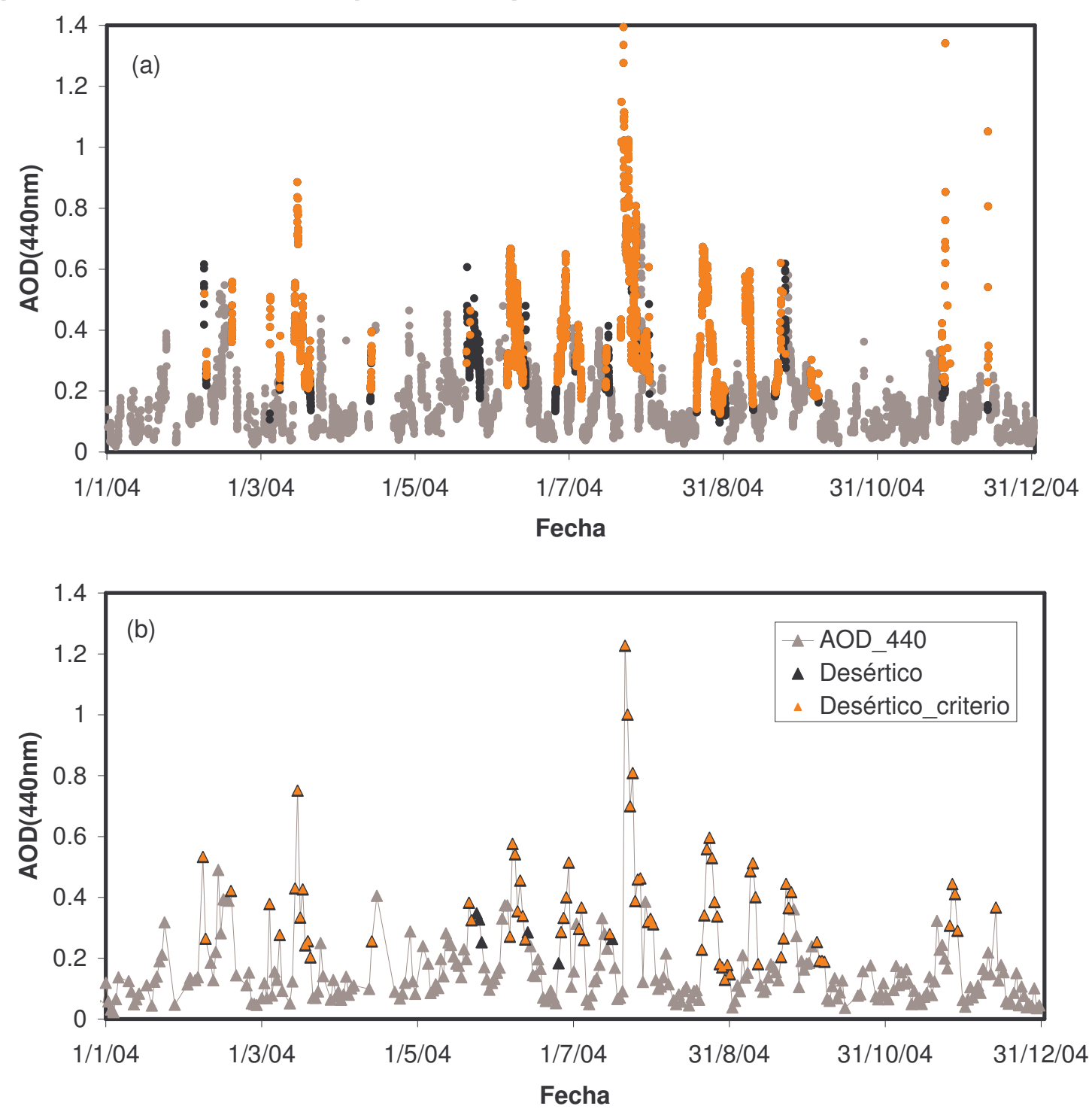

Figura VII-9. Resultado de la aplicación del criterio automático para la evaluación de episodios desérticos. Se representan en gris todas las medidas, en negro las correspondientes al inventario desértico y sobre éstas en naranja las que identifica el criterio automático: (a) para todas las medidas del año 2004; (b) promedios diarios del año 2004. 
Se podría definir un criterio análogo a partir de $\mathrm{AOD}(440 \mathrm{~nm})$ y alfa, que da como resultado $\mathrm{AOD}(440 \mathrm{~nm})>0.20$ y el mismo alfa $<1.05$. La región espectral quedaría definida de diferente manera, y el acierto global en cuanto a días identificados es algo menor (87\%). También hemos considerado más interesante definir nuestro criterio para medidas individuales que para los promedios diarios. Los días con fuerte variabilidad dan como resultado unos promedios diarios difíciles de clasificar. En todo caso se puede plantear también un criterio para los aerosoles desérticos a partir de los promedios diarios. El criterio debe ser menos restrictivo, ya que tomando los percentiles 20 y 80 de los promedios de $\mathrm{AOD}(870 \mathrm{~nm})$ y alfa el acierto sobre los días del inventario es tan solo del $70 \%$. Por ello hemos tomado los percentiles 10 y 90 para dar este criterio sobre los promedios diarios: $\mathrm{AOD}(870 \mathrm{~nm})>0.10 \mathrm{y}$ alfa $<1.16$. Con estos límites identificamos el $82 \%$ de los días.

En resumen nuestro criterio con $\mathrm{AOD}(870 \mathrm{~nm})$ y alfa aplicado sobre las medidas individuales nos va a permitir identificar un alto porcentaje de los días con aerosoles desérticos, en torno al 90\%. Es recomendable en una segunda fase analizar con cuidado los días que son identificados por el criterio automático, bien mediante imágenes de satélite o mediante retrotrayectorias, para fijar el comienzo y final del episodio y también de cara a descartar posibles errores.

\subsubsection{Comparación entre la evaluación de episodios de aerosoles desérticos y el análisis de masas de aire}

Consideramos que la comparación entre este inventario de días con aerosol desértico y las dos clasificaciones propuestas mediante las masas de aire es un estudio novedoso dentro de la investigación de aerosoles desérticos, y nos va a servir para comprobar hasta qué punto las retrotrayectorias son útiles para identificar los episodios de aerosoles desérticos. Para realizar esta comparación, hemos buscado a qué cluster pertenece cada día que hemos catalogado como desértico. Esto se ha realizado tanto para la clasificación por sectores como para la clasificación mediante el algoritmo de cluster no jerárquico (ver capítulo 6), y se recoge en la Tabla VII-IV.

\begin{tabular}{l|c|c||l|c|c}
\hline \hline $\begin{array}{l}\text { Clasificación por } \\
\text { sectores en 1500m }\end{array}$ & Días (\%) & $\begin{array}{l}\text { Presencia en } \\
\text { el sector (\%) }\end{array}$ & $\begin{array}{l}\text { Clasificación por algoritmo no } \\
\text { Jerárquico en 1500m }\end{array}$ & Días (\%) & $\begin{array}{l}\text { Presencia en } \\
\text { el cluster (\%) }\end{array}$ \\
\hline MP & $33(11.6)$ & 5.9 & Cluster 1 (NW lejanas) & $0(0.0)$ & 0.0 \\
MT & $59(20.1)$ & 26.6 & Cluster 2 (Atlánticas W) & $8(2.7)$ & 4.1 \\
A & $4(1.4)$ & 3.3 & Cluster 3 (Norteafricanas) & $177(60.4)$ & 62.5 \\
C & $12(4.1)$ & 11.9 & Cluster 4 (N-NE cercanas) & $12(4.1)$ & 7.4 \\
CT & $131(44.7)$ & 74.0 & Cluster 5 (Ártico) & $12(4.4)$ & 6.5 \\
L & $24(8.2)$ & 30.8 & Cluster 6 (Atlánticas curvadas) & $1(0.3)$ & 0.6 \\
Me & $29(9.9)$ & 38.2 & Cluster 7 (SW cercanas) & $82(28.0)$ & 27.9 \\
\hline \hline
\end{tabular}

Tabla VII-IV. Clasificación de los días con aerosol desértico según las dos clasificaciones propuestas para las masas de aire en el capítulo 5, por sectores (columnas 1-3) y mediante el algoritmo de cluster no jerárquico (columnas 4-6). Para cada cluster se da el número de días de desértico (y porcentaje sobre el total de días desérticos), y también el porcentaje que esos días representan sobre el número total de casos del cluster, que nos da la presencia de aerosol desértico en el mismo.

En la clasificación por sectores, el grueso de los días que hemos inventariado como desérticos pertenecen a los cluster Continental Tropical (CT) y Marítimo Tropical (MT), sumando entre ambos el $65 \%$ de los días. Es lógico que un porcentaje significativo quede catalogado como trayectorias Locales (L) o Mediterráneas (Me), bien por la permanencia 
de polvo en suspensión mientras no se renueve la masa de aire, bien por los episodios desérticos que nos llegan a través del Mediterráneo. Con esto ya agrupamos el $83 \%$ de los casos. Este análisis conlleva el error de que las trayectorias se calculan a las 12GMT para cada día, lo que puede suponer una discrepancia con el tipo de aerosol por ejemplo si la masa de aire con polvo desértico entra por la tarde, o también si se mantiene polvo en suspensión aun cuando la masa de aire ya ha empezado a renovarse. Esto puede explicar el $11.6 \%$ de casos de desértico que pertenecen al sector Marítimo Polar (MP), y también los casos aislados en los sectores Continental (C) y Ártico (A).

De esta manera hemos visto en qué sectores encontramos los días de aerosol desértico. Si miramos en sentido contrario, es decir, de la clasificación por masas de aire hacia el inventario, podemos evaluar la presencia de aerosol desértico en cada uno de los sectores. En primer lugar tenemos que el $74 \%$ de los días del cluster CT han sido catalogados como desérticos en el inventario, lo cual confirma que la mayoría de trayectorias desde el norte de África (sector CT) van a traer aerosol desértico, pero también que hay un $25 \%$ de trayectorias norteafricanas que no han traído aerosol desértico. La presencia de aerosol desértico en el sector MT es del $26 \%$. Aunque este porcentaje no es muy alto, el MT agrupa intrusiones desérticas muy importantes, con masas que viajan sobre el océano pero cuyo origen es claramente desértico, con AOD muy altos como pudimos ver por ejemplo en la Figura VI-7. La presencia de aerosol desértico en los sectores L y Me es del $31 \%$ y $38 \%$ respectivamente, también relevante, y en los clusters restantes la presencia de desértico es pequeña.

En cuanto a las medidas del Cimel, podemos comprobar en la Figura VI-8 que los días que hemos catalogado como desérticos, y que en el diagrama AOD-alfa ocupan esa rama de la parte inferior, se encuentran claramente en los sectores CT y MT. Para el sector CT el promedio de AOD era 0.30 y el de alfa era 0.69 , es decir, casi idénticos a los que hemos obtenido para los días desérticos, en los que el promedio de AOD(440nm) es 0.32 y el promedio de alfa es 0.69. También el histograma de alfa en el sector CT (Figura VI-15b) muestra dos modos en 0.45 y 0.90 , tal y como hemos encontrado también aquí para los desérticos (Figura VII-6c). En el sector MT los desérticos sólo representan el $26 \%$ de los casos por lo que en los histogramas no se ve una analogía tan clara como en el caso CT.

En cuanto a la segunda clasificación de masas de aire, según el algoritmo de cluster no jerárquico, el $60 \%$ de nuestros casos desérticos están en el cluster norteafricano, y el $28 \%$ en el de trayectorias del SW cercanas, es decir, quedan muy bien delimitados en esos dos cluster, que agrupan casi el $90 \%$ de los casos. La contribución de los restantes cluster es pequeña, lo que consideramos dentro de los márgenes de error comentados, y en algunos casos nula o casi nula, en concreto para las trayectorias atlánticas más largas del NW y las 'atlánticas curvadas'. Los clusters atlánticos quedan en esta clasificación bien separados de los casos desérticos, como sería lógico a priori.

De nuevo podemos evaluar la presencia de casos de aerosol desértico en cada uno de los clusters de la clasificación por masas de aire. El cluster norteafricano tiene en este caso un $62 \%$ de días evaluados como desérticos, menor que en la anterior clasificación. Este cluster se etiquetó como 'norteafricano', aunque ciertamente hay una parte de trayectorias de este cluster que no son norteafricanas (ver apartado 6.5 y Figura V-20). Por tanto no es de extrañar que no traigan aerosol desértico. En esta clasificación sólo el cluster 'SW cercanas' tiene también un número relevante de casos de desértico, con un $28 \%$. Al igual que sucede con el sector MT en la clasificación por sectores, estas trayectorias del sudoeste con aerosol desértico reúnen episodios muy importantes. En el resto de clusters no hay apenas casos de desértico, y podemos asimilarlos como transiciones entre las masas de aire en las que todavía permanece el polvo en suspensión. Aquí también entran los casos 
en los que los métodos de clasificación de masas de aire mostraban sus limitaciones, por ejemplo asociando una trayectoria del Ártico con aerosol claramente desértico (ver el ejemplo del capítulo anterior, Figura VI-9).

Las medias de AOD y alfa del Cimel para el cluster 3 (norteafricanas), en el que tenemos ese $62 \%$ de días con desértico, muestran también esta clara influencia, con un AOD promedio de 0.29 y alfa de 0.86 . Creemos que el promedio de alfa es más alto por las masas de aire de este cluster que no son africanas sino trayectorias cercanas, sobre todo mediterráneas. En el histograma de alfa de nuevo aparecen los dos modos, en torno a 0.5 y 1 , aunque en este caso la presencia de las trayectorias no africanas hace que el modo centrado en valores bajos de alfa no predomine en la distribución de frecuencias, sino que ambos sean similares (ver Figura VI-26b).
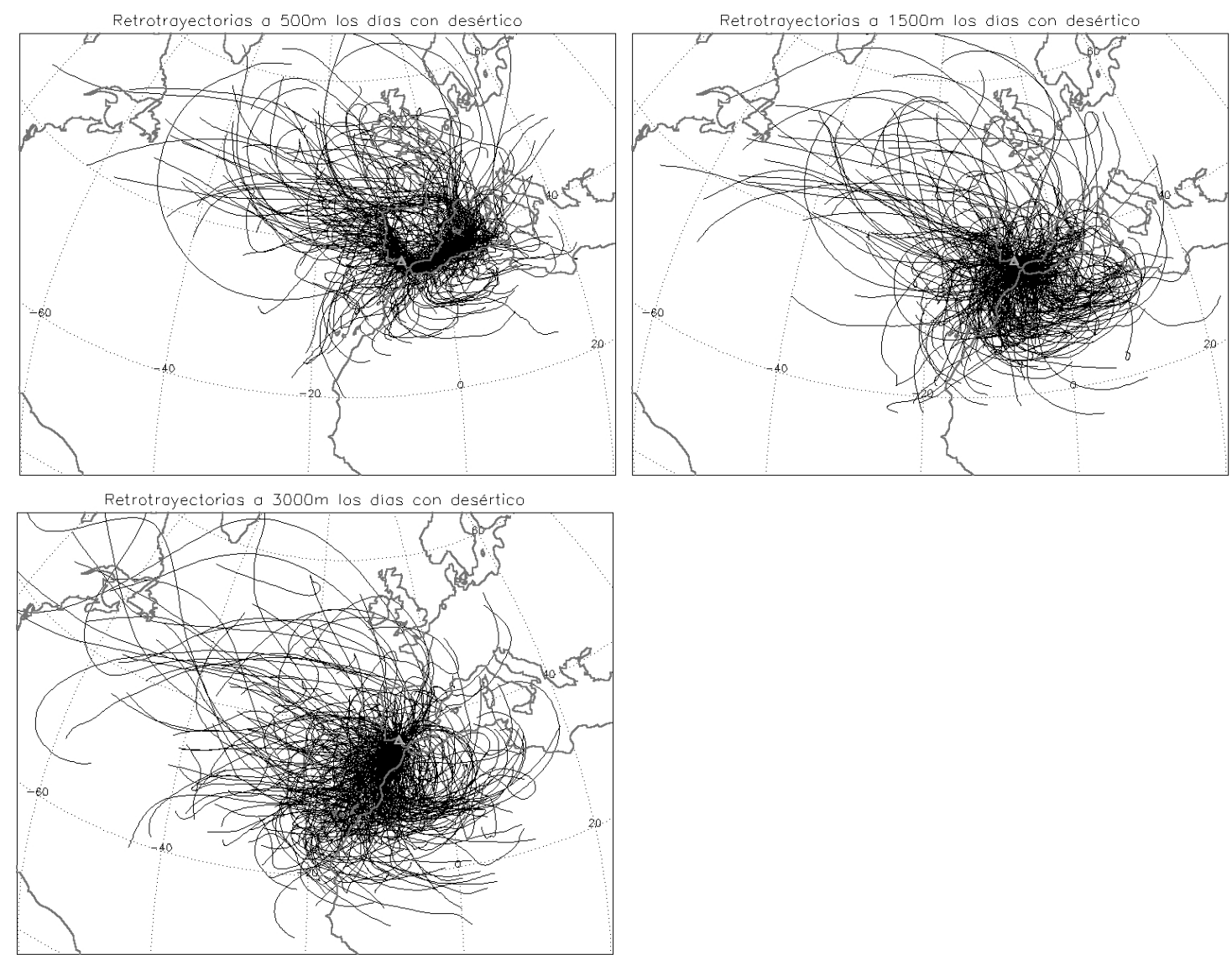

Figura VII-10. Retrotrayectorias de masas de aire del modelo HYSPLIT para los días con aerosol desértico en El Arenosillo.

La conclusión general es que las masas de aire cargadas con polvo desértico nos llegan desde el sur y el suroeste, a la vez que podemos afirmar que la gran mayoría de masas norteafricanas traen polvo desértico, aunque no en todos los casos. Podemos finalmente pintar las retrotrayectorias correspondientes a los días catalogados como desérticos en el inventario realizado, lo que mostramos en la Figura VII-10. Se trata por tanto de los mismos días pero en los tres niveles. Las trayectorias provienen mayoritariamente del sur en los niveles altos, aunque con matices. En $1500 \mathrm{~m}$ las retrotrayectorias provienen del sur y sureste, en muchos casos describiendo una curva de movimiento anticiclónico. En $3000 \mathrm{~m}$ predomina la dirección suroeste de procedencia, y además las trayectorias son algo más largas, por lo que su origen se sitúa en general algo más al sur y al oeste que en $1500 \mathrm{~m}$. En $500 \mathrm{~m}$ las masas de aire parecen recircular y moverse lentamente. Aunque hay también trayectorias que provienen del sur, existe un gran número que provienen del este o noreste (mar Mediterráneo) y desde el noroeste, pero son todas ellas trayectorias muy 
cortas y por tanto lentas. Esto parece relacionado con la situación típica de verano (NAHA), ya que en esos casos la baja térmica norteafricana nos hace llegar el flujo de aire lentamente desde el Mediterráneo en los niveles bajos de la atmósfera. También destaca el hecho de que apenas llegan trayectorias desde el norte sobre la Península frente a la gran cantidad de casos que llegan desde el noreste y noroeste, como se observa en el mapa. Por otra parte hay un número de trayectorias de otras procedencias que hacen menos claros estos mapas, y que se corresponden con las transiciones entre masas de aire ya comentadas. Constituyen menos del $10-12 \%$ de las trayectorias según hemos visto en la Tabla VII-IV. 


\subsection{Casos de estudio}

Hemos seleccionado como casos de estudio cuatros episodios con aerosol desértico en El Arenosillo, para realizar sobre ellos un análisis más detallado. Se trata de intrusiones excepcionales, pero que dan la medida de la intensidad que pueden alcanzar estos episodios y sus características. Analizaremos dos episodios del año 2000, que tuvieron lugar en febrero-marzo y julio-agosto. Un tercer episodio tuvo lugar el verano de 2003, y su peculiaridad es la detección simultánea del aerosol desértico junto con aerosoles debidos a incendios forestales en Portugal y la sierra de Huelva. Por último analizaremos el evento más fuerte registrado en nuestra base de datos y que alcanzó toda la Península Ibérica en julio de 2004.

\subsubsection{Episodio del 26 de febrero al 15 de marzo de 2000}

Durante el final del mes de febrero de 2000 se produjo un fuerte episodio desértico sobre las islas Canarias que inmediatamente alcanzó también la Península Ibérica. Ello corresponde a una situación anómala sobre el Sahara, casi propia del verano, con fuertes vientos del este en superficie que lanzaron varias oleadas consecutivas de polvo desértico sobre el Atlántico. Este episodio de carácter extraordinario ha sido estudiado por varios autores (Falke et al., 2001; Cana, 2002; Vergaz et al., 2003; Cachorro et al., 2005). La intensa nube de polvo, perfectamente observable desde las imágenes de satélite que mostramos a continuación (ver Figura VII-11a y también Figura VI-10), ha permitido verificar modelos de transporte de partículas desérticas que predicen los niveles de AOD (ver Figura VII-11b) y han ayudado a delimitar la capacidad de los sensores de satélite para realizar el seguimiento de este tipo de eventos.

La elección de este episodio se debe a que ha sido el segundo más fuerte registrado, y el primer episodio severo que tenemos en nuestra base de datos. Se detectó el 26 de Febrero (de acuerdo al criterio adoptado anteriormente) con su máxima intensidad el día 28 de febrero y 6-7-8 de marzo, y podemos considerar que duró hasta mediados de marzo. La cuestión de considerar un solo episodio o dos no es fácil. Si nos referimos a la medidas del Cimel habría que contabilizar dos episodios diferentes, ya que los niveles de AOD (y valores asociados de alfa) indican valores corrientes o bajos los días 1 al 4 de marzo. Sin embargo analizando estos días en conjunto, y con la ayuda de las imágenes de satélite, podemos decir que se produce una emisión más o menos continua desde las fuentes desérticas de enormes cantidades de polvo sobre el Atlántico durante un extenso conjunto de días, en los que resaltamos la enorme variabilidad, como muestran las imágenes del TOMS (Figura VII-15).

La base para la caracterización de este evento sobre El Arenosillo va a ser el análisis de datos del fotómetro Cimel. En la Figura VII-12 tenemos las medidas de este fotómetro en El Arenosillo entre los días 24 de febrero y 16 de marzo de 2000. La gran nube de polvo ha salido hacia el Atlántico durante los últimos días de Febrero, afectando de lleno a las islas Canarias, como muestra la imagen SeaWIFS para el día 26 de febrero (Figura VII11a). La primera oleada alcanza débilmente la estación de El Arenosillo entre los días 26 y 29 de febrero, en los que se alcanza un AOD (440nm) de 0.48 con alfa de 0.47 (Figura VII-12). También las retrotrayectorias muestran la llegada de una masa de aire norteafricana desde el día 25 de febrero (Figura VII-14a) hasta el 29 de febrero. Sin embargo el día 1 de marzo las retrotrayectorias indican la llegada de una masa atlántica (Figura VII-14b), lo que tiene su reflejo en el descenso de AOD del Cimel entre los días 1 y 4 de marzo. 


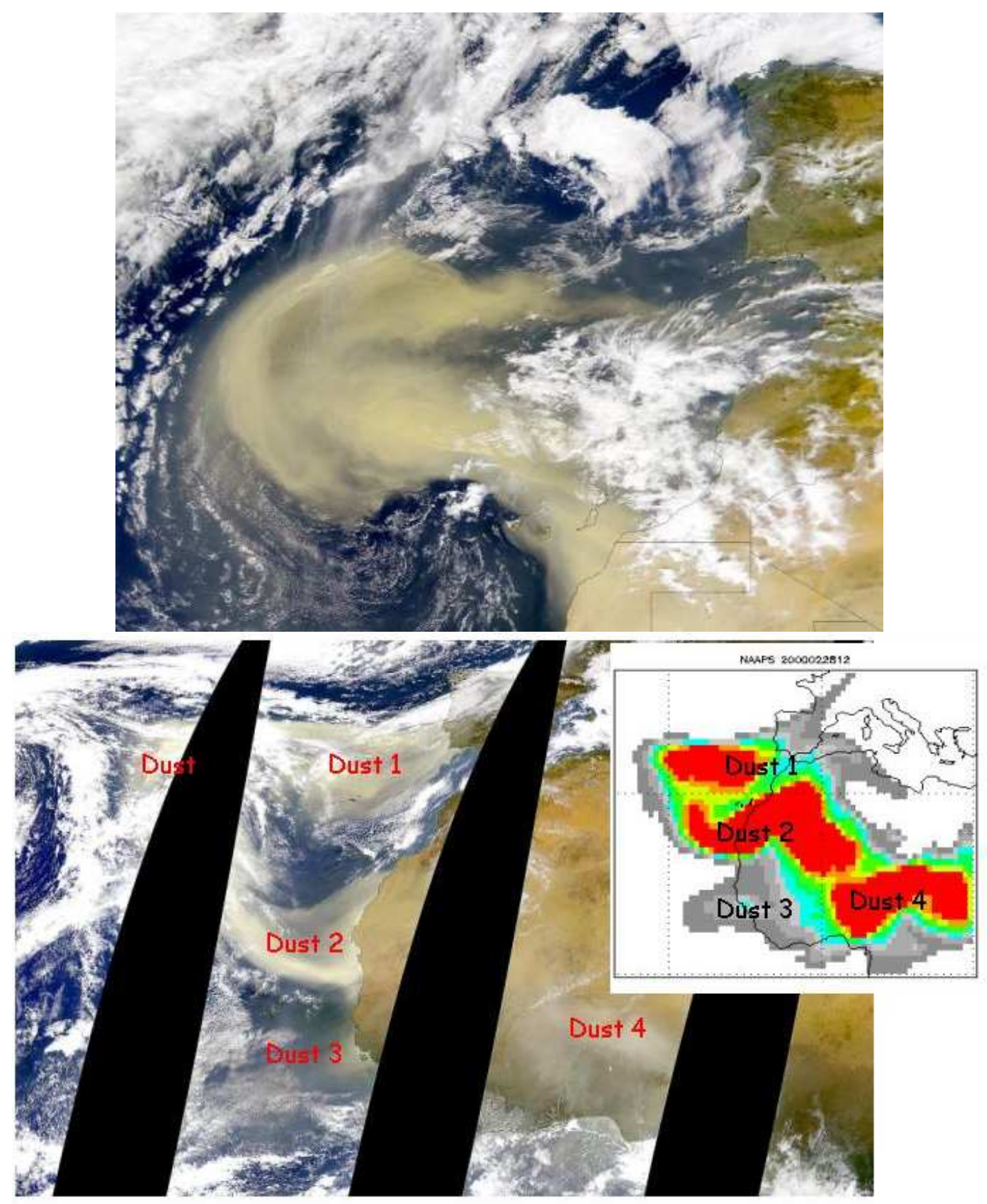

Figura VII-11. (a) Imagen del sensor SeaWIFS para el día 26 de febrero de 2000 ;(b) Comparación entre el modelo NAAPS y la imagen SeaWIFS para el día 28 de febrero de 2000 (tomado de http://www.nrlmry.navy.mil/aerosol).

La gran cantidad de polvo que hay sobre el Atlántico llega por segunda vez a la estación el 6 de marzo, como indican las medidas del Cimel. Según el inventario de Sánchez de la Campa (2004) basado en la visualización de retrotrayectorias, la segunda parte del episodio comienza el día 5, pero debido a la presencia de nubosidad el Cimel no pudo realizar medidas la tarde del día 5 y realizó muy pocas durante el día 6 . Sin embargo este día se alcanza el 1.05 de $\mathrm{AOD}(440 \mathrm{~nm})$, nivel que se mantiene con fuertes subidas y bajadas hasta el día 8. A partir de ese día el AOD va a ir disminuyendo lentamente, y no recuperamos los niveles anteriores al episodio hasta el día 16, aun cuando la masa de aire empieza a cambiar el día 14. El citado trabajo de Sánchez de la Campa (2004) da el final del episodio para el día 13, pero según las medidas del Cimel y nuestro criterio éste continúa 2 días más, puesto que los días 14 y 15 todavía hay valores de AOD(440nm) muy por encima de 0.3 y también AOD alto para las longitudes de onda largas (0.20 el día 
14 y 0.15 el día 15 de AOD promedio en $870 \mathrm{~nm}$ ). El día 15 el parámetro alfa ya muestra un promedio por encima de 1, indicando el final del episodio.

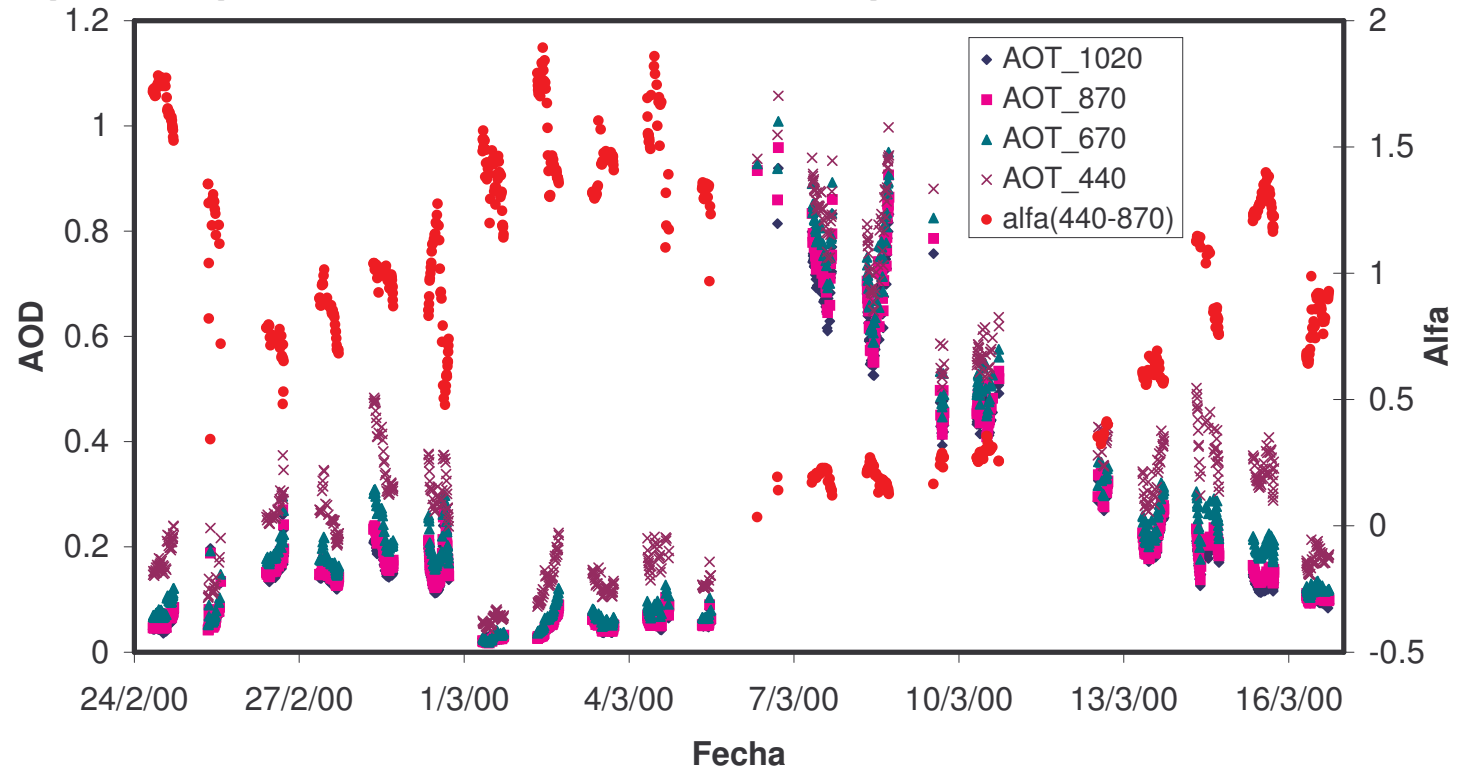

Figura VII-12. Registro del fotómetro Cimel en El Arenosillo entre los días 24 de febrero y 16 de marzo de 2000, con el AOD en los cuatro canales (eje izquierdo) y en rojo el parámetro alfa (eje derecho).
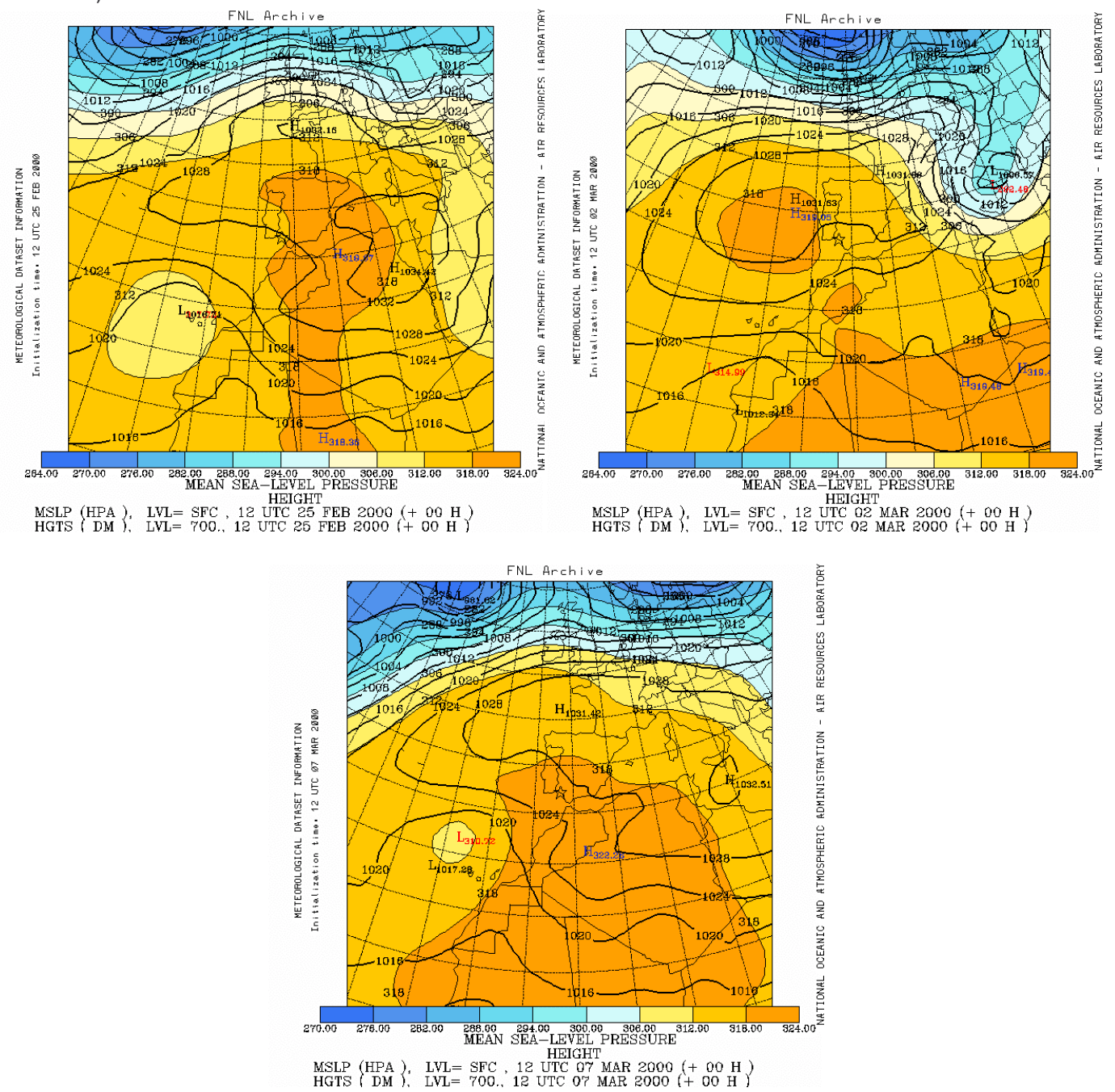

Figura VII-13. Situación sinóptica (base de datos FNL) dada por la presión en superficie y altura geopotencial en 700hPa para los días 25 de febrero, 3 de marzo y 7 de marzo de 2000. 
El análisis del parámetro alfa durante el episodio nos muestra valores muy bajos durante los primeros días del episodio, con 0.12 el día 6 y 0.19 en promedio los días 7 y 8 , mientras que a partir del día 10 comienzan a recuperarse, hasta recuperar el día 15 los valores altos. Por otra parte la alta turbiedad el día 15, con 0.34 en $440 \mathrm{~nm}$ y alfa alto de 1.27, puede ser también debida a fuentes de tipo local que se mezclan con el aerosol desértico. Durante este largo episodio tuvieron lugar algunos días con nubosidad como puede observarse en las imágenes de satélite o bien imágenes Meteosat, así como en los propios datos del Cimel los días 5 y 6 de marzo. Estas nubes perturban la detección de este evento al no poder apreciarse bien la subida del AOD. Las medidas afectadas por nubosidad también presentan AOD alto con alfas próximos a cero, como se puede comprobar analizando datos de AERONET en nivel 1.0, antes del cloud-screening. Hemos revisado dichas medidas brutas durante este episodio, en particular los días 5, 6 y 11 de marzo, que son los más afectados por nubosidad, junto con los mapas del Meteosat e imágenes SeaWIFS, llegando a la conclusión de que el algoritmo automático ha funcionado correctamente durante el episodio. Normalmente la rápida variabilidad del $\mathrm{AOD}$ en presencia de nubes es suficiente para esta discriminación.
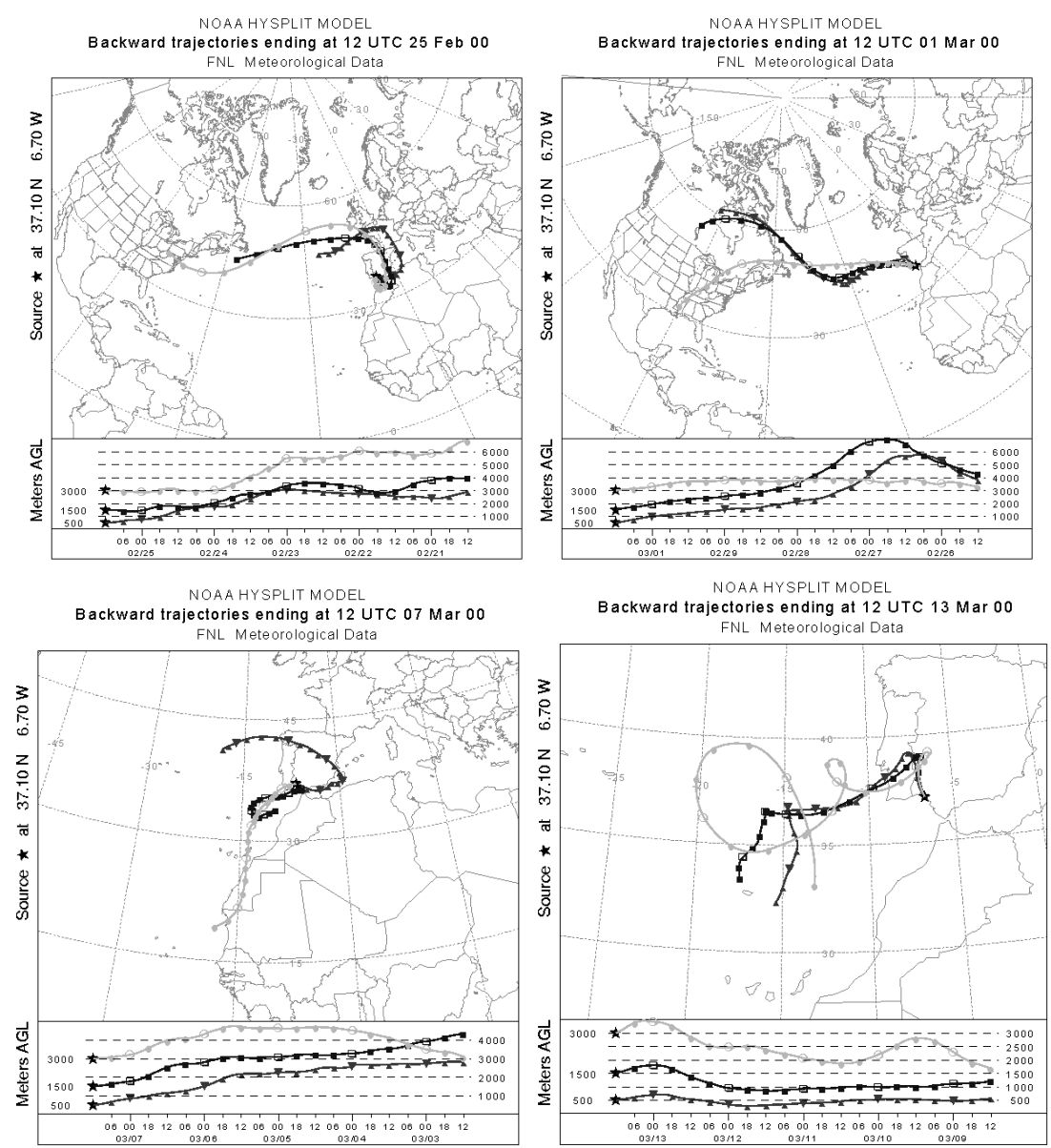

Figura VII-14. Retrotrayectorias a 5 días del modelo HYSPLIT en El Arenosillo para los días: (a) 7 de marzo y (b) 13 de marzo de 2000.

La situación sinóptica que da lugar al episodio hay que comenzar a analizarla los últimos días de febrero. En la Figura VII-13 vemos el mapa de presión en superficie y altura geopotencial en $700 \mathrm{hPa}$. El día 25 de febrero tenemos altas presiones sobre el norte de África, con una situación que catalogamos como tipo 1 (NAH-S). Sin embargo el intenso gradiente de presión sobre el Sahara, más propio del verano, junto con la presencia de una pequeña vaguada en altura al oeste del Sahara, refuerzan los vientos del este sobre el Sahara y lanza una gran cantidad de polvo hacia el Atlántico, lo que da lugar a la 
formación de una pluma o arco atlántico. Esta situación se mantiene durante muchos días, aunque con variaciones, como vemos en el mapa correspondiente al día 3 de marzo, en el que la posición del anticiclón ha variado, alejando momentáneamente la nube de polvo de la Península. Sin embargo el día 7 tenemos de nuevo una situación muy similar a la del inicio del episodio, y las masas de aire cargadas de polvo son transportadas en dirección noroeste sobre el Atlántico hasta llegar a la Península.

La situación anticiclónica provoca que las masas de aire recirculen y se renueven muy poco, lo que explica la lentitud en el descenso del AOD y el final poco claro del episodio. Las retrotrayectorias para el día 13 de marzo (Figura VII-14d) son una muestra de esta situación, con una masa de aire que se ha movido por las cercanías durante muchos días, perdiéndose el origen del polvo desértico. Las retrotrayectorias para el día 7 de marzo (Figura VII-14c) también muestran estas recirculaciones en $500 \mathrm{~m}$ y $1500 \mathrm{~m}$, aunque en este caso la trayectoria en $3000 \mathrm{~m}$ sí es de origen sahariano. Los mapas de índice de aerosoles (AI) del TOMS también muestran que los días de mayor intensidad son el 6, 7 y 8 de marzo, disminuyendo a partir del día 9, en el que la masa de aire se extiende por una amplia zona (Figura VII-15).
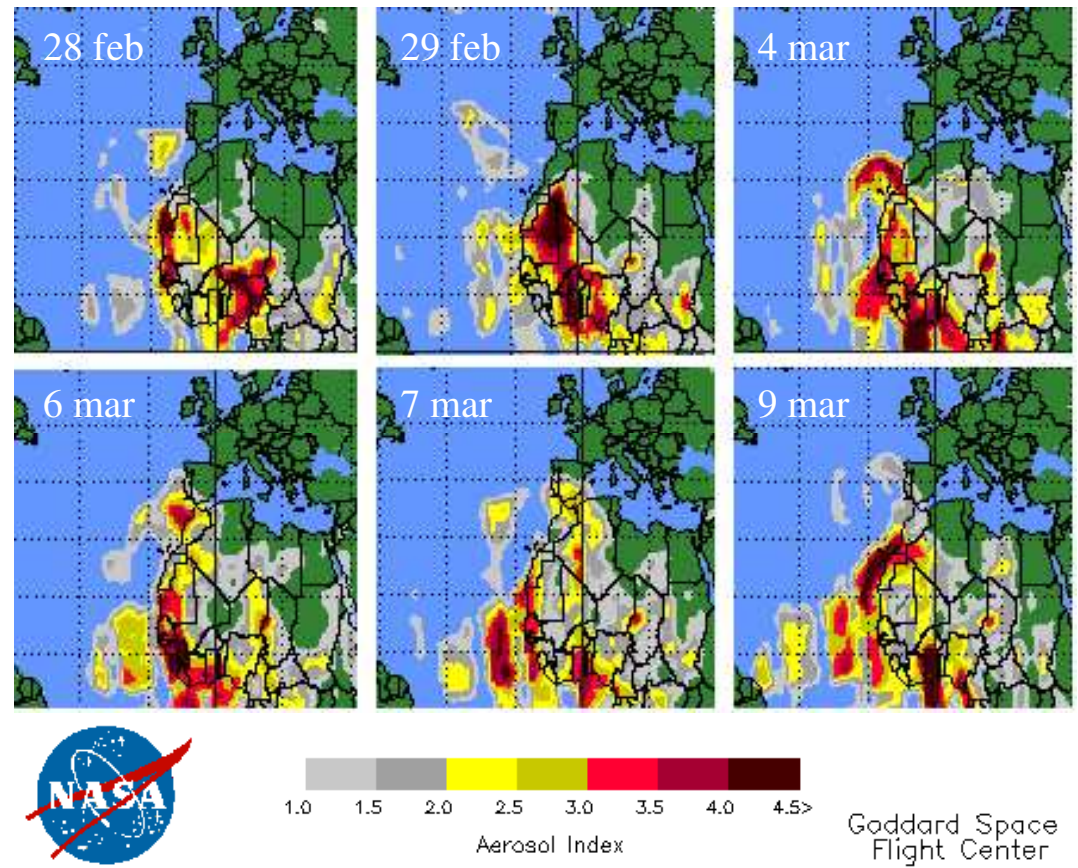

Gaddard Space
Flight Center

Figura VII-15. Mapas de índice de aerosoles (AI) del TOMS sobre la zona de estudio para 6 días seleccionados entre los días 28 de febrero y 9 de marzo.

\subsubsection{Episodio del 30 de julio al 3 de agosto de 2000}

Durante el año 2000 se contabilizaron numerosos episodios de aerosol desértico, especialmente durante el verano en los meses de julio y agosto. Uno de ellos tuvo lugar entre los días 30 de julio y 3 de agosto, con una duración de 5 días. Es un episodio que destacamos por ser muy claro, cercano a los promedios de duración e intensidad, y que por tanto podemos poner como ejemplo de típica entrada de aerosol desértico en la estación. Las medidas del fotómetro Cimel durante este episodio se encuentran en la Figura VII-17. 


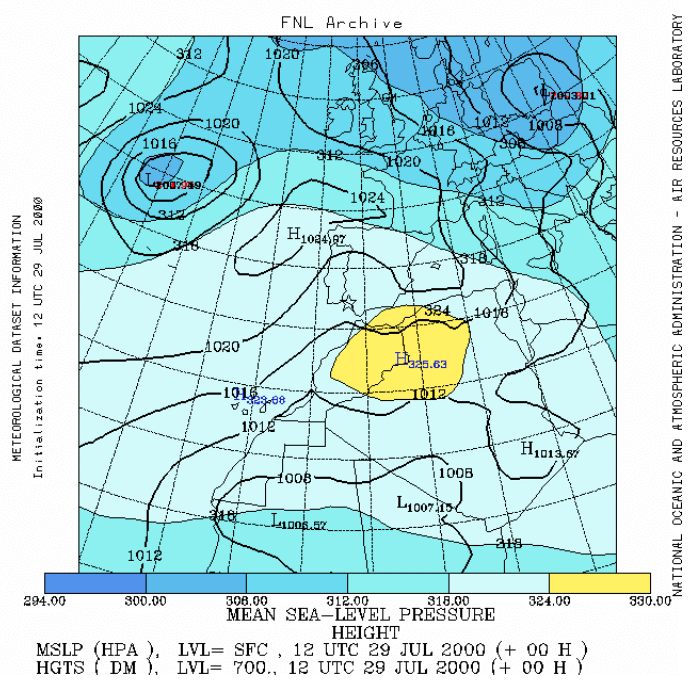

Figura VII-16. Situación sinóptica (base de datos FNL) dada por la presión en superficie y altura geopotencial en 700hPa para el día 29 de julio de 2000.

La situación sinóptica que da lugar a este episodio es también clara, correspondiendo con la clásica situación de verano (tipo NAH-A) que podemos observar en la Figura VII-16. La situación en superficie corresponde con la baja térmica norteafricana, mientras que en los niveles altos tenemos altas presiones situadas al sudeste de la Península, lo que permite la llegada del aerosol desértico. La retrotrayectoria para el día 1 de agosto, el día central del episodio (Figura VII-18b), confirma la entrada de una masa de aire sahariana en los niveles altos (1500m y $3000 \mathrm{~m})$, mientras que en $500 \mathrm{~m}$ la retrotrayectoria muestra una recirculación sobre la zona de Andalucía y el estrecho de Gibraltar.

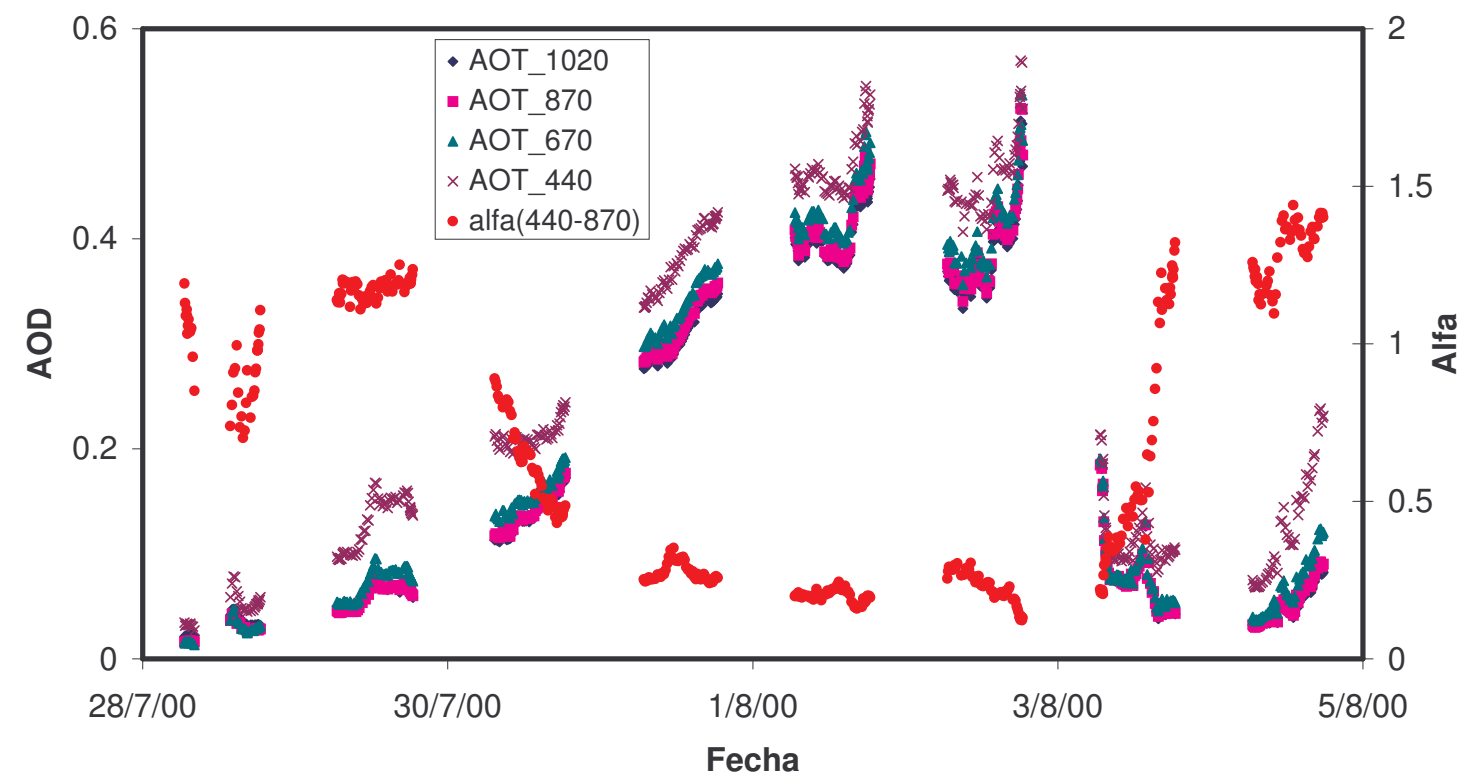

Figura VII-17. Registro del fotómetro Cimel en El Arenosillo entre los días 28 de julio y 4 de agosto de 2000, con el AOD en los cuatro canales (eje izquierdo) y parámetro alfa (eje derecho).

El día de comienzo del episodio es algo dudoso. Podemos considerar que éste se produce el día 30, pues aunque el $\mathrm{AOD}(440 \mathrm{~nm})$ se sitúa sólo alrededor de 0.2 (obsérvese el aumento al final de la tarde) el parámetro alfa ha ido bajando todo el día, desde 0.8 hasta 0.5, es decir, presenta unos valores que podemos considerar propios de los aerosoles desérticos. El mapa del modelo NAAPS (Figura VII-20a) indica que el aerosol desértico ya 
ha llegado el día 29, así como el AI del TOMS, que mostramos en la Figura VII-19. En los datos del Cimel se aprecia una pequeña subida del AOD ya este día 29, también en los canales del infrarrojo, aunque el parámetro alfa no desciende. La retrotrayectoria a las $12 \mathrm{~h}$ no indica una procedencia norteafricana, pero por la noche las trayectorias parecen indicar ya una transición por la llegada de una nueva masa de aire. El inventario de Sánchez de la Campa (2004) da el comienzo para el día 30. El día 30 y sobre todo el día 31 la masa de aire cargada de polvo ha entrado en la zona de estudio. El mapa de concentración de polvo en superficie del modelo NAAPS para el día 31 de julio también muestra esta entrada de polvo desértico (Figura VII-20b).

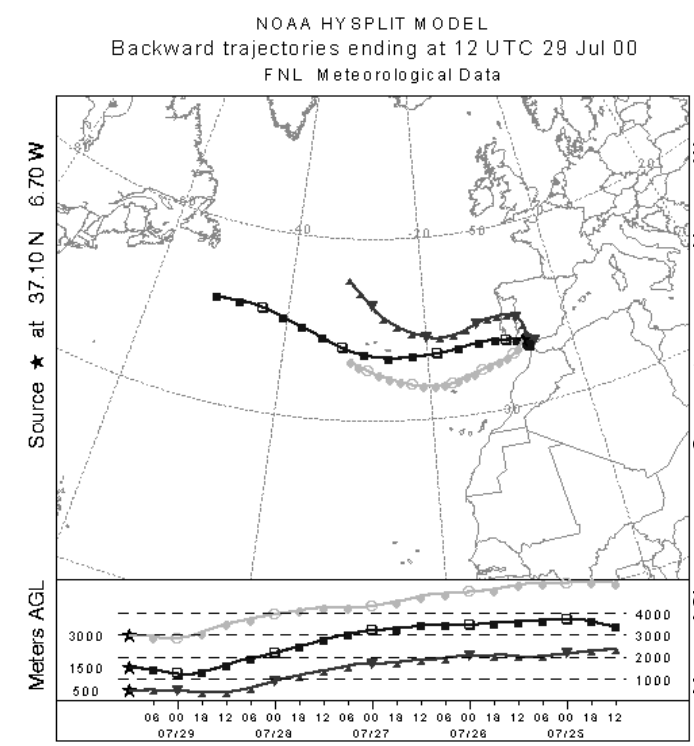
Backward trajectories ending at 06 UTC 30 Jul 00 FNL Meteorological Data

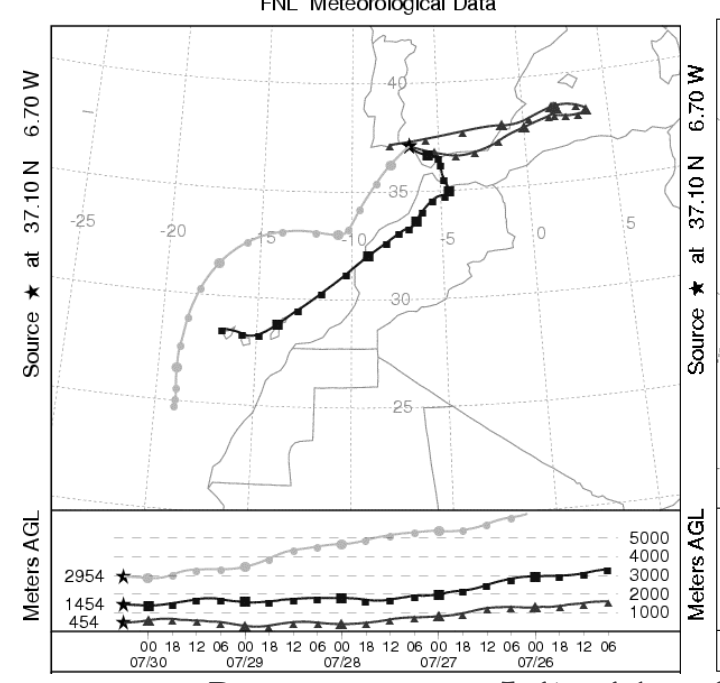

NUAA HYSPLII MUUEL

Backward trajectories ending at 22 UTC 29 Jul 00 FNL Meteorological Data

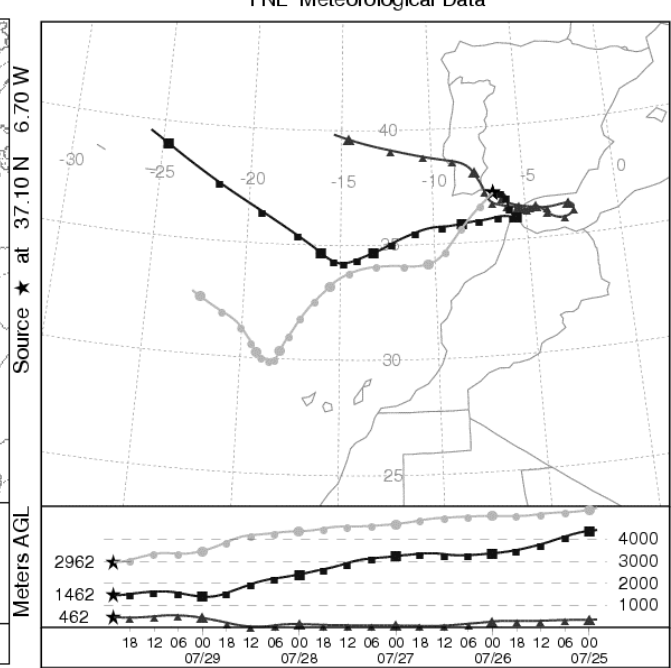

NOAAHYSPLIT MODEL

Backward trajectories ending at 12 UTC 01 Aug 00 FNL Meteorological Data

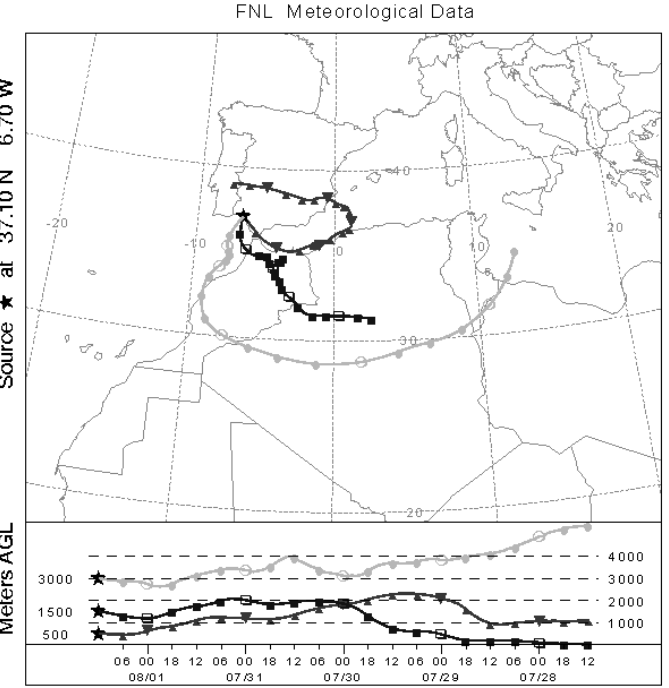

Figura VII-18. Retrotrayectorias a 5 días del modelo HYSPLIT en El Arenosillo para los días: (a) 29 de julio de 2000 a las $12 \mathrm{~h}$; (b) ) 29 de julio de 2000 a las $22 \mathrm{~h}$; (c) 30 de julio de 2000 a las $12 \mathrm{~h}$; (d) día 1 de agosto de 2000 a las $12 \mathrm{~h}$.

La turbiedad ha aumentado durante los días 29 y 30, y se alcanza un AOD(440nm) máximo de 0.53 durante el día 1 de agosto, con un alfa que ha descendido hasta un mínimo de 0.17. El día 3 de agosto la subida del parámetro alfa hasta 1.32, junto con el descenso en el AOD marcan el final de episodio. Este último día la imagen del TOMS nos indica que la masa de polvo se desplaza hacia el Mediterráneo, empujada por una masa de aire de procedencia atlántica. 

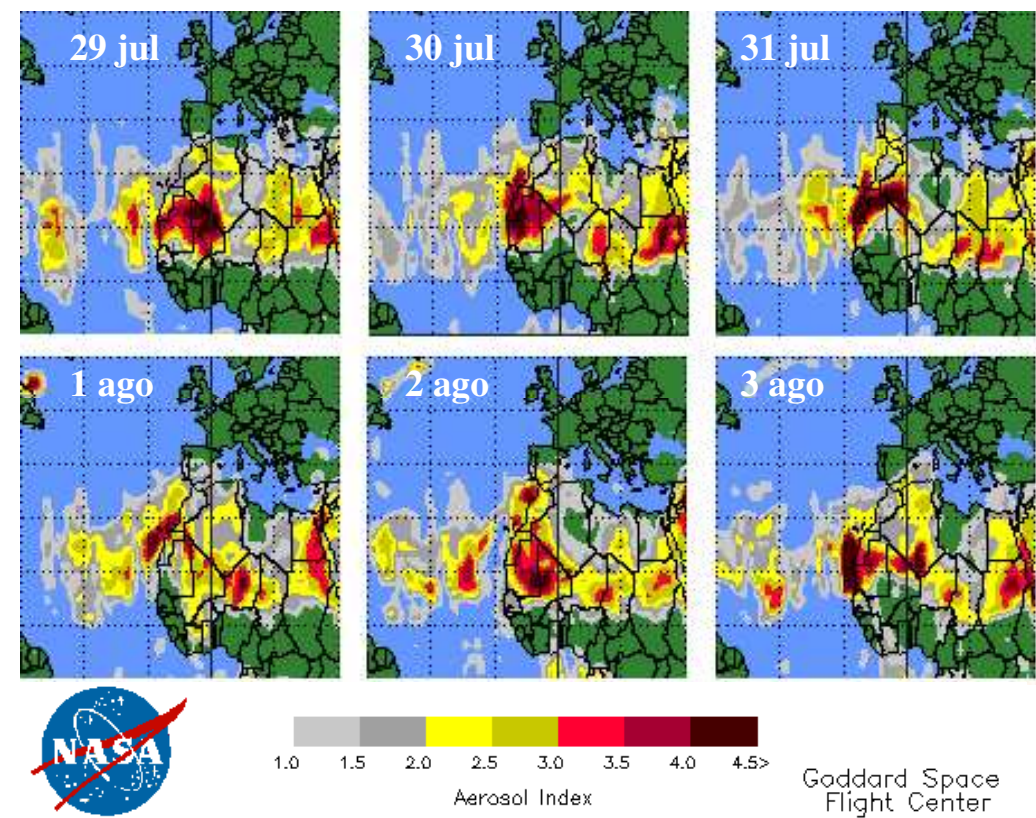

Gaddard Space

Figura VII-19. Mapas de índice de aerosoles del TOMS sobre la zona de estudio entre los días 29 de julio y 3 de agosto de 2000.

En resumen se trata de un evento de intensidad y duración medias, sin presencia de nubosidad, y cuyo seguimiento realiza el Cimel a través del AOD espectral, mostrando un aumento del AOD en la entrada del episodio, con un descenso de alfa, y la situación inversa una vez que la masa de polvo se va retirando.

Nh.APS Optical Depth for 12:00z 29 Jul zo00

Sulfate: Orange/Red, Dust: Green/Yellow, Smoke: Blue

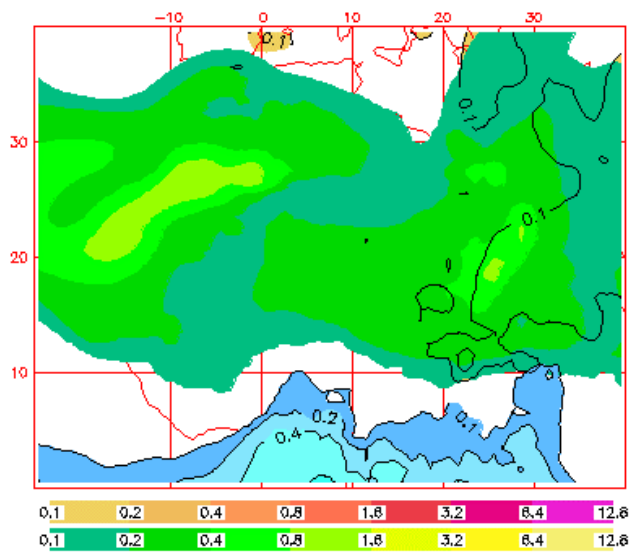

Nh.APS Surface Concentration (ug $-\mathrm{m}^{* *}$ ) for 12:002 31 Jul 2000 Dust

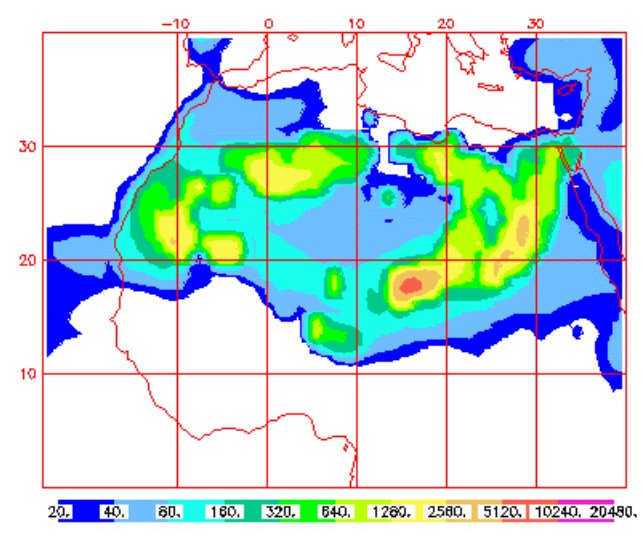

Figura VII-20. Mapas del modelo NAAPS sobre el Sahara y sur de la Península: (a) Espesor óptico el día 29 de julio, en escala de verdes; (b) Concentración de polvo en superficie el día 31 de julio de 2000.

\subsubsection{Episodio del 29 de julio al 15 de agosto de 2003}

Este episodio de aerosol desértico tiene dos particularidades que nos hacen destacarlo como caso de estudio. En primer lugar se trata del episodio más largo, con 18 días de permanencia sobre la zona de estudio. En segundo lugar porque simultáneamente se produjeron fuertes episodios de combustión de biomasa por los numerosos incendios en Portugal y la sierra de Huelva durante ese verano (Figura VII-21). En la imagen de la izquierda, correspondiente al día 4 de agosto, podemos observar numerosos incendios en Portugal y 2 focos en Huelva, cerca de nuestra estación, todos ello marcados en rojo, mientras que en la zona del golfo de Cádiz se puede observar la presencia de polvo 
desértico, que provoca esa turbiedad marrón sobre el mar. En la imagen de la derecha, correspondiente al día 13 de agosto, observamos una enorme turbiedad sobre toda la Península, y una concentración de incendios en el extremos suroeste que inyectan una gran cantidad de humo en la atmósfera. Para analizar este complejo episodio nos vamos a basar de nuevo en las medidas del Cimel durante el episodio (Figura VII-22), y nos apoyaremos en las retrotrayectorias del modelo HYSPLIT, imágenes MODIS y en las predicciones dadas por los mapas del modelo NAAPS.
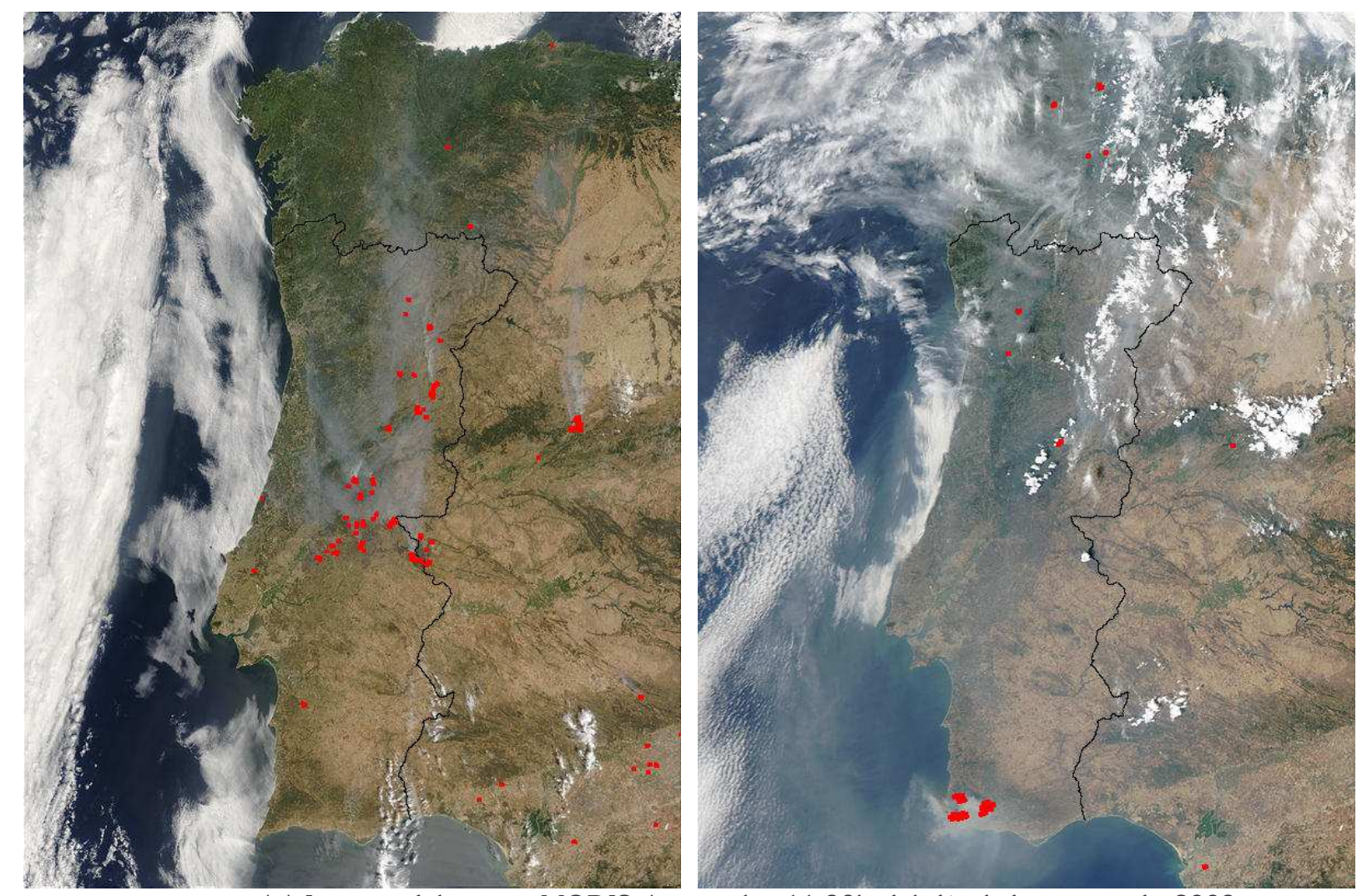

Figura VII-21. (a) Imagen del sensor MODIS-Aqua a las 11:30h del día 4 de agosto de 2003, en la que se indican en rojo las zonas de incendios. El tamaño del pixel es $1 \mathrm{~km}$; (b) Imagen MODIS-Aqua para el 13 de agosto a las 13h.

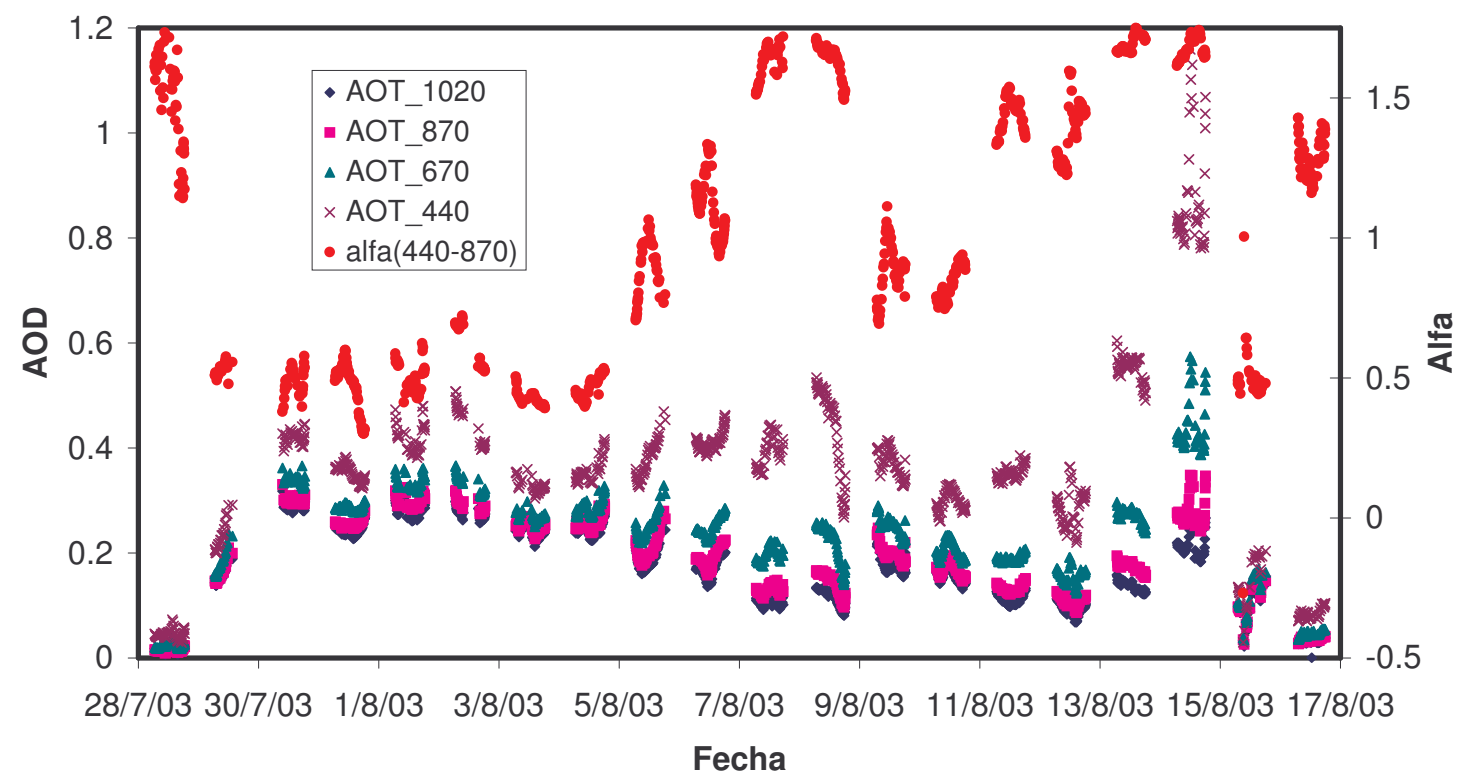

Figura VII-22. Registro del fotómetro Cimel en El Arenosillo entre los días 28 de julio y 16 de agosto de 2003, con el AOD en los cuatro canales (eje izquierdo) y parámetro alfa (eje derecho). 
Las medidas del Cimel durante los primeros días del episodio se corresponden con una entrada habitual de aerosol desértico. La situación sinóptica (no mostrada) es la clásica del verano, es decir, una situación del tipo 4 (NAH-A), con la llegada de polvo desde el sur. Las retrotrayectorias para el día 30 de julio (Figura VII-24a) y el mapa del modelo NAAPS para el día 1 de agosto (Figura VII-23a) así nos lo confirman. El espesor óptico sube durante el día 29 de julio y alcanza 0.44 (en 440nm) mientras que alfa desciende hasta 0.39. En los días siguientes el AOD llega a 0.50 (día 2 de agosto) y luego comienza a descender lentamente. Sin embargo a partir del día 5 se produce un nuevo escenario, que corresponde con el inicio de la presencia del humo de los incendios. En efecto el AOD en 440nm mantiene un valor en torno a 0.40 mientras que en las longitudes de onda largas el AOD continúa disminuyendo. Esto hace que el parámetro alfa aumente bruscamente, desde 0.5 el día anterior hasta valores superiores a 1. Esta tendencia se mantiene hasta el día 8, en el que podemos observar la enorme diferencia entre el $\mathrm{AOD}(440 \mathrm{~nm})$, que vale 0.52 al principio del día, y en $670 \mathrm{~nm}$, que vale 0.25 . El valor de alfa es 1.69 , lo cual tiene muy poco que ver con el aerosol desértico. Las retrotrayectorias el día 5 (Figura VII-24b) también confirman esta nueva situación, pues ya no muestran una masa de aire que llega directamente desde el sur sino que la masa de aire de procedencia norteafricana sufre una recirculación sobre la Península en los niveles de 1500 y 3000m.

Se trata por tanto de alta turbiedad, pero no con un espectro de AOD muy plano (como el aerosol desértico), sino con un espectro AOD que tiene una fuerte pendiente, es decir, con predominio de extinción debida a partículas finas. En realidad tenemos una mezcla de ambos tipos de aerosol, que da lugar a subidas y bajadas de alfa según predomina un tipo $\mathrm{u}$ otro de aerosol. El aerosol por combustión de biomasa es más intenso durante los días 13 y 14, como vemos por la alta turbiedad, alcanzándose 1.15 de AOD en 440nm el día 14 (0.25 tan solo en $1020 \mathrm{~nm}$ ), con un alfa de 1.72, es decir, un espectro de AOD con fuerte pendiente. Estas medidas son únicas durante los 5 años de la serie de datos. Podemos ver en el mapa del modelo NAAPS para el día 13 (Figura VII-23b) el espesor óptico debido a humos, en escala de azules, que se sobrepone a la escala de verdes que da el espesor óptico debido a polvo. Las retrotrayectorias tienen procedencia norteafricana los días 29 de julio al 5 de agosto, 8 al 11 (Figura VII-24d) y el 15 de agosto, mientras que las retrotrayectorias muestran procedencia mediterránea durante los días 6 y 7 de agosto (Figura VII-24c), y recirculaciones durante los días 12 al 14 de agosto. La correlación entre la procedencia dada por las retrotrayectorias y el parámetro alfa en la Figura VII-22 es muy clara, con bajadas cuando la procedencia es norteafricana, mientras que los valores son altos durante los días con procedencia mediterránea y en los días con recirculaciones.

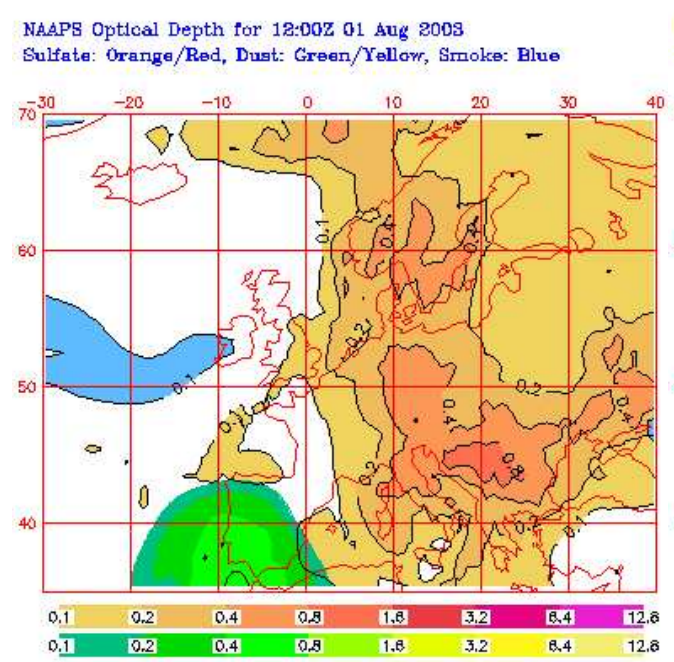

NhAPS Optical Depth for 12:002 19 Aug 2003 Sulfate: Orange/Red, Dust: Green/Yellow, Smoke: Blue

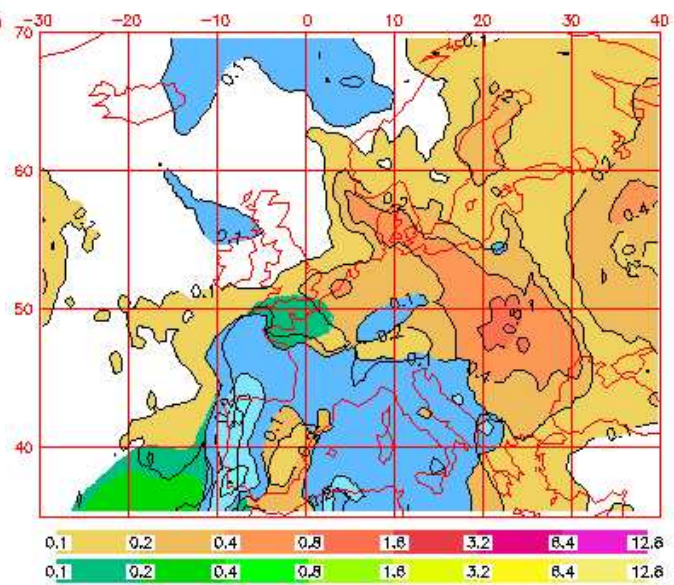

Figura VII-23. Mapas espesor óptico del modelo NAAPS para los días: (a) 1 de agosto; (b) 13 de agosto de 2003. 
Las retrotrayectorias durante el día 14 agosto (Figura VII-24e) son claramente locales, y nos indican que en los últimos 5 días la masa de aire apenas se ha movido. Además en los niveles bajos llega directamente desde Portugal. Sin embargo el día 15 la situación empieza a cambiar, y las retrotrayectorias ya empiezan a llegar desde el Atlántico, aunque dibujando un movimiento anticiclónico desde el Sahara (Figura VII-24f). Parece que este cambio retira el aerosol de combustión de biomasa y trae de nuevo aerosol desértico, pues la extinción por aerosoles se vuelve mucho más plana y el alfa disminuye. Finalmente el día 16 entra una masa de aire atlántica limpia, con la que recuperamos los valores de fondo de AOD y alfa.

En resumen hemos tenido una masa de aire con polvo en suspensión que se ha mantenido en la zona de estudio durante 18 días, con recirculaciones y mezcla con aerosol local, como confirman las retrotrayectorias. En este caso la turbiedad por humo de incendios forestales ha sido mucho más fuerte que el propio aerosol desértico. Queremos destacar la capacidad del fotómetro Cimel a través de las medidas continuas de AOD espectral, para realizar un seguimiento de estos eventos y distinguir tipos de aerosol. El parámetro alfa resulta muy útil para este análisis, y al respecto hay que tener en cuenta que los altos valores de AOD hacen que alfa tenga un error absoluto pequeño, y que por tanto es un parámetro fiable para este tipo de eventos con alta turbiedad.
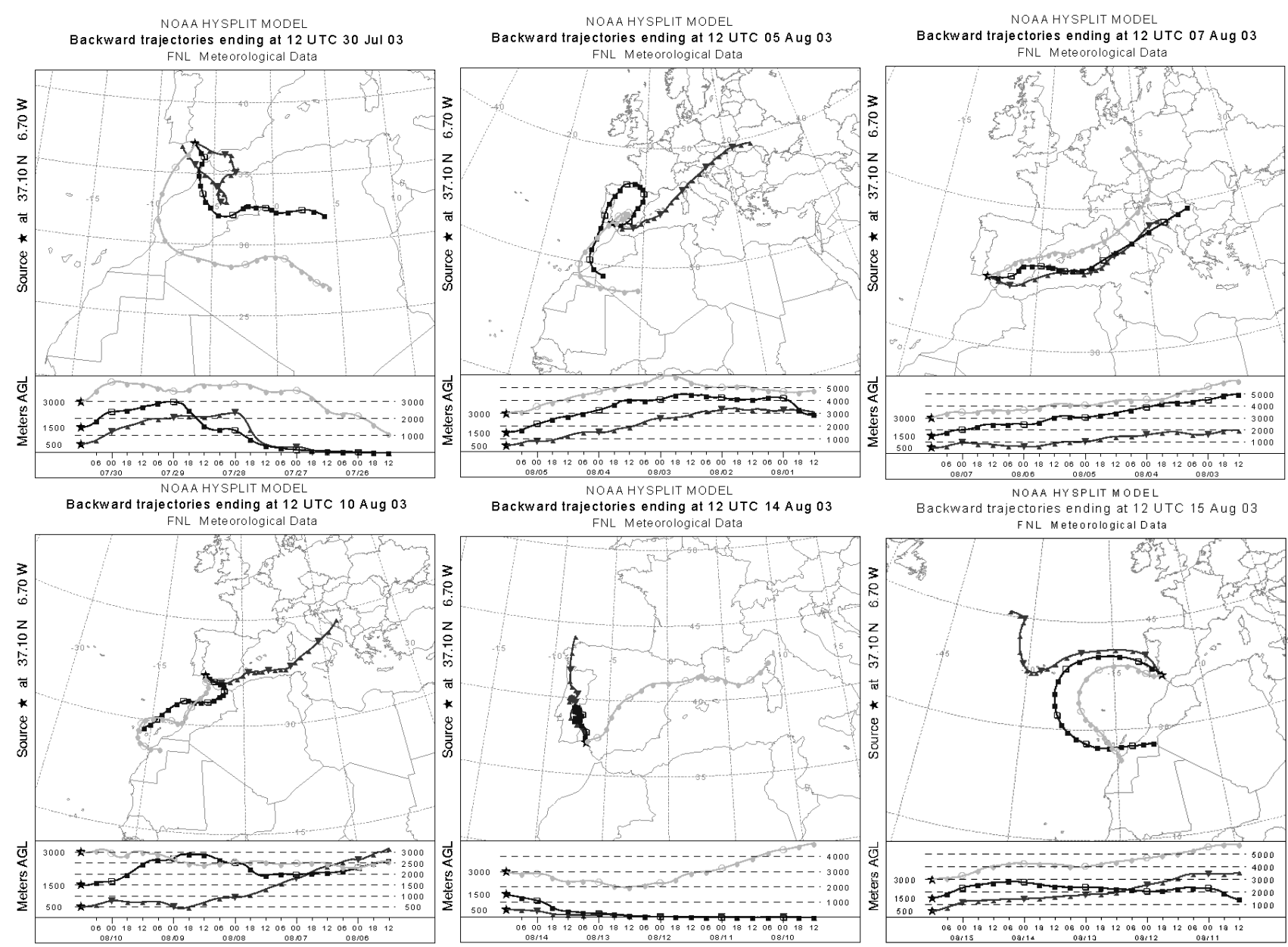

Figura VII-24. Retrotrayectorias a 5 días del modelo HYSPLIT en El Arenosillo para los días: (a) 30 de julio; (b) 5 de agosto; (c) 7 de agosto; (d) 10 de agosto; (e) 14 de agosto; y (f) 15 de agosto de 2003. 


\subsubsection{Episodio del 22 al 28 de julio de 2004}

Este episodio de aerosol desértico durante los días 22 al 28 de julio de 2004 se ha seleccionado ya que es el más fuerte registrado en la estación de El Arenosillo para la serie de datos de 5 años del Cimel. Tuvo una duración de 7 días, no excesivamente larga, pero se registró un AOD en 440nm de 2.7, el máximo de la serie de datos hasta el momento, y se midieron valores superiores a 0.75 durante 4 días consecutivos. Las medidas del Cimel durante el episodio completo están en la Figura VII-26. Un gran incendio en la sierra de Huelva entre los días 25 y 29 coincide con este episodio, aunque sus efectos en las medidas del Cimel no son tan severos como en el episodio de incendios en agosto de 2003. Este episodio desértico afectó a toda la Península Ibérica y extensas zonas de Europa en los días posteriores. En la estación de Palencia se alcanzó durante el día 23 un $\mathrm{AOD}(440 \mathrm{~nm})$ de 1.5. Como vemos en la imagen SeaWIFS del día 22 de julio (Figura VII25) el polvo desértico llegó en forma de arco atlántico.

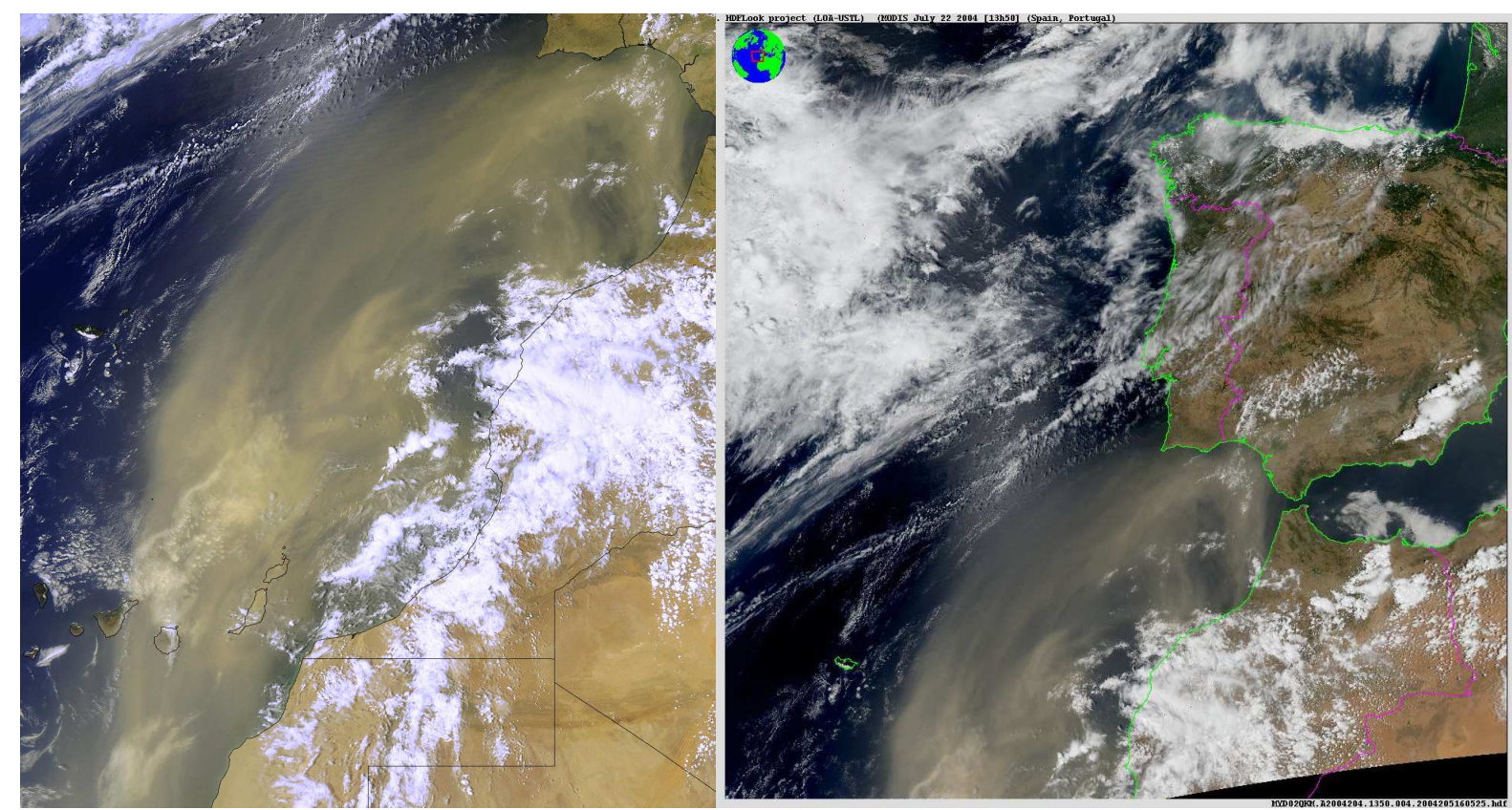

Figura VII-25. Imagen del sensor MODIS-Aqua para las 13:50h del día 22 de julio de 2004. El tamaño del pixel es $1 \mathrm{~km}$. A la izquierda, detalle de la zona entre Canarias y el golfo de Cádiz. A la derecha, imagen completa de la Península.

El episodio comienza en la madrugada del día 22 de julio, y cuando el fotómetro Cimel comienza a medir a las 7:50GMT el AOD(440nm) es ya de 0.4. La presencia de nubes hace que no se disponga de medidas del Cimel a primera hora de la mañana ni entre las 9.45 y las 12:45GMT. Este día el $\mathrm{AOD}(440 \mathrm{~nm})$ registra una fuerte subida desde 0.4 a 2.7 en pocas horas. De hecho la entrada del episodio se produce con una pluma estrecha de polvo que hace subir el $\mathrm{AOD}(440 \mathrm{~nm})$ de 1.01 a las $12: 45 \mathrm{~h}$ hasta 2.7 a las $16: 30 \mathrm{y}$ otro fuerte descenso hasta 1.4 a las 18:00h. Esta pluma con fuerte concentración de aerosol atravesó la estación en tan solo 5 horas. Los días siguientes hasta el día 25, registran AOD entre 0.6 y 1.4 , y como se puede ver en la gráfica el valor del AOD es muy similar en los cuatro canales del Cimel: el espectro de AOD es prácticamente plano y los valores de alfa son muy próximos a 0, con un mínimo de 0.02 durante el día 23. Entre los días 26 y 28 la contaminación por fuentes locales, en concreto por incendios en Portugal y la sierra de Huelva, se mezcla con el aerosol desértico que continúa entrando desde el Atlántico en forma de arco, dando lugar a subidas y bajadas del parámetro alfa, señal de la presencia de partículas finas de aerosol por combustión de biomasa. En concreto el día 28 se produce otra subida del AOD hasta 0.8 en $440 \mathrm{~nm}$ y con fuerte bajada de alfa, lo que indica la predominancia de aerosol desértico ese día. 


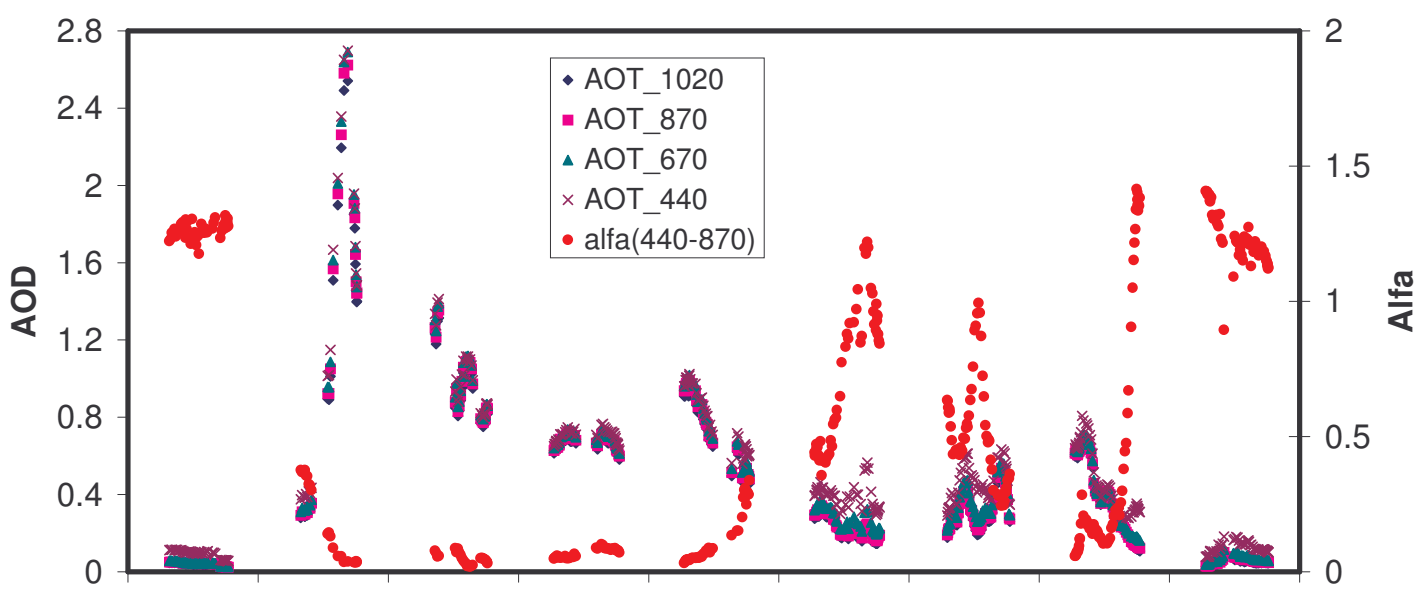

21/7/04 22/7/04 23/7/04 24/7/04 25/7/04 26/7/04 27/7/04 20/7/04

Fecha

Figura VII-26. Registro del fotómetro Cimel en El Arenosillo entre los días 21 y 29 de julio de 2004, con el AOD en los cuatro canales (eje izquierdo) y parámetro alfa (eje derecho).

Los datos de PM10 $10^{1}$ registrados durante el evento se sitúan por encima de $200 \mu \mathrm{g} / \mathrm{m}^{3}$ entre los días 23 y 26 , con un máximo de $450 \mu \mathrm{g} / \mathrm{m}^{3}$ el día 25 , siendo los más altos al menos durante los últimos 10 años. De estas medidas sólo queremos destacar que en superficie llegó la masa cargada de polvo a partir del mediodía del día 23, esto es, un día después de que comenzase el evento, tal y como podemos observar en las medidas del Cimel y en las imágenes de satélite, lo que sugiere que el episodio comenzó primero con la llegada de aerosoles desérticos en los niveles altos. Esto viene confirmado por la retrotrayectoria del día 22 (Figura VII-28a) en la que sólo proviene de la zona del Sahara la trayectoria en $3000 \mathrm{~m}$. El día siguiente, día 23 (Figura VII-28b) la masa de aire proviene del Sahara en los tres niveles. El día 26 (Figura VII-28c) las retrotrayectorias provienen todavía del Sahara occidental en los tres niveles, dibujando un claro movimiento anticiclónico y entrando a la zona de estudio desde el noroeste. Este paso sobre la mitad sur de Portugal está en concordancia con la mezcla del aerosol desértico con el humo de incendios que se registraron en esa zona, dando lugar a un aerosol mezclado y con alta turbiedad.

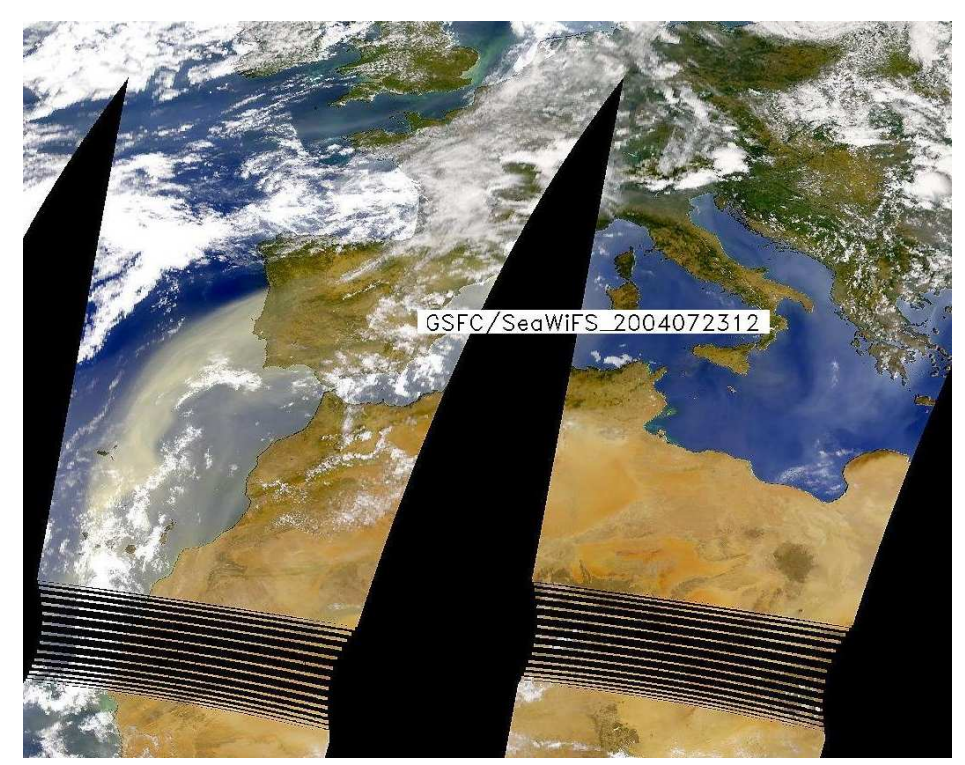

Figura VII-27. Imagen Seawifs para el día 23 de julio de 2004 a las $12 \mathrm{~h}$.

\footnotetext{
${ }^{1}$ Datos de PM10 promedio entre las estaciones de Campus el Carmen, Palos y Punta Umbría, proporcionados por la Consejería de Medio Ambiente de la Junta de Andalucía.
} 
Mostramos en la Figura VII-29a al mapa del modelo NAAPS para el día 23 de julio. En este caso hemos preferido el mapa de AOD debido a aerosol desértico (en escala de verdes) que también proporciona el modelo, en lugar de la concentración en superficie. Este modelo predice para el día 23 un AOD entre 0.8 y 1.8 para una estrecha franja (en color más amarillo), de acuerdo con los valores medidos por el Cimel. También podemos ver que el aerosol llega a la estación desde el suroeste por el Atlántico, pero tiene su origen en el Sahara occidental justo en la vertical de la Península. El mapa de índice de aerosoles del TOMS (Figura VII-29c) tampoco deja lugar a dudas sobre la intensidad del episodio. El día 23 se alcanza un valor cercano a 4, es decir, un índice de aerosoles similar al de las zonas fuente de polvo desértico en el Sahara, como podemos ver en el mismo mapa. También nos muestra la llegada del aerosol sobre el Atlántico desde el sudoeste.
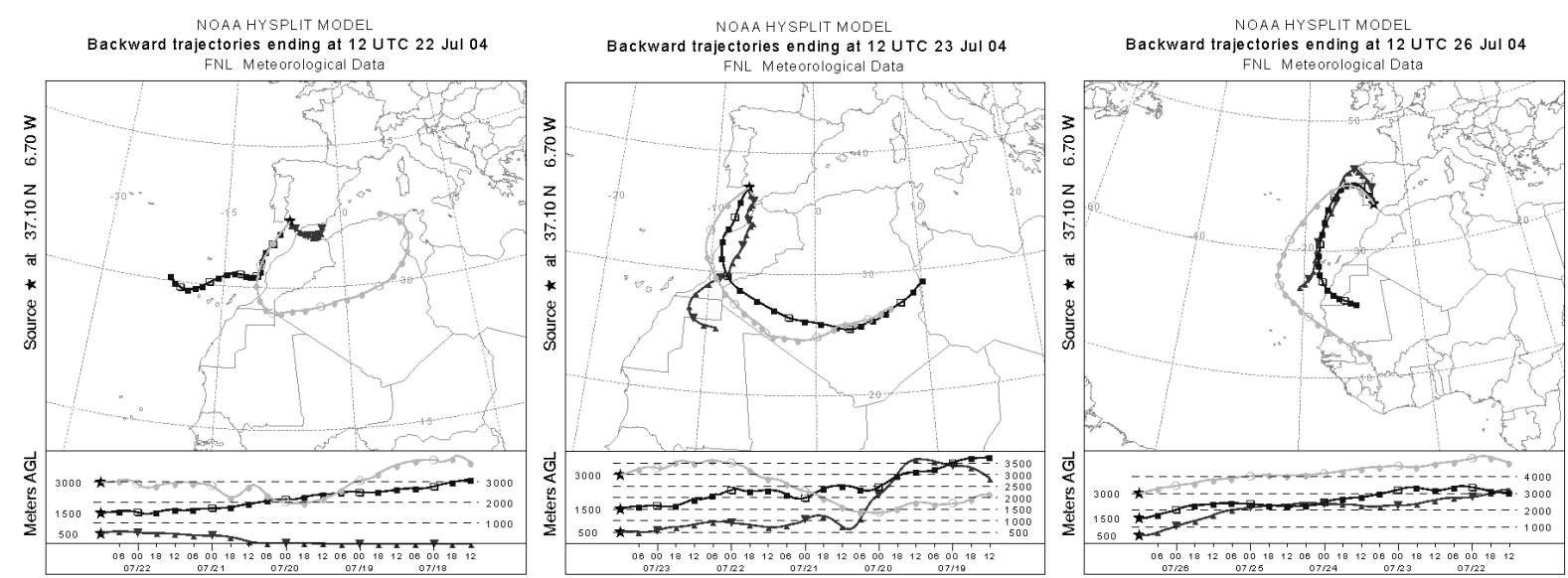

Figura VII-28. Retrotrayectorias en El Arenosillo para los días: (a) 22 de julio; (b) 23 de julio; y (c) 26 de julio de 2004.

Esto nos lleva a analizar con cuidado la situación sinóptica que da lugar a este evento excepcional (Figura VII-29b). La situación en superficie es la habitual del verano cuando hay entradas de aerosol desértico, es decir, bajas presiones relativas sobre el norte de África debido a la baja térmica. Sin embargo en los niveles altos la situación no es la clásica del tipo NAH-A (ver Figura VII-1d), puesto que las altas presiones en altura no están situadas al sudeste Peninsular sino sobre el norte de África. Desde esta posición obliga a la masa de aire a realizar un movimiento anticiclónico y da lugar al clásico arco atlántico, que es propio de las intrusiones de invierno o primavera (las que clasificamos como tipo NAH-S).

La imagen SeaWIFS para el día 23 (Figura VII-27) no deja lugar a dudas sobre el arco atlántico de polvo. Probablemente los grandes niveles de polvo en suspensión durante el verano sobre el Sahara occidental, obligados a viajar en una estrecha pluma, sean los responsables de los altos espesores ópticos medidos durante el primer día de este episodio. La situación sinóptica por tanto debe ser tenida en cuenta para posteriores eventos, de cara a confirmar si esta posición más al oeste de las altas presiones en los niveles altos da lugar de nuevo a arco atlántico de polvo y a intrusiones tan fuertes.

Aunque las medidas de radiancia del Cimel y todos los productos que se derivan de ellas han quedado fuera de los objetivos de esta tesis doctoral, queremos señalar que se están analizado en otros estudios que se llevan a cabo dentro del Grupo de Óptica Atmosférica. Además el estudio se completa con todo el conjunto de medidas que se realizan para el estudio de los aerosoles con otro tipo de instrumentación en El Arenosillo, las medidas in situ. Destacamos también que durante el verano-otono del año 2004 se realizó en El Arenosillo una extensa campaña (del 15 julio al 15 de Octubre) para el estudio de aerosoles. Esta campaña coincidió con el evento que aquí estamos analizando, por lo que 
a las medidas anteriores y habituales podemos añadir las de concentración másica de partículas con un impactor en cascada de 3 niveles (PM10, PM2.5, PM1) y un captador de alto volumen que da la masa total y PM10 (de estas últimas se realiza un extenso análisis químico). La medida de las distribuciones de tamaños de partículas (y por ende sus concentraciones) en superficie se registraron con dos espectrómetros de partículas, y se realizó la medida del coeficiente de absorción para los aerosoles recogidos en los filtros del impactor de 3 niveles (Mogo et al., 2005).

(a)

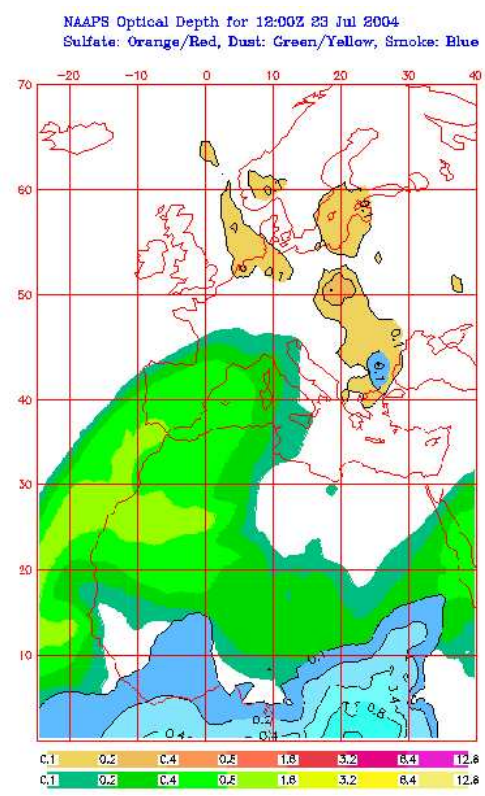

(b)

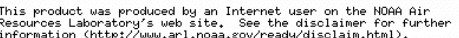

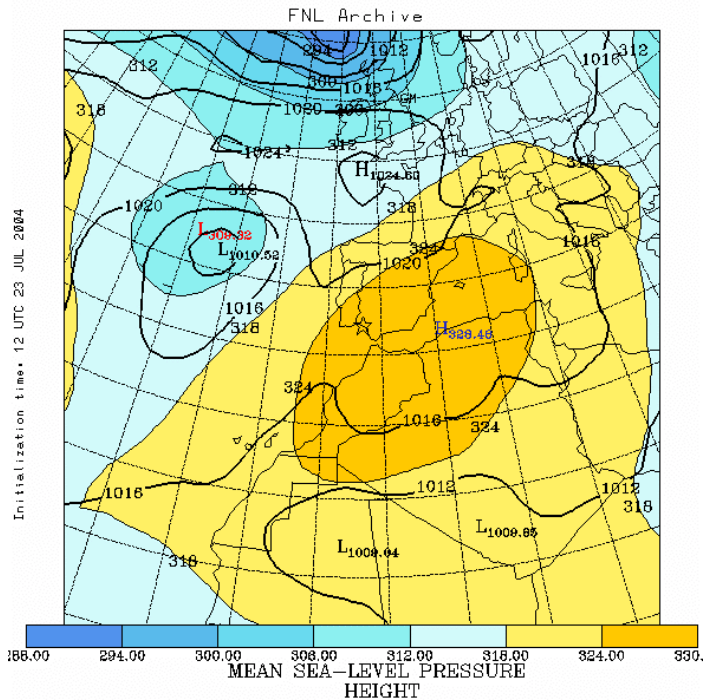

(c)
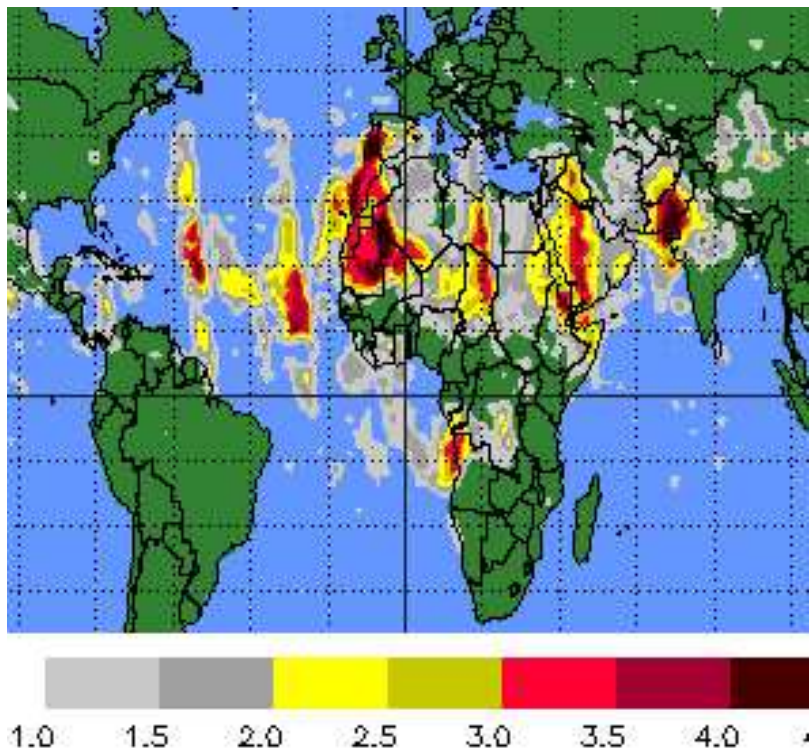

Aerosol Index

Figura VII-29. Día 23 de julio de 2004: (a) Mapa de espesor óptico debido a polvo desértico dado por el modelo NAAPS; (b) Situación sinóptica en superficie y $700 \mathrm{hPa}$; (c) Índice de Aerosoles del TOMS. 


\section{Conclusiones y líneas futuras}

La estación de El Arenosillo ha sido la primera estación en España incluida en la red AERONET, y en este momento posee una base de datos suficientemente extensa, más de 5 años, y de calidad contrastada para permitir un estudio climatológico de las propiedades de los aerosoles en esta región.

Debemos destacar la importancia de la red AERONET a nivel mundial, ya que actualmente es la red de referencia para el estudio de los aerosoles atmosféricos. Esta red, gestionada por NASA y la Universidad de Lille, tiene como objetivos la caracterización de las propiedades de los aerosoles a nivel global con fines climatológicos, así como validar las medidas que, desde sensores de satélite, se realizan sobre los aerosoles. Detrás de estos objetivos está la necesidad de determinar el papel de los aerosoles en el clima del planeta.

El trabajo realizado se puede recoger en los siguientes puntos:

1. Por una parte se ha desarrollado un trabajo de evaluación de la calidad de la serie datos, debido a problemas de calibración que se detectaron en las medidas. Estos problemas se resumen en la aparición de un ciclo diurno sistemático en las medidas del espesor óptico de aerosoles, que en ciertos casos da lugar a valores negativos del AOD, que no son admisibles en este tipo de medidas.

2. Esto ha concluido en el desarrollo del método KCICLO de calibración in situ, que nos ha permitido corregir el problema del falso ciclo diurno en nuestros datos a partir de la dependencia con la masa óptica del error del AOD. Se ha realizado también una evaluación de la precisión del método así como una comparación entre los datos corregidos y la serie de datos de AERONET.

3. Por otra parte se ha realizado un análisis sobre las propiedades de los aerosoles en la zona de estudio, mediante los parámetros AOD y alfa de la serie de datos corregida con el método KCICLO. En primer lugar se ha realizado una evaluación estadística de la serie, con análisis de evoluciones temporales, promedios y frecuencias. Este análisis revela la enorme variabilidad de los aerosoles presentes en la estación, con diferentes escenarios de alta y baja turbiedad que han sido analizados en base a los tipos básicos de aerosoles que encontramos en estaciones clave de AERONET. El promedio del espesor óptico de aerosoles en 440nm es de 0.18 (STD 0.14), aunque se llega a superar valores de 1 durante episodios de aerosol desértico, mientras que el promedio del parámetro alfa en el rango 440-870nm es de 1.05 (STD 0.43).

4. La necesidad de una clasificación de los tipos de aerosoles ha motivado el estudio de las propiedades de AOD y alfa en función de los tipos de masas de aire presentes en la estación. El estudio de las masas de aire se ha basado en el cálculo de retrotrayectorias con el modelo HYSPLIT. Las retrotrayectorias han sido clasificadas mediante dos metodologías diferentes y complementarias, una basada en la definición de sectores geográficos y la segunda mediante un algoritmo de cluster. El objetivo es relacionar cada tipo de masa de aire con las medidas de AOD y alfa de los aerosoles. A partir de este análisis se ha podido establecer los diferentes tipos de masas de aire y la frecuencia con que llegan a la estación. 
5. Las medidas del fotómetro Cimel han permitido establecer unas características de AOD espectral que podemos asociar a cada grupo de masas de aire. Las masas de aire más frecuentes son de procedencia atlántica, con una frecuencia del 68\%, y presentan cargas bajas de aerosol en general (AOD en 440nm en torno a 0.11), excepto en las masas que llegan del suroeste, en las que llega con cierta frecuencia aerosol de origen desértico. En segundo lugar se encuentran las masas de aire norteafricanas y mediterráneas (20\%), relacionadas con la llegada de aerosol de origen desértico, y que muestran por tanto turbiedad alta (AOD promedio de 0.29). Por último un $12 \%$ de masas de aire tienen su origen en el continente europeo, y presentan una turbiedad media pero debida a aerosoles de tipo antropogénico (AOD en 440nm de 0.19).

6. El análisis de la serie de datos se finaliza con la evaluación de los episodios de aerosol desértico registrados en la estación. A partir de las medidas del fotómetro Cimel se ha realizado un inventario de entradas de aerosol desértico durante el período 2000-2004. Se ha podido determinar la frecuencia y la estacionalidad de estos episodios, así como las características espectrales del espesor óptico de aerosoles para el aerosol desértico que llega desde el norte de África.

7. La presencia de aerosoles desérticos condiciona y modula las propiedades y la estacionalidad del AOD en la estación. Los episodios de polvo desértico tienen una frecuencia superior al $17 \%$ de los días, con un promedio de 64 días al año, y predominancia de episodios durante los meses de verano y los meses de febrero y marzo. En segundo lugar se ha podido constatar la enorme variabilidad de unos años a otros, desde 51 hasta 77 días dependiendo del año.

8. Los episodios de aerosol desértico se caracterizan por un aumento hasta un orden de magnitud del espesor óptico de aerosoles (AOD promedio en 440nm de 0.32), así como por un descenso en el parámetro alfa, con valores por debajo de 0.5 en los días centrales de los episodios (alfa en promedio 0.69). Esto ha permitido plantear también un criterio sobre las observaciones del fotómetro Cimel: $\operatorname{AOD}(870 \mathrm{~nm})>0.11 \mathrm{y}$ alfa $<1.05$, el cual permitirá en adelante la detección y evaluación de forma automática de los episodios desérticos en la región.

De todo lo expuesto, queremos destacar las siguientes aportaciones y conclusiones:

- Se ha desarrollado el método KCICLO para la corrección de los datos de espesor óptico de aerosoles. Este método se ha aplicado con éxito a la serie de datos del fotómetro Cimel en El Arenosillo, corrigiéndose los problemas detectados y estableciendo una base de datos consistente.

- Se ha realizado el primer análisis climatológico de una serie de datos larga sobre aerosoles atmosféricos en la Península, obtenida mediante las medidas del fotómetro Cimel de la red AERONET/PHOTONS en la estación de El Arenosillo.

- El estudio y la clasificación de las masas de aire en la zona de estudio nos ha permitido establecer una relación entre las masas de aire y el tipo de aerosol, que se ha cuantificado mediante los parámetros AOD y alfa.

- Se ha realizado por primera vez en España un inventario de episodios de aerosol desértico basado en datos de AOD, evaluando la frecuencia y la variabilidad interanual de los mismos. Se ha establecido un criterio para la detección y evaluación de estos episodios desérticos. 


\section{Líneas futuras}

A lo largo de la memoria ya se han apuntado algunas de las líneas de trabajo que consideramos se pueden abordar a partir de este trabajo. Una de ellas es la necesidad de evaluar con el máximo detalle el parámetro alfa en la red AERONET, mediante simulaciones, para establecer qué información es posible obtener y con qué fiabilidad a partir de este parámetro.

El método KCICLO puede ser mejorado de cara a aumentar la precisión en condiciones atmosféricas que no sean óptimas, según diversas propuestas que se pueden encontrar en la bibliografía. De cara a la red RIMA (Red Ibérica de Medida de Aerosoles), actualmente en desarrollo, el método KCICLO se puede establecer como una comprobación rutinaria de la calibración, con el objeto de detectar problemas y, en caso necesario, proporcionar una corrección.

El trabajo fundamental que se plantea ahora mismo es el análisis de la serie completa de medidas del Cimel en El Arenosillo, es decir, no sólo de las características de AOD y alfa, sino de todo el conjunto de productos que ofrece AERONET: parámetros microfísicos y radiativos que se obtienen mediante los algoritmos de inversión de la red (distribución de tamaños, albedo de scattering simple, función de fase, índice de refracción...), así como el vapor de agua en columna y los productos de polarización. Estos productos, en particular los microfísicos, pueden ser comparados y validados con la instrumentación para el muestreo de aerosoles in situ que está operativa en El Arenosillo. Por otra parte se puede extender el estudio en función de las masas de aire para todos estos productos.

La metodología de análisis de la serie de datos del fotómetro Cimel, el método KCICLO y el estudio de las masas de aire, pueden ser extendidos a otras estaciones de medida, como la estación de Palencia o Andenes, en las que nuestro grupo realiza medidas rutinarias. Esto permitirá poner a prueba y mejorar la metodología aquí desarrollada.

Otra línea de trabajo será la continuación del inventario de episodios desérticos en El Arenosillo y su extensión a otras zonas de la Península, así como la caracterización de las propiedades ópticas de estos aerosoles desérticos.

Finalmente creemos necesario seguir trabajando en la línea de construir bases de datos de aerosoles largas y de la máxima calidad, con instrumentos ubicados en redes como AERONET. Un ejemplo de ello es la creación de la Red Ibérica de Medida de Aerosoles (RIMA), iniciativa liderada por la Universidad de Valladolid. Esto permitirá construir una climatología de los aerosoles a escala local y nacional, en primer término, y después global, pues la red nace integrada en AERONET. 


\section{Bibliografía}

Ångström, A. On the atmospheric transmission of sun radiation an on dust in the air. Geograf. Ann. Deut., 11:156-166, 1929.

Ångström, A. The parameters of atmospheric turbidity. Tellus, 16, 64-76, 1964.

Ávila, A., Queralt, I., Alarcón, M.. Mineralogical composition of African dust delivered by red rains over north-eastern Spain. Journal of Geophysical Research 102, 21977-21996, 1997.

Ávila, A., Alarcón, .M., Queralt, I. The chemical composition of dust transported in red rains-its contribution to the biochemical cycle of a holm oak forest in Catalonia (Spain). Atmospheric Environment 32 (2),179-191, 1998.

Bahrmann, C.P. and Saxena, V.K. Influence of air mass history on black carbon concentrations and regional climate forcing in southeastern United States. J. Geophys. Res., 103 (D18), 2315323161, 1998.

Bokoye A. I., Royer A., O'Neill N.T., Fedosejevs G., Teillet P.M., McArthur B. Characterization of atmospheric aerosols across Canada. Assessment from a ground-based Sun-photometer network: AEROCAN. Atmosphere-Ocean. 39(4), 429-456, 2001.

Bonasoni P., F. Calzolari, U. Bonafè, P. Cristofanelli, F. Evangelisti, M.C. Facchini, S. Fuzzi, R. Van Dingenen, J. P. Putaud, M. Hanke, J. Uecker, H. Fischer, R. Kormann, F. Barnaba, M. Campanelli G. P. Gobbi, T. Colombo, M. Schulz, Y. Balkanski. The Mt. Cimone MINATROC field campaign European Geophysic Symposium, Nizza, 2001.

Cachorro, V. E., P. M. Romero, C. Toledano, E. Cuevas, and A. M. de Frutos, The fictitious diurnal cycle of aerosol optical depth: A new approach for "in situ" calibration and correction of AOD data series, Geophys. Res. Lett., 31, L12106, doi:10.1029/2004GL019651, 2004.

Cachorro, V.E., Vergaz, R., A. M. De Frutos, J. M. Vilaplana, D. Henriques, C. Toledano, N. Laulainen. Characterization of two Saharan desert dust events in the Southwestern of the Iberian Peninsula. Submitted to Annales Geophysicae, 2005.

Cana L. The Saharan dust episode of 26 February 2000 over the Canary archipelago: A synoptic overview. Weather, 57(10), 385-389(5), 2002.

Cape J.N., Methven J. and Hudson L.E. The use of trajectory cluster analysis to interpret trace gas measurements at Mace Head, Ireland, Atmospheric Environment, 34, 3651-3663, 2000.

Capel Molina, J.J. Los climas de España. Barcelona, Oikos-Tau (ISBN 8428104921), 1981.

Capel Molina, J.J. El clima de la Península Ibérica. Ed. Ariel Geografía, Barcelona, 2000.

Carlson, T.N., J.M. Prospero. The large-scale movement of Saharan air outbreaks over the Northern equatorial Atlantic. J. Appl. Meteorol., 11, 283-297, 1972.

Cimel-Electronique. Sunphotometer User manual version 4.6, 2004.

Cuevas, E. Estudio del comportamiento del ozono troposférico en el observatorio de Izaña (Tenerife) y su relación con la dinámica atmosférica. Tesis doctoral. Madrid, 1995. 
D'Almeida, G. A., P. Koepke, E. P. Shettle. Atmospheric Aerosol: Global Climatology and Radiative Characteristics, A. Deepak, Hampton, Va., 1991.

Dorling, S.R., Davies, T.D. and C. E. Pierce. Cluster analysis: A technique for estimating the synoptic meteorological controls on air and precipitation chemistry-Method and applications. Atmos. Environ., 26, 2575-2581, 1992.

Draxler, R. R. Boundary layer isentropic and kinematic trajectories during the August 1993 North Atlantic Regional Experiment Intensive. J. Geophys. Res., 101, 29 255-29 268, 1996.

Draxler, R. and Rolph, G. HYSPLIT (HYbrid Single-Particle Lagrangian Integrated Trajectory) Model access via NOAA ARL READY Website (http://www.arl.noaa.gov/ready/hysplit4.html) NOAA Air Resources Laboratory, Silver Spring, MD, 2003..

Dubovik, O. and M. D. King. A flexible inversion algorithm for retrieval of aerosol optical properties from Sun and sky radiance measurements. J. Geophys. Res., 105, 20673-20696, 2000a.

Dubovik, O., A. Smirnov, B.N. Holben, M.D. King, Y.J. Kaufman, T.F. Eck, I. Slutsker. Accuracy assessments of aerosol optical properties retrieved from AERONET Sun and sky-radiance measurements. J. Geophys. Res., 105, 9791-9806, 2000 b.

Dubovik, O., B.N.Holben, T.F. Eck, A. Smirnov, Y.J. Kaufman, M.D. King, D. Tanre, and I. Slutsker. Variability of absorption and optical properties of key aerosol types observed in worldwide locations, J. Atm. Sci., 59, 590-608, 2002.

Durán, P. Medidas espectrorradiométricas para la determinación de componentes atmosféricos (ozono, vapor de agua y aerosoles) y modelización del intercambio radiativo en la atmósfera, Tesis Doctoral, Universidad de Valladolid, 1997.

Eck, T.F., B.N.Holben, J.S. Reid, O. Dubovik, A. Smirnov, N.T. O'Neill, I. Slutsker, and S. Kinne. The wavelength dependence of the optical depth of biomass burning, urban and desert dust aerosols, J. Geophys. Res., 104, 31 333-31 350, 1999.

Escudero, M., S. Castillo, X. Querol, A. Ávila, M. Alarcón, M. M. Viana, A. Alastuey, E. Cuevas, and S. Rodríguez. Wet and dry African dust episodes over eastern Spain, J. Geophys. Res., 110, D18S08, 2005.

Falke, S.R., Husar, R.B., Schichtel, B.A. Fusion of SeaWiFS and TOMS satellite data with surface observations and topographic data during extreme aerosol events. Journal Of The Air \& Waste Management Association, 51(11), 1579-1585, 2001.

Font Tullot, I. Climatología de España y Portugal. 2ª edición. Ed. Universidad, Salamanca, 2000.

Forgan, B.W. "Bias in solar constant determination by the Langley method due to structured aerosol: Comment, Appl. Opt., 27, 2546-2548, 1988.

Forgan, B.W. General method for calibrating Sun photometers. Applied Optics, 33, 21, 4841-4850, 1994 (también comunicación privada, 2003).

Heintzenberg, J., T. Tuch, B. Wehner, A. Wiedensohler, H. Wex, A. Ansmann, I. Mattis, D. Mller, M. Wendisch, S. Eckhardt, and A. Stohl. Arctic haze over central Europe. Tellus B, 55,796-807, 2003.

Herman, B.M., M.A. Box, J.A. Reagan and C.M. Evans. Alternate approach for the analysis of solar photometer data. Applied Optics 20, 2925-2928, 1981. 
Herman, J.R., P.K. Bhartia, O. Torres, N.C. Hsu, C.J. Seftor, E. Celarier. Global distribution of UVabsorbing aerosols from Nimbus-7/TOMS data. Journal of Geophysical Research, 102, 1691116923, 1997.

Hess, M., Koepke, P. and Schult, I. Optical properties of aerosols and clouds: The software package OPAC. Bulletin of the American Meteorological Society, vol. 79, 5, 831-844, 1998.

Holben, B. N., Eck, T. F., Slutsker, I., Tanré, D., Buis, J. P., Setzer, A., Vermote, E. F., Reagan, J. A., Kaufman, Y. J., Nakajima, T., Lavenu, F., Jankowiak, I., \& Smirnov, A. "AERONET - A federated instrument network and data archive for aerosol characterization". Remote Sensing of Environment, 66(1), 1-16, 1998.

Holben, B.N., D. Tanre, A. Smirnov, T.F. Eck, I. Slutsker, N. Abuhassan, W.W. Newcomb, J. Schafer, B. Chatenet, F. Lavenue, Y.J. Kaufman, J. Vande Castle, A. Setzer, B. Markham, D. Clark, R. Frouin, R. Halthore, A. Karnieli, N.T. O'Neill, C. Pietras, R.T. Pinker, K. Voss, and G. Zibordi. An emerging ground-based aerosol climatology: Aerosol Optical Depth from AERONET, J. Geophys. Res., 106, 12067-12097, 2001.

Jorba, O., Pérez, C., Rocadenbosch, F., and Baldasano, J.M. Cluster analysis of 4-day back trajectories arriving in the Barcelona area, Spain, from 1997 to 2002. Journal of Applied Meteorology, 43, 887-901, 2004.

Jorba, O. Simulación de los campos de viento de la Península Ibérica y el área geográfica de Catalunya con alta resolución espacial para distintas situaciones meteorológicas típicas. Tesis doctoral, Universidad Politécnica de Cataluña, Marzo de 2005.

Kallos, G. V. Kotroni, K. Lagouvardos. "The regional weather forecasting system SKIRON: an overview". In: Proceedings of the symposium on regional weather prediction on parallel computer environments, University of Athens, Greece, 109-122, 1997.

Killing, A., A.F. Bais, M. Blumthaler, J. Schreder, C.S. Zerefos, and E. Kosmidis. Effect of aerosols on solar UV irradiances during the Photochemical Activity and solar Ultraviolet Radiation campaign, J. Geophys. Res., 103, 26051-26060, 1998.

King, M. V., D. M. Byrne, B.M. Herman, J.A. Reagan. Aerosol size distribution obtained by inversion of spectral optical depth measurements, J. Atmos. Sci., 35, 2153-2167, 1978.

Korotaev, G, K., S. M. Sakerin, A. M. Ignatov, L. L. Stowe and E. P. McClain, Sun-photometer observations of aerosol optical thickness over the North Atlantic from a Soviet research vessel for validation of satellite measurements, J. Atmos. Ocean. Tech. 10, 725-735, 1993.

Kremser, H., P. Koepke and H. Quenzel. Aerosol optical thickness from direct solar radiation: improved Langley method applied to measured data. IRS'84: Current Problems in Atmospheric Radiation. Ed. G. Fiocco. A. Deepak Publ. S46-49. Hampton, Virginia USA, 1984.

Martin, D., G. Bergametti, and B. Strauss. On the use of the synoptic vertical velocity in trajectory model: Validation by geo-chemical tracers. Atmos. Environ., 24, 2059-2069, 1990.

Martiny, N., Validation des corrections atmospheriques: application au capteur SEAWIFS en milieu côtier, Tesis doctoral, Université du Littoral, 2001.

Methven, J.; Evans, M.; Simmonds, P. and Spain, G. Estimating relationships between air mass origin and chemical composition. Journal of Geophysical Research, 106 , 5005-5019, 2001.

Mie, G. Beiträge zur Optik trüber Medien, speziell kolloidaler Metallösungen. Ann. Physik., 25, 377445, 1908. 
Mie, G. Tratado de Electricidad y Magnetismo (Traducción de la $2^{\mathrm{a}}$ edición alemana), Ed. Manuel Marín, Barcelona, 1944.

Miller, J. M. A five-year climatology of back trajectories from the Mauna Loa observatory, Hawaii. Atmospheric Environment, 15, 1553-1558, 1981.

Mogo S, Cachorro VE, Sorribas M, de Frutos A, Fernandez R. Measurements of continuous spectra of atmospheric absorption coefficients from UV to NIR via optical method. Geophys. Res. Lett., 32 (13), L13811, 2005.

Nakajima, T., M. Tanaka, and T. Yamauchi, Retrieval of the optical properties of aerosols from aureole and extinction data, Appl. Opt., 22, 2951-2959, 1983.

Nickovic, S., T. Papadopoulos, O. Kakaliagou, G. Kallos, 1988: Model for Prediction of Desert Dust Cycle in the Atmosphere. J. Geophys. Res., 106, 18113-18129, 2001.

Nieke, J., Pflug, B., Zimmermann, G. An aureole-corrected Langley-plot method developed for the calibration of HiRES grating spectrometers. Journal of Atmospheric and Solar-Terrestrial Physics, Volume 61, 10, 739-744(6), 1999.

Peña, Daniel. Análisis de datos multivariantes. McGraw Hill, D.L., Madrid, 2002.

Pérez, C., S. Nickovic, M. Sicard, C. Toledano, V. E. Cachorro, J. M. Baldasano. Saharan dust events over the Iberian Peninsula. LIDAR and sun photometer measurements. Third Workshop Lidar Measurements in Latin-America. Popayán, Colombia, Julio de 2005.

Prospero, J.M. Long range transport of mineral dust in the global atmosphere: impact of African dust on the environment of the south-eastern United States. Proc. Natl. Acad. Sci. USA, 96, 3396-3403, 1999.

Querol, Xavier, Sergio Rodríguez, Emilio Cuevas, Maria del Mar Viana y Andrés Alastuey. Intrusiones de masas de aire africano sobre la Península Ibérica y Canarias: Mecanismos de transporte y variación estacional. $3^{\mathrm{a}}$ Asamblea Hispano Portuguesa de Geodesia y Geofísica. Proceedings Tomo II (S13) 1284-1288, Valencia, España, 2002.

Querol, X., A. Alastuey, S. Rodríguez, M.M. Viana, B. Artiñano, P. Salvador, E. Mantilla, S. García do Santos, R. Fernandez Patier, J. de La Rosa, A. Sánchez de la Campa, M. Menéndez, J.J. Gil. Levels of particulate matter in rural, urban and industrial sites in Spain. Science of the Total Environment 334-335,359-376, 2004.

Raes, F., T. Bates, F. McGovern and M. Liederkeke. The second aerosol characterization experiment (ACE2): general context and main results. Tellus 52B, 111-126, 2000.

Reagan, J. A., Thomason, L. W., Herman, B. M., and Palmer, J. M. Assessment of atmospheric limitations on the determination of the solar spectral constant from ground-based spectroradiometer measurements. IEEE Trans. Geosci. Remote Sens. GE-24, 258-265, 1986.

Robinson, N. Solar Radiation. Elsevier, Amsterdam, 1966.

Rodríguez, S., X. Querol, A. Alastuey, G. Kallos, and O. Kakaliagou. Saharan dust contributions to PM10 and TSP levels in southern and E. Spain, Atmos. Environ., 35, 2433-2447, 2001.

Rolph, G. Real-time Environmental Applications and Display sYstem (READY) Website (http://www.arl.noaa.gov/ready/hysplit4.html) NOAA Air Resources Laboratory, Silver Spring, MD, 2003. 
Romero, P.M. y E. Cuevas. Variación diurna del espesor óptico de aerosoles: ¿Ficción o realidad?. $3^{\text {a }}$ Asamblea Hispano Portuguesa de Geofísica y Geodesia. Valencia, 2002.

Sánchez de la Campa, Ana. Contribución de fuentes al material particulado atmosférico en un área urbana altamente industrializada. Tesis doctoral, Universidad de Huelva, 2004.

Sánchez, J. Situaciones atmosféricas en España. Ministerio de Obras Públicas, Transporte y Medio Ambiente. Centro de Publicaciones, 1993.

Sánchez, M.L., Pascual, D., Ramos, C. and Pérez, I. Forecasting particulate pollutant concentrations in a city from meteorological variables and regional weather patterns. Atmospheric Environment, 24a, 6, 1509-1519, 1990.

Sancho, J.M and Romero, M.C. Programa de ozonosondeos del Observatorio Atmosférico de Izaña: el ozono sobre Tenerife y su relación con la procedencia de las masas de aire. Instituto Nacional de Meteorología, 2002.

Schmid, B. and Wehrli, Ch. "Comparison of sunphotometer calibration by Langley technique and standard lamp". Appl Opt., 34, 21, 1995.

Shettle, E.P. and R.W. Fenn. AFGL-TR-79-0214. Models for the aerosol of the lower atmosphere and the effects of humidity variations on their optical properties. Environ. Res. Paper, 676. Hanscom AFL Mass, 1979.

Slusser, J., J. Gibson, D. Bigelow, D. Kolinski, P. Disterhoft, K. Lantz, and A. Baubien. Langley method of calibrating UV filter radiometers. J. Geophys. Res., 105, D4, 4841-4849, 2000.

Smirnov, A., Yu. Villevalde, N.T. O'Neill, A. Royer, and A. Tarussov: Aerosol optical depth over the oceans: analysis in terms of synoptic air mass types, J. Geophys. Res., 100, 16 639-16 650, 1995.

Smirnov, A., B.N. Holben, T.F. Eck, O. Dubovik, I. Slutsker (2000): "Cloud Screening and quality control algorithms for the AERONET database. Rem. Sens. Environ., 73, 337-349, 2000.

Smirnov, A., B.N.Holben, Y.J. Kaufman, O. Dubovik, T.F. Eck, I. Slutsker, C. Pietras, and R. Halthore. Optical Properties of Atmospheric Aerosol in Maritime Environments, J. Atm. Sci., 59, 501-523, 2002.

Smirnov, A, Holben, B.N., Lyapustin, A., Slutsker, I. and Eck, T.F., AERONET processing algorithms refinement, AERONET Workshop, El Arenosillo, Spain, May 10-14, 2004.

Soufflet, V., C. Devaux, D. Tanré. A modified Langley plot method for measuring the spectral aerosol optical thickness and its daily variations. Appl. Opt., 31, 2154-2162, 1992.

Spencer J. W. Fourier series representation of the position of the Sun. Search 2(5), 172, 1971.

Stohl, A. Computation, accuracy and applications of trajectories: A review and bibliography, Atmospheric Environment, 32, 947 - 966, 1998.

Stunder, B. NCEP Model Output -- FNL ARCHIVE DATA. NOAA-Air Resources Laboratory. Disponible en http://www.arl.noaa.gov/ss/transport/archives.html. Silver Spring, MD, 1997.

Tanaka, M., T. Nakajima, and M Shiobara. Calibration of a sunphotometer by simultaneous measurements of direct-solar and circumsolar radiations. Applied Optics, 25, 1170-1176, 1986.

Takamura, T., T. Nakajima and SKYNET community group. Overview of SKYNET and its Activities. Proceedings of AERONET Workshop. Óptica Pura y Aplicada, 37 (3), 3303-3308, 2004 
Terez, E. I., and G. A. Terez, A method to determine atmospheric optical depth using observations of direct solar radiation, J. Geophys. Res., 108(D22), 4704, 2003.

Thomason, L.W. B.M. Herman, J.A. Reagan. The effect of atmospheric attenuators with structured vertical distributions on air mass determination and Langley-plot analysis. J. Atmos. Sci., 40, 1851-1854, 1983.

Toledano, C. Validación y análisis de serie de datos de espesor óptico de aerosoles del fotómetro Cimel en El Arenosillo. Trabajo de suficiencia investigadora. Universidad de Valladolid, 2003.

Toledano, C., V.E. Cachorro, R. Vergaz, A.M. de Frutos, M. Sorribas, J.M. Vilaplana and B. De la Morena. Analysis and correction of a fictitious diurnal cycle in the aerosol optical depth: the KCICLO method. Opt. Pur. y Apl., Vol. 37, pp. 49-60, 2004.

Torres, O., P. K. Bhartia, J. R. Herman, A. Sinyuk, P. Ginoux, and B. Holben. A long-term record of aerosol optical depth from TOMS observations and comparison to AERONET measurements, $J$. Atmos. Sci., 59, 389- 413, 2002.

Vergaz, R. Propiedades ópticas de los aerosoles atmosféricos. Caracterización del área del Golfo de Cádiz. Tesis doctoral, Universidad de Valladolid, Septiembre de 2001.

Vergaz, R., Cachorro, V.E. y de Frutos, A.M. Comparación entre clasificaciones de los tipos de aerosoles en el área del Golfo de Cádiz, basadas en su comportamiento espectral y en el cálculo de retrotrayectorias. $3^{a}$ Asamblea Hispano Portuguesa de Geodesia y Geofísica. Proceedings Tomo II (S13) pp.1229-1233, Valencia, España, 2002.

Vergaz, R., V.E. Cachorro, A.M. De Frutos, D.V. Henriquez, . J.M. Vilaplana. Analysis of desert dust events over the west Iberian peninsula in the year 2000. International Geoscience \& Remote Sensing Symposium IGARSS 2003. Toulouse, France, 2003.

Vergaz, R., V. E. Cachorro, A. M. de Frutos, J. M. Vilaplana, and B. A. de la Morena. Columnar characteristics of aerosols in the maritime area of the Cadiz Gulf (Spain). Int. J. Climatol. 25, 1781-1804, 2005

WCP-55, Deepak, A., and H. E. Gerber, Eds. Report of the experts meeting on aerosols and their climatic effects, 107 pp., 1983.

WCP-112, World Climate Program. WMO/TD, 389. WMO, Geneva, 1986.

Wehrli, Ch. "PFR Precision Filter Radiometer". Documentation, version 3.0 World Radiation Center. Observatorium Davos. Switzerland, 2000a.

Wehrli, Ch. Calibration of filter radiometers for determination of atmospheric optical depth. Metrologia, 37, 419-422, 2000b.

Wehrli, Ch. "Calibration of Filter Radiometers for the GAW Aerosol Optical Depth network at Jungfraujoch and Mauna Loa". ARJ Workshop, SANW Davos, 2002.

Willeke, K. and P.A. Baron, Editors. Aerosol Measurement. Principles, Techniques and Applications. John Wiley \& Sons, 1993. 


\section{ANEXO 1. Ajustes del método KCICLO para los diferentes fotómetros Cimel instalados en El Arenosillo}

En este anexo se recogen las tablas con los resultados de los ajustes del método KCICLO para cada fotómetro y para cada uno de los cuatro canales de AOD. En ellas se indica el canal y el fotómetro al que nos referimos, la fecha, si el ajuste se realiza con datos de la mañana o la tarde (AM ó PM), el número de puntos, el coeficiente de correlación $\mathrm{R}^{2}$, la desviación estándar (STD), el valor de LnK, con su error, el valor de $\tau$ también con su error, y finalmente $\mathrm{K}$ con su error. Los valores de $\mathrm{K}$ que aparecen en blanco en algunos ajustes corresponden con valores que no han superado el último filtro, es decir, que estaban más de 2 STD alejados del promedio de K para ese canal.

\begin{tabular}{lcccccccccc}
\hline \hline Fecha & $\mathbf{4 4 0}$ & $\mathbf{N}$ & $\mathbf{R}^{\mathbf{2}}$ & $\mathbf{S T D}$ & $\mathbf{l n K}$ & error $\mathbf{L n K}$ & Tau & error Tau & $\mathbf{K}$ & error K \\
\hline $01 / 03 / 00$ & AM & 22 & 0.8099 & 0.0013 & 0.0165 & 0.0018 & 0.0519 & 0.0007 & 1.0166 & 0.0018 \\
$05 / 03 / 00$ & AM & 21 & 0.9577 & 0.0017 & 0.0521 & 0.0025 & 0.1094 & 0.0010 & 1.0534 & 0.0026 \\
$26 / 03 / 00$ & AM & 19 & 0.9395 & 0.0015 & 0.0346 & 0.0021 & 0.0666 & 0.0009 & 1.0352 & 0.0022 \\
$24 / 04 / 00$ & PM & 19 & 0.9601 & 0.0027 & 0.0722 & 0.0036 & 0.0623 & 0.0014 & 1.0749 & 0.0038 \\
$29 / 05 / 00$ & PM & 22 & 0.8959 & 0.0030 & 0.0486 & 0.0037 & 0.0957 & 0.0015 & 1.0498 & 0.0039 \\
$04 / 06 / 00$ & PM & 20 & 0.7345 & 0.0061 & 0.0557 & 0.0079 & 0.1446 & 0.0031 & 1.0573 & 0.0083 \\
$07 / 06 / 00$ & PM & 19 & 0.9885 & 0.0022 & 0.1145 & 0.0030 & 0.1268 & 0.0012 & & \\
$10 / 06 / 00$ & AM & 17 & 0.8627 & 0.0051 & 0.0602 & 0.0062 & 0.0761 & 0.0048 & 1.0620 & 0.0066 \\
$10 / 06 / 00$ & PM & 24 & 0.8093 & 0.0033 & 0.0282 & 0.0029 & 0.0873 & 0.0017 & 1.0286 & 0.0030 \\
$12 / 06 / 00$ & PM & 18 & 0.6671 & 0.0017 & 0.0131 & 0.0023 & 0.1452 & 0.0009 & 1.0132 & 0.0023 \\
$16 / 06 / 00$ & PM & 20 & 0.9797 & 0.0016 & 0.0634 & 0.0022 & 0.1100 & 0.0009 & 1.0655 & 0.0023 \\
$21 / 06 / 00$ & PM & 17 & 0.7441 & 0.0065 & 0.0718 & 0.0109 & 0.1065 & 0.0089 & & \\
$22 / 06 / 00$ & PM & 19 & 0.7458 & 0.0052 & 0.0413 & 0.0058 & 0.0733 & 0.0029 & 1.0421 & 0.0061 \\
$29 / 06 / 00$ & AM & 19 & 0.9090 & 0.0040 & 0.0685 & 0.0053 & 0.0971 & 0.0043 & 1.0709 & 0.0056 \\
$29 / 06 / 00$ & PM & 15 & 0.9830 & 0.0026 & 0.1258 & 0.0046 & 0.0420 & 0.0020 & & \\
$04 / 07 / 00$ & PM & 21 & 0.9573 & 0.0017 & 0.0460 & 0.0022 & 0.0823 & 0.0009 & 1.0471 & 0.0023 \\
\hline \hline
\end{tabular}

Tabla A1-I. Ajustes KCICLO para el Cimel \#48. Filtro de 440nm.

\begin{tabular}{lcccccccccc}
\hline \hline Fecha & $\mathbf{6 7 0}$ & $\mathbf{N}$ & $\mathbf{R}^{\mathbf{2}}$ & $\mathbf{S T D}$ & $\mathbf{l n K}$ & error $\mathbf{L n K}$ & Tau & error Tau & $\mathbf{K}$ & error K \\
\hline $01 / 03 / 00$ & AM & 22 & 0.8336 & 0.0008 & 0.0113 & 0.0011 & 0.0283 & 0.0004 & 1.0113 & 0.0011 \\
$05 / 03 / 00$ & AM & 21 & 0.9741 & 0.0008 & 0.0312 & 0.0012 & 0.0616 & 0.0004 & 1.0317 & 0.0012 \\
$26 / 03 / 00$ & AM & 19 & 0.9102 & 0.0013 & 0.0237 & 0.0018 & 0.0373 & 0.0007 & 1.0240 & 0.0018 \\
$24 / 04 / 00$ & PM & 19 & 0.9603 & 0.0018 & 0.0475 & 0.0023 & 0.0383 & 0.0009 & 1.0486 & 0.0025 \\
$29 / 05 / 00$ & PM & 22 & 0.9422 & 0.0019 & 0.0430 & 0.0024 & 0.0479 & 0.0010 & 1.0439 & 0.0025 \\
$04 / 06 / 00$ & PM & 20 & 0.9216 & 0.0032 & 0.0598 & 0.0041 & 0.0976 & 0.0016 & & \\
$07 / 06 / 00$ & PM & 19 & 0.9836 & 0.0017 & 0.0717 & 0.0022 & 0.0739 & 0.0009 & & \\
$10 / 06 / 00$ & AM & 17 & 0.9352 & 0.0027 & 0.0486 & 0.0033 & 0.0284 & 0.0026 & 1.0498 & 0.0035 \\
$10 / 06 / 00$ & PM & 24 & 0.8965 & 0.0021 & 0.0263 & 0.0019 & 0.0507 & 0.0011 & 1.0267 & 0.0020 \\
$12 / 06 / 00$ & PM & 18 & 0.9414 & 0.0011 & 0.0248 & 0.0015 & 0.0667 & 0.0006 & 1.0251 & 0.0016 \\
$16 / 06 / 00$ & PM & 20 & 0.9664 & 0.0017 & 0.0506 & 0.0022 & 0.0657 & 0.0009 & 1.0519 & 0.0023 \\
$21 / 06 / 00$ & PM & 17 & 0.8739 & 0.0032 & 0.0547 & 0.0054 & 0.0502 & 0.0044 & & \\
$22 / 06 / 00$ & PM & 19 & 0.8277 & 0.0033 & 0.0339 & 0.0037 & 0.0419 & 0.0019 & 1.0344 & 0.0039 \\
$29 / 06 / 00$ & AM & 19 & 0.9507 & 0.0022 & 0.0530 & 0.0029 & 0.0504 & 0.0024 & & \\
$29 / 06 / 00$ & PM & 15 & 0.9902 & 0.0014 & 0.0891 & 0.0025 & 0.0184 & 0.0011 & & \\
$04 / 07 / 00$ & PM & 21 & 0.9640 & 0.0012 & 0.0371 & 0.0016 & 0.0459 & 0.0006 & 1.0378 & 0.0017 \\
\hline \hline
\end{tabular}

Tabla A1-II. Ajustes KCICLO para el Cimel \#48. Filtro de 670nm. 


\begin{tabular}{lcccccccccc}
\hline \hline Fecha & $\mathbf{8 7 0}$ & $\mathbf{N}$ & $\mathbf{R}^{\mathbf{2}}$ & $\mathbf{S T D}$ & $\mathbf{l n K}$ & error LnK & Tau & error Tau & $\mathbf{K}$ & error K \\
\hline $01 / 03 / 00$ & AM & 22 & 0.9518 & 0.0008 & 0.0217 & 0.0011 & 0.0215 & 0.0004 & 1.0220 & 0.0011 \\
$05 / 03 / 00$ & AM & 21 & 0.9761 & 0.0008 & 0.0314 & 0.0011 & 0.0485 & 0.0004 & 1.0319 & 0.0012 \\
$26 / 03 / 00$ & AM & 19 & 0.9247 & 0.0012 & 0.0236 & 0.0016 & 0.0281 & 0.0007 & 1.0239 & 0.0017 \\
$24 / 04 / 00$ & PM & 19 & 0.9519 & 0.0014 & 0.0342 & 0.0019 & 0.0328 & 0.0007 & 1.0348 & 0.0019 \\
$29 / 05 / 00$ & PM & 22 & 0.9015 & 0.0015 & 0.0245 & 0.0018 & 0.0351 & 0.0007 & 1.0248 & 0.0019 \\
$04 / 06 / 00$ & PM & 20 & 0.9236 & 0.0024 & 0.0451 & 0.0031 & 0.0847 & 0.0012 & 1.0462 & 0.0032 \\
$07 / 06 / 00$ & PM & 19 & 0.9836 & 0.0010 & 0.0416 & 0.0013 & 0.0617 & 0.0005 & 1.0425 & 0.0014 \\
$10 / 06 / 00$ & AM & 17 & 0.9384 & 0.0020 & 0.0376 & 0.0025 & 0.0216 & 0.0019 & 1.0383 & 0.0026 \\
$10 / 06 / 00$ & PM & 24 & 0.7387 & 0.0018 & 0.0124 & 0.0016 & 0.0438 & 0.0009 & 1.0125 & 0.0016 \\
$12 / 06 / 00$ & PM & 18 & 0.8761 & 0.0007 & 0.0104 & 0.0010 & 0.0481 & 0.0004 & 1.0104 & 0.0010 \\
$16 / 06 / 00$ & PM & 20 & 0.8993 & 0.0011 & 0.0191 & 0.0015 & 0.0574 & 0.0006 & 1.0193 & 0.0015 \\
21/06/00 & PM & 17 & 0.8940 & 0.0025 & 0.0472 & 0.0042 & 0.0290 & 0.0034 & 1.0484 & 0.0044 \\
22/06/00 & PM & 19 & 0.6890 & 0.0022 & 0.0152 & 0.0025 & 0.0373 & 0.0012 & 1.0153 & 0.0025 \\
29/06/00 & AM & 19 & 0.9038 & 0.0021 & 0.0349 & 0.0028 & 0.0433 & 0.0022 & 1.0356 & 0.0029 \\
29/06/00 & PM & 15 & 0.9784 & 0.0013 & 0.0556 & 0.0023 & 0.0176 & 0.0010 & 1.0572 & 0.0024 \\
04/07/00 & PM & 21 & 0.8785 & 0.0011 & 0.0178 & 0.0015 & 0.0400 & 0.0006 & 1.0180 & 0.0015 \\
\hline \hline
\end{tabular}

Tabla A1-III. Ajustes KCICLO para el Cimel \#48. Filtro de 870nm.

\begin{tabular}{lcccccccccc}
\hline \hline Fecha & $\mathbf{1 0 2 0}$ & $\mathbf{N}$ & $\mathbf{R}^{\mathbf{2}}$ & $\mathbf{S T D}$ & $\mathbf{l n K}$ & error $\mathbf{L n K}$ & Tau & error Tau & K & error K \\
\hline $01 / 03 / 00$ & AM & 22 & 0.9613 & 0.0011 & 0.0351 & 0.0016 & 0.0243 & 0.0006 & 1.0357 & 0.0016 \\
$05 / 03 / 00$ & AM & 21 & 0.9687 & 0.0010 & 0.0377 & 0.0016 & 0.0478 & 0.0006 & 1.0384 & 0.0016 \\
$26 / 03 / 00$ & AM & 19 & 0.9295 & 0.0014 & 0.0302 & 0.0020 & 0.0321 & 0.0008 & 1.0306 & 0.0021 \\
$24 / 04 / 00$ & PM & 19 & 0.9723 & 0.0012 & 0.0380 & 0.0016 & 0.0347 & 0.0006 & 1.0387 & 0.0016 \\
$29 / 05 / 00$ & PM & 22 & 0.9405 & 0.0012 & 0.0264 & 0.0015 & 0.0383 & 0.0006 & 1.0267 & 0.0015 \\
04/06/00 & PM & 20 & 0.9368 & 0.0024 & 0.0518 & 0.0032 & 0.0825 & 0.0013 & 1.0531 & 0.0033 \\
$07 / 06 / 00$ & PM & 19 & 0.9775 & 0.0011 & 0.0398 & 0.0015 & 0.0614 & 0.0006 & 1.0406 & 0.0015 \\
$10 / 06 / 00$ & AM & 17 & 0.9229 & 0.0022 & 0.0354 & 0.0026 & 0.0310 & 0.0021 & 1.0361 & 0.0027 \\
$10 / 06 / 00$ & PM & 24 & 0.8386 & 0.0022 & 0.0206 & 0.0019 & 0.0456 & 0.0011 & 1.0209 & 0.0020 \\
$12 / 06 / 00$ & PM & 18 & 0.9183 & 0.0008 & 0.0158 & 0.0012 & 0.0462 & 0.0005 & 1.0159 & 0.0012 \\
$16 / 06 / 00$ & PM & 20 & 0.5923 & 0.0030 & 0.0205 & 0.0040 & 0.0580 & 0.0016 & 1.0207 & 0.0041 \\
$21 / 06 / 00$ & PM & 17 & 0.9462 & 0.0020 & 0.0542 & 0.0033 & 0.0342 & 0.0027 & 1.0557 & 0.0035 \\
$22 / 06 / 00$ & PM & 19 & 0.8525 & 0.0024 & 0.0264 & 0.0027 & 0.0409 & 0.0013 & 1.0268 & 0.0027 \\
$29 / 06 / 00$ & AM & 19 & 0.8913 & 0.0023 & 0.0351 & 0.0030 & 0.0500 & 0.0024 & 1.0357 & 0.0031 \\
$29 / 06 / 00$ & PM & 15 & 0.9900 & 0.0010 & 0.0650 & 0.0018 & 0.0216 & 0.0008 & & \\
04/07/00 & PM & 21 & 0.9648 & 0.0011 & 0.0333 & 0.0015 & 0.0409 & 0.0006 & 1.0339 & 0.0015 \\
\hline \hline
\end{tabular}

Tabla A1-IV. Ajustes KCICLO para el Cimel \#48. Filtro de 1020nm. 


\begin{tabular}{|c|c|c|c|c|c|c|c|c|c|c|}
\hline Fecha & 4440 & $\overline{2} \mathbf{N}$ & R2 & $\overline{~ S T D ~}$ & $\ln K$ & error LnK & Tau & error Tau & $\overline{\mathbf{K}}$ & error $K$ \\
\hline$\overline{11 / 07 / 00}$ & AM & 11 & 0.8435 & 0.0013 & 0.0209 & 0.0030 & 0.0445 & 0.0016 & 1.0211 & 0.0031 \\
\hline $13 / 07 / 00$ & $\mathrm{AM}$ & 10 & 0.2218 & 0.0031 & 0.0133 & 0.0094 & 0.1499 & 0.0053 & 1.0134 & 0.0095 \\
\hline $13 / 07 / 00$ & PM & 16 & 0.8601 & 0.0045 & -0.0685 & 0.0074 & 0.2495 & 0.0034 & & \\
\hline 26/07/00 & $\mathrm{PM}$ & 16 & 0.2536 & 0.0023 & 0.0076 & 0.0035 & 0.0471 & 0.0015 & 1.0076 & 0.0035 \\
\hline $14 / 08 / 00$ & $\mathrm{AM}$ & 17 & 0.8259 & 0.0015 & 0.0201 & 0.0024 & 0.0892 & 0.0010 & 1.0203 & 0.0024 \\
\hline $14 / 08 / 00$ & PM & 19 & 0.7849 & 0.0031 & 0.0325 & 0.0041 & 0.1097 & 0.0017 & 1.0330 & 0.0043 \\
\hline $21 / 08 / 00$ & $\mathrm{AM}$ & 21 & 0.8885 & 0.0029 & 0.0463 & 0.0038 & 0.0271 & 0.0015 & 1.0474 & 0.0039 \\
\hline $24 / 08 / 00$ & $\mathrm{AM}$ & 16 & 0.9320 & 0.0022 & 0.0503 & 0.0036 & 0.0213 & 0.0017 & 1.0516 & 0.0038 \\
\hline $24 / 08 / 00$ & PM & 21 & 0.1801 & 0.0015 & 0.0040 & 0.0020 & 0.0477 & 0.0008 & 1.0041 & 0.0020 \\
\hline 07/09/00 & PM & 20 & 0.0017 & 0.0040 & 0.0009 & 0.0054 & 0.2131 & 0.0021 & 1.0009 & 0.0054 \\
\hline 08/09/00 & $\mathrm{PM}$ & 19 & 0.7113 & 0.0026 & 0.0222 & 0.0034 & 0.1949 & 0.0014 & 1.0225 & 0.0035 \\
\hline $21 / 09 / 00$ & $\mathrm{AM}$ & 22 & 0.8558 & 0.0037 & 0.0490 & 0.0045 & 0.0455 & 0.0019 & 1.0502 & 0.0047 \\
\hline 03/10/00 & $\mathrm{AM}$ & 24 & 0.8023 & 0.0031 & 0.0344 & 0.0036 & 0.0477 & 0.0016 & 1.0350 & 0.0038 \\
\hline $21 / 10 / 00$ & PM & 25 & 0.6485 & 0.0040 & 0.0300 & 0.0046 & 0.0524 & 0.0020 & 1.0305 & 0.0047 \\
\hline 01/11/00 & $\mathrm{AM}$ & 24 & 0.4294 & 0.0041 & 0.0209 & 0.0051 & 0.0524 & 0.0022 & 1.0211 & 0.0052 \\
\hline $20 / 01 / 01$ & $\mathrm{AM}$ & 23 & 0.5331 & 0.0040 & 0.0312 & 0.0064 & 0.0460 & 0.0024 & 1.0317 & 0.0066 \\
\hline $30 / 01 / 01$ & $\mathrm{AM}$ & 21 & 0.9557 & 0.0018 & 0.0539 & 0.0027 & 0.0176 & 0.0011 & 1.0554 & 0.0028 \\
\hline $11 / 02 / 01$ & $\mathrm{AM}$ & 15 & 0.7198 & 0.0021 & 0.0304 & 0.0053 & 0.0367 & 0.0028 & 1.0309 & 0.0054 \\
\hline $12 / 02 / 01$ & $\mathrm{AM}$ & 17 & 0.9462 & 0.0027 & 0.0747 & 0.0046 & 0.0117 & 0.0020 & 1.0775 & 0.0050 \\
\hline $13 / 03 / 01$ & $\mathrm{AM}$ & 23 & 0.9731 & 0.0020 & 0.0643 & 0.0023 & 0.0163 & 0.0010 & 1.0664 & 0.0025 \\
\hline $13 / 03 / 01$ & PM & 19 & 0.1873 & 0.0035 & 0.0088 & 0.0044 & 0.0559 & 0.0019 & 1.0088 & 0.0045 \\
\hline $10 / 04 / 01$ & PM & 21 & 0.0397 & 0.0041 & -0.0045 & 0.0050 & 0.1072 & 0.0021 & 0.9955 & 0.0050 \\
\hline $11 / 04 / 01$ & $\mathrm{AM}$ & 20 & 0.9802 & 0.0012 & 0.0435 & 0.0015 & 0.0568 & 0.0006 & 1.0445 & 0.0015 \\
\hline $30 / 05 / 01$ & $\mathrm{AM}$ & 19 & 0.9405 & 0.0030 & 0.0661 & 0.0040 & 0.1107 & 0.0016 & 1.0684 & 0.0043 \\
\hline
\end{tabular}

Tabla A1-v. Ajustes KCICLO para el Cimel \#114. Filtro de 440nm.

\begin{tabular}{|c|c|c|c|c|c|c|c|c|c|c|}
\hline Fecha & (670 & $\overline{\mathbf{N}}$ & R2 & STD & $\operatorname{lnK}$ & error LnK & Tau & error Tau & $\overline{\bar{K}}$ & error $K$ \\
\hline $11 / 07 / 00$ & AM & 11 & 0.8156 & 0.0008 & -0.0120 & 0.0019 & 0.0249 & 0.0010 & 0.9881 & 0.0019 \\
\hline 13/07/00 & $\mathrm{AM}$ & 10 & 0.6251 & 0.0012 & -0.0127 & 0.0037 & 0.0785 & 0.0021 & 0.9874 & 0.0037 \\
\hline $13 / 07 / 00$ & $\mathrm{PM}$ & 16 & 0.9058 & 0.0024 & -0.0451 & 0.0039 & 0.1223 & 0.0018 & & \\
\hline 26/07/00 & $\mathrm{PM}$ & 16 & 0.8278 & 0.0016 & -0.0190 & 0.0023 & 0.0287 & 0.0010 & 0.9812 & 0.0023 \\
\hline $14 / 08 / 00$ & $\mathrm{AM}$ & 17 & 0.5950 & 0.0010 & -0.0078 & 0.0017 & 0.0436 & 0.0007 & 0.9923 & 0.0016 \\
\hline $14 / 08 / 00$ & $\mathrm{PM}$ & 19 & 0.1476 & 0.0018 & 0.0042 & 0.0024 & 0.0574 & 0.0010 & 1.0042 & 0.0024 \\
\hline 21/08/00 & $\mathrm{AM}$ & 21 & 0.3193 & 0.0014 & 0.0053 & 0.0018 & 0.0109 & 0.0007 & 1.0053 & 0.0018 \\
\hline $24 / 08 / 00$ & $\mathrm{AM}$ & 16 & 0.2310 & 0.0017 & 0.0056 & 0.0027 & 0.0097 & 0.0013 & 1.0056 & 0.0027 \\
\hline $24 / 08 / 00$ & $\mathrm{PM}$ & 21 & 0.8617 & 0.0009 & -0.0131 & 0.0012 & 0.0218 & 0.0005 & 0.9870 & 0.0012 \\
\hline 07/09/00 & $\mathrm{PM}$ & 20 & 0.7831 & 0.0018 & -0.0194 & 0.0024 & 0.1071 & 0.0010 & 0.9808 & 0.0024 \\
\hline 08/09/00 & $\mathrm{PM}$ & 19 & 0.7121 & 0.0010 & -0.0090 & 0.0014 & 0.0954 & 0.0006 & 0.9910 & 0.0014 \\
\hline 21/09/00 & $\mathrm{AM}$ & 22 & 0.2330 & 0.0017 & 0.0051 & 0.0021 & 0.0230 & 0.0009 & 1.0051 & 0.0021 \\
\hline 03/10/00 & $\mathrm{AM}$ & 24 & 0.0053 & 0.0020 & -0.0008 & 0.0024 & 0.0181 & 0.0010 & 0.9992 & 0.0023 \\
\hline $21 / 10 / 00$ & $\mathrm{PM}$ & 25 & 0.0880 & 0.0024 & -0.0041 & 0.0028 & 0.0260 & 0.0012 & 0.9959 & 0.0027 \\
\hline 01/11/00 & $\mathrm{AM}$ & 24 & 0.1468 & 0.0034 & -0.0082 & 0.0042 & 0.0370 & 0.0018 & 0.9918 & 0.0042 \\
\hline 20/01/01 & $\mathrm{AM}$ & 23 & 0.1102 & 0.0023 & -0.0060 & 0.0037 & 0.0248 & 0.0014 & 0.9941 & 0.0037 \\
\hline $30 / 01 / 01$ & $\mathrm{AM}$ & 21 & 0.3646 & 0.0011 & 0.0051 & 0.0015 & 0.0080 & 0.0006 & 1.0051 & 0.0016 \\
\hline $11 / 02 / 01$ & $\mathrm{AM}$ & 15 & 0.2605 & 0.0013 & -0.0069 & 0.0032 & 0.0180 & 0.0017 & 0.9931 & 0.0032 \\
\hline $12 / 02 / 01$ & $\mathrm{AM}$ & 17 & 0.5869 & 0.0017 & 0.0131 & 0.0028 & 0.0053 & 0.0012 & 1.0132 & 0.0029 \\
\hline 13/03/01 & $\mathrm{AM}$ & 23 & 0.1671 & 0.0010 & 0.0025 & 0.0012 & 0.0134 & 0.0005 & 1.0025 & 0.0012 \\
\hline 13/03/01 & $\mathrm{PM}$ & 19 & 0.5228 & 0.0030 & -0.0161 & 0.0037 & 0.0275 & 0.0016 & 0.9840 & 0.0037 \\
\hline $10 / 04 / 01$ & $\mathrm{PM}$ & 21 & 0.5592 & 0.0020 & -0.0122 & 0.0025 & 0.0515 & 0.0010 & 0.9878 & 0.0025 \\
\hline $11 / 04 / 01$ & $\mathrm{AM}$ & 20 & 0.0135 & 0.0013 & -0.0008 & 0.0016 & 0.0313 & 0.0007 & 0.9992 & 0.0016 \\
\hline $30 / 05 / 01$ & $\mathrm{AM}$ & 19 & 0.1596 & 0.0017 & 0.0041 & 0.0023 & 0.0664 & 0.0009 & 1.0041 & 0.0023 \\
\hline
\end{tabular}

Tabla A1-VI. Ajustes KCICLO para el Cimel \#114. Filtro de 670nm. 


\begin{tabular}{|c|c|c|c|c|c|c|c|c|c|c|}
\hline Fecha & 870 & 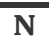 & R2 & 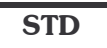 & $\bar{l} \operatorname{lnK}$ & error LnK & Tau & error Tau & $\overline{\mathrm{K}}$ & error $K$ \\
\hline $11 / 07 / 00$ & $\mathrm{AM}$ & 11 & 0.9767 & 0.0008 & -0.0387 & 0.0020 & 0.0228 & 0.0011 & 0.9621 & 0.0019 \\
\hline $13 / 07 / 00$ & $\mathrm{AM}$ & 10 & 0.9649 & 0.0011 & -0.0441 & 0.0032 & 0.0629 & 0.0018 & 0.9569 & 0.0030 \\
\hline $13 / 07 / 00$ & $\mathrm{PM}$ & 16 & 0.9683 & 0.0018 & -0.0625 & 0.0030 & 0.0913 & 0.0014 & 0.9394 & 0.0028 \\
\hline $26 / 07 / 00$ & $\mathrm{PM}$ & 16 & 0.9721 & 0.0015 & -0.0481 & 0.0022 & 0.0273 & 0.0009 & 0.9531 & 0.0021 \\
\hline $14 / 08 / 00$ & $\mathrm{AM}$ & 17 & 0.9858 & 0.0009 & -0.0456 & 0.0014 & 0.0343 & 0.0006 & 0.9554 & 0.0013 \\
\hline $14 / 08 / 00$ & $\mathrm{PM}$ & 19 & 0.9465 & 0.0014 & -0.0336 & 0.0019 & 0.0459 & 0.0008 & 0.9669 & 0.0019 \\
\hline $21 / 08 / 00$ & $\mathrm{AM}$ & 21 & 0.9748 & 0.0009 & -0.0327 & 0.0012 & 0.0107 & 0.0005 & 0.9679 & 0.0012 \\
\hline $24 / 08 / 00$ & $\mathrm{AM}$ & 16 & 0.9533 & 0.0013 & -0.0353 & 0.0021 & 0.0119 & 0.0010 & 0.9653 & 0.0020 \\
\hline $24 / 08 / 00$ & $\mathrm{PM}$ & 21 & 0.9898 & 0.0007 & -0.0427 & 0.0010 & 0.0183 & 0.0004 & 0.9582 & 0.0010 \\
\hline 07/09/00 & $\mathrm{PM}$ & 20 & 0.9881 & 0.0011 & -0.0578 & 0.0015 & 0.0798 & 0.0006 & 0.9438 & 0.0014 \\
\hline 08/09/00 & $\mathrm{PM}$ & 19 & 0.9931 & 0.0007 & -0.0491 & 0.0010 & 0.0699 & 0.0004 & 0.9520 & 0.0009 \\
\hline 21/09/00 & $\mathrm{AM}$ & 22 & 0.9623 & 0.0013 & -0.0360 & 0.0016 & 0.0230 & 0.0007 & 0.9647 & 0.0015 \\
\hline 03/10/00 & $\mathrm{AM}$ & 24 & 0.9556 & 0.0016 & -0.0407 & 0.0019 & 0.0160 & 0.0008 & 0.9601 & 0.0018 \\
\hline $21 / 10 / 00$ & $\mathrm{PM}$ & 25 & 0.9312 & 0.0017 & -0.0358 & 0.0020 & 0.0210 & 0.0009 & 0.9649 & 0.0020 \\
\hline 01/11/00 & $\mathrm{AM}$ & 24 & 0.8603 & 0.0031 & -0.0442 & 0.0038 & 0.0389 & 0.0016 & 0.9568 & 0.0036 \\
\hline 20/01/01 & $\mathrm{AM}$ & 23 & 0.9110 & 0.0018 & -0.0412 & 0.0028 & 0.0247 & 0.0011 & 0.9597 & 0.0027 \\
\hline $30 / 01 / 01$ & $\mathrm{AM}$ & 21 & 0.9762 & 0.0008 & -0.0338 & 0.0012 & 0.0117 & 0.0005 & 0.9668 & 0.0012 \\
\hline $11 / 02 / 01$ & $\mathrm{AM}$ & 15 & 0.9729 & 0.0008 & -0.0424 & 0.0020 & 0.0177 & 0.0010 & 0.9585 & 0.0019 \\
\hline $12 / 02 / 01$ & $\mathrm{AM}$ & 17 & 0.9517 & 0.0010 & -0.0289 & 0.0017 & 0.0100 & 0.0007 & 0.9715 & 0.0016 \\
\hline $13 / 03 / 01$ & $\mathrm{AM}$ & 23 & 0.9854 & 0.0008 & -0.0359 & 0.0010 & 0.0155 & 0.0004 & 0.9647 & 0.0009 \\
\hline $13 / 03 / 01$ & $\mathrm{PM}$ & 19 & 0.9407 & 0.0023 & -0.0463 & 0.0028 & 0.0215 & 0.0012 & 0.9547 & 0.0027 \\
\hline $10 / 04 / 01$ & $\mathrm{PM}$ & 21 & 0.9595 & 0.0017 & -0.0439 & 0.0021 & 0.0395 & 0.0009 & 0.9570 & 0.0020 \\
\hline $11 / 04 / 01$ & $\mathrm{AM}$ & 20 & 0.9771 & 0.0011 & -0.0387 & 0.0014 & 0.0260 & 0.0006 & 0.9620 & 0.0013 \\
\hline $30 / 05 / 01$ & $\mathrm{AM}$ & 19 & 0.9733 & 0.0012 & -0.0409 & 0.0016 & 0.0542 & 0.0006 & 0.9599 & 0.0016 \\
\hline
\end{tabular}

Tabla A1-VII. $\quad$ Ajustes KCICLO para el Cimel \#114. Filtro de 870nm.

\begin{tabular}{|c|c|c|c|c|c|c|c|c|c|c|}
\hline Fecha & 1020 & $\overline{\mathbf{N}}$ & R2 & STD & $\overline{l n K}$ & error LnK & $\overline{\text { Tau }}$ & error Tau & $\bar{K}$ & error $K$ \\
\hline$\overline{11 / 07 / 00}$ & AM & 11 & 0.9878 & 0.0008 & -0.0513 & 0.0019 & 0.0270 & 0.0010 & 0.9500 & 0.0018 \\
\hline $13 / 07 / 00$ & $\mathrm{AM}$ & 10 & 0.9801 & 0.0010 & -0.0557 & 0.0030 & 0.0621 & 0.0017 & 0.9458 & 0.0028 \\
\hline $13 / 07 / 00$ & PM & 16 & 0.9701 & 0.0020 & -0.0707 & 0.0033 & 0.0856 & 0.0015 & 0.9317 & 0.0031 \\
\hline $26 / 07 / 00$ & PM & 16 & 0.9806 & 0.0015 & -0.0583 & 0.0022 & 0.0295 & 0.0010 & 0.9434 & 0.0021 \\
\hline $14 / 08 / 00$ & $\mathrm{AM}$ & 17 & 0.9816 & 0.0013 & -0.0588 & 0.0021 & 0.0372 & 0.0009 & 0.9429 & 0.0020 \\
\hline $14 / 08 / 00$ & $\mathrm{PM}$ & 19 & 0.9718 & 0.0013 & -0.0436 & 0.0018 & 0.0460 & 0.0007 & 0.9573 & 0.0017 \\
\hline $21 / 08 / 00$ & AM & 21 & 0.9827 & 0.0010 & -0.0437 & 0.0013 & 0.0137 & 0.0005 & 0.9573 & 0.0013 \\
\hline $24 / 08 / 00$ & $\mathrm{AM}$ & 16 & 0.9737 & 0.0013 & -0.0484 & 0.0021 & 0.0175 & 0.0010 & 0.9527 & 0.0020 \\
\hline $24 / 08 / 00$ & PM & 21 & 0.9926 & 0.0008 & -0.0523 & 0.0010 & 0.0212 & 0.0004 & 0.9491 & 0.0010 \\
\hline 07/09/00 & PM & 20 & 0.9927 & 0.0010 & -0.0684 & 0.0014 & 0.0720 & 0.0006 & 0.9339 & 0.0013 \\
\hline 08/09/00 & PM & 19 & 0.9961 & 0.0007 & -0.0614 & 0.0009 & 0.0633 & 0.0004 & 0.9404 & 0.0009 \\
\hline $21 / 09 / 00$ & $\mathrm{AM}$ & 22 & 0.9840 & 0.0011 & -0.0481 & 0.0014 & 0.0262 & 0.0006 & 0.9530 & 0.0013 \\
\hline 03/10/00 & $\mathrm{AM}$ & 24 & 0.9598 & 0.0020 & -0.0522 & 0.0023 & 0.0169 & 0.0010 & 0.9492 & 0.0022 \\
\hline $21 / 10 / 00$ & PM & 25 & 0.9662 & 0.0014 & -0.0428 & 0.0017 & 0.0208 & 0.0007 & 0.9581 & 0.0016 \\
\hline 01/11/00 & $\mathrm{AM}$ & 24 & 0.9066 & 0.0029 & -0.0527 & 0.0036 & 0.0413 & 0.0015 & 0.9487 & 0.0034 \\
\hline 20/01/01 & AM & 23 & 0.9656 & 0.0013 & -0.0501 & 0.0021 & 0.0269 & 0.0008 & 0.9511 & 0.0020 \\
\hline $30 / 01 / 01$ & AM & 21 & 0.9846 & 0.0009 & -0.0438 & 0.0013 & 0.0157 & 0.0005 & 0.9571 & 0.0012 \\
\hline $11 / 02 / 01$ & $\mathrm{AM}$ & 15 & 0.9807 & 0.0009 & -0.0549 & 0.0021 & 0.0215 & 0.0011 & 0.9466 & 0.0020 \\
\hline $12 / 02 / 01$ & $\mathrm{AM}$ & 17 & 0.9896 & 0.0007 & -0.0424 & 0.0011 & 0.0144 & 0.0005 & 0.9585 & 0.0011 \\
\hline $13 / 03 / 01$ & $\mathrm{AM}$ & 23 & 0.9390 & 0.0017 & -0.0371 & 0.0021 & 0.0115 & 0.0009 & 0.9636 & 0.0020 \\
\hline $13 / 03 / 01$ & $\mathrm{PM}$ & 19 & 0.9137 & 0.0033 & -0.0556 & 0.0041 & 0.0215 & 0.0018 & 0.9459 & 0.0039 \\
\hline $10 / 04 / 01$ & PM & 21 & 0.9798 & 0.0032 & -0.1203 & 0.0040 & 0.0382 & 0.0017 & $*$ & \\
\hline $11 / 04 / 01$ & AM & 20 & 0.9982 & 0.0001 & -0.1099 & 0.0027 & 0.0300 & 0.0005 & $*$ & \\
\hline $30 / 05 / 01$ & $\mathrm{AM}$ & 19 & 0.9918 & 0.0021 & -0.1278 & 0.0028 & 0.0588 & 0.0011 & $*$ & \\
\hline
\end{tabular}

Tabla A1-VIII. Ajustes KCICLO para el Cimel \#114. Filtro de 1020nm.

*Algunos de los ajustes del filtro de $1020 \mathrm{~nm}$ del final del período han sido desechados por un problema en la corrección por temperatura en ese filtro. El Cimel no registró correctamente los datos de temperatura de esos días, y por tanto la señal en el canal de $1020 \mathrm{~nm}$ no se puede corregir adecuadamente. 


\begin{tabular}{lcccccccccc}
\hline \hline Fecha & $\mathbf{4 4 0}$ & $\mathbf{N}$ & $\mathbf{R 2}$ & $\mathbf{S T D}$ & $\mathbf{l n K}$ & error LnK & Tau & error Tau & K & error K \\
\hline $24 / 10 / 01$ & AM & 20 & 0.3192 & 0.0020 & 0.0101 & 0.0035 & 0.0538 & 0.0015 & 1.0101 & 0.0035 \\
$24 / 10 / 01$ & PM & 25 & 0.0321 & 0.0044 & -0.0055 & 0.0063 & 0.0895 & 0.0024 & 0.9945 & 0.0063 \\
$10 / 11 / 01$ & PM & 16 & 0.5012 & 0.0015 & 0.0206 & 0.0055 & 0.0491 & 0.0015 & 1.0208 & 0.0056 \\
$13 / 11 / 01$ & AM & 24 & 0.0054 & 0.0037 & 0.0018 & 0.0052 & 0.0583 & 0.0020 & 1.0018 & 0.0052 \\
$28 / 11 / 01$ & AM & 26 & 0.1227 & 0.0034 & -0.0095 & 0.0052 & 0.1051 & 0.0020 & 0.9905 & 0.0051 \\
$04 / 12 / 01$ & AM & 24 & 0.0305 & 0.0047 & -0.0069 & 0.0083 & 0.2055 & 0.0032 & 0.9931 & 0.0082 \\
$27 / 01 / 02$ & AM & 15 & 0.1167 & 0.0023 & -0.0092 & 0.0070 & 0.0726 & 0.0033 & 0.9908 & 0.0070 \\
$24 / 02 / 02$ & AM & 28 & 0.0000 & 0.0028 & 0.0000 & 0.0031 & 0.0651 & 0.0014 & 1.0000 & 0.0031 \\
$02 / 03 / 02$ & AM & 28 & 0.0051 & 0.0028 & -0.0011 & 0.0029 & 0.0699 & 0.0014 & 0.9989 & 0.0029 \\
$14 / 05 / 02$ & AM & 17 & 0.8274 & 0.0028 & 0.0372 & 0.0044 & 0.0855 & 0.0019 & 1.0379 & 0.0045 \\
$10 / 09 / 02$ & AM & 14 & 0.4669 & 0.0020 & 0.0235 & 0.0073 & 0.0468 & 0.0031 & 1.0238 & 0.0074 \\
$10 / 09 / 02$ & PM & 21 & 0.8814 & 0.0031 & 0.0490 & 0.0041 & 0.0168 & 0.0016 & 1.0502 & 0.0043 \\
25/10/02 & AM & 30 & 0.3486 & 0.0027 & 0.0121 & 0.0031 & 0.0616 & 0.0016 & 1.0122 & 0.0032 \\
26/10/02 & PM & 13 & 0.2467 & 0.0019 & 0.0124 & 0.0065 & 0.0643 & 0.0029 & 1.0125 & 0.0066 \\
$06 / 12 / 02$ & PM & 28 & 0.6584 & 0.0014 & 0.0154 & 0.0022 & 0.0434 & 0.0009 & 1.0155 & 0.0022 \\
$13 / 01 / 03$ & AM & 14 & 0.0320 & 0.0027 & 0.0063 & 0.0100 & 0.0541 & 0.0028 & 1.0063 & 0.0100 \\
\hline \hline
\end{tabular}

Tabla A1-IX. Ajustes KCICLO para el Cimel \#45. Filtro de 440nm.

\begin{tabular}{ccccccccccc}
\hline \hline Fecha & $\mathbf{6 7 0}$ & $\mathbf{N}$ & $\mathbf{R 2}$ & $\mathbf{S T D}$ & $\mathbf{l n K}$ & error LnK & Tau & error Tau & $\mathbf{K}$ & error K \\
\hline $24 / 10 / 01$ & AM & 20 & 0.9758 & 0.0009 & 0.0404 & 0.0015 & 0.0248 & 0.0006 & 1.0412 & 0.0016 \\
$24 / 10 / 01$ & PM & 25 & 0.7475 & 0.0023 & 0.0276 & 0.0033 & 0.0480 & 0.0013 & 1.0280 & 0.0034 \\
$10 / 11 / 01$ & PM & 16 & 0.9150 & 0.0006 & 0.0263 & 0.0021 & 0.0325 & 0.0006 & 1.0267 & 0.0022 \\
$13 / 11 / 01$ & AM & 24 & 0.8445 & 0.0022 & 0.0342 & 0.0031 & 0.0230 & 0.0012 & 1.0347 & 0.0032 \\
$28 / 11 / 01$ & AM & 26 & 0.8004 & 0.0018 & 0.0274 & 0.0028 & 0.0456 & 0.0011 & 1.0278 & 0.0029 \\
$04 / 12 / 01$ & AM & 24 & 0.2847 & 0.0023 & 0.0120 & 0.0041 & 0.1143 & 0.0016 & 1.0121 & 0.0041 \\
$27 / 01 / 02$ & AM & 15 & 0.8704 & 0.0011 & 0.0304 & 0.0033 & 0.0302 & 0.0015 & 1.0309 & 0.0034 \\
$24 / 02 / 02$ & AM & 28 & 0.8696 & 0.0023 & 0.0325 & 0.0025 & 0.0292 & 0.0011 & 1.0330 & 0.0025 \\
$02 / 03 / 02$ & AM & 28 & 0.9163 & 0.0019 & 0.0331 & 0.0020 & 0.0309 & 0.0009 & 1.0337 & 0.0020 \\
$14 / 05 / 02$ & AM & 17 & 0.9669 & 0.0018 & 0.0609 & 0.0029 & 0.0451 & 0.0013 & 1.0627 & 0.0031 \\
$10 / 09 / 02$ & AM & 14 & 0.9671 & 0.0010 & 0.0666 & 0.0035 & 0.0208 & 0.0015 & 1.0689 & 0.0038 \\
$10 / 09 / 02$ & PM & 21 & 0.9855 & 0.0016 & 0.0756 & 0.0021 & 0.0071 & 0.0008 & & \\
$25 / 10 / 02$ & AM & 30 & 0.9792 & 0.0016 & 0.0668 & 0.0018 & 0.0280 & 0.0010 & 1.0691 & 0.0020 \\
$26 / 10 / 02$ & PM & 13 & 0.9798 & 0.0008 & 0.0640 & 0.0028 & 0.0294 & 0.0012 & 1.0661 & 0.0030 \\
$06 / 12 / 02$ & PM & 28 & 0.9907 & 0.0008 & 0.0663 & 0.0013 & 0.0191 & 0.0005 & 1.0686 & 0.0013 \\
$10 / 01 / 03$ & PM & 19 & 0.8636 & 0.0021 & 0.0535 & 0.0052 & 0.0305 & 0.0017 & 1.0549 & 0.0054 \\
$12 / 01 / 03$ & PM & 14 & 0.9761 & 0.0010 & 0.0558 & 0.0025 & 0.0287 & 0.0010 & 1.0573 & 0.0027 \\
$13 / 01 / 03$ & AM & 14 & 0.8929 & 0.0014 & 0.0540 & 0.0054 & 0.0233 & 0.0015 & 1.0555 & 0.0057 \\
$15 / 01 / 03$ & PM & 19 & 0.8622 & 0.0023 & 0.0528 & 0.0051 & 0.0507 & 0.0017 & 1.0542 & 0.0054 \\
\hline \hline
\end{tabular}

Tabla A1-X. Ajustes KCICLO para el Cimel \#45. Filtro de 670nm. 


\begin{tabular}{lcccccccccc}
\hline Fecha & $\mathbf{8 7 0}$ & $\mathbf{N}$ & $\mathbf{R 2}$ & STD & lnK & error LnK & Tau & error Tau & K & error K \\
\hline $24 / 10 / 01$ & AM & 20 & 0.5916 & 0.0009 & 0.0081 & 0.0016 & 0.0233 & 0.0007 & 1.0081 & 0.0016 \\
$24 / 10 / 01$ & PM & 25 & 0.0035 & 0.0016 & 0.0006 & 0.0022 & 0.0402 & 0.0009 & 1.0006 & 0.0022 \\
$10 / 11 / 01$ & PM & 16 & 0.2806 & 0.0006 & -0.0049 & 0.0021 & 0.0292 & 0.0006 & 0.9951 & 0.0021 \\
$13 / 11 / 01$ & AM & 24 & 0.1977 & 0.0014 & 0.0046 & 0.0020 & 0.0206 & 0.0008 & 1.0046 & 0.0020 \\
$28 / 11 / 01$ & AM & 26 & 0.3154 & 0.0009 & 0.0047 & 0.0014 & 0.0302 & 0.0005 & 1.0047 & 0.0014 \\
$04 / 12 / 01$ & AM & 24 & 0.6233 & 0.0011 & -0.0122 & 0.0020 & 0.0845 & 0.0008 & 0.9879 & 0.0020 \\
$27 / 01 / 02$ & AM & 15 & 0.0047 & 0.0008 & -0.0006 & 0.0023 & 0.0250 & 0.0011 & 0.9994 & 0.0023 \\
$24 / 02 / 02$ & AM & 28 & 0.0551 & 0.0014 & -0.0019 & 0.0016 & 0.0266 & 0.0007 & 0.9981 & 0.0016 \\
$02 / 03 / 02$ & AM & 28 & 0.1751 & 0.0015 & 0.0037 & 0.0016 & 0.0258 & 0.0007 & 1.0037 & 0.0016 \\
$14 / 05 / 02$ & AM & 17 & 0.7593 & 0.0015 & 0.0161 & 0.0023 & 0.0384 & 0.0010 & 1.0162 & 0.0024 \\
$10 / 09 / 02$ & AM & 14 & 0.4835 & 0.0008 & 0.0093 & 0.0028 & 0.0195 & 0.0012 & 1.0094 & 0.0028 \\
$10 / 09 / 02$ & PM & 21 & 0.8758 & 0.0012 & 0.0190 & 0.0016 & 0.0078 & 0.0006 & 1.0191 & 0.0017 \\
$25 / 10 / 02$ & AM & 30 & 0.3793 & 0.0011 & 0.0054 & 0.0013 & 0.0268 & 0.0007 & 1.0054 & 0.0013 \\
$26 / 10 / 02$ & PM & 13 & 0.4152 & 0.0007 & 0.0066 & 0.0024 & 0.0236 & 0.0011 & 1.0066 & 0.0024 \\
$06 / 12 / 02$ & PM & 28 & 0.7621 & 0.0006 & 0.0091 & 0.0010 & 0.0170 & 0.0004 & 1.0092 & 0.0010 \\
$13 / 01 / 03$ & AM & 14 & 0.1963 & 0.0009 & 0.0055 & 0.0032 & 0.0155 & 0.0009 & 1.0055 & 0.0032 \\
\hline \hline
\end{tabular}

Tabla A1-XI. $\quad$ Ajustes KCICLO para el Cimel \#45. Filtro de 870nm.

\begin{tabular}{lcccccccccc}
\hline \hline Fecha & $\mathbf{1 0 2 0}$ & $\mathbf{N}$ & R2 & STD & lnK & error LnK & Tau & error Tau & K & error K \\
\hline $24 / 10 / 01$ & AM & 20 & 0.7426 & 0.0011 & 0.0132 & 0.0018 & 0.0243 & 0.0008 & 1.0133 & 0.0019 \\
$24 / 10 / 01$ & PM & 25 & 0.4635 & 0.0014 & 0.0090 & 0.0020 & 0.0378 & 0.0008 & 1.0090 & 0.0020 \\
$10 / 11 / 01$ & PM & 16 & 0.3259 & 0.0006 & 0.0057 & 0.0022 & 0.0284 & 0.0006 & 1.0057 & 0.0022 \\
$13 / 11 / 01$ & AM & 24 & 0.6454 & 0.0014 & 0.0130 & 0.0020 & 0.0215 & 0.0008 & 1.0130 & 0.0021 \\
$28 / 11 / 01$ & AM & 26 & 0.8675 & 0.0007 & 0.0142 & 0.0011 & 0.0266 & 0.0004 & 1.0143 & 0.0012 \\
$04 / 12 / 01$ & AM & 24 & 0.2044 & 0.0010 & -0.0043 & 0.0018 & 0.0747 & 0.0007 & 0.9957 & 0.0018 \\
$27 / 01 / 02$ & AM & 15 & 0.5246 & 0.0006 & 0.0070 & 0.0019 & 0.0238 & 0.0009 & 1.0070 & 0.0019 \\
$24 / 02 / 02$ & AM & 28 & 0.2874 & 0.0012 & 0.0042 & 0.0013 & 0.0261 & 0.0006 & 1.0042 & 0.0013 \\
$02 / 03 / 02$ & AM & 28 & 0.6484 & 0.0016 & 0.0115 & 0.0017 & 0.0257 & 0.0008 & 1.0115 & 0.0017 \\
$14 / 05 / 02$ & AM & 17 & 0.7586 & 0.0015 & 0.0166 & 0.0024 & 0.0374 & 0.0010 & 1.0167 & 0.0025 \\
$10 / 09 / 02$ & AM & 14 & 0.7190 & 0.0007 & 0.0132 & 0.0024 & 0.0238 & 0.0010 & 1.0133 & 0.0024 \\
$10 / 09 / 02$ & PM & 21 & 0.9130 & 0.0012 & 0.0225 & 0.0016 & 0.0123 & 0.0006 & 1.0228 & 0.0016 \\
$25 / 10 / 02$ & AM & 30 & 0.7888 & 0.0010 & 0.0115 & 0.0011 & 0.0295 & 0.0006 & 1.0116 & 0.0011 \\
$26 / 10 / 02$ & PM & 13 & 0.6469 & 0.0009 & 0.0138 & 0.0031 & 0.0229 & 0.0014 & 1.0139 & 0.0031 \\
$06 / 12 / 02$ & PM & 28 & 0.9389 & 0.0007 & 0.0208 & 0.0010 & 0.0167 & 0.0004 & 1.0211 & 0.0011 \\
$13 / 01 / 03$ & AM & 14 & 0.8027 & 0.0008 & 0.0205 & 0.0029 & 0.0146 & 0.0008 & 1.0207 & 0.0030 \\
\hline \hline
\end{tabular}

Tabla A1-XII. Ajustes KCICLO para el Cimel \#45. Filtro de 1020nm. 


\begin{tabular}{lcccccccccc}
\hline \hline Fecha & $\mathbf{4 4 0}$ & $\mathbf{N}$ & $\mathbf{R 2}$ & $\mathbf{S T D}$ & $\mathbf{l n K}$ & error LnK & Tau & error Tau & K & error $\mathbf{K}$ \\
\hline $01 / 02 / 03$ & AM & 14 & 0.7703 & 0.0015 & 0.0234 & 0.0012 & 0.0269 & 0.0037 & 1.0237 & 0.0012 \\
$01 / 02 / 03$ & PM & 22 & 0.4373 & 0.0017 & 0.0116 & 0.0011 & 0.0474 & 0.0029 & 1.0117 & 0.0011 \\
$02 / 02 / 03$ & AM & 19 & 0.8989 & 0.0020 & 0.0356 & 0.0012 & 0.0232 & 0.0029 & 1.0362 & 0.0012 \\
$27 / 06 / 03$ & AM & 13 & 0.8802 & 0.0019 & 0.0262 & 0.0013 & 0.0866 & 0.0029 & 1.0265 & 0.0013 \\
$16 / 07 / 03$ & PM & 13 & 0.6644 & 0.0013 & 0.0120 & 0.0011 & 0.0548 & 0.0026 & 1.0121 & 0.0011 \\
25/07/03 & AM & 18 & 0.8475 & 0.0017 & 0.0247 & 0.0011 & 0.0756 & 0.0026 & 1.0250 & 0.0011 \\
$27 / 07 / 03$ & PM & 18 & 0.9638 & 0.0019 & 0.0561 & 0.0011 & 0.0836 & 0.0027 & 1.0577 & 0.0012 \\
$28 / 07 / 03$ & AM & 18 & 0.8649 & 0.0018 & 0.0294 & 0.0011 & 0.0424 & 0.0029 & 1.0298 & 0.0012 \\
$16 / 08 / 03$ & AM & 14 & 0.9825 & 0.0018 & 0.0791 & 0.0013 & 0.0568 & 0.0031 & & \\
$02 / 12 / 03$ & AM & 15 & 0.9002 & 0.0019 & 0.0568 & 0.0015 & 0.0255 & 0.0052 & 1.0585 & 0.0016 \\
$12 / 12 / 03$ & PM & 17 & 0.9667 & 0.0012 & 0.0589 & 0.0009 & 0.0168 & 0.0028 & 1.0607 & 0.0009 \\
$13 / 12 / 03$ & AM & 14 & 0.8041 & 0.0015 & 0.0236 & 0.0012 & 0.0564 & 0.0034 & 1.0239 & 0.0012 \\
$04 / 01 / 04$ & PM & 16 & 0.4183 & 0.0020 & 0.0116 & 0.0014 & 0.0301 & 0.0037 & 1.0117 & 0.0014 \\
$05 / 01 / 04$ & AM & 15 & 0.8848 & 0.0019 & 0.0526 & 0.0020 & 0.0293 & 0.0053 & 1.0540 & 0.0021 \\
$12 / 01 / 04$ & AM & 17 & 0.9050 & 0.0020 & 0.0547 & 0.0016 & 0.0303 & 0.0046 & 1.0562 & 0.0017 \\
$19 / 01 / 04$ & PM & 18 & 0.8889 & 0.0020 & 0.0371 & 0.0012 & 0.0459 & 0.0033 & 1.0378 & 0.0012 \\
$02 / 03 / 04$ & AM & 17 & 0.9692 & 0.0019 & 0.0685 & 0.0014 & 0.0332 & 0.0032 & 1.0709 & 0.0014 \\
$23 / 03 / 04$ & AM & 14 & 0.9688 & 0.0017 & 0.0479 & 0.0012 & 0.0512 & 0.0025 & 1.0491 & 0.0013 \\
$06 / 04 / 04$ & AM & 18 & 0.8323 & 0.0020 & 0.0256 & 0.0011 & 0.1051 & 0.0029 & 1.0260 & 0.0011 \\
$25 / 04 / 04$ & PM & 19 & 0.9085 & 0.0018 & 0.0351 & 0.0010 & 0.0724 & 0.0027 & 1.0358 & 0.0010 \\
$26 / 04 / 04$ & AM & 20 & 0.9801 & 0.0013 & 0.0504 & 0.0007 & 0.0634 & 0.0017 & 1.0517 & 0.0007 \\
$27 / 04 / 04$ & AM & 15 & 0.9460 & 0.0020 & 0.0469 & 0.0013 & 0.1029 & 0.0031 & 1.0480 & 0.0013 \\
$08 / 05 / 04$ & PM & 13 & 0.9773 & 0.0019 & 0.0735 & 0.0011 & 0.0942 & 0.0034 & 1.0763 & 0.0012 \\
\hline \hline Tabla A1 & & & & & & & &
\end{tabular}

Tabla A1-XIII. Ajustes KCICLO para el Cimel \#50. Filtro de 440nm.

\begin{tabular}{lcccccccccc}
\hline \hline Fecha & $\mathbf{6 7 0}$ & $\mathbf{N}$ & $\mathbf{R 2}$ & $\mathbf{S T D}$ & $\mathbf{l n K}$ & error LnK & Tau & error Tau & $\mathbf{K}$ & error $\mathbf{K}$ \\
\hline $01 / 02 / 03$ & AM & 14 & 0.0355 & 0.0010 & 0.0017 & 0.0008 & 0.0144 & 0.0026 & 1.0017 & 0.0008 \\
$01 / 02 / 03$ & PM & 22 & 0.4412 & 0.0009 & -0.0062 & 0.0006 & 0.0252 & 0.0016 & 0.9938 & 0.0006 \\
$02 / 02 / 03$ & AM & 19 & 0.3502 & 0.0025 & 0.0111 & 0.0015 & 0.0099 & 0.0037 & 1.0111 & 0.0015 \\
$27 / 06 / 03$ & AM & 13 & 0.6887 & 0.0010 & 0.0080 & 0.0007 & 0.0447 & 0.0016 & 1.0081 & 0.0007 \\
$16 / 07 / 03$ & PM & 13 & 0.5292 & 0.0009 & 0.0062 & 0.0007 & 0.0320 & 0.0018 & 1.0062 & 0.0007 \\
$25 / 07 / 03$ & AM & 18 & 0.8313 & 0.0010 & 0.0140 & 0.0006 & 0.0365 & 0.0016 & 1.0141 & 0.0007 \\
$27 / 07 / 03$ & PM & 18 & 0.9431 & 0.0010 & 0.0238 & 0.0006 & 0.0402 & 0.0015 & 1.0241 & 0.0006 \\
$28 / 07 / 03$ & AM & 18 & 0.8187 & 0.0014 & 0.0189 & 0.0009 & 0.0172 & 0.0022 & 1.0191 & 0.0009 \\
$16 / 08 / 03$ & AM & 14 & 0.9661 & 0.0015 & 0.0456 & 0.0010 & 0.0269 & 0.0025 & 1.0467 & 0.0011 \\
$02 / 12 / 03$ & AM & 15 & 0.7160 & 0.0011 & 0.0164 & 0.0008 & 0.0176 & 0.0029 & 1.0166 & 0.0009 \\
$12 / 12 / 03$ & PM & 17 & 0.9586 & 0.0005 & 0.0229 & 0.0004 & 0.0068 & 0.0012 & 1.0231 & 0.0004 \\
$13 / 12 / 03$ & AM & 14 & 0.8470 & 0.0007 & 0.0119 & 0.0005 & 0.0222 & 0.0015 & 1.0120 & 0.0005 \\
$04 / 01 / 04$ & PM & 16 & 0.0998 & 0.0009 & 0.0021 & 0.0006 & 0.0110 & 0.0017 & 1.0021 & 0.0006 \\
$05 / 01 / 04$ & AM & 15 & 0.7960 & 0.0010 & 0.0192 & 0.0010 & 0.0100 & 0.0027 & 1.0194 & 0.0010 \\
$12 / 01 / 04$ & AM & 17 & 0.8683 & 0.0013 & 0.0292 & 0.0010 & 0.0091 & 0.0029 & 1.0296 & 0.0011 \\
$19 / 01 / 04$ & PM & 18 & 0.8250 & 0.0008 & 0.0122 & 0.0005 & 0.0234 & 0.0014 & 1.0123 & 0.0005 \\
$02 / 03 / 04$ & AM & 17 & 0.9023 & 0.0011 & 0.0220 & 0.0008 & 0.0101 & 0.0019 & 1.0222 & 0.0008 \\
23/03/04 & AM & 14 & 0.8632 & 0.0017 & 0.0223 & 0.0012 & 0.0231 & 0.0026 & 1.0226 & 0.0013 \\
$06 / 04 / 04$ & AM & 18 & 0.6404 & 0.0016 & 0.0126 & 0.0009 & 0.0608 & 0.0024 & 1.0127 & 0.0009 \\
$25 / 04 / 04$ & PM & 19 & 0.9516 & 0.0008 & 0.0223 & 0.0004 & 0.0358 & 0.0012 & 1.0225 & 0.0005 \\
$26 / 04 / 04$ & AM & 20 & 0.9673 & 0.0008 & 0.0263 & 0.0004 & 0.0335 & 0.0011 & 1.0267 & 0.0005 \\
$27 / 04 / 04$ & AM & 15 & 0.9455 & 0.0011 & 0.0267 & 0.0007 & 0.0571 & 0.0018 & 1.0271 & 0.0007 \\
$08 / 05 / 04$ & PM & 13 & 0.9672 & 0.0012 & 0.0365 & 0.0006 & 0.0553 & 0.0020 & 1.0372 & 0.0007 \\
\hline \hline Tabla A1 & & & & & & & & &
\end{tabular}

Tabla A1-XIV. Ajustes KCICLO para el Cimel \#50. Filtro de 670nm. 


\begin{tabular}{|c|c|c|c|c|c|c|c|c|c|c|}
\hline Fecha & 870 & $\mathbf{N}$ & R2 & 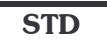 & $\overline{l n K}$ & error LnK & Tau & error Tau & $\overline{\mathrm{K}}$ & error $K$ \\
\hline $01 / 02 / 03$ & $\mathrm{AM}$ & 14 & 0.4429 & 0.0011 & -0.0090 & 0.0009 & 0.0145 & 0.0029 & 0.9911 & 0.0009 \\
\hline 01/02/03 & PM & 22 & 0.5424 & 0.0010 & -0.0089 & 0.0007 & 0.0212 & 0.0018 & 0.9912 & 0.0007 \\
\hline 02/02/03 & $\mathrm{AM}$ & 19 & 0.1936 & 0.0014 & 0.0041 & 0.0008 & 0.0100 & 0.0020 & 1.0041 & 0.0009 \\
\hline 27/06/03 & $\mathrm{AM}$ & 13 & 0.8800 & 0.0010 & -0.0142 & 0.0007 & 0.0361 & 0.0016 & 0.9859 & 0.0007 \\
\hline 16/07/03 & PM & 13 & 0.7868 & 0.0009 & -0.0115 & 0.0008 & 0.0287 & 0.0018 & 0.9886 & 0.0008 \\
\hline 25/07/03 & $\mathrm{AM}$ & 18 & 0.7652 & 0.0008 & -0.0090 & 0.0005 & 0.0297 & 0.0013 & 0.9910 & 0.0005 \\
\hline 27/07/03 & $\mathrm{PM}$ & 18 & 0.7227 & 0.0008 & -0.0074 & 0.0005 & 0.0323 & 0.0012 & 0.9926 & 0.0005 \\
\hline 28/07/03 & $\mathrm{AM}$ & 18 & 0.6113 & 0.0008 & -0.0061 & 0.0005 & 0.0155 & 0.0012 & 0.9939 & 0.0005 \\
\hline $16 / 08 / 03$ & $\mathrm{AM}$ & 14 & 0.8507 & 0.0015 & 0.0205 & 0.0010 & 0.0216 & 0.0025 & 1.0207 & 0.0010 \\
\hline 02/12/03 & $\mathrm{AM}$ & 15 & 0.2368 & 0.0012 & 0.0063 & 0.0009 & 0.0146 & 0.0031 & 1.0063 & 0.0009 \\
\hline $12 / 12 / 03$ & PM & 17 & 0.8097 & 0.0004 & 0.0075 & 0.0003 & 0.0080 & 0.0009 & 1.0075 & 0.0003 \\
\hline 13/12/03 & $\mathrm{AM}$ & 14 & 0.0158 & 0.0006 & -0.0006 & 0.0005 & 0.0178 & 0.0013 & 0.9994 & 0.0005 \\
\hline 04/01/04 & PM & 16 & 0.5071 & 0.0009 & -0.0060 & 0.0006 & 0.0117 & 0.0016 & 0.9940 & 0.0006 \\
\hline 05/01/04 & $\mathrm{AM}$ & 15 & 0.6464 & 0.0007 & 0.0090 & 0.0007 & 0.0105 & 0.0018 & 1.0090 & 0.0007 \\
\hline $12 / 01 / 04$ & $\mathrm{AM}$ & 17 & 0.5690 & 0.0008 & 0.0088 & 0.0007 & 0.0114 & 0.0020 & 1.0088 & 0.0007 \\
\hline $19 / 01 / 04$ & PM & 18 & 0.1760 & 0.0006 & 0.0019 & 0.0004 & 0.0191 & 0.0010 & 1.0019 & 0.0004 \\
\hline 02/03/04 & $\mathrm{AM}$ & 17 & 0.5615 & 0.0012 & 0.0083 & 0.0008 & 0.0102 & 0.0019 & 1.0083 & 0.0008 \\
\hline 23/03/04 & $\mathrm{AM}$ & 14 & 0.8076 & 0.0009 & 0.0093 & 0.0006 & 0.0222 & 0.0013 & 1.0094 & 0.0006 \\
\hline 06/04/04 & $\mathrm{AM}$ & 18 & 0.2704 & 0.0013 & -0.0047 & 0.0007 & 0.0543 & 0.0019 & 0.9954 & 0.0007 \\
\hline $25 / 04 / 04$ & PM & 19 & 0.0055 & 0.0006 & -0.0003 & 0.0004 & 0.0309 & 0.0010 & 0.9997 & 0.0004 \\
\hline 26/04/04 & $\mathrm{AM}$ & 20 & 0.6155 & 0.0005 & 0.0036 & 0.0003 & 0.0314 & 0.0007 & 1.0036 & 0.0003 \\
\hline 27/04/04 & $\mathrm{AM}$ & 15 & 0.5172 & 0.0012 & 0.0071 & 0.0008 & 0.0498 & 0.0019 & 1.0071 & 0.0008 \\
\hline 08/05/04 & PM & 13 & 0.8828 & 0.0010 & 0.0163 & 0.0006 & 0.0487 & 0.0018 & 1.0165 & 0.0006 \\
\hline
\end{tabular}

Tabla A1-XV. $\quad$ Ajustes KCICLO para el Cimel \#50. Filtro de 870nm.

\begin{tabular}{|c|c|c|c|c|c|c|c|c|c|c|}
\hline Fecha & 1020 & $\mathbf{N}$ & R2 & 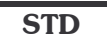 & $\overline{l n K}$ & error LnK & Tau & error Tau & $\overline{\mathrm{K}}$ & error $K$ \\
\hline$\overline{01 / 02 / 03}$ & AM & 14 & 0.4263 & 0.0010 & -0.0073 & 0.0008 & 0.0157 & 0.0024 & 0.9927 & 0.0008 \\
\hline 01/02/03 & $\mathrm{PM}$ & 22 & 0.5899 & 0.0011 & -0.0109 & 0.0007 & 0.0201 & 0.0020 & 0.9892 & 0.0007 \\
\hline 02/02/03 & $\mathrm{AM}$ & 19 & 0.4868 & 0.0012 & -0.0068 & 0.0007 & 0.0144 & 0.0017 & 0.9932 & 0.0007 \\
\hline $27 / 06 / 03$ & AM & 13 & 0.9542 & 0.0008 & -0.0199 & 0.0006 & 0.0336 & 0.0013 & 0.9803 & 0.0006 \\
\hline $16 / 07 / 03$ & PM & 13 & 0.8866 & 0.0010 & -0.0184 & 0.0008 & 0.0303 & 0.0020 & 0.9818 & 0.0008 \\
\hline 25/07/03 & $\mathrm{AM}$ & 18 & 0.8499 & 0.0013 & -0.0191 & 0.0008 & 0.0325 & 0.0020 & 0.9811 & 0.0008 \\
\hline $27 / 07 / 03$ & PM & 18 & 0.9123 & 0.0011 & -0.0208 & 0.0007 & 0.0348 & 0.0016 & 0.9794 & 0.0007 \\
\hline 28/07/03 & $\mathrm{AM}$ & 18 & 0.9176 & 0.0009 & -0.0193 & 0.0006 & 0.0191 & 0.0014 & 0.9809 & 0.0006 \\
\hline $16 / 08 / 03$ & AM & 14 & 0.4219 & 0.0018 & 0.0089 & 0.0012 & 0.0232 & 0.0030 & 1.0089 & 0.0013 \\
\hline 02/12/03 & AM & 15 & 0.1055 & 0.0010 & 0.0035 & 0.0008 & 0.0189 & 0.0028 & 1.0035 & 0.0008 \\
\hline $12 / 12 / 03$ & PM & 17 & 0.4703 & 0.0007 & 0.0061 & 0.0005 & 0.0101 & 0.0017 & 1.0061 & 0.0005 \\
\hline $13 / 12 / 03$ & $\mathrm{AM}$ & 14 & 0.0127 & 0.0049 & -0.0043 & 0.0039 & 0.0212 & 0.0109 & 0.9957 & 0.0039 \\
\hline 04/01/04 & $\mathrm{PM}$ & 16 & 0.3078 & 0.0013 & -0.0059 & 0.0009 & 0.0120 & 0.0024 & 0.9941 & 0.0009 \\
\hline 05/01/04 & $\mathrm{AM}$ & 15 & 0.0112 & 0.0011 & 0.0011 & 0.0011 & 0.0137 & 0.0030 & 1.0011 & 0.0011 \\
\hline $12 / 01 / 04$ & AM & 17 & 0.0238 & 0.0009 & 0.0013 & 0.0007 & 0.0150 & 0.0021 & 1.0013 & 0.0007 \\
\hline $19 / 01 / 04$ & PM & 18 & 0.2603 & 0.0007 & 0.0029 & 0.0004 & 0.0181 & 0.0012 & 1.0029 & 0.0004 \\
\hline 02/03/04 & $\mathrm{AM}$ & 17 & 0.4049 & 0.0011 & 0.0056 & 0.0008 & 0.0127 & 0.0018 & 1.0056 & 0.0008 \\
\hline $23 / 03 / 04$ & $\mathrm{AM}$ & 14 & 0.4313 & 0.0007 & 0.0030 & 0.0005 & 0.0250 & 0.0010 & 1.0031 & 0.0005 \\
\hline 06/04/04 & AM & 18 & 0.6690 & 0.0016 & -0.0130 & 0.0009 & 0.0548 & 0.0023 & 0.9871 & 0.0009 \\
\hline $25 / 04 / 04$ & PM & 19 & 0.7840 & 0.0009 & -0.0113 & 0.0005 & 0.0297 & 0.0014 & 0.9887 & 0.0005 \\
\hline $26 / 04 / 04$ & $\mathrm{AM}$ & 20 & 0.5191 & 0.0008 & -0.0047 & 0.0004 & 0.0327 & 0.0011 & 0.9953 & 0.0004 \\
\hline $27 / 04 / 04$ & $\mathrm{AM}$ & 15 & 0.0971 & 0.0015 & -0.0028 & 0.0010 & 0.0503 & 0.0024 & 0.9972 & 0.0010 \\
\hline 08/05/04 & PM & 13 & 0.7059 & 0.0010 & 0.0089 & 0.0006 & 0.0475 & 0.0017 & 1.0090 & 0.0006 \\
\hline
\end{tabular}

Tabla A1-XVI. Ajustes KCICLO para el Cimel \#50. Filtro de 1020nm. 


\section{ANEXO 2. Inventario de entradas de aerosol desértico en El Arenosillo medidas con el fotómetro Cimel.}

En este apartado incluimos el inventario de episodios de aerosol desértico que hemos identificado con el Cimel según la metodología descrita en la sección 7.2. Este inventario consta de los días de inicio y fin del episodio, la duración, el AOD máximo alcanzado, el alfa mínimo y el tipo de escenario sinóptico durante el episodio, así como un apartado de comentarios. Tipos sinópticos: NAH-S (1), AD (2), NAD (3) y NAH-A (4) (ver sección 7.1).

\begin{tabular}{ccccccc}
\hline \hline Inicio & Fin & $\begin{array}{c}\text { Duración } \\
\text { (días) }\end{array}$ & $\begin{array}{c}\text { AOD(440) } \\
\text { max }\end{array}$ & Alfa min & $\begin{array}{c}\text { Tipo } \\
\text { sinóptico }\end{array}$ \\
\hline $26 / 02 / 00$ & $29 / 02 / 00$ & 4 & 0.48 & 0.47 & 1 & \\
$06 / 03 / 00$ & $15 / 03 / 00$ & 10 & 1.05 & 0.03 & 1 & \\
$14 / 05 / 00$ & $17 / 05 / 00$ & 4 & 0.45 & 0.39 & 4 & \\
$03 / 06 / 00$ & $04 / 06 / 00$ & 2 & 0.36 & 0.67 & 4 & \\
$08 / 06 / 00$ & $08 / 06 / 00$ & 1 & 0.27 & 0.48 & 4 & \\
$23 / 06 / 00$ & $26 / 06 / 00$ & 4 & 0.6 & 0.4 & 4 & \\
$05 / 07 / 00$ & $09 / 07 / 00$ & 5 & 0.38 & 0.3 & 4 & \\
$21 / 07 / 00$ & $21 / 07 / 00$ & 1 & 0.48 & 0.22 & 4 & \\
$30 / 07 / 00$ & $03 / 08 / 00$ & 5 & 0.56 & 0.13 & 4 & \\
$07 / 08 / 00$ & $11 / 08 / 00$ & 5 & 0.63 & 0.28 & 4 & \\
$15 / 08 / 00$ & $17 / 08 / 00$ & 3 & 0.3 & 0.74 & 4 & \\
$20 / 08 / 00$ & $20 / 08 / 00$ & 1 & 0.25 & 0.19 & 4 & \\
$28 / 08 / 00$ & $02 / 09 / 00$ & 6 & 0.62 & 0.49 & 4 & \\
$11 / 09 / 00$ & $14 / 09 / 00$ & 4 & 0.43 & 0.37 & 4 & \\
$22 / 09 / 00$ & $27 / 09 / 00$ & 6 & 0.45 & 0.5 & 4 & \\
$22 / 10 / 00$ & $28 / 10 / 00$ & 7 & 0.6 & 0.26 & 3 & \\
$11 / 11 / 00$ & $12 / 11 / 00$ & 2 & 0.29 & 0.31 & 3 & \\
$19 / 12 / 00$ & $19 / 12 / 00$ & 1 & 0.22 & 0.32 & 2 & No llega al mínimo en AOD \\
\hline \hline
\end{tabular}

Tabla A1-XVII. Episodios de aerosol desértico en El Arenosillo medidos con el fotómetro Cimel durante el año 2000.

\begin{tabular}{|c|c|c|c|c|c|c|}
\hline Inicio & Fin & $\begin{array}{c}\text { Duración } \\
\text { (días) }\end{array}$ & $\begin{array}{c}\mathrm{AOD}(440) \\
\max \end{array}$ & Alfa min & $\begin{array}{c}\text { Tipo } \\
\text { sinóptico }\end{array}$ & Comentarios \\
\hline $21 / 01 / 01$ & $22 / 01 / 01$ & 2 & 0.7 & -0.09 & 1 & Sólo en nivel 1.0 \\
\hline $14 / 02 / 01$ & $14 / 02 / 01$ & 1 & 0.73 & 0.15 & 3 & Sólo 1 dato \\
\hline 20/02/01 & $26 / 02 / 01$ & 7 & 0.65 & 0.21 & 3 & \\
\hline 22/03/01 & 24/03/01 & 3 & 0.26 & 0.32 & 1 & \\
\hline 18/06/01 & 23/06/01 & 6 & 0.57 & 0.28 & 4 & \\
\hline $11 / 07 / 01$ & $12 / 07 / 01$ & 2 & 0.44 & 0.39 & 4 & \\
\hline 20/07/01 & $23 / 07 / 01$ & 4 & 0.72 & 0.22 & 4 & \\
\hline $30 / 07 / 01$ & 03/08/01 & 5 & 0.75 & 0.54 & 4 & \\
\hline 10/08/01 & $14 / 08 / 01$ & 5 & 0.55 & 0.45 & 4 & \\
\hline 24/08/01 & $25 / 08 / 01$ & 2 & 0.36 & 0.32 & 4 & \\
\hline 02/09/01 & 04/09/01 & 3 & 0.42 & 0.54 & 4 & \\
\hline $15 / 09 / 01$ & $17 / 09 / 01$ & 3 & 0.76 & 0.5 & 4 & \\
\hline $17 / 10 / 01$ & $17 / 10 / 01$ & 1 & 0.26 & 0.41 & 2 & \\
\hline $25 / 10 / 01$ & 08/11/01 & 15 & 0.51 & 0.23 & 2 & Hay 5 días sin datos. \\
\hline 23/11/01 & $25 / 11 / 01$ & 3 & 0.4 & 0.23 & 3 & \\
\hline 07/12/01 & 08/12/01 & 2 & 0.34 & 0.61 & 1 & \\
\hline
\end{tabular}

Tabla A1-XVIII. Episodios de aerosol desértico en El Arenosillo medidos con el fotómetro Cimel durante el año 2001. 


\begin{tabular}{|c|c|c|c|c|c|c|}
\hline Inicio & Fin & $\begin{array}{l}\text { Duración } \\
\text { (días) }\end{array}$ & $\begin{array}{l}\mathrm{AOD}(440) \\
\max \end{array}$ & Alfa min & $\begin{array}{c}\text { Tipo } \\
\text { sinóptico }\end{array}$ & Comentarios \\
\hline $05 / 01 / 02$ & $07 / 01 / 02$ & 3 & 0.62 & -0.01 & 2 & Dudoso por nubes \\
\hline 09/01/02 & $12 / 01 / 02$ & 4 & 0.83 & 0.11 & 1 & Posible continuación del anterior \\
\hline $12 / 02 / 02$ & $16 / 02 / 02$ & 5 & 0.57 & 0.41 & 1 & \\
\hline Marzo-02 & & 6 & & & & $\begin{array}{l}\text { Datos del Cimel no disponibles. } \\
\text { Según otras fuentes hay } 6 \text { días }\end{array}$ \\
\hline $15 / 05 / 02$ & $16 / 05 / 02$ & 2 & 0.4 & 0.2 & 4 & \\
\hline $30 / 05 / 02$ & $01 / 06 / 02$ & 3 & 0.37 & 0.2 & 4 & \\
\hline $12 / 06 / 02$ & $21 / 06 / 02$ & 10 & 0.42 & 0.29 & 4 & Hay 1día sin datos \\
\hline Julio-02 & & 10 & & & & $\begin{array}{l}\text { Campaña Veleta. Según otras } \\
\text { fuentes hay } 10 \text { días }\end{array}$ \\
\hline $28 / 07 / 02$ & 29/07/02 & 2 & 0.71 & 0.19 & 4 & \\
\hline $13 / 08 / 02$ & $13 / 08 / 02$ & 1 & 0.35 & 0.24 & 4 & \\
\hline 03/10/02 & 06/10/02 & 4 & 0.43 & 0.47 & 4 & \\
\hline 29/10/02 & 29/10/02 & 1 & 0.24 & 0.29 & 2 & No llega al mínimo de AOD \\
\hline
\end{tabular}

Tabla A1-XIX. Episodios de aerosol desértico en El Arenosillo medidos con el fotómetro Cimel durante el año 2002.

\begin{tabular}{|c|c|c|c|c|c|c|}
\hline Inicio & Fin & $\begin{array}{c}\text { Duración } \\
\text { (días) }\end{array}$ & $\begin{array}{c}\mathrm{AOD}(440) \\
\max \end{array}$ & Alfa min & $\begin{array}{c}\text { Tipo } \\
\text { sinóptico }\end{array}$ & Comentarios \\
\hline $12 / 03 / 03$ & $15 / 03 / 03$ & 4 & 0.46 & 0.11 & 2 & \\
\hline $22 / 03 / 03$ & $25 / 03 / 03$ & 4 & 0.63 & 0.15 & 3 & \\
\hline $09 / 04 / 03$ & 09/04/03 & 1 & 0.44 & 0.38 & 3 & \\
\hline $29 / 05 / 03$ & $01 / 06 / 03$ & 4 & 0.57 & 0.31 & 4 & \\
\hline 06/06/03 & $15 / 06 / 03$ & 10 & 0.5 & 0.22 & 4 & Hay 1 día sin datos \\
\hline $18 / 06 / 03$ & $21 / 06 / 03$ & 4 & 0.42 & 0.19 & 4 & Posible continuación del anterior \\
\hline $11 / 07 / 03$ & $11 / 07 / 03$ & 1 & 0.38 & 0.45 & 4 & \\
\hline $18 / 07 / 03$ & $20 / 07 / 03$ & 3 & 0.36 & 0.28 & 4 & \\
\hline $21 / 07 / 03$ & $22 / 07 / 03$ & 2 & 0.25 & 0.16 & 4 & \\
\hline $29 / 07 / 03$ & $15 / 08 / 03$ & 18 & 0.95 & 0.3 & 4 & $\begin{array}{l}\text { Hay } 1 \text { día sin datos. Mezclado con } \\
\text { los incendios de Portugal. }\end{array}$ \\
\hline $19 / 09 / 03$ & 20/09/03 & 2 & 0.3 & 0.3 & 2 & \\
\hline $18 / 11 / 03$ & $20 / 11 / 03$ & 3 & 0.28 & 0.2 & 3 & \\
\hline
\end{tabular}

Tabla A1-XX. Episodios de aerosol desértico en El Arenosillo medidos con el fotómetro Cimel durante el año 2003. 


\begin{tabular}{ccccccl}
\hline \hline Inicio & Fin & $\begin{array}{c}\text { Duración } \\
\text { (días) }\end{array}$ & $\begin{array}{c}\text { AOD(440) } \\
\text { max }\end{array}$ & Alfa min & $\begin{array}{c}\text { Tipo } \\
\text { sinóptico }\end{array}$ & Comentarios \\
\hline $08 / 02 / 04$ & $09 / 02 / 04$ & 2 & 0.6 & 0.64 & 1 & \\
$19 / 02 / 04$ & $19 / 02 / 04$ & 1 & 0.56 & 0.25 & 2 & Dudoso por nubes \\
$05 / 03 / 04$ & $05 / 03 / 04$ & 1 & 0.51 & 0.096 & 1 & \\
$09 / 03 / 04$ & $09 / 03 / 04$ & 1 & 0.38 & 0.43 & 1 & \\
$15 / 03 / 04$ & $21 / 03 / 04$ & 7 & 0.89 & 0.12 & 3 & \\
$14 / 04 / 04$ & $14 / 04 / 04$ & 1 & 0.4 & 0.52 & 3 & \\
$22 / 05 / 04$ & $27 / 05 / 04$ & 6 & 0.61 & 0.7 & 3 & Alfa muy alto, dudoso. \\
$07 / 06 / 04$ & $14 / 06 / 04$ & 8 & 0.67 & 0.29 & 4 & \\
$26 / 06 / 04$ & $30 / 06 / 04$ & 5 & 0.65 & 0.22 & 4 & \\
$04 / 07 / 04$ & $06 / 07 / 04$ & 3 & 0.42 & 0.3 & 4 & \\
$16 / 07 / 04$ & $17 / 07 / 04$ & 2 & 0.41 & 0.84 & 4 & Alfa fuera de criterio, dudoso. \\
$22 / 07 / 04$ & $28 / 07 / 04$ & 7 & 2.7 & 0.024 & 4 & \\
$31 / 07 / 04$ & $02 / 08 / 04$ & 3 & 0.39 & 0.44 & 4 & Posible recirculación del anterior \\
$21 / 08 / 04$ & $01 / 09 / 04$ & 12 & 0.67 & 0.12 & 4 & \\
$09 / 09 / 04$ & $12 / 09 / 04$ & 4 & 0.59 & 0.17 & 4 & \\
$21 / 09 / 04$ & $25 / 09 / 04$ & 5 & 0.61 & 0.32 & 4 & \\
$05 / 10 / 04$ & $08 / 10 / 04$ & 4 & 0.3 & 0.27 & 4 & \\
$26 / 11 / 04$ & $29 / 11 / 04$ & 4 & 0.85 & 0.01 & 2 & Nubes \\
$14 / 12 / 04$ & $14 / 12 / 04$ & 1 & 1.05 & -0.009 & 2 & Nubes \\
\hline \hline
\end{tabular}

Tabla A1-XXI. Episodios de aerosol desértico en El Arenosillo medidos con el fotómetro Cimel durante el año 2004. 